C A L I F O R N I A I N S T I T U T E O F T E C H N O L O G Y
DOE CONTRACT DE-FC26-01B15362

DR. WILLIAM GODDARD III, PRINCIPAL

INVESTIGATOR, CALTECH

\title{
LOWER COST METHODS FOR IMPROVED OIL RECOVERY (IOR) VIA SURFACTANT FLOODING
}

DOE Project: DE-FC26-01BC15362

\author{
PI: William A. Goddard III \\ Co-PI: Yongchun Tang \\ Senior Staff: Patrick Shuler, Mario Blanco \\ Postdoctoral Scholars: Seung Soon Jang, Shiang-Tai Lin, Prabal Maiti, \\ Yongfu Wu, Stefan Iglauer, Xiaohang Zhang \\ California Institute of Technology
}

Final Report

September, 2001 - September, 2004

- Covering the period from September, 2001 - September, 2003 -- 3 Year Report Compiled by Patrick Shuler and Mario Blanco

- Sections written by Mario Blanco, Seung Soon Jang, Shiang-Tai Lin, and Patrick Shuler.

- Completed September, 2004 
Disclaimer

This report was prepared as an account of work sponsored by an agency of the United States Government. Neither the United States Government nor any agency thereof, nor any of their employees, makes any warranty, express or implied, or assumes any legal liability or responsibility for the accuracy, completeness, or usefulness of any information, apparatus, product, or process disclosed, or represents that its use would not infringe privately owned rights. Reference herein to any specific commercial product, process, or service by trade name, trademark, manufacturer, or otherwise does not necessarily constitute or imply its endorsement, recommendation, or favoring by the United States Government or any agency thereof. The views and opinions of authors expressed herein do not necessarily state or reflect those of the United States Government or any agency thereof. 


\title{
Lower Cost Methods for Improved Oil Recovery via Surfactant Flooding DOE Project: DE-FC26-01BC15362
}

\author{
PI: William A. Goddard III Co-PI: Yongchun Tang
}

Senior Staff: Patrick Shuler, Mario Blanco.
Post doctoral Scholars: Seung Soon Jang, Shiang-Tai Lin, Prabal Maiti, Stefan Iglauer, Yongfu Wu, Xiaohang Zhang

California Institute of Technology

Final Report for Project

September, 2001 - September, 2004

\section{ABSTRACT}

This report provides a summary of the work performed in this 3-year project sponsored by DOE. The overall objective of this project is to identify new, potentially more costeffective surfactant formulations for improved oil recovery (IOR). The general approach is to use an integrated experimental and computational chemistry effort to improve our understanding of the link between surfactant structure and performance, and from this knowledge, develop improved IOR surfactant formulations.

Accomplishments for the project include: 1) completion of a literature review to assemble current and new surfactant IOR ideas, 2) Development of new atomistic-level MD (molecular dynamic) modeling methodologies to calculate IFT (interfacial tension) rigorously from first principles, 3) exploration of less computationally intensive mesoscale methods to estimate IFT, Quantitative Structure Property Relationship (QSPR), and cohesive energy density (CED) calculations, 4) experiments to screen many surfactant structures for desirable low IFT and solid adsorption behavior, and 5) further experimental characterization of the more promising new candidate formulations (based on alkyl polyglycosides (APG) and alkyl propoxy sulfate surfactants).

Important findings from this project include: 1) the IFT between two pure substances may be calculated quantitatively from fundamental principles using Molecular Dynamics, the same approach can provide qualitative results for ternary systems containing a surfactant, 2) low concentrations of alkyl polyglycoside surfactants have potential for IOR (Improved Oil Recovery) applications from a technical standpoint (if formulated properly with a cosurfactant, they can create a low IFT at low concentration) and also are viable economically as they are available commercially, and 3) the alkylpropoxy sulfate surfactants have promising IFT performance also, plus these surfactants can have high optimal salinity and so may be attractive for use in higher salinity reservoirs. Alkylpropoxy sulfate surfactants are not yet available as large volume commercial products. The results presented herein can provide the needed industrial impetus for extending application (alkyl polyglycoside) or scaling up (alkylpropoxy sulfates) of these two promising surfactants for enhanced oil recovery. Furthermore, the advanced simulations tools presented here can be used to continue to uncover new types of surfactants with promising properties such as inherent low IFT and biodegradability. 


\section{TABLE OF CONTENTS}

1. Abstract

2. Table of Contents

3. List of Graphical Materials and Tables

4. Executive Summary 14

5. Introduction 16

5.1 Project Description 16

5.2 Literature review highlights 18

6. Experimental and Numerical Methods 20

6.1 Computational Chemistry Methods 20

6.2 Experimental Procedures $\quad 27$

7. Results and Discussion 31

7.1 Theoretical Calculations 31

7.1.1 Summary of key findings from theoretical study 31

7.1.2 MD Model Validation and Calculation Sensitivity
Analysis

7.13 MD Model Calculations of IFT for Alkyl Benzene

Sulfonate Surfactants 36

7.14 Salinity Effects Investigated in Molecular Dynamic (MD) Simulations

7.15 Molecular Modeling Study of Two Model Alkyl

Polyglycosides (APG) -- Water/n-Octanol Cosurfactant with n-Octane

7.16 Molecular Modeling Study of Two Model Alkyl Polyglycosides (APG) -- Small Isomeric Difference in Structure Creates Large Change in Behavior

7.17 Study of IFT of alkyl propoxylated sulfate surfactants via MD simulations

7.1.8 QSPR (Quantitative Structure Property Relationship) 74

7.1.9 Mesoscale Model -- Dependence of Interfacial Tension on Surface Architecture

7.1.10 Cohesive Energy Density in the Water-Surfactant-Oil System -- Molecular Origin of IFT

7.1.11 Hildebrand and Hansen Parameters - Insights from Solubility Parameters $\quad 82$

7.2 Experimental Investigation 84

7.2.1 Initial Screening of Surfactants for Low IFT 84

7.2.2 Initial Screening of Surfactants for Solid Adsorption $\quad 89$

7.2.3 More Detailed Study for Calamide CW-100 92

7.2.4 Alkyl Polyglycoside (APG) Surfactants -- Background 96 
7.2.5 Alkyl Polyglycosides (APG) Formulated with n-Alcohols as Cosurfactant 97

7.2.6 Alkly Polyglycosides (APG) Formulated with Aromatic Alcohols as Cosurfactants 108

7.2.7 Alkly Polyglycosides (APG) Formulated with Sorbitan-Type and Other Surfactants 115

7.2.8 Alkyl Polyglycosides (APG) Formulations_-Effect of Oil Phase Composition 120

7.2.9. Pure APG Formulations - Study of Structure Effects 122

7.2.10 Alkyl Propoxy Sulfate Surfactants 131

$\begin{array}{ll}7.3 \text { Project Implications/Ideas for Improved Surfactant Formulations } & 145 \\ \text { 8. Conclusions } & 145\end{array}$

9. References 147

10. List of Acronyms and Abbreviations $\quad 150$

$\begin{array}{ll}\text { Appendices } & 152\end{array}$

A -- Further Surfactant Adsorption Experimental Results 153

\section{Attachments}

1. Published papers and presentations

2. Literature review report (previously provided as supplement to second annual report) 


\section{LIST OF GRAPHICAL MATERIAL AND TABLES}

\section{LIST OF FIGURES}

Figure 5.1.1. The residual oil trapped in a porous medium after water flooding may be reduced if the ratio of viscous to capillary forces $\left(\mathrm{N}_{\mathrm{c}}\right)$ becomes large enough. Typical curves are shown for a low- and high- permeability sandstone.

Figure 5.1.2. Schematic of the surfactant flooding process applied to a field.

Figure 6.1.2.1 Example MD result showing distribution of atoms between a liquid and gas phase. The surface tension may be calculated by applying the Kirkwood-Buff formula.

Figure 6.1.3.1. Different model surfactants used in predicting their interfacial surface tension.

Figure 7.1.2.1 Molecular description of a liquid/gas system. Individual molecules of argon at $57^{\circ} \mathrm{K}$ are shown as purple dots. (a) Initial and (b) Final configuration of gas/liquid described via a Lennard Jones Fluid Model. Calculated surface tension computed from the difference in normal and tangential stress at interface via the Kirkwood-Buff formula (Equation 4).

Figure 7.1.2.2. This shows the variability in calculated IFT values from atomistic constant pressure (NPT) simulations versus the size of the simulated cell. As the size (indicated by the $\mathrm{n} \times \mathrm{n}$ Miller indices of the cell base, e.g., $1 \times 1,2 \times 1,2 \times 2,3 \times 3$ ) increases the IFT values fluctuate less and become mostly positive.

Figure 7.1.2.3 The figure shows the time averaged IFT for the same surfactant system as a function of time. Value appears to converge after $30 \mathrm{ps.}$

Figure 7.1.2.4 The integration time step, typically $1.0 \mathrm{fs}$, has a measurable but small effect on the calculated IFT values over short dynamics ( 5 ps). For longer dynamics (not shown), 1.0 fs time steps are adequate to estimate IFT.

Figure 7.1.2.5 The figure shows no difference in increasing the number of slabs from 100 to 800 in the integration of Kirkwood-Buff IFT formula. Most runs use 100 to 200 .

Figure 7.1.2.6 IFT values are averaged over a period of time before being printed out for further analysis.

Figure 7.1.2.7 The standard deviation in IFT decreases greatly when the averaging period is changed to 500 fs or longer. The effect of using only half of the simulation for calculating IFT is also shown. 
Figure 7.1.3.1. Branched alkyl benzene sulfonates. $(\mathrm{m}=1,2,3,4,5,6,7)$.

Figure 7.1.3.2. Density profile and stress profile for C16_4 (isomeric substitution of benzosulfonate on $4^{\text {th }}$ position of a $\mathrm{C} 16$ carbon chain).

Figure 7.1.3.3. Interfacial tension profile for C16_4. The integrated profile leads to a interfacial tension of $\gamma=11.03$ dynes $/ \mathrm{cm}$.

Figure 7.1.3.4. Change of interfacial tension as a function of attachment position of benzosulfonate group as calculated by MD simulations.

Figure 7.1.3.5. Comparable experimental results of interfacial tension as a function of attachment position of benzosulfonate group (Doe and Wade, 1977). (IFT measured at $25 \mathrm{C}$, iso-pentanol $2 \mathrm{wt} \%, 3 \mathrm{~g} / \mathrm{l} \mathrm{NaCl}, 0.7 \mathrm{~g} / \mathrm{l}$ surfactant).

Figure 7.1.3.6. Full set of experimental data reported for interfacial tension for water/n-alkane for isomers of C16-benzosulfonates. Note that the No. 4 isomer gives the lowest IFT for n-decane as the oil phase.

Figure 7.1.4.1. Running average of IFT between liquid/vacuum interface for pure water and $6.3 \% \mathrm{NaCl}$ solution.

Figure 7.1.4.2. Density profile for pure water.

Figure 7.1.4.3. Calculated IFT versus salinity for number 4 isomer of $\mathrm{C} 16$ linear alkyl benzene sulfonates

Figure 7.1.4.4. Stress profiles associated with calculations containing salt

Figure 7.1.4.5. Interfacial tension profile for molecular dynamic cases that include salt.

Figure 7.1.4.6. Fluid density profiles for molecular dynamic calculations containing salt

Figure 7.1.5.1. Schematic molecular structure of a typical APG surfactant.

Figure 7.1.5.2 Schematic Chemical Structure of HBDM

Figure 7.1.5.3. (a) Absolute Stereochemistry of the Decyl version of HBDM surfactant. (b) Molecular model of head group displaying the full stereochemistry for HNDM. Each color line is one atom.

Figure 7.1.5.4. ESP Charges for head group of HBDM Each colored line is one atom.

Figure 7.1.5.5. Binding energy distributions for 100,000 pairs of surfactant/cosurfactant. Surfactant is HBDM in the trans conformation, cosurfactant is n-octanol. 
Figure 7.1.5.6. Four most binding conformations for pairs of surfactant/cosurfactant. HBDM is in the gauche conformation; cosurfactant is n-octanol. Each ball is one atom.

Figure 7.1.5.7. Four most binding conformations for pairs of surfactant/cosurfactant. Surfactant is HBDM in the trans conformation. cosurfactant is n-octanol. Each ball is one atom.

Figure 7.1.5.8. a) Octane/Octanol/HBDM/water interface model. Left side is n-octane, Middle is interfacial region, Right size is bulk aqueous phase. b) Top view of the noctanol layer. Shows only n-octanol molecules to simplify the picture.

Figure 7.1.5.9. Octane/water interfacial structure of HBDM after 500 ps of NPT molecular dynamics at $300 \mathrm{~K}$. Both, all-trans and S-shaped molecular conformations were observed.

Figure 7.1.5.10. Top and bottom views of Octane/water interfacial structure of HBDM/n-octanol.

Figure 7.1.5.11. (a) Octane/water interfacial structure of OBDM after 3250 picoseconds of NVT molecular dynamics (only OBDM surfactant structure shown). Formulation contains n-octanol:OBDM in a 30:1 molar ratio. (b). Octane/water interfacial structure of HBDM after 500 ps of NPT molecular dynamics.

Figure 7.1.5.12. Initial structure of a single OBDM molecule shown on the left. On the right is shown the unit cell used in MD simulations of n-octanol:OBDM calculations.

Figure 7.1.5.13. (a) Parallel and transverse components of the pressure tensor in OBDM 3.25 ns simulation at room temperature. (b) Calculated instantaneous (blue) and cumulative (pink) IFT values (left scale is IFT in dyne/cm).

Figure 7.1.6.1. Molecular structure of two subject glucosides

Figure 7.1.6.2. Changes in molecular structure of n-Octyl-D-Glucoside.

Figure 7.1.7.1. Structure of a alkyl propoxylated sulfate surfactant used in MD simulations.

Figure 7.1.7.2. A typical structure of the alkyl propoxy sulfate systems. Aqueous surfactant layer between two oil layers.

Figure 7.1.7.3. Final structure for each alkyl propoxylated sulfate system. Aqueous surfactant layer between two oil layers.

Figure 7.1.7.4. Density profile for each system. The slab ID is scaled z coordinate. The aqueous phase is in the middle, between oil phases sitting on both ends. 
Figure 7.1.7.5. Stress profiles and tension profiles for each system.

Figure 7.1.7.6. Comparison of IFT between surfactant molecules with different number of propylene oxide group.

Figure 7.1.7.7. Final structure for each system as a function of temperature. Aqueous surfactant layer between two oil layers.

Figure 7.17.8. Density profile for each system studied here. The slab ID is a scaled $\mathrm{z}$ coordinate. The middle phase is water and both sides correspond to oil. The parts shown as peaks in these profiles come from the surfactant.

Figure 7.1.7.9. Stress profiles and tension profiles for each temperature, 280,300 , and $350 \mathrm{~K}$, from top to bottom.

Figure 7.1.7.10. Change in IFT as a function of temperature.

Figure 7.1.7.11. Typical structure for oil/surfactant/water system. Alkyl propoxy sulfate surfactant solution and n-decane.

Figure 7.1.7.12. Change in IFT as a function of concentration.

Figure 7.1.9.1. Snapshots of initial and final configuration for liquid-vapor interface.

Figure 7.1.9.2. Density profile for the vapor-liquid interface.

Figure 7.1.9.3 Stress profile (both tangential and normal components) for the vaporliquid interface.

Figure 7.1.9.4. Variations of IFT as a function of hydrocarbon tail length for a single chain surfactant.

Figure 7.1.9.5. Correlation function between the polar head group and oil beads for different positions of this polar head group.

Figure 7.1.9.6. Variation of IFT as a function of position of the polar group along the surfactant backbone. The solid line is a guide to eye only.

Figure 7.1.9.7. Density profile for different positions of the polar group.

Figure 7.1.9.8. Snapshots of final configurations for various positions of the polar group $(1,2,4$, and 6 positions).

Figure 7.1.10.1a. Spatial distribution of atomic strain energy in the z-direction for water-decane mixture. 
Figure 7.1.10.1b. Molecular model of the water-decane mixture.

Figure 7.1.10.2a. Spatial distribution of atomic strain energy in the z-direction for water-surfactant(C16_8)-decane mixture.

Figure 7.1.10.2b. Molecular model of the water-surfactant (C16_8)-decane mixture.

Figure 7.1.10.3a. Spatial distribution of atomic strain energy in the z-direction for watersurfactant(C16_4)-decane mixture.

Figure 7.1.103b. Molecular model of the water-surfactant (C16_4)-decane mixture.

Figure 7.1.11.1. Calculated Hansen parameters for water, PG 2062, n-octane, and several alcohols.

Figure 7.2.1.1. Comparison of IFT for octyl and dodecyl sulfate surfactants

Figure 7.2.1.2. Octyl sulfate surfactant IFT versus salinity for different n-alkane hydrocarbons as the oil phase.

Figure 7.2.1.3. Structure of Aerosol series of surfactants

Figure 7.2.1.4. IFT for a series of esters of sulfosuccinic acid versus n-octane.

Figure 7.2.1.5. Structure of Zonyl flourosurfactnts and Neodol surfactant

Figure 7.2.1.6. Structure of commercial gemini surfactants evaluated

Figure 7.2.1.7. Measured IFT for different commercial gemini surfactants versus salinity. The oil phase is n-octane in all cases.

Figure 7.2.2.1. Measured retention of SDBS onto kaolinite clay

Figure 7.2.2.2. Details of structure for Pluronic series of nonionic surfactants

Figure 7.2.2.3. Increase in mass adsorption of Pluoronic products with an increase in the $\mathrm{PO} / \mathrm{EO}$ ratio.

Figure 7.2.24. Structure details for the Igepal series of surfactants studied.

Figure 7.2.2.5. Solid adsorption of a series of Igepal surfactants onto kaolinite clay.

Figure 7.2.3.1. Adsorption isotherm for Calamide CW-100 onto kaolinite clay at $25 \mathrm{C}$. 
Figure 7.2.3.2. Tertiary oil recovery for $\mathrm{CW}-100$ and TR-70 in Berea sandstone coreflood experiments.

Figure 7.2.4.1. Molecular structure of a typical alkyl polyglycoside (APG)

Figure 7.2.5.1a. IFT measured for equilibrated samples containing PG 2062 and smaller n-alcohols versus n-octane as the hydrocarbon phase.

Figure 7.2.5.1b. IFT measured for equilibrated samples containing PG 2062 and larger n-alcohols versus n-octane as the hydrocarbon phase.

Figure 7.2.5.2. Increasing the alkyl chain length of the commerical APG surfactants decreases the IFT when blended with a 1-alcohol cosurfactant.

Figure 7.2.5.3. Experimental data of the minimum IFT measured for selected APG/alcohol formulations.

Figure 7.2.5.4. Molecular structure of mannose surfactant tested that has a 16 alkyl chain hydrophobic tail

Figure 7.2.5.5. Measured IFT results for n-hexadecyl-beta-D-mannose (HBDM) surfactant with a series of different alcohol cosurfactants

Figure 7.2.5.6. Data illustrating IFT is nearly independent of temperature for a mixture of APG surfactant/alcohol versus n-octane.

Figure 7.2.5.7. Data illustrating IFT is nearly independent of the salinity for an APG surfactant/alcohol formulation versus n-octane.

Figure 7.2.5.8. Adsorption of APG products increases with an increase in their average alkyl chain length.

Figure 7.2.5.9 Berea Coreflood oil recovery test result - PG 2062/n-octanol formulation

Figure 7.2.5.10. IFT data versus mole fraction of n-octanol in a butanol/octanol/dodecanol cosurfactant blend.

Figure 7.2.6.1. Comparison of IFT behavior for different alcohol cosurfactants, all containing 6 carbons.

Figure 7.2.6.2. Measured IFT with different aromatic alcohols as APG cosurfactants

Figure 7.2.6.3. 1-naphthol as a cosurfactant with APG surfactants may create a low IFT condition at a low chemical concentration. 
Figure 7.2.6.4. Tertiary oil recovery for Berea coreflood test using a formulation containing PG 2062 and 1-butanol/1-naphthol.

Figure 7.2.6.5. Effect of functional group in cosurfactant on the IFT when formulated with PG 2062 and measured versus n-octane.

Figure 7.2.6.6. IFT measurements for formulations containing PG 2062 and diethylene mono glycol butyl ether as cosurfactant

Figure 7.2.7.1. Example of a SPAN surfactant

Figure 7.2.7.2. Example of a TWEEN surfactant

Figure 7.2.7.3. IFT measured for different mixtures of PG 2067 APG and some SPAN surfactants.

Figure 7.2.7.4. Greater oil recovery occurs in a sand pack experiment when injecting a PG 2067 / SPAN 20 chemical solution versus a waterflood.

Figure 7.2.8.1. Effect of oil phase composition on the IFT with PG 2062/n-butanol formulations at room temperature.

Figure 7.2.8.2. Effect of oil phase composition on the IFT with PG 2062/n-octanol formulations at room temperature.

Figure 7.2.9.1. Five different pure APG structures included in IFT experimental study with n-octanol as cosurfactant

Figure 7.2.9.2. Structures of surfactants containing an aromatic function

Figure 7.2.9.3. Structure of alpha- and beta- forms of a surfactant

Figures 7.2.10.1 - 7.2.10.9. IFT for 9 different Alfoterra

Figure 7.2.10.10. IFT results for $0.2 \mathrm{wt} \%$ Alfoterra 13,15 , and 18 versus salinity

Figure 7.2.10.11. IFT results for $0.2 \mathrm{wt} \%$ Alfoterra 63,65 , and 68 versus salinity

Figure 7.2.10.12. IFT measured for blends of alkyl propoxy sulfates and APG commercial surfactants

Figure 7.2.10.13. Tertiary oil recovery results from injection of a Alfoterra 38 chemical formulation.

Figure 7.2.10.14. Tertiary oil recovery results from injection of a Alfoterra 23 chemical formulation. 


\section{LIST OF TABLES}

Table 7.1.4.1. Comparison of the change of water/vacuum IFT at different salt $(\mathrm{NaCl})$ concentrations.

Table 7.1.5.1. Properties of n-Decyl $\beta$-D-maltoside

Table 7.1.7.1. Density and dimensions of whole system for alkyl propoxylated sulfates

Table 7.1.7.2. The cell parameters and the densities

Table 7.1.11.1. Calculated Values of Hansen Parameters for Components in Figure 7.1.11.1

Table 7.2.1.1 Representative list of surfactants in the initial IFT screening evaluation

Table 7.2.1.2. Measured IFT results with flourosurfactants

Table 7.2.3.1 Measured IFT Between CW-100 Surfactant Formulation and n-Octane

Table 7.2.5.1. Replicate Measurements at a Low IFT Condition

Table 7.2.5.2. Effect of Salinity and Temperature on IFT for a Near Optimum Formulation

Table 7.2.5.3. IFT Values at $25^{\circ} \mathrm{C}$ for a Series of APG/n-Alcohols in Fresh Water

Table 7.2.5.4. Selected adsorption results for APG/cosurfactant formulations

Table 7.2.5.5. IFT changes versus blends of cosurfactant n-alcohols with PG 2062 surfactant.

Table 7.2.6.1 IFT values for PG 2062/1-naphthol formulations versus n-octane, with the initial values of 1-naphthol of about $600 \mathrm{ppm}$ in the aqueous phase.

Table 7.2.6.2. IFT for PG 2062/ethanol/1-naphthol formulations versus n-octane.

Table 7.2.6.3. IFT for PG 2062/alcohol/1-naphthol formulations versus n-octane.

Table 7.2.7.1. Chemical structure information for SPAN and TWEEN surfactants.

Table 7.2.7.2. Measured IFT for APG / SPAN surfactant mixtures in a $2 \% \mathrm{NaCl}$ brine versus n-octane as the hydrocarbon phase. 
Table 7.2.7.3. Measured IFT for APG / TWEEN surfactant mixtures in a $2 \% \mathrm{NaCl}$ brine versus n-octane as the hydrocarbon phase at room temperature.

Table 7.2.7.4. Selected adsorption results for APG/cosurfactant formulations

Table 7.2.9.1. IFT results for various pure APG surfactants in fresh water with n-octanol as a cosurfactant

Table 7.2.9.2. IFT results for various pure APG surfactants with an aromatic function

Table 7.2.9.3a. IFT Results for Mixtures of Maltoside and Sorbitan Surfactants; Equilibrated Samples

Table 7.2.9.3b. IFT Results for Mixtures of Maltoside and Sorbitan Surfactants Nonequilibrated Samples

Table 7.2.9.4 IFT results for n-Octyl-apha-D- and n-Octyl-beta-Glucoside surfactants

Table 7.2.10.1. Effect of number of average PO groups and temperature

Table 7.2.10.2. IFT measurements at $0.2 \mathrm{wt} \%$ for selected Alfoterra products

Table 7.2.10.3. IFT measurements at $0.1 \mathrm{wt} \%$ for selected Alfoterra products

Table 7.2.10.4. Effect of Alfoterra concentration on IFT for selected formulations

Table 7.2.10.5. Alfoterra surfactant IFT versus two crude oils with $0.3 \mathrm{wt} \% \mathrm{NaCl}$

Table 7.2.10.6. Alfoterra 28 surfactant IFT versus GOM crude oil and n-octane

Table 7.2.10.7. Adsorption of Alfoterra surfactants onto kaolinite clay

Table 7.2.10.8. Rank order of Alfoterra surfactants by solid adsorption onto kaolinite. 


\section{EXECUTIVE SUMMARY}

This report provides a summary of the work performed for this 3-year project. The overall objective of this project is to apply a combination of new and existing computational/theoretical chemistry and experimental methods to identify new, potentially more cost-effective surfactant formulations for improved oil recovery (IOR). The general approach is to improve our understanding of the linkage between surfactant structure and performance, and from this knowledge, develop improved surfactant formulations.

A literature review was performed to collect current and some new surfactant EOR ideas, plus assemble a suite of interfacial tension (IFT) and solid adsorption data. These data are organized so that they are readily accessible for future reference such as to test proposed theoretical models for prediction of surfactant performance.

Accomplishments for this project for the theoretical efforts include:

1) A novel application of atomistic-level MD (molecular dynamic) modeling and the Kirkwood-Buff formula to calculate interfacial tension (IFT) for aqueous surfactant/hydrocarbon systems from first principles. Studies include a series of branched alkyl benzosulfonates, alkyl polyglycosides, and branched polypropylene sulfate surfactants. The simulation IFT values are in qualitative agreement with IFT data trends reported in literature and measured in this project regarding the effect of surfactant structure (e.g. branching of alkyl chain, alkyl chain length, system temperature) on the IFT.

2) Calculated IFT values approach quantitative agreement to experimental data for pure systems (e.g. IFT for brine/n-alkane systems).

3) Mesoscale modeling of oil/surfactant/water interfaces has also been conducted with comparable results to full atomistic simulations at a fraction of the cost. These larger size-scale, simpler models are not yet able to account for the effect of salinity on IFT, but provide insights concerning linkages between surfactant structure and IFT, as well as the impact of cosurfactants.

We investigated the molecular origin of interfacial (or surface) tension. This is often attributed to the difference of cohesive energy density (CED) of the two phases in contact. The CED, defined as the energy of interaction per unit volume, is a measure of the extent of molecular interactions in a system. The inhomogeneity in CED leads to an excess of free energy and a tensile net force at the interface, which are related to the IFT. We find that the main effect of the surfactant is to disperse the sharp changes in CED between a pure water/oil interface. We also find that the coulomb and van der Waals component forces are important across the interface. This theoretical work led to one published (Jang SS, Lin ST, Maiti PK, Blanco M, Goddard WA, Shuler P, Tang YC; Molecular dynamics study of a surfactant-mediated decane-water interface: Effect of molecular architecture of alkyl benzene sulfonate, JOURNAL OF PHYSICAL CHEMISTRY B, 108 (32): 12130-12140 AUG 12 2004) and two additional "in preparation" scientific publications. 
A related concept to the cohesive energy is the relative miscibility of the components in a water/surfactant/cosurfactant/oil system. The miscibility properties of the individual components may be expressed quantitatively via Hildebrand and Hansen parameters. These parameters, taken together, provide another perspective to match the selection of surfactant/cosurfactant combinations for a particular oil and process conditions.

Progress in the experimental portion of this project include:

1) Laboratory experimental measurements have been performed for selected surfactant formulations with regards to their phase behavior/IFT, solid adsorption, and efficiency of displacing residual oil from Berea sandstone cores. Based on literature and initial modeling efforts, these surfactant formulations were thought to have a possibility of improved or unique IOR performance behavior. For example, encouraging IFT results were noted for a non-ionic diethanoloamine surfactant among the many surfactant ideas screened experimentally early in the project.

2) The experimental program focused in large part on characterizing the properties and performance of alkyl polyglycoside (APG) surfactants. Theoretical work, the literature, and our own experiments demonstrate that nonionic surfactants such as APG's that have a large hydrophilic head group offer several advantages as IOR candidates. In particular:

a) Microemulsions formed with APG may be largely independent of both temperature and salinity. This makes formulation for low IFT easier, and suggests chemistries that may have low IFT in higher salinity brines.

b) Literature data and our measurements show IFT values less than 0.01 dyne/cm of APG-based surfactant formulations versus simple n-alkanes. Furthermore, our measurements indicated that some APG formulations (when include an alcohol cosurfactant or a second surfactant) can achieve low IFT with low surfactant concentration.

c) APG chemistry is based on saccharides and fatty alcohols and so has no toxicity issues. APG has become a large volume commercial product for household and cosmetics in the 1990's. Thus it has become a practical surfactant option for IOR applications only recently.

d) Experimental results have been presented at three different presentations at American Chemistry Society meetings and at the Society of Petroleum Engineers IOR Meeting (paper SPE 89472).

3) Alkyl propoxy sulfate surfactants also were the subject of more intense experimental study in this project. These surfactants have been applied successfully in displacement of hydrocarbons, but so far only as agents for the near sub-surface remediation of aquifers contaminated with spilled hydrocarbons. Our studies suggest these surfactants may be good technical candidates for some oilfield IOR situations. In particular, we found that some of these surfactants can create low-IFT conditions. The optimal salinity for some of these alkyl propoxy sulfate surfactants is several percent salt, and hence these may be suitable candidates for incremental recovery from higher salinity reservoirs. 


\section{INTRODUCTION}

\subsection{Project Description}

This report provides a summary of the entire 3-year project. The overall objective of this project is to integrate the latest computational chemistry and experimental methods to identify new, surfactant for improved oil recovery (IOR) and thus to conduct a first principle's based search for cost-effective formulations.

The basic physics behind the surfactant flooding EOR process is that the residual oil dispersed as micron-size ganglia is trapped by high capillary forces within the porous media. Increasing the fluid flow viscous forces or decreasing the forces holding the oil in place are required before the oil can be pushed through the pore throats within the reservoir rock and mobilized to reach a production well.

Petroleum engineers have found a simple dimensionless number $\left(\mathrm{N}_{\mathrm{c}}=\right.$ Capillary Number $)$ is a useful means to correlate the process conditions and the mobilization of the waterflood residual oil, $\mathrm{N}_{\mathrm{c}}=\mathrm{v} * \mu / \gamma$.

Here $\mathrm{v}$ is the fluid velocity, $\mu$ the fluid viscosity, and $\gamma$ the interfacial tension. The velocity and viscosity refer to the injected aqueous phase. The figure below gives typical results (Lake, 1989).

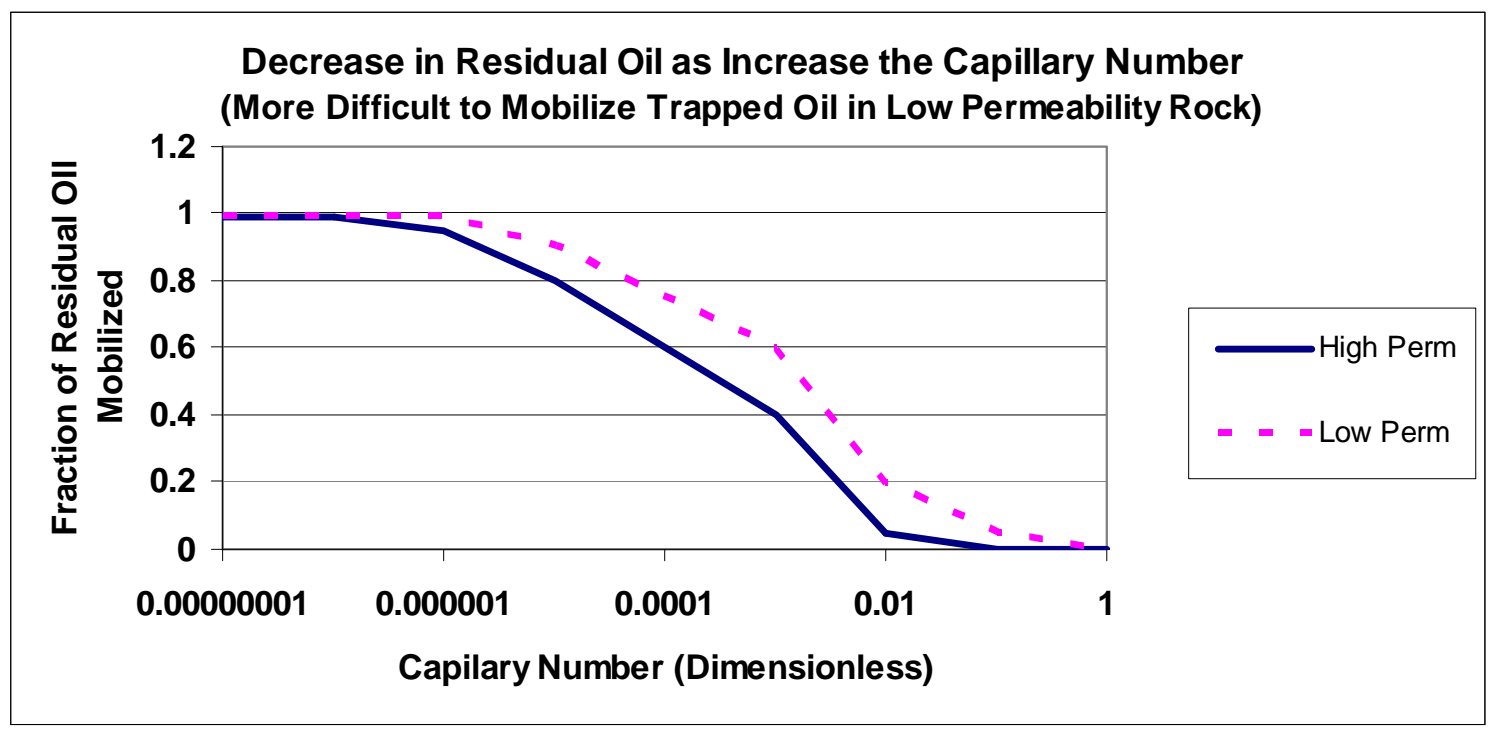

Figure 5.1.1. The residual oil trapped in a porous medium after water flooding may be reduced if the ratio of viscous to capillary forces $\left(\mathrm{N}_{\mathrm{c}}\right)$ becomes large enough. Typical curves are shown for a low- and high- permeability sandstone.

Curves such as shown in Figure 5.1.1 have been generated in other laboratories via controlled tests varying systematically water velocity, viscosity, and water/oil IFT. 
- The $\mathrm{N}_{\mathrm{c}}$ is only around $10^{-7}$ for a typical water flood.

- The $\mathrm{N}_{\mathrm{c}}$ must be increased by a few orders-of-magnitude to mobilize significant trapped oil. It is not practical to do this via increasing water injection rate.

- It is even more difficult to mobilize the oil if the reservoir rock is low permeability and thus the pores are smaller size (increases capillary trapping of the residual oil).

The most practical means to reach the required large Capillary Numbers in the oil field is to reduce the interfacial tension, IFT, $\gamma$ between the injected aqueous and residual oil droplets to ultra-low values (typical target of $0.001 \mathrm{dyne} / \mathrm{cm}$ ), as compared to the water/oil IFT values of $20-50$ dyne $/ \mathrm{cm}$.

The figure below is a schematic of the surfactant flood process as it has been applied in the field. The injected surfactant solution will create the very low IFT required to mobilize the oil trapped in pore spaces left behind after the waterflood. It is common practice to maintain a favorable mobility ratio by including a polymer in the surfactant formulation slug, and even more common to include a polymer in the drive solution. The mobilized oil then forms a bank and is driven to a nearby production well for recovery.

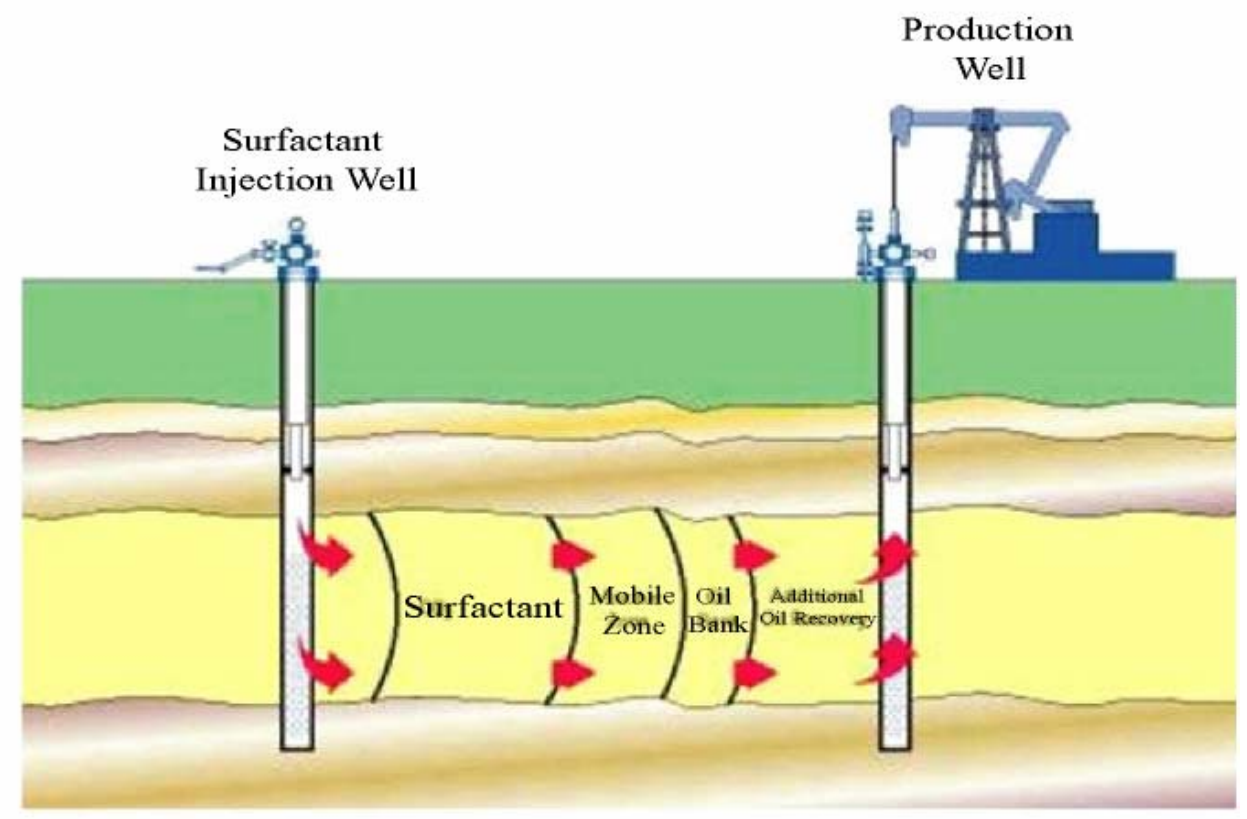

Figure 5.1.2. Schematic of the surfactant flooding process applied to a field.

Besides the requirement to achieve a low in-situ IFT, a second major factor that determines the technical and economic success of a surfactant flood project is the depletion of the injected surfactant, with the major sink usually from solid adsorption onto minerals and clays in the reservoir. Identifying surfactants or processes that will decrease significantly such chemical losses would improve the economics of the EOR approach greatly (Wu 1996, Taber 1996). 
Laboratory studies and surfactant flood field tests have demonstrated this improved oil recovery (IOR) method can mobilize and produce significant post-waterflood residual oil Green and Willhite, 1998). There are several major economic hurdles to widespread commercial deployment of this technology to the oil field, however. Perhaps the largest impediment is the relatively high chemical cost for this process. In addition, field tests of surfactant flooding have identified several technical barriers. These include problems such as less than anticipated reduction in the interfacial tension (IFT) and high retention of the surfactant in the reservoir (Lake, 1989, Wu 1996, Taber 1996).

Our general study approach is to improve our understanding of the linkage between surfactant structure and performance, and from this, develop improved surfactant formulations. Literature and laboratory data provide test cases to tune the theoretical models and help develop correlations between the surfactant structure and their IFT and adsorption behavior. Additional experiments then have been performed to quantify the potential improvement in performance of surfactant structures suggested by this computational chemistry effort.

Desirable characteristics of alternate chemical formulations include those: 1) able to create ultra-low IFT over a substantial range of low and moderate concentrations, 2) suffering minimal loss in the reservoir due to mineral and clay adsorption, 3) with inherently lower unit chemical cost, and 4) exhibiting desirable IFT and adsorption behavior for more technically challenging conditions such as higher salinity reservoirs.

This integrated theoretical and experimental study in particular emphasizes Point 1), that is, identifying chemical systems that may attain low IFT versus hydrocarbons, while keeping in mind the other 3 objectives. The experimental work also addresses the other desirable chemical performance characteristics.

This report primarily is divided between a presentation of the theoretical and experimental work. Within each part we include a discussion of how the two parallel efforts are integrated together, plus at the end there is a general discussion of results and conclusions that ties everything together.

\subsection{Literature review highlights}

A literature review (Task 1 of the project) was included as a supplement to the second Annual report and appears as a supplement also in this final project report.

One purpose of the literature review is to identify data sets that can provide good test cases for the theoretical effort to develop prediction methods for surfactant IFT and surfactant adsorption. In particular, we have organized data for a series of alkylbenzene sulfonates for purposes of initial model development.

In addition, the survey of the literature indicated a number of classes of surfactants as potential candidates for EOR besides alkyl benzene sulfonates, such as alpha-olefin 
sulfonates, diphenyl ether sulfonate, alkyl propoxy ethoxy sulfates and sulfonates, alkyl ethoxylated carboxylates, betain and sulfo-betaines, and alkyl polyglycosides and other carbohydrate/bio-based surfactants.

Published papers reviewed show the evolution of the surfactant flooding EOR technology over the last 30 years, with the literature review summarizing more recent innovations such as: combining caustic with surfactant, development of surfactants for mobilizing organic compounds, but for purposes of remediation of contaminated aquifers, carbohydrate/bio-based surfactants, and particular anionic/cationic surfactant blends.

Three types of surfactants in particular have emerged from the literature search as being newer, intriguing ideas for EOR applications. First, sulfonated alkyl aromatics have been reported as being suitable for EOR just recently, and in fact these have seen use in some small field projects (Berger, 2002). These are different structures in that the sulfonate group is attached to the alkyl chain as opposed to the benzene ring. In contrast to the usual alkyl benzene sulfonates, alkyl groups are coupled to a sulfonated aromatic ring. One advantage claimed for this newer type of surfactant is that it is easier to manufacture because it can be synthesized as a one step process.

Alkyl propoxylated sulfates are an interesting surfactant idea as they have become popular for remediation of spilled hydrocarbons (so-called NAPL, nonaqueous phase liquids) from aquifers in recent years, but this chemistry has not been exploited recently for oil field EOR. Workers have developed surfactant formulations to mobilize NAPL's that can achieve low IFT, require minimal addition of co-surfactant, and suffer only low losses due to solid adsorption (Jayanti, 2001). These NAPL' s studied for these remediation projects may be quite different in character than typical crude oils, but the alkyl propxylated sulfates likely can be adapted for EOR purposes in many oil reservoirs.

Alkyl polyglucosides (APG, and possibly other carbohydrate/sugar based surfactants) are a third, intriguing surfactant type that has come from the literature search so far.

Published reports suggest these surfactants can be formulated to have some unusual characteristics such as having the ability to formulate a middle-phase, low IFT condition that is not sensitive to changes in salt or temperature (Forster, 1996). One patent from 1991 (U.S. 4,985,154) claims the application of APG surfactants for EOR, but there does not seem to have been follow-up studies in the published literature in this area. We show that these compounds offer the ability to generate ultra-low IFT at very low concentrations once a feasible cosurfactant is properly identified

Other ideas that are even more speculative include the newer versions of the so-called "dimeric" or "gemini" surfactants. These molecules have two identical tail structures connected at the head group by a spacer group. Some of the potential advantages of these gemini surfactants indicated by the literature are 1) lower surface tension, 2), lower cmc, so that the minimum surface tension occurs at a lower concentration, and 3 ) better solubilizing, wetting, foaming properties. The gemini version of surfactants may have a tendency for less adsorption as compared to the monomeric version of the same surfactant molecule. Finally, some dimeric surfactants show remarkable rheological 
properties (viscoelasticity, gelification, shear thickening) at relatively low concentrations, which mean that they also may provide mobility control (Zana, 1998). The knowledge gained by the combined computational/experimental effort documented here indicate that Gemini surfactants have structures that help "anchor" the hydrophobic end of the surfactant to the oil phase, while surfactants with large hydrophilic heads, such as APG surfactants, are able to do the same at the aqueous end of the interface.

Finally, the recent literature has paid more attention to the role of formulations and mixtures to create target low IFT conditions. For example, Sabatini (2003) advocates the use of a combination of hydrophilic and hydrophobic linker molecules. Properly selected, these compounds might segregate neat the oil/water interface and increase the interaction energies there. Sabatini mentioned specifically six to nine carbon tail surfactant-like molecules as candidate hydrophobic linkers. An interesting offshoot of this concept is the recent observation that some amphiphilic block copolymers can boost the IFT reducing efficiency of the normal surfactant. One author (Endo, 2002) speculates the effect is related to the ability of the block copolymer to extend further into the adjacent sub phases. Furthermore, from analysis of high-precision neutron scattering data and theoretical calculations of the phase diagram suggest that these block copolymers are incorporated into the surfactant layer. Our computational/experimental approach could potentially yield sufficient information to create highly targeted surfactant formulations, including preferential extraction of a hydrocarbon with a given range of carbon numbers.

We evaluated several of these ideas during the course of this project. Results and observations are summarized in previous project reports as well this final report to DOE, plus scientific papers and presentations. Of these surfactant types, we studied the alkyl polyglycoside (APG) and the alkylpropoxy sulfate surfactants the most intensely.

\section{THEORETICAL AND EXPERIMENTAL METHODS}

\subsection{Computational Chemistry Methods}

\subsubsection{Overview}

The purpose of this work effort is to develop atomistic/molecular level models and other computational methods for predicting surfactant IFT and solid adsorption characteristics. A major effort has been to develop successfully the proper force field descriptions and detailed methods to compute surfactant IFT from first principles (Molecular Dynamics, MD). In MD approaches a so called "force field" describes the strength of molecular interactions at the atomic level with mathematical expressions referred to as "potential energy functions". These potential energy functions are nowadays extracted from first principles Quantum Mechanics. We used published force fields in all the MD calculations presented here. Other theoretical efforts focused on developing calculation tools that are more approximate, non-atomistic, in estimating surfactant performance based on compilations of experimental data to extract parameters for simulations of structure and process conditions. These other approaches are easier to implement and 
require less intensive calculations. We also explore here with qualitative success some of these so called "meso-scale" or Quantitative Structure Property Relationship (QSPR) approaches. We now examine the first principle MD approach referred to above.

\subsubsection{Molecular Dynamics Approach -- Calculation of IFT}

We have implemented a computational module to estimate Interfacial Tension (IFT) from first principles Molecular Dynamics (MD).

The density profile is given by

$$
\rho(z)=\frac{\langle n(z)\rangle}{V_{s}} \quad V_{s}=L_{x} L_{y} \Delta z
$$

The normal and transverse pressures are given by

$$
\begin{gathered}
P_{N}(z)=\rho(z) k_{B} T-\frac{1}{V_{s}}\left\langle\sum_{(i, j)} \frac{z_{i j}^{2}}{r_{i j}} \frac{d u\left(r_{i j}\right)}{r_{i j}}\right\rangle \\
P_{T}(z)=\rho(z) k_{B} T-\frac{1}{V_{s}}\left\langle\sum_{(i, j)} \frac{x_{i j}^{2}+y_{i j}^{2}}{2 r_{i j}} \frac{d u\left(r_{i j}\right)}{r_{i j}}\right\rangle
\end{gathered}
$$

where $\mathrm{k}_{B}$ is the Bolztman's constant and $\mathrm{T}$ is the absolute temperature. The differential terms in Equations (2) and (3) represent the change in interaction energies, $u$, with the inter particle distances, $r_{i j}$. The IFT is computed from the Kirkwood-Buff formula

$$
\gamma=1 / 2 \int d z\left[P_{N}(z)-P_{T}(z)\right]
$$

Where the normal, $\mathrm{P}_{\mathrm{N}}$, and transverse stresses, $\mathrm{P}_{\mathrm{T}}$, are given by the stress tensor components. The interface plane is perpendicular to $\mathrm{z}$ and it has a particle density $\rho(\mathrm{z})$, temperature $\mathrm{T}$, and volume $\mathrm{V}_{\mathrm{s}}$. It has been customary to neglect the long range interactions in the estimation of IFT because of the difficulties in portioning these into local contributions to the stress tensor. We take into account long range electrostatics (Ewald sums) with a new formulation of the virial contribution by each atom. 

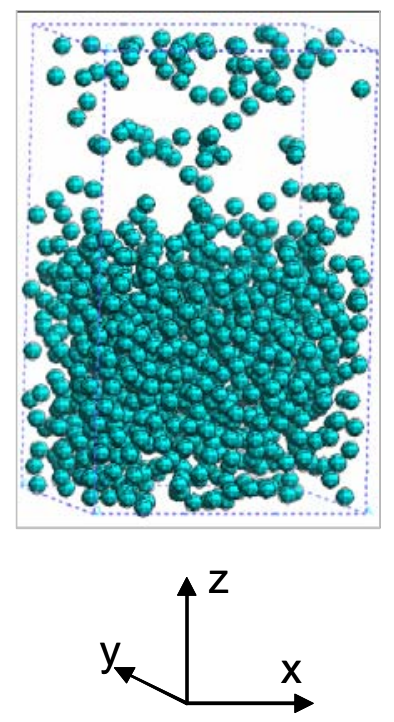

Figure 6.1.2.1 Example MD result showing distribution of atoms between a liquid and gas phase. The surface tension may be calculated by applying the Kirkwood-Buff formula.

As shown in Figure 6.1.2.1, the MD calculations involve placing a population of atoms into a "calculation box". The movement of these atoms are dictated by Newton's equations of motion, and the quantum mechanical potential energy rules based on first principles that are contained in the MD simulation code developed specifically for this project. The configuration of the atoms and the forces allows the calculation of physical properties (such as density, IFT, etc.) and also provides a useful "picture" of the state of the matter that can offer some additional physical insights.

First, this approach was validated by matching for several simple, "pure" chemistry cases the surface tension or interfacial tension reported in the literature data. Next, we carried out full atomistic simulations to calculate the aqueous/hydrocarbon phase IFT for some more complicated, but more interesting chemical systems:

- A series of alkyl benzene sulfonates

- Effect of salinity

- Alkly polyglycoside surfactant formulations

- A series of alkyl propoxlated sulfate surfactants

\subsubsection{Mesoscale Modeling}

Mesoscale models refers to the technique of modeling chemical systems not at an atomistic scale, but at one size level greater where groups of atoms lumped together for purposes of simulating their behavior. The simulations do not have as much of the chemical detail and energetics as a full-blown MD simulation, and thus the results for a mesoscale simulation are somewhat generic in nature. These calculations are faster than MD and are being used to explore the trends in IFT behavior as a function of the main features of the surfactant architecture. 
In our model, surfactants, water and oil molecules are represented using the simple model that we have developed for studying surfactant self assembly (Maiti, 2002). The model is similar in spirit to the model earlier introduced by Telo de Gamma and Gubbins (1986), Smit et. al. (1990), and Grest et. al (1986). Water and oil molecules are represented by one water-like and oil-like particle respectively. Surfactant molecules are represented by a combination of water-like ("hydrophilic") and oil-like (hydrophobic") particles connected by harmonic springs and is represented by TmHp, where T denotes the tail and $\mathrm{H}$ denotes the head part of the surfactants, $\mathrm{m}$ and $\mathrm{p}$ being integers denoting the number of particles in the tail and head, respectively. Thus, each single-chain surfactant is a chain of length $l_{a}=(m+p)$. We shall refer to each particle on the surfactants as a monomer. In terms of the same symbols, the microscopic model of a dimeric and trimeric surfactant, which we propose here, can be represented by the symbol TmHqSnHqTm and TmHqSnHqTmSnHqTm respectively where $n$ is the number of sites constituting the spacer represented by the symbol $\mathrm{S}$. We have carried out simulations for $\mathrm{q}=1, \mathrm{n}=2$ and $\mathrm{m}=4$. The model surfactants are shown in Figure 6.1.31.

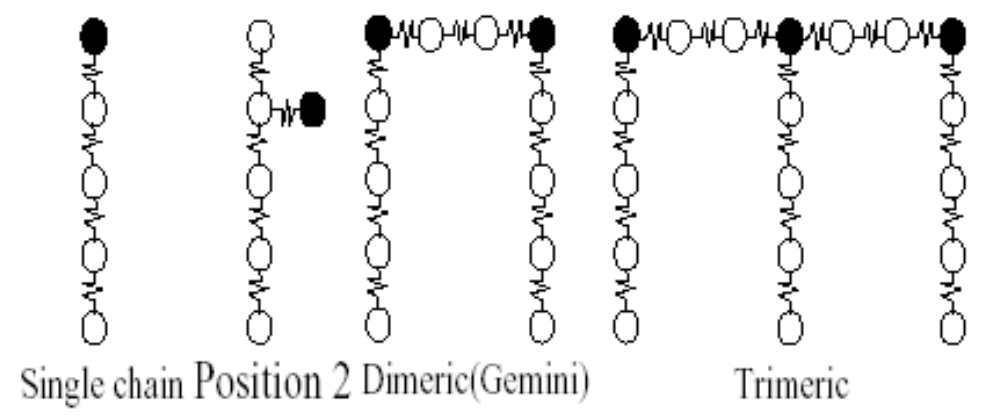

Figure 6.1.3.1. Different model surfactants used in predicting their interfacial surface tension.

Attractive interaction between like particles (water-water, hydrophilic-water, and hydrophilic-hydrophilic) is modeled via a Lennard-Jones (LJ) potential:

$$
V_{L J}=4 \epsilon\left\{\left(\frac{\sigma}{r}\right)^{12}-\left(\frac{\sigma}{r}\right)^{6}\right\}
$$

where $r$ is the distance between the two interacting particles. The parameter $\varepsilon$ (a binding energy) governs the strength of interaction and $\sigma$ (a measure of the equilibrium distance between the particles) defines a length scale. We have assumed that $\varepsilon=1$ and $\sigma=1$ for all interactions. We have taken a cut-off of $r_{c}=2: 5 \sigma$, large enough to include excluded volume effects and attractive forces.

Repulsive hydrophobic-hydrophilic and water-hydrophobic interactions are modeled by truncated and shifted LJ potential (WCA potential): 


$$
\begin{array}{rlrl}
V_{L J} & =4 \epsilon\left\{\left(\frac{\sigma}{r}\right)^{12}-\left(\frac{\sigma}{r}\right)^{6}\right\}+\epsilon \quad r \leq r_{c}, \\
& =0 & \text { otherwise. } &
\end{array}
$$

where $r_{c}=2^{1 / 6} \sigma$.

Neighboring particles of the chain are connected by a harmonic spring and interact via a harmonic potential given by:

$$
V(r)=\frac{1}{2} k\left(r-r_{0}\right)^{2}
$$

Here $\mathrm{k}$ is the harmonic spring constant and $\mathrm{r}_{0}$ is the equilibrium bond length. The values of the parameter are chosen as $r_{0}=1: 2 \sigma$ and $\mathrm{k}=30$. The bond bending potential is given by

$$
V(\theta)=\frac{1}{2} k_{\theta}\left(\cos \theta-\cos \theta_{0}\right)^{2}
$$

where $\mathrm{k}_{\theta}$ is the bond bending force constant, $\theta_{0}$ is the equilibrium bond angle, and $\theta$ is the actual bond angle. For the simulation presented here we have assumed the chains to be completely flexible i.e. $\mathrm{k}_{\theta}=0$. The effect of rigidity of the chain as well as the spacer on the resulting supramolecular structure is the subject of a future publication. For simplicity all the monomers are assumed to have same mass $m$.

The $\sigma$. and $\varepsilon$ values for the interaction between unlike atoms are calculated using the standard geometrical mean 'combining rules' in terms of those between identical atoms, i.e. $\sigma_{\mathrm{ij}}=\left(\sigma_{\mathrm{ii}} \sigma_{\mathrm{jj}}\right)^{1 / 2}$ and $\varepsilon_{\mathrm{ij}}=\left(\varepsilon_{\mathrm{ii}} \varepsilon_{\mathrm{jj}}\right)^{1 / 2}$ We have used conventional MD simulations, numerically solving Newton's equations of motion for all of the particles. The trajectories are integrated using the velocity Verlet algorithm with a time step of $0.01 \tau_{\mathrm{o}}=$ $\left(m \sigma^{2} / \varepsilon\right)^{1 / 2}$ 
The non-bonded interactions were computed using cell list with phantom atoms. This simplifies the computation of forces across periodic boundaries by introducing copies of all atoms that are within a distance $r_{c}$ of any region boundary and placing them just outside the simulation regime adjacent to the opposite boundary.

\subsubsection{QSPR}

A number of surfactants have been screened for low IFT behavior, some types not considered for EOR applications previously, through a traditional Quantitative Structure Property Relationship expression (QSPR). The concept behind this method is to relate the structure of the molecules and its solution properties to its behavior (e.g. interfacial tension). The motivation for exploring this approach is that if it is successful, it is a calculation method that is much faster than full, molecular-level simulation and can provide semi-quantitative predictions of its behavior (e.g. IFT) based on the molecular structure and process conditions.

Because this approach relies heavily on experimental data to "tune" the model, it has limited value with regards to gaining fundamental understanding of cause/effect and in extrapolating the observations to the behavior of significantly different surfactant structures.

\subsubsection{Cohesive Energy -- Origin of IFT}

The molecular origin of interfacial (or surface) tension is often attributed to the difference of cohesive energy density (CED) of the two phases in contact. The CED, defined as the energy of interaction per unit volume, is a measure of the extent of molecular interactions in a system. The motivation for these series of calculations is to ascertain if CED simulations would aid in understanding the particular details of the different energetic components as to how they relate to IFT behavior. One advantage of this approach is that the computational effort is less intense than full MD simulations.

Molecules in a bulk phase experience the same the forces (and interactions) from all directions. However, molecules at an interface would experience different molecular interactions from the two phases, each of which has a specific value of CED. Such inhomogeneity in CED leads to an excess of free energy and a tensile net force at the interface. The interfacial tension is directly related to the interface excess free energy and the tensile force.

Here we perform an analysis on the spatial distribution (along the z-direction) of the atomic strain energy of the water-surfactant-oil. The atomic strain energy, when summed over all the atoms in a system gives the total energy of the system. This is a good measure of local molecular interactions and is identical to the cohesive energy for systems whose intramolecular interactions are negligible. 


\subsubsection{Solubility Characteristics - Hildebrand and Hansen Parameters}

The solubility behavior of the components in multi-phase system of interest water (brine), surfactant, co-surfactant, and oil are of interest. In particular, the relative solubility among the various components is one determining factor in the phase behavior exhibited in these multi-phase liquid systems. Because the IFT is in turn related to the phase behavior, it would seem that having values of parameters that quantify the relative solubility of each component could be useful. Also, these solubility parameters are related to the cohesive energy density (CED) concept discussed in the previous section. In 1936 Joel H. Hildebrand proposed a simple definition for a "solubility parameter" that would provide a systemic description of the miscibility behavior of solvents and which subsequently has found multiple uses in chemistry. System components with similar solubility parameters are expected to be miscible with one another.

This solubility parameter $\delta$ is defined as square root of the cohesive energy density, that is, the heat of vaporization divided by the molar volume.

$$
\delta=\left[\left(\Delta H_{V}-R T\right) / V_{m}\right]^{1 / 2}
$$

where

$$
1 \text { hidebrand }=1 \mathrm{cal}^{1 / 2} \mathrm{~cm}^{-3 / 2}=2.046 \times \mathrm{MPa}^{1 / 2}=2.4542 \times 10^{-2}(\mathrm{Kcal} / \mathrm{mol})^{1 / 2} \mathrm{~A}^{-3 / 2}
$$

Hansen went one step further, and developed individual solubility parameters that when taken together are equivalent to the Hildebrand parameter:

$$
\delta^{2}=\delta_{d}^{2}+\delta_{p}^{2}+\delta_{h}^{2}
$$

where $\delta_{\mathrm{d}}, \delta_{\mathrm{p}}$, and $\delta_{\mathrm{h}}$ are the dispersion, electrostatic (or polar), and hydrogen bond components of $\delta$, respectively.

Solvents with similar Hansen solubilities are miscible in most proportions; dissimilar values yield limited solubilities. Hildebrand and Hansen solubility parameters are useful for selecting solvents and additives in formulations, for the blending of polymers, for the control of kinetics and monomer sequence distributions in copolymers, and for the proper selection of time-release formulations in the delivery of pharmaceuticals. Again, in our study, we are testing the idea that these parameters for all of the system components taken together could provide some insights relating to the phase behavior and IFT of aqueous based chemical mixture formulations versus hydrocarbons. 
The Hildebrand parameter can be calculated easily for compounds with known heats of vaporization and densities. The Hansen parameters may be measured with difficulty, but their values for the chemical formulations in our study may be most revealing when coupled with the experimental observations of phase behavior/IFT. Our strategy is to calculate these Hansen parameters for the relevant compounds because of the experimental difficulties to generate these solubility parameters.

Recent work at the California Institute of Technology in fact already considered molecular modeling approaches to calculate Hansen parameters (M. Belmares, M. Blanco, W. A Goddard, R. B Ross, et-al, "Hildebrand and Hansen Solubility Parameters from Molecular Dynamics with Applications to Electronic Nose Polymer Sensors”, in press, Journal of Computational Chemistry). This other study used calculated Hansen values as a tool in a study of the swelling behavior of polymers when exposed to different organic vapors. A Cohesive Energy Density (CED) computational method was used that offers consistency throughout the various organic compounds of interest in formulation work. CED is a multiple sampling Molecular Dynamics method that estimates Hildebrand and Hansen solubility parameters with good precision (ca. 0.44 hildebrands). The use of multiple sampling techniques allows for the precise determination of solubility parameters in a systematic way. The CED method, when combined with a generic force field and quantum mechanically determined atomic charges yields first-principles hildebrand parameter predictions in good agreement with experiment (rms 1.1 hildebrands). The CED method overcomes the common equilibration problems with condensed phase molecular dynamics, i.e., how to choose initial molecular configurations not far from equilibrium at normal densities.

\subsection{Experimental Procedures}

\subsubsection{Phase Behavior and IFT}

The purpose of these measurements is to determine for aqueous solutions with candidate surfactant formulations the phase behavior and IFT versus various hydrocarbons. Data from these tests are used to compare against and validate theoretical model predictions. Other tests focus on screening new ideas for surfactant formulations to determine their potential suitability for the more serious study as EOR agents.

The basic procedure for these experiments is to prepare test tube size samples that contain brine, surfactant, cosurfactant, and a hydrocarbon. A common screening test recipe was:

- Surfactant concentration of $2 \mathrm{wt} \%$

- Co-surfactant, (optional)

- Salt solutions, $\mathrm{NaCl}$, usually 1 or $2 \mathrm{wt} \%$

- Hydrocarbon phase, various n-alkanes, most often n-octane

- Water to oil ratio, usually 1:1

More intense study of phase behavior and IFT was performed for formulations containing alkyl polyglycosides (APG) surfactant. These candidate formulations tested a large 
number of different chemical combinations APG/cosurfactants and chemical concentrations. A significant number of tests also were performed for formulations containing alkyl propoxysulfate surfactants.

The test tubes are mixed well for several hours and allowed to stand for several days or as much as a few weeks to allow the fluids to come to phase equilibrium at ambient conditions. The physical appearance of the phases are noted, such as the relative volumes of the aqueous and oleic phases, and if any third, so-called middle-phase forms. Other qualitative information collected are the color or opacity/clarity of the various phases.

The interfacial tension (IFT) was determined for selected phase equilibrated samples by using a spinning drop tensiometer method (from Temco, Inc.) as detailed elsewhere (Cayais, 1977). For our samples we loaded the glass tube with the aqueous phase, followed by injection of a few microliters of the uppermost oleic phase. The glass tube was spun in the instrument and the IFT determined from the oil drop geometry. Because the samples already come from fluids at phase equilibrium, typically it required less than 2 hours for the measured IFT to stabilize to a final value.

This same procedure was used whether the sample had only 2 phases, or a third middlephase. For the case of 3 phase samples, it is much easier to measure IFT between the excess phases than to attempt to measure the IFT that includes the middle-phase because of the opacity of the middle-phase emulsion. The IFT of the excess phases are still representative of the behavior of the IFT with the middle-phases because of Antov's rule:

$$
\text { IFT (aq-oil) }<\text { or }=\text { IFT (aq-middle) }+ \text { IFT (middle-oil) }
$$

For middle-phase systems, the greater of the IFT (aq-middle) or the IFT (middle-oil) is the "controlling one" that determines the mobilization of residual oil. One implication is that the IFT (aq-oil) will be its lowest under the same process conditions as the controlling IFT's associated with middle-phase. Also, the reported IFT (aq-oil) may overestimate the actual, controlling IFT, but at the very worst by a factor of two.

In some instances we measured the "fresh" IFT values, by which we mean the IFT between the aqueous formulation and an oil drop that had never been exposed previously to an aqueous solution. These measurements are more convenient because they bypass the lengthy procedure to create phase behavior tubes, followed by waiting some days for the phases to approach equilibrium. On the other hand, at least several hours time usually is required to attain a steady-state IFT due to the fluids not starting from a mutual equilibration. Also, our intuition is that the IFT from the phase-equilibrated samples is more representative of actual conditions where the aqueous and oil phases have a long contact time.

The rationale for using n-alkanes as the hydrocarbon phase is that previous studies by other workers demonstrate they are a good proxy for real crude oils for trends in IFT behavior. In fact the EACN (Equivalent Alkane Carbon Number) concept (see for example Cash, 1976) says that for most crude oils there is a single average n-alkane that 
best mimics its phase behavior and IFT trends versus surfactant formulations. One may then screen a number of surfactants using a simple n-alkane (or appropriate mixture) as a good proxy for that crude oil. In particular, previous studies indicate that for several crude oils investigated, that these oils have an EACN that may be modeled by alkanes ranging between $\mathrm{n}$-hexane to $\mathrm{n}$-decane. Thus, this current study has selected n-octane as a "typical" representative hydrocarbon. That is to say, surfactant formulations that are effective in reducing IFT versus n-octane are likely good candidates also for mobilizing crude oils.

In addition, using $n$-alkanes as the hydrocarbon phases offers the advantages of being a low cost material that can be obtained easily with a consistent quality, thereby improving the probability of obtaining reproducible results. Yet another reason to use simple alkanes is that many previous investigations have also used these materials as test hydrocarbons. Hence it is easier to compare our results to some of those reported in the literature.

\subsubsection{Surfactant Adsorption onto Kaolinite Clay}

The purpose of this study effort is to quantify and compare the adsorption tendency of the various surfactant formulations we evaluate. A number of commercial surfactants have been evaluated for their tendency to adsorb onto kaolinite clay. All of these tests were conducted at $25^{\circ} \mathrm{C}$ with a weight ratio of liquid/solid of 20 , and for an exposure period of 8 hours.

The rationale for using a clay in the adsorption portion of the study is that it is the clays with their relatively huge surface area that dominate chemical adsorption (at least in sandstone reservoirs). Kaolinite is selected (obtained from the University of Missouri) as the adsorbent of choice because 1) it is among the most common clays found in oil reservoirs, 2) it may be obtained in a fairly reproducible form, 3) it is inexpensive, and 4) it is a stable material (e.g., will not swell when immersed in fresh water).

The composition provided by the supplier for the kaolinite has the following major components (weight percents):

$$
\begin{array}{llllllll}
\mathrm{SiO}_{2} & 44.2 & \mathrm{Al}_{2} \mathrm{O}_{3} & 39.7 & \mathrm{TiO}_{2} & 1.39 & \mathrm{Fe}_{2} \mathrm{O}_{3} & 0.13
\end{array}
$$

with trace amounts of sodium, manganese, calcium, potassium, phosphorous, and fluorine. They also provide a measured specific surface area of about 10 square meter/gram.

The general procedure in these adsorption experiments includes

- Prepare surfactant solutions in $\mathrm{NaCl}$ brines (default is $2 \mathrm{wt} \%$ ).

- Use kaolinite clay with surfactant solution in test tubes at a weight ratio of 1:20 ( 0.5 g clay and $9.5 \mathrm{~g}$ solution).

- The sample is shaken at room temperature for 8 hours. 
- Centrifuge the test tubes for at least 1 hour to separate the solid material and the solution. In some cases the sample is centrifuged twice.

- Transfer the separated solution for analysis to determine the surfactant concentration remaining in the supernatant solution.

- From the reduction in the surfactant concentration in the supernatant solution, one may calculate the amount of surfactant transferred to the kaolinite solid phase.

Analysis of the remaining, equilibrium surfactant bulk concentration following exposure to the test solid material (kaolinite in this case) is a key to being able to measure surfactant adsorption accurately. The surfactant analytical method (for example, UV or IR) selected depends upon the chemistry of the subject surfactant.

More recently we developed a gravimetric method to screen samples for solid adsorption. The basic procedure is to measure carefully on an analytical balance the starting weights of the dish, kaolinite, and starting surfactant solution. Per the step-by-step procedure listed above, the disaggregated kaolinite clay is exposed to the surfactant solution and the equilibrated supernatant solution is removed from the test tube. An aliquot of this supernatant solution is evaporated to dryness. Knowing the activity of the starting surfactant material and brine salinity, one can calculate the mass of surfactant that is left in the supernatant solution. Hence, these data provide the quantitative value of the reduction in the amount of the surfactant in solution and thereby the total surfactant retention. An advantage of this approach is that it avoids the complication of having to perform an analytical chemical assay test of the supernatant solution.

\subsubsection{Coreflood Tests of Residual Oil Displacement}

Common laboratory procedures were used to test mobilization of residual oil from Berea sandstone cores.

The experimental steps include:

- Saturate a Berea sandstone core (1"x 12") with a brine

- Pump brine through the core to condition it to the water chemistry and establish the initial permeability by measurement of rate and pressures.

- Displace the brine with the test oil (an n-alkane) until reaching an irreducible water condition.

- Water flood with a brine until reach residual oil saturation.

- Inject the candidate surfactant formulation for a target pore volume.

- Inject the polymer chaser slug/water drive until obtain no further tertiary oil recovery.

These flow experiments were performed at a nominal superficial velocity of about 3 feet/day during the chemical injection steps. Higher velocities were used during the flow stapes to introduce brine and oil. All oil displacement tests reported here were performed at room temperature. 


\section{RESULTS AND DISCUSSION}

\subsection{Theoretical Calculations}

\subsubsection{Summary of key findings from theoretical study}

Some of the key findings from the theoretical investigation include:

- The MD simulation methods employed for calculating IFT are validated for simple one and two component systems.

- The MD IFT calculations for ternary complex chemical system that include surfactants and other components are more useful for predicting IFT trends rather than exact values. The limitations are not of the method but of computational resources (cpu time) needed to carry out the calculations to reach convergence. It is expected that as additional processors are made available ternary systems will also yield quantitative (sub dyne $/ \mathrm{cm}$ ) accurate results.

This detailed, molecular-level analysis provides additional insights as to the characteristics associated with low IFT conditions. For example:

- MD results have shown the structure that surfactants adopt at the interface between water and oil. In turn this elicited additional directions for research, such as the need to add larger hydrophilic head groups to the surfactant

- Molecular simulations are able to provide information of the association between surfactant and cosurfactant. For example, we ran extensive MD simulations where cosurfactants coexisted in a 30:1 mole ratio with surfactant molecules for long periods of time. We surmise that the cosurfactant is able to keep surfactant molecules from overcrowding the interface, spreading their effect over a larger surface area while, achieving ultra low IFT with minimal concentrations. Such layers are shown to be thermally stable by the MD simulation.

- The MD tools used here also inspire new models of the oil/water interface. For example, one conceptual model is that the interface consists of a single layer of surfactant between oil and water. This is not supported by the experimental evidence. Oil/Surfactant/water systems of low IFT typically have a large third phase (interface). This interface is optically turbid. Our simulations indicate that multi-layers (oil/surfactant/water/surfactant/oil) are quite stable and may be responsible for the third phase in such formulations. We surmise that this phase consists of an intimate folding of water/surfactant/oil layers, an indication that the interfacial tension is low enough to allow such complex, flexible, layers to fold on themselves over and over.

Less complicated theoretical tools have the potential to provide other useful information with a reduced computation effort. For example:

- We were able to extract similar information from the fully atomistic simulations using "bead" simulation approach where entire chemical groups are represented by a single bead. In this case we were able to identify that alkylbenzosulfonate $(\mathrm{Cn}, \mathrm{n}=16)$ substituted in the $4^{\text {th }}$ carbon were optimal structures to yield low IFT. The results are corroborated by the experiments and the more advanced fully 
atomistic simulations. However, such "bead" models are of limited use since the "bead-bead" interactions cannot be estimated from first principles. Such interactions are typically set arbitrarily by the modeler, typically to fit a given observed experimental data. This defeats the purpose of studying systems de nove, i.e., without the need for chemical synthesis and laboratory measurements.

\subsubsection{Model Validation and Calculation Sensitivity Analysis}

\subsubsection{Pure Component Surface Tension or Interfacial Tension}

A few validation examples were performed to test the accuracy of the IFT molecular models. For example, these include water/vacuum interface (water/air) and the decane/water IFT.

First, consider simple liquid complicated cases of several surface tension (liquid/vacuum) systems:

\begin{tabular}{lcc}
$\frac{\text { Liquid }}{\text { Argon }\left(57^{\circ} \mathrm{K}\right)}$ & Experimental $($ dynes $/ \mathrm{cm})$ & Calculated $($ dynes $/ \mathrm{cm})$ \\
\cline { 2 - 3 } & 14.5 & 15.5 \\
Water $\left(298^{\circ} \mathrm{K}\right)$ & 72 & 69.5 \\
Cyclohexane $\left(298^{\circ} \mathrm{K}\right)$ & 23 & 33 \\
n-Decane & 23.4 & 16.6
\end{tabular}

The agreement is mite onod sonsiderino the calculation of IFT came from a first principle,
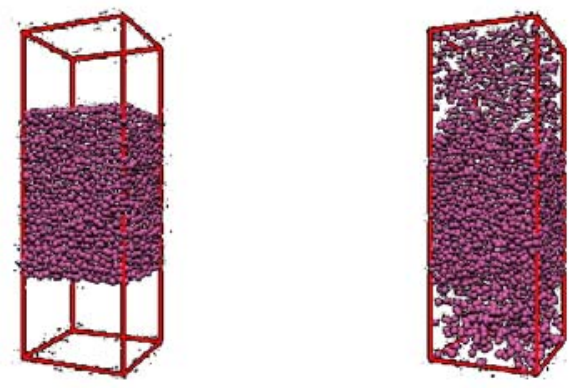

Figure 7.1.2.1 Molecular description of a liquid/gas system. Individual molecules of argon at $57^{\circ} \mathrm{K}$ are shown as purple dots. (a) Initial and (b) Final configuration of gas/liquid described via a Lennard Jones Fluid Model. Calculated surface tension computed from the difference in normal and tangential stress at interface via the Kirkwood-Buff formula (Equation 4).

Now, consider the more complicated, but more interesting situation where we wish to predict the IFT between an aqueous and hydrocarbon liquid. 
NPT (constant pressure) MD (Molecular Dynamics)

Decane (60 molecules)/Water NPT MD:

$48.29 \pm 52.20$ dynes $/ \mathrm{cm}$

Experimental:

$51.51 \mathrm{dynes} / \mathrm{cm}$

Again, the agreement between the theory and the reported IFT are quite close. The large uncertainty in the IFT calculation is related to its value being very sensitive to minor fluctuations in the position of the atoms in the interfacial region. This is discussed in more detail below where we present a sensitivity analysis of the effect of some of the input parameters on the calculated IFT.

\subsubsection{Sensitivity Analysis of IFT Calculation from MD Simulations}

We conducted various sensitivity tests to ascertain the level of precision for estimating IFT values through atomistic simulations, and to help guide in selection of parameters for IFT calculation studies. The main results are presented in this section. We employed a simple oil/water interface. We use n-decane as a model oil. The unit cell (1x1) contains 60 oil molecules and 400 water molecules respectively.

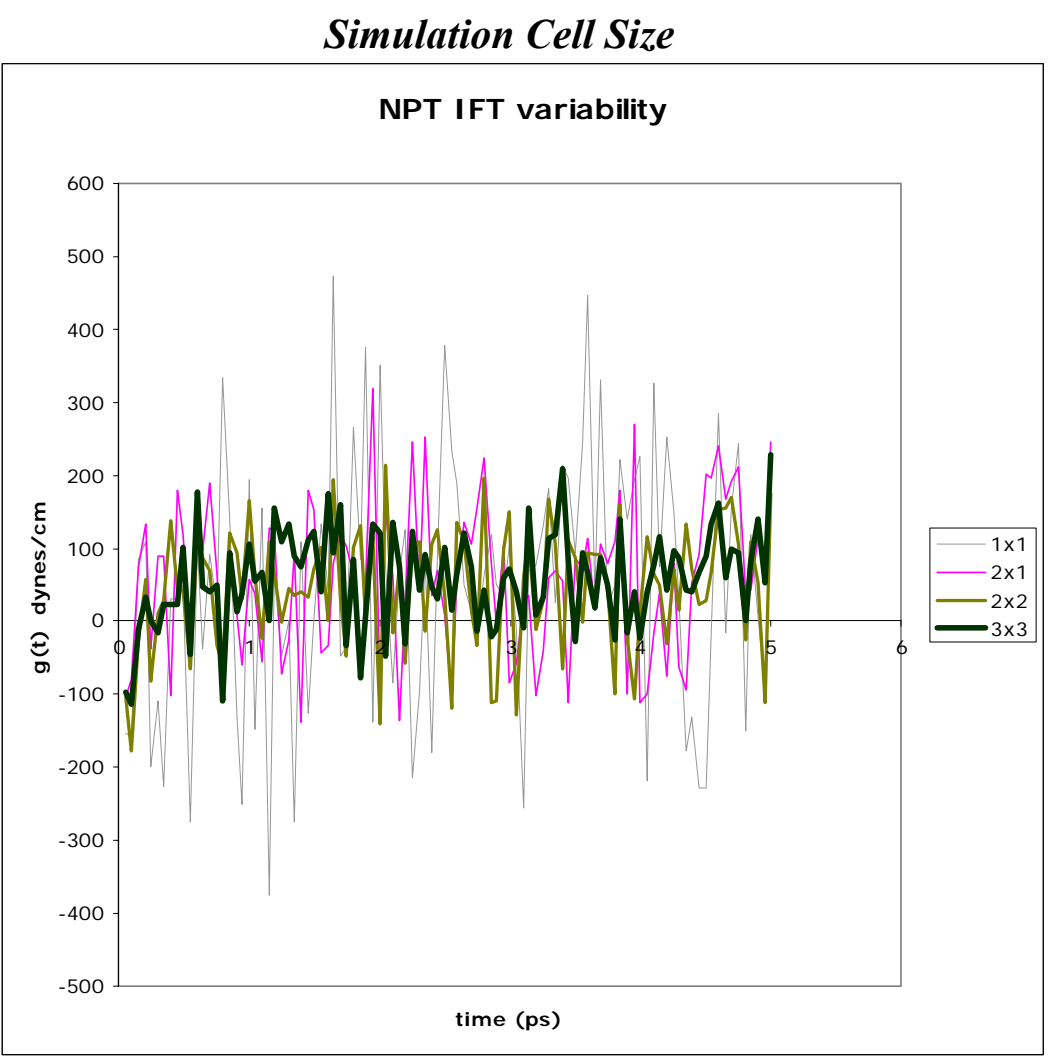

Figure 7.1.2.2. This shows the variability in calculated IFT values from atomistic constant pressure (NPT) simulations versus the size of the simulated cell. As the size (indicated by the $\mathrm{n} \times \mathrm{n}$ Miller indices of the cell base, e.g., $1 \mathrm{x} 1,2 \times 1,2 \times 2,3 \times 3$ ) increases the IFT values fluctuate less and become mostly positive. 


\section{Total Simulation Time}

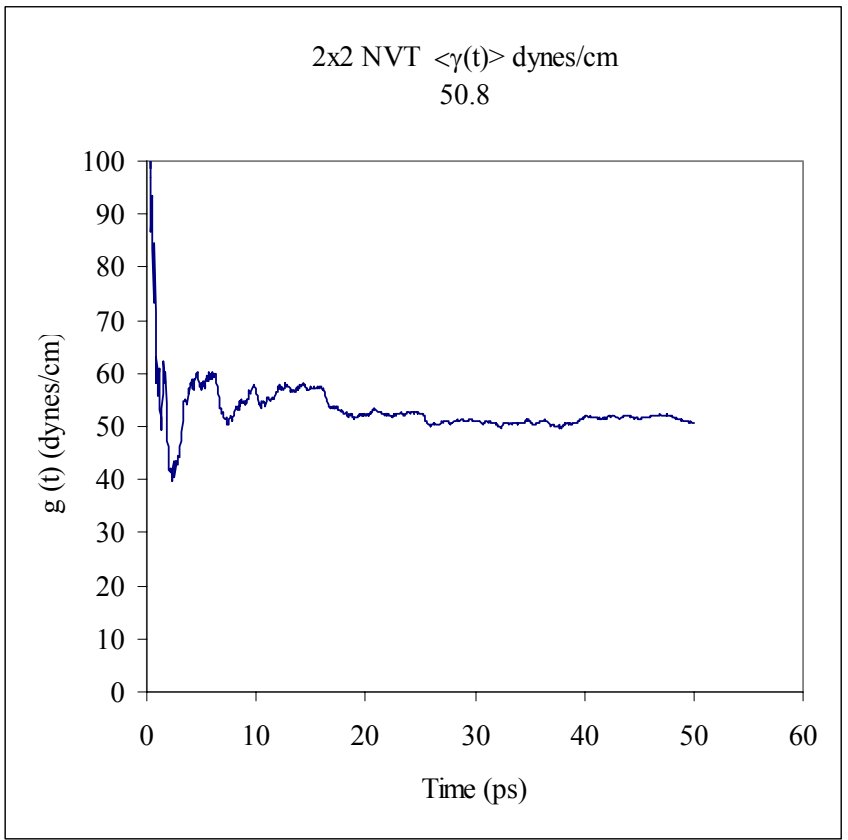

Figure 7.1.2.3 The figure shows the time averaged IFT for the same surfactant system as a function of time. Value appears to converge after $30 \mathrm{ps.}$

\section{Molecular Dynamics Integration Time Step}

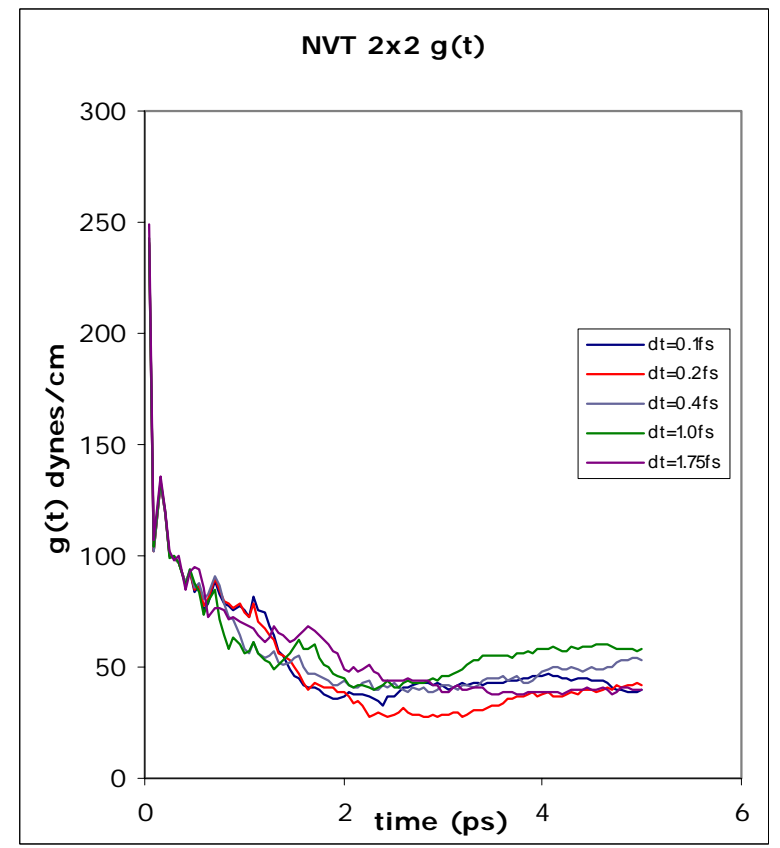

Figure 7.1.2.4 The integration time step, typically $1.0 \mathrm{fs}$, has a measurable but small effect on the calculated IFT values over short dynamics ( $5 \mathrm{ps}$ ). For longer dynamics (not shown), $1.0 \mathrm{fs}$ time steps are adequate to estimate IFT. 


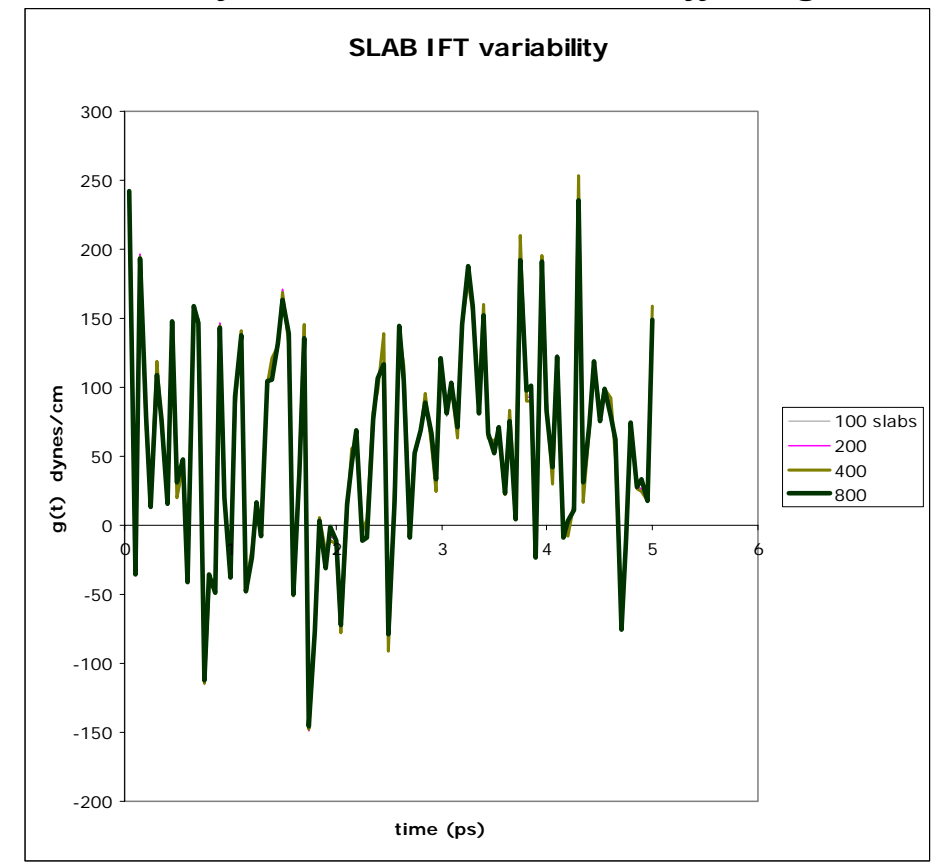

Figure 7.1.2.5 The figure shows no difference in increasing the number of slabs from 100 to 800 in the integration of Kirkwood-Buff IFT formula. Most runs use 100 to 200.

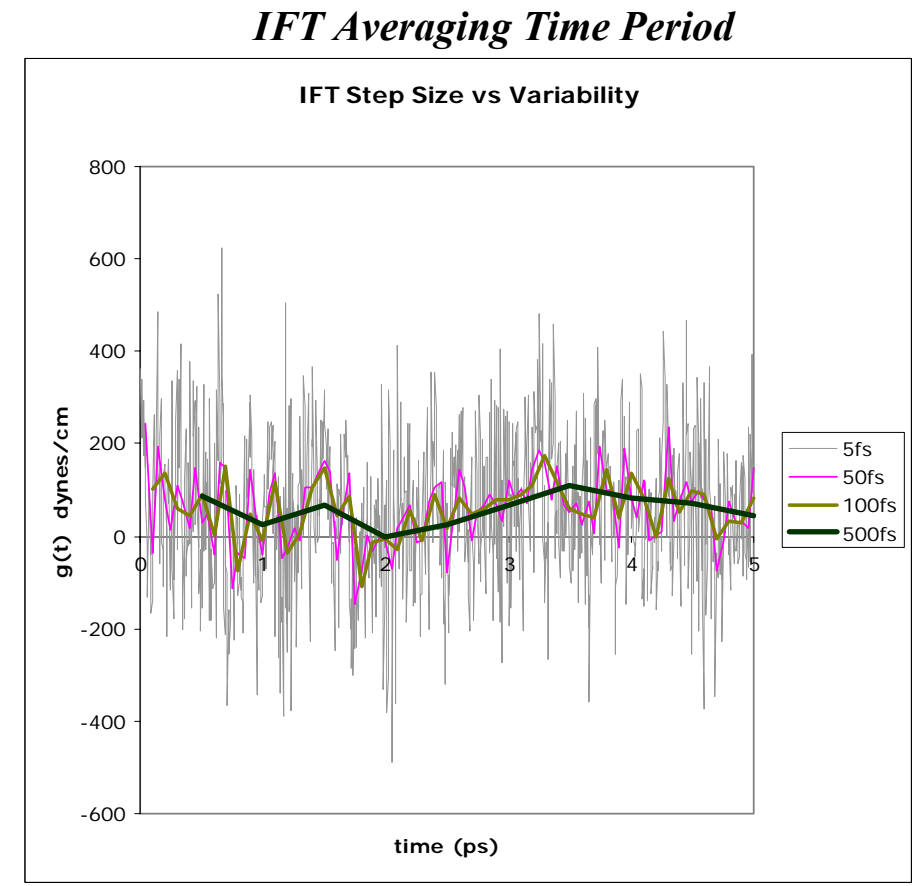

Figure 7.1.2.6 IFT values are averaged over a period of time before being printed out for further analysis. The figure shows that there is great variability in the individual values of IFT. For short averaging steps (5fs) IFT varies between large positive and negative unphysical IFT values. For longer averaging periods ( $500 \mathrm{fs})$ the values are always positive. We have adopted an averaging period of $200 \mathrm{fs}$ ( $2 \mathrm{ps}$ ) or 20 Nose cycles to compute IFT on a routine basis. 


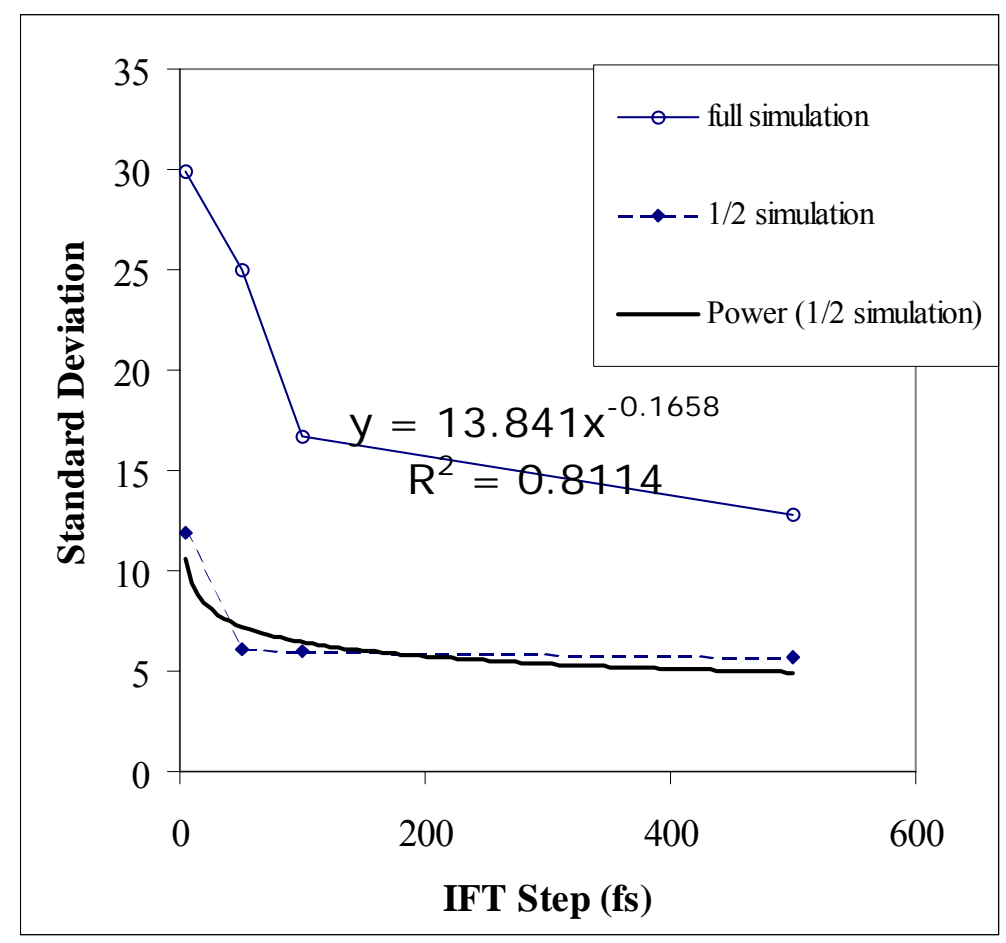

Figure 7.1.2.7 The standard deviation in IFT decreases greatly when the averaging period is changed to $500 \mathrm{fs}$ or longer. The effect of using only half of the simulation for calculating IFT is also shown. This indicates that the first half is not yet fully equilibrated. The protocol now includes avoiding this transient values and using averaging periods of at least $2 \mathrm{ps}$.

Overall the conclusion is that we need to carry out MD simulations with larger systems, for longer periods of time using typically integration time steps (1fs), averaging over longer block units of time to get instantaneous IFT values that fluctuate less and converge faster.

\subsubsection{Model Calculations of IFT for Alkyl Benzene Sulfonate Surfactants}

We chose to focus the first MD simulations that include a surfactant on branched alkyl benzene sulfonates, a commercially available class of surfactants for producing low interfacial tensions between hydrocarbons and water. One reason is that alkyl benzene sulfonates have been the most common type of surfactant chemistry employed for IOR in field applications. Another is that there is a significant body of literature data about their behavior and characteristics.

The particular suite of branched surfactants included in this study are of interest also because of their strong sensitivity (3-4 orders of magnitude change in IFT) to isomeric chemical structural changes. We anticipate that having a molecular level understanding of why fairly subtle changes in the surfactant structure produce profound changes in the 
IFT will be very valuable for calculations to project IFT behavior for other, novel surfactant concepts.

These IFT molecular simulations were performed under the following conditions of a water/surfactant/oil interface.

Surfactant: C16_2, C16_3, C16_4, C16_5, C16_6, and C16_8. This nomenclature denotes a benzene sulfonyl type of $\mathrm{C} 16$ straight chain alkyl sulfonate, with the second number giving the differing alkyl chain point of attachment. Structure:

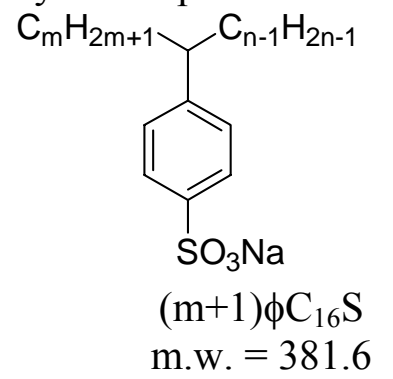

Figure 7.1.3.1. Branched alkyl benzene sulfonates. $(\mathrm{m}=1,2,3,4,5,6,7)$.

Model: full atomistic description using Dreiding FF, alkyl tail: united atom description

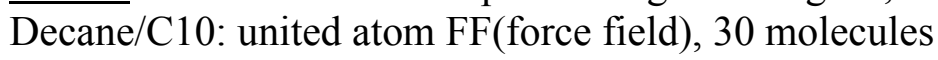

Number of discrete slabs for IFT integration: 105, Average block sums at every $1.0 \mathrm{ps}$

Water: F3C Model FF, 400 molecules

Temperature $299.15 \mathrm{~K}$ (26 C experimental temperature)

The water and surfactant are placed between two layers of C10 (n-decane) as the oil phase. Hence, in the plots immediately below, the oil layers are on the left and right side of the aqueous layer. Results are shown below:

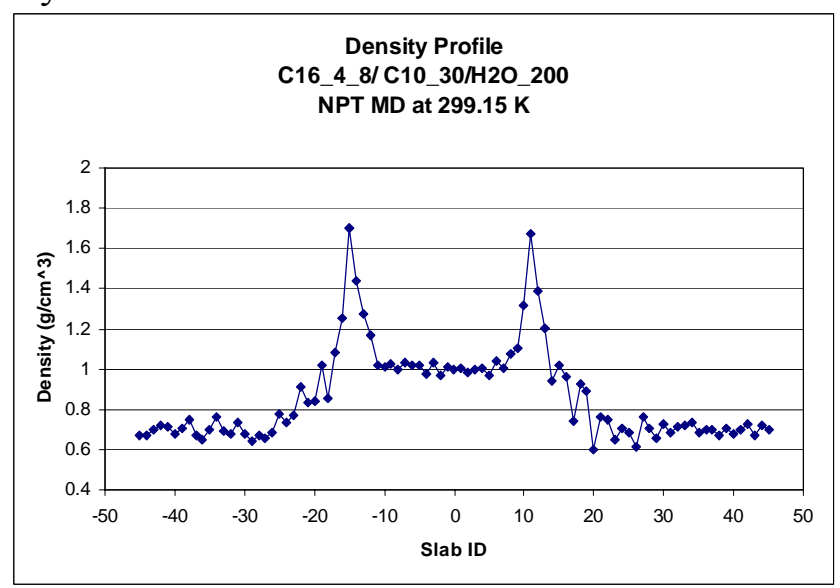




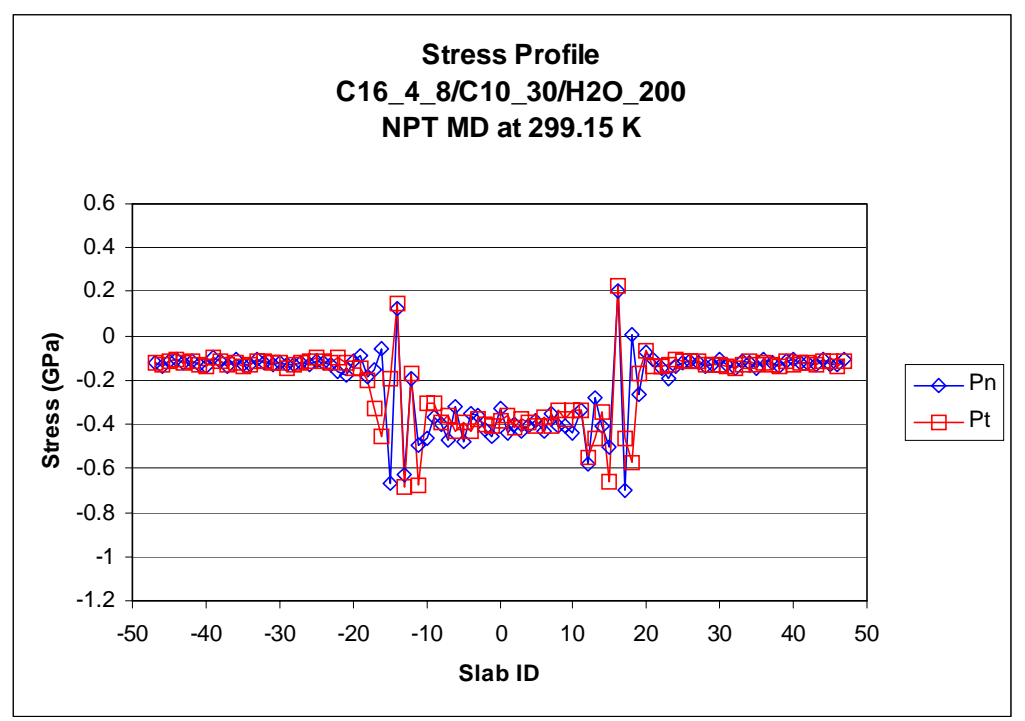

Figure 7.1.3.2. Density profile and stress profile for C16_4 (isomeric substitution of benzosulfonate on $4^{\text {th }}$ position of a $\mathrm{C} 16$ carbon chain).

Figure 7.1.3.2 describes the interface generated by surfactant between water and oil (C10) phase. Each phase has correct density, which means that our force field for oil and water can simulate this system properly. Additionally, surfactant molecules are located stably at the interface. The interfacial tension profile resulted from the above stress profile is shown in Figure 7.1.3.3. Interfacial tension is observed at only the interface region and its integration along the axis normal to the surface leads to IFT $=11.03 \mathrm{dyne} / \mathrm{cm}$, a reduced value when compared to the bare interface between water and oil (C10, IFT $=51.51$ dyne $/ \mathrm{cm})$. Clearly, our atomistic simulations are able to capture the interfacial-tensionreducing effect of surfactant.

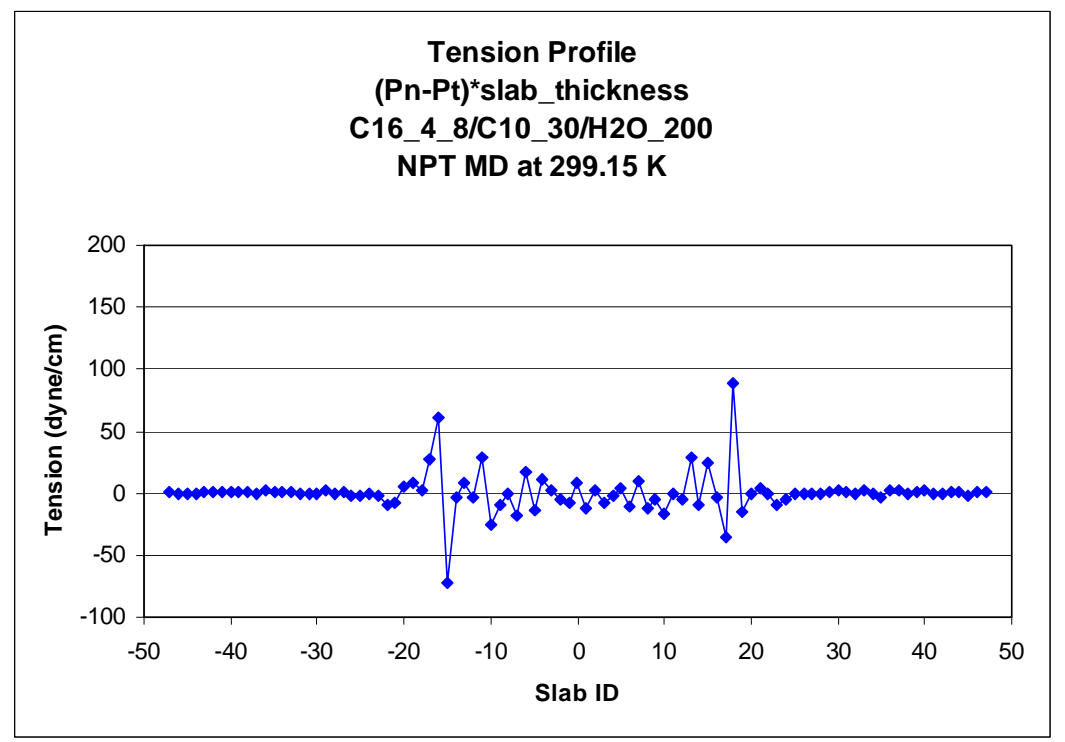

Figure 7.1.3.3. Interfacial tension profile for $\mathrm{C} 16 \_4$. The integrated profile leads to a interfacial tension of $\gamma=11.03 \mathrm{dynes} / \mathrm{cm}$. 
Next, the performance of $n$-alkylbenzosulfonate $(n=16)$ surfactant with various benzosulfonate branching positions from 2 to 8 is shown in Figure 7.1.3.4. The atomistic model implementing Equation (4) reproduce the non-monotonic behavior of interfacial tension as a function of benzosulfonate substitution position along the backbone.

Furthermore, the prediction that the attachment position with the $4^{\text {th }}$ carbon leads to the minimum $\gamma$ value agrees with the published results (Figure 7.1.3.5 and 7.1.3.6, see Doe and Wade, 1977). While the calculated IFT values do reflect the correct trend, they do not provide a quantitative prediction of IFT (IFT experimental results report some low values). (Note that salinity effects, which can have large effects on IFT values, were not included in this series of atomistic simulations.)

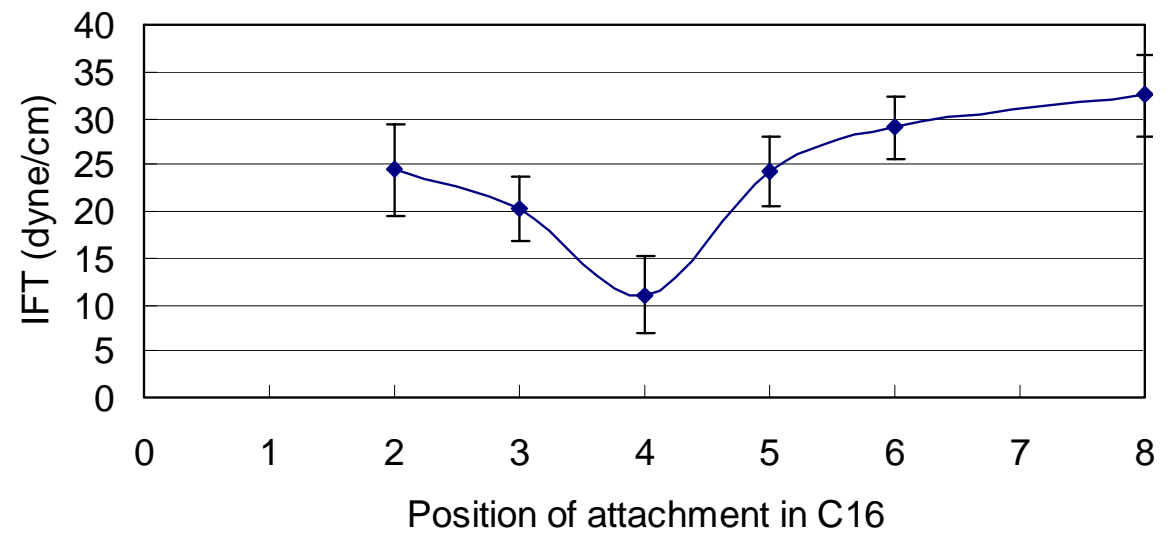

Figure 7.1.3.4. Change of interfacial tension as a function of attachment position of benzosulfonate group as calculated by MD simulations.

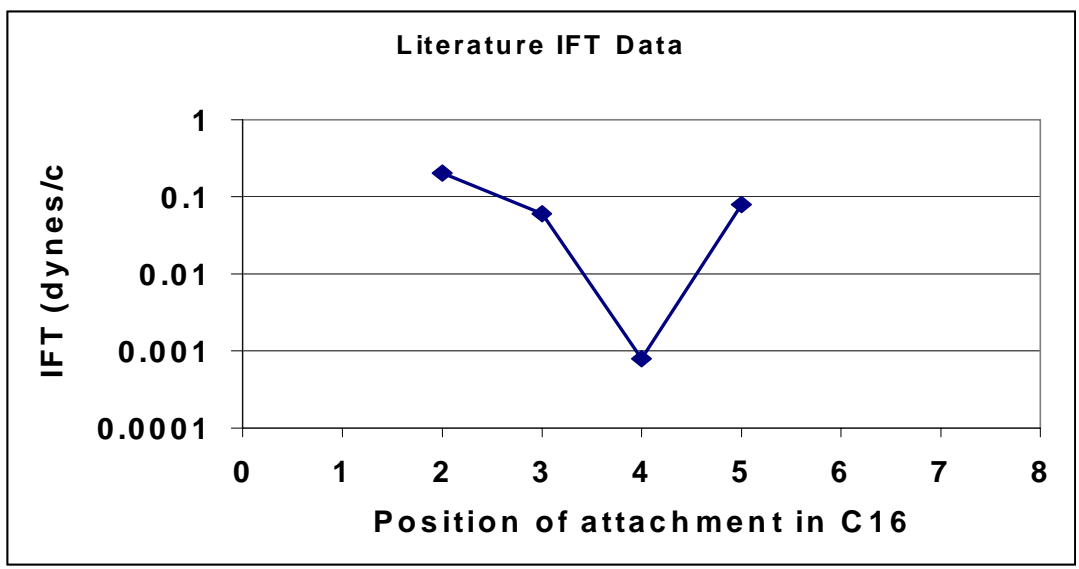

Figure 7.1.3.5. Comparable experimental results of interfacial tension as a function of attachment position of benzosulfonate group (Doe and Wade, 1977). (IFT measured at $25 \mathrm{C}$, iso-pentanol $2 \mathrm{wt} \%, 3 \mathrm{~g} / 1 \mathrm{NaCl}, 0.7 \mathrm{~g} / 1$ surfactant) 


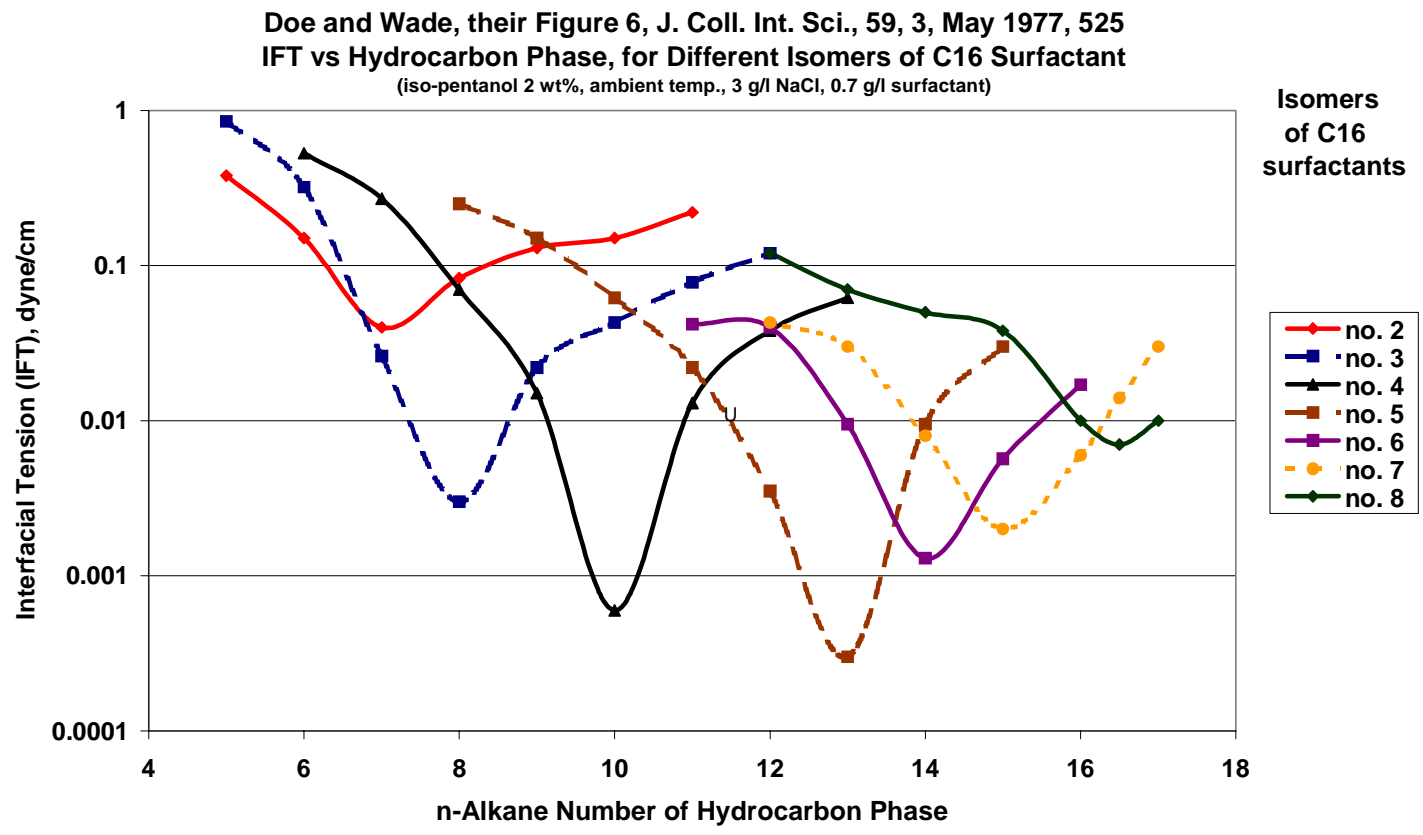

Figure 7.1.3.6. Full set of experimental data reported for interfacial tension for water/n-alkane for isomers of C16-benzosulfonates. Note that the No. 4 isomer gives the lowest IFT for n-decane as the oil phase.

These series of simulations are discussed in significantly greater detail in the paper written for publication. This and other publications from this project are attached to this final report.

\subsubsection{Salinity Effects Investigated in Molecular Dynamic (MD) Simulations}

\subsubsection{Salt Effect with Water (no surfactant) on Interfacial Tension}

First, simulations for IFT were performed with water only (no surfactant) and n-decane as the hydrocarbon phase.

Table 7.1.4.1 summarizes the salt effects on the water/vacuum IFT. Our simulations found that the IFT values increase from about $65 \mathrm{dyne} / \mathrm{cm}$ for pure water to about 72 dyne/cm for $6.3 \mathrm{wt} \% \mathrm{NaCl}$ solution, in agreement with experimental trend of actual values of 72 for pure water and 74 for the $\mathrm{NaCl}$ solution. An example of the time running average of the IFT calculation for pure water and the $\mathrm{NaCl}$ solution is given in Figure 7.1.4.1. The IFT values have converged after about $70 \mathrm{ps}$. The density profiles along the z-direction for pure water are shown in Figure 7.1.4.2. The wavy shape of density profile for $\mathrm{NaCl}$ solution (not shown) suggests layering of the sodium and 
chlorine atoms in water. This is consistent with studies of Jungwirth and Tobias (2001) that there is an excess chlorine concentration near the water/vacuum interface.

Furthermore, the smooth density profile for the $\mathrm{NaCl}$ solution at the interface indicates that there is a region void of ions at the interface, as predicted by the Gibbs adsorption equation (Chattoraj , 1984). Physically this is a result of the poor hydration of ions at the interface.

Table 7.1.4.1. Comparison of the change of water/vacuum IFT at different salt $(\mathrm{NaCl})$ concentrations

\begin{tabular}{|c|c|c|c|c|c|}
\hline Concentration & $\begin{array}{l}\mathrm{x}, \mathrm{y} \text { dimension } \\
(\AA \hat{x} \AA \overline{)})\end{array}$ & $\begin{array}{l}\text { \# water } \\
\text { molecules }\end{array}$ & $\begin{array}{l}\# \\
\mathrm{NaCl}\end{array}$ & $\begin{array}{l}\text { Simulation } \\
\text { IFT } \\
\text { (dyne/cm) }\end{array}$ & $\begin{array}{l}\text { Expt IFT } \\
\text { (dyne/cm) }\end{array}$ \\
\hline Pure water & $39.4 \times 39.4$ & 800 & 0 & 65.72 & \multirow{2}{*}{72} \\
\hline Pure water & $21.2 \times 21.2$ & 800 & 0 & 64.16 & \\
\hline 3.3 wt $\%$ & $39.4 \times 39.4$ & 784 & 8 & 65.88 & 73 \\
\hline 6.3 wt $\%$ & $39.4 \times 39.4$ & 768 & 16 & 66.03 & \multirow{3}{*}{74} \\
\hline 6.3 wt $\%$ & $21.2 \times 21.2$ & 768 & 16 & 75.14 & \\
\hline 6.3 wt $\%$ & $42.4 \times 42.4$ & 3072 & 64 & 71.67 & \\
\hline
\end{tabular}

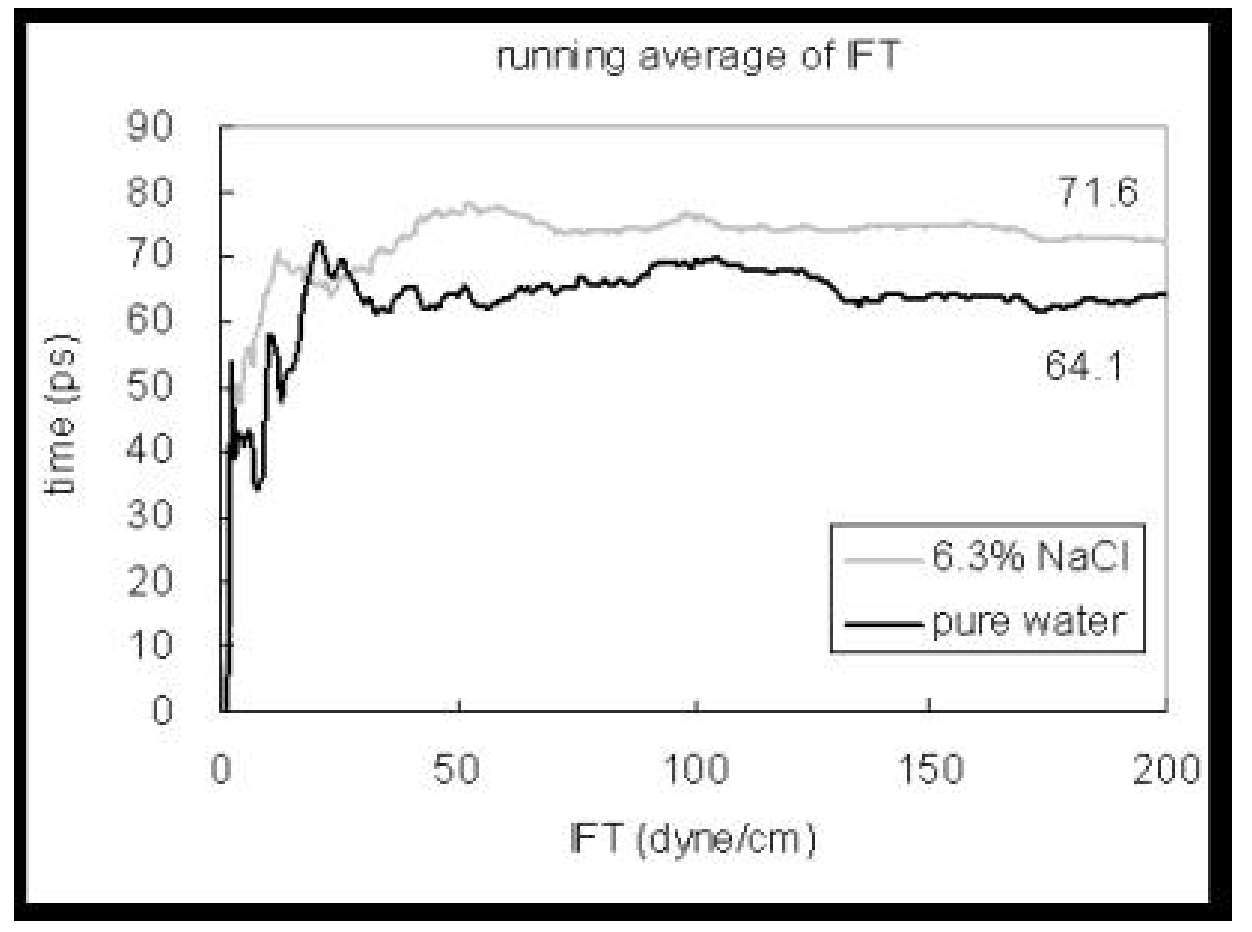

Figure 7.1.4.1. Running average of IFT between liquid/vacuum interface for pure water and $6.3 \% \mathrm{NaCl}$ solution. 


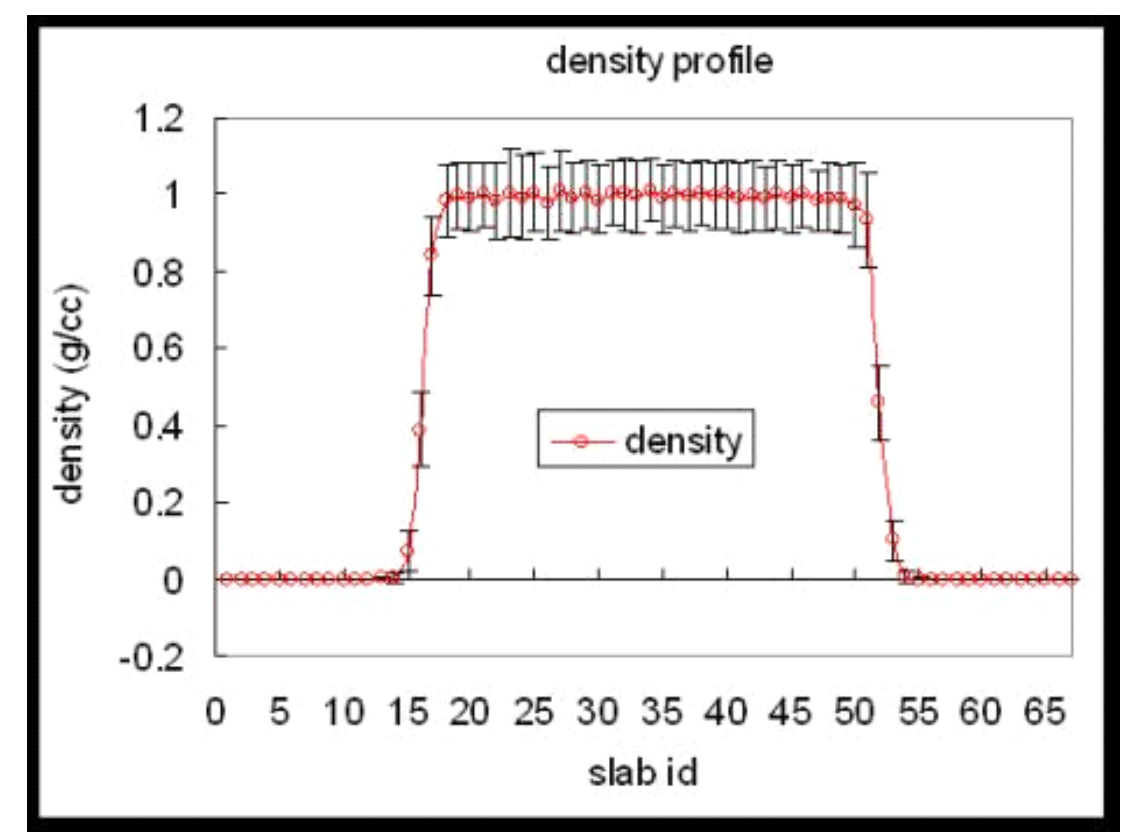

Figure 7.1.4.2. Density profile for pure water.

\subsubsection{Salt Effect on the Surfactant Solution Interfacial Tension (IFT)}

The surfactant considered is an alkyl benzosulfonate with a $\mathrm{C} 16$ alkyl tail branching at the $8^{\text {th }}$ or $4^{\text {th }}$ position, and the hydrocarbon phase is n-decane.

The calculated effect of including salinity in the simulation is to increase the IFT. This trend is consistent with the experimental data if in fact all these salt calculations are at an "over optimum" salinity condition where water in oil emulsions would dominate. (Low IFT data was reported at $0.3 \%$ salt, and model calculations are at $0.82 \mathrm{wt} \%$ salt and higher). A concern is if the structure that we have chosen to model (surfactant monolayer) does not capture the physics of the effects of salinity properly. We expect that adding salt concentration changes the phase behavior of the surfactant/water mixture, driving the surfactant towards the interface. It is plausible that the interface consists of multiple layers, or that the measured IFT values correspond to a bicontinuous water/oil/surfactant interface rather than the monolayer in our models. Studied Systems:

\begin{tabular}{|c|c|l|l|l|l|}
\hline NaCl wt\% & $\begin{array}{l}\text { No. of Salt } \\
\text { and Water } \\
\text { molecules }\end{array}$ & $\begin{array}{l}\text { No. } \\
\text { Surfactant } \\
\text { Molecules }\end{array}$ & $\begin{array}{l}\text { No. of n- } \\
\text { Decane, oil } \\
\text { Molecules }\end{array}$ & $\begin{array}{l}\text { Calculated } \\
\text { IFT } \\
(\text { dyne/cm })\end{array}$ & Comments \\
\hline 0 & 0,200 & 8 & 30 & 11.03 & Base Case \\
\hline 0.82 & 1,398 & 16 & 60 & 12.68 & \\
\hline 1.64 & 1,198 & 8 & 30 & 22.99 & \\
\hline 3.31 & 2,196 & 8 & 30 & 27.45 & \\
\hline 6.76 & 4,192 & 8 & 30 & 37.81 & \\
\hline
\end{tabular}


Model: full atomistic description using Dreiding FF, alkyl tail: united atom description Decane/C10: united atom FF; Number of discrete slabs for IFT integration: 105, Average block sums at every 1.0 ps; Water: F3C Model FF, 400 molecules Temperature $299.15 \mathrm{~K}$ (26 C experimental temperature)

The figure below shows the calculated IFT results versus salinity.

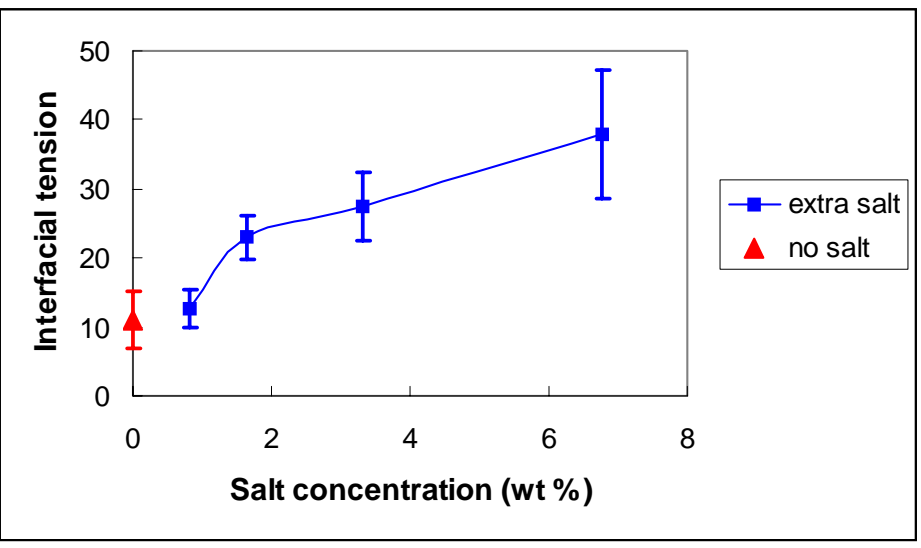

Figure 7.1.4.3. Calculated IFT versus salinity for number 4 isomer of $\mathrm{C} 16$ linear alkyl benzene sulfonates

\section{Stress profile}

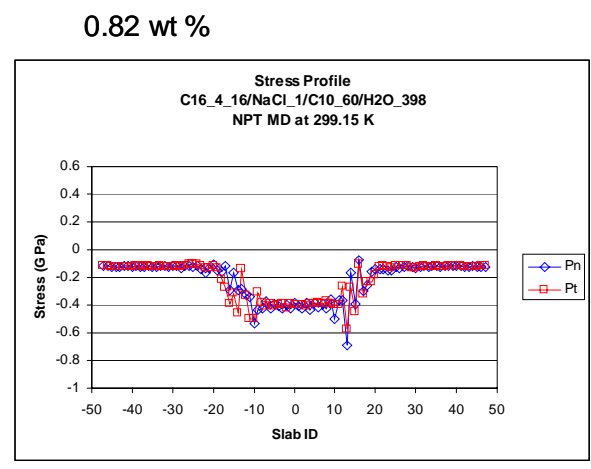

3.31 wt $\%$

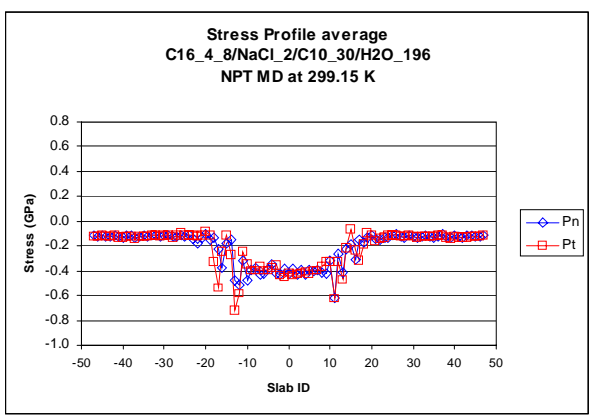

1.64 wt \%

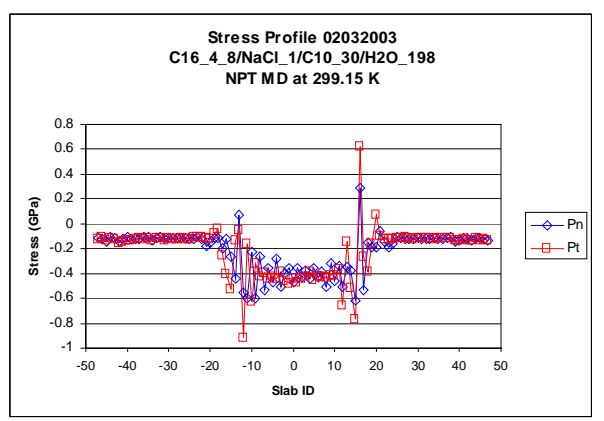

6.76 wt $\%$

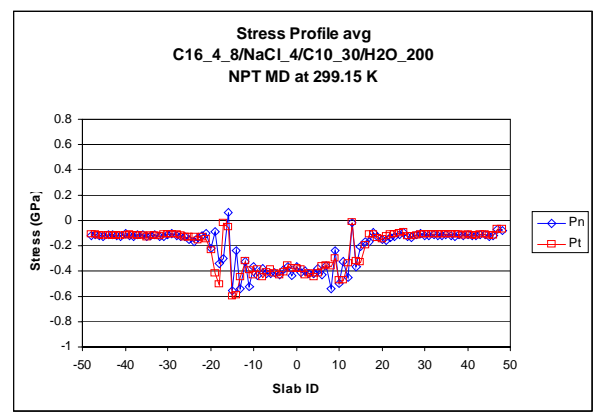

Figure 7.1.4.4. Stress profiles associated with calculations containing salt 


\section{Tension profile}

0.82 wt \% (12.68 \pm 2.84 dyne/cm)

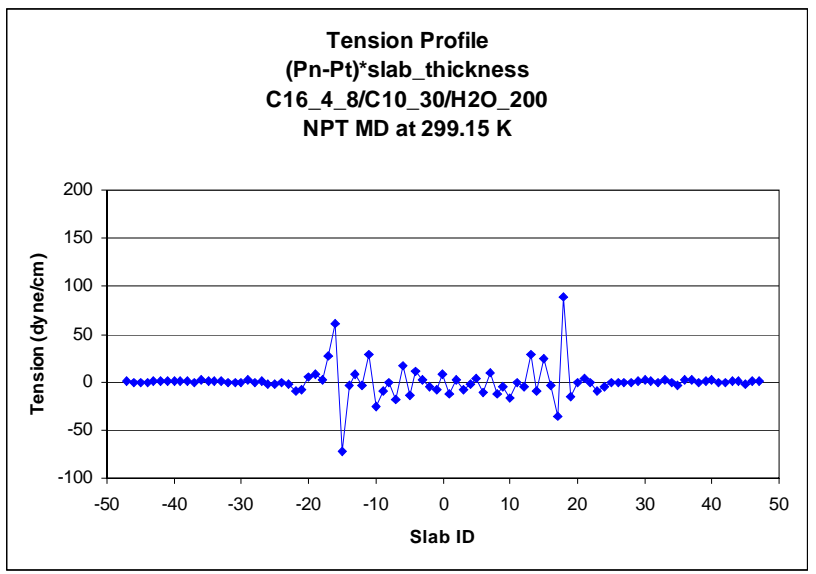

3.31 wt \% (27.45 \pm 4.96 dyne/cm)

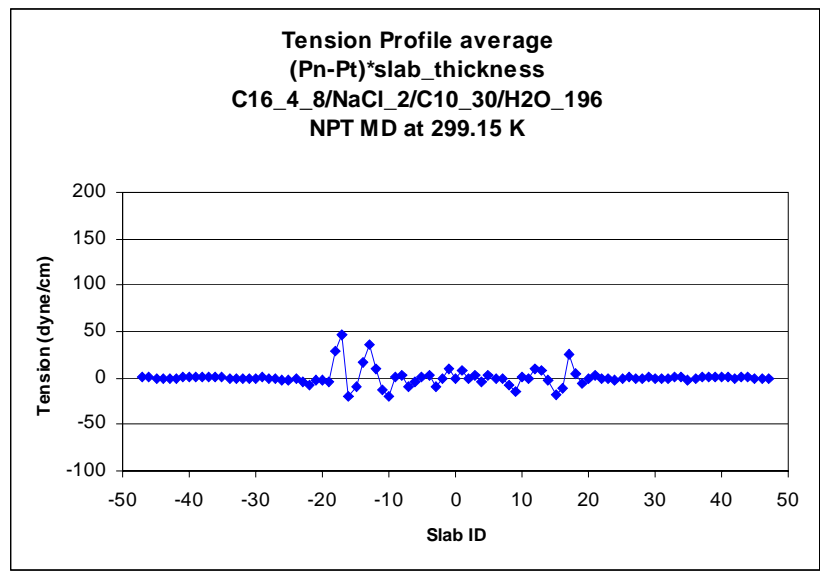

1.64 wt \% (22.99 \pm 3.16 dyne/cm)

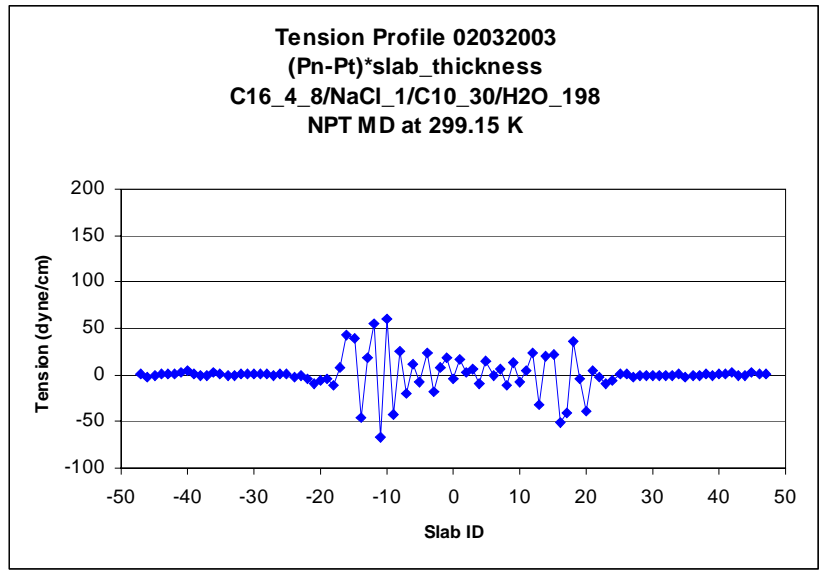

\subsection{6 wt \% (37.81 \pm 9.35 dyne/cm)}

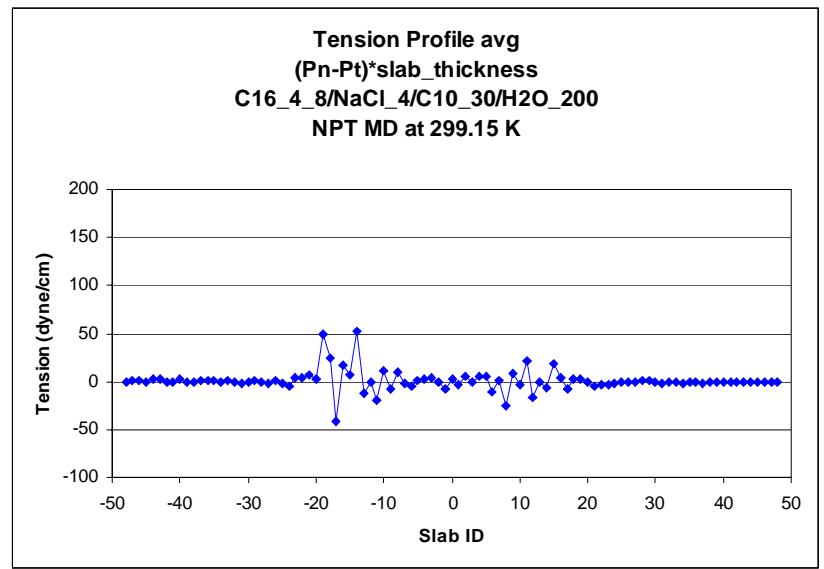

Figure 7.1.4.5. Interfacial tension profile for molecular dynamic cases that include salt. 


\section{Density profile}
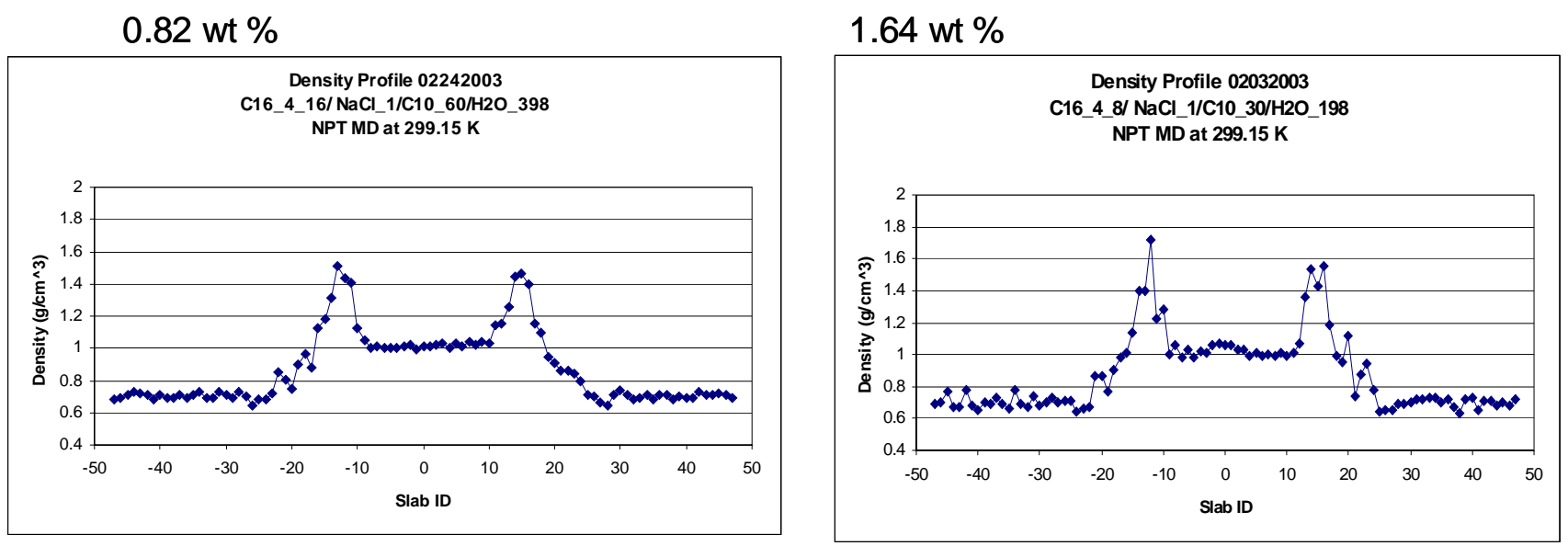

\subsection{1 wt \%}

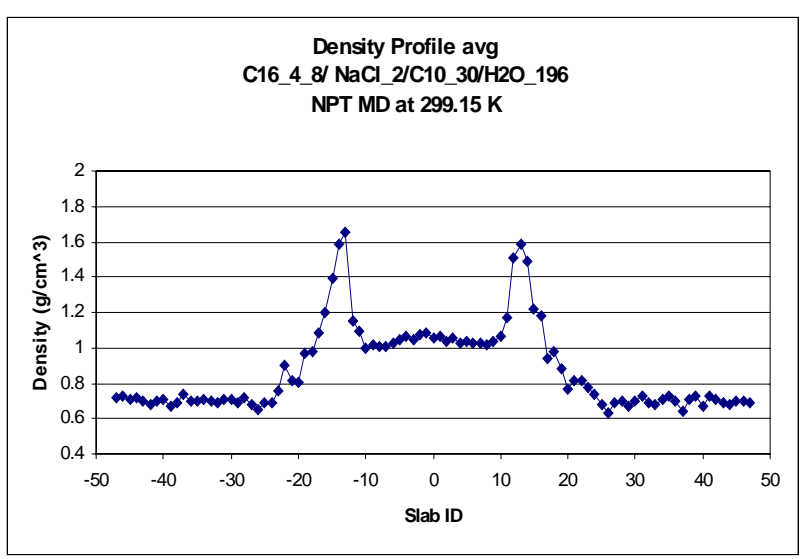

6.76 wt \%

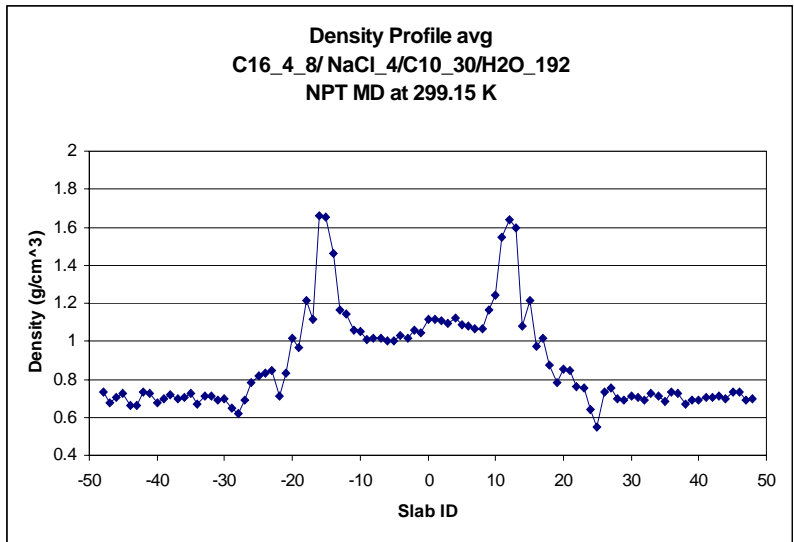

Figure 7.1.4.6. Fluid density profiles for molecular dynamic calculations containing salt

In conclusion, we can estimate salinity effects on IFT from MD simulations. However, we typically run the simulations at concentrations higher than what is found in oil field applications. Lower concentrations require larger models and longer computations. 


\subsubsection{Molecular Modeling Study of Two Model Alkyl Polyglycosides (APG) - Water/n-Octanol Cosurfactant/Octane Interfacial Structure and IFT}

\subsubsection{Motivation for Modeling - Favorable IFT Results with Alkyl Polyglycoside (APG) Surfactants}

This class of surfactants was discussed briefly in the Literature Review Summary (Section 5.2) and in greater detail in the whole Literature Review (attached to report). Also detailed later in this report are the extensive experimental IFT results with this class of nonionic surfactant. A typical alkyl polyglycoside (APG) surfactant is shown below

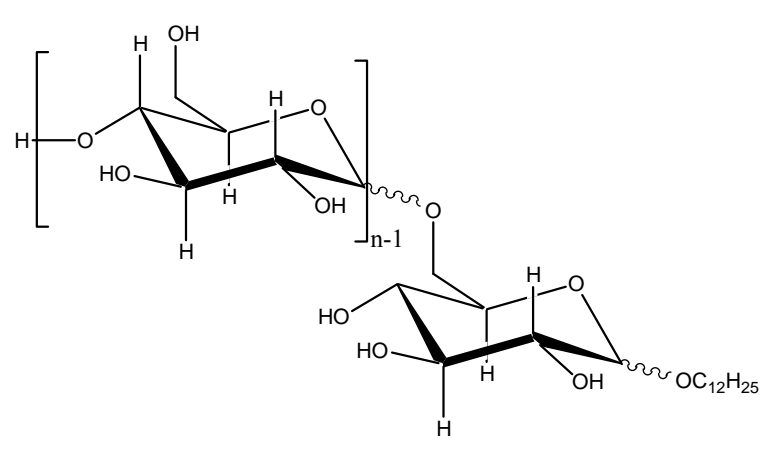

Figure 7.1.5.1. Schematic molecular structure of a typical APG surfactant.

We focused on this compound since we suspected that a large head group may yield some of the benefits that a larger tail group gives to Gemini surfactants. The enhanced stability would be at the aqueous rather than the oil phase. We were further encouraged to take on this system since we were fortunate to have the force field parameters in place for the simulation and modeling of glycosides. All we required were a few extra Quantum Mechanical simulations to obtain atomic charges and structures for these surfactants. We were further intrigued by the possibility a large number of stereoisomers (so called anomeric isomers) of the various polyglycosides may have identical chemical formulas but drastically different IFT values. In all, this seems like a good benchmark to undertake to link structure with interfacial tension effects.

For inexpensive, commercial versions of these surfactants the product will contain a range of number of head groups (mostly a mixture of mono- and di-, trace amounts of larger) and a range of length of the alkyl chain tail. As reported in the literature and observed from our laboratory studies, some combinations of commercial APG products and cosurfactants or other surfactants in aqueous solution can create low IFT versus a hydrocarbon. One of the more intriguing experimental results found is low IFT can occur between an aqueous formulation containing a low concentration of a commercial APG and an alcohol cosurfactant (e.g. n-octanol) versus a hydrocarbon (e.g. n-octane). Hence, one target simulation study is to model the IFT and interfacial structure of such aqueous solution formulations equilibrated with a hydrocarbon phase. 
If we were to select at random an "average" APG structure there is the concern about the validity of the theoretical modeling results. We know that the presence of small amounts of a surfactant $(<0.2 \%)$ influence the IFT values by often several orders of magnitude $(<0.01 \mathrm{dynes} / \mathrm{cm})$, and thus ignoring the low concentration surfactant components may not be a suitable first order approximation.

\subsubsection{Molecular Modeling Study with a Pure APG Example (HBDM)}

As an alternative approach, we did select a single, pure compound, n-Hexadecyl- $\beta$-Dmaltopyranose (HBDM) for theoretical study, but importantly, we also generated experimental data for this single surfactant (obtained at a 97\% purity) so as to have a direct physical comparison between theoretical and experimental results. Another important point is that and that the trends of these data with a pure APG surfactant are consistent with that measured for commercial APG products. Again, one of the more intriguing experimental results found is low IFT can occur between an aqueous formulation containing a low concentration of HBDM and a high molar excess of an alcohol cosurfactant (e.g. n-octanol) versus a hydrocarbon (e.g. n-octane).

Thus, this HBDM surfactant should in fact serve as a valid proxy for the commercial APG product so that the physical insights gained from the molecular modeling study results (which now can be performed in a practical amount of time) can be tied to a real physical system that behaves similarly to the commercial system of economic interest. The structure of HBDM is shown below.

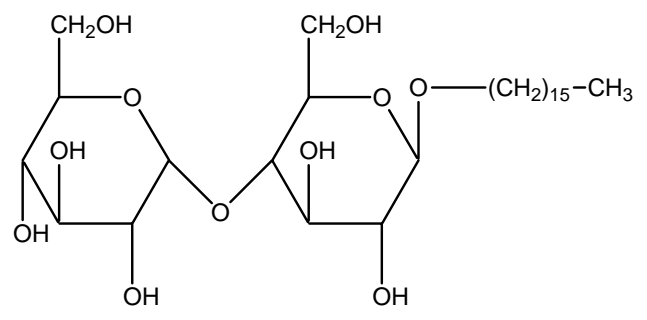

n-Hexadecyl-beta-D-maltopyranoside

Figure 7.1.5.2 Schematic Chemical Structure of HBDM

HBDM is a dissacharide with $\mathrm{C} 16$ chain, but the structure as shown in Figure 7.1.5.2 is not sufficient to reproduce the correct chirality of each chiral center. We conducted a literature SciSearch and found the absolute chirality for the Decyl- $\beta$-D-maltopyranose analogue shown in Figure 7.1.5.3 (Formula: C22 H42 O11, CA Registry Number: 8249409-5, Index Name: $\beta$-D-Glucopyranoside, decyl 4-O- $\beta$-D-glucopyranosyl-). Other names include 1-Decyl $\beta$-D-maltoside; 1 -O-Decyl- $\beta$-D-maltoside; Decyl $\beta$-Dmaltopyranoside; Decyl $\beta$-D-maltoside; Decyl $\beta$-maltopyranoside; Decyl- $\beta$-maltoside; Decylmaltoside; n-Decyl $\beta$-D-maltoside. Physical properties for the Decyl compound are included in Table 7.1.5.1 for completeness. Care was exercised to model the correct stereoisomer of the dissacharide. 
The absolute structure assumed for these theoretical studies is given below

(a)

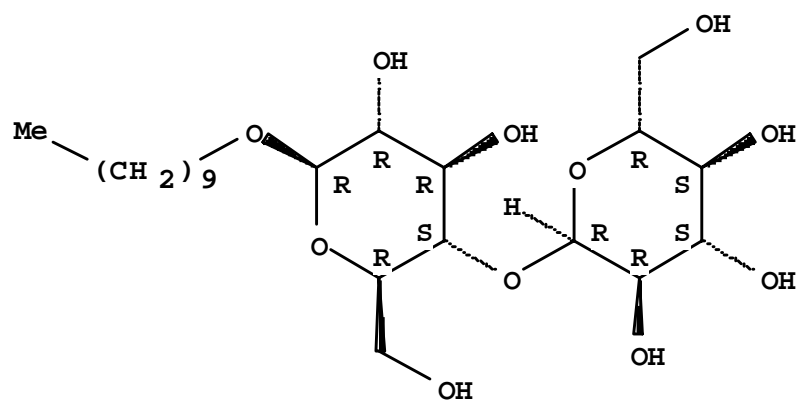

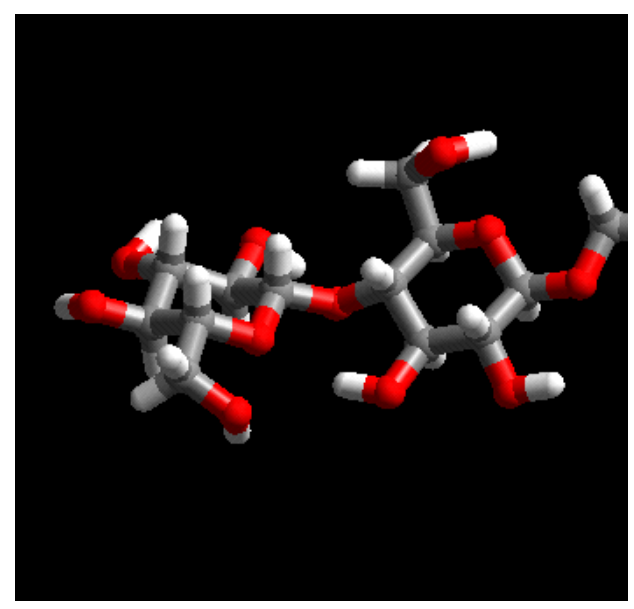

(b)

Figure 7.1.5.3. (a) Absolute Stereochemistry of the Decyl version of HBDM surfactant.

(b) Molecular model of head group displaying the full stereochemistry for HNDM. Each color line is one atom.

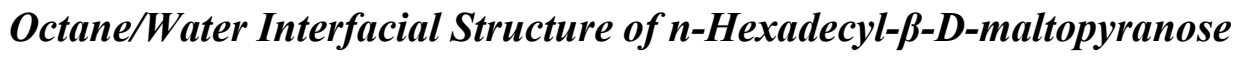
and n-octanol cosurfactant formulation

It is important to understand how it is that a formulation consisting of low concentrations $(<0.2 \%)$ of glycoside surfactants in the presence of a cosurfactant such as n-octanol (noctanol+HDBM 2wt $\%$ ) can yield low IFT values, even approaching $0.001 \mathrm{dynes} / \mathrm{cm}$. We surmise the following hypothesis:

1) Excess surfactant $(>0.2 \mathrm{wt} \%)$ may sequester the co-surfactant to the water phase, in the form of mixed micelles. The large polar group requires the additional strong packing of n-octanol to create a micelle layer.

2) Polyglycoside surfactants in low concentrations help stabilize the presence of n- 
octanol at the oil/water interface.

3) The structure of the polyglycoside at the interface adopts a specific conformation at the oil/water interface

To gain some insight into these ideas we conducted quantum mechanical and molecular dynamics simulations.

Figure 7.1.5.4 shows the calculated quantum electrostatic potential (ESP) charges (B3LYP DFT 6-31g**) for the head group of HBDM. Charges for the C2-C16 CH2 and the terminal $\mathrm{CH} 3$ group in the alkyl chain were set to zero to be consistent with the same choice made for n-octane (modeled oil). In the interface simulations we use ESP B3LYP DFT 6-31g** charges for $n$-octanol. In all cases we restrict the charges to reproduce the quantum dipole moment. Water charges and force field follow the $\mathrm{F} 3 \mathrm{C}$ model $(\mathrm{qH}=0.41$, $\mathrm{qO}=-0.82$ ). We use explicit hydrogens in all alkyl chains, including the n-octane liquid phase.

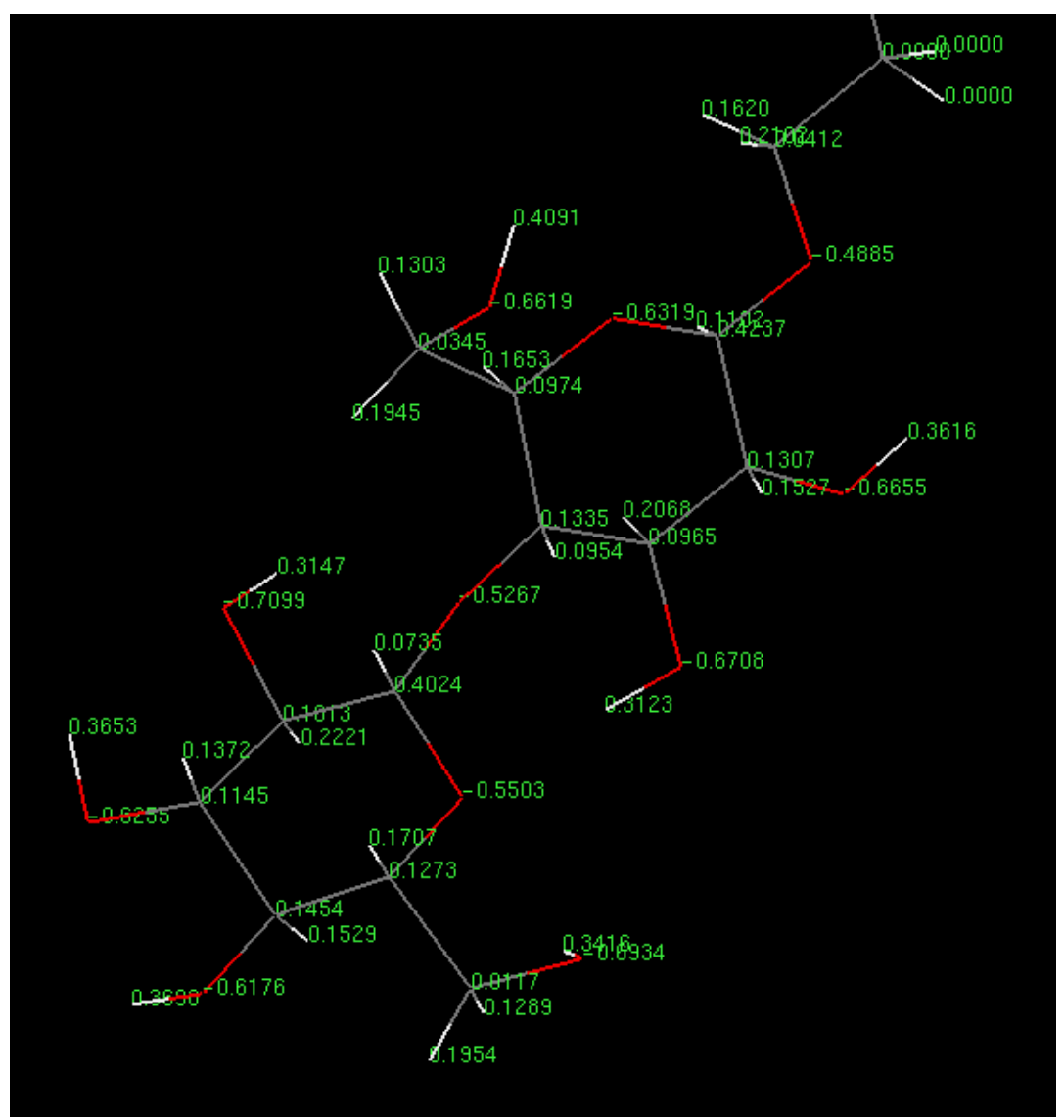

Figure 7.1.5.4. ESP Charges for head group of HBDM

Each colored line is one atom.

The interactions of a single n-octanol molecule with HBDM were investigated using the BLENDS module. We chose two conformations of HBDM for study, a trans and a gauche conformation at the 2 and 3 carbon atoms in the alkyl chain. For each of the initial structures of HBDM we sampled 100,000 configurations of n-octanol. Figure 7.1.5.5 displays the full distribution of n-octanol/HBDM binding energetics. 


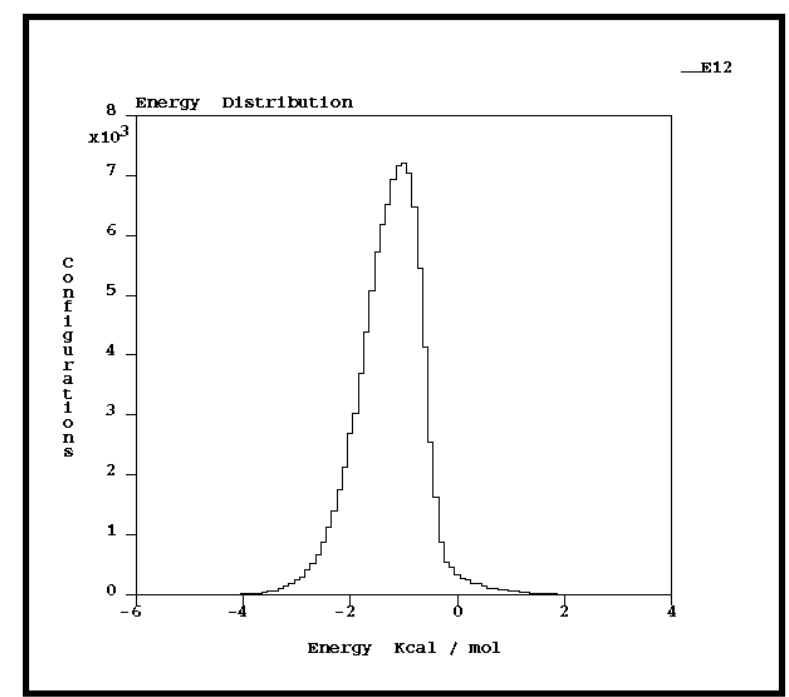

Figure 7.1.5.5. Binding energy distributions for 100,000 pairs of surfactant/cosurfactant. Surfactant is HBDM in the trans conformation, cosurfactant is n-octanol.

The 4 most binding configurations are displayed in Figure 7.1.5.6 for the gauche and in Figure 7.1.5.7 for the trans HBDM conformations. The most binding conformation $\left(E_{\text {bind }}=5.1 \mathrm{Kcal} / \mathrm{mol}\right)$ was found to be the trans conformation shown in the lower left corner of Figure 7.1.5.7.

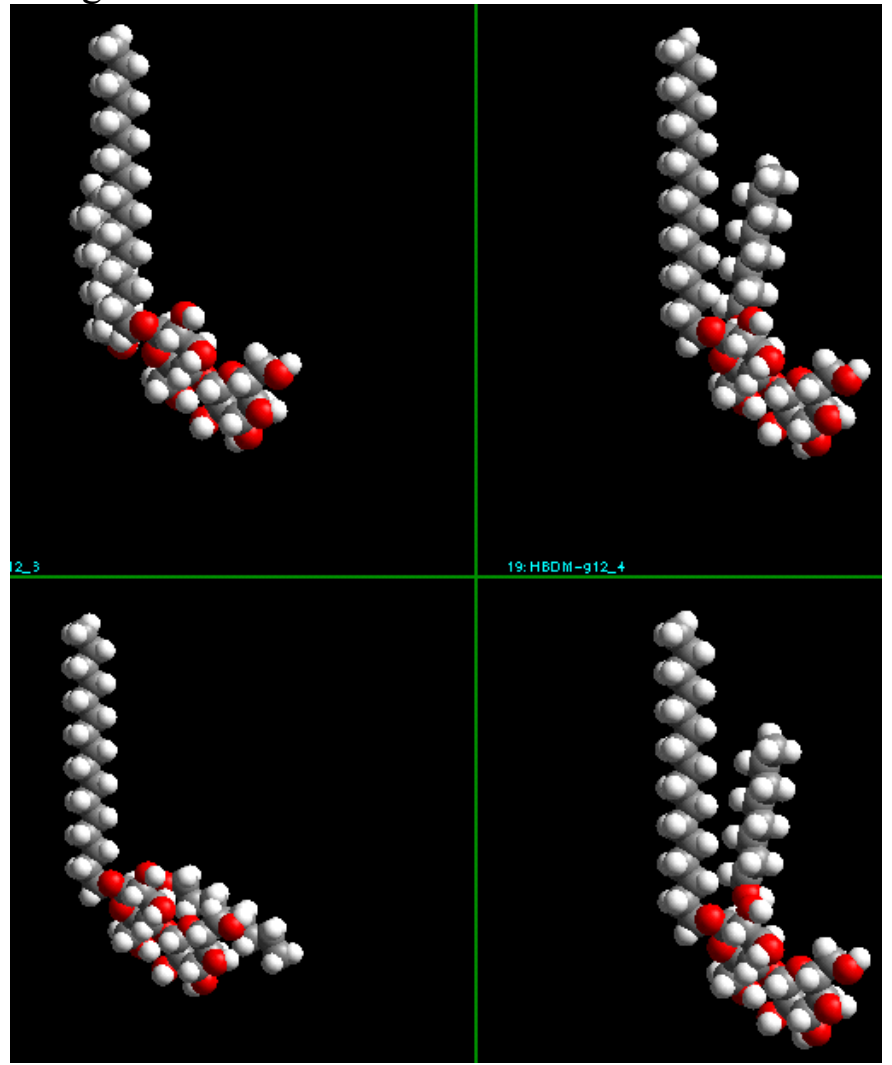

Figure 7.1.5.6. Four most binding conformations for pairs of surfactant/cosurfactant. 
HBDM is in the gauche conformation; cosurfactant is n-octanol. Each ball is one atom.

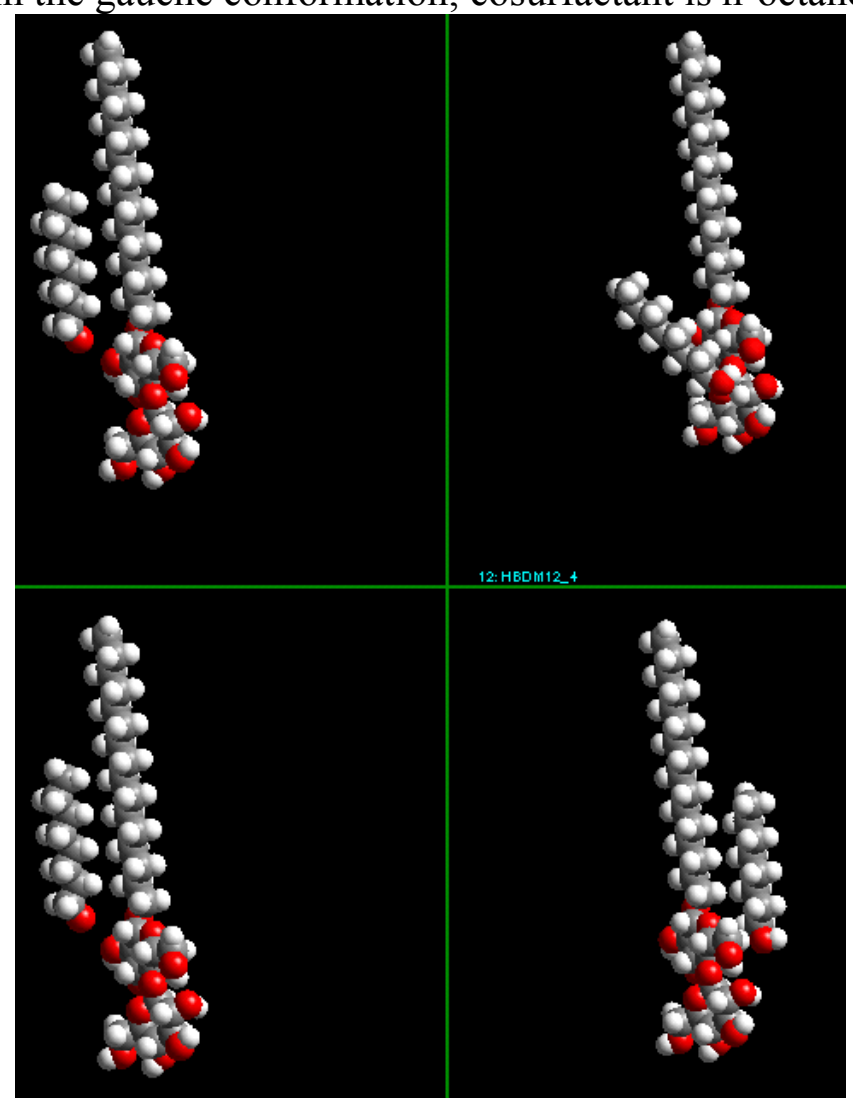

Figure 7.1.5.7. Four most binding conformations for pairs of surfactant/cosurfactant. Surfactant is HBDM in the trans conformation. cosurfactant is n-octanol. Each ball is one atom.

A 6x6 SAM of n-octanol was prepared and minimized to less than $0.1 \mathrm{kcal} / \mathrm{mol}-\mathrm{A}$ (rootmean-square) rms force. One of the n-octanol molecules was then deleted and a surfactant molecule in the trans conformation was inserted in its place. This yields a molar ratio of 1:35 surfactant:n-octanol, close to the experimental ratio of 1:39 that yielded low IFT in our experiments. The structure was then minimized and 500 picoseconds of NPT molecular dynamics were run in the LAMMPS software ( S. J. Plimpton, R. Pollock, M. Stevens, "Particle-Mesh Ewald and rRESPA for Parallel Molecular Dynamics Simulations", in Proc of the Eighth SIAM Conference on Parallel Processing for Scientific Computing, Minneapolis, MN, March 1997.

S. J. Plimpton, "Fast Parallel Algorithms for Short-Range Molecular Dynamics", J Comp Phys, 117, 1-19 (1995). )

The overall oil/surfactant/cosurfactant/water model is shown in Figure 7.1.5.8a. The noctanol phase developed into a hexagonal closed packed structure within 20 ps (Figure 7.1.5.8b). 


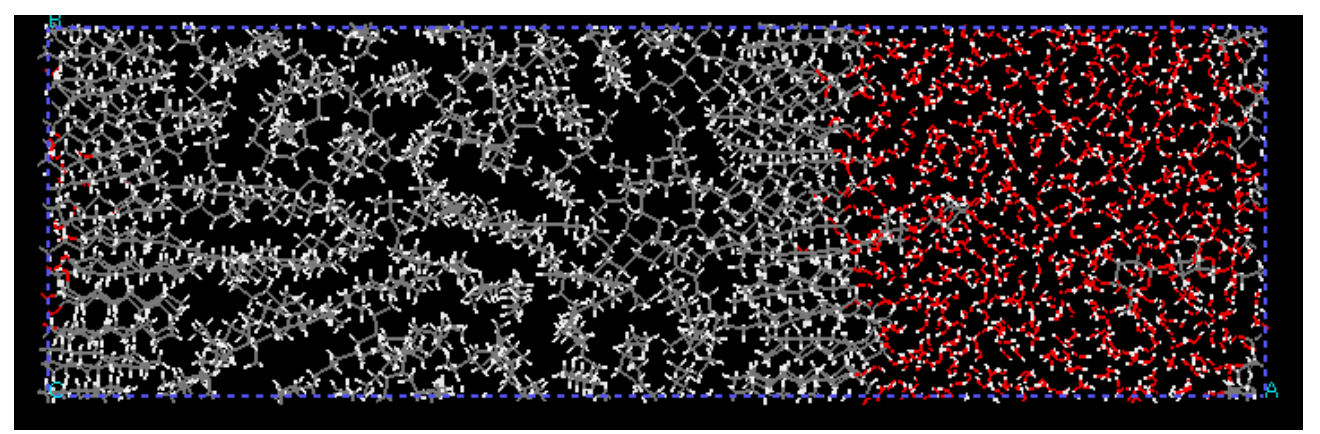

(a)

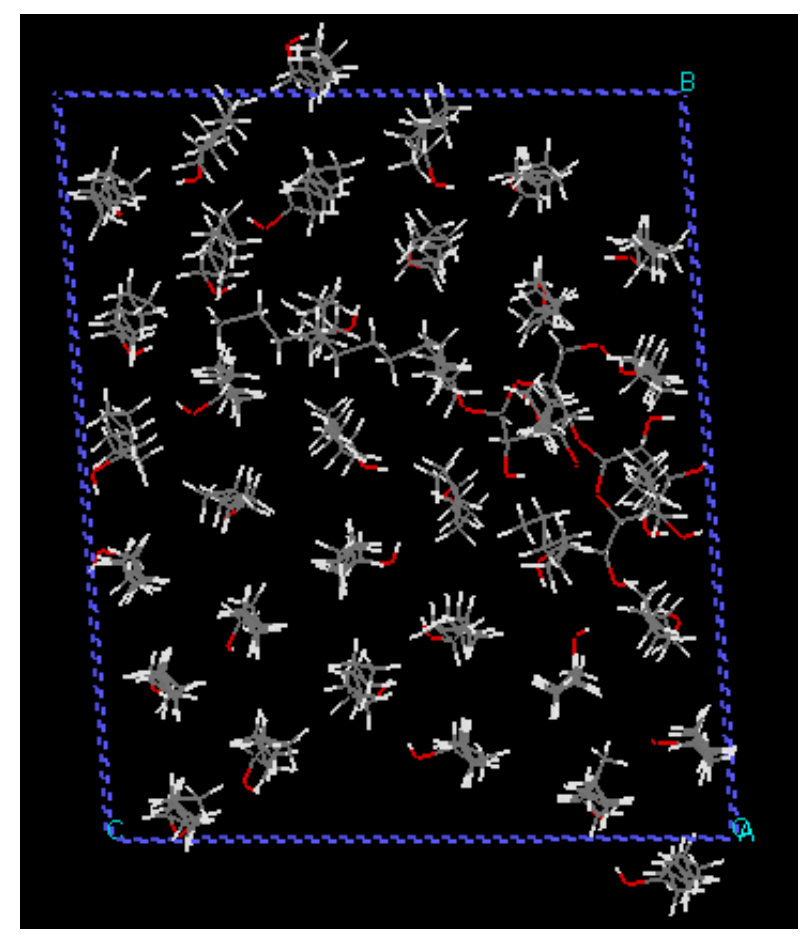

(b)

Figure 7.1.5.8. a) Octane/Octanol/HBDM/water interface model. Left side is n-octane, Middle is interfacial region, Right size is bulk aqueous phase.

b) Top view of the n-octanol layer. Shows only n-octanol molecules to simplify the picture.

The resulting HBDM structure after $500 \mathrm{ps}$ is shown in Figure 7.1.5.9. The HBDM structure oscillates between an s-shaped and an all trans conformation during the dynamics. The locations of the three segments (sugar, $\mathrm{c} 8+\mathrm{c} 8$ ) follows that of the three phases (water/n-octanol/octane) at all times. It is perhaps this feature that makes this surfactant such a good performer at such a low concentrations in the presence of a cosurfactant such as n-octanol. 


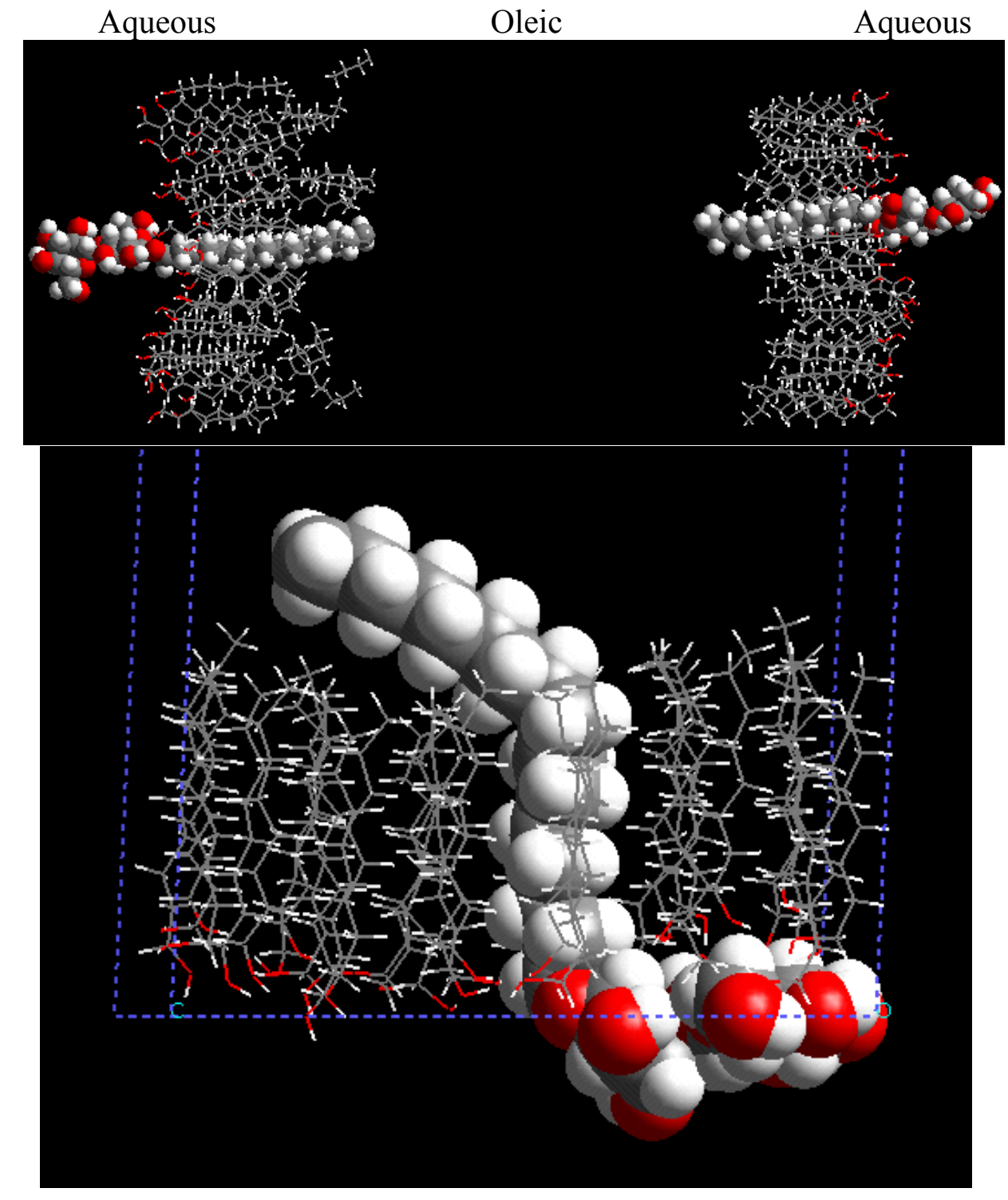

Figure 7.1.5.9. Octane/water interfacial structure of HBDM after 500 ps of NPT molecular dynamics at $300 \mathrm{~K}$. Both, all-trans and S-shaped molecular conformations were observed.

The upper diagram in Figure 7.1.5.9 shows a single molecule of HBDM surrounded by noctanol molecules penetrating into the oleic phase (oil molecules not shown). The lower diagram is a closer view of the interfacial region where a HBDM penetrates upward through the n-octanol into the oleic phase (n-octanol molecules not shown).

A top and bottom view of the surfactant/cosurfactant layer is shown in Figure 7.1.5.10. 

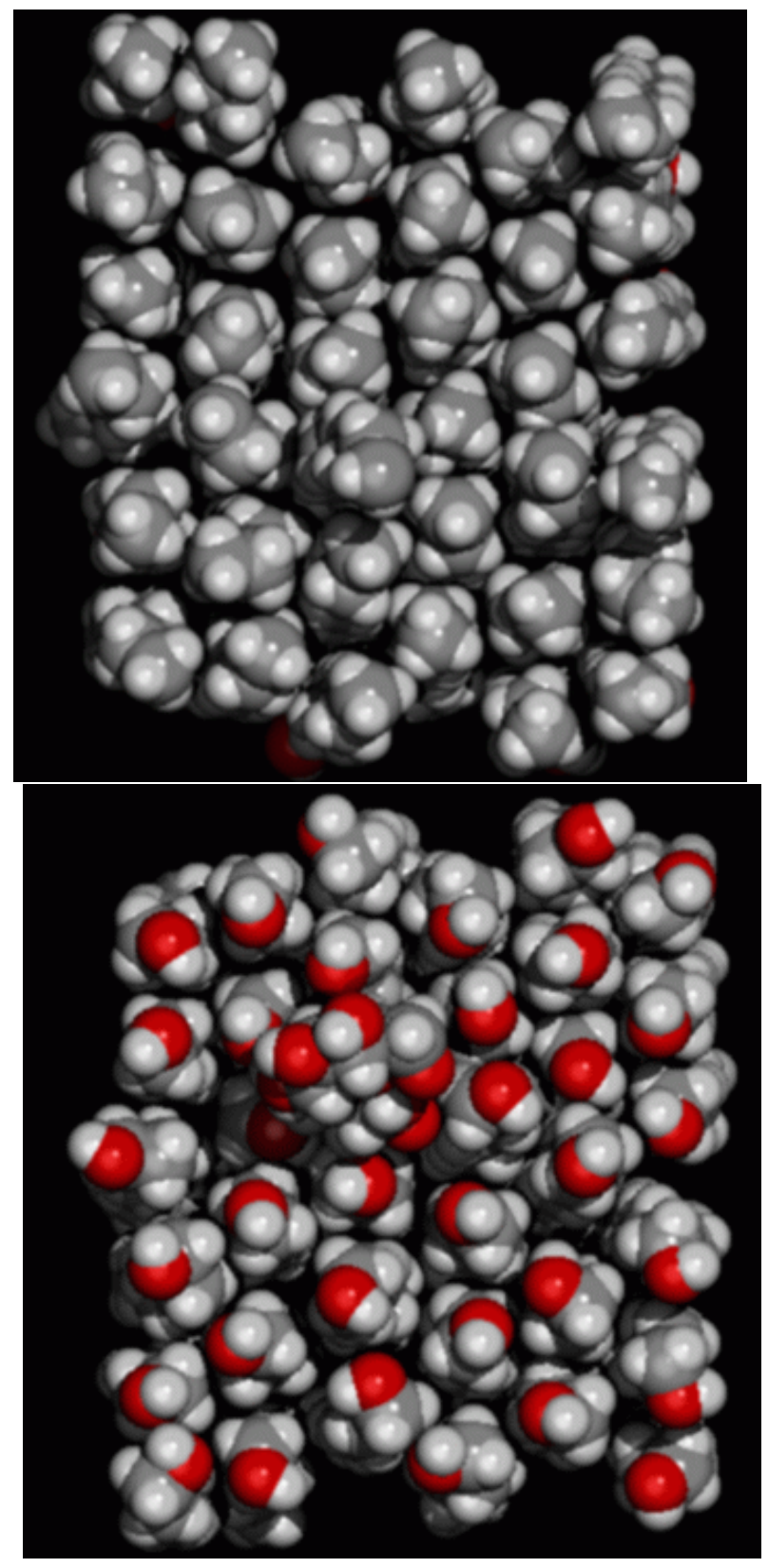

Figure 7.1.5.10. Top and bottom views of Octane/water interfacial structure of HBDM/n-octanol. Top view is from the oil side, so hydrocarbon chain appears. The bottom view from the aqueous phase shows the hydroxyl group of the n-octanol dominates.

The calculated IFT for this HBDM/n-octanol/n-octane system (other constraints are no salt and at $25 \mathrm{C}$ temperature) is $21 \mathrm{dyne} / \mathrm{cm}$. The experimental IFT value measured is lower, 0.5 dyne $/ \mathrm{cm}$. 
Table 7.1.5.1. Properties of n-Decyl $\beta$-D-maltoside

\begin{tabular}{|c|c|c|c|}
\hline Property & $\begin{array}{l}\text { Calculated } \\
\text { Value }\end{array}$ & Condition & Note \\
\hline$\overline{\text { Bioconc. Factor }}$ & $\overline{10.1}$ & $\overline{\mathrm{pH} 1}$ & $\overline{(1) \mathrm{A}} \mathrm{CD}$ \\
\hline Bioconc. Factor & 10.1 & $\mathrm{pH} 4$ & (1) $\mathrm{ACD}$ \\
\hline Bioconc. Factor & 10.1 & $\mathrm{pH} 7$ & (1) $\mathrm{ACD}$ \\
\hline Bioconc. Factor & 10.1 & $\mathrm{pH} 8$ & (1) $\mathrm{ACD}$ \\
\hline Bioconc. Factor & 10.1 & $\mathrm{pH} 10$ & (1) ACD \\
\hline Boiling Point & $689.4 \pm 55.0^{\circ} \mathrm{C}$ & $\begin{array}{l}\text { Press: } 760.0 \\
\text { Torr }\end{array}$ & (1) $\mathrm{ACD}$ \\
\hline Enthalpy of Vap. & \multicolumn{3}{|c|}{$115.56 \pm 6.0 \mathrm{~kJ} / \mathrm{mol}(1) \mathrm{ACD}$} \\
\hline Flash Point & \multicolumn{2}{|c|}{$370.8 \pm 56.7^{\circ} \mathrm{C}$} & (1) $\mathrm{ACD}$ \\
\hline H acceptors & \multicolumn{2}{|l|}{11} & (1) $\mathrm{ACD}$ \\
\hline H donors & \multicolumn{2}{|l|}{7} & (1) $\mathrm{ACD}$ \\
\hline Koc & 182 & $\mathrm{pH} 1$ & (1) $\mathrm{ACD}$ \\
\hline Koc & 182 & $\mathrm{pH} 4$ & (1) ACD \\
\hline Koc & 182 & $\mathrm{pH} 7$ & (1) $\mathrm{ACD}$ \\
\hline Koc & 182 & $\mathrm{pH} 8$ & (1) $\mathrm{ACD}$ \\
\hline Koc & 181 & $\mathrm{pH} 10$ & (1) $\mathrm{ACD}$ \\
\hline $\log D$ & 1.62 & $\mathrm{pH} 1$ & (1) $\mathrm{ACD}$ \\
\hline $\log D$ & 1.62 & $\mathrm{pH} 4$ & (1) ACD \\
\hline $\log D$ & 1.62 & $\mathrm{pH} 7$ & (1) $\mathrm{ACD}$ \\
\hline $\log D$ & 1.62 & $\mathrm{pH} 8$ & (1) $\mathrm{ACD}$ \\
\hline $\log D$ & 1.62 & $\mathrm{pH} 10$ & (1) ACD \\
\hline $\log P$ & $1.625 \pm 0.492$ & & (1) $\mathrm{ACD}$ \\
\hline Molar Solubility & Sparingly Solub & $\mathrm{pH} 1$ & (1) $\mathrm{ACD}$ \\
\hline Molar Solubility & Sparingly Solub & $\mathrm{pH} 4$ & (1) $\mathrm{ACD}$ \\
\hline Molar Solubility & Sparingly Solub & $\mathrm{pH} 7$ & (1) $\mathrm{ACD}$ \\
\hline Molar Solubility & Sparingly Solub & $\mathrm{pH} 8$ & (1) ACD \\
\hline Molar Solubility & Sparingly Solub & $\mathrm{pH} 10$ & (1) $\mathrm{ACD}$ \\
\hline Molecular Weight & 482.56 & & (1) $\mathrm{ACD}$ \\
\hline pKa & $12.82 \pm 0.70$ & Most Acidic & (1) $\mathrm{ACD}$ \\
\hline Vapor Pressure & 5.36E-22 Torr & Temp: $25.0^{\circ} \mathrm{C}$ & (1) $\mathrm{ACD}$ \\
\hline
\end{tabular}

Notes:

(1) Calculated using Advanced Chemistry Development (ACD) Software Solaris V4.67 


\subsubsection{Molecular Modeling Study with a Pure APG Example (OBDM) -- Effect of alkly chain length of surfactant}

Next, we carried out computer simulations of n-Octyl-beta-D-Maltopyranoside (OBDM), again with n-octanol as a cosurfactant at a molar ratio surfactant:cosurfactant of 1:30. This is to examine the effects of the shorter alkyl chain length (C8) of the surfactant with the simulations above with the hexadecyl case (hexadecyl-beta-D-maltopyranoside, HBDM, C16 alkyl chain.) The experimental data presented later indicate that the C8 chain length is too short to generate low IFT with this system.

\section{Octane/Water Interfacial Structure of n-Octyl-beta-D-Maltopyranoside Surfactant and n-Octanol Formulation}

The structure of OBDM in the 1:30 formulation with n-octanol after 3.25 nanosecond NVT (constant volume) simulation at $300 \mathrm{~K}$ is shown in Figure 7.1.5.11.

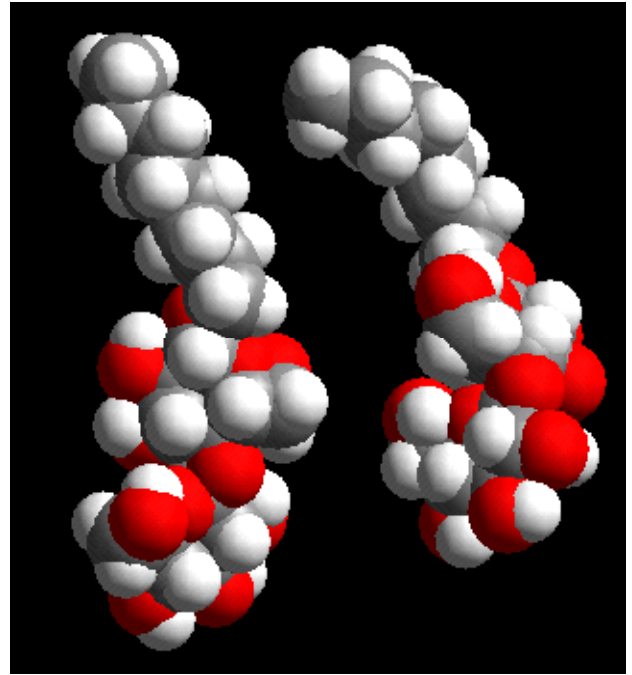

(a)

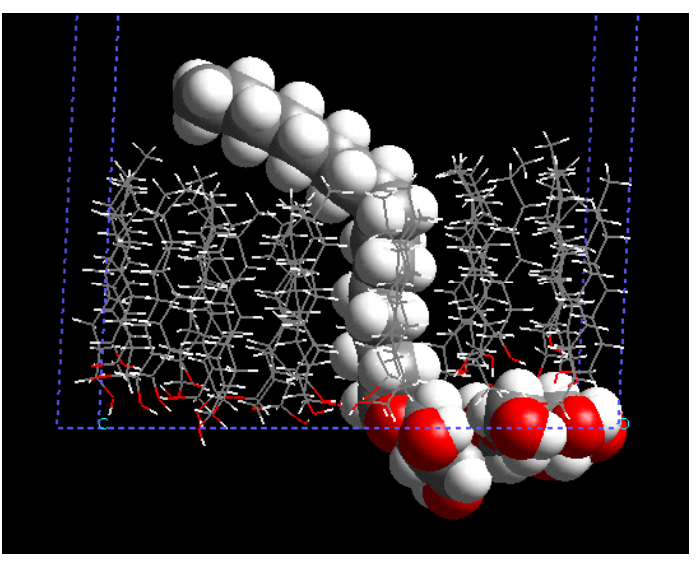

(b)

Figure 7.1.5.11. (a) Octane/water interfacial structure of OBDM after 3250 picoseconds of NVT molecular dynamics (only OBDM surfactant structure shown). Formulation contains n-octanol:OBDM in a 30:1 molar ratio.

(b) Octane/water interfacial structure of HBDM after 500 ps of NPT molecular dynamics at $300 \mathrm{~K}$ (same as lower portion Figure 7.1.5.9). The diagram shows a HBDM molecule penetrating upward through the n-octanol into the oleic phase (n-octanol molecules not shown).

Both, all-trans and S-shaped molecular conformations were observed in HBDM case while mostly all-trans molecular conformations are observed for OBDM.

The structure of OBDM should be compared with the structure of its hexadecyl, C16 cousin in Figure 7.1.5.11(b). In contrast to n-hexadecyl, the n-octyl surfactant adopts a more trans-like conformation (stretched out) than the longer n-hexadecyl surfactant. OBDM then lacks the ability to lock with the oil phase (n-octane) the way HBDM is 
capable of via the bent alkyl chain. This may help explain the lower IFT values observed with HBDM experimentally. The ability to reside at the interface, and any factors such as favorable conformations, greatly affect the interfacial tension. After certain alkyl chain-lengths, other factors, such as high oil solubility, become more important. Thus, for a given oil phase, such as n-octane, there is an optimal chain length, longer than $\mathrm{C} 8$ but less than $\mathrm{C} 20$, that may favorably affect IFT and provide low values in conjunction with a co-surfactant such as n-octanol.

\section{IFT Predictions of n-octanol:n-Octyl-beta-maltopyranose (OBDM) (30:1)}

We carried out NVT molecular dynamics simulations on this surfactant. The full structure of OBDM and the unit cell used in the simulation are shown in Figure 7.1.5.12.
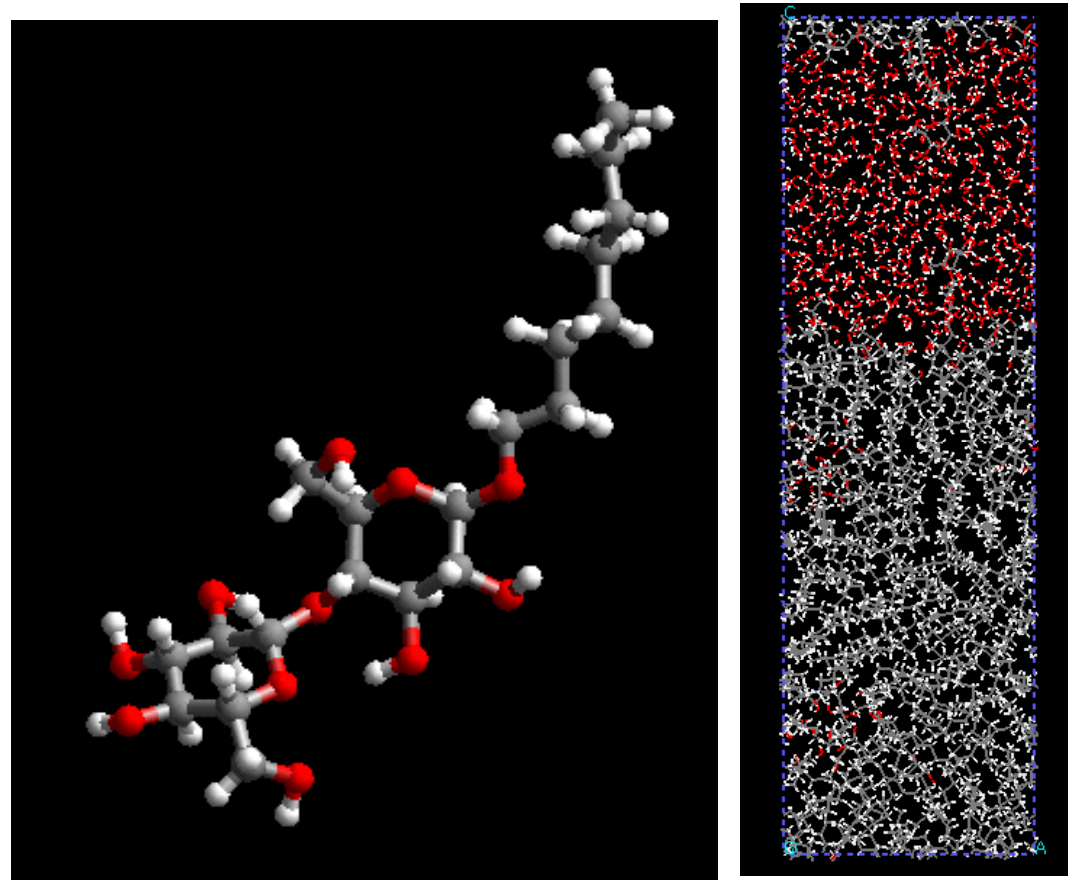

Figure 7.1.5.12. Initial structure of a single OBDM molecule shown on the left. On the right is shown the unit cell used in MD simulations of n-octanol:OBDM calculations. Aqueous phase overlays the oleic phase.

After 3.25 nanoseconds simulations the water/surfactant/oil interfaces remain well differentiated. A few water molecules (lower left corner of unit cell) have become immersed in the oil phase, and no molecules of n-octanol molecule have become embedded in the water phase. Arguably the system has not yet reached complete equilibrium even after such long computer simulation (typically these simulations take 24 weeks to complete on a $2.5 \mathrm{GHz}$ Linux processor). However, instabilities in the interface can be observed when present in as little as 0.1 nanoseconds (100 picoseconds). We conclude that this interface is quite stable.

Results of the calculated IFT for n-octanol:OBDM (30:1 molar ratio) with n-octane as the modeled oil phase are shown in Figure 7.1.5.13. 
(a)

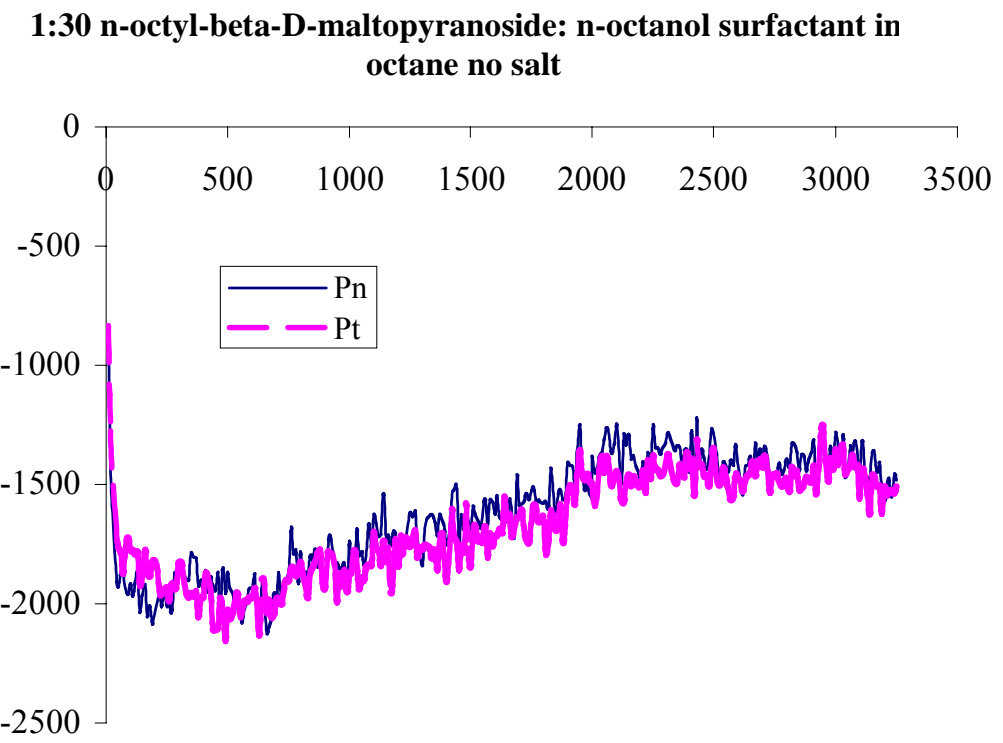

Time (ps)

(b)

\section{1:30 n-octyl-beta-D-maltopyranoside: n-octanol surfactant in octane no salt $\gamma=35$ dynes $/ \mathrm{cm}$}

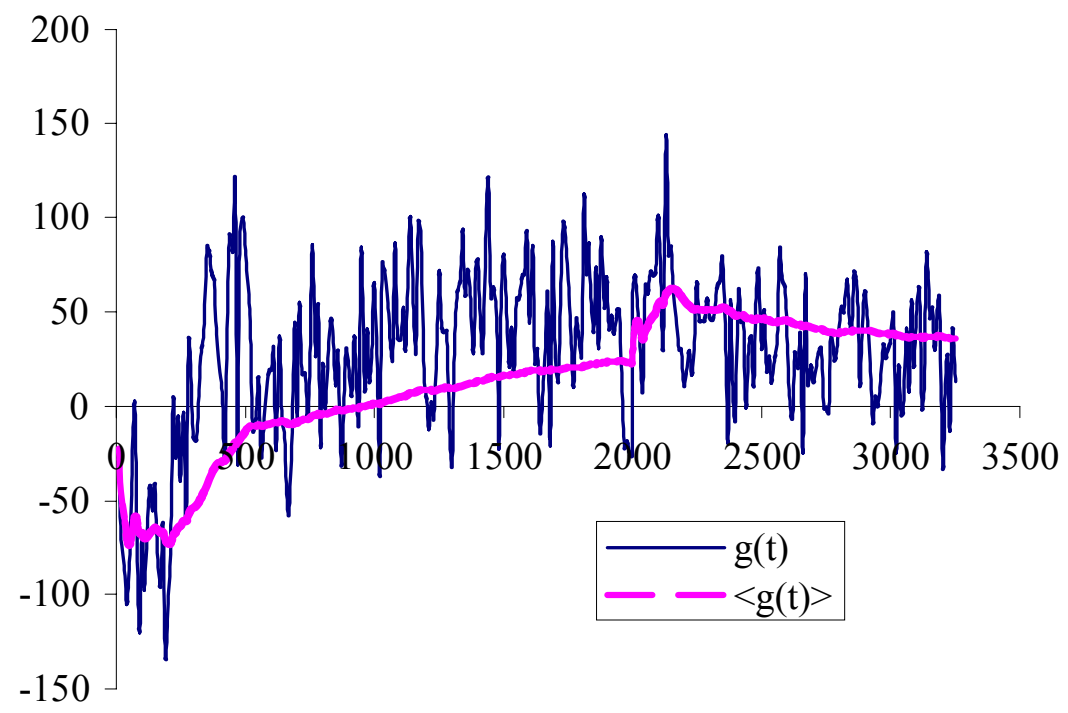

Time (ps)

Figure 7.1.5.13. (a) Parallel and transverse components of the pressure tensor in OBDM 3.25 ns simulation at room temperature. (b) Calculated instantaneous (blue) and cumulative (pink) IFT values (left scale is IFT in dyne/cm). 
The calculated IFT value is 35 dynes/cm. The previous calculated values for the nhexadecyl compound (HBDM) were 21 dynes $/ \mathrm{cm}$. Although the absolute values are not obtained with these MD simulations the relative ordering of the values compare well with the experimentally measured value of 8.5 and 0.5 dynes $/ \mathrm{cm}$, respectively.

Significant aspects of these calculations include:

- The modeling results are consistent with the experimental observation that the lower IFT occurs with the longer alkyl chain version of the pure APG type surfactant.

- The MD simulations accounted for the presence of an alcohol cosurfactant.

- From the atomistic-level picture of the surfactant conformations at the interface, we speculate that their stabilizing the n-octanol at the oil interface aids in reducing IFT. Further, the ability of the longer alkyl chain OBDM to penetrate into the oil phase reduces the IFT even further.

\subsubsection{Molecular Modeling Study of Two Model Alkyl Polyglycosides (APG) -- Small Isomeric Difference in Structure Creates Large Change in Behavior}

One other detailed MD simulation investigation centered about two pure APG surfactants with almost the same structure, but with the intriguing observation that they have vastly differently CMC (critical micelle concentration) values.

Specifically, consider the disaccharide glucoside type surfactants: n-Octyl- $\alpha-D-$ Glucoside and n-Octyl- $\beta$-D-Glucoside

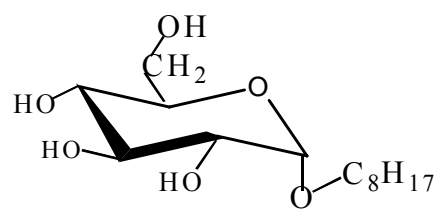

n-Octyl- $\alpha$-D-Glucoside FW=294.4 CMC 0.01 mM

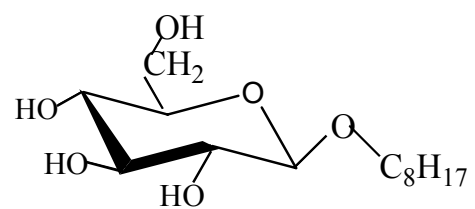

n-Octyl- $\beta$-D-Glucoside FW=294.4 CMC 20 mM

Figure 7.1.6.1. Molecular structure of two subject glucosides

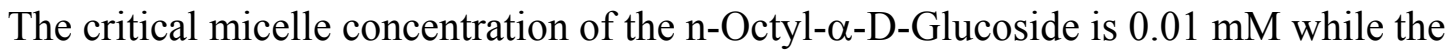
value for $n-O c t y l-\beta-D-G l u c o s i d e$ is $20 \mathrm{mM}$. This is a difference of a factor of 2000 due to a single change in internal hydrogen bonding caused by the anomeric change, all other features of the surfactant being the same. The $\alpha$ anomer has a significantly higher 
tendency towards micellization than the $\beta$.

It is important to understand how it is that a subtle change (say a change in $\alpha$ to $\beta$ linkage in a disaccharide glucoside type surfactant, so called "anomeric isomers"), can produce such a marked difference in behavior. We hypothesize the following:

- Changes in internal covalent structure of the head group may have significant effects on the water solubility of the surfactant head group

- Different patterns of internal hydrogen bonds might be observed

- These changes may affect the IFT as well as the CMC of these surfactants, most of the effect due to changes in solubility.

To gain some insight into these ideas we conducted quantum mechanical solvation calculations of these two surfactants. The large difference in CMC between these isomers was investigated in regards to the relative predicted solubility free energies in water for these compounds. We conducted Quantum Mechanical calculations (B3LYP DFT 6-31g**) solvation calculations for these two compounds. A full geometry optimization was performed in a high dielectric medium $(\mathrm{d}=80)$ representing water. The structures significant differences are shown in Figure 18. The $\alpha$ glucoside has a more bent structure than the $\beta$ glucoside, which remains more open in solution. The $\alpha$ glucoside hydrogen bonds internally at the 6-4 position and at the 3-2 position while the $\beta$ glucoside hydrogen bonds at the 1 '-2 position ( 1 '=alkyl ether tail oxygen) and at 34 ring positions. The net effect is as follows:

1. The 6-4 internal hydrogen bond in the $\alpha$ glucoside anomer is responsible for tying the $\mathrm{CH} 2 \mathrm{OH}$ group

2. The $\mathrm{CH} 2 \mathrm{OH}$ group is free to be solvated by water in the $\beta$ glucoside anomer

3. The extra solvation of the $\mathrm{CH} 2 \mathrm{OH}$ group leads to a more open structure and to a more favorable solvation free energy $(-14.3 \mathrm{Kcal} / \mathrm{mol})$ for the $\beta$ glucoside anomer

4. These results have the following implications:

a. the $\alpha$ glucoside anomer has a much lower critical micelle concentration (CMC) because is significantly less soluble in water

b. the $\beta$ glucoside anomer is more water-soluble and thus requires higher concentrations to reach its CMC.

c. Provided we choose concentrations above the $\mathrm{CMC}$ of these two surfactants we expect the $\alpha$ glucoside anomer to have a lower IFT by virtue of its lower water solubility.

d. Co-solvents that "push" the surfactant out of aqueous solution can enhance the IFT of the $\beta$ glucoside anomer more than the $\alpha$ glucoside anomer.

Note in the figure below the alpha anomer $(-3 \mathrm{kcal} / \mathrm{mol})$ is not as soluble and the beta $(-14 \mathrm{kcal} / \mathrm{mol})$. This helps explain the significant difference in the CMC fortwo very similar structures.

These observations are consistent with the measured results of an IFT of 6.6 versus 9.4 dyne $/ \mathrm{cm}$ for the $\alpha$ versus the $\beta$ glucoside anomer $\left(25^{\circ} \mathrm{C}, .3 \mathrm{wt} \%\right.$ surfactant, fresh water). 


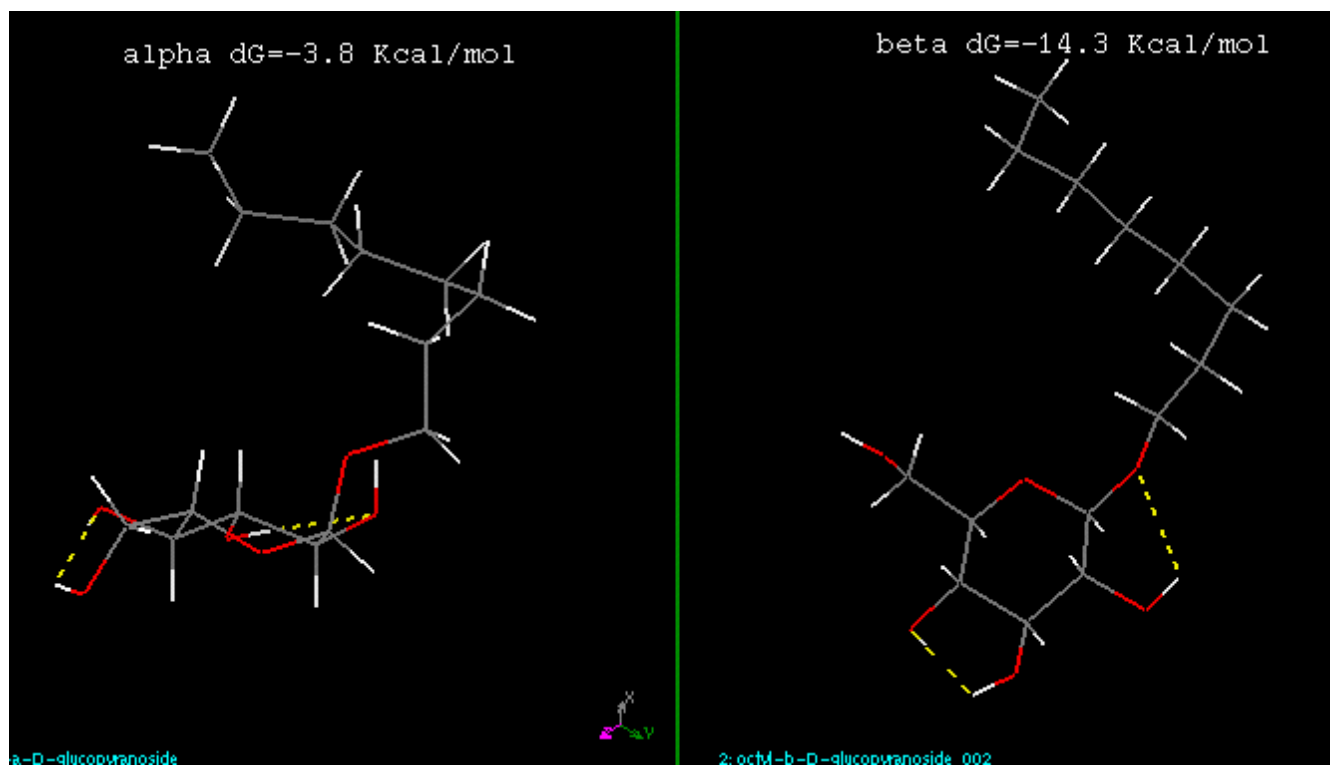

Figure 7.1.6.2. Changes in molecular structure of n-Octyl-D-Glucoside. The $\alpha$-glucoside anomer and the n-Octyl- $\beta$-D-Glucoside anomeric structures were calculated in aqueous solution from full quantum mechanical geometry optimizations.

\subsubsection{Study of IFT of alkyl propoxylated sulfate surfactants via MD}

\subsubsection{Motivation for theoretical study of alkly propoxy surfactants}

We carried out the full atomistic simulations of water/oil interface mediated by alkyl propoxlated sulfate surfactants. The molecular structure used in our simulation is

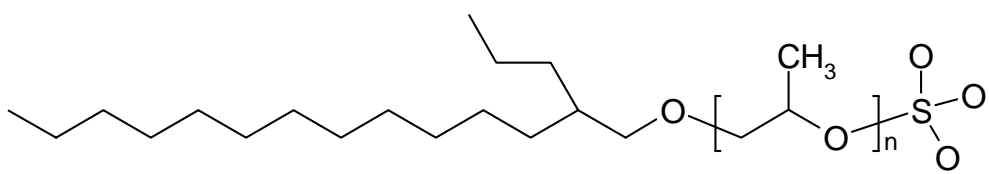

Figure 7.1.7.1. Structure of a alkyl propoxylated sulfate surfactant used in MD simulations.

The characteristic feature of this surfactant's molecular architecture is that it integrates an ionic surfactant (sulfonic anionic surfactant with branched alkyl tail) and a non-ionic surfactant (propylene ether with branched alkyl tail) together. As mentioned in the summary of the Literature Review, these surfactants are of interest because they are a newer type of material, and they have been shown be to be efficient in mobilizing and recovering hydrocarbons that are spilled near the surface. Their use in so-called SEAR (Surfactant Enhanced Aquifer Remediation -- injecting a surfactant solution to recover spilled hydrocarbons) is roughly the same process as used for IOR in oil reservoirs.

The laboratory results (IFT and oil displacement tests) in this project and summarized in this report confirm that this class of surfactant has potential as an effective candidate for 
IOR. The theoretical MD simulations investigate the expected trends in IFT with changes in 1) the number of propoxy groups, 2) temperature, and 3) surfactant concentration. All of the MD simulations used fresh water (no salinity).

\subsubsection{Effect of number of propylene oxide units on IFT}

The goal of this simulation is to investigate the effect of the number of propylene oxide units, $n$, in the surfactant molecule on the interfacial tension (IFT) between water and oil which is decane in this study). We expect to impact overall surfactant solubility and its interfacial activity.

The system in our simulation was constructed as shown in Figure 7.1.7.2.

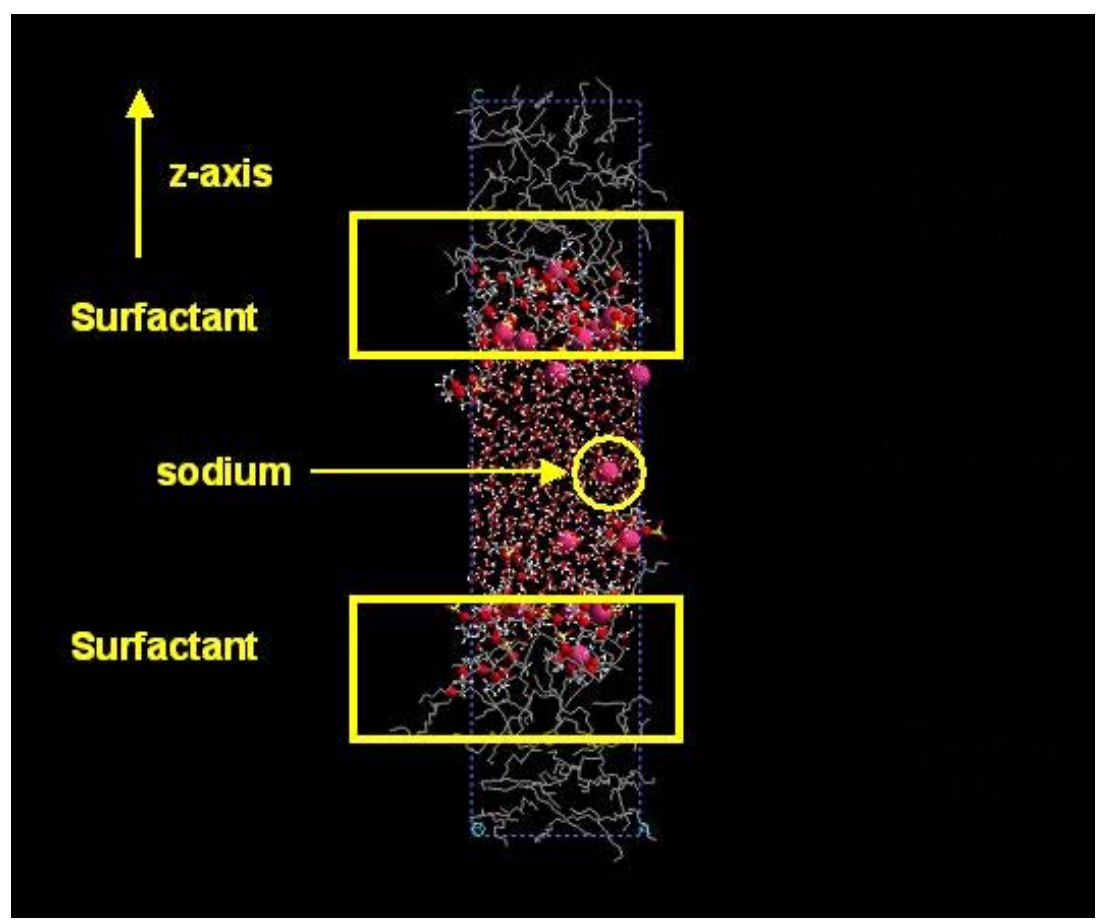

Figure 7.1.7.2. A typical structure of the alkyl propoxy sulfate systems.

Aqueous surfactant layer between two oil layers.

Each system has the same composition in which 600 water molecules, 60 decane (C10) molecules and 16 surfactant molecules were simulated using F3C water FF (Force Field), the united atom FF, and the Dreiding FF, respectively. The number of propylene oxide groups we considered was 1 (ppo_1), 3 (ppo_3), 5 (ppo_5) and 8 (ppo_8). To equilibrate the system, we used NPT molecular dynamics (MD) simulation at $300 \mathrm{~K}$ under $1 \mathrm{~atm}$ for 400 ps. After equilibration, subsequent NPT MD simulation was performed to collect data for IFT calculation. Figure 7.1.7.3 shows the side view of the final structure for each system. In addition, from the trajectories of MD simulations, we obtained the time averaged density profile along $\mathrm{z}$-axis as shown in Figure 7.1.7.4. From these structures and density profiles, it is observed that all the alkyl propylene sulfate molecules remain stably at the interface between water and oil. 


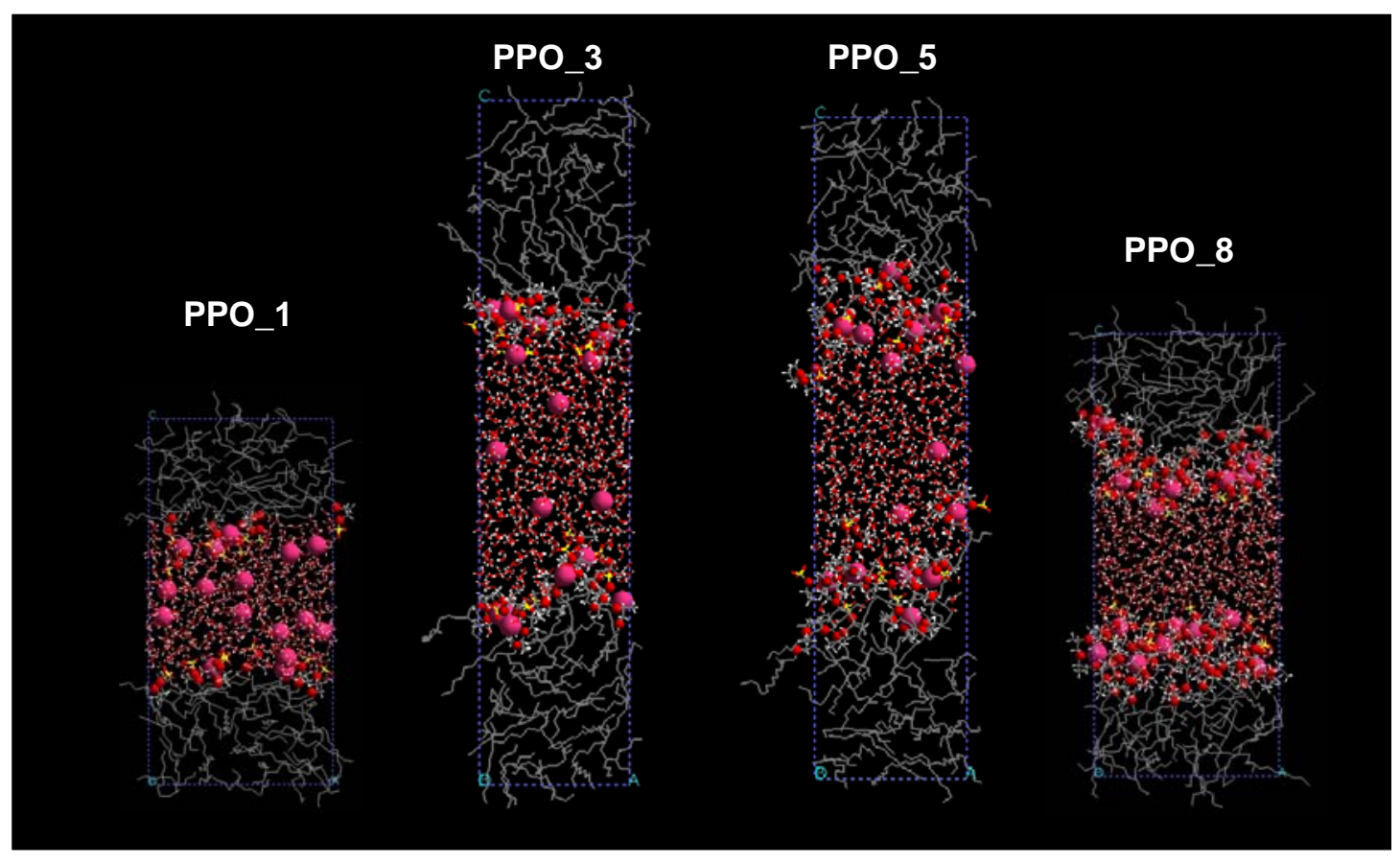

Figure 7.1.7.3. Final structure for each alkyl propoxylated sulfate system. Aqueous surfactant layer between two oil layers.

A noteworthy point in Figure 7.1.7.3 at the densities of water phase $\left(\sim 1.0 \mathrm{~g} / \mathrm{cm}^{3}\right)$ and oil phase $\left(\sim 0.7 \mathrm{~g} / \mathrm{cm}^{3}\right)$ in the systems including surfactant PPO_3 and PPO_5 have the similar value of pure bulk phase of each material at the same temperature and pressure condition. On the other hand, the density of water and oil phase in the system including PPO_1 and PPO_8 shows some offset from the value of pure phase. In the case of PPO_1, the density of oil phase is slightly larger than that of pure oil, and in the case of PPO_8, water has a smaller density and the oil has a larger density.

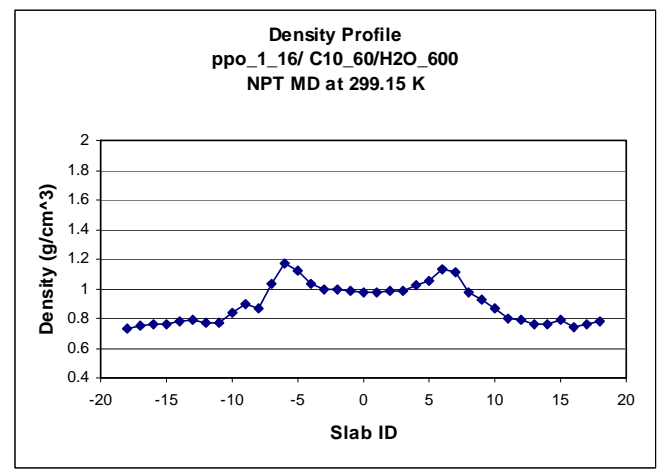

(a) PPO_1

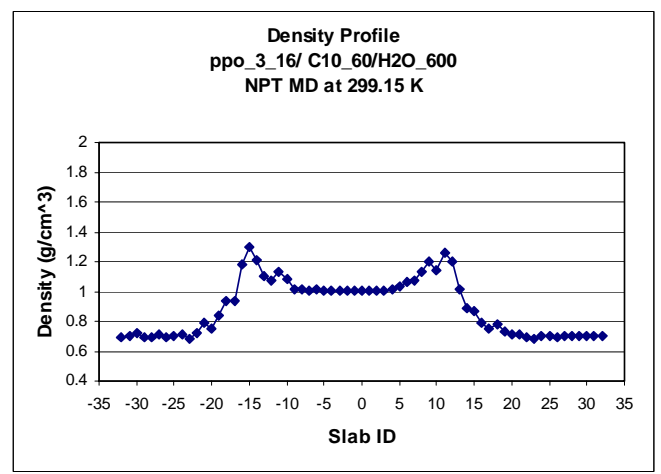

(b) PPO_3 


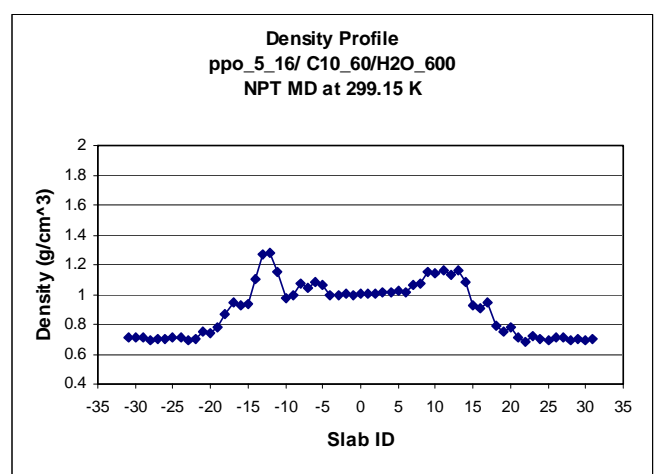

(c) PPO_5

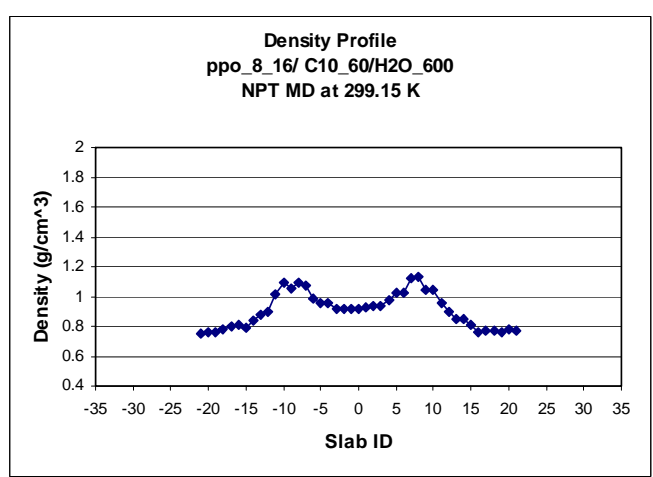

(d) $\mathrm{PPO} \_8$

Figure 7.1.7.4. Density profile for each system. The slab ID is scaled $\mathrm{z}$ coordinate. The aqueous phase is in the middle, between oil phases sitting on both ends. The peaks in these density profiles come from the surfactant.

In addition, the difference among the various surfactants is more clearly observed in the equilibrated system as a whole in Table 7.1.7.1.

Table 7.1.7.1. Density and dimensions of whole system for alkyl propoxylated sulfates

\begin{tabular}{|c|c|c|c|c|c|c|c|c|c|c|}
\hline \multirow{2}{*}{$\mathbf{N}$} & \multicolumn{2}{|c|}{ density } & \multicolumn{2}{|c|}{$\mathbf{a}$} & \multicolumn{2}{|c|}{ b } & \multicolumn{2}{|c|}{ C } & \multicolumn{2}{|c|}{ cross-sectional area } \\
\hline & avg & std & avg & std & avg & std & avg & std & avg & std \\
\hline 1 & 0.8977 & 0.0008 & 26.94 & 0.01 & 32.92 & 0.01 & 54.23 & 0.01 & 886.8648 & 0.0001 \\
\hline 3 & 0.8968 & 0.0015 & 20.70 & 0.10 & 25.88 & 0.13 & 96.02 & 0.19 & 535.7678 & 0.0137 \\
\hline 5 & 0.9027 & 0.0012 & 21.53 & 0.01 & 26.91 & 0.01 & 94.40 & 0.04 & 579.3339 & 0.0001 \\
\hline 8 & 0.9083 & 0.0011 & 26.99 & 0.01 & 33.74 & 0.01 & 65.29 & 0.03 & 910.5655 & 0.0002 \\
\hline
\end{tabular}

In this table, the total densities of equilibrated system are more or less similar to each other, but their dimensions are quite different. Compared to PPO_1 and PPO_8, surfactant PPO_3 and PPO_5 induced smaller cross-sectional area, indicating the area occupied by individual surfactant molecule of PPO_3 and PPO_5 is 38\% smaller than PPO_1 and PPO_8.

The stress profiles and tension profile along z-axis are shown in Figure 7.1.7.5. All the profiles seem to have the similar feature that the profile is symmetric and that in each phase of water and oil, the value of stress components $\left(\mathrm{P}_{t}\right.$ for transverse direction and $\mathrm{P}_{\mathrm{n}}$ for normal direction) are almost same. As a result, the non-zero value of interfacial tension appears at the interface occupied by the surfactant molecules. The IFT values were calculated as shown in Figure 7.1.7.6. Through our simulations, it is predicted that the IFT of PPO_3 and PPO_5 are smaller than the others for water/decane interface, which is thought to be consistent with the results shown above.

The following observations are pertinent:

First, the surfactants PPO_3 and PPO_5 do not affect the water and oil phase as much as the others (PPO_1 and PPO_8) and therefore, the each phase is expected to have their own thermodynamic properties. Second, the interfacial area occupied by individual surfactant 
molecule is smaller in the case of PPO_3 and PPO_5 than PPO_1 and PPO_8, which means surfactants PPO_3 and PPO_5 efficiently reduce the interfacial area and thereby contribute to a smaller IFT value in comparison with the PPO_1 and PPO_8.
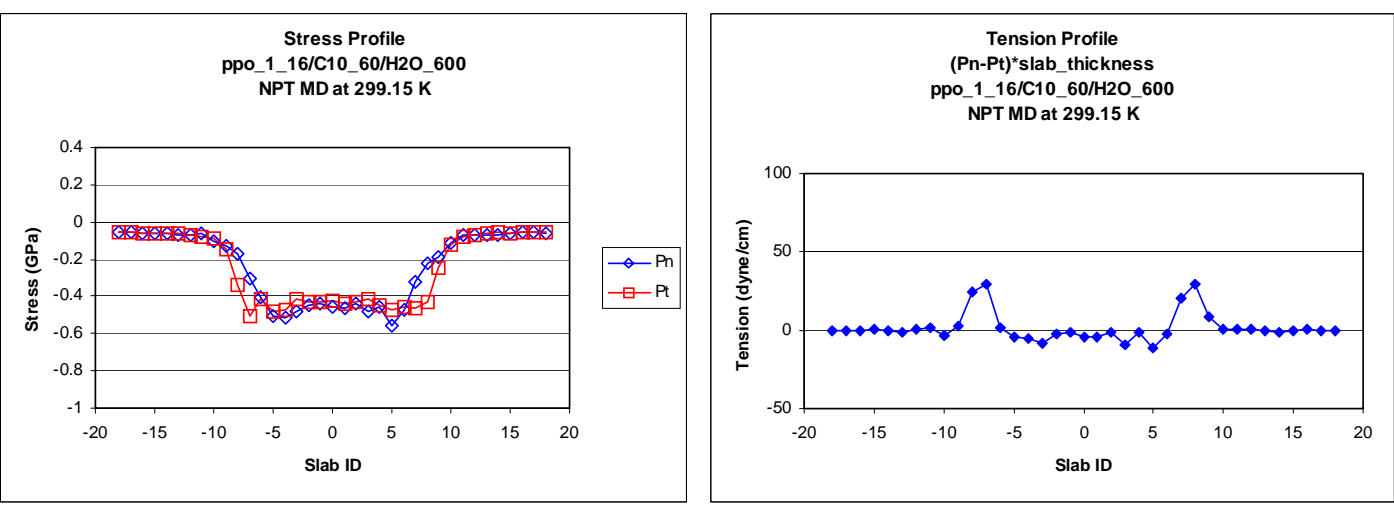

(a) $\mathrm{PPO}_{-} 1$
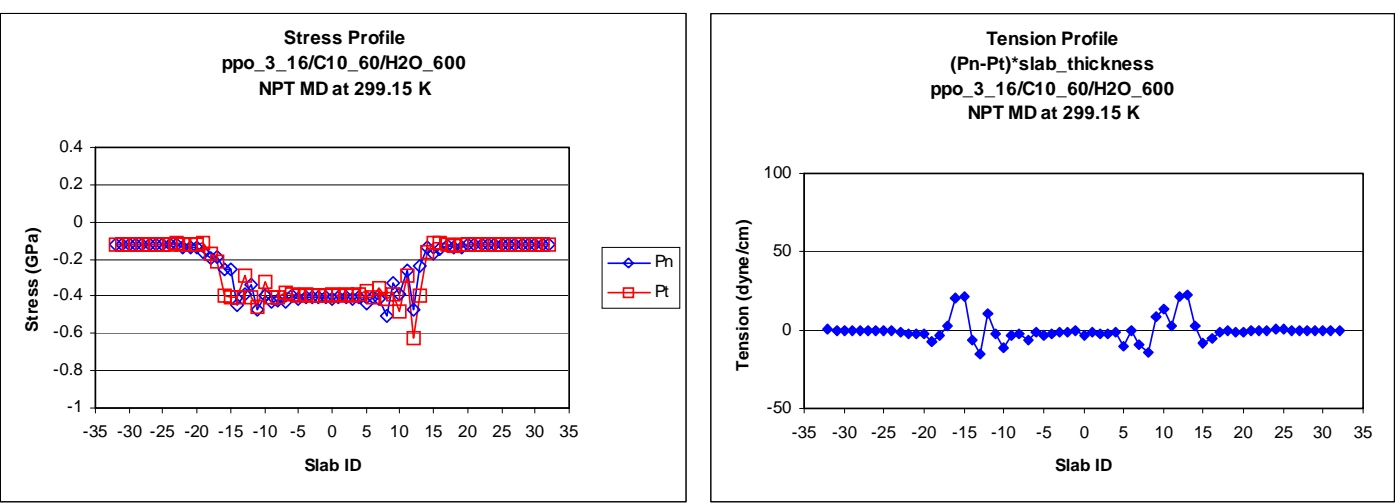

(b) PPO_3
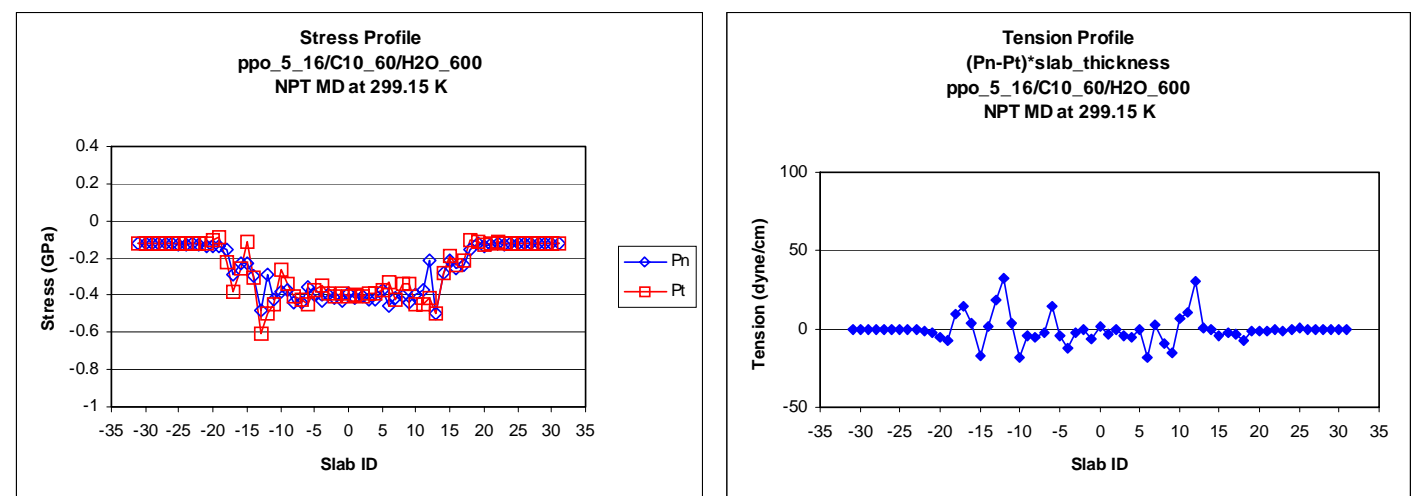

(c) PPO_5 

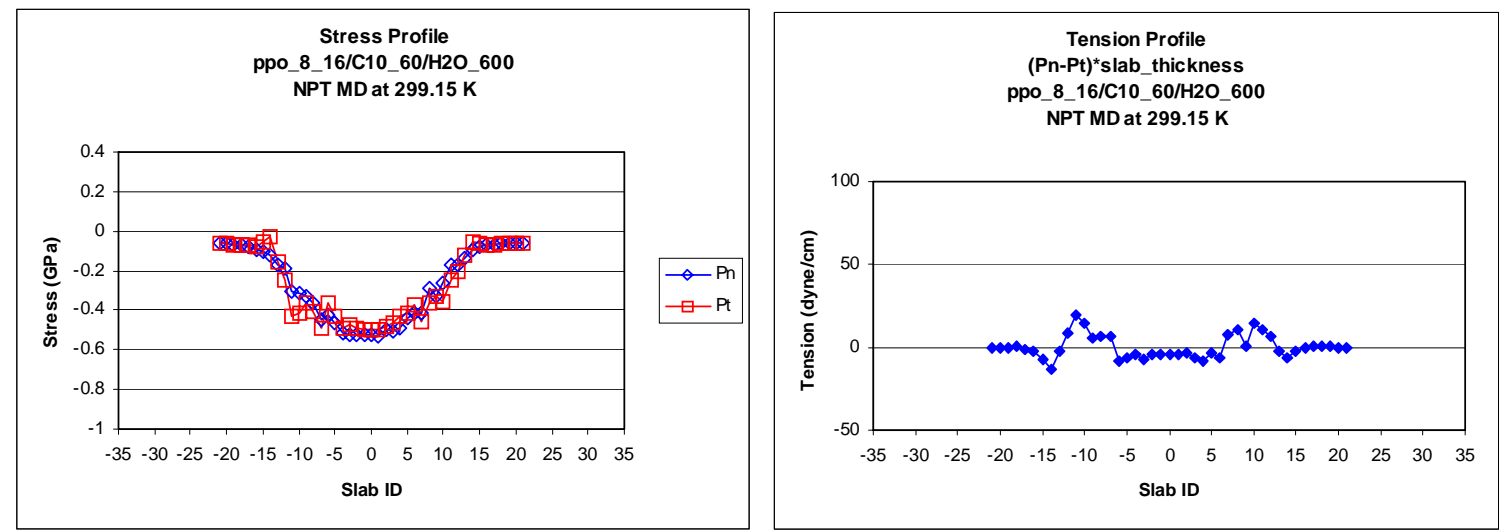

Figure 7.1.7.5. Stress profiles and tension profiles for each system.

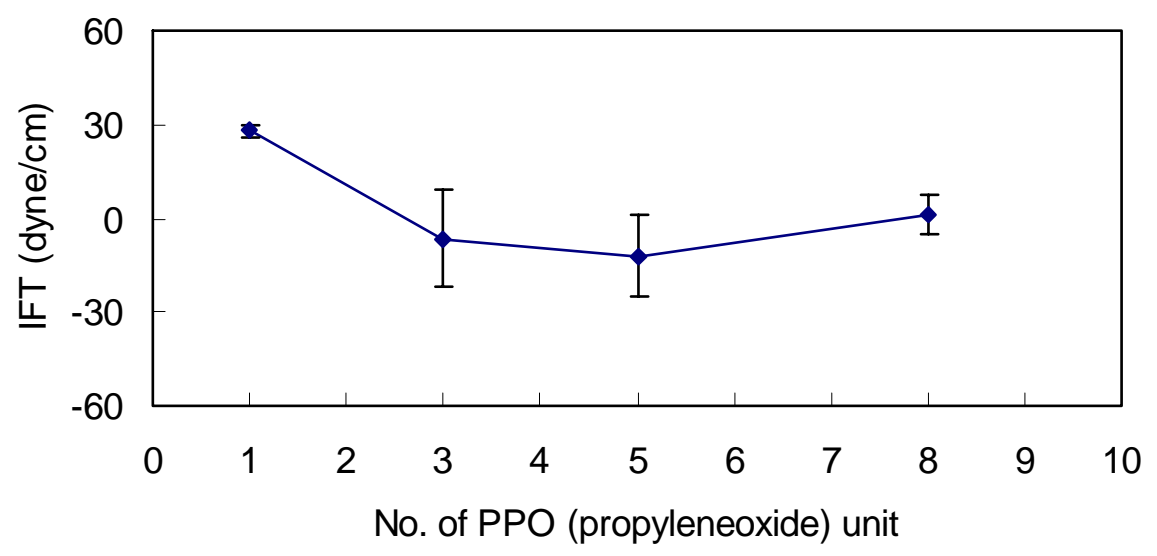

Figure 7.1.7.6. Comparison of IFT between surfactant molecules with different number of propylene oxide group.

Parallel experimental results tests performed in distilled water and n-decane as the oil phase had these results (more details appear in the experimental portion of the report):

Sample

Alfoterra-33

Alfoterra-35

PO Groups IFT (dyne/cm)

Alfoterra-38

$\begin{array}{ll}3 & 1.4 \\ 5 & 2.2 \\ 8 & 1.9\end{array}$

In this case, the experimental results are in fact the opposite of the simulation predictions, with the surfactant with 5 PO groups actually having a slightly greater IFT than that for 3 or 8 PO groups. Note, however, that the experiments are not an exact duplication of the theoretical calculations. The experimental materials are commercially made and so have not only impurities (such as unreacted products), but also a distribution of alkyl and propoxy group structures. This Alfoterra surfactant has alkly chains from $\mathrm{C} 12$ to $\mathrm{C} 16$,and so on average shorter than the $\mathrm{C} 16$ chain used as a single structure in the MD simulations. In addition there is uncertainty in the theoretical values as the calculated IFT does depend on details of the simulation procedure (step size, etc.) 


\subsubsection{Effect of temperature on IFT of alkyl propoxylated sulfates}

We carried out full atomistic simulations of water/oil interfaces for these surfactants to consider the effect of temperature. In particular, this study focused on the molecule shown in Figure 7.1.7.1, where now the number of propoxy groups is 5.

The system consisting of 600 water molecules, 60 decane (C10) molecules and 16 surfactant molecules was simulated using the F3C water Force Field of Levitt et-al, the united atom FF, and the Dreiding FF, respectively. To equilibrate the system, we used NPT molecular dynamics (MD) simulations at $300 \mathrm{~K}$ under 1 atm for $400 \mathrm{ps}$. After equilibration, subsequent NPT MD simulation was performed to collect data for IFT calculation for $2 \mathrm{~ns}$. Figure 7.1.7.7 shows a side view of the final structure for each system. The averaged cell parameters and total densities are summarized in Table 7.1.7.2. It was found that the volume of the system expands with increasing temperature, which is expected of a general liquid system.

Table 7.1.7.2. The cell parameters and the densities

\begin{tabular}{c|cc|cc|ccc|cc}
\hline \multirow{2}{*}{$T$} & \multicolumn{2}{|c|}{ a } & \multicolumn{3}{c|}{ b } & \multicolumn{3}{c|}{ C } & \multicolumn{2}{c}{ density } \\
& avg & std & avg & std & avg & std & avg & std \\
\hline 280.00 & 21.42 & 0.01 & 26.77 & 0.02 & 93.92 & 0.07 & 0.9168 & 0.0020 \\
300.00 & 21.53 & 0.01 & 26.91 & 0.01 & 94.40 & 0.04 & 0.9027 & 0.0010 \\
350.00 & 21.91 & 0.05 & 27.35 & 0.01 & 95.96 & 0.03 & 0.8592 & 0.0007 \\
\hline
\end{tabular}

In addition, from the trajectories of MD simulations, we obtained the time averaged density profile along z-axis as shown in Figure 7.1.7.8. From these structures and density profiles, it is observed that all this surfactant molecules remain stable at the interface between water and oil over this temperature range.

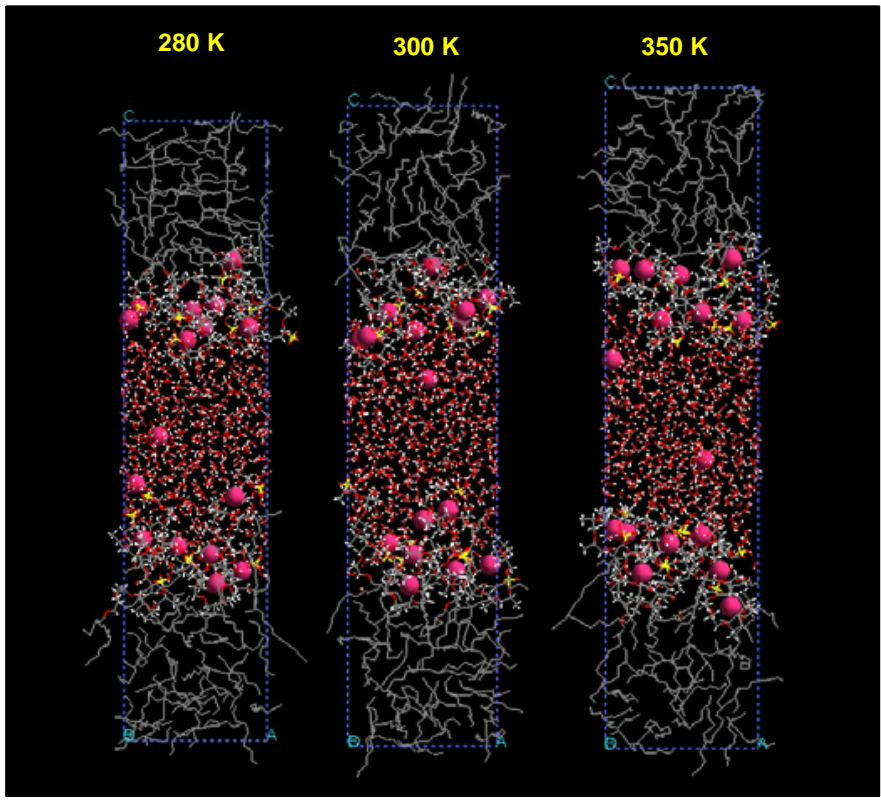

Figure 7.1.7.7. Final structure for each system as a function of temperature. Aqueous surfactant layer between two oil layers. 
In Figure 7.1.7.8, the density profile shows a peak at the interface between water and decane, which is due to the presence of surfactant molecules. A noteworthy point is that this peak becomes broader as the simulated temperature is increased.

(a) $280 \mathrm{~K}$
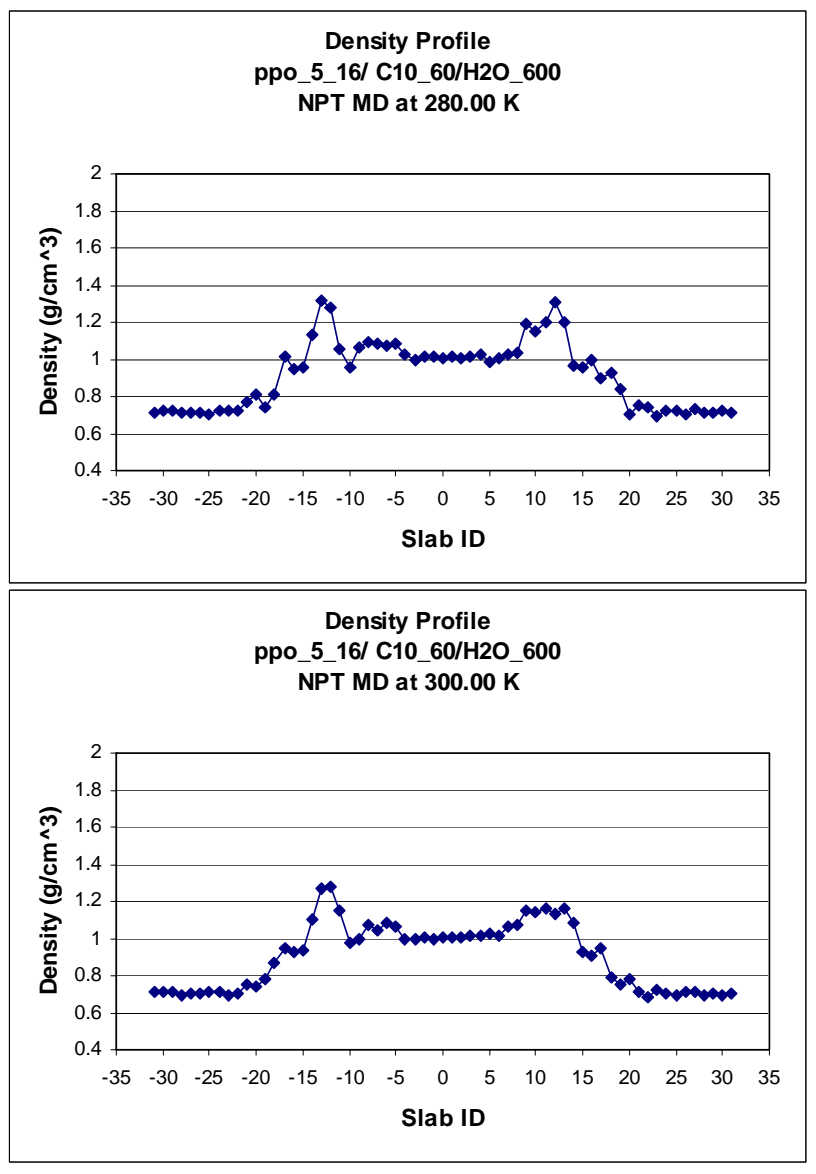

(b) $300 \mathrm{~K}$

(c.) $350 \mathrm{~K}$

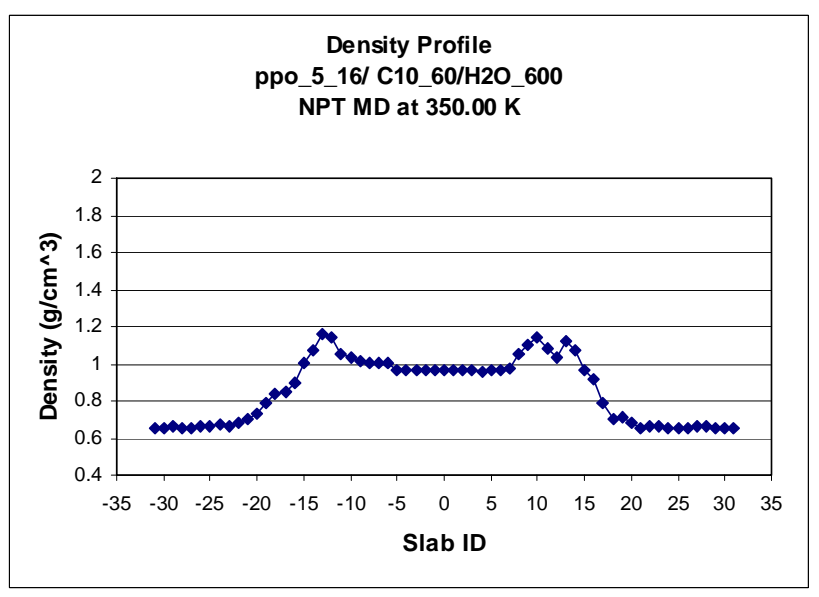

Figure 7.17.8. Density profile for each system studied here. The slab ID is a scaled $\mathrm{z}$ coordinate. The middle phase is water and both sides correspond to oil. The parts shown as peaks in these profiles come from the surfactant. 
The stress profile and tension profile along z-axis is shown in Figure 7.1.7.9. All the profiles seem to have similar features. The profile is more or less symmetric. The value of stress components $\left(\mathrm{P}_{\mathrm{t}}\right.$ for transverse direction and $\mathrm{P}_{\mathrm{n}}$ for normal direction) in each separate phase are closely the same. It should be noted that the non-zero value of Kirkwood-Buff tension appears only at the interface occupied by the surfactant molecules. By integrating the tension profile according to the equation (4), the IFT values were calculated as shown in Figure 7.1.7.10. As we expected, the IFT values are decreased as the temperature is increased. This adds further support to the validity of our atomistic description for an interface between water and oil mediated by ionic/non-ionic surfactants of this type.
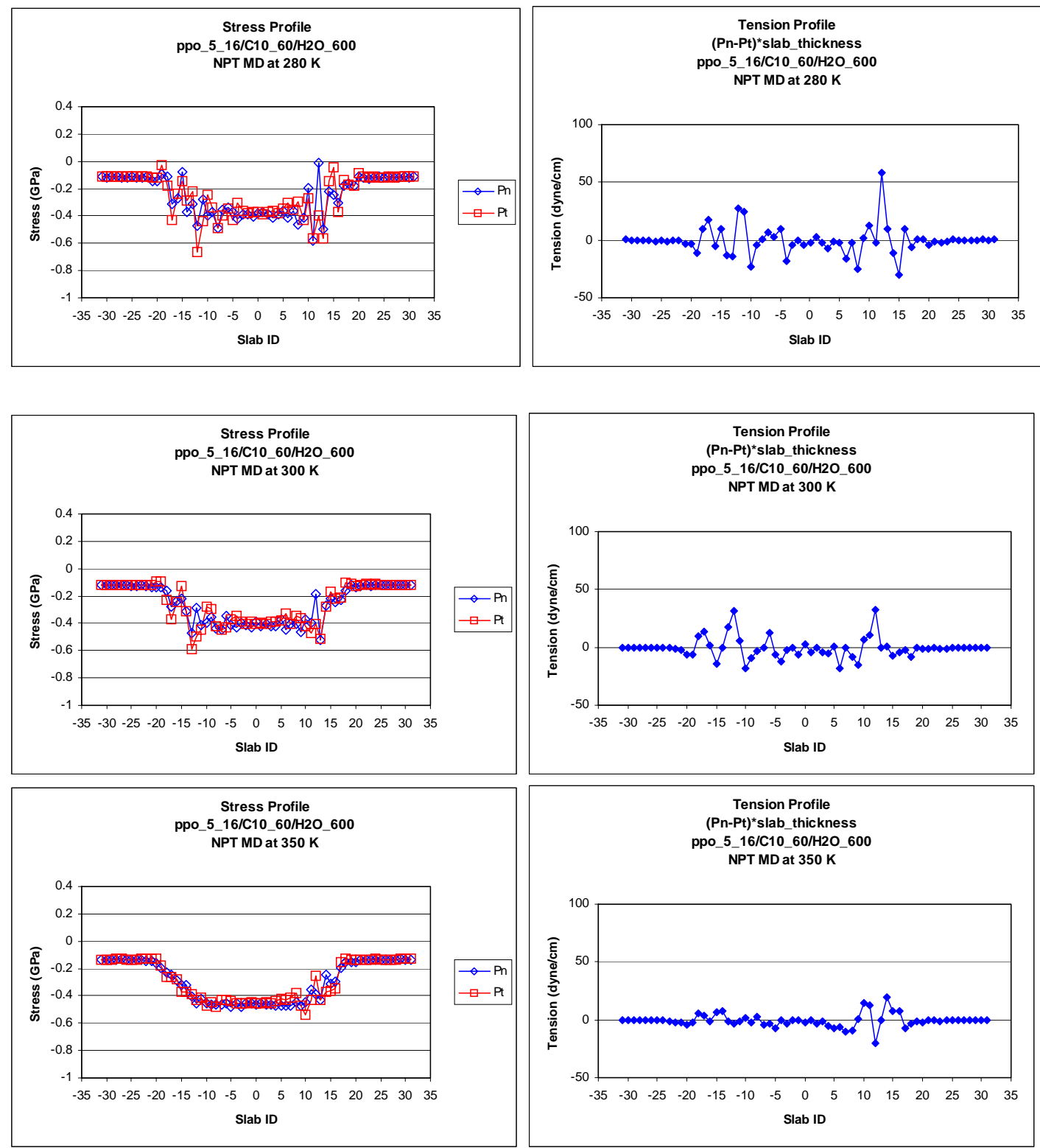

Figure 7.1.7.9. Stress profiles and tension profiles for each temperature, 280,300, and $350 \mathrm{~K}$, from top to bottom. 


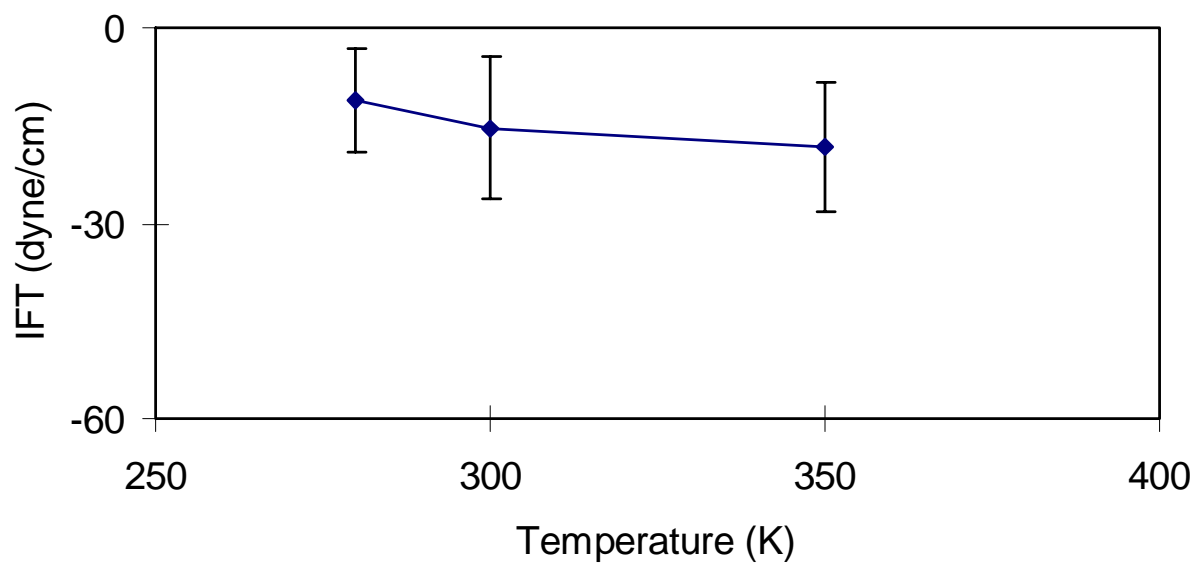

Figure 7.1.7.10. Change in IFT as a function of temperature.

Parallel experimental results tests performed in distilled water and n-decane as the oil phase had these results (more details appear in the experimental portion of the report):

$\begin{array}{ccc} & \text { Temperature } & \text { IFT (dyne/cm) } \\ \text { Alfoterra-35 } & 25^{\circ} \mathrm{C} & 2.2 \\ \text { Alfoterra-35 } & 77^{\circ} \mathrm{C} & 1.6\end{array}$

In this case, the experimental results follow the trend predicted by the simulation, with a mild decrease in IFT at a higher temperature. The MD simulations actually all show a negative IFT. As demonstrated in the next subsection, this effect is probably related to using an unrealistically high interfacial surfactant concentration (assumed 16 surfactant molecules in the unit cell) in the MD simulations.

\subsubsection{Effect of alkyl propoxylated sulfate concentration on on the calculated IFT}

We carried out the full atomistic simulations of water/oil interface mediated by this surfactant. The purpose of these simulations was to evaluate the effect of surfactant concentration ("interface crowding") on the interfacial tension. The molecular structure of surfactant used in this series of MD simulations was shown in Figure 7.1.7.1, with 5 PO groups $(n=5)$.

The system consists of 600 water molecules, 60 decane $(\mathrm{C} 10)$ molecules, and surfactant molecules whose number was controlled from 8 to 16 in this system to simulate various concentrations. F3C water force field (FF), the united atom FF, and the Dreiding FF were used for water, oil and surfactant, respectively. To equilibrate the system, we used NPT molecular dynamics (MD) simulation at $300 \mathrm{~K}$ under 1 atm for 400 ps. After equilibration, subsequent NPT MD simulation was performed to collect data for IFT calculation for $2 \mathrm{~ns}$. Figure 7.1.7.11 shows the side view of a typical structure of system with 16 surfactant molecules. Table 7.1.7.3 summarizes the system specifications. 
Table 7.1.7.3. System specifications

\begin{tabular}{cccc}
\hline No. surfactant & area (anstrom^2) & volume (angstrom^3) & conc (mol/cc) \\
\hline 8 & 438.0 & 45692.8 & $2.91 \mathrm{E}-04$ \\
10 & 453.6 & 48156.6 & $3.45 \mathrm{E}-04$ \\
12 & 467.0 & 50306.6 & $3.96 \mathrm{E}-04$ \\
14 & 479.6 & 52344.3 & $4.44 \mathrm{E}-04$ \\
16 & 579.2 & 54674.6 & $4.86 \mathrm{E}-04$ \\
\hline
\end{tabular}

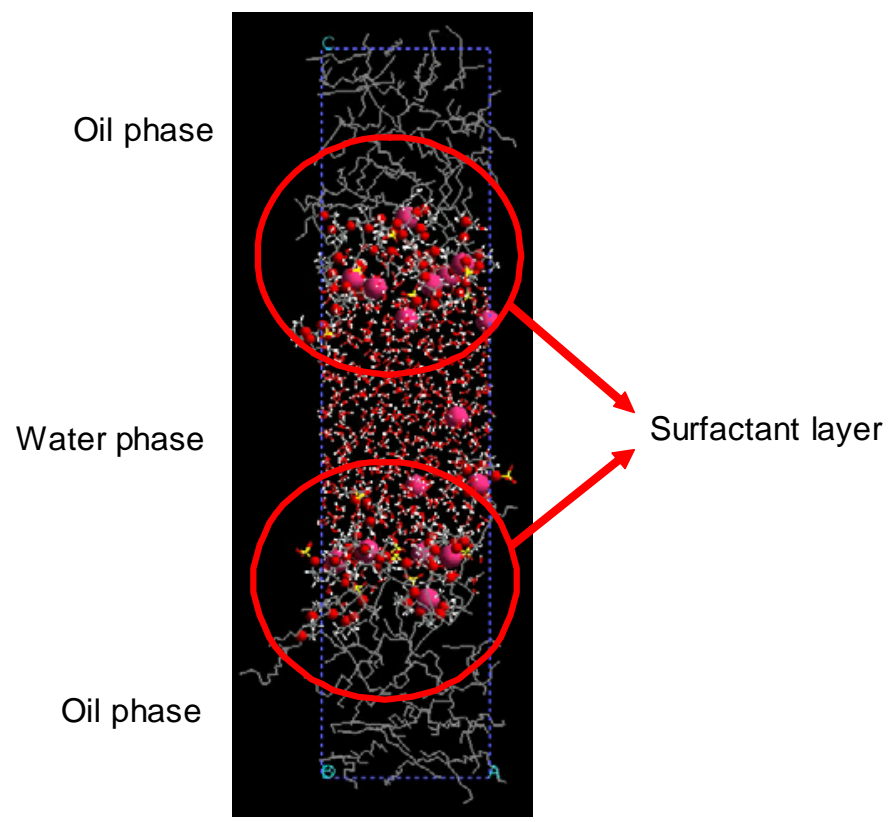

Figure 7.1.7.11. Typical structure for oil/surfactant/water system. Alkyl propoxy sulfate surfactant solution and n-decane

The stress profile and tension profile along z-axis is shown in Figure 7.1.7.12. All the profiles seem to have similar features. The profile is more or less symmetric, in each water and oil phases the value of the stress components $\left(P_{t}\right.$ for transverse direction and $P_{n}$ for normal direction) are almost same. It should be noted that the non-zero value of tension appears only at the interface occupied by the surfactant molecules.

The IFT values were calculated as shown in Figure 7.1.7.13. It is clear that the IFT values are decreased as the concentration is increased because the surfactant molecules reduce the IFT between the oil/water phases. Another noteworthy point is that the IFT becomes negative beyond a certain level of concentration, which means the interface between oil and water phase tends to expand. At large concentrations this results in the repulsion of surfactant molecules from the interface, a process that has not been simulated here. 
Only at the equilibrium concentration we can be sure that the calculated IFT corresponds to that measured experimentally. The experimental setup usually has a "maturation" period, often several days, before values are measured. Similarly, we need to make sure that the concentrations we simulate are at thermodynamic equilibrium.
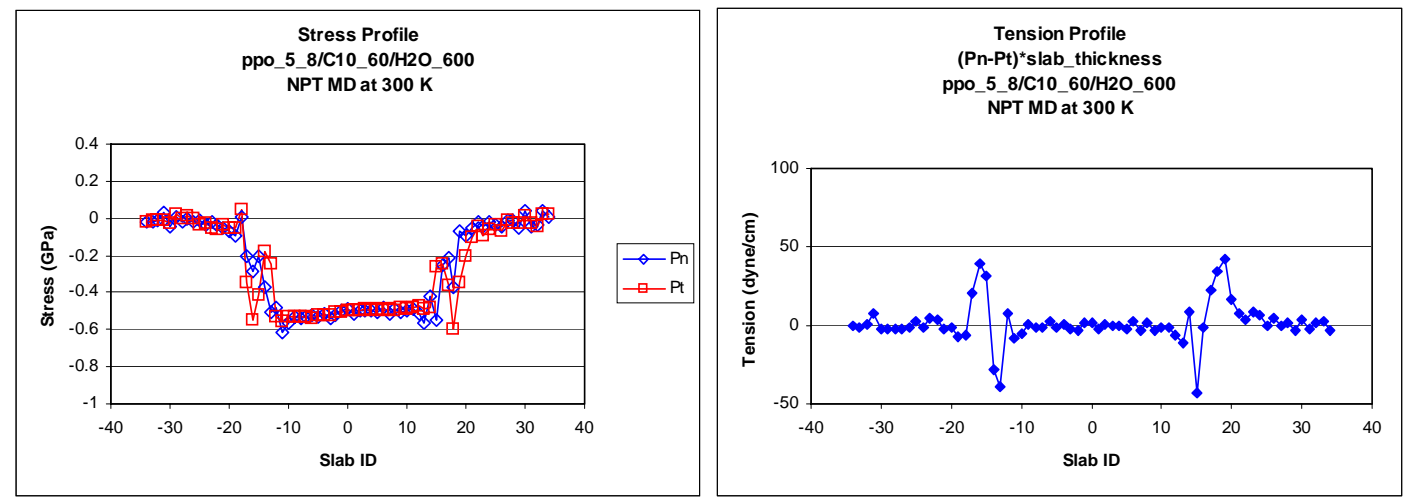

(a) 8 surfactant molecules
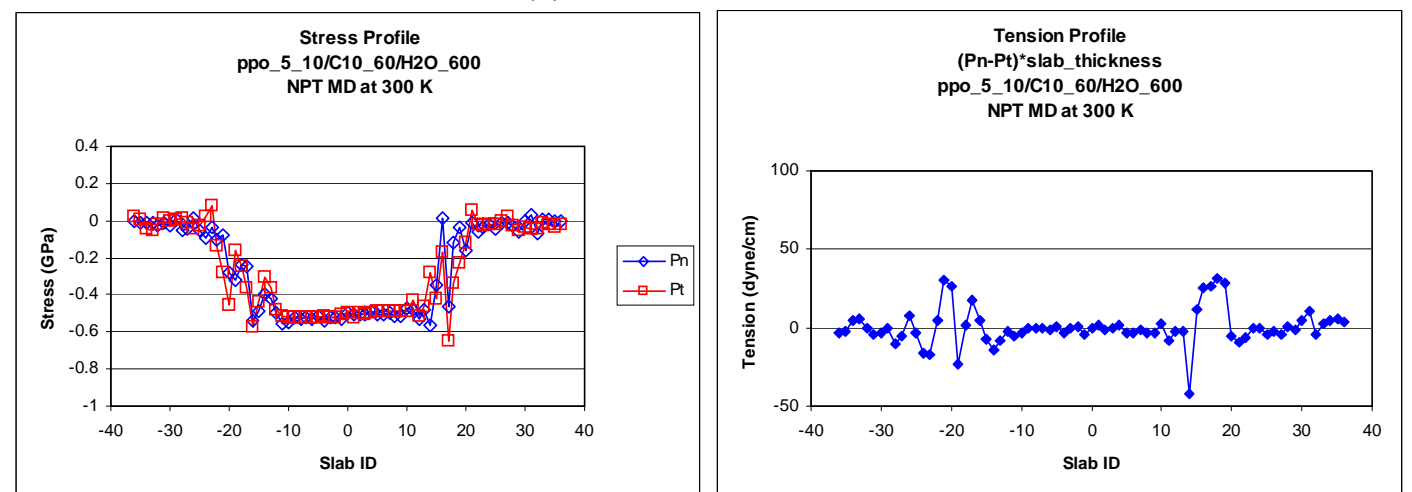

(b) 10 surfactant molecules
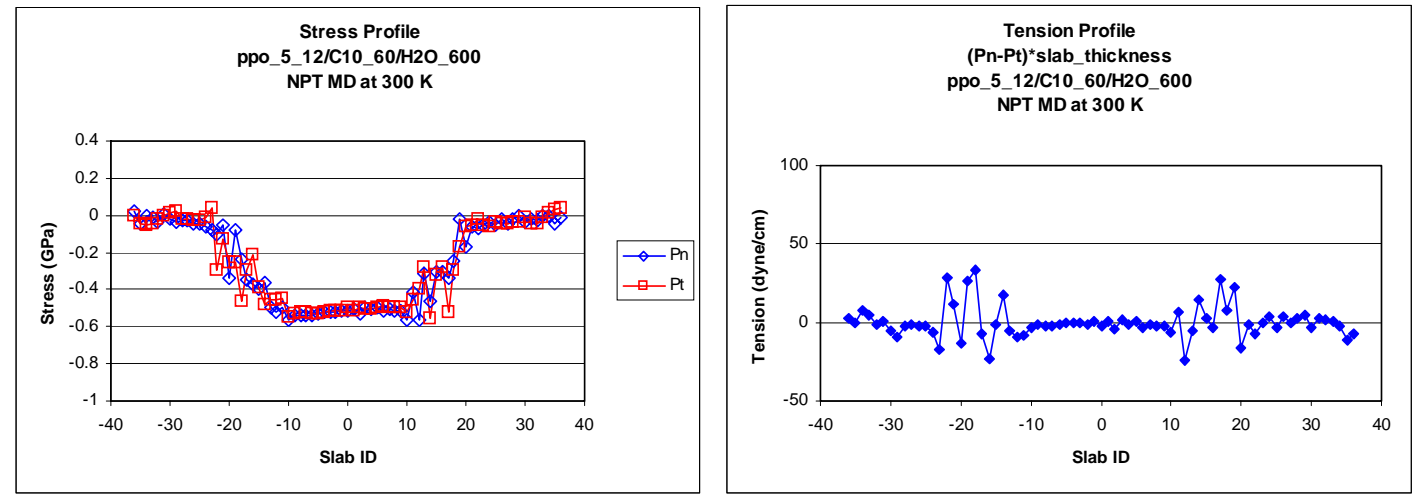

(c) 12 surfactant molecules 

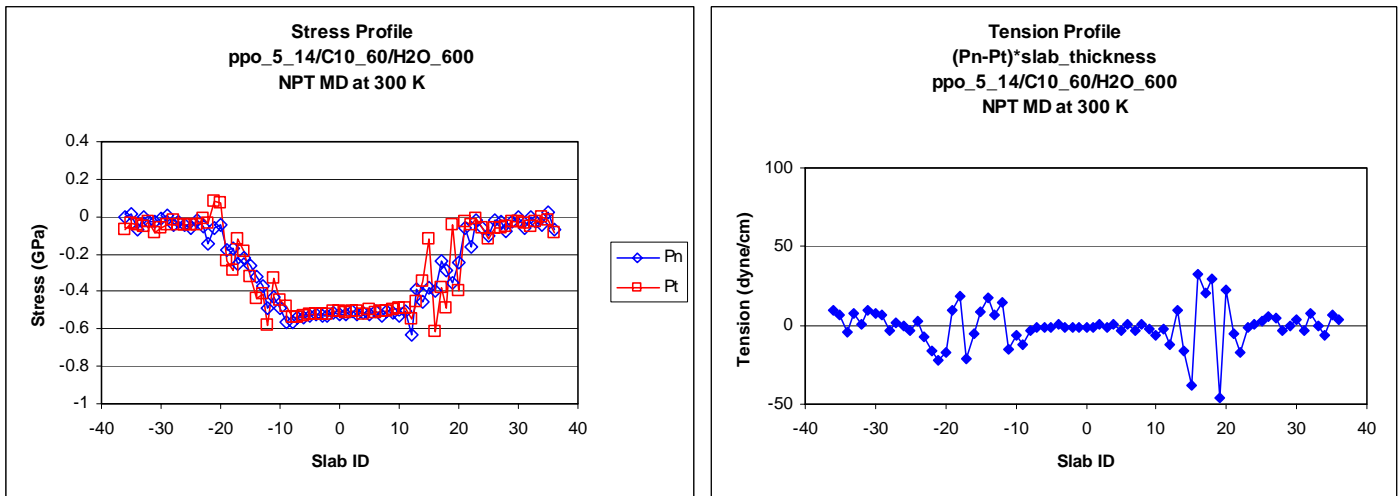

(d) 14 surfactant molecules
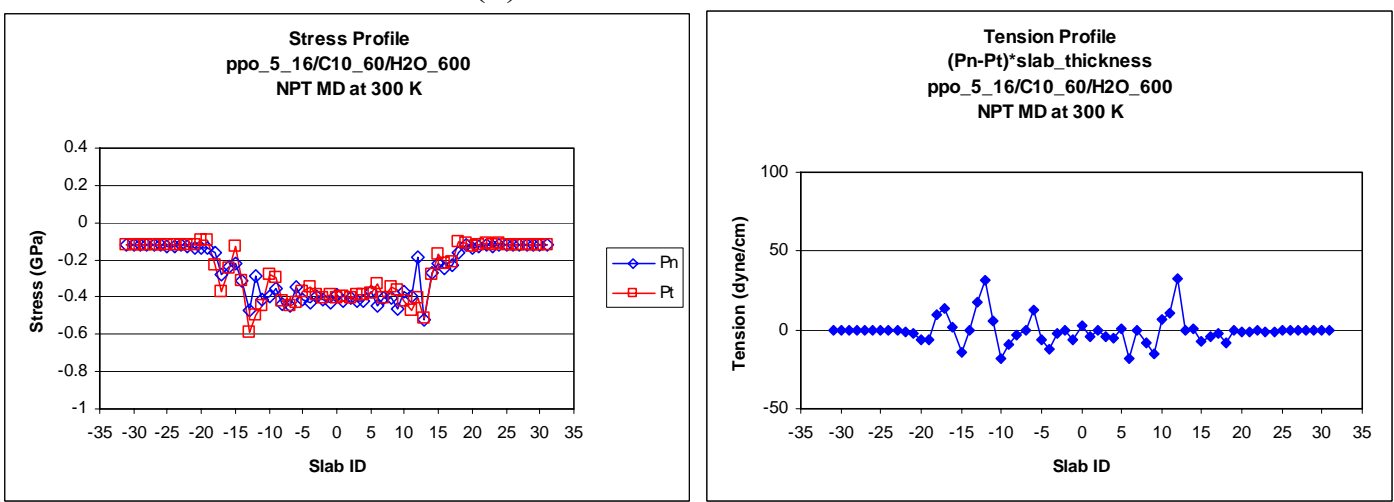

(e) 16 surfactant molecules

Figure 7.1.7.12. Stress profiles and tension profiles for each concentration. of alkyl propoxy sulfate surfactant.

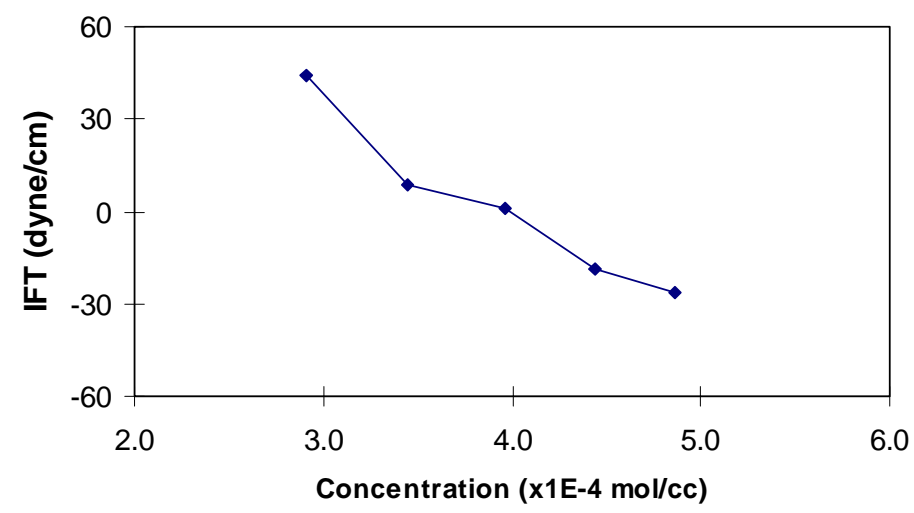

Figure 7.1.7.12. Change in IFT as a function of concentration.

Again, the IFT actually is calculated to become negative at high surfactant concentrations. The most sensible result is for case of 10 surfactant molecules placed at the interface in the simulation unit cell. Here the calculated IFT is about 5 dyne $/ \mathrm{cm}$, close to the corresponding experimental value of $2.2 \mathrm{dyne} / \mathrm{cm}$. Note that this corresponds to an interfacial concentration of 45 Angstroms $^{\wedge} 2 /$ molecule; a reasonable value for a surfactant of this type and size. The simulations with more surfactants begin to have surfactant 
interfacial areas that are unrealistically small (tighter than realistic packing). This artifact is related to the physics in the simulations not having an allowance for moving any excess surfactant from the interface.

\subsubsection{QSPR (Quantitative Structure Property Relationship) Model}

A number of surfactants have been screened for low IFT behavior, some types not considered for EOR applications previously, through a traditional Quantitative Structure Property Relationship expression (QSPR). The concept behind this method is to relate the structure of the molecules and its solution properties to its behavior (e.g. interfacial tension). The motivation for exploring this approach is that if it is successful, it is a calculation method that is much faster than full, molecular-level simulation and can provide semi-quantitative predictions of its behavior (e.g. IFT) based on the molecular structure and process conditions. Based on the initial attempts to apply this method, however, we conclude that QSPR are of limited usefulness in correlating structure/concentration or co-surfactants/oil type to IFT, and of some utility in correlating the effect of salinity on interfacial tension. No correlation with Persons' correlation coefficient greater than 0.6 was uncovered after extensive analysis of the existing literature values.

\subsubsection{Mesoscale Model -- Dependence of Interfacial Tension on Surface Architecture}

To validate our mesoscale modeling methodology described in Section 6.1, first we have computed the surface tension of liquid-vapor interface using simple LJ particles. We have done simulations for two different system sizes at two different temperatures. One for $\mathrm{N}$ $=10000$ at $\mathrm{T}^{*}=1.0$ and one for $\mathrm{N}=4000$ at $\mathrm{T}=* 0.72$. All the simulations were carried out in NVT (constant volume) ensemble.

For the case $\mathrm{T}^{*}=1$, we get a liquid density $\rho^{*}=0.6374$ and density in gas phase $\rho^{*}=$ 0.0474 . We get a surface tension value of $\gamma^{*}=0.2125+0: 011$ (Figure 7.1.9.1). For the case $\mathrm{T}^{*}=0.72$ the density in liquid phase was $\rho^{*}=0.7984$ and the density in gas phase was $\rho^{*}=0.0049$. In this case we found a surface tension value $\gamma^{*}=0.7236+0: 013$.

Both these values are in quantitative agreement with available simulation results on similar systems. This validates our methods as well as the software program. 

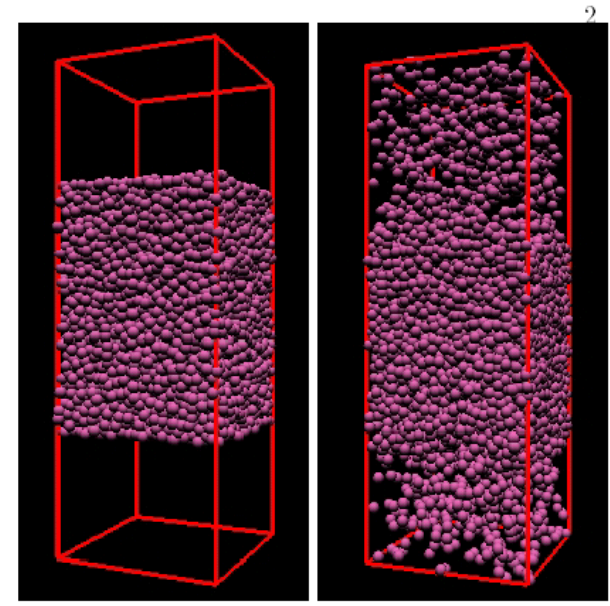

Figure 7.1.9.1. Snapshots of initial and final configuration for liquid-vapor interface.

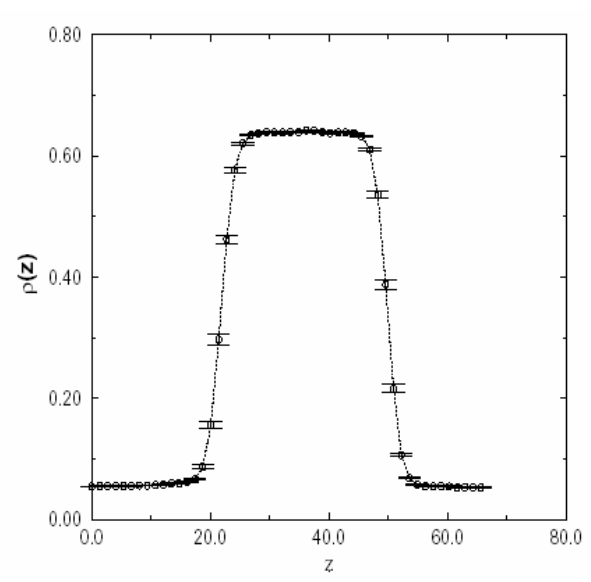

Figure 7.1.9.2. Density profile for the vapor-liquid interface.

We have calculated IFT values for several surfactant architectures. To begin with we have studied the interfacial properties of conventional single chain surfactants. We have calculated the IFT as a function of the tail length of the hydrocarbon tail. From Figure 7.1.9.4 we see that the IFT decreases as a function of the tail length. This trend of is generally consistent with available experimental data.

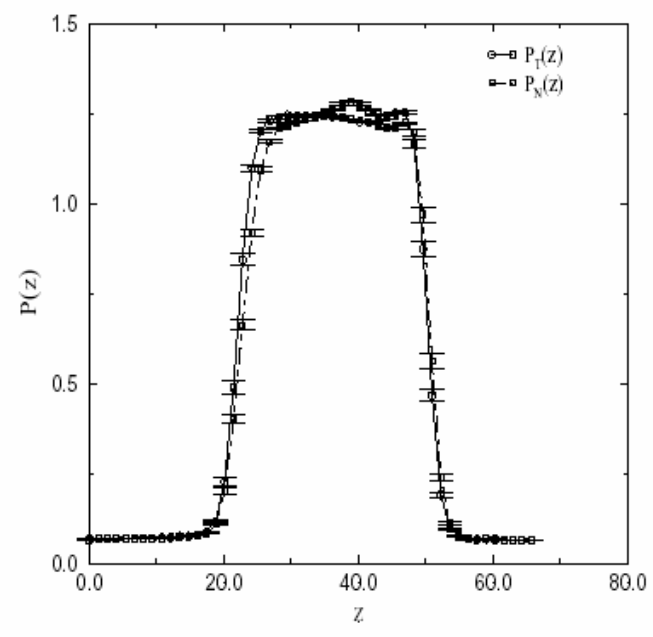

Figure 7.1.9.3 Stress profile (both tangential and normal components) for the vapor-. liquid interface

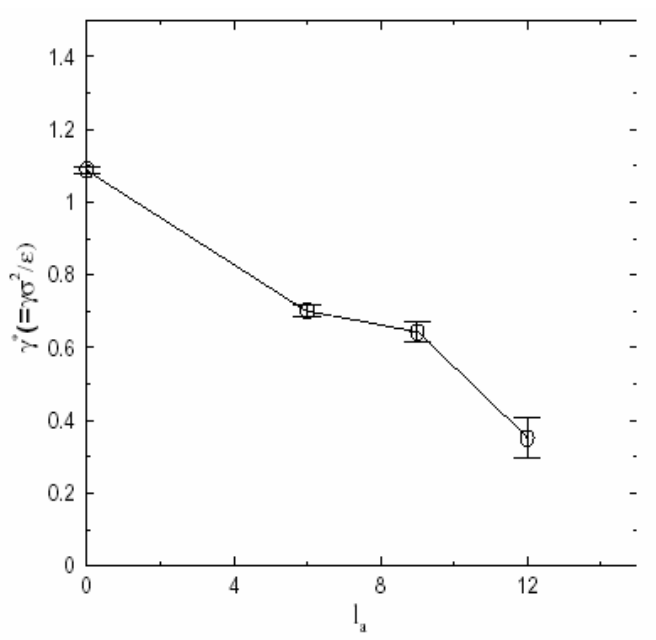

Figure 7.1.9.4. Variations of IFT as a function of hydrocarbon tail length for a single chain surfactant. (The solid line is a guide to eye only.) 
To understand the origin of the minima in the IFT behavior with the isomer number (carbon attachment point, see for example Figure 7.1.3.4), we have calculated the pair correlation between the polar head group and the oil beads. This is a measure of how soluble is the surfactant in oil as we change the position of the polar head group along the backbone of the chain. From Figure 7.1.9.5 we see that the position 4 is the most soluble in this 12 carbon-length alkyl chain, giving rise to the lowest IFT (Figure 7.1.9.6). In fact, the sequence of calculated IFT from lowest to highest (Figure 7.1.9.6) is in the same sequence as the value of the pair correlation going from the highest to lowest (Figure 7.1.9.5).

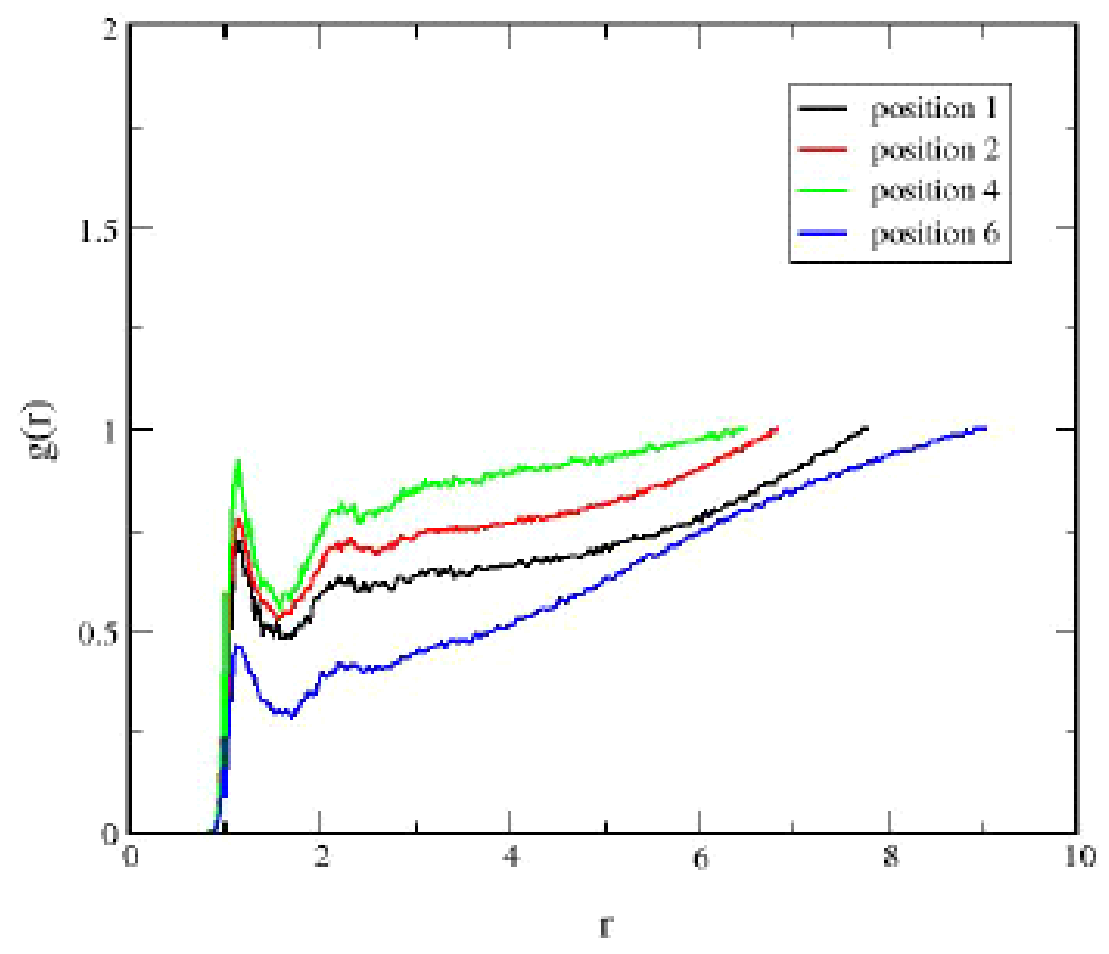

Figure 7.1.9.5. Correlation function between the polar hear group and oil beads for different positions of this polar head group. 


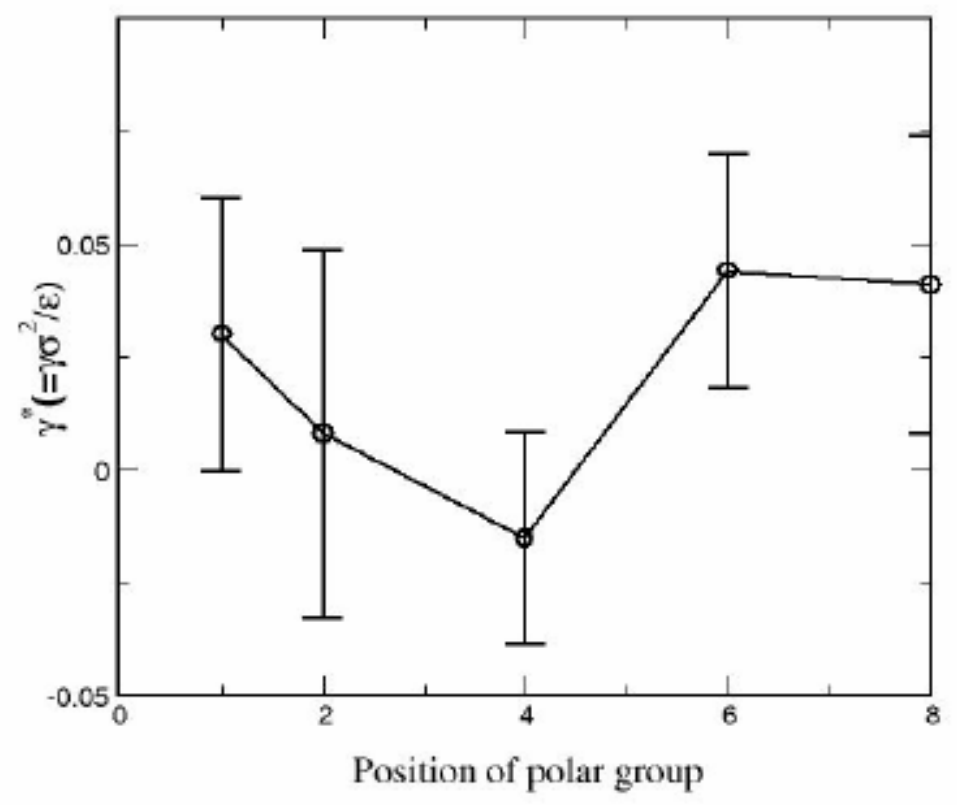

Figure 7.1.9.6. Variation of IFT as a function of position of the polar group along the surfactant backbone. The solid line is a guide to eye only.

In Figure 7.1.9.7 we have plotted the density profile to illustrate how that changes with a change in the position of the polar head group

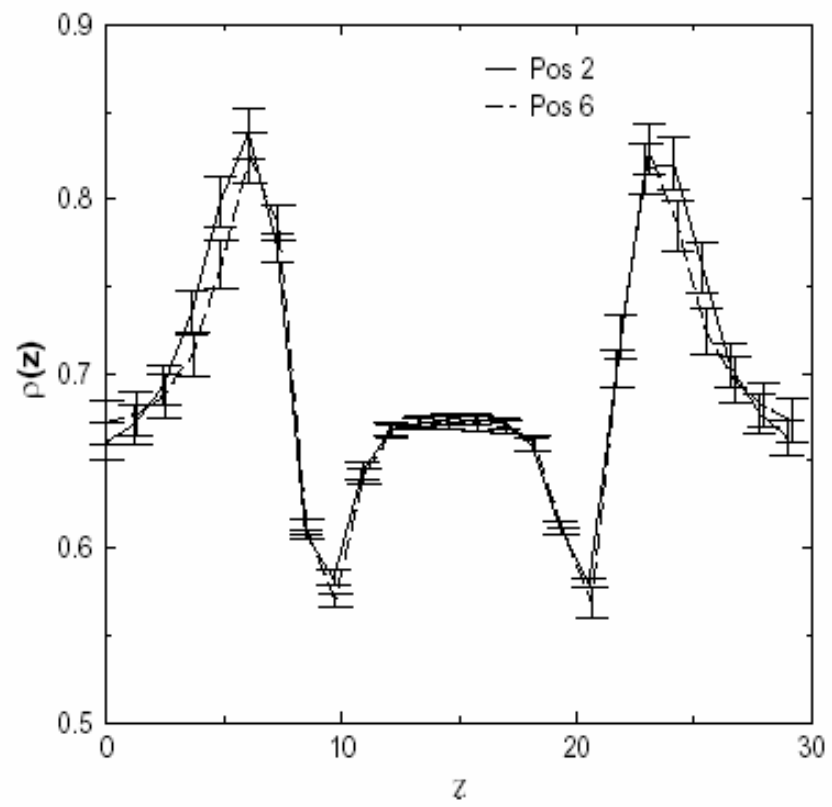

Figure 7.1.9.7. Density profile for different positions of the polar group. 

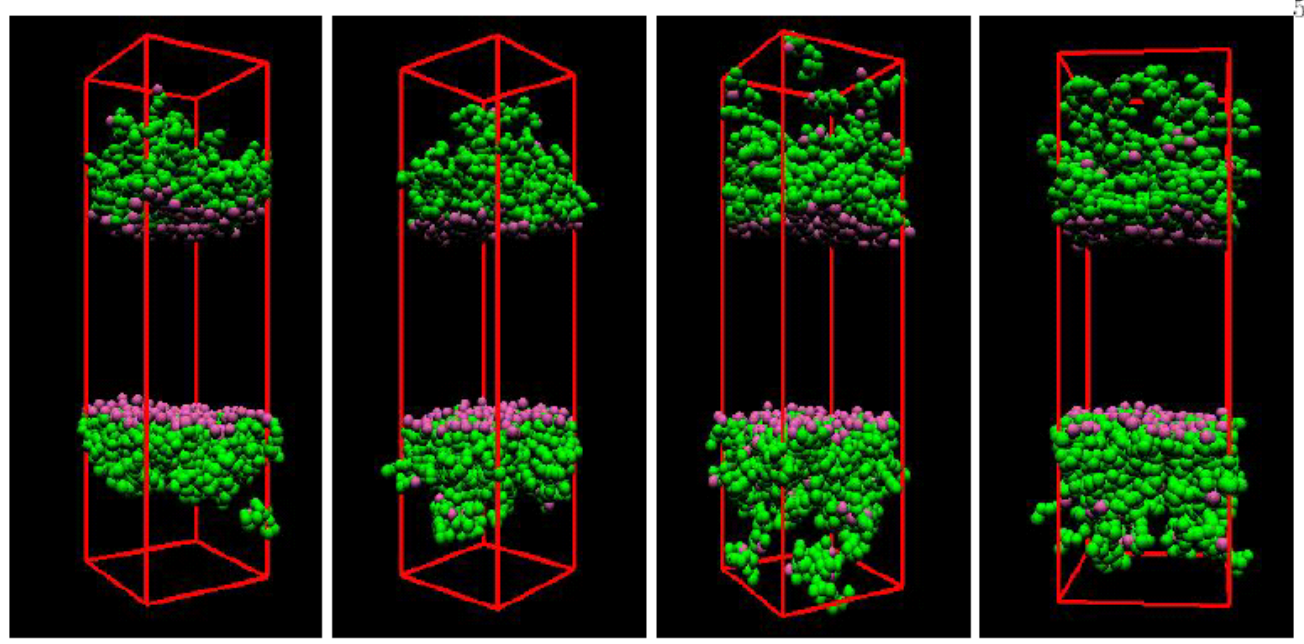

Figure 7.1.9.8. Snapshots of final configurations for various positions of the polar group $(1,2,4$, and 6 positions).

\subsubsection{Cohesive Energy Density in the Water-Surfactant-Oil System -- Molecular Origin of IFT}

The molecular origin of interfacial (or surface) tension is often attributed to the difference of cohesive energy density (CED) of the two phases in contact. The CED, defined as the energy of interaction per unit volume, is a measure of the extent of molecular interactions in a system. Molecules in a bulk phase experience the same the forces (and interactions) from all directions. However, molecules at an interface would experience different molecular interactions from the two phases, each of which has a specific value of CED. Such inhomogeneity in CED leads to an excess of free energy and a tensile net force at the interface. The interfacial tension is directly related to the interface excess free energy and the tensile force.

Here we perform an analysis on the spatial distribution (along the z-direction) of the atomic strain energy of the water-surfactant-oil. The atomic strain energy, when summed over all the atoms in a system gives the total energy of the system. This is a good measure of local molecular interactions and is identical to the cohesive energy for systems whose intramolecular interactions are negligible.

Figure 7.1.10.1a shows the strain energy profile in the z-direction obtained from averaging over 20 ps NPT molecular dynamics simulation for a water-decane binary mixture. For clarity, a snapshot of the system taken from the simulation is shown in Figure 7.1.10.1b. The strain energy profile is distinctively different in the water and the decane regimes. Because of the stronger interactions between water molecules, the magnitude of atomic strain energy is larger in the water phase $\left(\sim-0.0016 \mathrm{kcal} / \AA^{3}\right)$, whereas the interaction is only $1 / 8$ in the oil phase $\left(\sim-0.0002 \mathrm{kcal} / \AA^{3}\right)$. Furthermore, the change of the strain energy is quite sharp at the interface (thickness $\sim 5 \AA$ ), indicating a large interfacial tension between the water and the decane phase. 
Figures 7.1.10.2 and 7.1.10.3 show similar graphics for the water-surfactant-decane three-component system, for the so-called (C16_8) and (C16_4) surfactants, respectively. These surfactants are linear alkyl benzene sulfonates that have been already modeled in this project (see Figure 7.1.3.1). The C16_8 surfactant has a tail with 16 carbons, and the number 8 carbon is attached to the benzene ring. The C16-4 surfactant also has an alkyl chain length of 16 carbons, but now the $4^{\text {th }}$ carbon is attached to the benzene ring.

Our previous theoretical studies and literature data indicate the $\mathrm{C} 16 \_4$ creates a lower IFT versus decane than does the C16-8 surfactant.

There are noticeable differences in Figures 7.1.10.2 and 7.1.10.3 as compared to the surfactant-free system (Figure 7.1.10.1), especially the broadening of the interface and the magnitude of water strain energy. The size of the interface increases to about $13 \AA$ with the presence of surfactants. Such broadening of the interface is also observed in experiment for lower interfacial tension systems. The origin of the variation in the magnitude of atomic strain energy in the water phase regime requires more research. We suspect that there is compression in the z-direction.

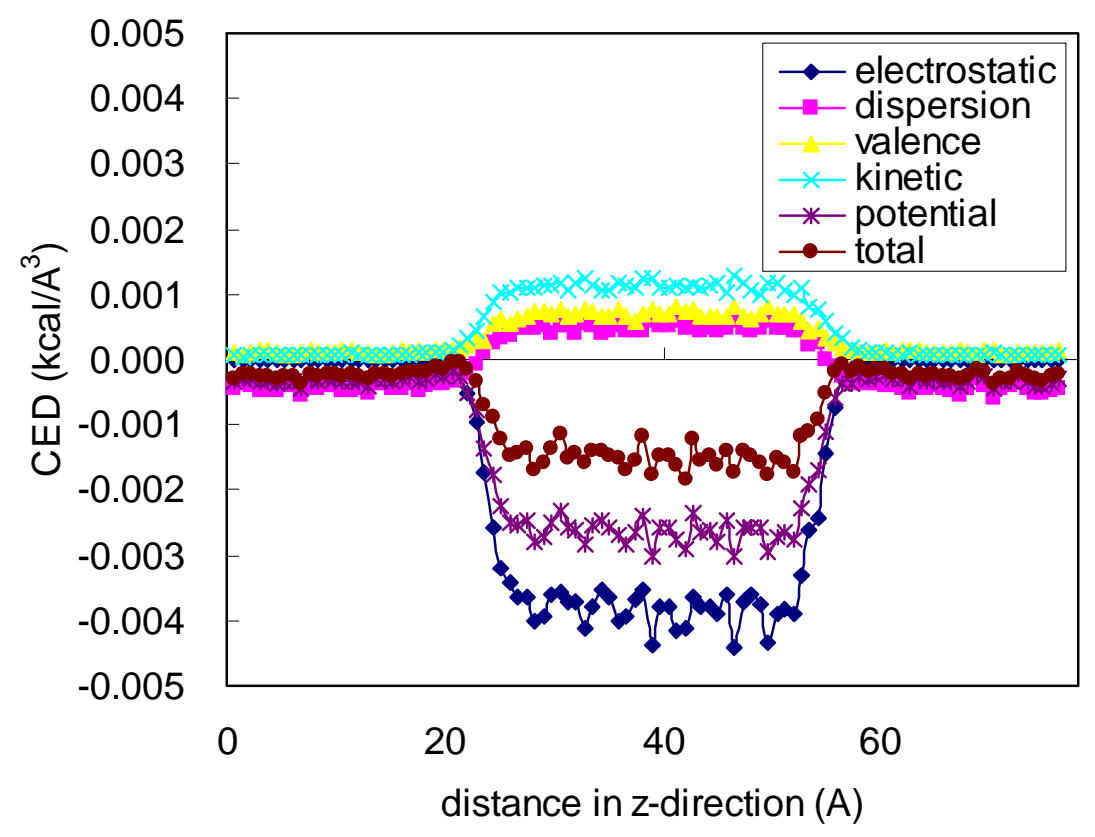

Figure 7.1.10.1a. Spatial distribution of atomic strain energy in the z-direction for water-decane mixture. 


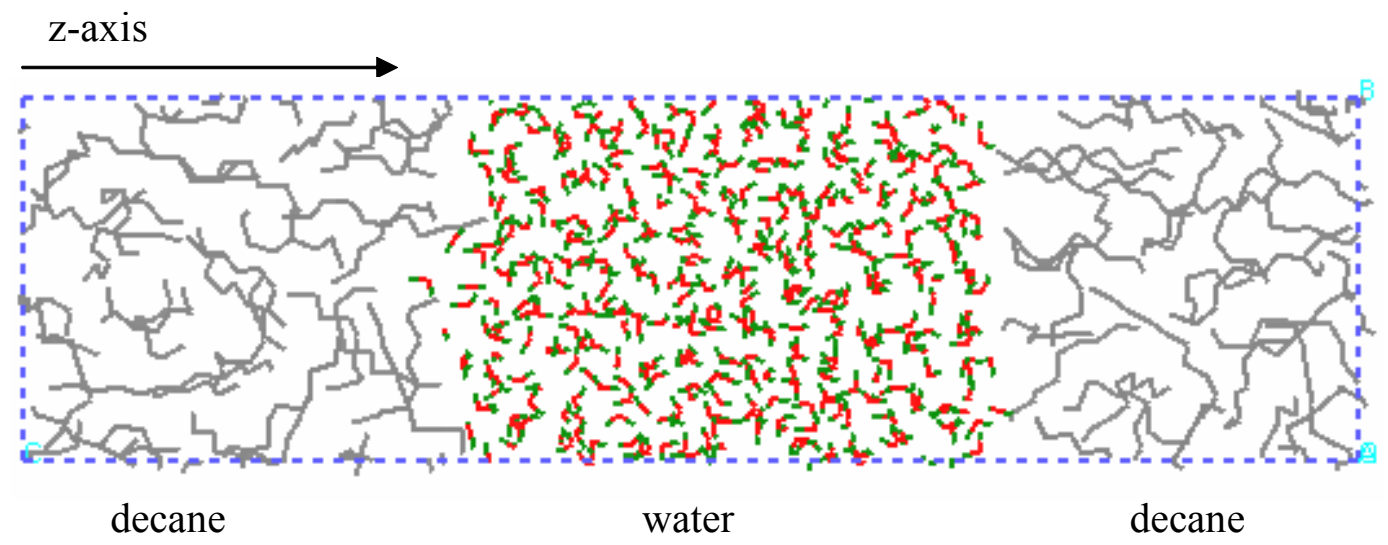

Figure 7.1.10.1b. Molecular model of the water-decane mixture.

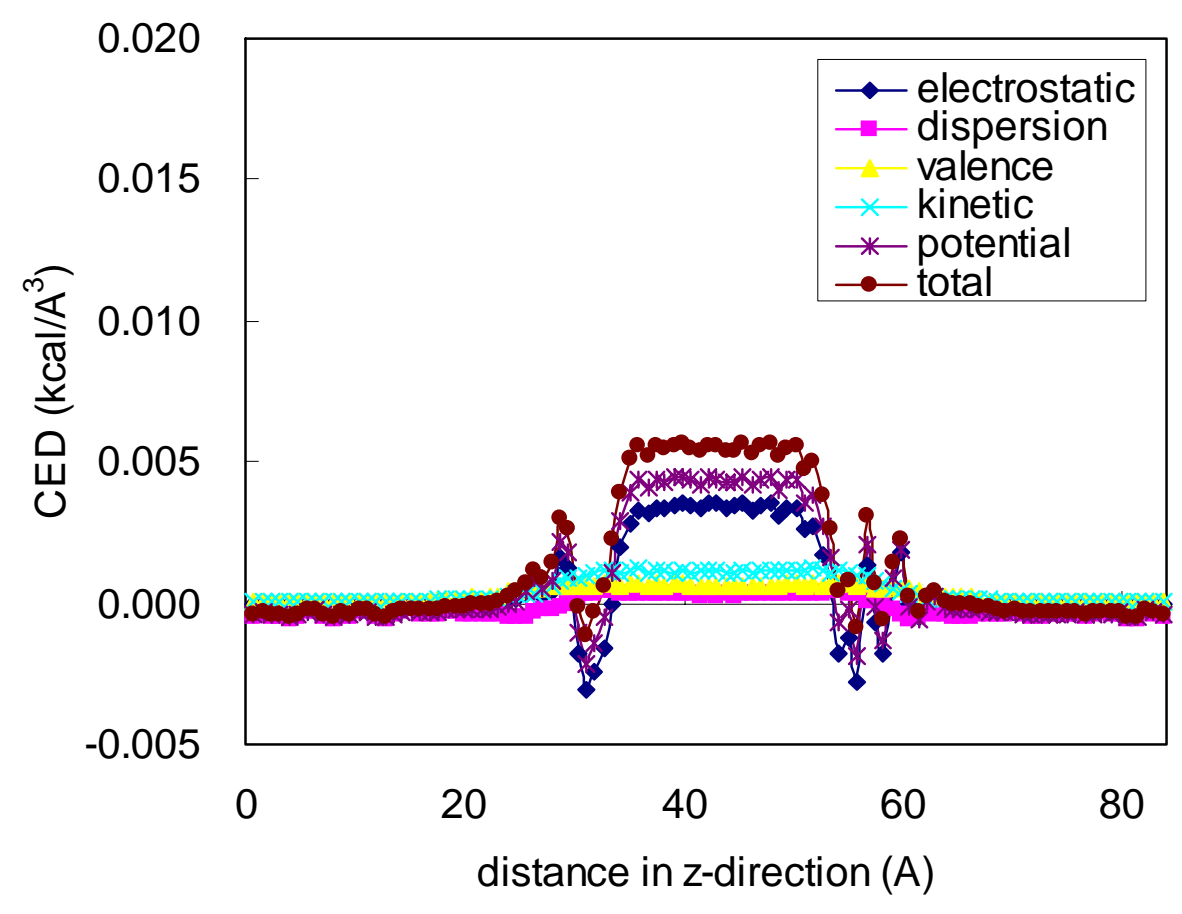

Figure 7.1.10.2a. Spatial distribution of atomic strain energy in the z-direction for water-surfactant(C16_8)-decane mixture. 


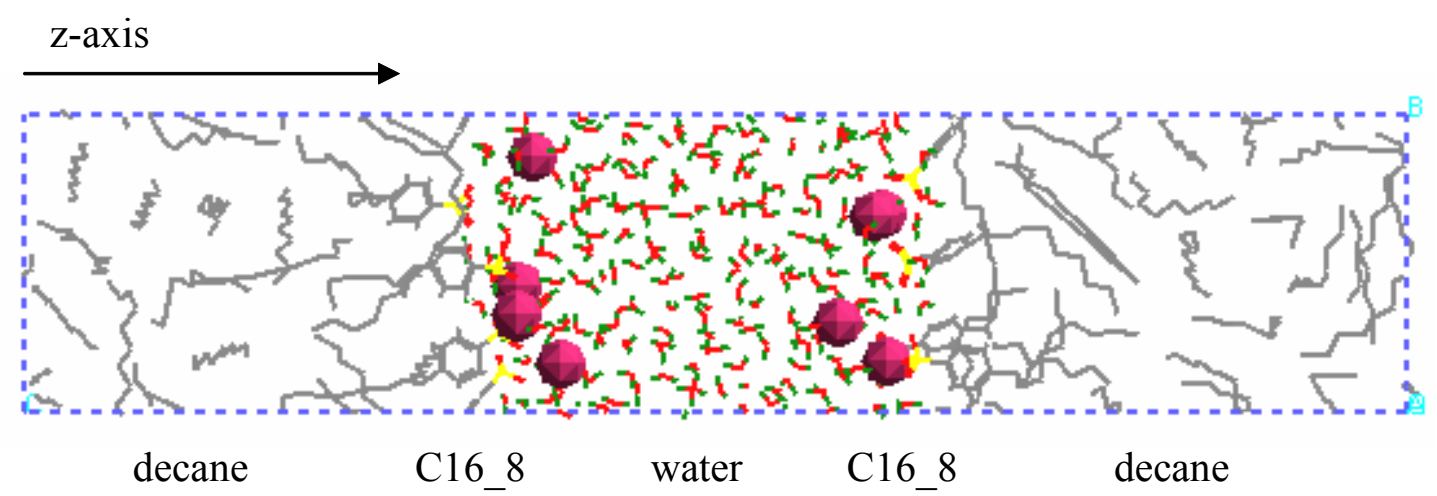

Figure 7.1.10.2b. Molecular model of the water-surfactant (C16_8)-decane mixture.

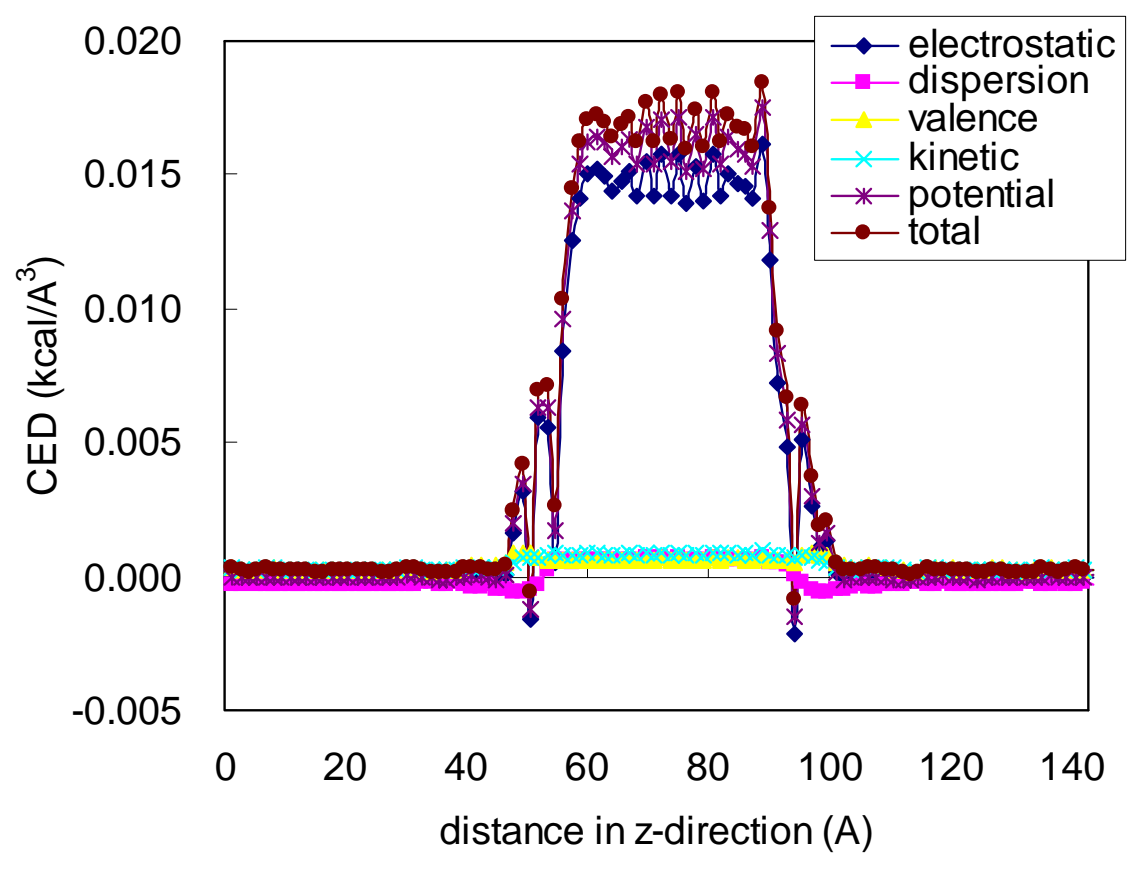

Figure 7.1.10.3a. Spatial distribution of atomic strain energy in the z-direction for water-surfactant C16_4)-decane mixture.

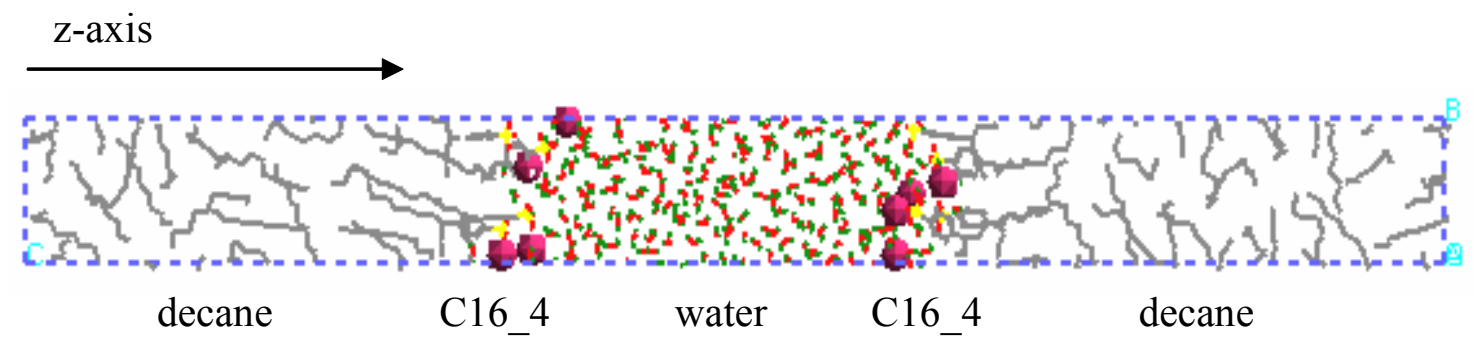

Figure 7.1.103b. Molecular model of the water-surfactant (C16_4)-decane mixture. 


\subsubsection{Hildebrand and Hansen Parameters -- Insights from Solubility Properties}

Hansen parameters as determined by experiment are reported in the literature for a number of compounds. Values are given for many of the materials of direct interest to our study, including water, smaller n-alkanes, and many of the alcohol based cosurfactants. However, there are few published experimental Hansen values reported for surfactants, especially the ones included in our study. In addition, the experimental details in measuring these Hansen parameters often produce some inconsistencies. As described in the theoretical methods section, calculations were performed to generate Hansen solubility parameters for the alkyl polyglycoside surfactants under investigation. We also calculated these parameters for the other components of interest (water, oils, and cosurfactants) so as to have an internally consistent set of Hansen parameters for all of the subject components.

We compare below the 3 Hansen parameters for water, n-octane, APG surfactant PG 2062 and several alcohols that have been studied experimentally as cosurfactants. Figure 7.1.11.1 is a triangular plot showing normalized values for the Hansen parameters. The actual parameter values are given in Table 7.1.11.1.

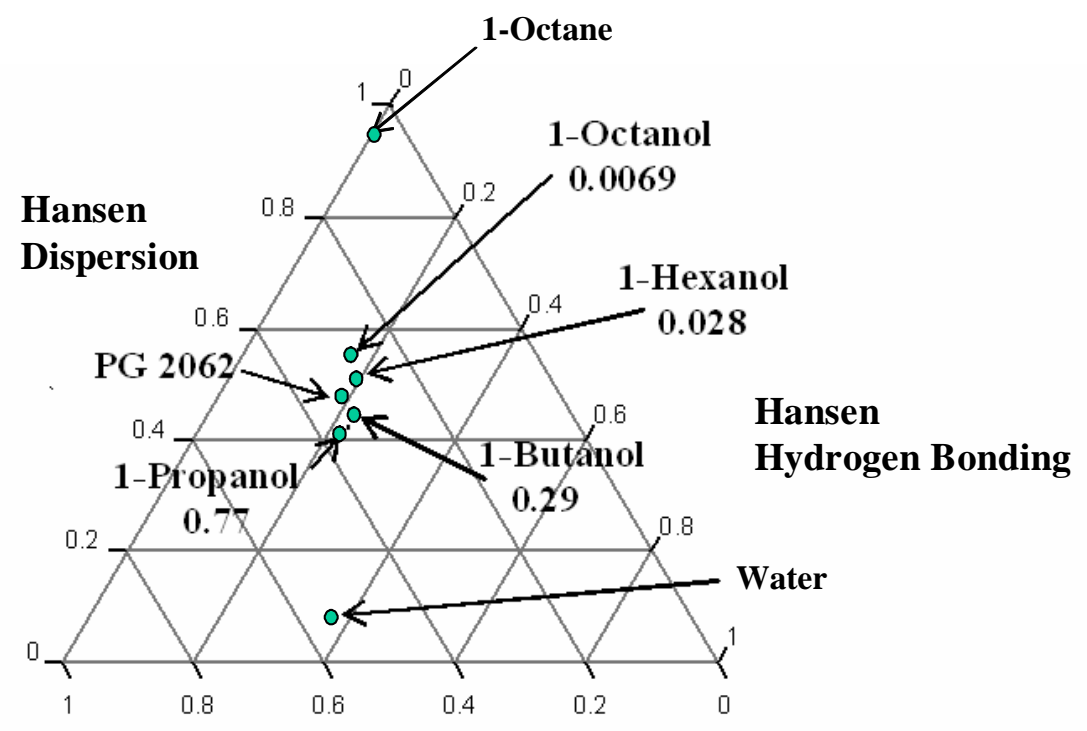

Hansen Polarization

Figure 7.1.11.1. Calculated Hansen parameters for water, PG 2062, n-octane, and several alcohols. The IFT value associated with that alcohol as cosurfactant is given below its label (IFT for 0.8\% PG 2062/1.2\% n-alcohol, n-octane, $25 \mathrm{C}$ ). 
Table 7.1.11.1. Calculated Values of Hansen Parameters for Components in Figure 7.1.11.1

Solubility Parameters $\left(\mathrm{Mpa}^{\wedge} 0.5\right)$

\begin{tabular}{lcccc} 
Component & Hildebrand* & Dispersion & Polarization & Hydrogen Bonding \\
\hline Water & 49.1 & 6.48 & 40.4 & 27.6 \\
n-octane & 16.8 & 16.6 & 1.5 & 0.0 \\
PG 2062 & 19.9 & 15.5 & 10.8 & 6.1 \\
1-propanol & 20.7 & 15.3 & 12.5 & 8.0 \\
1-butanol & 20.3 & 15.7 & 11.4 & 7.0 \\
1-hexanol & 19.3 & 16.1 & 9.0 & 5.9 \\
1-octanol & 19.1 & 16.5 & 8.0 & 5.0
\end{tabular}

* Hildebrand - (cohesive energy density $)^{\wedge} 0.5$; hlidebrand ${ }^{\wedge} 2=$ sum of squares of 3 Hansen parameters

The concept of this approach is that by knowing the combinations of component Hansen parameters that makes a low IFT, one may gain some guidance with respect to creating new formulations for low IFT. From the results shown in Figure 7.1.11.1 we conclude that as the cosurfactant Hansen parameters, particularly dispersion, approach those of the idealized oil in question (n-octane) the IFT values decrease significantly. That is, a lower IFT versus n-octane occurs for aqueous PG 2062 APG surfactant/alcohol formulations as the alcohol Hansen parameters for dispersion increases, polarization decreases, and hydrogen bonding decreases.

A future strategy then would be to calculate the Hansen parameters for a number of new compounds and focus on those chemical combinations with follow-up experimental studies that exhibit a favorable pattern of Hansen values. Of course, these estimates should also be supplemented with atomistic MD simulations, but they can provide a valuable screening tool to focus on most promising formulations and avoid the cost of long computer simulations. 


\subsection{Experimental Investigation}

\subsection{Initial Screening of Surfactants for Low IFT}

The focus of the phase behavior/IFT experiments at the beginning of the project was to explore some different chemistries for their potential as EOR surfactants. Many of these tests also provide phase behavior and IFT data for a series of surfactants within which there is a systematic variation in the chemical structure. Such data add to the test cases to test and tune the theoretical models.

A significant number of surfactants were screened for their ability to depress the IFT. This first set of surfactants selected included those that are commonly reported in the literature, and a number of commercially available products. The table below is a representative list of surfactants where we have performed an initial IFT screening test. Again, the default conditions are $2 \mathrm{wt} \%$ surfactant $/ 1 \mathrm{wt} \%$, isopropanol at $25^{\circ} \mathrm{C}$ versus an n-octane oil phase.

Table 7.2.1.1 Representative list of surfactants in the initial IFT screening evaluation

\begin{tabular}{|c|c|c|}
\hline$\underline{\text { Surfactant(s) }}$ & Source & Comments \\
\hline Sodium dodecyl sulfonate (SDS) & Aldrich & \\
\hline $\begin{array}{l}\text { Sodium dodecyl benezene sulfonate } \\
\text { (SDBS) }\end{array}$ & Aldrich & \\
\hline Sodium octyl sulfate & Witco, product NAS-8 & \\
\hline Sodium dodecyl sulfate & Aldrich & \\
\hline Aerosol MA-80 & American Cyanamid & Ester of sulfosuccinic acid; di-hexyl \\
\hline Aerosol OT-B & American Cyanamid & Ester of sulfosuccinic acid; di-octyl \\
\hline Aerosol TR-70 & American Cyanamid & Ester of sulfosuccinic acid; tri-decyl \\
\hline Aristonate L-LF & Pilot Chemical & $\begin{array}{l}\text { Low molecular weight alky aryl } \\
\text { sulfonate blend }\end{array}$ \\
\hline Aristonate H-LF & Pilot Chemical & $\begin{array}{l}\text { Low molecular weight alky aryl } \\
\text { sulfonate blend }\end{array}$ \\
\hline Petrostep 465 & Stepan Chemical & Petroleum sulfonate \\
\hline Witcolate- 1247 & Witco & C8 - C12 alkyl ether sulfate \\
\hline Dynol-604 & Air Products & $\begin{array}{l}\text { "gemini" surfactant } \\
\text { Polyethylene glycol 2,5,8,11- } \\
\text { tetramethyl-6-dodecyne-5,8-diol } \\
\text { ether }\end{array}$ \\
\hline Surfynol-440 & Air Products & $\begin{array}{l}\text { “gemini” surfactant } \\
\text { 2,4,7,9-TETRAMETHYL-5- } \\
\text { DECYNE-4,7-DIOL } \\
\text { ETHOXYLATE }\end{array}$ \\
\hline
\end{tabular}




\begin{tabular}{|l|l|l|}
\hline Surfynol-465 & Air Products & $\begin{array}{l}\text { "gemini" surfactant } \\
2,4,7,9-\text { TETRAMETHYL-5- } \\
\text { DECYNE-4,7-DIOL } \\
\text { ETHOXYLATE }\end{array}$ \\
\hline Zonyl FSG & DuPont & Fluorinated methacrylate copolymer \\
\hline Zonyl FSK & DuPont & $\begin{array}{l}\text { Perfluoro N-type betaine } \\
\text { (amphoteric) }\end{array}$ \\
\hline Zonyl FSN & DuPont & Perfluoro ethoxylate \\
\hline Zonyl FSO & DuPont & Perfluoro ethoxylate \\
\hline Neodol 11-5 & Shell & Alkyl (C11) Ethoxylate (5EO) \\
\hline Alcodet-MC-2000 & Rhone Poulenc & Mercaptan ethoxylate \\
\hline Calamide-C & Pilot Chemicals & Coconut diethanolamide \\
\hline Igepal DM-710 & GAF & Ethoxylated alkyl phenol \\
\hline Pluronic Series & BASF & $\begin{array}{l}\text { Block copolymers of ethylene and } \\
\text { propylene oxide }\end{array}$ \\
\hline
\end{tabular}

Some of the more interesting results from these tests are given below.

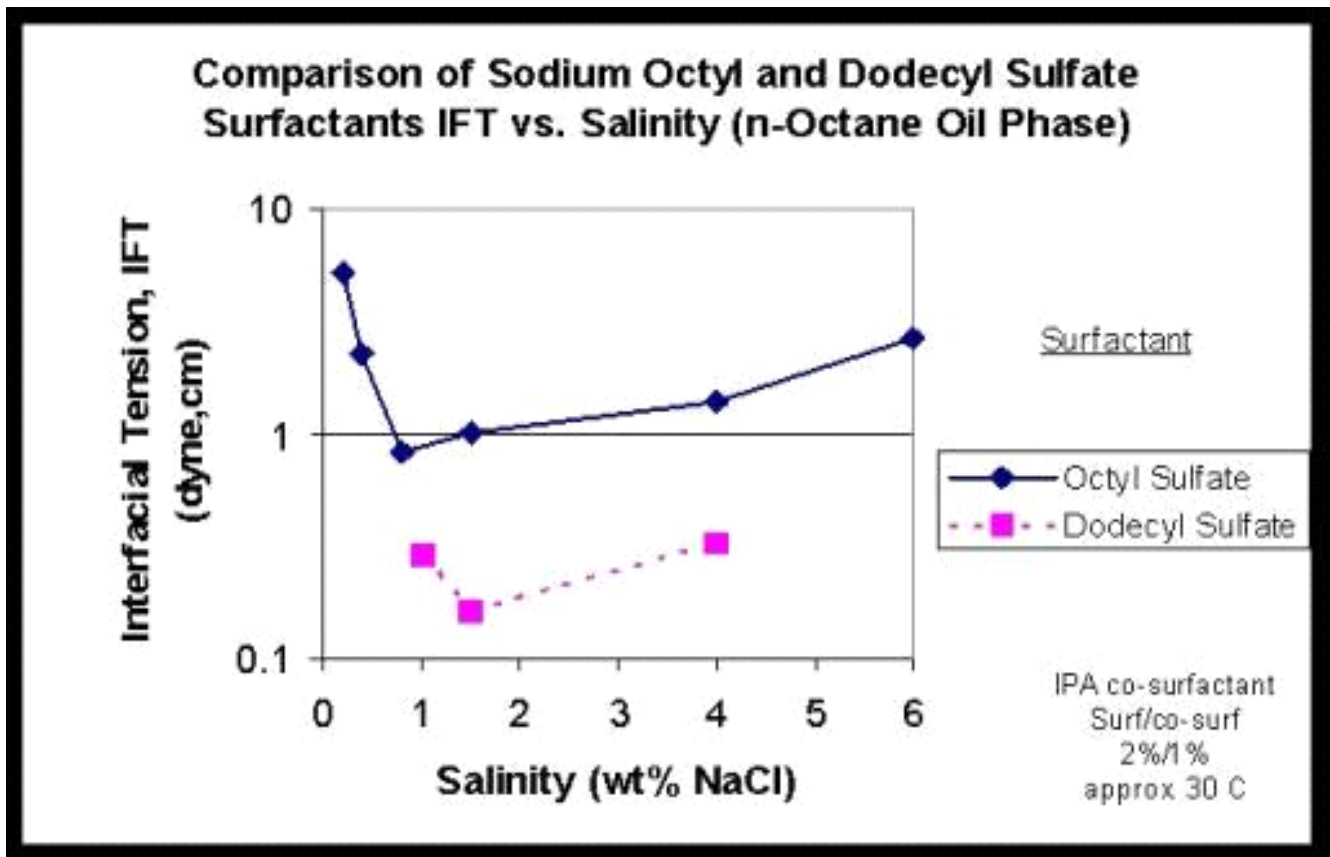

Figure 7.2.1.1. Comparison of IFT for octyl and dodecyl sulfate surfactants

These results indicate a lower IFT occurs with the surfactant with the longer alkyl chain. This is consistent with the literature data (Doe, 1977, 1978) for specific alkyl benzene sulfonate surfactants. These authors in fact maintain that low IFT is difficult to achieve with the alkyl chain being less than C12. Figure 7.2.1.1 results are consistent with the 
general "rule of thumb" that as you increase the molecular weight petroleum sulfonate from about 350 to 450, these products generate a lower IFT (Shah and Schechter, 1977b).

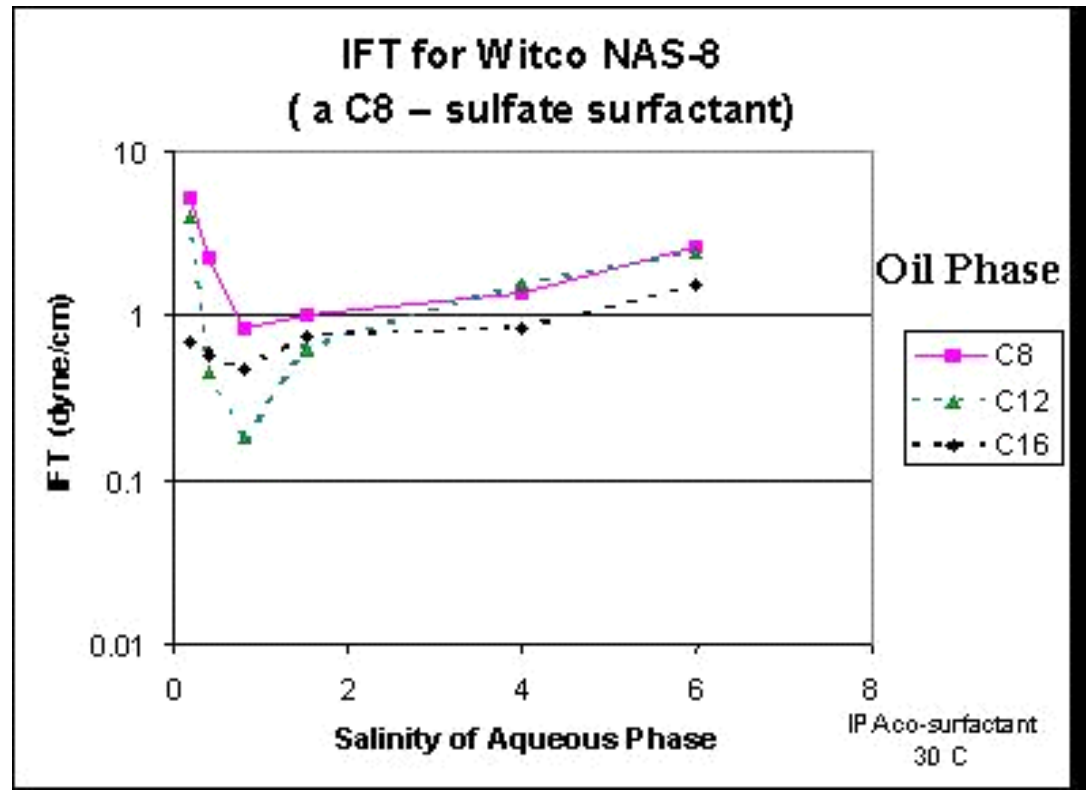

Figure 7.2.1.2. Octyl sulfate surfactant IFT versus salinity for different n-alkane hydrocarbons as the oil phase

Figure 7.2.1.2 illustrates that the IFT is sensitive to the choice of the hydrocarbon phase. In this case, the n-dodecane, the C12, hydrocarbon exhibits the lowest IFT for the measured data. It is somewhat surprising that "optimal salinity", the salinity at which there is a minimum in IFT, does not change obviously with a change in the oil phase composition $(0.8 \% \mathrm{NaCl}$ for all cases). Based on behavior of alkyl benzene sulfonate surfactants we would have expected to see an increase in the optimal salinity as the hydrocarbon phase increases in chain length from $\mathrm{C} 8$ to $\mathrm{C} 16$. Perhaps if there were more data around the $0.8 \% \mathrm{NaCl}$ samples we would see this effect.

The structures for three different esters of sulfosuccinic acid are shown below. The difference among these surfactants is the length of the alkyl chain.<smiles>[R]OC(=O)C(S)CC(O)O[R]</smiles>

Aerosol MA-80 R = branched C6 Aerosol OT-B $\mathrm{R}=$ branched C8 Aerosol TR-70 $\mathrm{R}=$ linear $\mathrm{C} 13$

Figure 7.2.1.3. Structure of Aerosol series of surfactants 


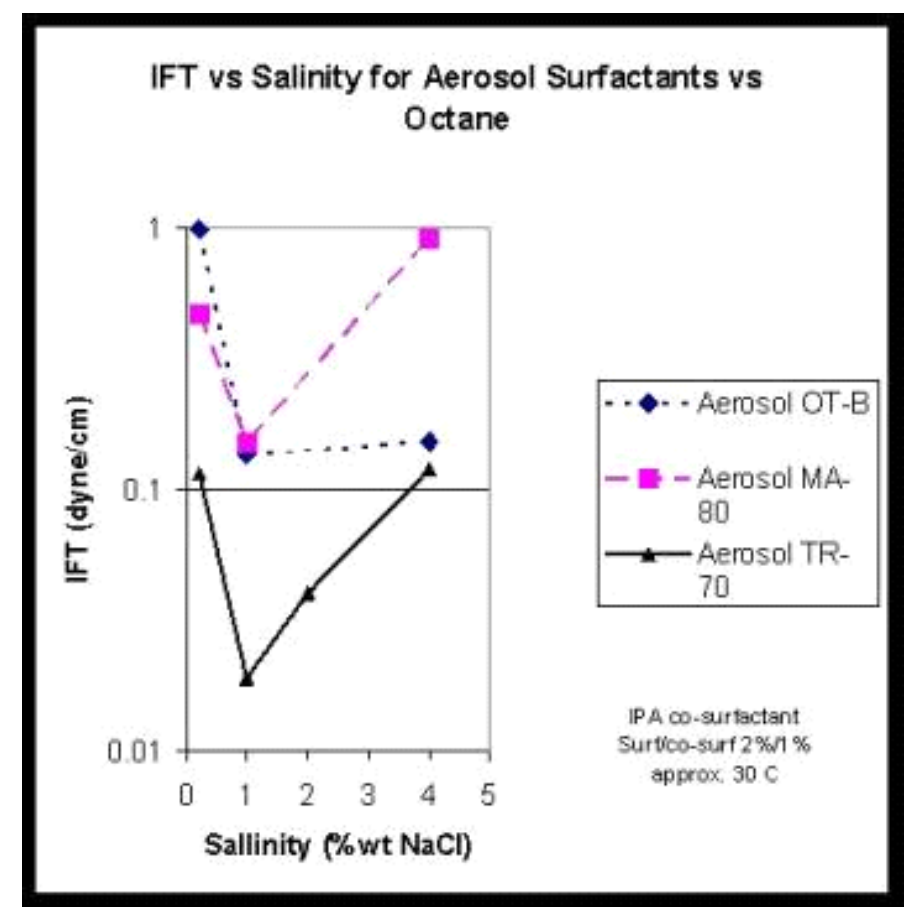

Figure 7.2.1.4. IFT for a series of esters of sulfosuccinic acid versus n-octane.

The Aerosol TR-70, the surfactant with the longest R group shows the lowest IFT. Again, additional IFT measurements could be beneficial to pinpoint if there is a difference in the optimal salinity and to locate better the true minimum IFT's.

The Zonyl series of surfactants are unusual because they have fluorine instead of hydrogen in the alkyl chains. The vendor claims these products can have a minimum in surface tension at very low concentration, and also will water wet surfaces very efficiently. These properties, plus the quite different chemical make up of these products make them intriguing from both an experimental and theoretical standpoint.

The structure for two Zonyl fluorosurfactants and an analog alkyl ethoxylate surfactant (Neodal 11-9) are shown below.

FSN:

$$
\mathrm{R}_{\mathrm{f}} \mathrm{CH}_{2}-\mathrm{CH}_{2}-\mathrm{O}-\left(\mathrm{CH}_{2} \mathrm{CH}_{2} \mathrm{O}\right)_{\mathrm{x}} \mathrm{H}
$$

$\mathrm{R}_{\mathrm{f}}$ - fluorinated alkyl chain: $\mathrm{F}\left(\mathrm{CF}_{2} \mathrm{CF}_{2}\right)_{\mathrm{y}} ; \mathrm{X}=0$ to about $25 ; \mathrm{Y}=1$ to about 9.

$$
\text { FSN: } \quad \mathrm{R}_{\mathrm{f}} \mathrm{CH}_{2}-\mathrm{CH}_{2}-\mathrm{O}-\left(\mathrm{CH}_{2} \mathrm{CH}_{2} \mathrm{O}\right)_{\mathrm{x}} \mathrm{H}
$$

$$
\mathrm{R}_{\mathrm{f}} \text { - fluorinated alkyl chain: } \mathrm{F}\left(\mathrm{CF}_{2} \mathrm{CF}_{2}\right)_{\mathrm{y}} ; \mathrm{X}=0 \text { to about } 15 ; \mathrm{Y}=1 \text { to about } 7 \text {. }
$$

Neodol 11-9:

$$
\mathrm{R}_{\mathrm{h}} \mathrm{CH}_{2}-\mathrm{CH}_{2}-\mathrm{O}-\left(\mathrm{CH}_{2} \mathrm{CH}_{2} \mathrm{O}\right)_{\mathrm{x}} \mathrm{H}
$$

Rh: $\mathrm{H}\left(\mathrm{CH}_{2} \mathrm{CH}_{2}\right)_{y}, \mathrm{X}=$ about $8, \mathrm{y}=$ about 5; average of 11 carbons in alkyl chain.

Figure 7.2.1.5. Structure of Zonyl flourosurfactnts and Neodol surfactant 
We find in fact though that the IFT values are quite high with the Zonyl products (see Table below). The IFT is a bit lower when we consider the analog "conventional" surfactant (Neodol 11-9) that has hydrogen in the alkyl chain instead of fluorine.

Table 7.2.1.2. Measured IFT results with flurosurfactants

\begin{tabular}{|c|c|c|}
\hline Surfactant (1 wt\%) & Salinity (wt\% NaCl) & IFT (dyne/cm) \\
\hline$\overline{\text { Zonyl-FSN }}$ & $\begin{array}{l}0 \\
0\end{array}$ & 9.5 \\
\hline Zonyl-FSN & 1 & 7.8 \\
\hline Zonyl-FSO & 0 & 4.5 \\
\hline Zonyl-FSO & 1 & 4.8 \\
\hline Neodol 11-9 & 0 & 2.4 \\
\hline Neodol 11-9 & 1 & 2.3 \\
\hline
\end{tabular}

Another feature is that the measured IFT for all of these nonionic surfactants is almost the same with the $1 \mathrm{wt} \%$ salinity as with fresh water.

Lastly in this initial screening study, we present some results from experiments performed with so-called "gemini" surfactants; these chemicals have two head and tails groups. These chemicals have become an active topic of research study recently (Zana, 2002). Gemini or (dimeric) surfactants have the interesting property of often having a CMC (critical micelle concentration) that is orders of magnitude lower than conventional surfactants and being much more efficient in reducing the surface tension of water. This may translate to low IFT at very low surfactant concentrations. Some gemini surfactants with short spacers may build a very high viscosity at relative low concentrations. This may be useful in chemical flood design where the conventional approach is to rely on added polymer to create a sufficient viscosity (circa $5-100 \mathrm{cp}$ ) to displace mobilized oil and not have undesirable viscous fingering. Thus, conceivably, the right gemini surfactant could create a target aqueous phase viscosity and not require adding (as much) polymer to the injected fluids.

We performed IFT experiments with some commercially available gemini surfactants manufactured by Air Products; the structures are given below:

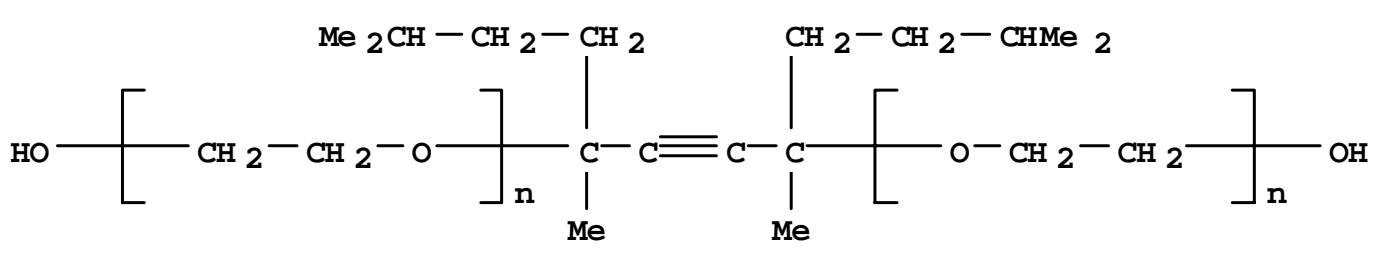

Dynol-604 Polyethylene glycol 2,5,8,11-tetramethyl-6-dodecyne-5,8-diol ether 


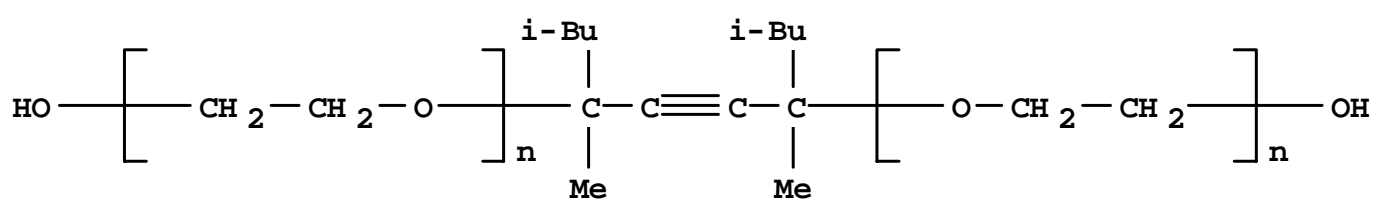

Surfynol -440 and Surfynol-465

2,4,7,9-TETRAMETHYL-5-DECYNE-4,7-DIOL ETHOXYLATE

Figure 7.2.1.6. Structure of commercial gemini surfactants evaluated

The Surfynol-440 and -465 differ by the number of ethoxylate groups. The Surfynol-440 is water dispersible, whereas the Surfynol-465 has more ethoxylate groups and is more water soluble.

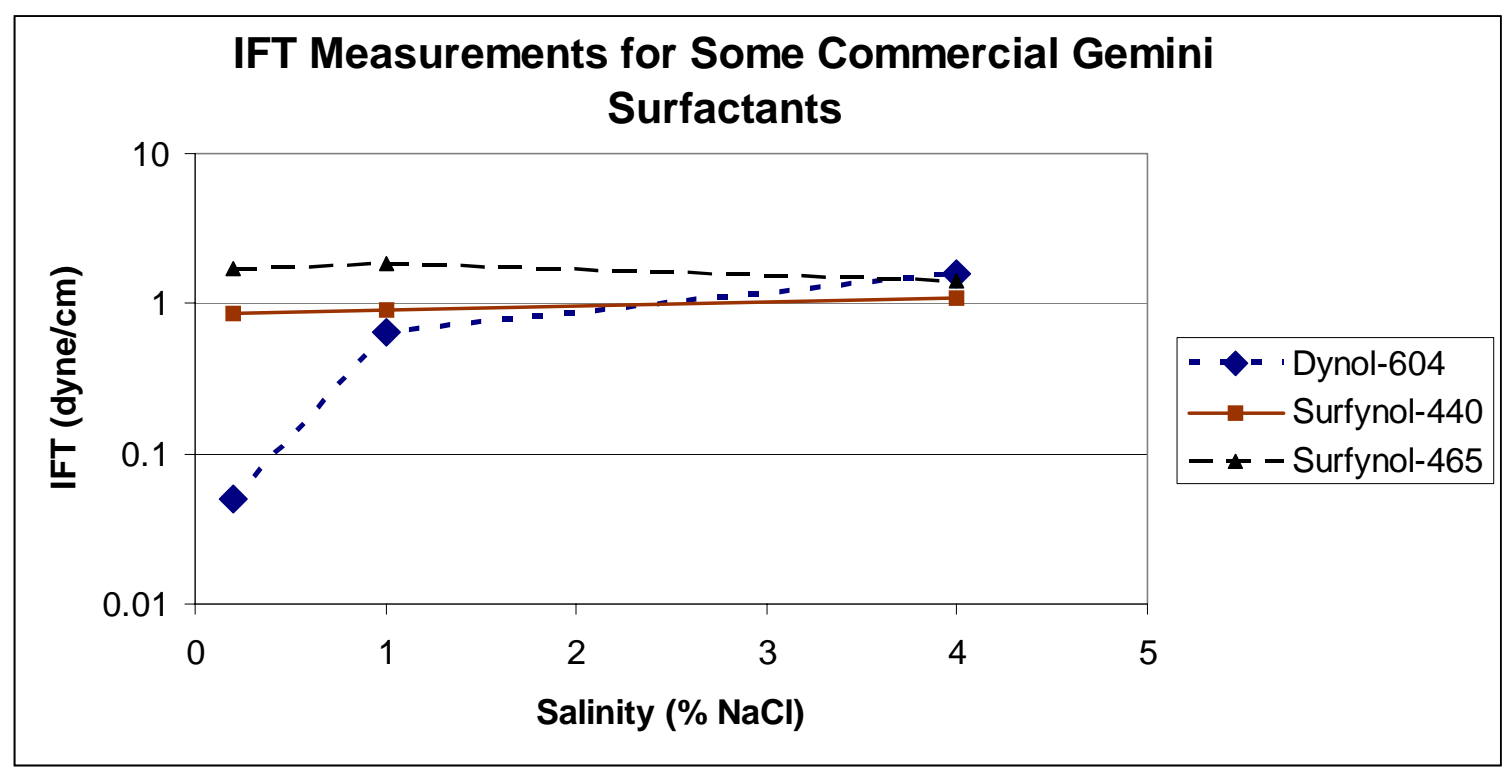

Figure 7.2.1.7. Measured IFT for different commercial gemini surfactants versus salinity. The oil phase is n-octane in all cases.

All of these products have some interfacial activity, and the Surfynol-440 shows some promising performance. Future studies could determine if the relatively low IFT's might still occur at very low Surfynol-440 concentrations. (The vendor claimed these products have low $\mathrm{CMC}$, but did not provide quantitative data.).

\subsubsection{Initial Screening of Surfactants for Solid Adsorption Behavior}

Data measured for SDBS (dodeycl benzene sulfonate) adsorption levels onto kaolinite clay as a benchmark case is reasonable (several $\mathrm{mg}$ surfactant/gram of clay), being 
comparable to that reported for commercial petroleum sulfonate surfactants (see Figure 7.2.2.1). The tremendous surface area of the clays dictate that these species control solid adsorption in sandstone reservoirs (surface area of quartz is much less). Because the actual concentrations of clays in oil reservoirs are typically just a few percent of the total solids composition, kaolinite adsorption of several $\mathrm{mg} / \mathrm{gm}$ would translate to be roughly $0.1-1 \mathrm{mg}$ surfactant/gram reservoir rock in most oil reservoirs.

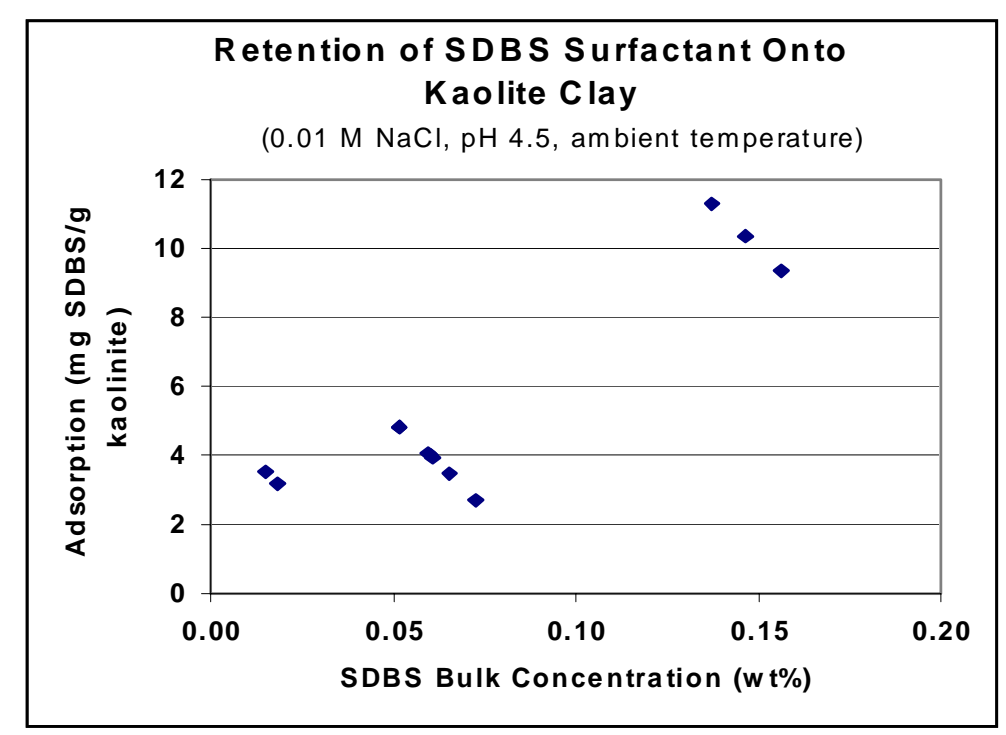

Figure 7.2.2.1. Measured retention of SDBS onto kaolinite clay.

Appendix A presents a summary list of adsorption results generated for a number of nonionic surfactants. These data demonstrate some trends concerning tendency for solid adsorption that relate the surfactant properties and structure to adsorption behavior.

The Pluronic series are block co-polymers of $\mathrm{EO}$ - $\mathrm{PO}$ - EO nonionic surfactants..

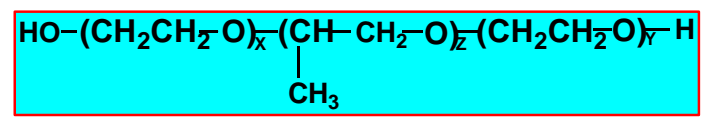

\begin{tabular}{|c|c|c|c|}
\hline Surfactants & $E O(X+Y)$ & $P O(Z)$ & $M . W$. \\
\hline Pluronic L-31 & 3 & 16 & 1078 \\
\hline Pluronic L-44 & 22 & 21 & 2204 \\
\hline Pluronic L-61 & 7 & 29 & 2008 \\
\hline Pluronic L-62 & 14 & 32 & 2409 \\
\hline Pluronic L-64 & 26 & 30 & 2902 \\
\hline Pluronic L-72 & 16 & 35 & 2752 \\
\hline Pluronic L-81 & 8 & 41 & 2748 \\
\hline
\end{tabular}

\begin{tabular}{|l|c|c|c|}
\hline Surfactants & $E O(X+Y)$ & $P O(Z)$ & $M . W$. \\
\hline Pluronic L-31 & 3 & 16 & 1078 \\
\hline Pluronic L-44 & 22 & 21 & 2204 \\
\hline Pluronic L-61 & 7 & 29 & 2008 \\
\hline Pluronic L-62 & 14 & 32 & 2409 \\
\hline Pluronic L-64 & 26 & 30 & 2902 \\
\hline Pluronic L-72 & 16 & 35 & 2752 \\
\hline Pluronic L-81 & 8 & 41 & 2748 \\
\hline
\end{tabular}

Figure 7.2.2.2. Details of structure for Pluronic series of nonionic surfactants 
The molecular weights vary from a few hundred to a few thousand. There is a general trend of increasing adsorption with an increase in the ratio of $\mathrm{PO} / \mathrm{EO}$ groups (an increase in hydrophobicity). Note that all of these adsorption tests were performed with the Pluronic nonionic surfactants dissolved in $2 \mathrm{wt} \% \mathrm{NaCl}$, and the weight ratio of surfactant solution/solid was 20 .

The figure below summarizes adsorption results for this series of Pluronic surfactants.

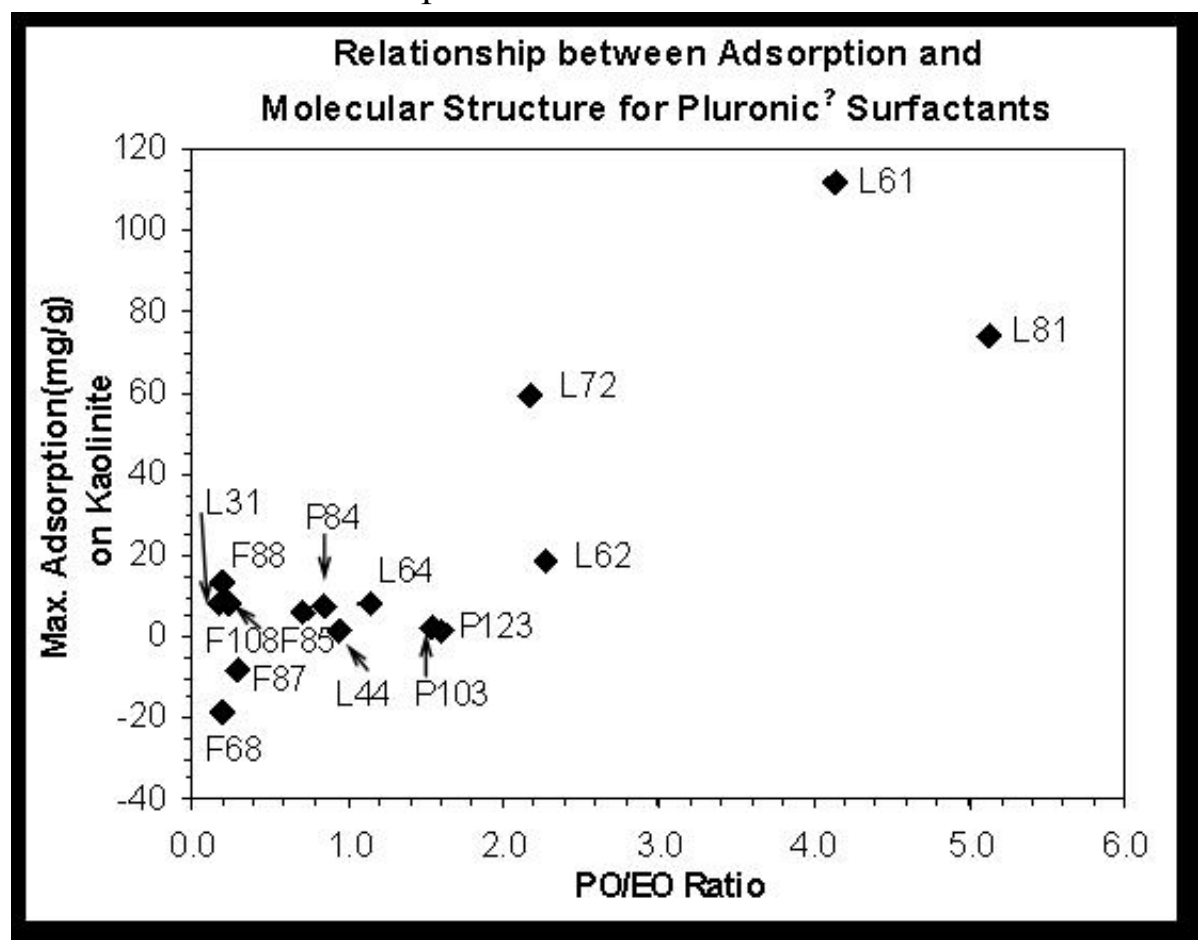

Figure 7.2.2.3. Increase in mass adsorption of Pluoronic products with an increase in the $\mathrm{PO} / \mathrm{EO}$ ratio. The symbols are marked with the product code of these nonionic surfactants.

Another example is shown below for the Igepal series of nonionic surfactants (ethoxylated nonylphenols). Their average structure may be represented by
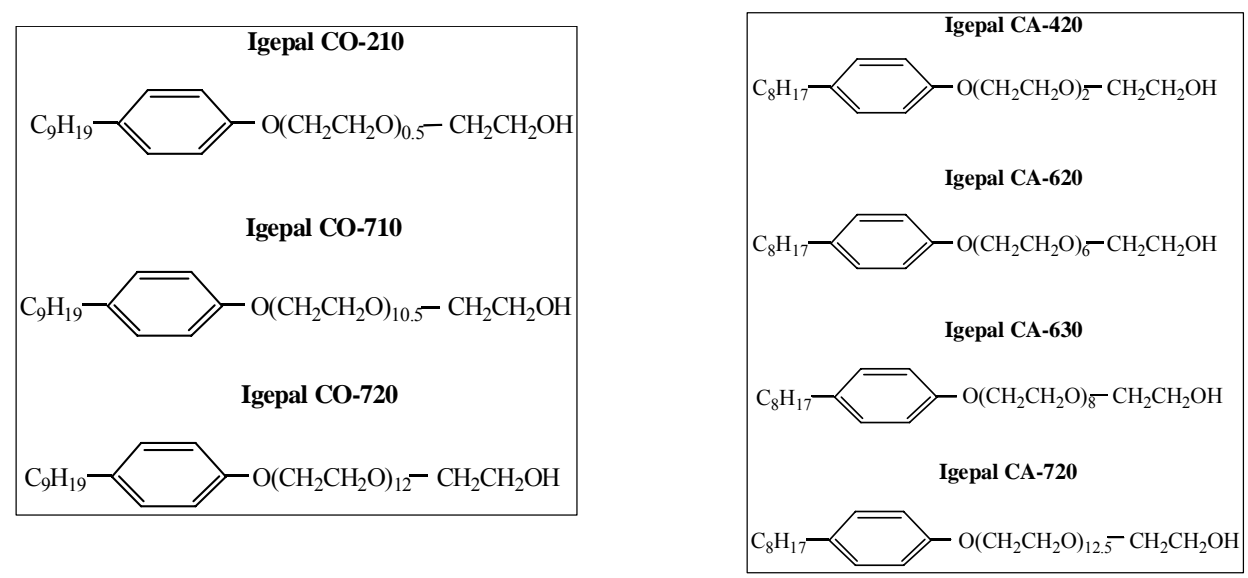

Figure 7.2.24. Structure details for the Igepal series of surfactants studied. 


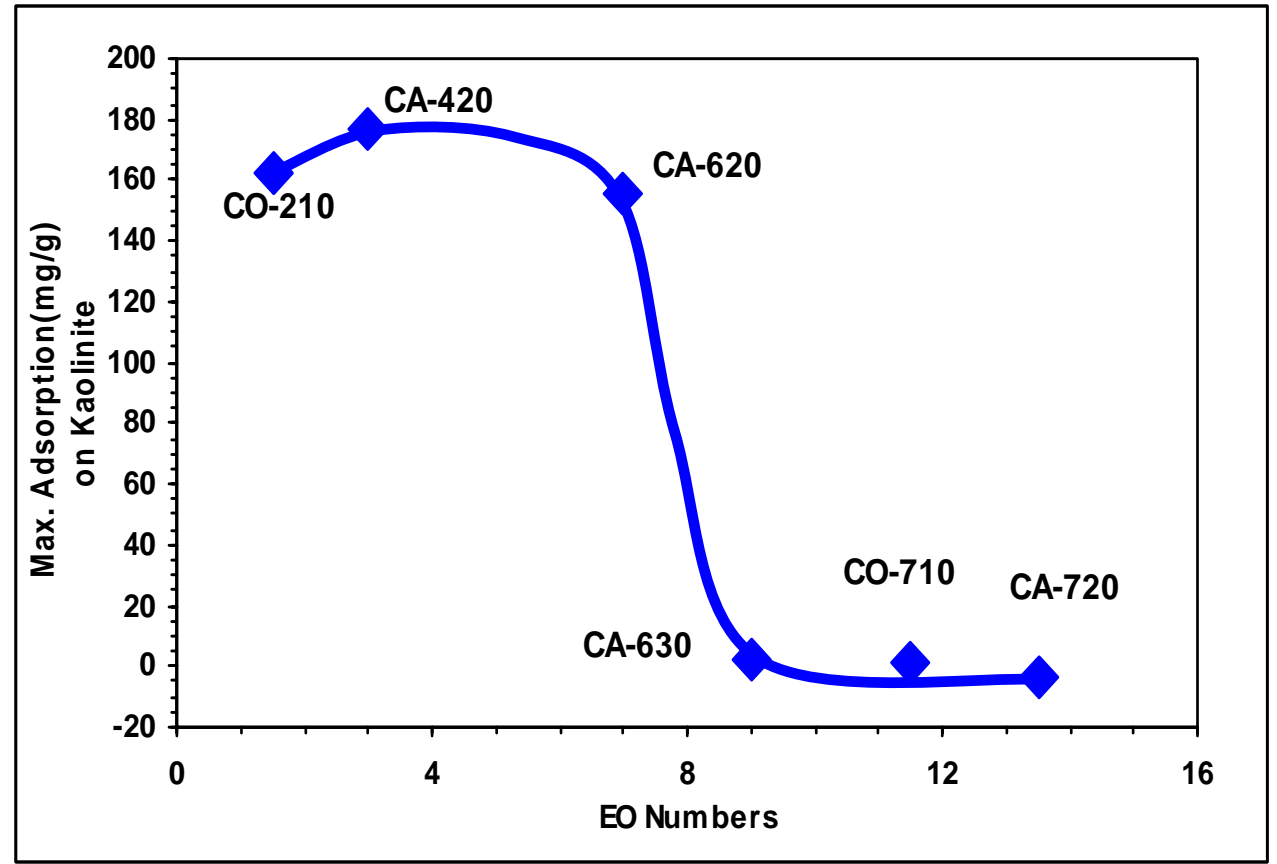

Figure 7.2.2.5. Solid adsorption of a series of Igepal surfactants onto kaolinite clay.

Presumably the decrease in solid adsorption with an increase in EO groups is related to its increasing brine solubility. The large drop in adsorption going from 7 to $9 \mathrm{EO}$ groups coincides with making the solution go below the cloud point (make a clear solution) under these test conditions.

\subsection{More Detailed Study for Calamide CW-100}

One of the better surfactants found in this initial screening process was Calamide CW100. This is a coconut diethanolamide supplied by Pilot Chemical.

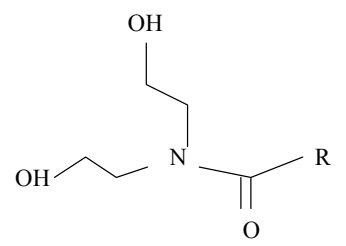

R predominantly $\mathrm{C} 12-\mathrm{C} 14$

In particular, the IFT test results are encouraging, as reported below. (These IFT measurements are with the default test conditions -- $2 \mathrm{wt} \% \mathrm{CW}-100$ surfactant and 1 $\mathrm{wt} \%$ isopropanol cosurfactant in a $\mathrm{NaCl}$ solution versus $\mathrm{n}$-octane $(\mathrm{W} / \mathrm{O}=1)$ after allowing a few weeks time for phase equilibrium. 
Table 7.2.3.1 Measured IFT Between CW-100 Surfactant Formulation and n-Octane

\begin{tabular}{cc} 
Salinity (wt \% NaCl) & IFT (dyne/cm) \\
\hline 0.2 & 0.05 \\
1 & 0.02 \\
4 & 0.003
\end{tabular}

The patent literature mentions using dialkanolamides as part of a surfactant EOR system, with the intention of improving performance of alkyl aryl sulfonate surfactant systems in higher salinity brines ( Braden, 1977). There does not seem to be mention, however, of using a dialkanolamide as the main surfactant for mobilizing residual oil.

Other surfactants besides the Calamide CW-100 manufactured by Pilot Chemical were screened for their IFT performance. These are listed below with measured IFT values for the test conditions -- $1 \mathrm{wt} \%$ surfactant in a $2 \mathrm{wt} \% \mathrm{NaCl}$, oil phase is n-octane, $25^{\circ} \mathrm{C}$--

1. Calfax 10L-45:

$\underline{\text { Surfactant/Description }}$

$\underline{\text { IFT }(\text { dyne } / \mathrm{cm})}$

Sodium Linear Decyl Diphenyl Oxide Disulfonate, CAS\#: 36445-71-3 1.5

2. Calfax 16L-35:

Sodium n-Hexadecyl Diphenyl Disulfonate, CAS\#: 65143-89-7

5

3. Calfax DB-45:

Sodium Dodecyl Diphenyl Oxide Disulfonate, CAS\#:119345-04-9

4. Calsoft LAS-99:

0.8

Linear Dodecyl Benzene Sulfonic Acid(97\%), CAS\#: 27176-87-0

5. Calamide C:

0.3

Cocamide DEA* Supermide, CAS\#: 61791-31-9

6. Calsoft T-60:

0.7

Triethanolamine dodecylbenzene Sulfonate (60\%), CAS\#: 248-406-9

7.. Calimulse EM-22:

0.5

Sodium Branched Dodecylbenzene Sulfonate (22\%)

Measured kaolinite adsorption results for the Calamide CW-100 surfactant are somewhat high, showing a maximum adsorption of about $17 \mathrm{mg} / \mathrm{gm}$. Typical petroleum sulfonates would be expected to be around $10 \mathrm{mg} / \mathrm{grm}$ in this adsorption test. The remaining surfactant concentration in solution, and hence the depletion of surfactant from solution (mass lost by adsorption), was determined via UV absorbance. 


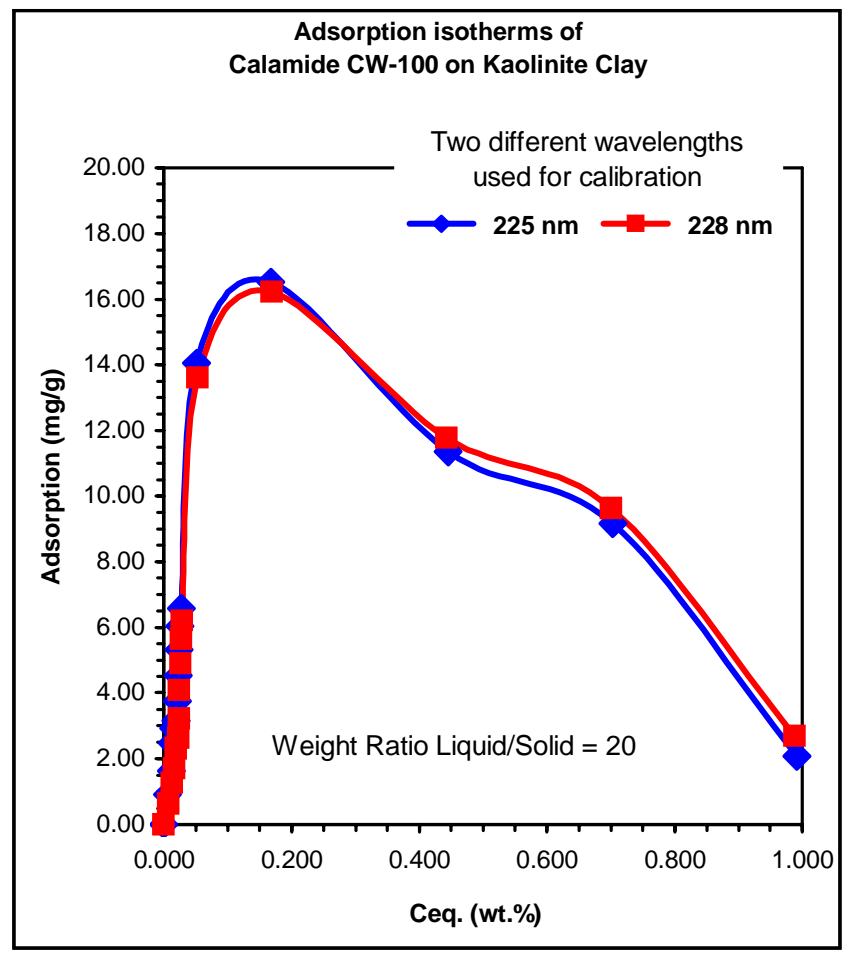

Figure 7.2.3.1. Adsorption isotherm for Calamide CW-100 onto kaolinite clay at $25 \mathrm{C}$.

Two oil displacement Berea coreflood experiments were performed to contrast the behavior of the Calamide CW-100 and one of the first promising surfactants screened for IFT behavior, the tri-deycl ester of sulfosuccinic acid, Aerosol TR-70.

The Calamide CW-100 surfactant exhibited lower IFT behavior in conjunction with test tube/phase behavior studies than the Aerosol TR-70 surfactant (minimum IFT of 0.002 versus 0.02 dyne $/ \mathrm{cm}$ ). The $\mathrm{CW}-100$ surfactant also is intriguing in that relatively low IFT was observed for higher salinity conditions $(4 \% \mathrm{NaCl})$ in test tube/phase behavior/IFT studies. Such products could be especially attractive as many reservoirs in the U.S. that might be good candidates for surfactant EOR that have a formation brine that has several percent or more concentration of dissolved salts.

Consistent with the measured IFT behavior, the Calamide CW-100 recovered about $75 \%$ of the waterflood residual oil (n-decane) from a 200 md Berea core, whereas the Aerosol TR-70 surfactant only recovered $15 \%$ of the trapped oil (Figure 7.2.3.2). 


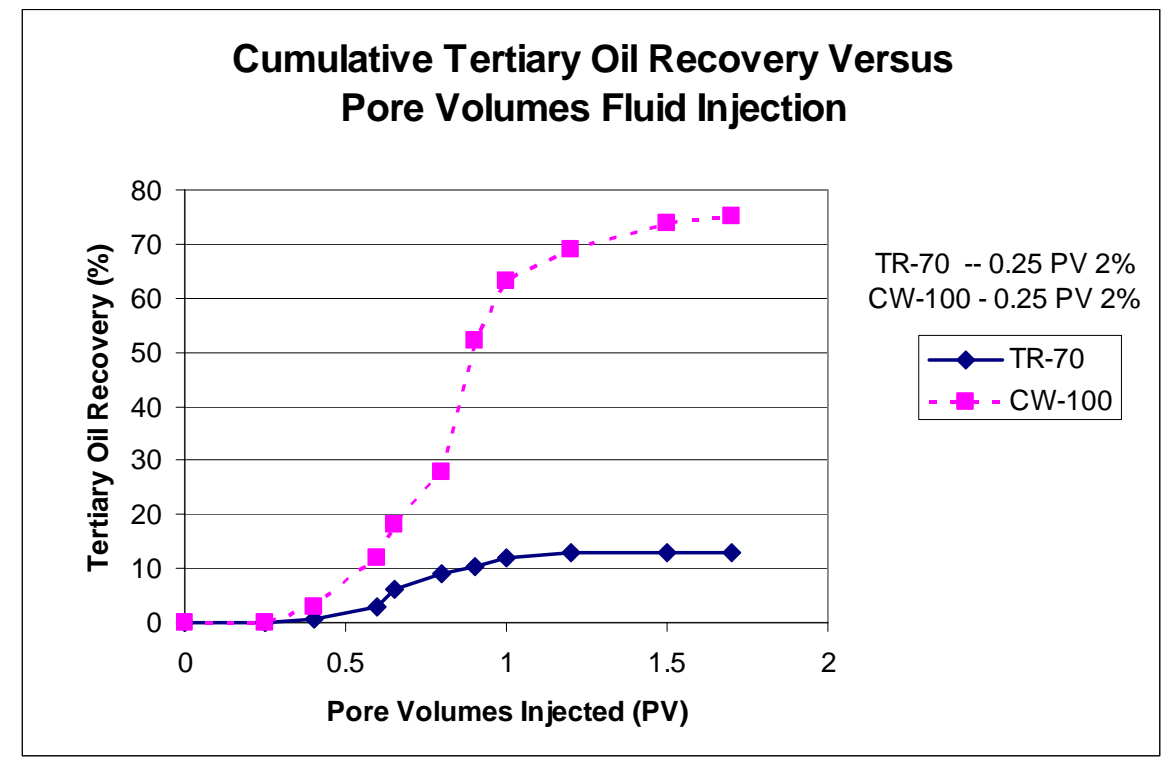

Figure 7.2.3.2. Tertiary oil recovery for CW-100 and TR-70 in Berea sandstone coreflood experiments.

Some details about the coreflood experiments (see above figure for results).

Berea sandstone cores; approximately $200 \mathrm{md}$ water permeability, 1" x 12" core

All steps at room temperature

Oil phase: n-decane

Waterflood residual oil saturation: 0.34 ;

Connate brine composition $1 \mathrm{wt} \% \mathrm{NaCl}, 10 \mathrm{ppm} \mathrm{Ca}$

Aerosol TR-70 or Calamide $\mathrm{CW}-100$ formulations in $1 \mathrm{wt} \% \mathrm{NaCl}$ :

$2 \%$ surfactant

$1 \%$ iso-propanol co-surfactant

0.25 PV slug

Drive polymer solution:

500 ppm Alcoflood 1235 (Ciba Corp.) in $1 \mathrm{wt} \% \mathrm{NaCl}$.

0.5 Pore Volume

Brine Drive:

$1 \mathrm{wt} \% \mathrm{NaCl}$

1 Pore Volume

Chemical injection at $0.06 \mathrm{ml} / \mathrm{min}$, or about a $3 \mathrm{ft} /$ Day frontal advance rate.

A second coreflood with Calamide $\mathrm{CW}-100$ was performed under identical conditions, except now the connate water salinity was increased to $4 \mathrm{wt} \% \mathrm{NaCl}$. Again, the tertiary recovery was $75-80 \%$ of the $n$-decane remaining trapped after a complete waterflood. 


\subsection{Alkyl Polyglycoside (APG) Surfactants - Background}

\subsubsection{Motivation for their study}

Based on our experimental results with $\mathrm{CW}$ and theoretical suggestions we decided to look into surfactants with large, non-ionic, polar head groups. Alkyl polyglycosides (APG) are nonionic surfactants with these characteristics. APG's are prepared with renewable raw materials, namely starch and fat or their derivative glucose and fatty alcohols. A typical APG structure is shown below:

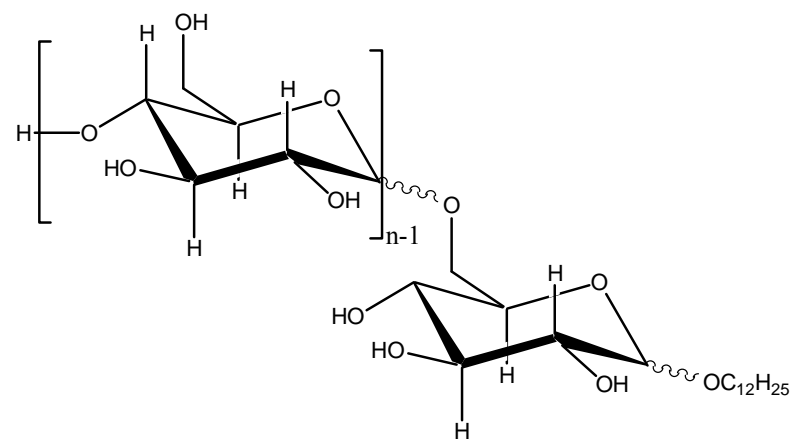

Figure 7.2.4.1. Molecular structure of a typical alkyl polyglycoside (APG)

The degree of polymerization is actually quite low, with $\mathrm{n}$ usually between 1.1 and 3 (n typically ranges from $1.2-1.5$, meaning that mono- and di-head groups predominate). Commercial APG products have a mixture of molecular structures, not only in terms of the number distribution of the head groups, but also in the number of alkyl groups in the hydrophobic tail.

APG surfactants were described initially over 100 years ago, first recognized as a potentially useful surfactant type in 1936, and then largely ignored until the 1980's. APG has gained favor as economical processes were developed to manufacture them, and there has been an increased drive to use surfactants with favorable, low toxicity like APG. This surfactant now sees widespread use in household detergents, cosmetics, and agricultural products (Balzer, 1991a). A recent (1999) estimate for worldwide capacity for APG surfactants is 80,000 tons/year (Hill and Rhode, 1999). APG has been considered riefly for EOR applications, with one U.S. patent issued (Balzer, 1991b).

\subsubsection{APG Surfactants Potential Advantages as EOR Agents}

The APG formulations have some interesting and potentially useful properties as EOR agents; literature reports state for APG formulations in contact with a hydrocarbon phase (Balzer, 1996):

1. APG do not seem to form middle-phase microemulsions by themselves.

2. However, when mixed with a hydrophobic cosurfactant (e.g. an alcohol or another surfactant), a middle-phase microemulsion may appear, and in some cases it may create a low IFT ( 0.01 dyne/cm or less). Some encouraging phase behavior/IFT 
data are reported with simple n-alkanes as the oil phase (Balzer, 1991, Balzer, 1996, Kutschmann, 1995, and Forster, T., 1996).

3. A remarkable property for these APG formulations is that they are reported to have a phase behavior and IFT that is largely independent of temperature and salinity. Surfactant formulations that create a low IFT irrespective of temperature and salinity would be a very useful property for oilfield EOR applications.

Theoretical/modeling aspects also indicate that having this large head group and the nonionic character of the APG molecule is consistent with the observation of the phase behavior and IFT being largely indifferent to changes in the temperature and the salinity.

Other motivations for having a focus on APG surfactants are:

- They are available as commercial products and used already in significant quantities for other industrial applications. Hence their cost and availability are reasonable.

- Because they may be formulated to have behavior not very dependent on temperature and salinity, their behavior should be less complicated to study with available theoretical tools.

- The behavior observed for the combination of APG surfactants and various cosurfactants offer trends and insights as to the chemical structure and ability to reduce IFT. This information provides test data for the theoretical models and suggests other surfactant variations.

- APG surfactants serve as a practical example of a sugar-based surfactant. There are of course, thousands of other possible sugar-based surfactant structures.

- Related to the above, APG products being sugar-surfactants, their behavior offer some insights into the best application of bio-surfactants (Peypoux, 1999). This work may suggest improvements in microbial enhanced oil recovery technology.

The interested reader may refer to the published papers in Appendix A that summarize the experimental studies performed with APG surfactants as part of this project.

\subsubsection{Alkyl Polyglycosides (APG) Formulated with n-Alcohols as Cosufactant}

\subsubsection{IFT of APG/n-Alcohol Formulations}

We used 3 different commercial APG products supplied by Cognis Corporation:

Average

\begin{tabular}{llcccc} 
Product & \multicolumn{2}{l}{ Alkyl Chain } & Chain Length & Average n & HLB \\
\hline PG 2067 & $8 / 10$ & $(45: 55)$ & 9.1 & 1.7 & 13.6 \\
PG 2069 & $9 / 10 / 11$ & $(20: 40: 40)$ & 10.1 & 1.6 & 13.1 \\
PG 2062 & $12 / 14 / 16(68: 26: 6)$ & 12.5 & 1.6 & 11.6
\end{tabular}

The HLB (hydrophile-lipophile balance) of a surfactant refers to its behavior in creating emulsions and is related to its oil/water solubility. Higher HLB products such as those found for these APG surfactants mean they tend to be relatively water soluble. Alcohol co-solvents evaluated include several n-alcohols ranging from C3 to C20. 
The base case for these series of phase behavior/IFT tests is n-octane as the hydrocarbon phase. As explained in the experimental procedure, we focus first on n-octane as the model hydrocarbon phase because it should be a good proxy for an "average" crude oil. The aqueous phase has $2 \mathrm{wt} \%$ combined APG/cosurfactant concentration and has a default brine salinity of $2 \mathrm{wt} \% \mathrm{NaCl}$. The oil and aqueous surfactant solutions are mixed at a $1 / 1$ volume ratio and equilibrated at ambient temperature. The graphs below show IFT results with the PG 2062 APG surfactant and different n-alcohols.

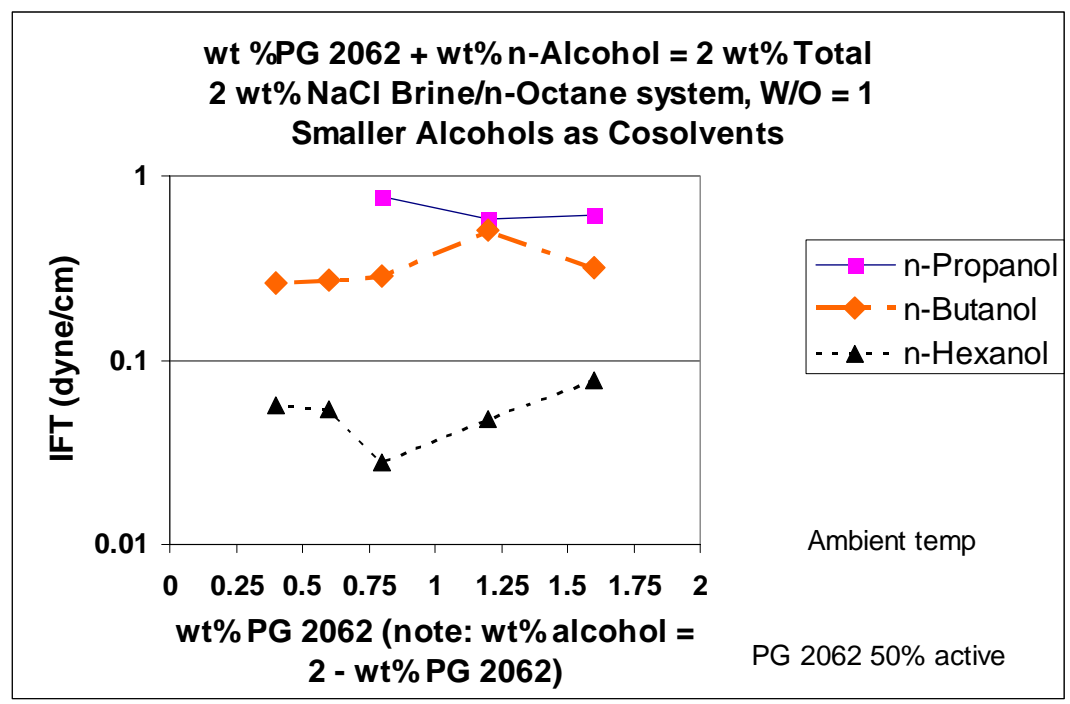

Figure 7.2.5.1a. IFT measured for equilibrated samples containing PG 2062 and smaller n-alcohols versus n-octane as the hydrocarbon phase.

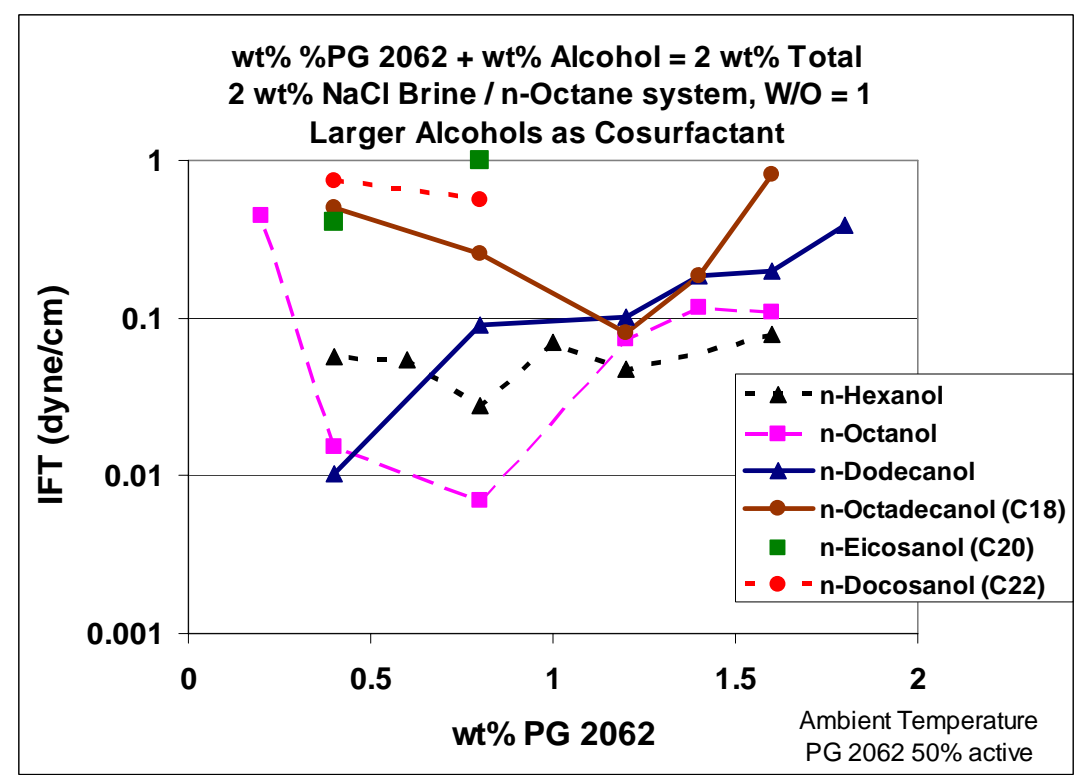

Figure 7.2.5.1b. IFT measured for equilibrated samples containing PG 2062 and larger n-alcohols versus n-octane as the hydrocarbon phase.

Note that the IFT for PG 2062 alone is about 2 dyne/cm, and for an n-alcohol alone the IFT is over several dynes $/ \mathrm{cm}$, perhaps even greater than $30 \mathrm{dynes} / \mathrm{cm}$. One explanation 
for the synergistic action of the added alcohols is that they pack at the interface so as to decrease the curvature of the interfacial layer and thereby reduce the IFT. Perhaps the notion of a "hydrophobic linker" is a good physical model for the action of these cosurfactants (Sabatini, 2001), that is, an additive for linking the oil and surfactant molecules better at the interface.

Some other comments about these results:

- There is an "optimal" alcohol cosurfactant (in this system n-octanol or perhaps n-decanol) that creates the lowest IFT condition. The lowest IFT measured was less than $0.01 \mathrm{dyne} / \mathrm{cm}$.

- Almost all of the samples indicated in Figure 7.2.5.1 had a third, middle-phase, even if only a small volume. Even the samples with PG 2062 mixed with the most unfavorable cosurfactant (n-propanol) has at least a small middle phase.

- The IFT behavior versus the amount of APG and n-alcohol are fairly constant. This suggests the desirable result that the low IFT condition may be attained with even a modest concentration of APG surfactant. In addition, we see that changes in the APG/cosurfactant ratio may not adversely impact the IFT. Note, for example, the results with n-dodecanol as a cosurfactant; extrapolation suggests even lower IFT might be found at a PG 2062 concentration below $0.25 \mathrm{wt} \%$ (less than $0.125 \mathrm{wt} \%$ on an active basis).

Replicate spinning drop measurements of these samples with one of the lowest IFT conditions (PG 2062 and n-octanol formulation) had good reproducibility.

Table 7.2.5.1. Replicate Measurements at a Low IFT Condition

$\begin{array}{ccc}\frac{\text { Sample }}{65} & & \text { IFT }(\text { dyne/cm) } \\ 142 & & 0.007 \\ 143 & & 0.004 \\ & 0.007\end{array}$

Test Conditions:

aqueous phase: PG $20620.8 \mathrm{wt} \%$ (as is) and $1.2 \mathrm{wt} \% \mathrm{n}$-octanol in a $2 \mathrm{wt} \% \mathrm{NaCl}$ oil phase: n-octane

$\mathrm{W} / \mathrm{O}$ ratio $=1$; Phases equilibrated and IFT measured at room temperature

Figure 7.2.5.2 summarizes data that compares the IFT measured among the 3 different commercial APG surfactants. The trend is that increasing the alkyl chain length of the of the hydrophobic surfactant tail provides a lower IFT. That is, with regards to IFT, the performance of APG commercial products follows the sequence (lower to higher IFT)

PG $2062<$ PG $2069<$ PG 2067 


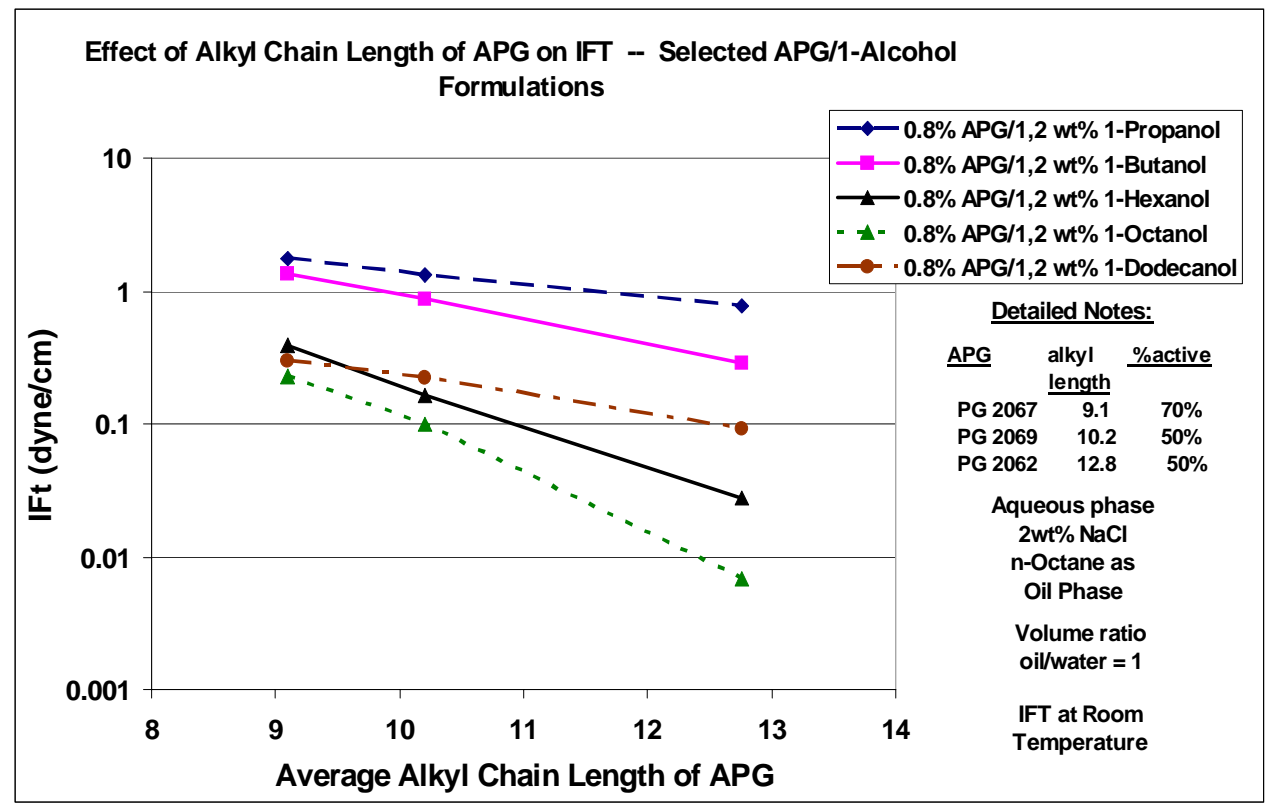

Figure 7.2.5.2. Increasing the alkyl chain length of the commerical APG surfactants decreases the IFT when blended with a 1-alcohol cosurfactant.

In addition, for all 3 of the APG surfactants, adding 1-octanol as the alcohol cosurfactant creates the lowest IFT for that APG product. The above plotted data are selected for only $\mathrm{APG} /$ alcohol blends with the weight concentration formulation of $0.8 \% / 1.2 \%$.

The results in Figure 7.2.5.3 present similar IFT data as above, but with a different perspective by plotting IFT against the alcohol carbon number. For the figure below we

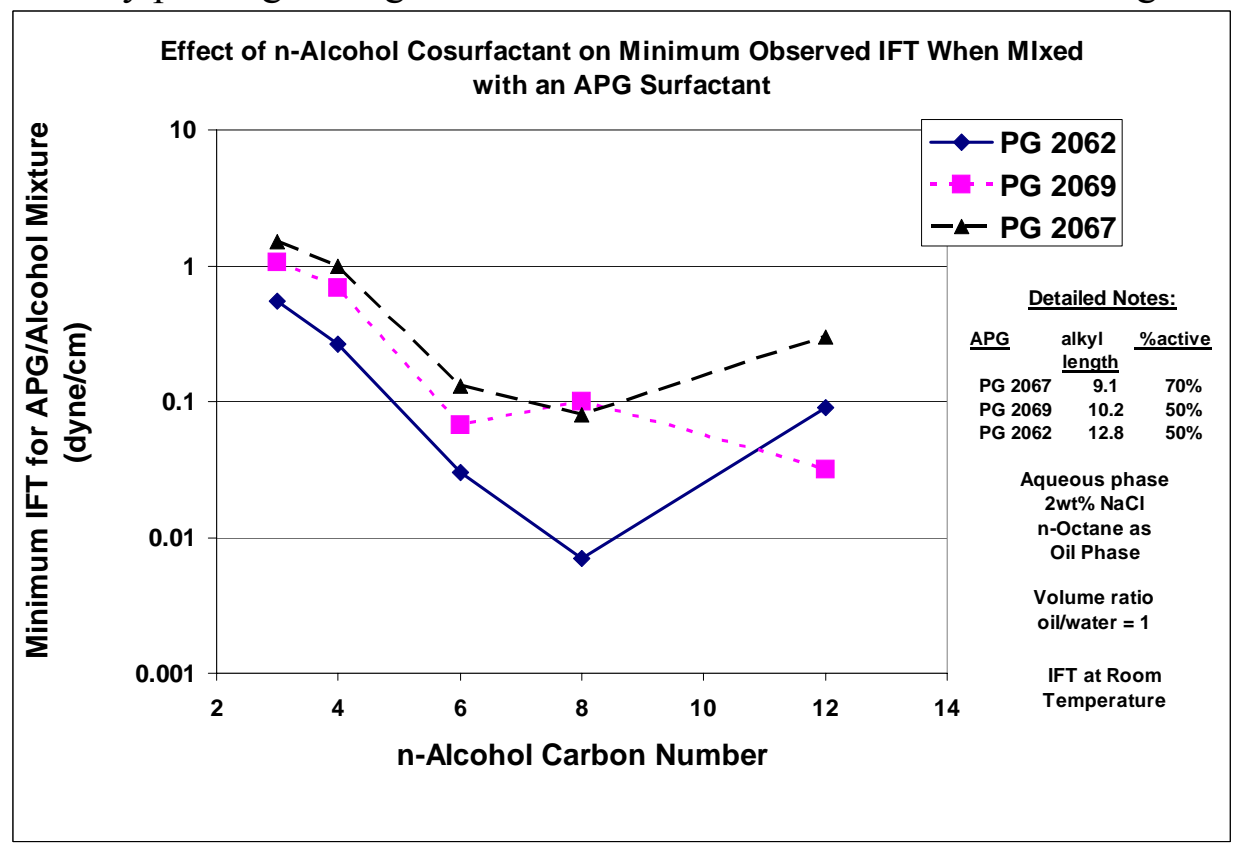

Figure 7.2.5.3. Experimental data of the minimum IFT measured for selected APG/alcohol formulations. 
plot the minimum IFT for each APG/alcohol blend. (That is, we report the minimum IFT we measured for that blend from the several mix ratios tested; some are not at the ratio 0.8/1.2 wt\%. we used for Figure 7.2.5.2). These IFT data in Figure 7.2.5.3 still indicate the longer alkyl chain APG product (PG 2062) provides the lower IFT, and that $\mathrm{n}$-octanol is about the best choice for a 1-alcohol cosurfactant at these process conditions.

Another variation of the APG chemistry we evaluated for phase behavior and IFT is the molecule shown below. The motivation for considering this n-hexadecyl-beata-Dmannose (HBDM) surfactant is that it has a longer (C16) alkyl chain than the commercial

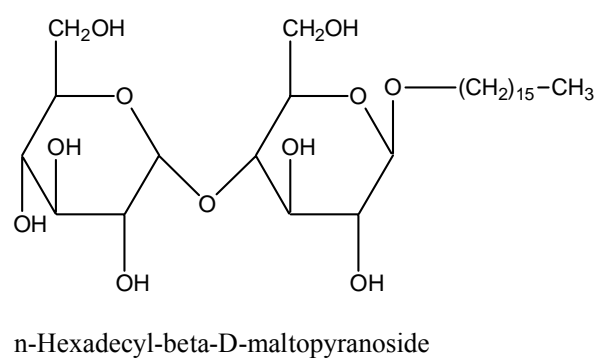

Figure 7.2.5.4. Molecular structure of mannose surfactant tested that has a C16 alkyl chain hydrophobic tail

APG products tested (mixtures with alkyl chains from $\mathrm{C} 8$ to $\mathrm{C} 14+$ ). The figure below shows the results of the IFT measurements for formulations with this HBDM surfactant and a series of $n$-alcohols included as a cosurfactants.

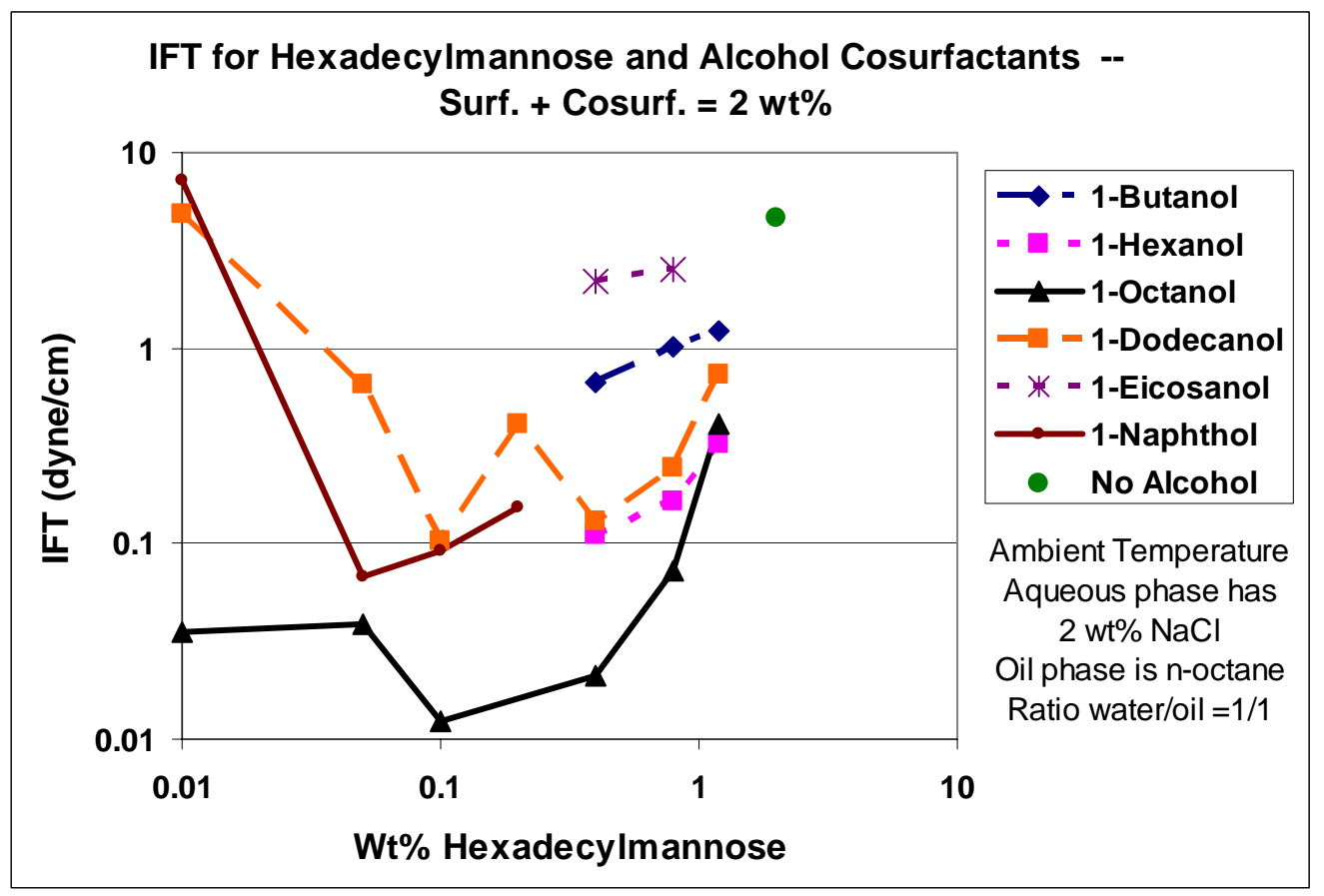

Figure 7.2.5.5. Measured IFT results for n-hexadecyl-beta-D-mannose (HBDM) surfactant with a series of different alcohol cosurfactants 
Comparing the above results for the hexadecylmannose surfactant (HBDM) to the commercial APG products (Figure 7.2.5.1) suggest the lowest IFT occurs still with the PG 2062 product (average alkyl chain of C12.5). It is conceivable that this chain length is about the optimum alkyl chain length for this surfactant and n-octane as the oil phase at these process conditions and that the $\mathrm{C} 16$ alkyl chain of the mannose surfactant molecule is too long. On the other hand, it could also be argued that this hexyldecylmannose surfactant head group is too different from the APG type to be able to base a comparison of differences on IFT behavior just on the effect of the alkyl chain length. Yet another difference is that the commercial APG products are a mixture of surfactants whereas the hexadecylmannose almost a single structure. It could be that the mixture of surfactants would be able to disrupt the water/oil interface better, and thereby reduce IFT more.

Figures 7.2.5.6 and 7.2.5.7 present results that are consistent with the reported literature trend the desirable attributes of maintaining nearly constant IFT in spite of significant changes in the temperature and the salinity. In particular, note that the IFT remains at the same low level in Figure 7.2.5.6, even if the salinity is increased to $10 \% \mathrm{NaCl}$ and includes significant hardness $\left(1 \% \mathrm{CaCl}_{2}\right.$.added $)$. This suggests APG's as candidates for higher salinity EOR applications.

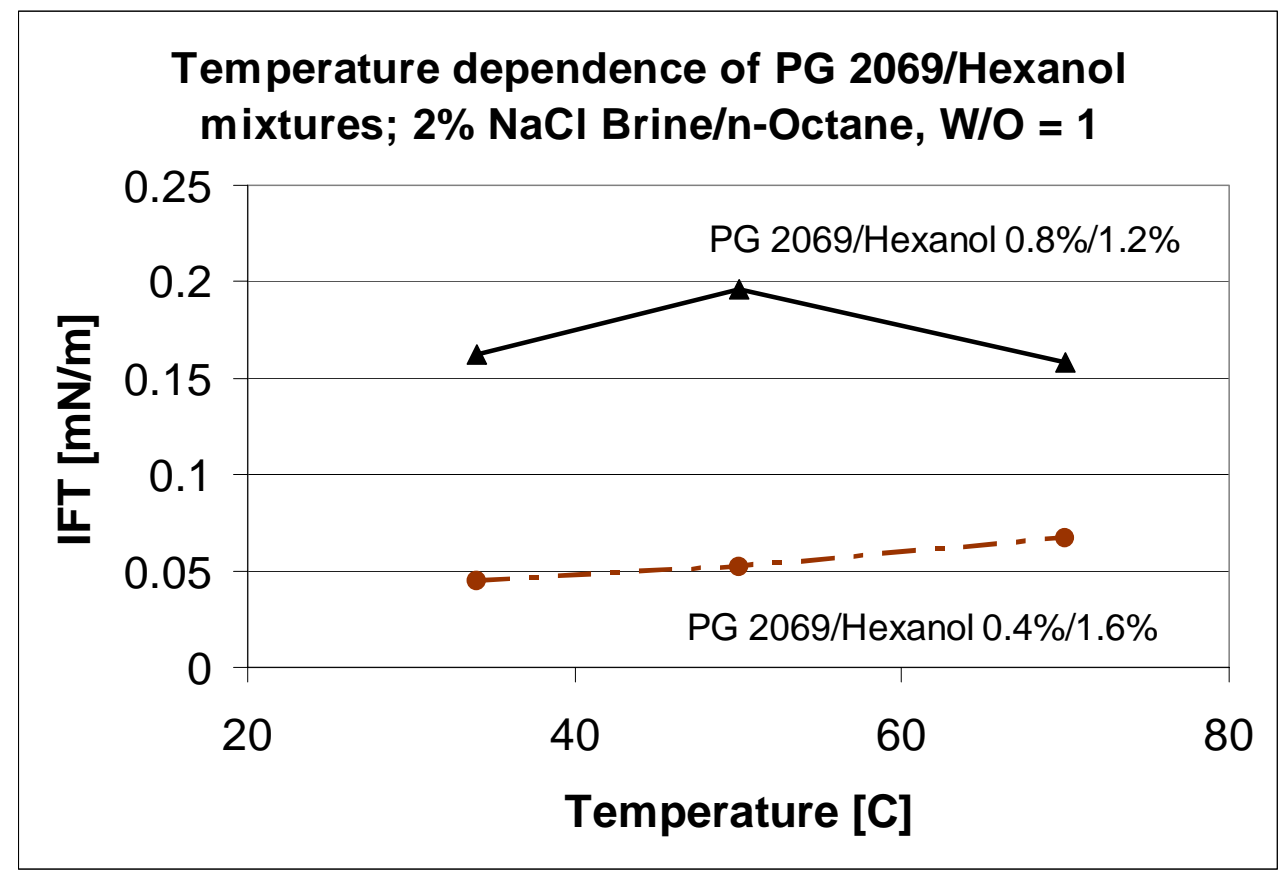

Figure 7.2.5.6. Data illustrating IFT is nearly independent of temperature for a mixture of APG surfactant/alcohol versus n-octane. 


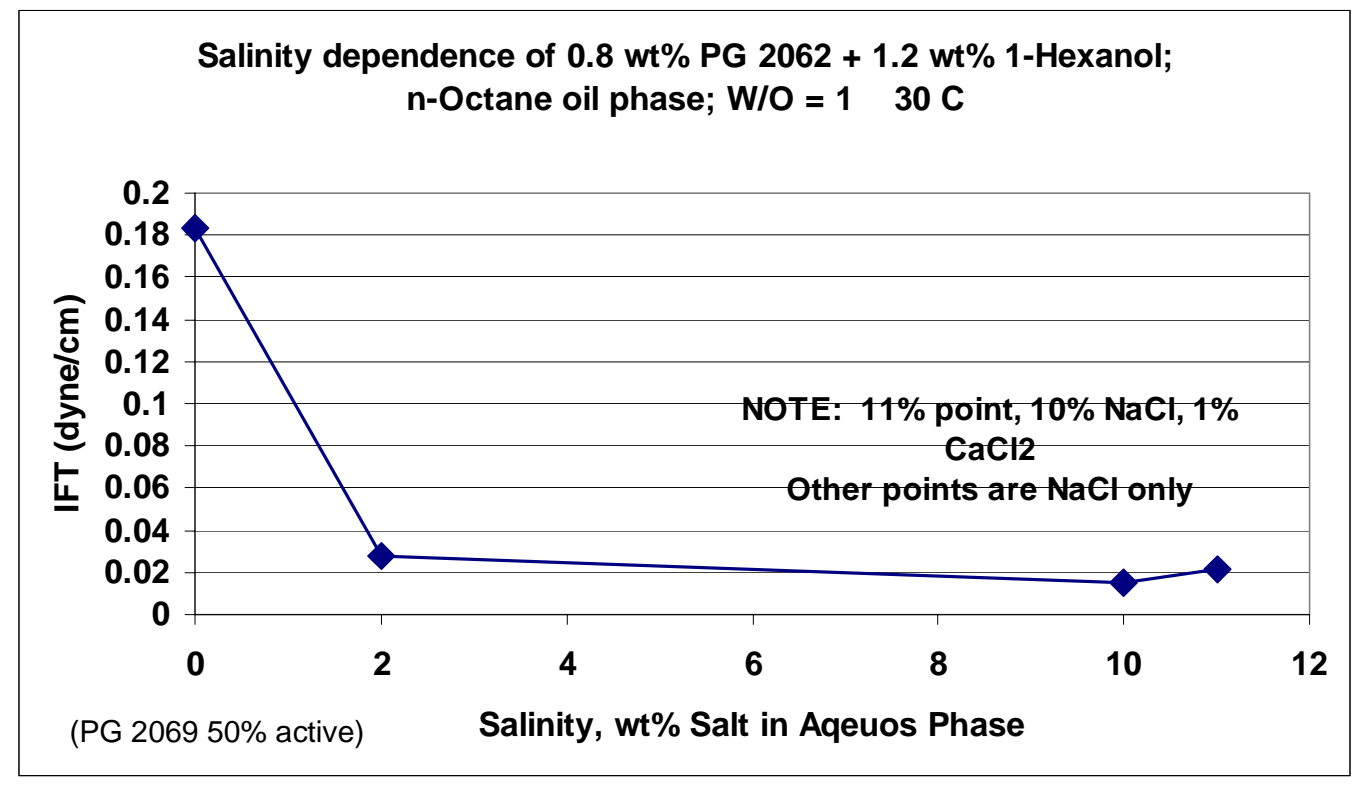

Figure 7.2.5.7. Data illustrating IFT is nearly independent of the salinity for an APG surfactant/alcohol formulation versus n-octane.

Other test samples were prepared to explore the variation in the IFT with changes in the aqueous phase salinity and the system temperature. These measurements centered around the low IFT condition of a PG 2062/n-octanol formulation versus n-octane as the oil phase. Increases in either the salinity or more so, the temperature, produce some increase in the IFT. Also the IFT is greater when the aqueous solution is at the other extreme of no salt (fresh water, sample F-4).

Table 7.2.5.2. Effect of Salinity and Temperature on IFT for a Near Optimum Formulation

\begin{tabular}{|c|c|c|c|}
\hline Sample(s) & $\begin{array}{c}\text { Salinity } \\
\text { (wt\% NaCl) }\end{array}$ & $\underline{\left.\text { Temperature }^{\mathbf{0}} \mathbf{C}\right)}$ & $\begin{array}{c}\text { IFT } \\
\text { (dyme/cm) }\end{array}$ \\
\hline F-4 & 0 & 25 & 0.13 \\
\hline $65,142,143$ & 2 & 25 & 0.006 \\
\hline 149 & 5 & 25 & 0.005 \\
\hline 152 & 10 & 25 & 0.23 \\
\hline 156 & 2 & 50 & 0.042 \\
\hline 150 & 5 & 50 & 0.024 \\
\hline 153 & 10 & 50 & 0.026 \\
\hline
\end{tabular}

Test Conditions: PG 2062 0.8\% (as received), $1.2 \mathrm{wt} \%$ n-octanol $\mathrm{W} / \mathrm{O}=1$ samples equilibrated at room temperature

The Table below presents the IFT results for various n-alcohols as cosurfactants, but now in fresh water. 
Table 7.2.5.3. IFT Values at $25{ }^{\circ} \mathrm{C}$ for a Series of APG/n-Alcohols in Fresh Water

\begin{tabular}{|c|c|c|c|c|}
\hline Sample & $\underline{\text { APG Product }}$ & $\underline{\text { n-alcohol }}$ & $\frac{\underline{\mathbf{n}-\mathbf{a l c o h o l}}}{\mathbf{\text { wt } \mathbf { 0 }}}$ & $\begin{array}{c}\underline{\mathbf{I F T}} \\
\mathbf{\text { dyne } / \mathbf { c m } )}\end{array}$ \\
\hline F-1 & PG 2062 & Propanol & 0.55 & 0.97 \\
\hline F-2 & PG 2062 & Butanol & 0.68 & 1.0 \\
\hline F-3 & PG 2062 & Hexanol & 0.94 & 0.12 \\
\hline F-4 & PG 2062 & Octanol & 1.2 & 0.13 \\
\hline F-5 & PG 2062 & Dodecanol & 1.72 & 3.1 \\
\hline F-6 & PG 2062 & NONE & & 1.7 \\
\hline & & & & 2.0 \\
\hline F-7 & PG 2067 & Propanol & 0.71 & 1.7 \\
\hline F-8 & PG 2067 & Butanol & 0.88 & 0.25 \\
\hline F-9 & PG 2067 & Hexanol & 1.21 & 0.007 \\
\hline F-10 & PG 2067 & Octanol & 1.54 & 6.6 \\
\hline F-11 & PG 2067 & Dodecanol & 2.20 & 2.4 \\
\hline F-12 & PG 2067 & NONE & & \\
\hline
\end{tabular}

Notes for data shown in Table above:

APG concentration: $0.4 \%$ active basis, with different $n$-alcohols as cosurfactants APG/alcohol molar ratio $=0.1$-- (previously found best ratio for PG 2062/n-octanol) Oil phase: n-decane Aqueous/Hydrocarbon phases initially $50 / 50$ by volume and allowed to equilibrate

Previous IFT results reported in this subsection used $2 \mathrm{wt} \% \mathrm{NaCl}$ as the default salinity for APG formulation tests. This is the same trend as found before with samples made up in $2 \mathrm{wt} \% \mathrm{NaCl}$, with the lowest IFT occurring when $\mathrm{n}$-hexanol or n-octanol are cosurfactants. Generally, the IFT values are somewhat greater when the aqueous formulation is in fresh water versus previous results performed in $2 \mathrm{wt} \% \mathrm{NaCl}$.

\subsubsection{APG/n-Alcohol Formulation in Salt Water -- Adsorption and Oil Displacement Experiments}

Adsorption measurements for the 3 commercial APG surfactants (PG 2067, PG 2069, and

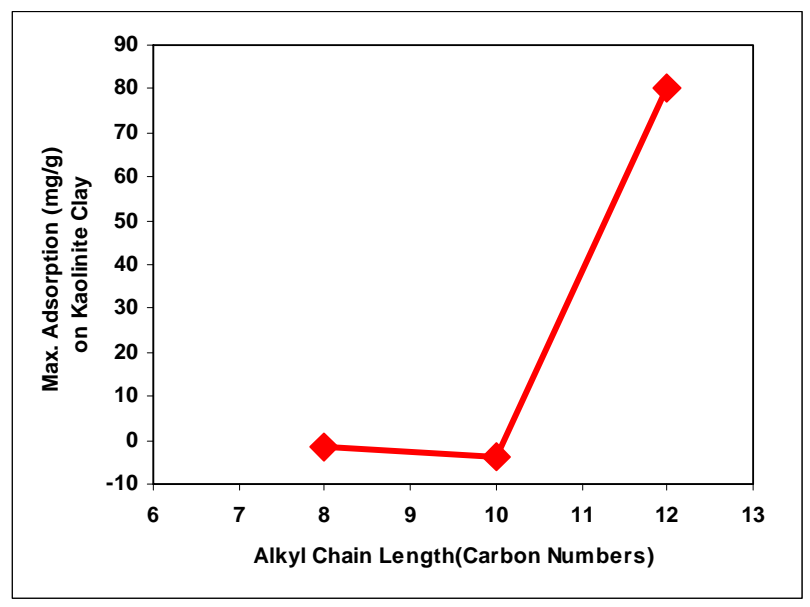

Figure 7.2.5.8. Adsorption of APG products increases with an increase in their average alkyl chain length. 
PG 2062, left right on the plot) from Cognis Corporation are given in the figure above. Similar to other adsorption tests, the aqueous surfactant solution has $2 \mathrm{wt} \% \mathrm{NaCl}$ and a ratio (by weight) of solution/solid of 20 .

Additional surfactant retention tests onto kaolinite clay were performed with APG mixed with n-alcohols as a cosurfactant. All tests used a make up solution of $2 \mathrm{wt} \% \mathrm{NaCl}$ and a ratio of solution/solid of 20 .

Table 7.2.5.4. Selected adsorption results for APG/cosurfactant formulations

$\underline{\text { Surfactant(s) }}$

PG $2067 \quad 0.5 \%$

PG $2069 \quad 0.5 \%$

PG $2062 \quad 0.5 \%$

SPAN $20 \quad 0.5 \%$

PG $20620.4 \%$ 1-propanol $1.2 \%$

PG $2062 \quad 0.4 \%$ 1-butanol $1.2 \%$

PG $20620.4 \%$ 1-hexanol $1.2 \%$

PG $2062 \quad 0.4 \% \quad 1$-octanol $1.2 \%$
Kaolinite Retention (mg surfactant/gm kaolinite) $($ dyne/cm) $* *$ negligible negligible

61

82

41

42

52

46
Approximate

$$
\underline{\text { IFT }}
$$

2

2

2

2
0.8
0.3
0.03
0.007

** IFT measured in separate experiment. IFT for surfactant formulation made up in a $2 \mathrm{wt} \% \mathrm{NaCl}$ brine after phase equilibration reached with n-octane at $25 \mathrm{C}$.

Some trends evident from these data:

- Low adsorption for APG product with shorter alkyl chains, but significant adsorption for the PG 2062 (longest alkyl chain with an average close to 12.5).

- The adsorption levels with mixtures of PG 2062 and 1-alcohols are almost independent of the specific alcohol cosurfactant selected.

- The adsorption levels seem to be independent of the measured IFT of the formulations (if equilibrated with n-octane at ambient temperature).

The figure below provides results about a Berea coreflood experiment using a PG 2062/n-octanol formulation. This combination was selected because the IFT studies indicate this provides the lowest IFT value versus an n-octane at room temperature. 


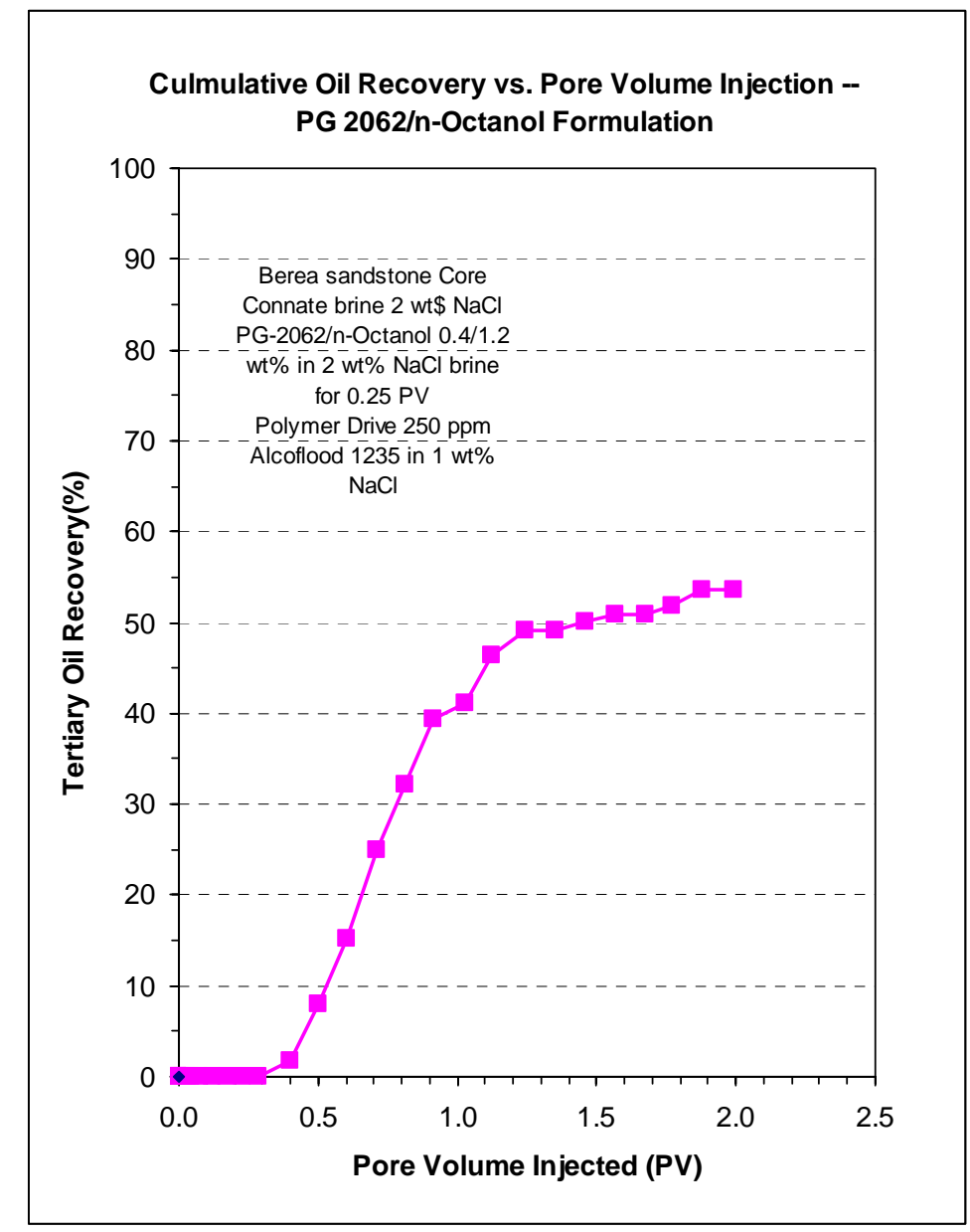

Figure 7.2.5.9 Berea Coreflood oil recovery test result PG 2062/n-octanol formulation

Some details about this coreflood:

Berea sandstone cores; approximately $200 \mathrm{md}$ water permeability, 1" x 12" core All steps at room temperature

Oil phase: n-octane

Waterflood residual oil saturation: 0.30 ;

Connate brine composition $2 \mathrm{wt} \% \mathrm{NaCl}$,

PG 2062 formulation in $2 \mathrm{wt} \% \mathrm{NaCl}$ :

$0.4 \%$ PG 2062 surfactant $(0.2 \%$ on an active basis $)$

$1.2 \%$ n-octanol co-surfactant

0.25 PV slug

Drive polymer solution:

250 ppm Alcoflood 1235 (Ciba Corp.) in $1 \mathrm{wt} \% \mathrm{NaCl}$.

1.5 Pore Volume

Chemical injection at $0.06 \mathrm{ml} / \mathrm{min}$, or about a $3 \mathrm{ft} /$ Day frontal advance rate. 


\subsubsection{IFT of Commercial APG/n-Alcohol Mixtures in Fresh Water}

This series of tests measured the IFT of mixtures of different $n$-alcohols (see table and the figure below). The purpose of these tests was to examine if "molar equivalent" mixtures of n-alcohol cosurfactants would have the same IFT as a single n-alcohol.

Table 7.2.5.5

IFT CHANGES VERSUS BLENDS OF COSURFACTANT n-ALCOHOLS WITH PG 2062 APG SURFACTANT

\begin{tabular}{|c|c|c|c|c|c|c|c|c|}
\hline \multirow[b]{2}{*}{ Sample } & \multicolumn{3}{|c|}{ mole\% of each alcohol } & \multicolumn{3}{|c|}{ wt\% of each alcohol } & \multirow[b]{2}{*}{ Total wt\% } & \multirow{2}{*}{$\begin{array}{c}\text { Equilibrium } \\
\text { IFT } \\
\text { (dyne/cm) }\end{array}$} \\
\hline & n-butanol & n-octanol & n-dodecanol & n-butanol & n-octanol & n-dodecanol & & \\
\hline$F-49$ & 50 & 0 & 50 & 0.34 & 0.00 & 0.86 & 1.2 & 0.49 \\
\hline$F-50$ & 40 & 20 & 40 & 0.27 & 0.24 & 0.69 & 1.2 & 0.56 \\
\hline F-51 & 33 & 33 & 33 & 0.23 & 0.40 & 0.57 & 1.2 & 0.69 \\
\hline F-52 & 25 & 50 & 25 & 0.17 & 0.60 & 0.43 & 1.2 & 0.91 \\
\hline F-53 & 10 & 80 & 10 & 0.07 & 0.960 & 0.17 & 1.2 & 0.054 \\
\hline $\mathrm{F}-4$ & 0 & 100 & 0 & 0 & 1.20 & 0 & 1.2 & 0.13 \\
\hline
\end{tabular}

RUN CONDITIONS FOR ALL SAMPLES:

PG 20620.4 wt\% active; Total Alcohol Cosurfactant Concentration 1.2 wt $\%$

Molar ratio for all $n$-alcohols/PG 2062 blends $=10: 1$

AVERAGE MOLECULAR WEIGHT of all alcohol blends are the SAME

NO SALT -- distilled water

$\mathrm{n}$-decane is oil phase

Temperarture $25 \mathrm{C}$

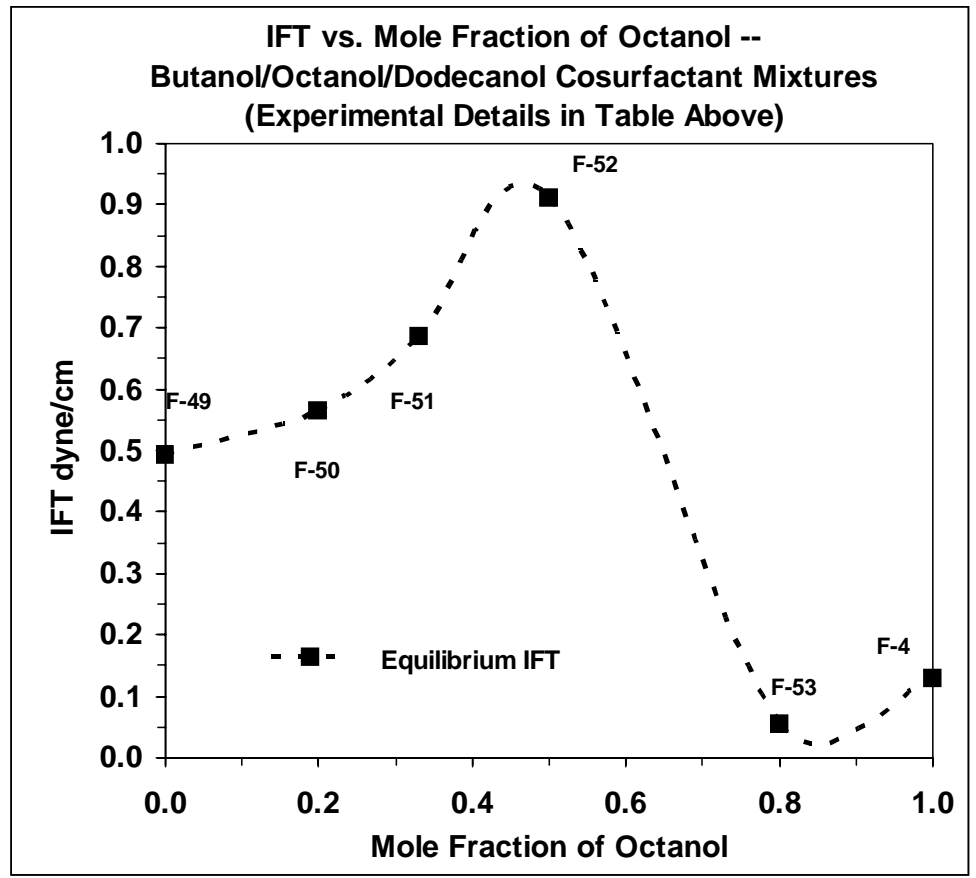

Figure 7.2.5.10. IFT data versus mole fraction of n-octanol in a butanol/octanol/dodecanol cosurfactant blend 
Specifically, we tested blends of C4, C8, and C12 alcohols, with the C8 (n-octanol) occupying the center point of the study because of previous results showing that being perhaps the optimum n-alcohol cosurfactant with the commercial APG products. All of formulations tested for IFT had the same average molecular weight and total weight percentage of n-alcohol cosurfactants. The results show that for these samples with molar cosurfactant blends that all "average" the same chain length of C8 that the IFT is in fact not the same. The IFT is lowest with the cosurfactant as a 10/80/10 butanol/octanol/dodecanol mixture. That is, including a small amount of "end members" n-butanol and n-dodecanol improve performance versus having n-octanol only. Mixtures with a greater percentage of n-butanol and n-dodecanol did not create as low of an IFT.

\subsubsection{Alkyl Polyglycosides (APG) Formulated with Aromatic Alcohols And Other Compounds as Cosufactant}

Other alcohol cosurfactants were investigated as possibly offering superior performance as cosurfactants compared to the n-alcohol cosurfactants already evaluated. In particular, another class of cosurfactants investigated were the aromatic alcohols. Note that we have submitted an invention disclosure for this idea as it seems not to have been considered in existing patents about APG surfactants. In particular, as detailed below, adding 1-naphthol as a cosurfactant is an intriguing idea because some formulations using this plus an APG surfactant created a low IFT even with very low APG concentrations.

One test series included the PG 2062 commercial APG surfactant and a series of alcohol cosurfactants, all with 6 carbons. These data show below the IFT is roughly similar for the 4 different cosurfactants tested, but the 1-alcohol structure has a lower IFT versus the branched, ring, and aromatic versions.

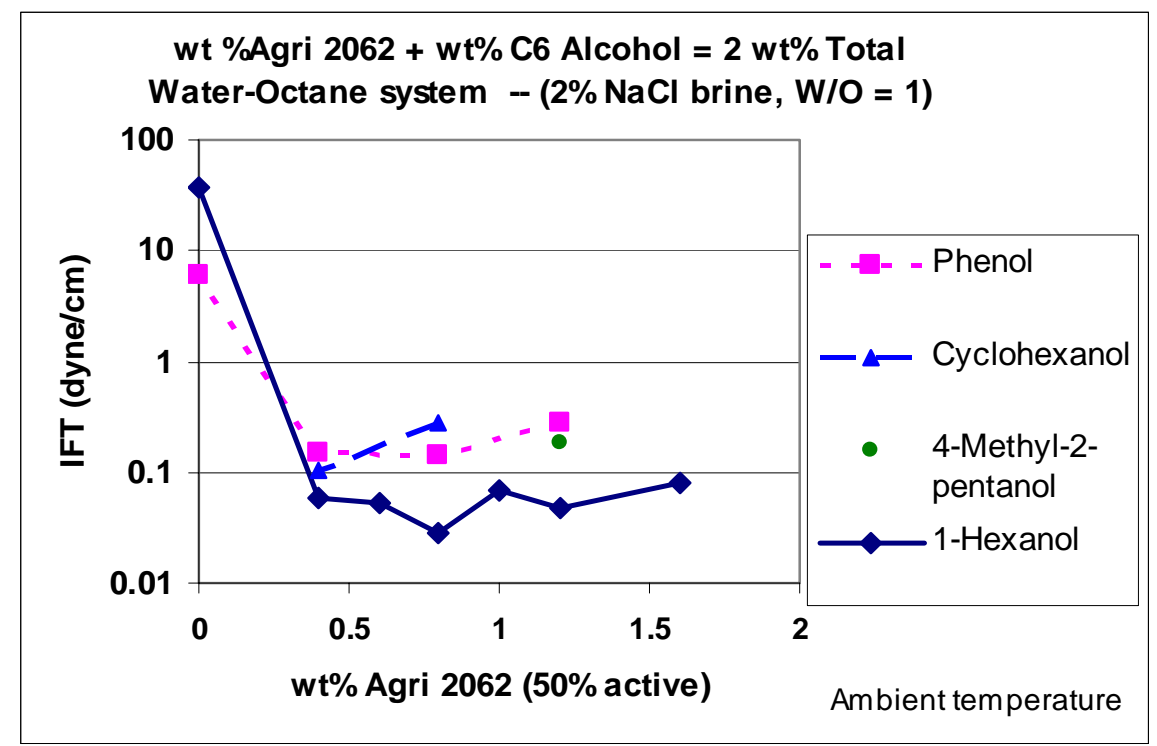

Figure 7.2.6.1. Comparison of IFT behavior for different alcohol cosurfactants, all containing 6 carbons. 
Another variation in cosurfactant structure explored is with a series of aromatic alcohols. Figure 7.2.6.2 shows that most of the IFT values are again about $0.2 \mathrm{dyne} / \mathrm{cm}$.

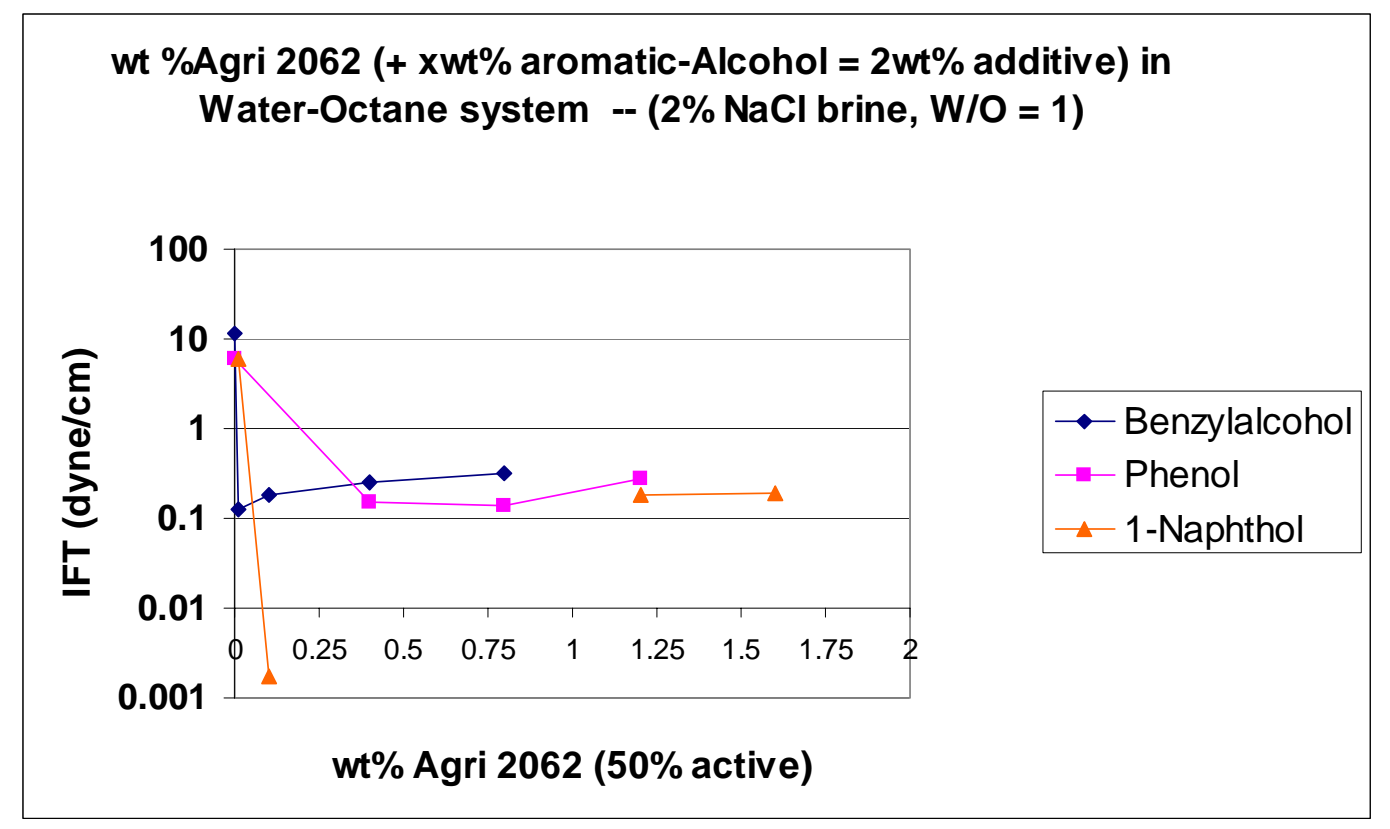

Figure 7.2.6.2. Measured IFT with different aromatic alcohols as APG cosurfactants

The interesting exception is the low IFT of $0.002 \mathrm{dyne} / \mathrm{cm}$ found with the very low concentration of $0.1 \mathrm{wt} \%$ PG 2062 (only $0.05 \mathrm{wt} \%$ on an active basis) and a greater concentration of 1-naphthol. (While the plot indicates the 1-napthol concentration is close to $2 \mathrm{wt} \%$, actually the dissolved concentration is much less due its limited solubility of this solid compound in water.). Very low IFT at low surfactant/cosurfactant concentrations has an obvious practical benefit if that condition can be propagated successfully in the reservoir.

The next figure compares the IFT response for both PG 2062 and the pure C16 version of (HBDM, Hexadecyl-beta-D-mannose, Figure 7.2.5.4) APG surfactants when formulated with 1-naphthol as a cosurfactant. The precise dissolved concentration of the cosurfactant in each sample is difficult to say; this material is only somewhat soluble in water and hydrocarbon (n-octane used here as the oil phase), and was added to excess. This was evidenced by an incomplete dissolution of the added cosurfactant solid particles. Tests with a GC analysis of the equilibrated fluids determined the actual dissolved concentration of 1 -naphthol to be several hundred ppm in the aqueous phase, and perhaps as high as a few thousand ppm in the n-octane hydrocarbon phase. 


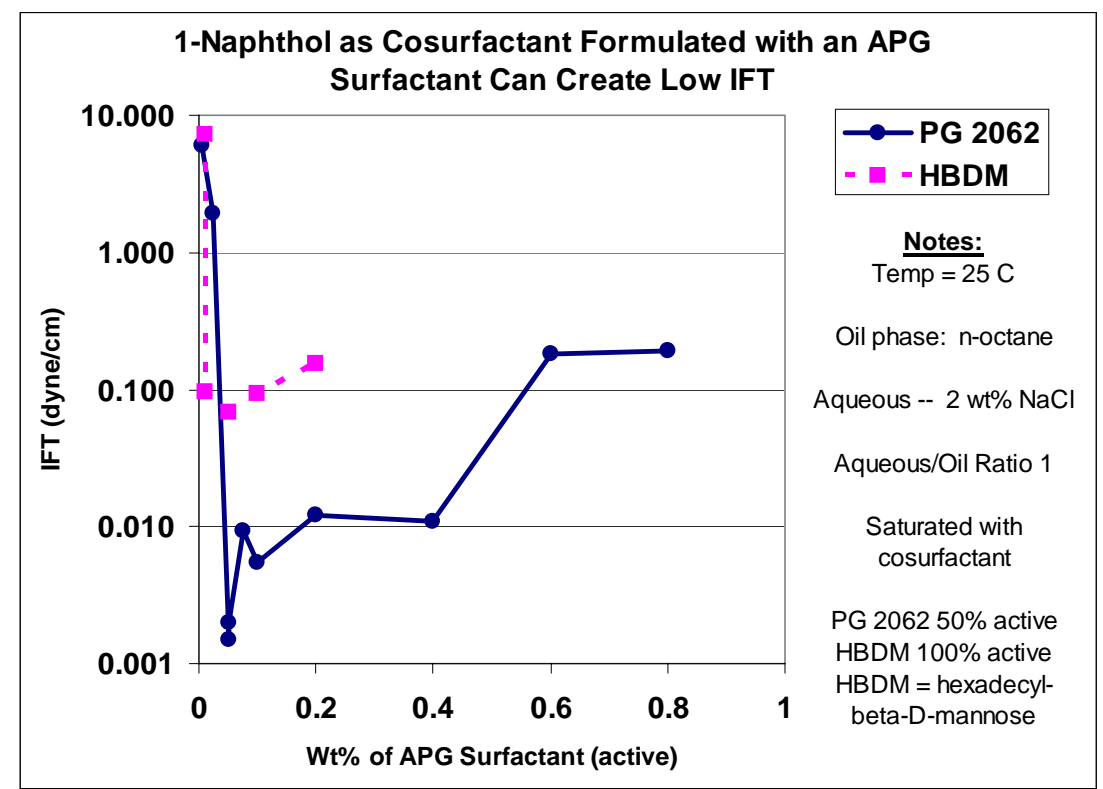

Figure 7.2.6.3. 1-naphthol as a cosurfactant with APG surfactants may create a low IFT condition at a low chemical concentration.

Follow-up studies included tests where the solid 1-naphthol is "packaged" different ways. First, there was a series of tests where the added 1-naphthol concentration is only several $\operatorname{ppm}(5-10 \mathrm{ppm})$ and the initial aqueous solution has PG 2062 concentrations ranging from $0.1-1.5 \mathrm{wt} \%$ in a $2 \mathrm{wt} \% \mathrm{NaCl}$ brine. The measured IFT versus $\mathrm{n}$-octane ranged from $0.4-0.7 \mathrm{dyne} / \mathrm{cm}$ at $25 \mathrm{C}$; this is a remarkable result in that PG 2062 surfactant solutions versus n-octane create IFT values of more than 2 dyne $/ \mathrm{cm}$; the IFT becomes less than 1 dyne/cm just with very low ppm concentration additions of the 1-napthol.

The next test series used a fresh water saturated 1-naphthol solution as the starting makeup water to create several APG/1-naphthol formulations. Because the 1-naphthol solubility in fresh water is several hundred ppm at ambient temperature, these aqueous formulations have a concentration of this cosurfactant that is about 100 times greater than the previous set of samples. The IFT values are about the same for these samples as the previous series with the very dilute 1-naphthol concentrations

Table 7.2.6.1 IFT values for PG 2062/1-naphthol formulations versus n-octane, with the initial values of 1-naphthol of about $600 \mathrm{ppm}$ in the aqueous phase.

PG 2062

Concentration

$\frac{(\mathrm{wt} \%)}{0.5}$
0.75

1

1.5

1.75
Measured IFT

$\frac{(\text { dyne/cm) }}{0.46}$

0.46

0.39

0.42

0.4

0.33 
Notes:

n-Octane as oil phase, $\quad \mathrm{W} / \mathrm{O}=1, \quad$ Brine of $2 \mathrm{wt} \% \mathrm{NaCl}, \quad$ Room Temperature Estimated starting concentration of added 1-naphthol is $600 \mathrm{ppm}$ in the aqueous phase.

The next test series has the 1-naphthol in even higher concentrations in the aqueous formulation; this is accomplished by first dissolving the 1-naphthol in a mutual solvent where it has very high solubility. The table below reports IFT results where the stock solution for adding the 1-naphthol is via a 90/10 by weight blend of ethanol/1-naphthol.

Table 7.2.6.2. IFT for PG 2062/ethanol/1-naphthol formulations versus n-octane.

$\begin{array}{cccc}\begin{array}{c}\text { PG 2062 } \\ \text { Concentration } \\ (\mathrm{wt} \%)\end{array} & \begin{array}{c}\text { Ethanol/1-naphthol } \\ \text { Mixture Concentration } \\ (\mathrm{wt} \%)\end{array} & \begin{array}{c}\text { 1-naphthol } \\ \text { Concentration } \\ (\mathrm{wt} \%)\end{array} & \begin{array}{c}\text { Measured IFT } \\ (\text { dyne/cm) }\end{array} \\ 0.1 & 1.7 & 0.17 & 0.12 \\ 0.25 & 1.6 & 0.16 & 0.16 \\ 0.75 & 1.4 & 0.14 & 0.30 \\ 1.0 & 1.1 & 0.11 & 0.30 \\ 1.5 & 0.9 & 0.09 & 0.35 \\ & 0.5 & 0.05 & 0.48\end{array}$

Notes:

n-Octane is oil phase, $\quad \mathrm{W} / \mathrm{O}=1, \quad$ Brine is $2 \mathrm{wt} \% \mathrm{NaCl}, \quad$ Room Temperature

These results above show the IFT actually decreases with lower concentrations of PG 2062 (and where the ratio of 1-napthol/PG2062 is greater).

The next series of phase behavior/IFT tests including 1-naphthol as a cosurfactant considers other alcohols as a carrier for the 1-napthol.

Table 7.2.6.3. IFT for PG 2062/alcohol/1-naphthol formulations versus n-octane.

Alcohol

Concentration

$\frac{(\mathrm{wt} \%)}{1.5}$

1.5

1.5

1.5
Alcohol

Diluent

ethanol

1-propoanol

cyclohexanol

1-butanol 1-naphthol

Concentration

(wt \%)

0.5

0.5

0.5

0.5
Measured IFT

(dyne/cm)

0.017

0.015

0.82

0.005

Notes:

PG 2062 is $0.1 \mathrm{wt} \%$ (0.05 wt $\%$ active);

n-Octane as oil phase, $\quad \mathrm{W} / \mathrm{O}=1, \quad$ Brine of $2 \mathrm{wt} \% \mathrm{NaCl}, \quad$ Room Temperature

A coreflood experiment was performed to determine how well a very low APG concentration formulation using 1-naphthol as a cosurfactant could displace residual oil. 
Based on the low IFT value shown in the table above we used an APG formulation with a 1-butanol/1-naphthol mixture. The tertiary oil recovery was about $40 \%$.

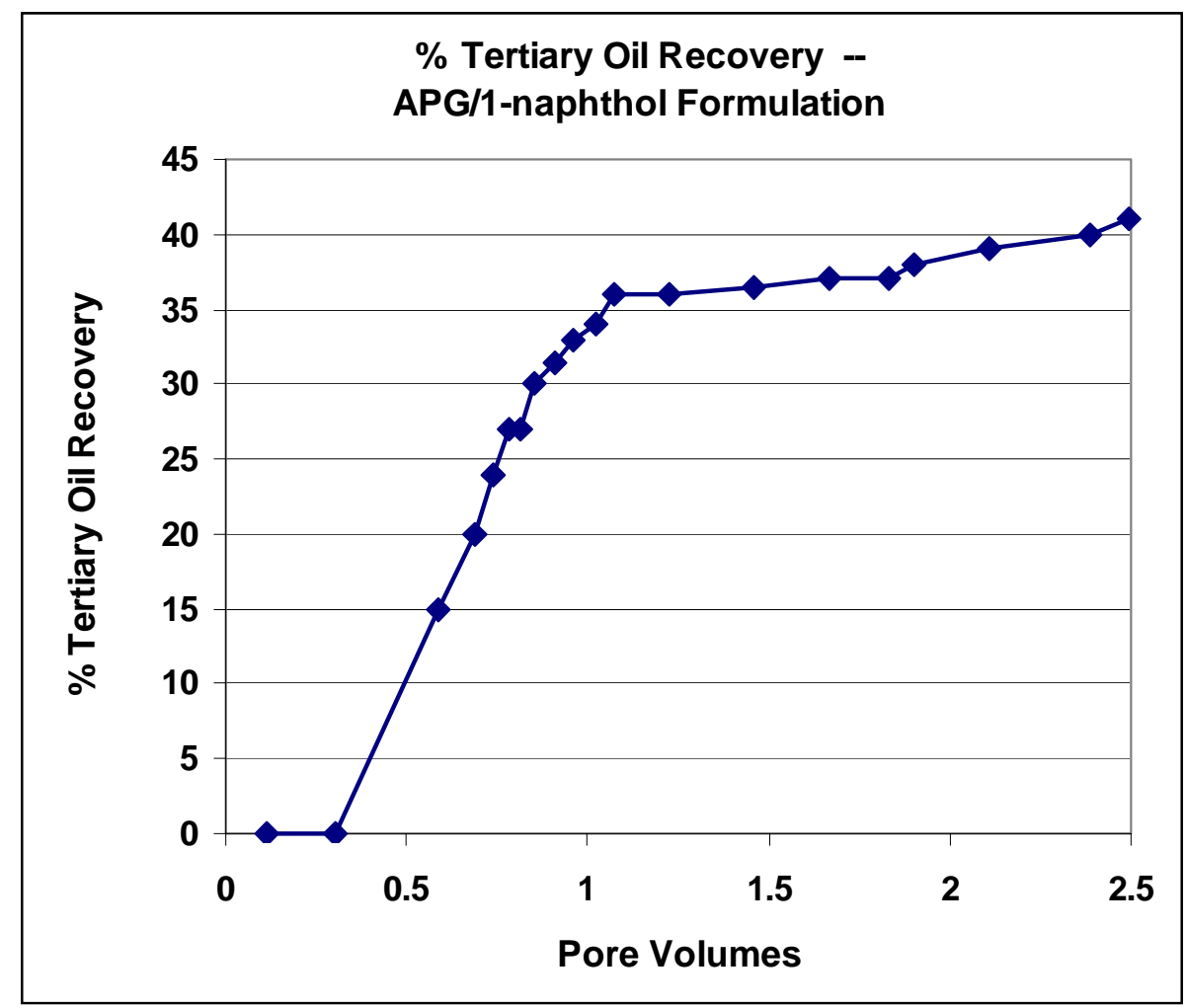

Figure 7.2.6.4. Tertiary oil recovery for Berea coreflood test using a formulation containing PG 2062 and 1-butanol/1-naphthol.

Some details about this coreflood (see above):

Berea sandstone core; approximately $300 \mathrm{md}$ water permeability, 1" x 12" core

Oil phase: n-octane

Waterflood residual oil saturation: 0.31 ;

Connate brine composition $2 \mathrm{wt} \% \mathrm{NaCl}$,

PG 2062 formulation in $2 \mathrm{wt} \% \mathrm{NaCl}$ :

$0.1 \mathrm{wt} \%$ PG 2062 surfactant $(0.05 \%$ on an active basis $)$

$2 \mathrm{wt} \%$ in-butanol/1-naphthol mixture; weight ratio of 75/25 n-butanol/1-naphthol

0.8 Pore Volume slug

Drive polymer solution:

350 ppm Alcoflood 1235 (Ciba Corp.) in 2 wt\% NaCl.

2 Pore Volume

Chemical injection at $0.05 \mathrm{ml} / \mathrm{min}$, or about a $3 \mathrm{ft} /$ Day frontal advance rate.

It is remarkable that with only $0.05 \mathrm{wt} \%$ (active) of the APG surfactant (PG 2062) in the injected chemical slug that there was significant tertiary oil recovery of about $40 \%$. 
Another coreflood experiment using the same basic system was repeated, but with now a higher concentration of the PG 2062 (2 wt\%). A spinning drop IFT measurement between this aqueous formulation and a fresh 2-microliter drop of n-octane quickly attained an IFT of less than $0.01 \mathrm{dyne} / \mathrm{cm}$.

Some details about this coreflood:

Berea sandstone core; approximately 300 md water permeability, 1" x 12" core

Oil phase: n-octane

Waterflood residual oil saturation: 0.30 ;

Connate brine composition $2 \mathrm{wt} \% \mathrm{NaCl}$,

PG 2062 formulation in $2 \mathrm{wt} \% \mathrm{NaCl}$ :

$2 \mathrm{wt} \%$ PG 2062 surfactant ( $1 \mathrm{wt} \%$ on an active basis)

$1.9 \mathrm{wt} \%$ in-butanol/1-naphthol mixture; weight ratio of 75/25 n-butanol/1-naphthol

0.25 Pore Volume slug

Drive polymer solution:

350 ppm Alcoflood 1235 (Ciba Corp.) in $2 \mathrm{wt} \% \mathrm{NaCl}$.

1 Pore Volume

Chemical injection at $0.05 \mathrm{ml} / \mathrm{min}$, or about a $3 \mathrm{ft} /$ Day frontal advance rate.

Result: Tertiary Oil Recovery of less than $10 \%$.

It is not clear why it is that the recovery is so poor with this second coreflood using the high concentration of PG 2062 (2 wt $\%$ ) versus the previous, successful coreflood that had only $0,05 \mathrm{wt} \%$ PG 2062. The IFT was shown to be low with the $2 \mathrm{wt} \%$ PG 2062 combined with the 1-butanol/1-naphthol cosurfactant (IFT measurement was just with fresh fluids, not with a phase equilibrated sample). Note that in Table 7.2.6.2 the equilibrium IFT is actually less with a lower concentration of PG 2062 having the ethanol/1-naphthol mixture added as a cosurfactant. Also note that the fresh fluid IFT for the first formulation (with the $0.1 \mathrm{wt} \%$ PG 2062) was quite high, around $5 \mathrm{dyne} / \mathrm{cm}$, whereas the equilibrated IFT was 0.005 dyne/cm (Table 7.2.6.3).

Another variation explored in this series of IFT experiments with APG formulations is the effect of changing the functional group in the cosurfactant. Namely, in the figure below there are results comparing the IFT as there is a change in the cosurfactant from an alcohol or an acid or an ester (cosurfactants variation of n-hexanol). The n-alcohol appears to be the best among this set of cosurfactants. 


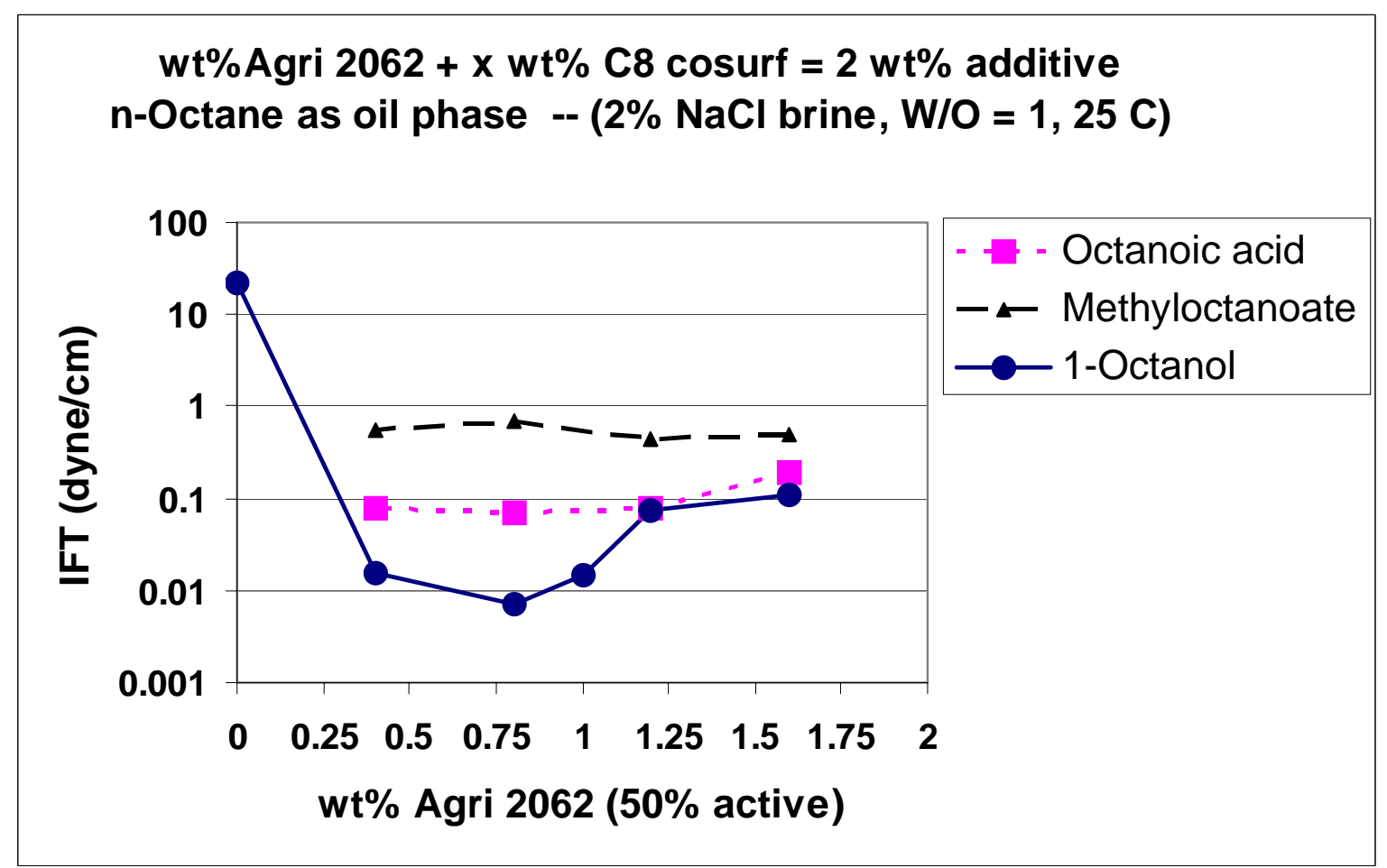

Figure 7.2.6.5. Effect of functional group in cosurfactant on the IFT when formulated with PG 2062 and measured versus n-octane.

Finally, one other chemical variation examined as a cosurfactant for the APG surfactants are glycol ethers. These are one of the classes of compounds included in the U.S. Patent $4,985,154$ as candidate cosurfactants with APG. In this investigation, we screened diethylene monoglycol butyl ether (DMBE) as a candidate cosurfactant. These results indicate the IFT is relatively high and not very dependent on the APG/DMBE ratio.

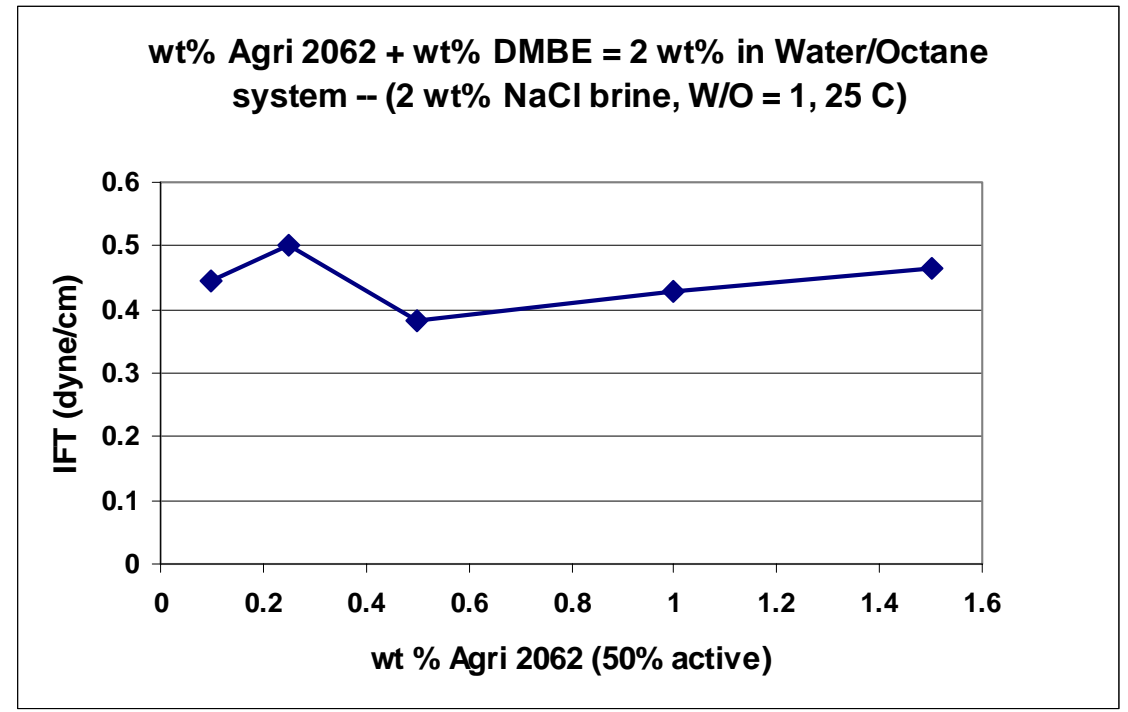

Figure 7.2.6.6. IFT measurements for formulations containing PG 2062 and diethylene mono glycol butyl ether as cosurfactant 


\subsubsection{Alkyl Polyglycosides (APG) Formulated with Sorbitan-Type and Other Surfactants}

\subsubsection{IFT of APG/Sorbitan Surfactant Blends}

Another approach to creating low IFT conditions suggested in the literature is the combination of an APG surfactant with a sorbitan type of surfactant. For this part of the project experimental investigation we considered mixtures of commercial APG products with a series of SPAN and TWEEN sorbitan-type commercial surfactants.

When another nonionic surfactant with a smaller head group than that of the APG (such as a sorbitan), synergistic mixtures may be found that form three-phase behavior and low IFT. Because both surfactants have largely temperature independent interfacial behavior, their blends are also expected to exhibit IFT that is temperature independent.

An example Sorbitan surfactant (SPAN 20 -- Sorbitan Monolaurate) is shown below

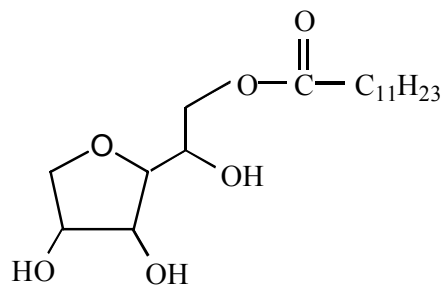

Figure 7.2.7.1. Example of a SPAN surfactant

A variation of the SPAN surfactants is the TWEEN product line of surfactants. An example molecular structure is shown below (Polyoxyethylene (20) Sorbitan Monolaurate, $\mathrm{X}+\mathrm{Y}+\mathrm{Z}=20$ ).

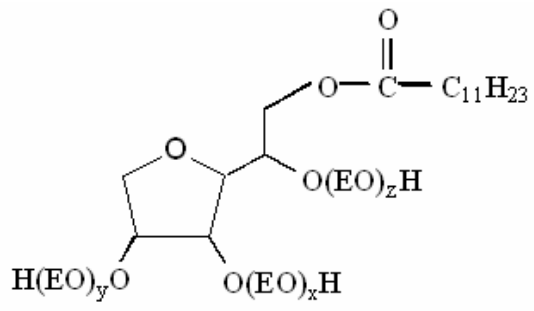

Figure 7.2.7.2. Example of a TWEEN surfactant

The table below provides chemical structure details for these two series of commercial surfactants. 
Table 7.2.7.1. Chemical structure information for SPAN and TWEEN surfactants.

\begin{tabular}{|c|c|c|}
\hline \multicolumn{2}{|c|}{ Length } & Average \\
\hline Product & Alkyl Chain & HLB \\
\hline SPAN 20 & $\mathrm{C} 12$ & 8.6 \\
\hline SPAN 40 & $\mathrm{C} 14$ & 6.7 \\
\hline SPAN 60 & $\mathrm{C} 18$ & 4.7 \\
\hline SPAN 80 & C18 (one double bond) & 4.3 \\
\hline SPAN 85 & $3 \mathrm{C} 18$ (each has double bond) & 1.8 \\
\hline
\end{tabular}

$\begin{array}{llcl}\text { Product } & \begin{array}{c}\text { Number } \\ \text { EO Groups }\end{array} & \begin{array}{c}\text { Length } \\ \text { Alkyl Chain }\end{array} & \begin{array}{c}\text { Average } \\ \text { HLB }\end{array} \\ \text { TWEEN 20 } & 20 & \text { C12 } & 16.7 \\ \text { TWEEN 40 } & 5 & \text { C12 } & 13.3 \\ \text { TWEEN 80 } & 20 & \text { C18 } & 15.0 \\ \text { TWEEN 81 } & 5 & \text { C18 } & 10.0 \\ \text { TWEEN 85 } & 20 & \text { 3 C18 chains } & 11.0\end{array}$

Table 7.2.7.2 lists some of the IFT results for different combinations of the longer alkyl chain APG products, PG 2069 and PG 2062, and various SPAN products. The default test conditions included using a $2 \mathrm{wt} \% \mathrm{NaCl}$ brine in the aqueous phase and measuring the IFT versus $\mathrm{n}$-octane as the oil phase at room temperature (also $\mathrm{W} / \mathrm{O}=1)$.

Table 7.2.7.2. Measured IFT for APG / SPAN surfactant mixtures in a $2 \% \mathrm{NaCl}$ brine versus n-octane as the hydrocarbon phase.

\begin{tabular}{|c|c|c|c|c|}
\hline & SPAN & weight & weight & IFT \\
\hline APG & Product & \% APG & \% SPAN & (dyne/cm) \\
\hline PG 2069 & 20 & 0.80 & 1.20 & 0.0035 \\
\hline PG 2069 & 40 & 0.40 & 1.60 & 1.40 \\
\hline PG 2069 & 60 & 0.40 & 1.60 & 0.33 \\
\hline PG 2069 & 85 & 0.40 & 1.60 & 1.55 \\
\hline PG 2069 & 85 & 1.50 & 0.50 & 0.8 \\
\hline PG 2069 & 85 & 1.60 & 0.40 & 1.2 \\
& & & & \\
\hline PG 2062 & 20 & 0.80 & 1.20 & 0.90 \\
\hline PG 2062 & 20 & 1.20 & 0.80 & 0.75 \\
\hline PG 2062 & 40 & 0.40 & 1.60 & 0.85 \\
\hline PG 2062 & 60 & 0.40 & 1.60 & 1.00 \\
\hline PG 2062 & 60 & 0.80 & 1.20 & 0.73 \\
\hline PG 2062 & 80 & 0.40 & 1.60 & 1.20 \\
\hline PG 2062 & 85 & 0.40 & 1.60 & 0.68 \\
\hline PG 2062 & 85 & 0.80 & 1.20 & 0.25 \\
\hline PG 2062 & 85 & 1.20 & 0.80 & 0.40 \\
\hline
\end{tabular}


Note that the IFT for the commercial APG products alone and these SPAN products by themselves is about 2 dyne $/ \mathrm{cm}$. In some cases there is an obvious strong synergistic effect with surfactant mixtures, with the IFT attaining very low values. The combinations (shown above) where all of the phases (aqueous, microemulsion, and oleic) appear to be fluid, the measured IFT results cover a wide range of values. The IFT value is especially low $(0.0035 \mathrm{dyne} / \mathrm{cm})$ for the first sample shown (the PG 2069 / SPAN 20 blend at $0.8 / 1.2 \mathrm{wt} \%$,), but the IFT exceeds $0.1 \mathrm{dyne} / \mathrm{cm}$ for the others.

Other combinations of these APG products and the SPAN surfactants we prepared created stiff gels in the oil phase. Those combinations exhibiting such unfavorable phase behavior are not viable as an EOR formulation and most were not measured for IFT.

The figure below plots some of the IFT results for the PG 2067/SPAN blends.

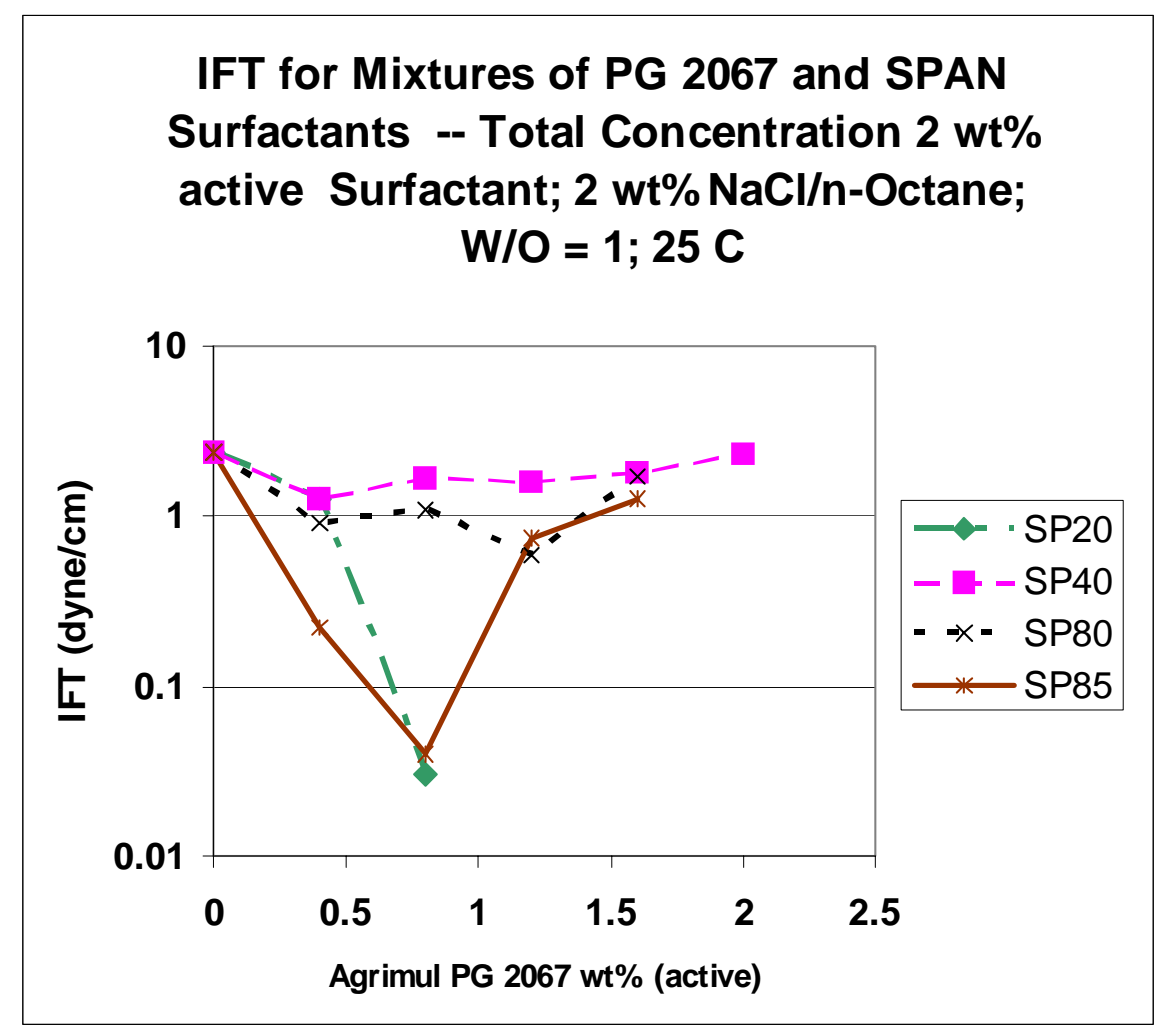

Figure 7.2.7.3. IFT measured for different mixtures of PG 2067 APG and some SPAN surfactants

It seems odd that the two "end members" of the SPAN series, SPAN 20 (HLB = 8.6) and the SPAN $85(\mathrm{HLB}=1.8)$ can create a low IFT when used in these APG formulations. In contrast, mixtures employing SPAN $40(\mathrm{HLB}=6.7)$ and SPAN $80(\mathrm{HLB}=4.3)$ never exhibit this synergistic, lower IFT effect. Also, preliminary data suggest a low IFT may occur with PG 2067 / SPAN 60 mixtures (data not shown). 
One available relevant set of data in the literature describes IFT for APG solutions mixed with SPAN 20 and equilibrated versus n-dodecane as the oil phase (Forster, 1996). Low IFT occurs, but only over a narrow range of APG / SPAN 20 mixture ratios. We observe this same general effect, with for example the PG 2067/SPAN 85 having a sharp minimum in IFT at a ratio of $0.75 \% / 1.25 \%$., but significantly greater IFT at other mixing ratios.

Results of IFT measurements for combinations of APG and TWEEN surfactants are summarized in the table below.

Table 7.2.7.3. Measured IFT for APG / TWEEN surfactant mixtures in a $2 \% \mathrm{NaCl}$ brine versus n-octane as the hydrocarbon phase at room temperature

\begin{tabular}{|c|c|c|c|c|}
\hline & TWEEN & & & IFT \\
\hline APG & Product & $\frac{\text { \% APG }}{\text { PG67 }}$ & 21 & $\frac{\text { \%TWEEN }}{\text { (dyne/cm) }}$ \\
\hline PG67 & 21 & 1.20 & 0.80 & 1.07 \\
\hline PG67 & 85 & 0.80 & 0.40 & 1.42 \\
\hline PG67 & 85 & 1.00 & 1.20 & 0.76 \\
\hline PG67 & 85 & 1.20 & 0.80 & 0.38 \\
\hline PG67 & 85 & 1.60 & 0.40 & 0.9 \\
\hline PG69 & 21 & 1.60 & 0.40 & 1.25 \\
\hline PG69 & 40 & 1.60 & 0.40 & 1.7 \\
\hline PG69 & 81 & 1.00 & 1.00 & 9.6 \\
\hline PG62 & 21 & 0.40 & 1.60 & 0.05 \\
\hline PG62 & 81 & 0.40 & 1.60 & 1.3 \\
\hline PG62 & 81 & 0.80 & 1.20 & 6.10 \\
\hline PG62 & 85 & 0.40 & 1.60 & 0.76 \\
\hline
\end{tabular}

Similar to the observations of the APG / SPAN phase behavior test tubes, most combinations of the APG and TWEEN surfactants created gels in the oil phase. The combinations (shown above) where all of the phases (aqueous, microemulsion, and oleic) are fluid, allowed a meaningful measurement of the IFT. None of these combinations employing the TWEEN surfactant appeared to be interesting as an EOR candidate except perhaps for the PG 2062 / TWEEN 21 at 0.4 / $1.6 \mathrm{wt} \%$ (IFT of $0.05 \mathrm{dyne} / \mathrm{cm}$ ).

Another idea briefly explored was to mix surfactants from the so-called Pluronic series with the APG surfactants. These Pluronic nonionic surfactants (supplied by BASF) has a general structure (shown before in 7.2.2.2) are, which are blocks of EO and PO groups. These Pluronic surfactants can have significant molecular weight (as much as a few thousand), and were thought to have potential to exhibit the beneficial effect reported (Endo, 2002) that low molecular weight amphiphilic block copolymers can boost the IFT reducing efficiency of the main surfactant. However, test tube samples made up to examine this idea in fact showed unfavorable phase behavior (all of the samples showed a strong gel formed in the oil phase); hence this idea was not pursued further in this project. 


\subsubsection{Adsorption and Oil Displacement Tests - APG/SPAN 20 Surfactant Blends}

Further work focused on blends of APG and the SPAN 20 surfactants as they had the most promising, lowest measured IFT values in this part of our investigation.

Table 7.2.7.4. Selected adsorption results for APG/cosurfactant formulations

$\underline{\text { Surfactant(s) }}$

PG $2067 \quad 0.5 \%$

PG $2059 \quad 0.5 \%$

PG $20620.5 \%$

SPAN $200.5 \%$

PG $20670.4 \%$ SPAN $200.6 \%$

PG $20690.4 \%$ SPAN $200.6 \%$

PG $20620.4 \%$ SPAN 20 0.6\%
Kaolinite Retention (mg surfactant/gm kaolinite) negligible

negligible

61

82

87

121

132
Approximate

IFT

$($ dyne $/ \mathrm{cm}) * *$ 2

2

2

2

** IFT measured in separate experiment. IFT for surfactant formulation made up in a $2 \mathrm{wt} \% \mathrm{NaCl}$ brine after phase equilibration reached with n-octane at $25 \mathrm{C}$.

Some trends from these data are: 1) adsorption levels are relatively high -- typical alkylbenzene sulfonates would have an adsorption level closer to $10 \mathrm{mg} / \mathrm{gr}$ kaolinite, 2) there is an increased total surfactant adsorption when mixing with the SPAN 20 Sorbitan surfactant - in fact, retention is likely from both surfactants, and 3) the adsorption levels seem to be independent of the IFT -- as measured with these formulation versus n-octane at room temperature.

The evaluation of APG/SPAN formulations includes an oil displacement experiment from a sand pack. We selected for this test the surfactant blend, $0.8 \mathrm{wt} \%$ PG2069 with $1.2 \mathrm{wt} \%$ SPAN 20 because it had the lowest measured IFT $(0.003 \mathrm{dyne} / \mathrm{cm})$.

In this case we performed the oil displacement test in a sand pack. We used a plastic tube about two-inches in diameter and nine-inches in length, packed with a clean sand that had been sieved and sized to be between 60 and 200 mesh.

Other details about the sand pack oil displacement tests:

Sand Pack -- 75 wt $\% 60$ - 100 mesh sand; 25 wt\% 100 - 200 mesh sand; Porosity $0.29 ; \mathrm{PV}=160 \mathrm{ml}$ Permeability about 1 Darcy

Oil phase: n-octane

Oil flood saturation: 0.285 ; or $46 \mathrm{ml}$; 
Connate brine composition $2 \mathrm{wt} \% \mathrm{NaCl}+20 \mathrm{ppm} \mathrm{Ca++}$,

Test 1: Waterflood alone:

Inject brine $-2 \mathrm{wt} \% \mathrm{NaCl}+20 \mathrm{ppm} \mathrm{Ca}++$ for $1.3 \mathrm{PV}$

Result: waterflood oil recovery -- $25 \mathrm{ml}$ or $53 \%$ of the oil in place

Inject n-octane to increase the oil saturation back to irreducible water saturation);

Final oil saturation 0.26 ; or $42.5 \mathrm{ml}$ of n-octane

Test 2: Inject chemical formulation into pack at irreducible water conditions:

PG 2062 formulation in $2 \mathrm{wt} \% \mathrm{NaCl}$ :

$0.8 \mathrm{wt} \%$ PG 2069 surfactant $(0.4 \%$ on an active basis $)$

$1.2 \mathrm{wt} \%$ SPAN 20 surfactant

0.25 Pore Volume slug

Drive polymer solution:

350 ppm Alcoflood 1235 (Ciba Corp.) in $2 \mathrm{wt} \% \mathrm{NaCl}$.

0.75 Pore Volume

Chemical injection at $0.20 \mathrm{ml} / \mathrm{min}$, or about a $0.4 \mathrm{ft} /$ Day frontal advance rate.

Results: Total recovery of $94 \%$ of oil in place (40 out of $42.5 \mathrm{ml}$ )

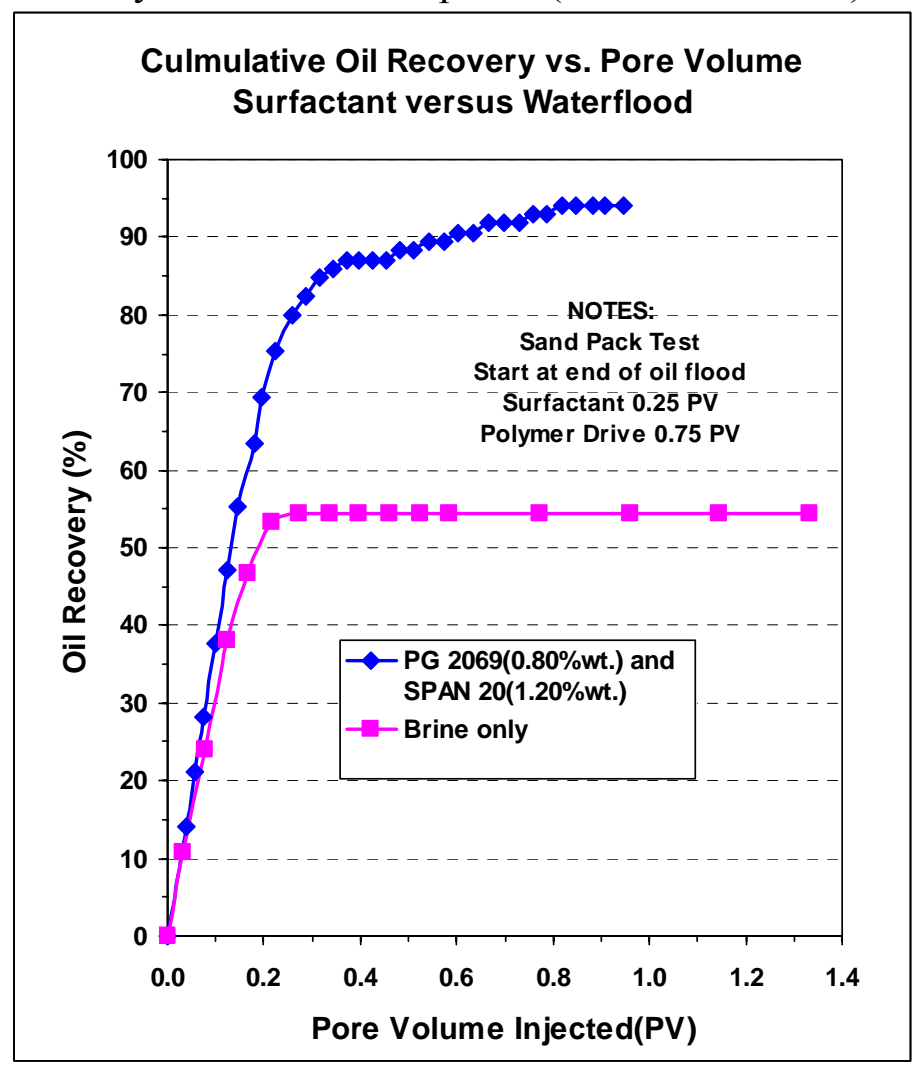

Figure 7.2.7.4. Greater oil recovery occurs in a sand pack experiment when injecting a PG 2067 / SPAN 20 chemical solution versus a waterflood. 
In this test series we contrast the amount of oil recovered by waterflood only versus injection of a chemical formulation having a blend of PG 2069/Span 20 surfactants. Both of these tests were performed starting at irreducible water saturation conditions (at the conclusion of an oil flood step). While the waterflood alone recovered a bit over half of the oil in place, the chemical injection test recovered almost all of the oil in place. This marked improvement in oil recovery is consistent with the formulation's low IFT $(0.003$ dyne $/ \mathrm{cm}$ ) measured versus n-octane in the previous test tube/phase behavior experiments.

\subsubsection{Alkyl Polyglycosides (APG) Formulations - Effect of the Oil Phase Composition}

The studies up to now have focused on n-octane as the hydrocarbon phase, again with the justification that n-octane should be a good model for IFT/phase behavior trends for a "typical" crude oil. The figures below, however, demonstrate that the IFT measurements change significantly upon changing the oil phase composition.

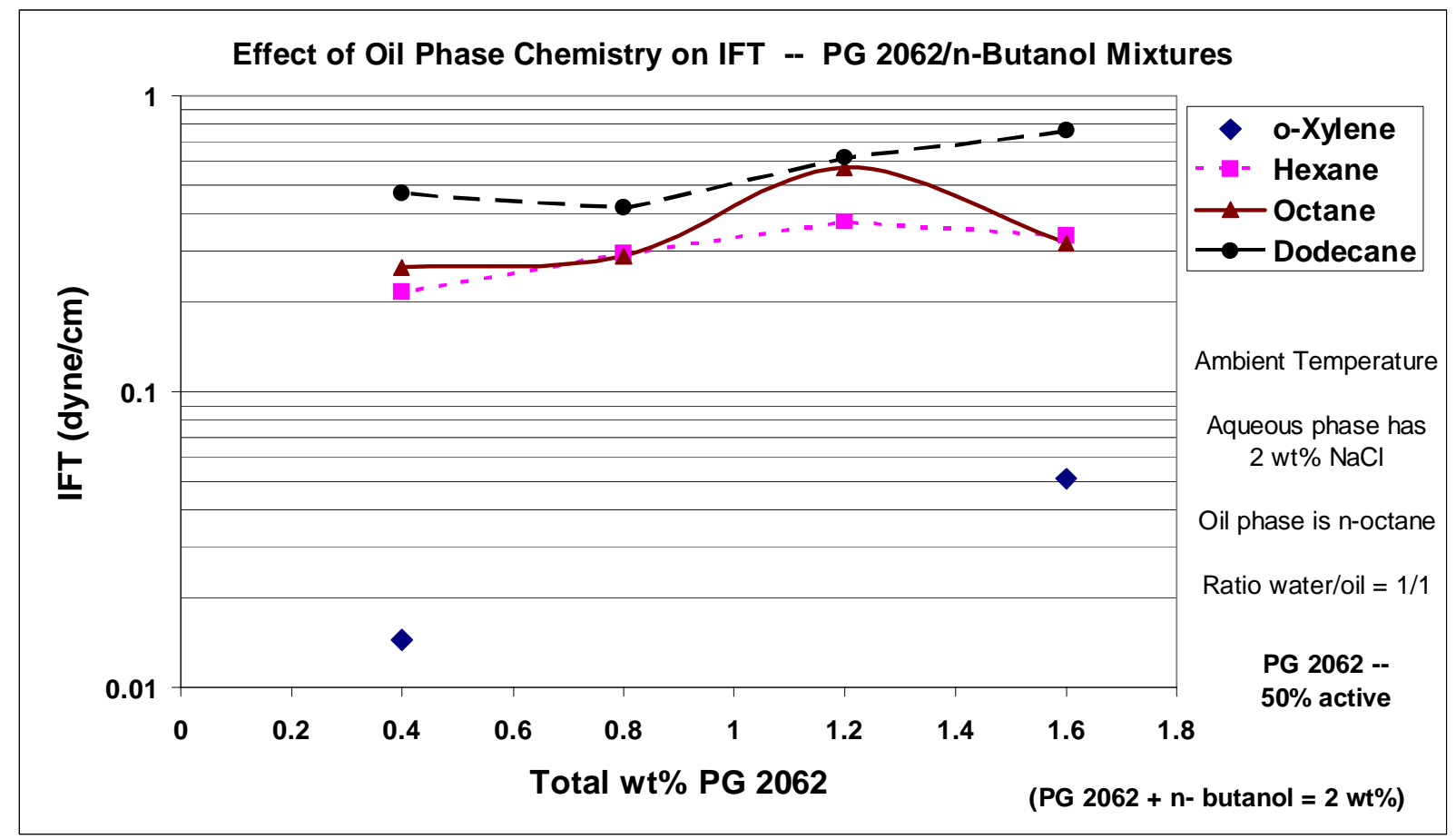

Figure 7.2.8.1. Effect of oil phase composition on the IFT with PG 2062/n-butanol formulations at room temperature. 


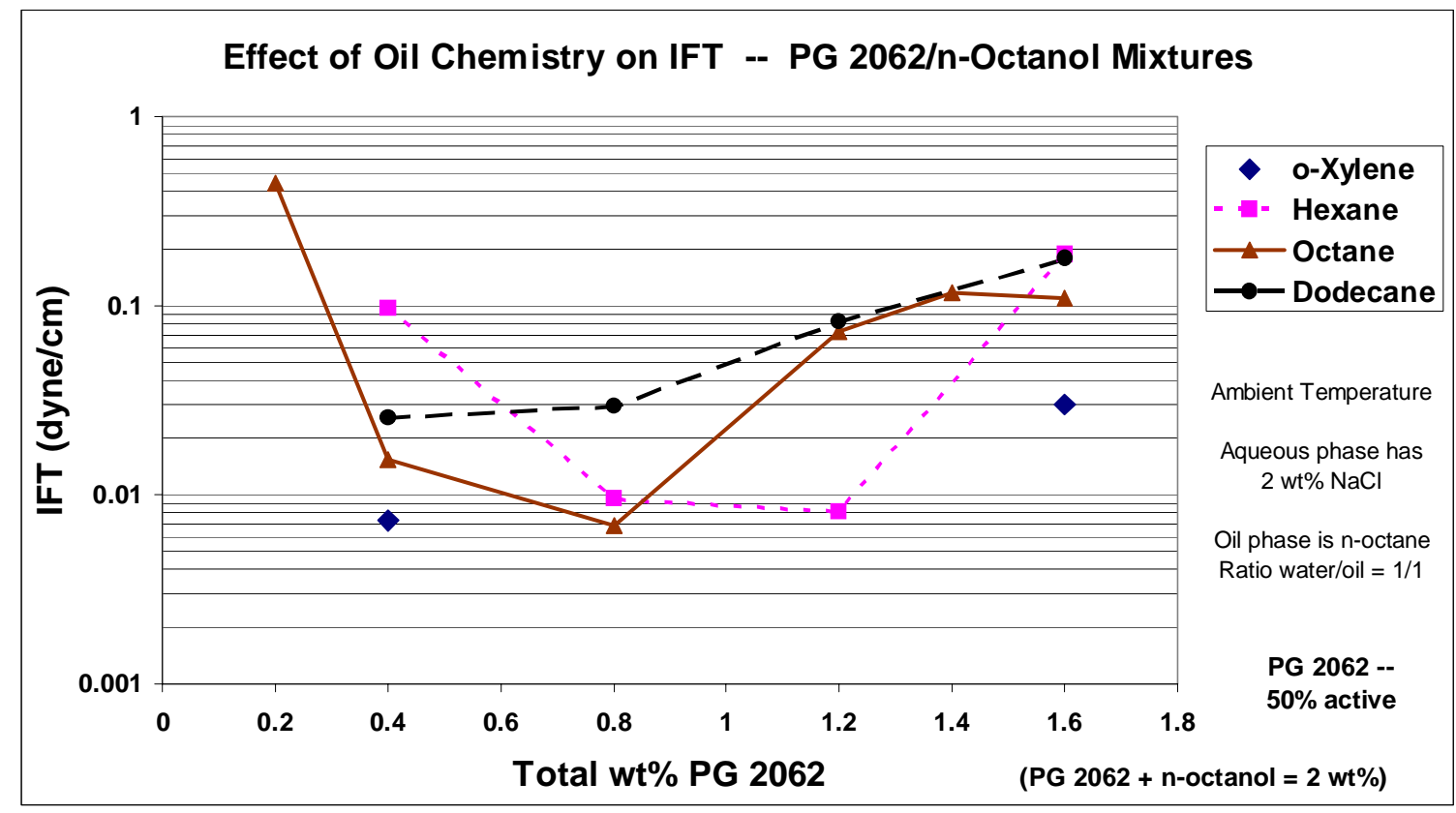

Figure 7.2.8.2. Effect of oil phase composition on the IFT with PG 2062/n-octanol formulations at room temperature.

Generally the IFT values are lower as the oil phase is a lighter alkane (from dodecane, to octane, to hexane), or is o-xylene, an aromatic compound. These trends in oil chemistry effect on the IFT generated are consistent with what is reported in the literature when the surfactant is alkyl benzene sulfonates, the conventional choice for EOR applications. The beneficial effect of having an aromatic oil is particularly pronounced for the PG 2062/ n-butanol blend. 


\subsubsection{Pure APG Surfactants - Effect of Chemical Structure}

\subsubsection{Background/Motivation for Investigation}

Further work focused on systematic studies of the effect of the structure for APG surfactants on their behavior, particularly regarding their performance in creating a low IFT versus hydrocarbons. We can glean some of this information from the performance of the various commercial APG products in the laboratory studies. However, because they are commercial products, they have a spectrum of number of head groups and hydrophobic alkyl chain length. Thus it is somewhat uncertain if one can simply compare "average" structures among the commercial versions of APG to infer the role of surfactant structure on performance.

Thus, the approach to investigate APG structure/behavior was to select a number of purified surfactants. These products will have some mixture of isomers, etc., but are far less complicated than a commercial APG product.

1. Testing surfactants with defined chemical structures. IFT performance data for these surfactants then can be related back directly to their structure. Such "clean" data where there are fewer surfactant structures makes it more feasible to conduct molecular modeling studies to cross-compare to the experimental findings.

2. Based on the experimental observations and results of the molecular simulations, have a better basis to understand what surfactant structure is preferred to create a low IFT, and from that, speculate what new molecular structures are likely to provide even better performance.

These data were generated with fresh water (zero added salt) to simplify further the physical system and make it even easier to perform the parallel molecular modeling studies. The pure APG surfactants (95-99\%pure) all were supplied by Anatrace.

\subsubsection{IFT Tests -- Pure APG with n-Octanol as Cosurfactant}

This series of tests included with the pure APG examples the best cosurfactant (n-octanol) for decreasing IFT found in the previous testing with commercial APG products.

The figure below shows the various chemical structures included in this portion of the investigation. These surfactants consider one- and two-head groups and alkyl tails that range from $\mathrm{C} 8$ to $\mathrm{C} 16$ to investigate the effects of these surfactant structure changes.

a)

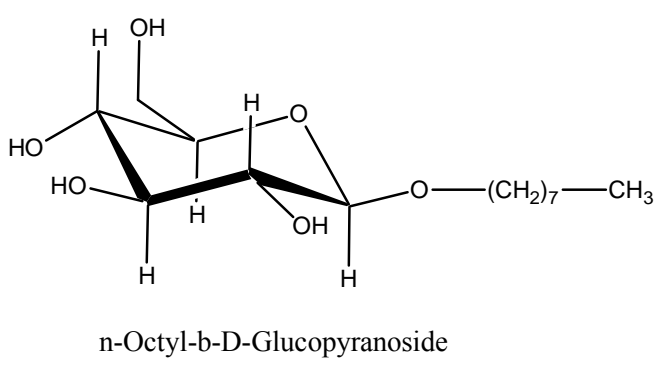


b)

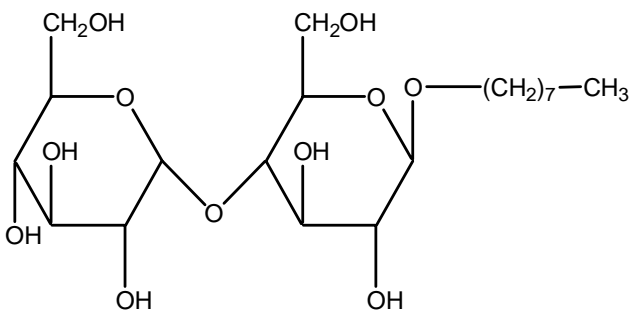

n-Octyl-beta-D-maltopyranoside

(OBDM)

c)

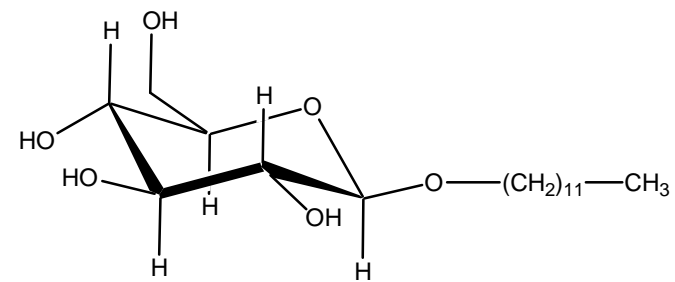

n-Dodecyl-b-D-Glucopyranoside

d)

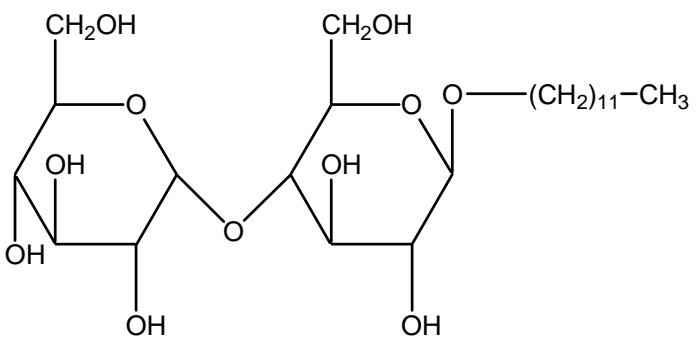

n-Dodecyl-beta-D-maltopyranoside

e)

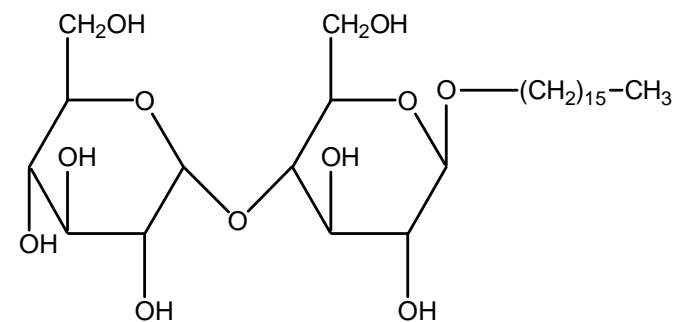

n-Hexadecyl-beta-D-maltopyranoside

(HBDM)

Figure 7.2.9.1. Five different pure APG structures included in IFT experimental study with n-octanol as cosurfactant 
We see from the results in the table below that the IFT generally is lower with increasing the ratio of n-octanol/APG and also by increasing the APG alkyl chain length. Recall that the low IFT conditions occurred with commercial APG products formulation with n-octanol at n-octanol/APG molar ratios around 30. These results also indicate that the IFT is slightly lower for the surfactants having one versus two head groups

Table 7.2.9.1. IFT results for various pure APG surfactants in fresh water with n-octanol as a cosurfactant

ONE HEAD GROUP

APG mole ratio IFT

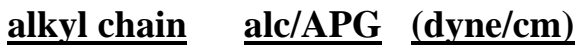

$\begin{array}{ccc} & \text { n-Octyl-glucopyranoside } \\ 8 & 80 & 7.1 \\ 8 & 30 & 5.4 \\ 8 & 10 & 11.3 \\ 8 & 0 & 15.2\end{array}$

n-Dodeycl-beta-D-glucopyranoside

$\begin{array}{lll}12 & 80 & 0.27\end{array}$

$\begin{array}{lll}12 & 30 & 0.27\end{array}$

$\begin{array}{lll}12 & 10 & 0.82\end{array}$

$\begin{array}{lll}12 & 0 & 0.57\end{array}$ $\underline{\text { TWO HEAD GROUPS }}$

\begin{tabular}{ccc}
$\begin{array}{c}\text { APG } \\
\text { alkyl chain }\end{array}$ & $\begin{array}{c}\text { mole ratio } \\
\text { alc/APG }\end{array}$ & $\begin{array}{c}\text { IFT } \\
\text { dyne/cm }\end{array}$ \\
\cline { 2 - 3 } n-Octyl-beta-D-maltopyranoside & (OBDM) \\
8 & 80 & 8.8 \\
8 & 30 & 8.5 \\
8 & 10 & 22.2 \\
8 & 0 & 22.9 \\
n-Dodecyl-beta-D-maltopyranoside \\
12 & 80 & 0.27 \\
12 & 30 & 0.69 \\
12 & 10 & 0.9 \\
12 & 0 & 3.1 \\
& & \\
n-Hexadeycl-beta-D-maltopyranoside (HBDM) \\
16 & 80 & 0.077 \\
16 & 30 & 0.52 \\
16 & 10 & 4.1 \\
16 & 0 & 4.2
\end{tabular}

\section{Test Conditions:}

Brine Composition: Distilled Water

Oil Phase: n-octane

Equal volumes of aqueous and oil phase

Room Temperature for equilibration of phases and IFT

ALL SAMPLES have $0.1 \mathrm{wt} \%$ Surfactant

Recall that more complete results are shown in Figure 7.2.5.5 and 7.2.6.3 for the n-Hexadeycl-beta-D-maltopyranoside (HBDM) surfactant for a number of different cosurfactants. These tests with HBDM presented earlier in this report were performed to determine if a C16 APG surfactant would be even better than the commercial APG product (PG 2062) with the longest chain length (average of C12.5).

Also, the theoretical analysis discussed elsewhere in this report focused on contrasting 
the simulated interfacial structure and IFT between the C8 surfactant -- n-Octyl-beta-Dmaltopyranoside (OBDM) and C16 surfactant -- n-Hexadeycl-beta-D-maltopyranoside (HBDM). These simulations included n-octanol as a cosurfactant with a molar ratio of n-octanol/surfactant of 30 ; this ratio is of interest because with this molar ratio for PG 2062/n-octanol created a low IFT (approaching $10^{-3}$ dyne $/ \mathrm{cm}$ ). The calculated IFT value via MD simulations is 35 dynes/cm for OBDM versus 21 dynes/cm for the HBDM. Although the absolute values are not obtained with these MD simulations the relative ordering of the values compare well with the experimentally measured value of 8.5 and 0.5 dynes $/ \mathrm{cm}$, respectively. Also, the theoretical work provided an atomistic-level picture of the surfactant/cosurfactant conformations at the interface. From this we speculate that the APG surfactant is stabilizing the n-octanol at the oil interface and thereby aids in reducing IFT. Furthermore, the ability of the longer alkyl chain HBDM to penetrate into the oil phase is probably a factor in reducing the IFT more than the OBDM.

Another series of "sugar" surfactants evaluated are related by their all having an aromatic functionality (structures shown below).

a)

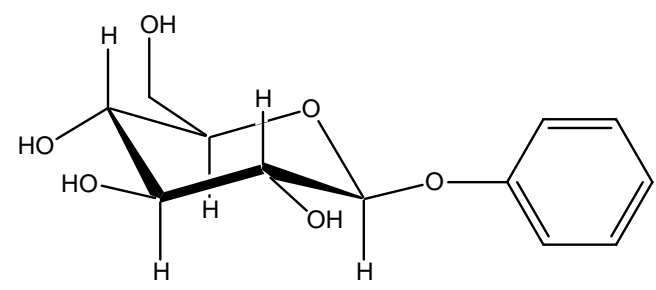

Phenyl-beta-D-glucopyranoside

b)

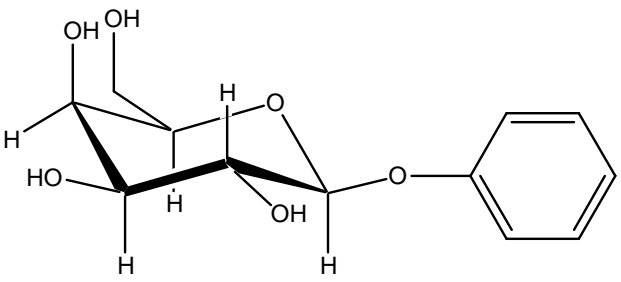

Phenyl-beta-D-galactopyranoside

c)

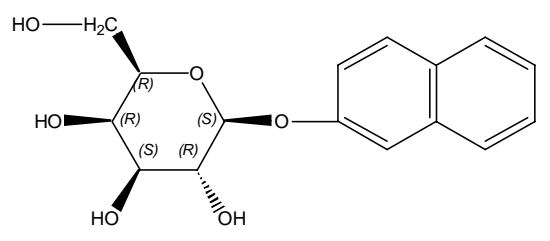

2-Naphthyl- $\beta$-D-galactopyranoside 
d)

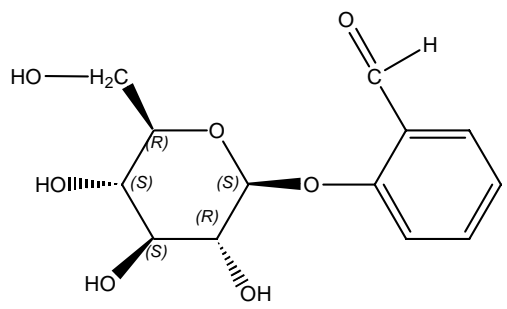

Helicin $=$ Salicylaldehyde- $\beta$-D-glucoside

Figure 7.2.9.2. Structures of surfactants containing an aromatic function

The rationale for testing these particular compounds is the observation that the 1-naphthol appears to be a very good cosurfactant with APG products. That is, why not try a surfactant that has features of both of these compounds. The structures in the above figure combine the sugar-type head group with a hydrophobic portion containing an aromatic function. The results of IFT measurements of these structures formulated with 1-octanol are given in the table below.

Table 7.2.9.2. IFT results for various pure APG surfactants with an aromatic function

\section{Compound}

Phenyl-beta-D-gluco-pyranoside +

Phenyl-beta-D-galacto-pyranoside + $\underline{\text { Salinity, } \mathrm{NaCl} w \mathrm{t} \%}$

0

2

IFT dyne/cm

14.3

6.3

$\begin{array}{ll}0 & 9.1 \\ 2 & 8.3\end{array}$

Naphthyl-beta-D-galacto-pyranoside +

$\begin{array}{ll}0 & 5.2 \\ 2 & 1.0\end{array}$

$\begin{array}{rrr}\text { Helicin }=\text { Salicylaldehyde- } \beta \text {-D-glucoside } & 0 & 4.1 \\ 2 & 16.1\end{array}$

\section{Test Conditions:}

Oil Phase: n-octane

Equal volumes of aqueous and oil phase

Room Temperature for equilibration of phases and IFT

ALL SAMPLES have $0.4 \mathrm{wt} \%$ Surfactant and $1.2 \mathrm{wt} \% \mathrm{n}$-octanol

None of the IFT values are low. We believe that is result mainly due to these surfactants not being sufficiently hydrophobic. We would speculate that adding an alkyl chain on to the aromatic portion would increase their efficiency as surfactants. The IFT is lowest among this series for the Naphthyl-beta-D-galacto-pyranoside + . The two aromatic rings 
would provide greater hydrophobicity than the other structures with a single ring structure. Finally, note that the IFT generally decrease with the addition of $2 \mathrm{wt} \% \mathrm{NaCl}$; a result consistent with the trends found for the commercial APG products.

\subsubsection{IFT Tests -- Pure APG with SPAN 20 as Cosurfactant}

This series of tests included with the pure APG examples, but now with SPAN 20 as an added surfactant to the formulation. Recall that the commercial product PG 2069 and SPAN 20 could be blended to create a low IFT, and further, that such a blend was shown to be effective in an oil displacement experiment.

Recall the chemical structure of the SPAN 20 is:<smiles>CCC(=O)OC[C@@H](O)[C@H]1OC[C@@H](O)[C@H]1O</smiles>

Sorbitan Monolaurate $\left(\mathrm{SPAN}^{\circledR} 20\right)$

F.W. $=346.5 ; \mathrm{HLB}=8.6$

The relevant pure APG structures are shown above in Figure 7.2.9.1.

Table 7.2.9.3a. IFT Results for Mixtures of Maltoside and Sorbitan Surfactants (Oil Phase: fresh n-Octane, Temperature: $30^{\circ} \mathrm{C}$; Fresh Water; Pre-equilibrated)

\begin{tabular}{|c|c|c|c|c|}
\hline Mixtures & $\begin{array}{l}\text { Conc. Of } \\
\text { Maltoside }\end{array}$ & $\begin{array}{l}\text { Conc. Of } \\
\text { Sorbitan }\end{array}$ & $\begin{array}{l}\text { Molar } \\
\text { Ratio }\end{array}$ & $\begin{array}{c}\text { IFT } \\
(\mathrm{mN} / \mathrm{m})\end{array}$ \\
\hline $\begin{array}{l}\text { n-Octyl- } \beta \text {-D-Maltoside and } \\
\text { Sorbitan Monolaurate(SPAN 20) }\end{array}$ & $\begin{array}{c}6.60 \times 10^{-3} \mathrm{M} \\
(0.30 \% \mathrm{wt} .)\end{array}$ & $\begin{array}{c}6.60 \times 10^{-3} \mathrm{M} \\
(0.24 \% \mathrm{wt} .)\end{array}$ & $1: 1$ & 4.19 \\
\hline $\begin{array}{l}\text { n-Decyl- } \beta \text {-D-Maltoside and } \\
\text { Sorbitan Monolaurate(SPAN 20) }\end{array}$ & $\begin{array}{c}6.60 \times 10^{-3} \mathrm{M} \\
(0.32 \% \mathrm{wt} .)\end{array}$ & $\begin{array}{c}6.60 \times 10^{-3} \mathrm{M} \\
(0.24 \% \mathrm{wt} .)\end{array}$ & $1: 1$ & 3.88 \\
\hline $\begin{array}{l}\text { n-Dodecyl- } \beta \text {-D-Maltoside and } \\
\text { Sorbitan Monolaurate(SPAN 20) }\end{array}$ & $\begin{array}{c}6.60 \times 10^{-3} \mathrm{M} \\
\text { (0.34\%wt.) }\end{array}$ & $\begin{array}{c}6.60 \times 10^{-3} \mathrm{M} \\
\text { (0.24\%wt.) }\end{array}$ & $1: 1$ & 3.57 \\
\hline $\begin{array}{l}\mathrm{n} \text {-Tridecyl- } \beta \text {-D-Maltoside and } \\
\text { Sorbitan Monolaurate(SPAN 20) }\end{array}$ & $\begin{array}{c}6.60 \times 10^{-3} \mathrm{M} \\
(0.35 \% \mathrm{wt} .)\end{array}$ & $\begin{array}{c}6.60 \times 10^{-3} \mathrm{M} \\
(0.24 \% \mathrm{wt} .)\end{array}$ & $1: 1$ & 3.18 \\
\hline $\begin{array}{l}\mathrm{n} \text {-Hexadecyl- } \beta-\mathrm{D}-\text { Maltoside and } \\
\text { Sorbitan Monolaurate(SPAN 20) }\end{array}$ & $\begin{array}{l}6.60 \times 10^{-3} \mathrm{M} \\
(0.37 \% w \mathrm{wt})\end{array}$ & $\begin{array}{l}6.60 \times 10^{-3} \mathrm{M} \\
\text { (0.24\%wt.) }\end{array}$ & $1: 1$ & 2.72 \\
\hline
\end{tabular}

Table 7.2.9.3b. IFT Results for Mixtures of Maltoside and Sorbitan Surfactants 
(Oil Phase: fresh n-Octane, Temperature: $30^{\circ} \mathrm{C}$; Fresh Water; Not-equilibrated)

\begin{tabular}{|c|c|c|c|c|}
\hline Mixtures & $\begin{array}{l}\text { Conc. of } \\
\text { Maltoside }\end{array}$ & $\begin{array}{l}\text { Conc. Of } \\
\text { Sorbitan }\end{array}$ & $\begin{array}{l}\text { Molar } \\
\text { Ratio }\end{array}$ & $\begin{array}{c}\text { IFT } \\
(\mathrm{mN} / \mathrm{m})\end{array}$ \\
\hline $\begin{array}{l}\text { n-Octyl- } \beta \text {-D-Maltoside and } \\
\text { Sorbitan Monolaurate(SPAN 20) }\end{array}$ & $\begin{array}{c}6.60 \times 10^{-3} \mathrm{M} \\
(0.30 \% \text { wt. })\end{array}$ & $\begin{array}{c}6.60 \times 10^{-3} \mathrm{M} \\
(0.24 \% \text { wt. })\end{array}$ & $1: 1$ & 4.15 \\
\hline $\begin{array}{l}\text { n-Decyl- } \beta \text {-D-Maltoside and } \\
\text { Sorbitan Monolaurate(SPAN 20) }\end{array}$ & $\begin{array}{c}6.60 \times 10^{-3} \mathrm{M} \\
(0.32 \% \mathrm{wt} .)\end{array}$ & $\begin{array}{c}6.60 \times 10^{-3} \mathrm{M} \\
(0.24 \% \text { wt. })\end{array}$ & $1: 1$ & 3.22 \\
\hline $\begin{array}{l}\text { n-Dodecyl- } \beta \text {-D-Maltoside and } \\
\text { Sorbitan Monolaurate(SPAN 20) }\end{array}$ & $\begin{array}{c}6.60 \times 10^{-3} \mathrm{M} \\
\text { (0.34\%wt.) }\end{array}$ & $\begin{array}{c}6.60 \times 10^{-3} \mathrm{M} \\
(0.24 \% \mathrm{wt} .)\end{array}$ & $1: 1$ & 2.13 \\
\hline $\begin{array}{l}\mathrm{n} \text {-Tridecyl- } \beta \text {-D-Maltoside and } \\
\text { Sorbitan Monolaurate(SPAN 20) }\end{array}$ & $\begin{array}{c}6.60 \times 10^{-3} \mathrm{M} \\
(0.35 \% \text { wt. })\end{array}$ & $\begin{array}{c}6.60 \times 10^{-3} \mathrm{M} \\
(0.24 \% \mathrm{wt})\end{array}$ & $1: 1$ & 2.24 \\
\hline 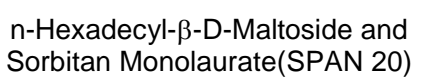 & $\begin{array}{l}6.60 \times 10^{-3} \mathrm{M} \\
\text { (0.37\%wt.) }\end{array}$ & $\begin{array}{l}6.60 \times 10^{-3} \mathrm{M} \\
(0.24 \% w t .)\end{array}$ & $1: 1$ & 3.73 \\
\hline
\end{tabular}

The upper, Table 7.2.9.3a presents IFT data for test tubes that have the aqueous and oleic phases pre-equilibrated. For the IFT measurement itself, we performed this by sampling the aqueous phase, adding a fresh drop of n-octane, and recording the IFT after allowing a couple of hours of spinning time to approach a steady-state value. The lower, Table 7.2.9.3b uses both aqueous and oleic phases that are prepared and then used to measure IFT without any previous exposure to the other fluid.

None of these IFT values are very low, suggesting perhaps that including $2 \mathrm{wt} \% \mathrm{NaCl}$ in the previous APG/SPAN formulations was an important factor to attain low IFT. In fact, we have evidence of that behavior, based on our previous experience with APG/alcohol formulations. We might conclude from these data that the $\mathrm{C} 12$ or $\mathrm{C} 13$ alkyl chain length APG surfactant are the optimum chain lengths (lowest IFT) with the SPAN 20 as a cosurfactant where n-octane is the oil phase, and the system is at room temperature. The IFT values are similar whether using an aqueous phase pre-equilibrated to the oil or one that never had been exposed to a hydrocarbon, suggesting mass transfer is not an issue for these samples.

\subsubsection{IFT Tests -- Pure APG, alpha-versus beta-anomers}

One other comparison is the effect of a subtle change in molecular structure on IFT. Namely we compared the IFT behavior for the alpha- and beta- configurations of n-Octyl-D-Glucoside. The molecular structures for these two surfactants are below: 


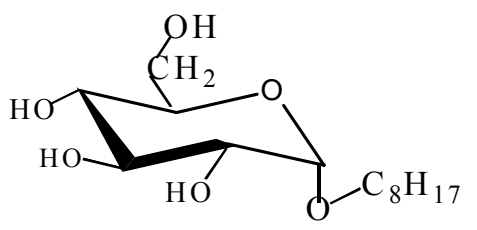

n-Octyl- $\alpha$-D-Glucoside, $\quad$ F.W. $=294.4$

Critical Micellar Concentration: $0.01 \mathrm{mM}$

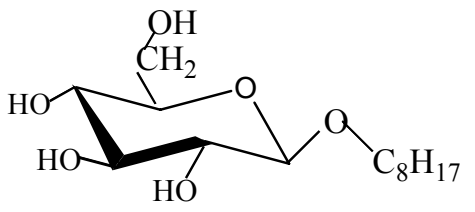

n-Octyl- $\beta$-D-Glucoside, $\quad$ F.W. $=294.4$

Critical Micellar Concentration: $20 \mathrm{mM}$

Figure 7.2.9.3. Structure of alpha- and beta- forms of a surfactant

There is a large difference in behavior between the two surfactants, given that the CMC (critical micelle concentration) of the alpha- $(0.0001 \mathrm{M})$ is $2000 \mathrm{x}$ less than for the beta$(0.02 \mathrm{M})$. The dramatic difference in the CMC between these two surfactants is striking, especially as they have only a subtle difference in their molecular structure. This report also contains a discussion in the theoretical analysis concerning calculated differences between these two anomers. The MD simulations indicate that for the alpha-structure there is more internal hydrogen bonding, whereas the beta-structure is more open so there is a more favorable solvation in water. This means the beta-structure is more water soluble and thus has a greater CMC.

\section{Table 7.2.9.4}

IFT results for n-Octyl-apha-D- and n-Octyl-beta-Glucoside surfactants

(Oil Phase: fresh n-Octane, Temperature: $30^{\circ} \mathrm{C}$ )

\begin{tabular}{|c||c|c|c|}
\hline Compound & Concentration & $\begin{array}{c}\text { Measurement } \\
\text { Condition }\end{array}$ & $\begin{array}{c}\text { IFT } \\
(\mathrm{mN} / \mathrm{m})\end{array}$ \\
\hline \hline n-Octyl- $\alpha-D-G l u c o s i d e$ & $\begin{array}{c}1.00 \times 10^{-2} \mathrm{M} \\
(0.30 \% \mathrm{wt} .)\end{array}$ & $\begin{array}{c}\text { Not equilibrated } \\
\text { with oil phase }\end{array}$ & $\mathbf{1 . 4 8}$ \\
\hline n-Octyl- $\beta-D-G l u c o s i d e$ & $\begin{array}{c}1.00 \times 10^{-2} \mathrm{M} \\
(0.30 \% \mathrm{wt} .)\end{array}$ & $\begin{array}{c}\text { Not equilibrated } \\
\text { with oil phase }\end{array}$ & $\mathbf{2 . 2 4}$ \\
\hline $\mathrm{n}$-Octyl- $\alpha-\mathrm{D}-$ Glucoside & $\begin{array}{c}1.00 \times 10^{-2} \mathrm{M} \\
(0.30 \% \mathrm{wt} .)\end{array}$ & $\begin{array}{c}\text { Equilibrated } \\
\text { with oil phase }\end{array}$ & $\mathbf{6 . 5 6}$ \\
\hline $\mathrm{n}$-Octyl- $\beta-\mathrm{D}-\mathrm{G}-\mathrm{Glucoside}$ & $\begin{array}{c}1.00 \times 10^{-2} \mathrm{M} \\
(0.30 \% \mathrm{wt})\end{array}$ & $\begin{array}{c}\text { Equilibrated } \\
\text { with oil phase }\end{array}$ & $\mathbf{9 . 4 4}$ \\
\hline
\end{tabular}

Results in the table above show that the IFT values are a bit lower with the alpha- versus the beta- form of the surfactant. This is consistent with our intuition of the lower CMC 
surfactant being more efficient in reducing IFT. (Note that here the surfactant concentration is above the $\mathrm{CMC}$ of the alpha-form and below that of the beta-form.) None of the IFT values are very low, probably in part because these surfactants have fairly short hydrophobic tails.

\subsubsection{Alkyl Propoxy Sulfate Surfactants}

\subsubsection{Background/Motivation for Investigation}

Further work focused on systematic studies of the effect of the structure for a series of alkyl propoxy sulfate surfactants (generously provided by Sasol Corporation).

An example structure of one of these surfactants is shown in the theoretical section, but is repeated here for the reader's convenience:

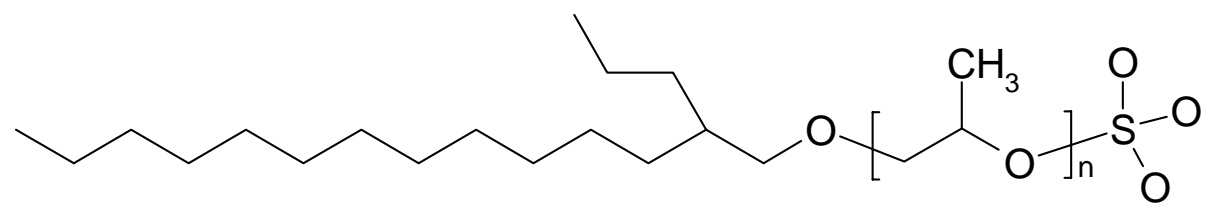

The experiments reported here used 18 surfactant samples supplied by Sasol Chemical.

Surfactant Sample

Alfoterra 13

Alfoterra 15

Alfoterra 18

Alfoterra 23

Alfoterra 25

Alfoterra 28

Alfoterra 33

Alfoterra 35

Alfoterra 38

Alfoterra 43

Alfoterra 45

Alfoterra 53

Alfoterra 55

Alfoterra 58

Alfoterra 48

Alfoterra 63

Alfoterra 65

Alfoterra 68

The second digit of the product code provides the average number of propoxy groups in the surfactant. The other variation in structure among the series of surfactants is 
associated with mainly with the size of the branched alkyl chain. Most, if not all, of these products have branched alkyl chains, as indicated in Figure 7.2.10.1.

As discussed in the summary of the Literature Review in Section 5, this type of surfactants are an interesting surfactant idea as they have become popular for remediation of spilled hydrocarbons (so-called NAPL, nonaqueous phase liquids) from aquifers in recent years, but this chemistry has not been exploited recently for oil field EOR.

Workers have developed surfactant formulations to mobilize NAPL's that can achieve low IFT, require minimal co-surfactant, and suffer only low losses due to solid adsorption (Jayanti, 2001). These NAPL' s studied for these remediation projects may be quite different in character than typical crude oils, but the alkyl propxylated sulfates likely can be adapted for some chemical EOR candidates.

\subsubsection{IFT measurements with alkyl propoxy sulfate surfactants}

The first IFT measurements with the Alfoterra series of surfactants were performed in distilled water in order to mimic the MD simulation studies with the structure shown in Figure 7.2.10.1. These MD simulations had two general findings: 1) lower IFT when the number of propxy groups (PO) was 3 or 5 versus the end members of PO groups of 1 or 8 , and 2) lower IFT with an increase in temperature.

Parallel experimental results tests performed with $1 \mathrm{wt} \%$ Alfoterra 35 in distilled at room temperature and n-decane as the oil phase. The Alfoterra 35 surfactant was selected as it has a longer alkyl chain than the other products, and so is more nearly like the structure used in the MD simulations. The experimental results are shown below.

Table 7.2.10.1. Effect of number of average PO groups and temperature

Sample

Alfoterra 33

Alfoterra 35

Alfoterra 38

$\begin{array}{cc}\text { PO Groups } & \text { IFT (dyne/cm) } \\ 3 & 1.4 \\ 5 & 2.2 \\ 8 & 1.7\end{array}$

These results actually show an IFT maximum at a mid-PO number of simulation results. groups, in contrast to the simulation results.
Alfoterra 35
Alfoterra 35
$25^{\circ} \mathrm{C}$
$77^{\circ} \mathrm{C}$
2.2
1.7

Temperature IFT $($ dyne $/ \mathrm{cm})$

The latter results are in agreement with the simulation, exhibiting a decrease in IFT with an increase in temperature.

The figures below present IFT data for 9 of these Alfoterra surfactants over a range of aqueous phase salinities versus n-octane as the oil phase. All samples contain $2 \mathrm{wt} \%$ (active) of the surfactant. The IFT was measured with formulations that contained only 
Alfoterra surfactant and also samples with $1 \mathrm{wt} \%$ isopropanol as a cosurfactant. In all cases n-octane is the oil phase and the temperature is about $25^{\circ} \mathrm{C}$.

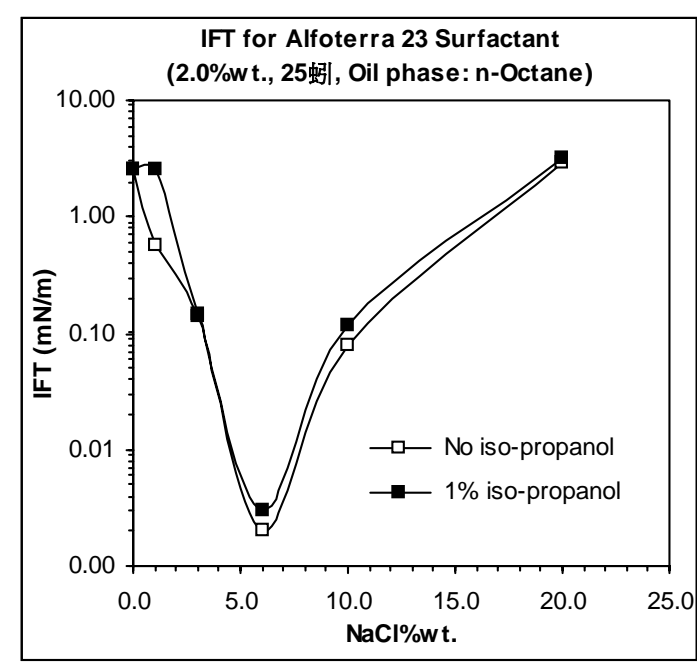

Figure 7.2.10.1 IFT for Alfoterra 23 versus salinity.

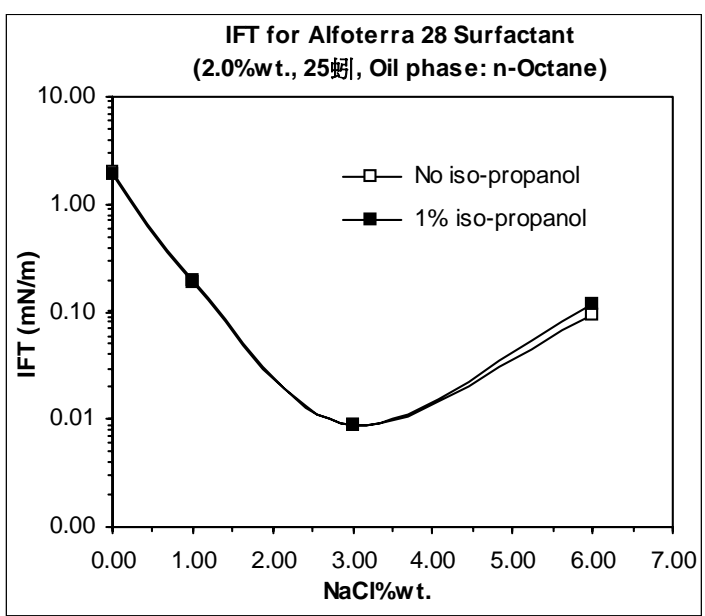

Figure 7.2.10.3 IFT for Alfoterra 28 versus salinity.

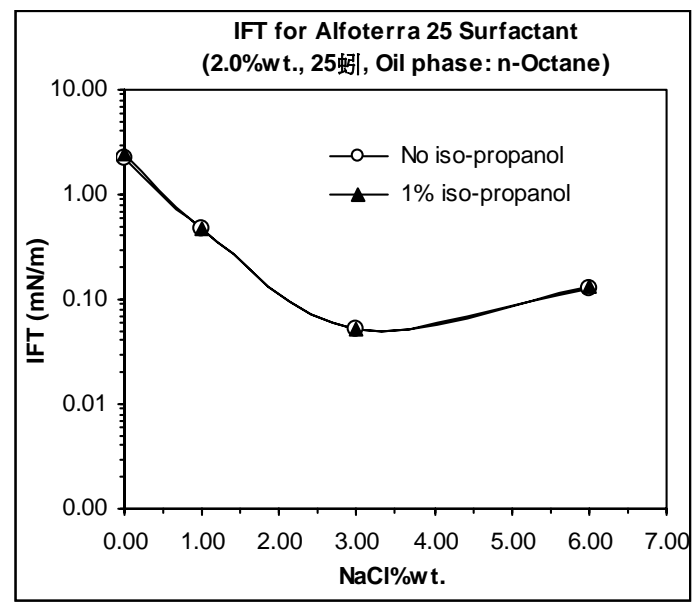

Figure 7.2.10.2 IFT for Alfoterra 25 versus salinity.

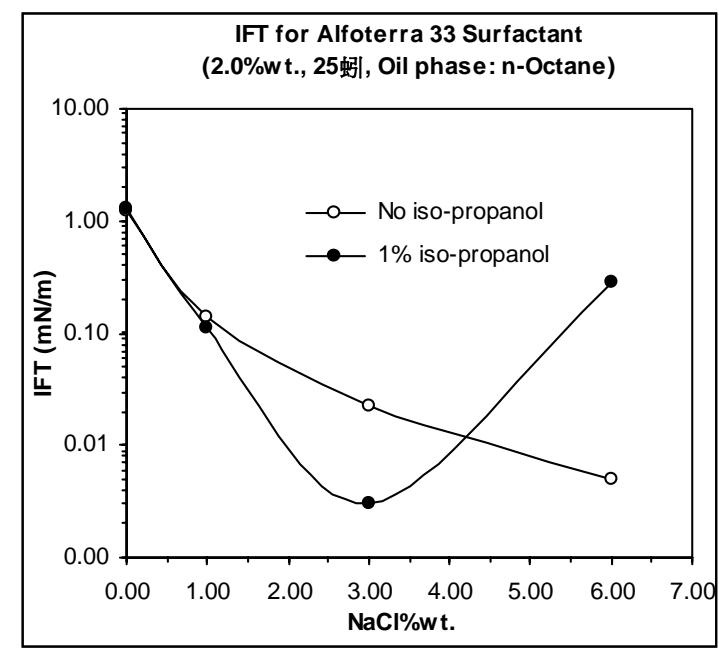

Figure 7.2.10.4 IFT for Alfoterra 33 versus salinity. 


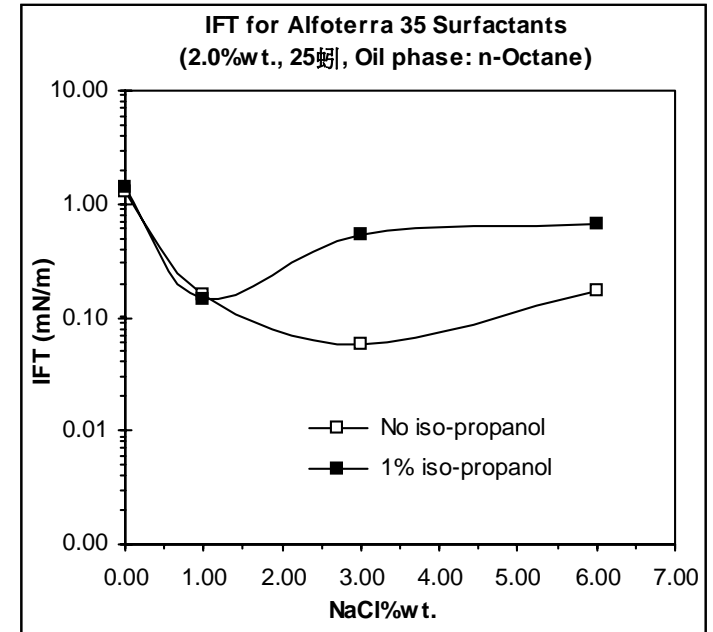

Figure 7.2.10.5 IFT for Alfoterra 35 versus salinity.

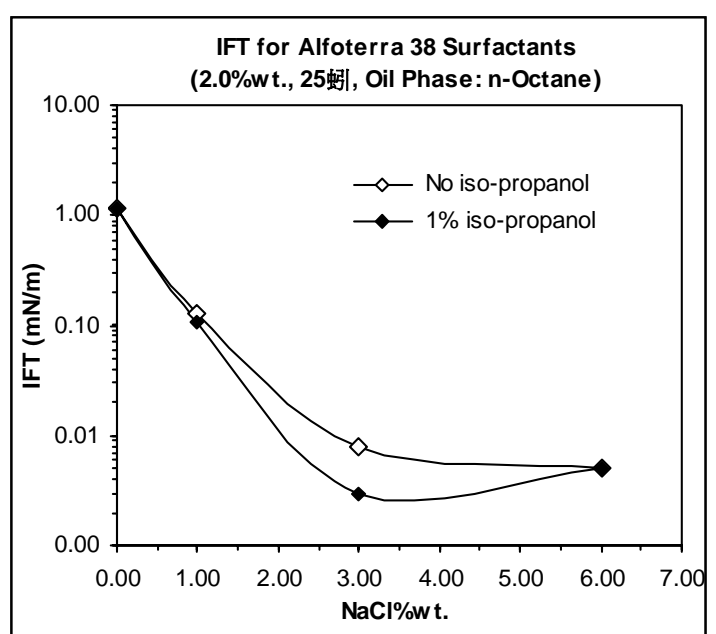

Figure 7.2.10.6 IFT for Alfoterra 38 versus salinity.

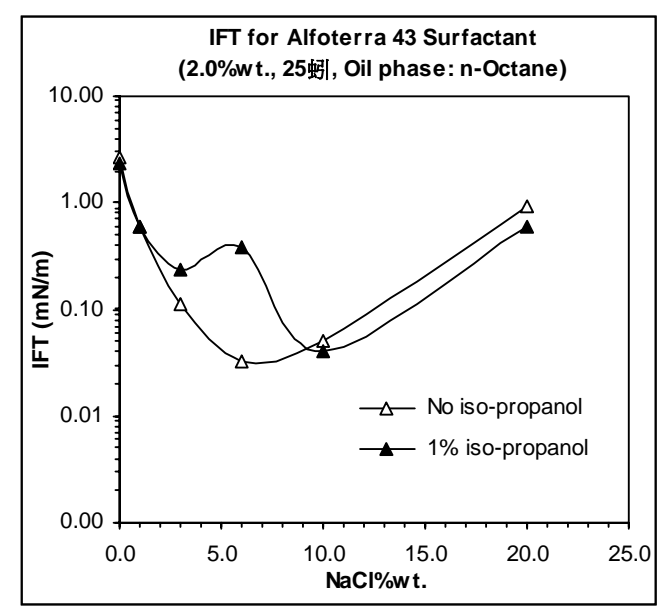

Figure 7.2.10.7 IFT for Alfoterra 43 versus salinity.

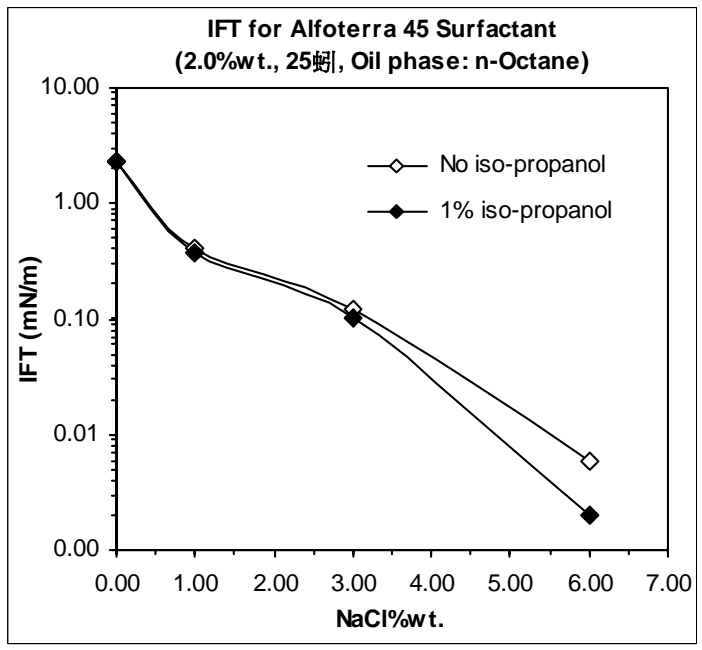

Figure 7.2.10.8 IFT for Alfoterra 45 versus salinity. 


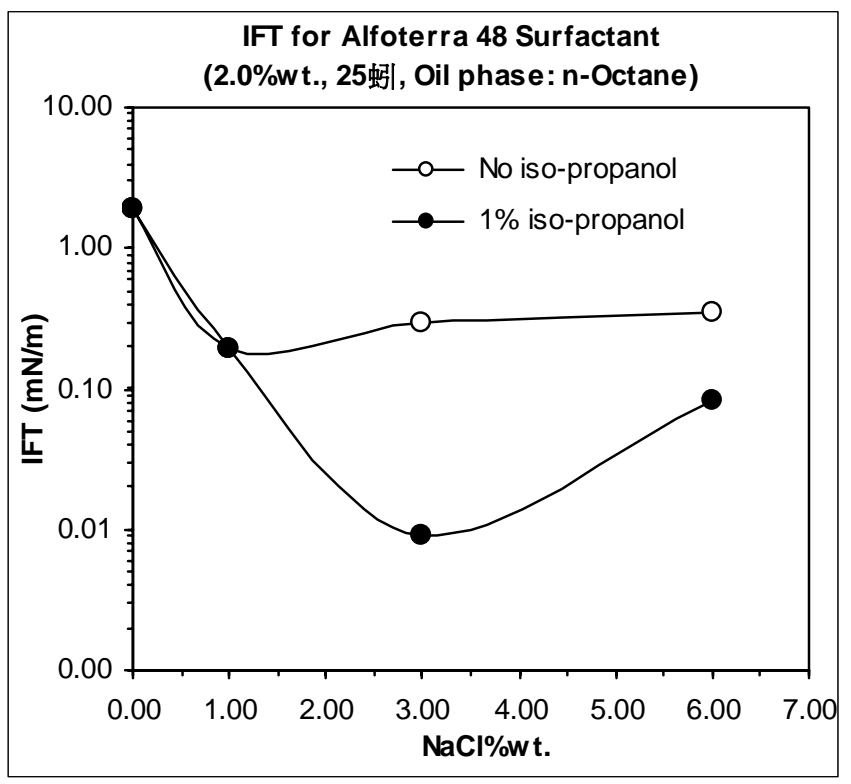

Figure 7.2.10.9 IFT for Alfoterra 48 versus salinity.

The strong sensitivity of IFT to salinity is not surprising given that these surfactants are anionic. These results suggest that the so-called "optimal salinity" (salinity where we observe the lowest IFT and where there is a balance of the surfactant for the aqueous and oleic phases) can be as high as several percent $\mathrm{NaCl}$ concentration. This behavior can be useful in the design of a chemical system that will mobilize oil efficiently in reservoirs that have an in-situ brine with this relatively high salinity.

At salinities significantly below the optimum, the surfactant will tend to remain in the aqueous phase, whereas when above the optimal salinity, the surfactant is driven into the bulk of the oil phase. One trend is that the surfactants with more propoxylated groups (the higher second number of the product code indicates more PO groups) have a lower optimal salinity. This is consistent with the notion that more PO groups make the surfactant more hydrophobic; less salt needs to be added to the system to move the bulk of the surfactant from the aqueous phase to the interface and create its lowest IFT condition.

The addition of iso-propanol as a cosurfactant appears to have little effect on the measured IFT with this type of surfactant. Exceptions in this test series are that some higher IFT's occur when this alcohol is included as a cosurfactant in the samples containing Alfoterra 35 surfactant, and lower IFT values for the surfactants Alfoterra 38 and 48 .

This initial screening produced a number of combinations of Alfoterra surfactant type, brine salinity, and cosurfactant that exhibited low IFT. For the more promising formulations we also measured the IFT at much lower surfactant concentrations, 0.2 or even $0.1 \mathrm{wt} \%$. at the optimal salinity indicated by the $2 \mathrm{wt} \%$ surfactant IFT

measurements. Again, the measurements used n-octane as the oil phase and were 
performed at ambient temperature. Low IFT's at these low surfactant concentrations would be attractive for further study towards a field application.

Table 7.2.10.2. IFT measurements at $0.2 \mathrm{wt} \%$ for selected Alfoterra products

\begin{tabular}{|c|cccc|}
\hline Surfactant & Surf(wt\%) & $\mathrm{IPA}(\mathrm{wt} \%)$ & $\mathrm{NaCl}(\% \mathrm{wt})$ & $\mathrm{IFT}(\mathrm{mN} / \mathrm{m})$ \\
\hline Alfo-23 & 0.2 & 0 & 6 & 0.009 \\
Alfo-23 & 0.2 & 0.1 & 6 & 0.006 \\
Alfo-28 & 0.2 & 0 & 3 & 0.04 \\
Alfo-28 & 0.2 & 0.1 & 3 & 0.019 \\
Alfo-33 & 0.2 & 0.1 & 3 & 0.006 \\
Alfo-33 & 0.2 & 0 & 6 & 0.111 \\
Alfo-38 & 0.2 & 0 & 3 & 0.081 \\
Alfo-38 & 0.2 & 0.1 & 3 & 0.121 \\
Alfo-38 & 0.2 & 0.1 & 6 & 0.249 \\
Alfo-45 & 0.2 & 0 & 6 & 0.012 \\
Alfo-45 & 0.2 & 0.1 & 6 & 0.011 \\
Alfo-48 & 0.2 & 0.1 & 3 & 0.014 \\
\hline
\end{tabular}

IPA $=$ isopropanol

For the lower IFT formulations found at $0.2 \mathrm{wt} \%$ (see table above), the IFT was measured then at an even lower $0.1 \mathrm{wt} \%$ Alfoterra concentration (results given below).

Table 7.2.10.3. IFT measurements at $0.1 \mathrm{wt} \%$ for selected Alfoterra products

\begin{tabular}{|c|cccc|}
\hline Surfactant & Surf(wt\%) & $\mathrm{IPA}(\mathrm{wt} \%)$ & $\mathrm{NaCl}(\% \mathrm{wt})$ & $\mathrm{IFT}(\mathrm{mN} / \mathrm{m})$ \\
\hline Alfo-23 & 0.1 & 0 & 6 & 0.018 \\
Alfo-23 & 0.1 & 0.05 & 6 & 0.017 \\
Alfo-28 & 0.1 & 0 & 3 & 0.009 \\
Alfo-28 & 0.1 & 0.05 & 3 & 0.008 \\
Alfo-33 & 0.1 & 0.05 & 3 & 0.018 \\
Alfo-33 & 0.1 & 0 & 6 & $\mathrm{ND}$ \\
Alfo-38 & 0.1 & 0 & 3 & $\mathrm{ND}$ \\
Alfo-38 & 0.1 & 0.05 & 3 & $\mathrm{ND}$ \\
Alfo-38 & 0.1 & 0.05 & 6 & $\mathrm{ND}$ \\
Alfo-45 & 0.1 & 0 & 6 & 0.008 \\
Alfo-45 & 0.1 & 0.05 & 6 & 0.043 \\
Alfo-48 & 0.1 & 0.05 & 3 & 0.064 \\
\hline
\end{tabular}

$$
\text { IPA }=\text { isopropanol } \quad(\mathrm{ND}=\text { not determined })
$$

The above data are organized below to show the change in IFT with Alfoterra concentration for the formulations selected for their better performance. 
Table 7.2.10.4. Effect of Alfoterra concentration on IFT for selected formulations

\begin{tabular}{|c|c|c|c|c|}
\hline Surfactant & Surf(wt\%) & IPA(wt\%) & $\mathrm{NaCl}(\% w t)$ & IFT(dyne/cm) \\
\hline Alfo-23 & 2 & 0 & 6 & 0.002 \\
\hline Alfo-23 & 0.2 & 0 & 6 & 0.009 \\
\hline Alfo-23 & 0.1 & 0 & 6 & 0.018 \\
\hline Alfo-23 & 2 & 1 & 3 & 0.003 \\
\hline Alfo-23 & 0.2 & 0.1 & 3 & 0.006 \\
\hline Alfo-23 & 0.1 & 0.05 & 3 & 0.017 \\
\hline Alfo-28 & 2 & 0 & 3 & 0.009 \\
\hline Alfo-28 & 0.2 & 0 & 3 & 0.04 \\
\hline Alfo-28 & 0.1 & 0 & 3 & 0.009 \\
\hline Alfo-28 & 2 & 1 & 3 & 0.009 \\
\hline Alfo-28 & 0.2 & 0.1 & 3 & 0.019 \\
\hline Alfo-28 & 0.1 & 0.05 & 3 & 0.008 \\
\hline Alfo-45 & 2 & 0 & 6 & 0.006 \\
\hline Alfo-45 & 0.2 & 0 & 6 & 0.012 \\
\hline Alfo-45 & 0.1 & 0 & 6 & 0.008 \\
\hline Alfo-45 & 2 & 1 & 6 & 0.002 \\
\hline Alfo-45 & 0.2 & 0.1 & 6 & 0.011 \\
\hline Alfo-45 & 0.1 & 0.05 & 6 & 0.043 \\
\hline Alfo-48 & 2 & 1 & 3 & 0.009 \\
\hline Alfo-48 & 0.2 & 0.1 & 3 & 0.014 \\
\hline Alfo-48 & 0.1 & 0.05 & 3 & 0.064 \\
\hline
\end{tabular}

It is encouraging that for several cases, the IFT is quite low at all three surfactant concentrations. The Alfoterra- 23 perhaps has the best results, followed by the Alfoterra45 at $6 \mathrm{wt} \% \mathrm{NaCl}$ and Alfoterra- 28 at $3 \mathrm{wt} \% \mathrm{NaCl}$ and no IPA. In some cases, the IFT does not increase monotonically with a decrease in the Alfoterra concentration. This behavior could be associated with experimental errors in the IFT measurement and/or reflect that the optimal salinity can shift with a change in surfactant concentration.

Some follow-up IFT studies considered 6 other surfactants from this series. Samples Alfoterra 13, 15, and 18 were included later because they showed relatively low adsorption in our kaolinite clay adsorption test (see next subsection). Samples Alfoterra 63,65 , and 68 were added because the supplier indicated that these 3 surfactants likely would be least expensive and easiest to manufacture if the Alfoterra surfactants were manufactured in significant commercial volumes.

These IFT tests screened the performance of these 6 particular products at only $0.2 \mathrm{wt} \%$ concentration (active) of each surfactant. Because previous results already demonstrated 
some of the other Alfoterra products could achieve quite low IFT at 0.2, or even at 0.1 $\mathrm{wt} \%$ (active) surfactant, we focused immediately on more severe test conditions for measuring IFT for this other group of Alfoterra surfactants. IFT measurements used an aqueous phase already equilibrated with n-octane and were done at room temperature.

The figures below show the IFT results for the Alfoterra surfactants $13,15,18,63,65$, and 68 .

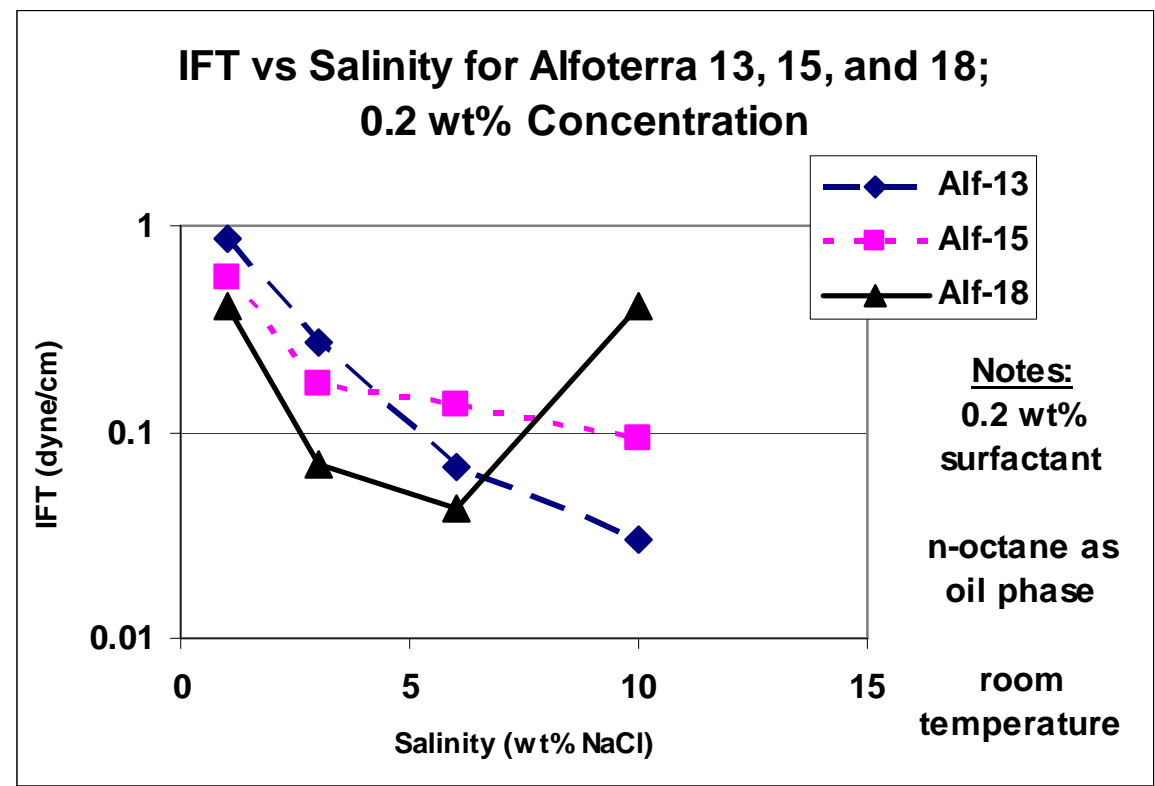

Figure 7.2.10.10. IFT results for $0.2 \mathrm{wt} \%$ Alfoterra 13,15 , and 18 versus salinity

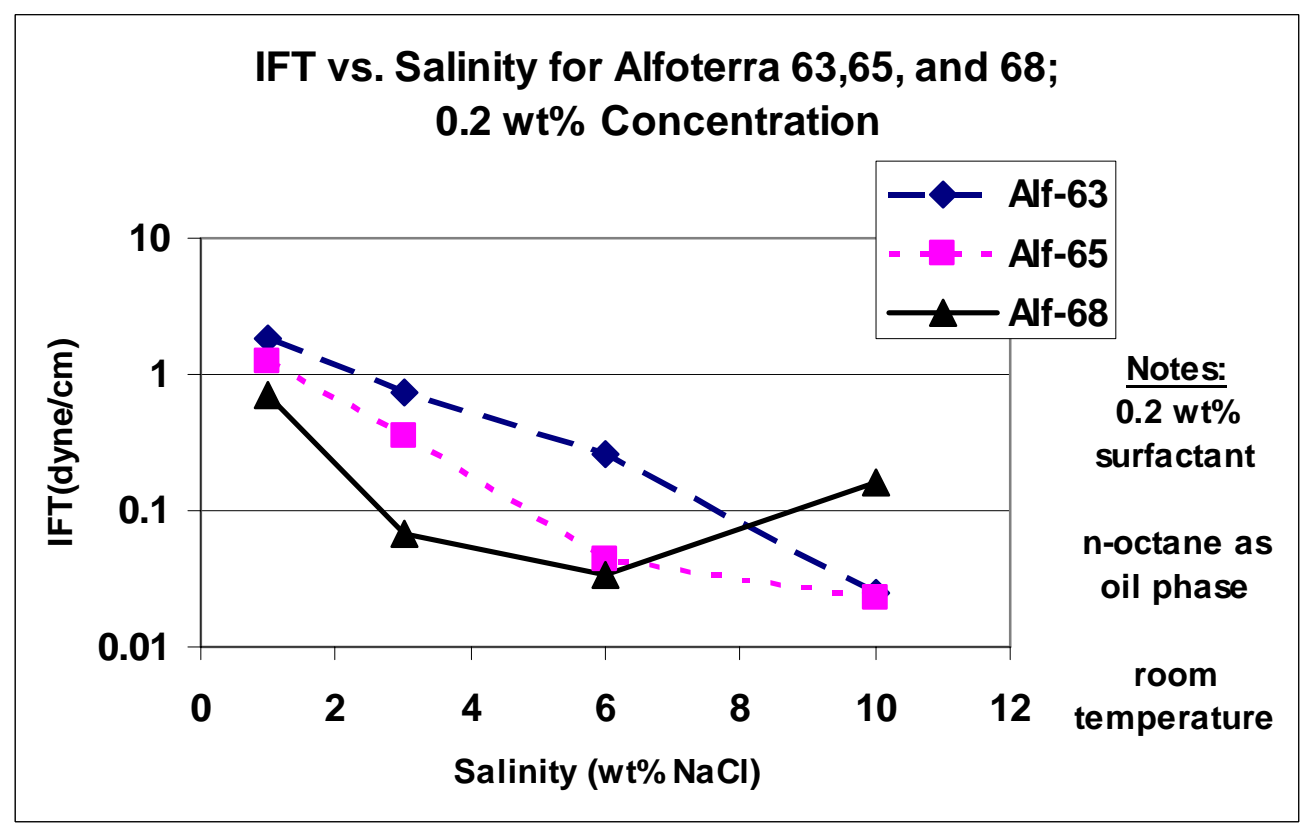

Figure 7.2.10.11. IFT results for $0.2 \mathrm{wt} \%$ Alfoterra 63 , 65, and 68 versus salinity 
One trend is that the optimal salinity (minimum IFT condition) in general increases with an increase in the number of propoxy groups (increase of hydrophobicity); this is the same behavior as found for the $2 \mathrm{wt} \%$ Alfoterra products screened previously. The IFT results indicate the optimal salinity for some of these 6 Alfoterra surfactants in Figures 7.2.10.10 and 7.2.10.11 may exceed even $10 \mathrm{wt} \%$ salinity; this suggests they are possible candidates for high salinity applications. None of the measured IFT values are especially low, as compared to the best Alfoterra systems reported above in Table 7.2.10.2.

The table below compares the measured IFT for different Alfoterra products and two crude oils at $45^{\circ} \mathrm{C}$. One crude (Oil \#4) has a density of $0.857 \mathrm{~g} / \mathrm{cc}$ and the other crude (GOM) density is $0.812 \mathrm{~g} / \mathrm{cc}$ at $45^{\circ} \mathrm{C}$. We selected this test temperature and a brine salinity of about $0.3 \mathrm{wt} \% \mathrm{NaCl} /$ liter as that is representative of conditions at the Oil \#4 reservoir. The second oil -- GOM -- from the Gulf of Mexico - is a lighter, somewhat waxy crude.

Table 7.2.10.5. Alfoterra surfactant IFT versus two crude oils with $0.3 \mathrm{wt} \% \mathrm{NaCl}$

\section{Different Hydrocarbons, IFT (dyne/cm)}

\begin{tabular}{cccl} 
Surfactant (0.2 wt\%) & Crude Oil \#4 & GOM Oil & n-Octane \\
\hline Alfoterra 23 & 0.94 & N/A & N/A \\
Alfoterra 28 & 0.22 & 0.17 & 0.49 \\
Alfoterra 38 & 0.23 & 0.10 & 0.72 \\
Alfoterra 45 & 0.65 & N/A & N/A \\
Alfoterra 48 & 0.31 & N/A & N/A \\
Alfoterra 68 & 0.69 & N/A & N/A
\end{tabular}

None of the IFT values are low, but at least these values are lower for the crude oils than the n-octane at these same conditions. We speculated that the IFT values could be significantly lower for a crude oil if the brine salinity was increased. This is based on the observation that previous results with n-octane and Alfoterra surfactants at ambient temperature indicate an optimal salinity of several percent $\mathrm{NaCl}$.

Results shown below it is possible to attain low IFT with an Alfoterra surfactant and the GOM crude oil, and again with an IFT even less than the n-octane as the oil phase.

Table 7.2.10.6. Alfoterra 28 surfactant IFT versus GOM crude oil and n-octane

\section{IFT (dyne/cm)}

\begin{tabular}{ccc} 
wt\% NaCl & GOM Crude & n-Octane \\
\cline { 1 - 2 } & 0.11 & 0.14 \\
3 & 0.003 & 0.007 \\
6 & 0.078 & 0.22
\end{tabular}

Notes: Surfactant $0.2 \mathrm{wt} \%, 45^{\circ} \mathrm{C}, \mathrm{W} / \mathrm{O}=1$, fresh fluids (non-equilibrated) 
Finally, in this portion of the test program we measured IFT for formulations that have a blend of both an alkyl propoxy sulfate and a commercial APG surfactant. This is to test the idea that perhaps these two surfactant types that by themselves can create low IFT conditions may in fact be a synergistic combination. The results indicate that mixing PG 2067 with the alkyl propoxy sulfates increases the IFT, whereas the mixture containing the PG 2062 has relatively little effect as compared to surfactants Alfoterra 23, 25 , and 28 by themselves. Test conditions included $1 \mathrm{wt} \%$ (active) total surfactant concentration, in a $2 \mathrm{wt} \% \mathrm{NaCl}$ brine, against an n-octane oil phase, and at ambient temperature.

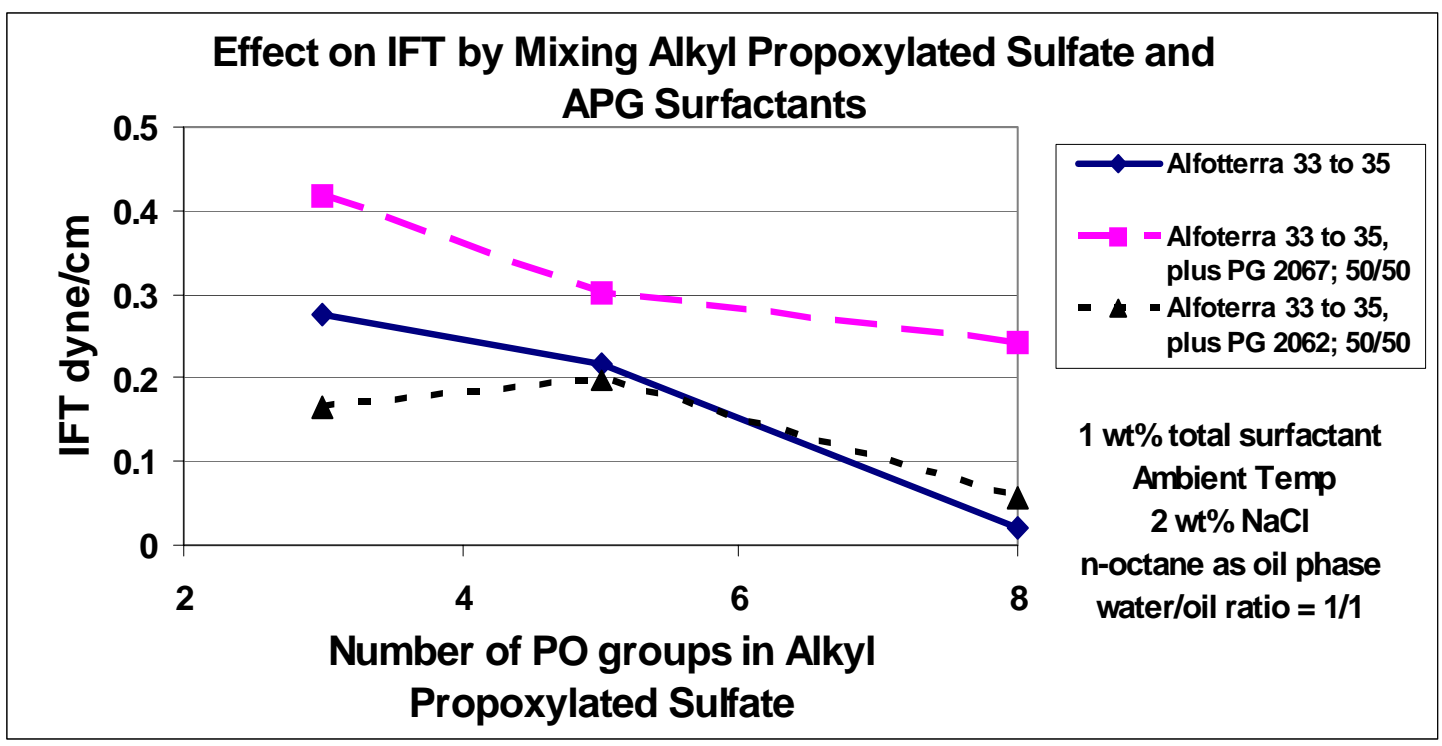

Figure 7.2.10.12. IFT measured for blends of alkyl propoxy sulfates and APG commercial surfactants

\subsubsection{Adsorption and oil displacement tests}

Results for solid adsorption screening tests for selected Alfoterra surfactants are given below. In this test series, as with other surfactants studies previously, surfactant solutions in $2 \mathrm{wt} \% \mathrm{NaCl}$ are exposed to kaolinite clay (weight ratio solution/clay is 20) at room temperature with mild agitation for a day to allow the system to approach equilibrium. This test series used starting Alfoterra concentrations of $0.5,1$, and $2 \mathrm{wt} \%$ surfactant. Results are summarized in the table below. 
Table 7.2.10.7. Adsorption of Alfoterra surfactants onto kaolinite clay

\section{Surfactant Final Concentrations: Solution and Surface Adsorption}

\begin{tabular}{|l|c|c|c|c|c|c|}
\hline \hline \multicolumn{1}{|c|}{ Name } & $\mathbf{C}_{\text {eq. }}(\mathbf{w t . \%})$ & $\Gamma$ (mg/g) & $\mathbf{C}_{\text {eq. }}(\mathbf{w t . \%})$ & $\Gamma(\mathbf{m g} / \mathbf{g})$ & $\mathbf{C}_{\text {eq. }}(\mathbf{w t . \% )}$ & $\Gamma$ (mg/g) \\
\hline Alfoterra 13 & 0.4513 & 8.9 & 0.9637 & 6.7 & 1.979 & 4 \\
\hline Alfoterra 15 & 0.4508 & 9.2 & 0.9275 & 14.3 & 2.017 & -3.4 \\
\hline Alfoterra 18 & 0.4843 & 3 & 0.9686 & 6.1 & 1.9621 & 7.2 \\
\hline Alfoterra 23 & 0.4231 & 14.5 & 0.8898 & 20.6 & 1.7829 & 42.5 \\
\hline Alfoterra 25 & 0.4839 & 3.2 & 0.9586 & 7.9 & 1.9682 & 5.4 \\
\hline Alfoterra 28 & 0.4991 & 0.4 & 1.0146 & -2.2 & 2.0295 & -5.4 \\
\hline Alfoterra 33 & 0.3902 & 20.8 & 0.7963 & 38.3 & 1.6974 & 55.7 \\
\hline Alfoterra 35 & 0.3602 & 26.1 & 0.7587 & 45.1 & 1.6381 & 66.1 \\
\hline Alfoterra 38 & 0.4375 & 11.9 & 0.8906 & 20.6 & 1.8278 & 30.1 \\
\hline Alfoterra 43 & 0.3941 & 20 & 0.8386 & 30.5 & 1.7523 & 46.7 \\
\hline Alfoterra 45 & 0.47 & 12 & 0.9436 & 23.6 & 1.9488 & 35.9 \\
\hline Alfoterra 48 & 0.4977 & 6.2 & 0.9126 & 12.6 & 1.8451 & 27.1 \\
\hline Alfoterra 53 & 0.3797 & 22.5 & 0.7859 & 40.1 & 1.6619 & 61.7 \\
\hline Alfoterra 55 & 0.4252 & 13.8 & 0.8755 & 22.6 & 1.7748 & 40 \\
\hline Alfoterra 58 & 0.4417 & 10.3 & 0.9047 & 15.2 & 1.796 & 33.6 \\
\hline Alfoterra 63 & 0.3943 & 20 & 0.7728 & 42.5 & 1.7478 & 47.7 \\
\hline Alfoterra 65 & 0.4523 & 9.1 & 0.9006 & 18.8 & 1.8533 & 27.6 \\
\hline Alfoterra 68 & 0.4672 & 6.2 & 0.9267 & 14.3 & 1.88 & 22.9 \\
\hline
\end{tabular}

If these results are ranked order by the Alfoterra surfactant with the lowest to the highest solid adsorption (sum of adsorption at 3 concentrations), the results are:

Table 7.2.10.8. Rank order of Alfoterra surfactants by solid adsorption onto kaolinite.

$\begin{array}{ll}1 & \text { Alfoterra 28 } \\ 2 & \text { Alfoterra 18 } \\ 3 & \text { Alfoterra 25 } \\ 4 & \text { Alfoterra 13 } \\ 5 & \text { Alfoterra 48 } \\ 6 & \text { Alfoterra 15 } \\ 7 & \text { Alfoterra 68 } \\ 8 & \text { Alfoterra 65 } \\ 9 & \text { Alfoterra 58 } \\ 10 & \text { Alfoterra 38 } \\ 11 & \text { Alfoterra 45 } \\ 12 & \text { Alfoterra 55 } \\ 13 & \text { Alfoterra 23 } \\ 14 & \text { Alfoterra 43 } \\ 15 & \text { Alfoterra 33 } \\ 16 & \text { Alfoterra 63 } \\ 17 & \text { Alfoterra 53 } \\ 18 & \text { Alfoterra 35 }\end{array}$


The general trend for adsorption sorted by Alfoterra surfactant series from lowest to highest solid adsorption is

$$
\text { Alfoterra 10's }<20 \text { 's }<60 \text { 's, }<50 \text { 's }<.40 \text { 's }<30 \text { 's }
$$

If we group the adsorption results by the number of $\mathrm{PO}$ groups in the Alfoterra surfactants, then these data further suggest the adsorption decreases with an increase in the average number of $\mathrm{PO}$ groups.

Based on early favorable IFT measurements with the Alfoterra surfactants, we selected the Alfoterra 38 for further investigation in a coreflood oil displacement experiment. It appears to have a broad region of low IFT versus salinity, with the optimum salinity at around $3 \mathrm{wt} \% \mathrm{NaCl}$.

Note that in this experiment the connate brine is quite high, $10 \mathrm{wt} \% \mathrm{NaCl}$, and the chemical slugs are formulated at a much lower $1 \mathrm{wt} \% \mathrm{NaCl}$, This was designed to test two aspects, 1) that an Alfoterra surfactant formulation could recover significant tertiary oil where the in-situ brine is fairly high salinity, and 2) that the so-called "salinity gradient" approach is a reasonable strategy. That is, the in-situ brine is above the optimal salinity (about $3 \mathrm{wt} \% \mathrm{NaCl}$ ) for the chemical formulation, and the injected solutions are below this optimum salinity. The tertiary oil recovery curve is presented below.

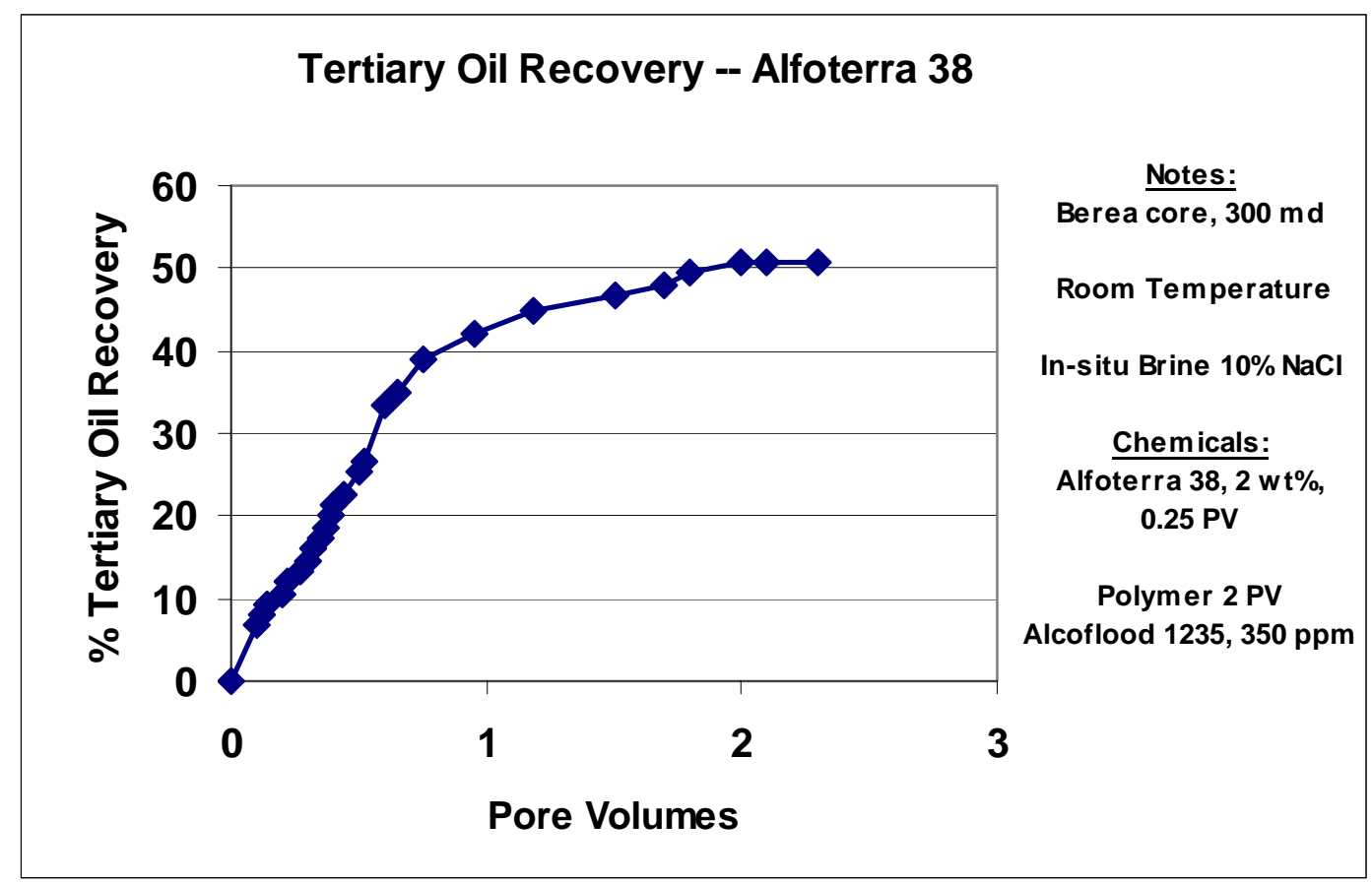

Figure 7.2.10.13. Tertiary oil recovery results from injection of a Alfoterra 38 chemical formulation. 
Some details about this coreflood:

All steps at room temperature.

Berea sandstone cores; approximately $300 \mathrm{md}$ water permeability, 1" x 12 " core

Oil phase: n-octane

Waterflood residual oil saturation: 0.25 ;

Connate brine composition $10 \mathrm{wt} \% \mathrm{NaCl}$,

Surfactant Slug:

$20 \mathrm{wt} \%$ (active) Alfoterra 38 in $1 \mathrm{wt} \% \mathrm{NaCl}$.

$0.25 \mathrm{PV}$ slug

Drive polymer solution:

350 ppm Alcoflood 1235 (Ciba Corp.) in $1 \mathrm{wt} \% \mathrm{NaCl}$.

2 Pore Volume

Chemical injection at $0.06 \mathrm{ml} / \mathrm{min}$, or about a $3 \mathrm{ft} /$ Day frontal advance rate.

Also based on early favorable IFT measurements with these surfactants, we selected the Alfoterra 23 for further investigation in a coreflood oil displacement experiment. This surfactant was found to have low IFT at a concentration of only $0.2 \mathrm{wt} \%$. It appears to have a broad salinity region of low IFT, with the optimum salinity at around $6 \mathrm{wt} \% \mathrm{NaCl}$.

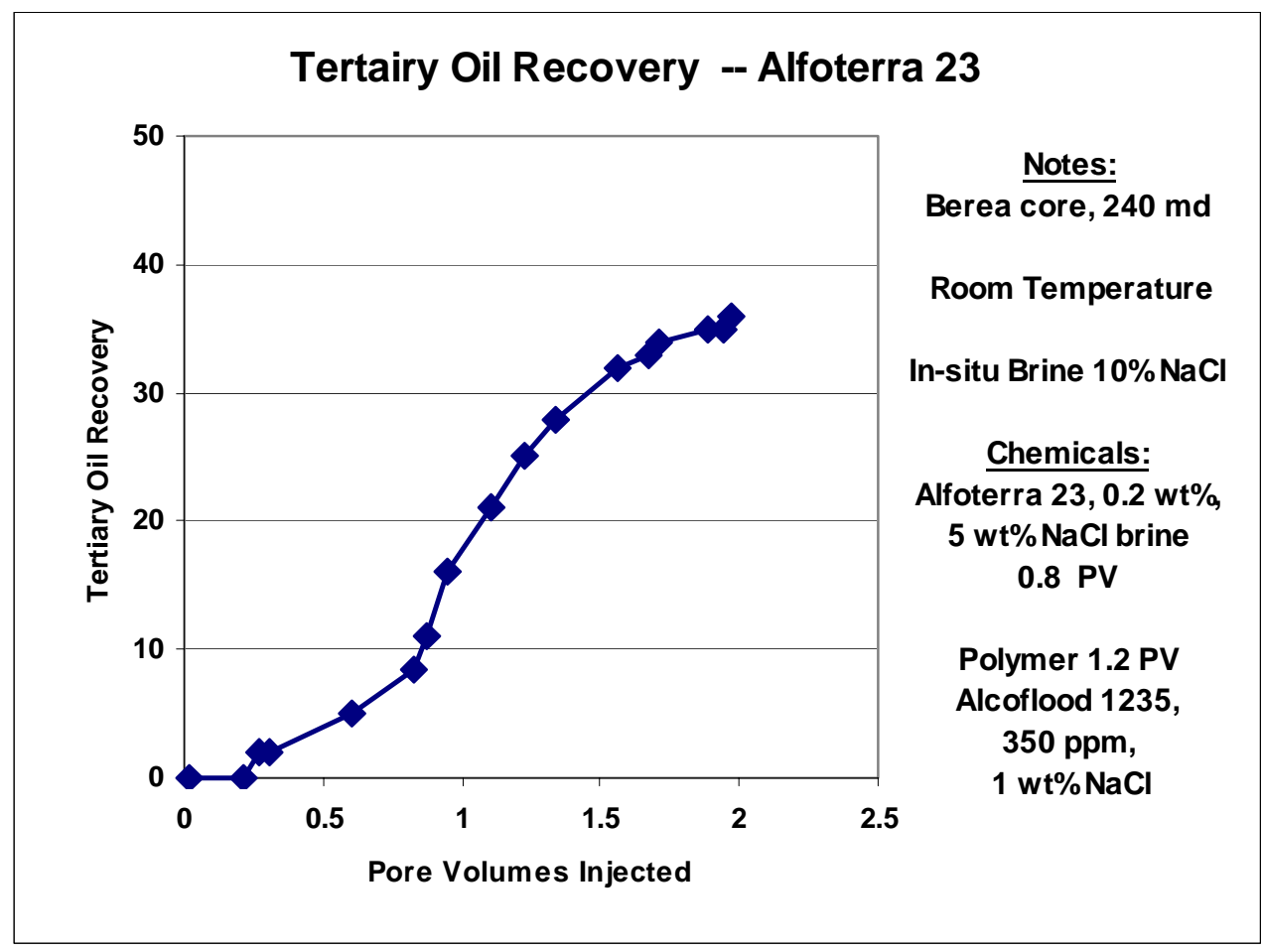

Figure 7.2.10.14. Tertiary oil recovery results from injection of a Alfoterra 23 chemical formulation. 
Some details about this coreflood:

All steps at room temperature.

Berea sandstone cores; approximately $250 \mathrm{md}$ water permeability, 1" x 12 " core

Oil phase: n-octane

Waterflood residual oil saturation: 0.25 ;

Connate brine composition $10 \mathrm{wt} \% \mathrm{NaCl}$,

Surfactant Slug:

$0.2 \mathrm{wt} \%$ (active) Alfoterra 38 in $\% \mathrm{wt} \% \mathrm{NaCl}$.

$0.8 \mathrm{PV}$ slug

Drive polymer solution:

350 ppm Alcoflood 1235 (Ciba Corp.) in $1 \mathrm{wt} \% \mathrm{NaCl}$.

1.2 Pore Volume

Chemical injection at $0.06 \mathrm{ml} / \mathrm{min}$, or about a $3 \mathrm{ft} /$ Day frontal advance rate.

The purpose of this coreflood was to confirm that a low concentration of Alfoterra 23 could recover significant tertiary oil. In particular, the design of the coreflood experiment was intended to have the displacement occur at a favorable, low IFT environment by having the large surfactant slug at $5 \mathrm{wt} \% \mathrm{NaCl}$, just below the optimal salinity. Finally, the chase, polymer slug, was made up in a low salinity $(1 \mathrm{wt} \% \mathrm{NaCl})$ brine so as to be well below optimum and complete an overall salinity gradient design.

While the tertiary oil recovery is only mediocre, the chemical efficiency of this coreflood experiment is relatively good. The mass of Alfoterra 23 surfactant injected is less than that used in the previous, Alfoterra 38 coreflood test, and the percent tertiary recovery being somewhat less with the Alfoterra 23 . As with the previous oil recovery experiment, significant residual oil recovery occurred even with the connate brine being a relatively high, $10 \mathrm{wt} \% \mathrm{NaCl}$. 


\subsection{Project Implications / Ideas for Improved Surfactant Formulations}

One general research direction would be further study of yet other nonionic surfactants with large polar hear groups in addition to the APG-type. One expectation is that these large-head nonionic structures are expected to function relatively independently of the salinity. This behavior has the advantage of making the design of the IOR process easier and more robust (choice of make-up and chase brine is less critical). Also, such behavior should lend itself to developing chemical formulations that can create a low IFT at relatively high salinity.

A related idea is the use of effective, moderately priced, surfactants that in combination with inexpensive cosurfacants can achieve extremely low IFT values. This was shown to be the case with the alkylpolyglycoside (APG) surfactants / alcohol mixtures presented here. On average we are able to create significantly low IFT values $(0.01 \mathrm{dynes} / \mathrm{cm}$ or less) with less than $20 \%$ of the customary surfactant concentration by weight.

One theme brought out by the modeling efforts is the concept of achieving a diffuse interface; this suggests creating longer surfactants built with a gradual transitions from very polar to very non polar behavior. The general idea is to create a "dumb bell" shaped surfactant to "lock" the large polar regions in water and the non-polar regions in the alkane, providing a more robust interface. This is consistent with the notion and observation of improved IFT performance (but normally a downside of increasing solid adsorption) with increasing the alkyl chain length.

Another idea would be to combine features of the APG and the alkyl propoxy sulfate surfactants. For example, the head group of an APG surfactant could be combined with a branched alkyl chain, perhaps thereby improving the water solubility of an APG product. Furthermore, this alkyl chain could feature ethoxyl (EO) or propoxy (PO) groups that can aid in adjusting the hydrophilic and hydrophobic balance.

\section{CONCLUSIONS}

Conclusions from this project include:

The rigorous calculations of interfacial tension (IFT) via atomistic level simulations are in quantitative agreement for pure component systems (e.g. water and a hydrocarbon). Including surfactant molecules complicates the simulation significantly; so far atomistic level simulations are successful in providing semi-quantitative agreement with water/surfactant/oil systems considered. Absolute values are typically higher than found experimentally. In addition, perhaps due to the limited sample sizes and simulation times, as well as the inherit numerical approximations in the calculation of pressures from molecular dynamics, the precision of the calculations is not high enough so far to distinguish surfactants with IFT differences of less than 1 dyne $/ \mathrm{cm}$.

In spite of the current limitations, the molecular dynamics simulations provided useful technical insights as to the molecular structure and sources of IFT water/surfactant/oil 
systems that translate into recommended surfactants for laboratory evaluation. Examples of this include

a) our ability to reproduce the effect of changing the substitution site for the polar (benzosulfonate) group along the alkyl backbone chain in this type of ionic surfactants, b) uncovering the correlation between a high interfacial-mixing of oil/surfactant and surfactant/water media and low surface tension

c) establishing the stability of interfaces for three families of surfactants i) ionic (alkylbenzosulfonates), ii) alkyl propoxylated surfactants, and iii) non-ionic alkyl polyglucoside surfactants. For the last surfactants, simulations also examined the interactions with a cosurfactant at an interface and found regular packing associated with a low IFT condition.

Testing of various simulation parameters has provided some guidelines as to the required values of numerical parameters (e.g. number of molecules) required to achieve a converged prediction of IFT, number of integration slabs, length of time of simulations, averaging step size, and thermodynamic ensembles.

The less rigorous simulation methods can aid in providing a qualitative picture of the desirable features of an effective surfactant. For example, surfactants that enhance the transition between the water and oil phases is desirable. Coulomb and van der Waals component forces are important across the interface.

The completed literature review suggests some of the newer ideas for IOR surfactants including: 1) sulfonated alkyl aromatics, 2) alkyl propoxylated sulfates, 3) alkyl polyglycosides, 4) more emphasis on formulation (synergistic cosurfactants), 5) "dumb bell" surfactants, and 6) polymeric surfactants. A substantial compilation of literature and new results are included in this report.

Laboratory measurements of adsorption of several homologous series of non-ionic surfactants onto kaolinite clay demonstrate their adsorption level generally decreases as the surfactant is more water soluble.

Supporting experimental studies also demonstrate that a non-ionic diethanolamine may be a good candidate as the main surfactant (previously considered as a cosurfactant).

Focused experimental studies of alkyl polyglycoside (APG) surfactant-based formulations confirm they can have the desirable attributes of creating a low IFT that is somewhat independent of salinity and temperature. Some formulations also had the beneficial attribute of creating a low IFT even with very low APG concentrations. It also was found there is an optimum co-linker additive with an APG surfactant to generate its minimum IFT. In particular, we uncovered a new result that aromatic based alcohols may be efficient co-linkers for APG surfactants. More specifically, the best APG formulations included:

PG 2062 / 1-octanol

PG 2062 / 1-naphthol

PG 2069 / SPAN 20 
Detailed study with a series of branched alkyl propoxy sulfate surfactants demonstrate these may create low IFT conditions, and at a low surfactant concentration. In addition, these low IFT values may occur at a salinity of several percent, suggesting these surfactants may be applicable for IOR in relatively higher salinity reservoirs. For this class of surfactants, those with good performance in our testing program are Alfoterra 23 and Alfoterra 38. The Alfoterra 28 also has promise given that It has relatively low solid adsorption, plus it can attain low IFT under certain conditions.

The data presented herein can provide the needed industrial impetus for extending the application (alkyl polyglycoside) or scaling up (alkylpropoxy sulfates) of promising surfactants for enhanced oil recovery. Furthermore, the advanced simulations tools presented here can be used to continue to uncover new types of surfactants with promising properties such as inherent low IFT and biodegradability and can be of further utility to the specialty chemical oil industry.

\section{REFERENCES}

Balzer, D.: "Alkylpolyglcosides, their Physico-chemical Properties and their Uses", Tenside Surf. Det., 38, 419 - 427, 1991a.

Balzer, D., et. al., U.S. Patent 4,985,154, "Process for the extraction of crude oil from an underground deposit using surfactants", 1991b.

Balzer, D., and Luders, H., editors.: Nonionic Surfactants, Alkyl Polyglycosides, Marcel Dekker, New York, p. 228 - 243, 1996.

Barakat, Y., et. al.: "Adsorption of alkylbenzene sulphonates onto mineral surfaces", Indian Journal of Chem. Tech., Vol. 2, 162-166, May 1995.

Belmares, M., Blanco, M., Goddard III, W.A., et. al.: "Hildebrand and Hansen Solubility Parameters from Molecular Dynamics with Applications to Electronic Nose Polymer Sensors", submitted for publication.

Berger, P.and Lee, C.H.: "Ultra-low Concentration Surfactants for Sandstone and Limestone Floods", paper SPE 75186, presented at SPE/DOE Symposium on Improved Oil Recovery, Tulsa, April 13-17, 2002.

Cash, R.L., et. al.: "Modeling Crude Oils for Low Interfacial Tension”, paper SPE 5813, presented at the SPE Symposium on Improved Oil Recovery, 22-24 March 1976.

Cayais, J.L., Schechter, R.S., and Wade, W.H.: "The Measurement of Low Interfacial Tension via the Spinning Drop Technique", section 17, Surfactant Applications, 1977.

Chattoraj, D.K. and K.S. Birdi, Adsorption and the Gibbs Surface Excess, Plenum: New York, 1984. 
Doe, P.H.,Wade, W.H, and Schechter, R.S.: “Alkyl Benzene Sulfonates for Producing Low Interfacial Tension between Hydrocarbons and Water", J. Coll. Int. Science, 59, $525,1977$.

Doe, P.H.,El-Emary, Wade, W.H, and Schechter, R.S.: "The Influence of Surfactant Structure on Low interfacial Tensions”, ACS Symposium Series 91, Chapter 2, 1978.

Endo, H., et. al.: "Amphiphilic block copolymers as efficiency boosters in microemulsions: a SANS investigation of the role of polymers", Appl. Phys. A [Suppl.], S392 - S395, 2002.

Forster, T., et. al.: "Physico-chemical basics of microemulsions with alkyl polyglycosides", Progr. Colloid Polym. Sci., 101, 105 - 112, 1996.

Forster, T., Guckenbiehl, B., Hensen, H., and von Rybinski.: "Physico-chemical basis of microemulsions with alkyl polyglycosides”, Progr. Colloid Polym Sci., 101, 105-112, 1996.

Grest, G. S.; Lacasse, M. D.; Kremer, K., Phy. Revf. A, 33, 3628, 1986.

Green, D.W. and Willhite, G.P., Enhanced Oil Recovery, pp 274-277, SPE Textbook Series Volume 6, Richardson, Texas, 1998.

Hansen, C.M., J. Paint Tech., 39, 511, 1967.

Hansen, C.M., Hansen Solubility Parameters, A User's Handbook, New York, CRC Press, 2000.

Hildebrand, J.H., The Solubility of Non-Electrolytes, New York, Reinhold, 1936.

Hill, K. and Rhode, O.: "Sugar-based surfactants for consumer products and technical applications", Fett/Lipid, 10, 25 - 33, 1999.

Iglauer, S., Wu, Y., Shuler, P., Tang, Y., Blanco, M., and Goddard, III, W., "The Influence of Alcohol Co-surfactants on the Interfacial Tensions of Alkylpolyglucoside Surfactant Formulations vs. n-Octane”, presented at Annual ACS meeting, Ananheim, CA, April 1, 2004.

Iglauer, S., Wu, Y., Shuler, P, Blanco, M., Tang, Y., and Goddard, III, W., "Alkylpolyglucoside Surfactants for Improved Oil Recovery, paper SPE/DOE 89472, SPE/DOE Improved Oil Recovery Symposium, Tulsa, OK, April 17 - 21, 2004.

Jang, S.S., Lin, S.T., Maita, P.K., Blanco, M., Goddard III, W.A., Shuler, P.J., and Tang, Y.: "Molecular Dynamics Study of a Surfactant-Mediated Decane-Water Interface: Effect 
of Molecular Architecture of Alkyl Benzene Sulfonate", J. Phys. Chem. B, 108, 1213012140, 2004.

Jayanti, Shekhar, et. al.: "Use of Surfactants to Recover Oils from Groundwater", SPE 66753, presented, SPE/EPA/DOE E\&P Environmental Conference, San Antonio, TX, 2628 February 2001.

Jungwirth, P. and D.J. Tobias, D.J.: "Molecular Structure of Salt Solutions: A New View of the Interface with Implications for Heterogeneous Atmospheric Chemistry", J. Phys. Chem., 105, 10468-10472, 2001.

Kluge, K., Stubenrauch, C., Sottmann, T., and Strey, R.: "Temperature-insensitive microemulsions formulated from octyl monoglucoside and alcohols: potential candidates for practical applications", Tenside Surf. Det., 38, 30 - 40, 2000.

Kutschmann, E.M., et. al.: "Interfacial tension of alkylglucosides in different APG/oil/water systems", Colloid Polym. Sci. 273, 565 - 571, 1995.

Lake, L.W.: Enhanced Oil Recovery, Prentice-Hall, Inc., 1989.

Maiti, P.K., Y. Lansac, M. A. Glaser, N. A. Clark, and Y. Rouault, Langmuir, 18, 1908, 2002.

Peypoux, F., Bonmatin, J.M., and Wallach, J.: "Recent trends in biochemistry of surfactin”, Appl Microbial Biotechnol, 51, 553 - 563, 1999.

Sabatini, D.A., Acosta, E., and Harwell, J.H.: "Linker Molecules in Surfactant Mixtures", submitted to Current Opinion in Colloid and Interface Science, February, 2003.

Shah, D.O. and Schechter, R.S.: Improved Oil Recovery by Surfactant and Polymer Flooding, pp. 205 - 252, Academic Press, New York, 1977a.

Smith, B.; Hilbers, P. A. J.; Esselink, K.; Rupert, L. A. M.; van Os, N. M.; Schlijper, A. G,. Nature, 348, 624, 1990.

Taber, J.J., Martin, F.D., and Seright, R.S.: "EOR Screening Criteia Revisited - Part 2. Application and Impact of Oil Prices", SPE Reservoir Engineering, August 1996.

Telo da Gama, M. M.; Gubbins, K. E. Molec. Phys., 59, 227, $1986 .$.

U.S. Patent 4,985,154, "Process for the extraction of crude oil from an underground deposit using surfactants", 1991.

Wu, W., et.al.: "Design and Optimization of Low-Cost Chemical Flooding", paper SPE/DOE 35355, presented at Symposium on Improved Oil Recovery, Tulsa, OK, 21-24 April, 1996. 
Wu, Y., Iglauer, S., Shuler, P., Tang, Y., Blanco, M., and Goddard, III, W., "Synergistic Effect of Alkyl Polyglycoside and Sorbitan Mixtures on Lowering Interfacial Tension and Enhancing Oil Recovery", presented at Annual ACS meeting, Ananheim, CA, April $1,2004$.

Zana, R., "Dimeric (Gemini) Surfactants", Chapter 8, Novel Surfactants, ed. K. Holmberg, Marcel Dekker, New York, 1998.

Zana, R.: "Dimeric and oligomeric surfactants. Behavior at interfaces and in aqueous solution: a review", Advances in Colloid and Interface Science, 97, 205-253, 2002.

\title{
10. LIST OF ACRONYMS AND ABBREVIATIONS
}

\author{
APG -- alkyl polyglycoside (surfactant) \\ CED -- cohesive energy density \\ CMC - critical micelle concentration \\ DMBE -- diethylene monoglycol butyl ether \\ EOR -- enhanced oil recovery \\ FF -- force field \\ HBDM -- n-Hexadeycl-beta-D-maltopyranoside \\ HLB -- hydrophile-lipophile balance \\ IOR -- improved oil recovery \\ IPA = isopropanol \\ LJ -- Lennard Jones \\ MD - molecular dynamics \\ $\mathrm{ND}=$ Not determined \\ NPT -- constant pressure simulation \\ NVT -- constant volume simulation \\ OBDM -- n-Octyl-beta-D-maltopyranoside
}


SEAR - surfactant enhanced aquifer remediation 


\section{APPENDIX A. \\ SOME DETAILS OF SURFACTANT ADSORPTION EXPERIMENTS}

A number of commercial nonionic surfactants were evaluated for their tendency to adsorb onto kaolinite clay. All of these tests were conducted at $25 \mathrm{C}$ with a weight ratio of liquid/solid of 20, and for an exposure period of 8 hours. The gravimetric method described in the Experimental Procedure section was used.

The following table summarizes the results from these adsorption tests of this study. The values reported are for 3 different initial bulk surfactant concentrations and the adsorption amounts are expressed in units of $\mathrm{mg}$ surfactant/gram kaolinite. 
Adsorption of Surfactants on Kaolinite

(Soln./Solid $=20: 1,25^{\circ} \mathrm{C}, 8$ hours)

Page: 1

\begin{tabular}{|c|c|c|c|c|c|c|c|c|}
\hline \multicolumn{3}{|c|}{ Surfactants } & \multicolumn{6}{|c|}{ Adsorption } \\
\hline Name & C.P. & HLB & $\mathrm{C}_{\text {eq. }}$ (wt.\%) & $\Gamma(\mathrm{mg} / \mathrm{g})$ & $\mathrm{C}_{\text {eq. }}(\mathrm{wt} . \%)$ & $\Gamma(\mathrm{mg} / \mathrm{g})$ & $\mathrm{C}_{\text {eq. }}(\mathrm{wt} . \%)$ & $\Gamma(\mathrm{mg} / \mathrm{g})$ \\
\hline Pluronic L-31 & $37^{\circ} \mathrm{C}$ & 5.0 & 0.4594 & 7.2 & 0.6912 & 6.5 & 0.9407 & 8.4 \\
\hline Pluronic L-44 & $65^{\circ} \mathrm{C}$ & 16.0 & 0.5083 & -1.8 & 0.7490 & -1.0 & 0.9890 & 1.2 \\
\hline Pluronic L-61 & $24^{\circ} \mathrm{C}$ & 16.0 & 0.2360 & 47.9 & 0.3227 & 75.9 & 0.3882 & 111.9 \\
\hline Pluronic L-62 & $32^{\circ} \mathrm{C}$ & 7.0 & 0.4819 & 1.4 & 0.7120 & 4.4 & 0.8863 & 18.5 \\
\hline Pluronic L-64 & $61^{\circ} \mathrm{C}$ & 15.0 & 0.4556 & 6.9 & 0.6810 & 9.1 & 0.9519 & 8.2 \\
\hline Pluronic L-72 & $25^{\circ} \mathrm{C}$ & 6.5 & 0.3476 & 26.7 & 0.5753 & 29.6 & 0.6791 & 59.1 \\
\hline Pluronic L-81 & $20^{\circ} \mathrm{C}$ & 2.0 & 0.2209 & 49.5 & 0.3596 & 66.9 & 0.5822 & 74.4 \\
\hline Pluronic F-68 & $>100^{\circ} \mathrm{C}$ & 29.0 & 0.5386 & -12.3 & 0.8093 & -18.6 & 1.0430 & -18.7 \\
\hline Pluronic F-87 & $>100^{\circ} \mathrm{C}$ & 24.0 & 0.5540 & -7.5 & 0.8000 & -8.9 & 1.0420 & -8.3 \\
\hline Pluronic F-88 & $>100^{\circ} \mathrm{C}$ & 28.0 & 0.2700 & 42.7 & 0.5815 & 31.6 & 0.9288 & 13.2 \\
\hline Pluronic F-108 & $>100^{\circ} \mathrm{C}$ & 27.0 & 0.3694 & 25.1 & 0.6375 & 21.3 & 0.9666 & 8.0 \\
\hline Pluronic P-84 & $74^{\circ} \mathrm{C}$ & 16.0 & 0.5070 & -1.5 & 0.7273 & 3.1 & 0.9713 & 7.3 \\
\hline Pluronic P-85 & $85^{\circ} \mathrm{C}$ & 16.0 & 0.4902 & 2.3 & 0.7217 & 4.9 & 0.9677 & 5.6 \\
\hline Pluronic P-103 & $86^{\circ} \mathrm{C}$ & 9.0 & 0.4934 & 1.5 & 0.7413 & 0.9 & 0.9917 & 2.3 \\
\hline Pluronic P-123 & $90^{\circ} \mathrm{C}$ & 8.0 & 0.4824 & 5.0 & 0.7295 & 3.0 & 0.9971 & 1.6 \\
\hline Pluronic 17R-2 & $39^{\circ} \mathrm{C}$ & 4.0 & 0.5130 & -1.7 & 0.7534 & -0.5 & 1.0069 & 2.0 \\
\hline Pluronic 25R-2 & $33^{\circ} \mathrm{C}$ & 4.0 & 0.3522 & 26.3 & 0.4241 & 58.5 & 0.5551 & 81.6 \\
\hline Tetronic 701 & $22^{\circ} \mathrm{C}$ & 3.0 & 0.2374 & 49.1 & 0.3256 & 78.1 & 0.4073 & 110.8 \\
\hline Surfynol 440 & $?$ & 8.0 & 0.6132 & -23.6 & 0.4748 & 47.9 & 0.4867 & 90.7 \\
\hline Surfynol 465 & $63^{\circ} \mathrm{C}$ & 13.0 & 0.5538 & -10.7 & 0.9612 & -41.3 & 1.2248 & -42.3 \\
\hline Surfynol 2502 & & $?$ & 0.3856 & 20.4 & 0.5469 & 36.6 & 0.8948 & 17.9 \\
\hline Surfynol SE-F & & 4.5 & 0.1048 & 45.3 & 0.1012 & 74.4 & 0.1986 & 90.3 \\
\hline
\end{tabular}


Adsorption of Surfactants on Kaolinite

(Soln./Solid $=20: 1,25^{\circ} \mathrm{C}, 8$ hours)

Page: 2

\begin{tabular}{|c|c|c|c|c|c|c|c|c|}
\hline \multicolumn{3}{|c|}{ Surfactants } & \multicolumn{6}{|c|}{ Adsorption } \\
\hline Name & C.P. & HLB & $\mathrm{C}_{\text {eq. }}($ wt. $\%)$ & $\Gamma(\mathrm{mg} / \mathrm{g})$ & $\mathrm{C}_{\text {eq. }}(\mathrm{wt} . \%)$ & $\Gamma(\mathrm{mg} / \mathrm{g})$ & $\mathrm{C}_{\text {eq. }}($ wt. $\%)$ & $\Gamma(\mathrm{mg} / \mathrm{g})$ \\
\hline Enviogem AD01 & $37^{\circ} \mathrm{C}$ & $?$ & $\begin{array}{c}\text { Not } \\
\text { Soluble }\end{array}$ & $\mathrm{N} / \mathrm{A}$ & $\begin{array}{c}\text { Not } \\
\text { Soluble }\end{array}$ & N/A & $\begin{array}{c}\text { Not } \\
\text { Soluble }\end{array}$ & N/A \\
\hline Dynol 604 & & $?$ & 0.1182 & 69.6 & 0.1494 & 110.1 & 0.1658 & 146.8 \\
\hline SIL WET L-77 & & 6.5 & 0.4421 & 11.3 & 0.6687 & 13.4 & 1.0245 & -4.7 \\
\hline SIL WET L-720 & & 9.5 & 0.5309 & -7.2 & 0.7018 & 6.5 & 0.9590 & 6.9 \\
\hline SIL WET L-722 & & 6.5 & 0.1281 & 67.8 & 0.1154 & 123.5 & 0.1358 & 162.3 \\
\hline SIL WET L-7001 & & 6.5 & 0.4667 & 6.2 & 0.4369 & 11.8 & 0.6626 & 16.0 \\
\hline SIL WET L-7500 & & 6.5 & 1.0016 & -1.0 & 0.0731 & 82.6 & 0.1155 & 124.9 \\
\hline SIL WET L-7600 & & 6.5 & 0.1674 & 156.5 & 0.5245 & -3.0 & 0.7644 & -0.7 \\
\hline SIL WET L-7602 & & 6.5 & 0.2100 & 54.6 & 0.2468 & 94.8 & 0.3029 & 129.0 \\
\hline SIL WET L-7605 & & 6.5 & 0.5456 & -8.4 & 0.7720 & -4.9 & 1.0945 & -16.7 \\
\hline SIL WET L-7607 & & 6.5 & 0.5065 & -2.1 & 0.7588 & -1.7 & 1.0104 & -1.2 \\
\hline SIL WET L-7614 & & $?$ & 0.5402 & -7.3 & 0.8056 & -10.8 & 1.0743 & -13.3 \\
\hline SurfadoneLP100 & & 6.0 & 0.0332 & 88.1 & 0.0866 & 122.8 & 0.1101 & 166.6 \\
\hline SurfadoneLP300 & & 3.0 & 0.0409 & 89.4 & 0.0429 & 135.0 & 0.0624 & 179.4 \\
\hline Aerosol GPG & & $?$ & 0.2416 & 51.1 & 0.3049 & 85.9 & 0.3653 & 123.4 \\
\hline Aerosol MA-80 & & $?$ & 0.4806 & 3.3 & 0.7197 & 5.6 & 1.0798 & -14.9 \\
\hline Aerosol OT-75 & & $?$ & 0.2570 & 46.8 & 0.4870 & 51.3 & 0.6955 & 59.1 \\
\hline Aerosol OT-B & & $?$ & 0.3991 & 19.6 & 0.6655 & 16.0 & 0.8807 & 22.4 \\
\hline Aerosol OT-S & & $?$ & 0.0971 & 77.8 & 0.4759 & 53.2 & 0.1515 & 162.6 \\
\hline Aerosol TR-70 & & $?$ & 0.0891 & 77.8 & 0.1040 & 124.4 & 0.0987 & 171.8 \\
\hline Igepal CA-420 & & 8.0 & 0.0465 & 86.3 & 0.0673 & 127.1 & 0.0644 & 177.2 \\
\hline Igepal CA-620 & & 12.0 & 0.0754 & 78.9 & 0.0754 & 127.2 & 0.1598 & 155.9 \\
\hline
\end{tabular}




\begin{tabular}{|c|c|c|c|c|c|c|c|c|}
\hline \multicolumn{9}{|c|}{$\begin{array}{l}\text { Adsorption of Surfactants on Kaolinite } \\
\text { (Soln./Solid }=20: 1,25^{\circ} \mathrm{C}, 8 \text { hours) }\end{array}$} \\
\hline \multicolumn{3}{|c|}{ Surfactants } & \multicolumn{6}{|c|}{ Adsorption } \\
\hline Name & C.P. & HLB & $\mathrm{C}_{\text {eq. }}$ (wt. \%) & $\Gamma(\mathrm{mg} / \mathrm{g})$ & $\mathrm{C}_{\text {eq. }}(\mathrm{wt} . \%)$ & $\Gamma(\mathrm{mg} / \mathrm{g})$ & $\mathrm{C}_{\text {eq. }}(\mathrm{wt} . \%)$ & $\Gamma(\mathbf{m g} / \mathbf{g})$ \\
\hline Igepal CA-630 & & 13.0 & 0.4849 & 2.9 & 0.7342 & 1.8 & 0.9931 & 1.8 \\
\hline Igepal CA-720 & & 14.6 & 0.5026 & 0.0 & 0.7444 & 1.5 & 1.0220 & -3.4 \\
\hline Igepal CO-210 & & 4.6 & 0.0716 & 79.4 & 0.0793 & 122.9 & 0.1095 & 162.2 \\
\hline Igepal CO-710 & & 13.6 & 0.4895 & 1.8 & 0.7334 & 3.4 & 0.9865 & 1.4 \\
\hline TWEEN 20 & & 16.7 & 0.4658 & 5.7 & 0.6776 & 11.3 & 0.9288 & 15.0 \\
\hline TWEEN 21 & & 13.3 & 0.4029 & 17.1 & 0.5305 & 39.2 & 0.7336 & 46.0 \\
\hline TWEEN 40 & & 15.6 & 0.4775 & 3.8 & 0.6713 & 12.8 & 0.9409 & 10.3 \\
\hline TWEEN 80 & & 15.0 & 0.4311 & 11.3 & 0.6625 & 13.2 & 0.9485 & 6.8 \\
\hline TWEEN 81 & & 10.0 & 0.1362 & 68.3 & 0.1780 & 109.4 & 0.2772 & 133.0 \\
\hline TWEEN 85 & & 11.0 & 0.2796 & 36.9 & 0.5050 & 40.3 & 0.7674 & 36.7 \\
\hline Calfax 10L-45 & & & 0.4971 & 1.1 & 0.7508 & 0.0 & 0.9941 & 1.3 \\
\hline Calsoft LAS-99 & & & 0.4185 & 15.0 & 0.6280 & 21.7 & 0.8584 & 26.9 \\
\hline Calamide C & & & 0.3199 & 32.0 & 0.6142 & 24.8 & 0.9213 & 14.8 \\
\hline Calamide CW-100 & & & 0.3918 & 20.5 & 0.6793 & 10.1 & 0.9296 & 8.1 \\
\hline Calfax 16L-35 & & & 0.4881 & 2.3 & 0.7260 & 5.3 & 1.1881 & -33.9 \\
\hline Calfax DB-45 & & & 0.4919 & 4.0 & 0.7306 & 7.0 & 1.3996 & -71.4 \\
\hline Calfoam ES-603 & & & 0.4203 & 15.5 & 0.7036 & 10.8 & 0.9674 & 7.8 \\
\hline Calfoam EA-603 & & & 0.4310 & 14.9 & 0.6835 & 15.2 & 1.3075 & -53.6 \\
\hline Calamide CWT & & & 0.2523 & 48.0 & 0.3341 & 80.9 & 0.4884 & 96.9 \\
\hline Callmulse PRS & & & 0.4656 & 7.2 & 0.9920 & -41.4 & 1.7634 & -140.1 \\
\hline Caloxylate N-9 & & & 0.4640 & 5.9 & 0.7358 & 2.9 & 1.0133 & -4.2 \\
\hline Calsoft T-60 & & & 0.5092 & 0.0 & 0.8953 & -27.9 & 1.7204 & -136.6 \\
\hline
\end{tabular}




\section{ATTACHMENT 1}

\section{PUBLISHED PAPERS AND PRESENTATIONS}

1. Yongfu Wu, Patrick J. Shuler, Yongchun Tang: “Adsorption of Novel Surfactants for Enhanced Oil Recovery onto Kaolinite Clay”, presented at ACS Meeting, New York, September 2003.

2. Iglauer, S., Wu, Y., Shuler, P., Tang, Y., Blanco, M., and Goddard, III, W., "The Influence of Alcohol Co-surfactants on the Interfacial Tensions of Alkylpolyglucoside Surfactant Formulations vs. n-Octane”, presented at Annual ACS meeting, Ananheim, CA, April 1, 2004.

3. Wu, Y., Iglauer, S., Shuler, P., Tang, Y., Blanco, M., and Goddard, III, W., "Synergistic Effect of Alkyl Polyglycoside and Sorbitan Mixtures on Lowering Interfacial Tension and Enhancing Oil Recovery”, presented at Annual ACS meeting, Ananheim, CA, April 1, 2004.

4. Wu, Y., Iglauer, S., Shuler, P., Tang, Y., Blanco, M., and Goddard, III, W., "Synergistic Effect of Alkyl Polyglycoside and Sorbitan Mixtures on Lowering Interfacial Tension and Enhancing Oil Recovery”, presented at Annual ACS meeting, Ananheim, CA, April 1, 2004.

5. Jang, S.S., Lin, S.T., Maita, P.K., Blanco, M., Goddard III, W.A., Shuler, P.J., and Tang, Y.: "Molecular Dynamics Study of a Surfactant-Mediated DecaneWater Interface: Effect of Molecular Architecture of Alkyl Benzene Sulfonate”, J. Phys. Chem. B, 108, 12130-12140, 2004. 
226th ACS National Meeting, New York, NY, September 7-11, 2003

\author{
Abstract \\ Adsorption of Novel Surfactants for Enhanced Oil Recovery onto Kaolinite Clay \\ Yongfu Wu, Patrick J. Shuler, Yongchun Tang \\ PEER Center, California Institute of Technology \\ 20970 Currier Road, Walnut CA 91789
}

The success of a surfactant EOR process depends on: (1) creating low interfacial tension and thereby displacing residual oil, (2) minimizing loss of surfactant. Surfactant adsorption onto reservoir clays is a major concern because with their high surface area they are capable of removing most of the surfactant from the injected solution, thereby making this EOR process ineffective.

We have measured adsorption of various surfactants from $2.0 \% \mathrm{NaCl}$ aqueous solution onto kaolinite, a common oil reservoir clay. For block polymer surfactants investigated, their adsorption maxima increase with an increase of the ratio of the number of oxypropylene (PO) groups to the number of oxyethylene (EO) groups. For ethoxylated alcohol surfactants, their adsorption maxima decrease with increase of the number of oxyethylene (EO) groups. Relatively lower adsorption was found for some novel EOR surfactants such as alkyl polyglucosides and their mixtures with sorbitan carboxylic ester surfactants. 


\section{The Influence of Alcohol Co-surfactants on the Interfacial Tensions of Alkylpolyglucoside Surfactant Formulations vs. n-Octane}

Stefan Iglauer, Yongfu Wu, Patrick Shuler, Yongchun Tang, Mario Blanco, and William Goddard

PEER Center, California Institute of Technology

738 Arrow Grand Circle, Covina CA 91722

\section{Introduction}

Alkyl polyglycosides (APG) are nonionic surfactants prepared with renewable raw materials, namely starch and fat or their components glucose and fatty alcohols. A typical APG structure is shown below:

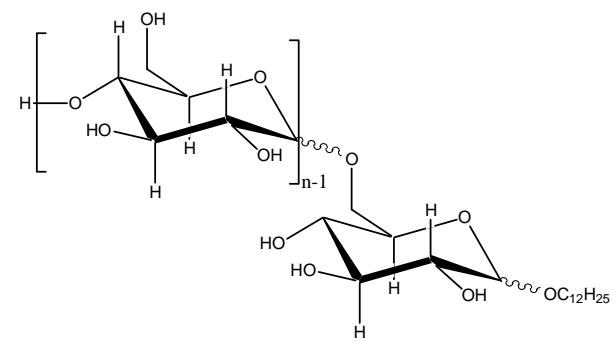

Figure 1. Molecular structure of an example alkyl polyglycoside (n-Dodecylglucopyranoside)

The degree of polymerization is actually quite low, with n usually between 1.1 and 3 (n typically ranges from $1.2-1.5$ ). Commercial APG products have a mixture of molecular structures, both in terms of the number distribution of the head groups and the length of the alkyl groups in the hydrophobic tail.

APG surfactants were described initially over 100 years ago, first recognized as a potentially useful surfactant type in 1936, and then largely ignored until the 1980's. APG has gained favor as economical processes were developed to manufacture them, and there has been an increased drive to use surfactants with favorable, low toxicity like APG. This surfactant now sees widespread use in household detergents, cosmetics, and agricultural products. ${ }^{1}$ A recent (1999) estimate for worldwide capacity for APG surfactants is 80,000 tons/year. ${ }^{2}$

In this study, we consider APG surfactants as candidates for enhanced oil recovery (EOR). In this application, surfactant formulations are injected into subsurface reservoirs containing crude oil. Provided the injected solution can reduce the interfacial tension (IFT) to very low values (below 0.01 dynes/cm) between the aqueous chemical solution and the crude oil, then this will mobilize and produce more oil than by conventional methods. Experience shows that injection of plain salt water alone, for example, may only recover half of the crude oil, with the remainder trapped as small oil droplets due high capillary forces in the micron-size pores in the reservoir rock. APG surfactants hardly have been considered as EOR applications previously, with just one U.S. patent issued more than a decade ago. ${ }^{3}$
The APG formulations have some interesting and potentially special properties as EOR agents; literature reports indicate: ${ }^{4}$

1. APG do not seem to have the capability form low IFT middle-phase microemulsions by themselves.

2. When mixed with a cosurfactant (e.g. an alcohol), a middle-phase microemulsion may appear, and in some cases it may create a low IFT (0.01 dyne/cm or less). Some encouraging phase behavior/IFT data are reported with simple n-alkanes as the oil phase. ${ }^{1,4,5,6}$

3. A remarkable property for these APG / alcohol formulations is that they are reported to have a phase behavior and IFT that is largely independent of temperature and salinity. Surfactant formulations that create a low IFT irrespective of temperature and salinity would be simpler to use in EOR applications and be a very useful property.

In this study alkyl polyglycosides (APG) surfactants were formulated with various alcohols as co-surfactants in aqueous salt solutions with the objective of identifying combinations that attain low interfacial tensions (IFT) versus n-octane.

\section{Experimental}

We have used 3 different commercial APG products supplied by Cognis Corporation (see Figure 1):

\begin{tabular}{lccrc} 
& Average & & & \\
Product & Alkyl Chain & Average n & HLB & Activity \\
\hline PG 2067 & 9.1 & 1.7 & 13.6 & $70 \%$ \\
PG 2069 & 10.1 & 1.6 & 13.1 & $50 \%$ \\
PG 2062 & 12.5 & 1.6 & 11.6 & $50 \%$
\end{tabular}

The HLB (hydrophile-lipophile balance) of a surfactant refers to its behavior in creating emulsions and is related to its oil/water solubility. Higher HLB products such as those found for these APG surfactants mean they tend to be relatively water soluble.

Several common alcohols were selected as co-solvents to create surfactant formulations with the APG surfactants. The alcohols were supplied by Aldrich. Most formulations included reagent grade sodium chloride, also supplied by Aldrich.

For the hydrocarbon phase n-octane was used (Aldrich) as a model compound. Other studies have shown that IFT and phase behavior of crude oils often is represented well by nalkanes ranging from n-hexane to n-decane. Thus, this current study has selected n-octane as a "typical” representative hydrocarbon. That is to say, surfactant formulations that are effective in reducing IFT versus n-octane are likely good candidates also for mobilizing crude oils.

Test tube samples were prepared with $5 \mathrm{ml}$ of aqueous surfactant/co-solvent/salt formulations and $5 \mathrm{ml}$ of n-octane. After mixing well for several hours, they were allowed to stand for a few weeks to allow the fluids to come to phase equilibrium at ambient conditions. The physical appearance of the phases was noted, such as the relative volumes of the aqueous and oleic phases, and if any third, so-called middle- 
phase forms. Other qualitative information collected is the color or opacity/clarity of the different liquid phases.

The interfacial tension (IFT) was determined for selected phase equilibrated test tube samples by using a spinning drop tensiometer (from Temco, Inc.) as detailed elsewhere. ${ }^{7}$ For our samples we loaded the glass tube with the aqueous phase, followed by injection of a few micro-liters of the uppermost oleic phase. The glass tube was spun in the instrument and the IFT determined from the oil drop geometry. Because the samples already come from fluids at phase equilibrium, typically it required less than 2 hours for the measured IFT to stabilize to a final value.

\section{Results and Discussion}

Alcohol co-solvents being evaluated include several nalcohols ranging from C3 to C20. The aqueous phase has 2 wt\% combined APG/Co-solvent concentration and has a default brine salinity of $2 \mathrm{wt} \% \mathrm{NaCl}$. The oil and aqueous surfactant solutions are mixed at a 1/1 volume ratio and equilibrated at ambient temperature. The graph below shows IFT results with the PG 2062 APG surfactant and n-alcohols.

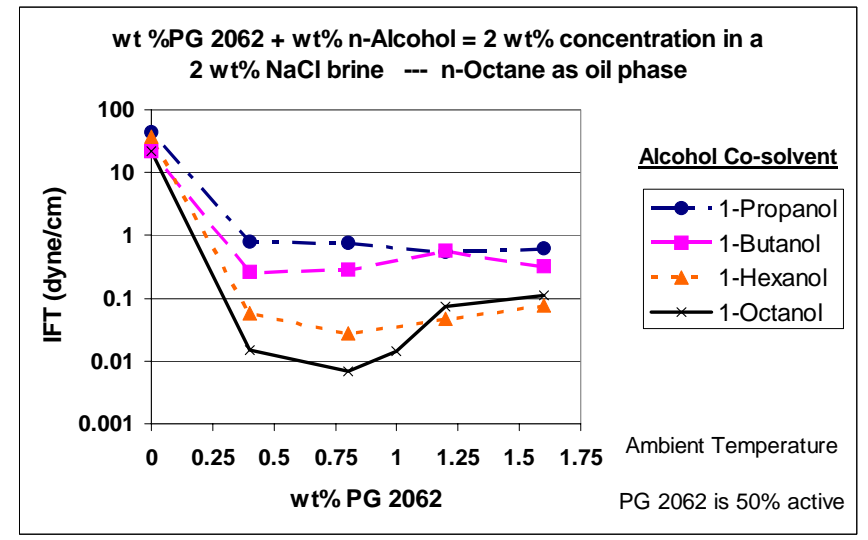

Figure 2. IFT measured for equilibrated samples containing PG 2062 and smaller n-alcohols versus n-octane as the hydrocarbon phase.

Note that the IFT for PG 2062 alone is about 2 dyne/cm, and for an alcohol alone the IFT is over several dynes/cm, perhaps even greater than 30 dynes $/ \mathrm{cm}$. One explanation for the synergistic action of the added alcohols is that they pack at the interface so as to decrease the curvature of the interfacial layer and thereby reduce the IFT. Perhaps the notion of a "hydrophobic linker" is a good physical model for the action of these cosurfactants. ${ }^{8}$ That is, an additive may work by linking the oil and surfactant molecules better at the interface. Our general observation is that virtually all alcohol cosolvents act to decrease the IFT of the main, APG surfactant.

Some other comments about these results:

- There is an "optimal” alcohol cosurfactant (in this system n-octanol) that creates the lowest IFT condition (less than 0.01 dyne/cm.). Larger nalcohols as co-solvents (not shown here) created only a higher IFT.
- Almost all of the samples indicated in Figure 2 had a third, middle-phase, if only a small volume. Even the samples with PG 2062 mixed with the most unfavorable cosurfactant (n-propanol) has at least a small middle phase.

- The IFT behavior versus the amount of APG and nalcohol are fairly constant. This suggests the desirable result that the low IFT condition may be attained with low concentrations of APG surfactant.

Figure 3 summarizes data that compares the IFT measured among the 3 different commercial APG surfactants. The trend is that increasing the alkyl chain length of the

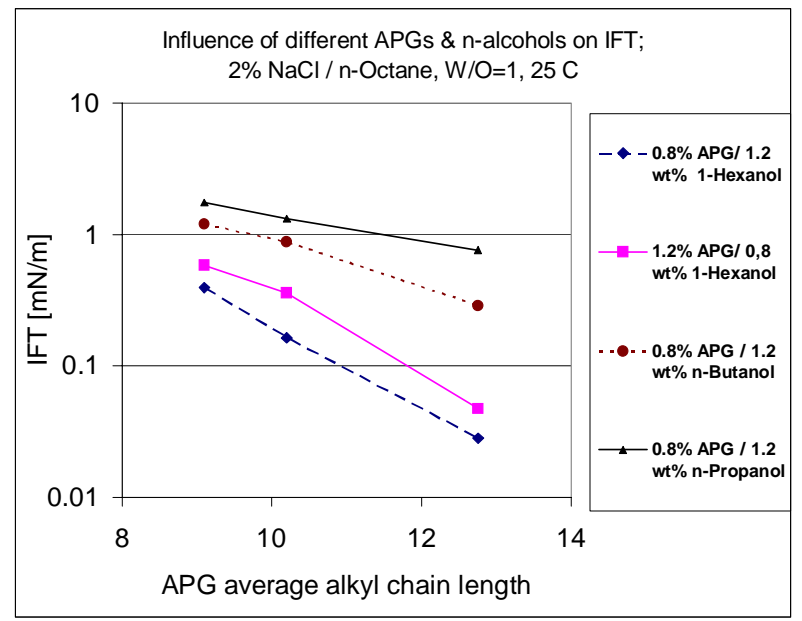

Figure 3. Increase of APG average alkyl chain length decreases the IFT.

APG surfactant decreases the IFT for the same APG/n-alcohol mixture.

Closer inspection of the data in Figure 3 indicates that the IFT for PG 2067 and PG 2069 (average alkyl chain lengths of 9.1 and 10.1, respectively) also have a lower IFT as the cosurfactant alcohol chain length increases from n-propanol to n-hexanol. (This is the same trend as shown for the PG 2062 surfactant in Figure 2.)

Other experiments examined the effect of yet other alcohols as co-solvents, focusing on the PG 2062 APG product as it had the lowest IFT among the commercial APG products studied. These other co-solvents included larger nalcohols, with the result showing n-octanol still as the best cosolvent. Another series of tests examined a series of C6 alcohols as co-solvents, with the variation being the alcohol structure as a straight chain aliphatic, branched chain alcohol, saturated ring, and as an aromatic ring structure. Results show the straight chain (n-hexane) structure provides the lowest IFT among this group of co-solvents. Yet another test series has different aromatic alcohol co-solvents, with low IFT occurring in some particular formulations.

One important feature of these APG formulations is that the IFT appears to be largely independent of the temperature. 


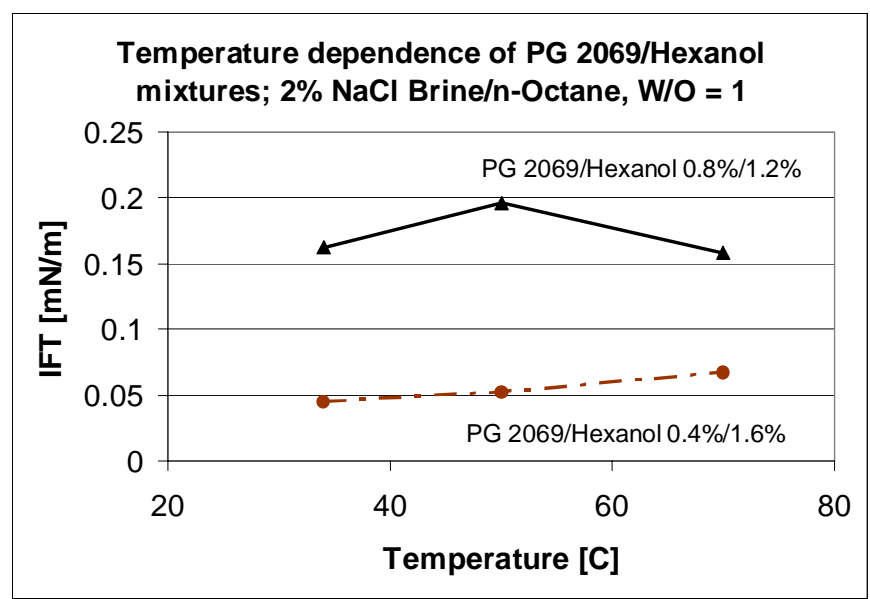

Figure 4. Data illustrating IFT is nearly independent of temperature for a mixture of APG surfactant/alcohol versus n-octane as the hydrocarbon phase.

This is desirable because in oil reservoirs, the temperature will vary from zone to zone, with higher temperatures occurring in deeper subsurface depths. This behavior means that one may formulate a solution that is able to mobilize the crude oil in spite of these temperature differences.

Similarly, our data confirm the reports in the literature that APG/alcohol formulations are also not very dependent on the salinity of the aqueous brine. This is also a desirable

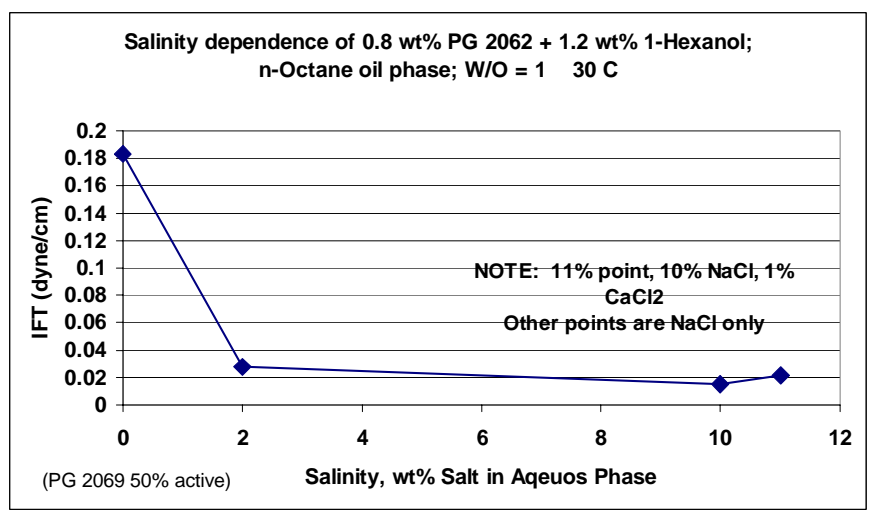

Figure 5. Data illustrating IFT is nearly independent of the salinity for an APG surfactant/alcohol formulation versus n-octane as the hydrocarbon phase.

feature for application as an EOR chemical system. The salinity in the brine in the subsurface oil reservoir may vary from zone to zone. This property of the surfactant solution means that one may formulate a solution that is able to mobilize the crude oil in spite of the differences in the salinity.

\section{Conclusions}

This study demonstrates that alkyl polyglycoside (APG) surfactants, when mixed with some alcohols as co-solvent may be effective formulations for purposes of enhanced oil recovery (EOR). Attractive features of these formulations include: 1) low interfacial tension (IFT) may be obtained with low concentrations of APG surfactant, 2) these formulations may be remain at low IFT conditions in spite of changes that may occur with temperature and salinity.

Acknowledgement. We wish to acknowledge the financial support from the Department of Energy for this study. We also wish to thank our industrial collaborators, ChevronTexaco and Akzo Nobel.

\section{References}

(1) Balzer, D. Tenside Surf. Det., 1991, 38, 419 - 427.

(2) Hill, K. and Rhode, O. Fett/Lipid, 1999, 10, 25 - 33.

(3) Balzer, D. 1991, U.S. Patent 4,985,154.

(4) Balzer, D., and Luders, H., editors.: Nonionic Surfactants, Alkyl Polyglycosides, 1996, Marcel Dekker, New York, 1996; pp. 228 - 243.

(5) Kutschmann, E.M. Colloid Polym. Sci., 1995, 273, 565 - 571.

(6) Forster, T. Progr. Colloid Polym. Sci., 1996, 101, $105-112$.

(7) Cayais, J.L., Schechter, R.S., and Wade, W.H. 1977, Surfactant Applications, Section 17.

(8) Sabatini, D.A., Acosta, E., and Harwell, J.H. 2003, submitted to Current Opinion in Colloid and Interface Science. 


\section{Synergistic Effect of Alkyl Polyglycoside and Sorbitan Mixtures on Lowering Interfacial Tension and Enhancing Oil Recovery}

Yongfu Wu, Stefan Iglauer, Patrick Shuler, Yongchun Tang, Mario Blanco, and William Goddard

PEER Center, California Institute of Technology

738 Arrow Grand Circle, Covina CA 91722

\section{Introduction}

Alkyl polyglycosides (APG) are nonionic surfactants prepared with renewable raw materials, namely starch and fat or their components glucose and fatty alcohols. A typical APG structure is shown below:

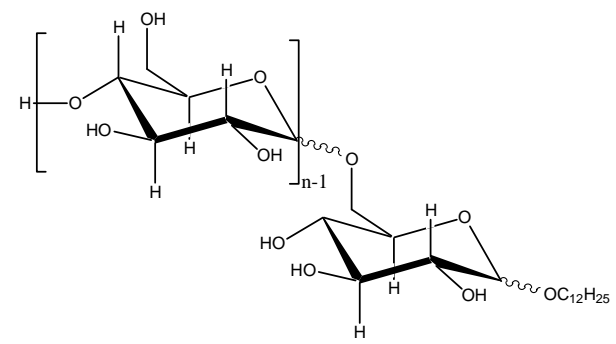

Figure 1. Molecular structure of an example alkyl polyglycoside (dodecylglucopyranoside)

The degree of polymerization is low, with n usually between 1.1 and 3 (n typically ranges from $1.2-1.5$ ). Commercial APG products have a mixture of many molecular structures, both in the number distribution of the head groups and the length of the alkyl groups in the hydrophobic tail.

APG surfactants were described initially over 100 years ago, first recognized as a potentially useful surfactant type in 1936, and then largely ignored until the 1980's. APG has gained market share as processes have been developed to manufacture them economically, and there has been an increased drive to use surfactants with favorable, low toxicity like APG. This surfactant now sees widespread use in household detergents, cosmetics, and agricultural products. ${ }^{1}$ A recent (1999) estimate for worldwide capacity for APG surfactants is 80,000 tons/year. ${ }^{2}$

In this study, we consider APG surfactants as candidates for enhanced oil recovery (EOR). In this application, surfactant formulations are injected into subsurface reservoirs containing crude oil. Provided the injected solution can reduce the interfacial tension (IFT) to very low values (below 0.01 dynes $/ \mathrm{cm}$ ) between the aqueous chemical solution and the crude oil, then this will mobilize and produce more oil than by conventional methods (e.g. injection of just a salt water). Injection of water alone, for example, may only recover half of the oil, with the remaining oil trapped as droplets due high capillary forces in the micron-size pores in the reservoir rock. APG surfactants hardly have been considered for EOR applications; there has been only one U.S. patent issued on this topic. ${ }^{3}$

The APG formulations may have some desirable properties as EOR agents; literature reports indicate: ${ }^{4}$
1. APG do not seem to have the capability form low IFT middle-phase microemulsions by themselves.

2. When mixed with a cosurfactant (e.g. an alcohol or another surfactant such as the Sorbitan types discussed here), a middle-phase microemulsion may appear, and in some cases it may create a low IFT (0.01 dyne/cm or less). Some encouraging phase behavior/IFT data are reported with simple n-alkanes as the oil phase. ${ }^{1,4,5,6}$

The structure shown below in Figure 2 is one of the common Sorbitan surfactants considered in this investigation.

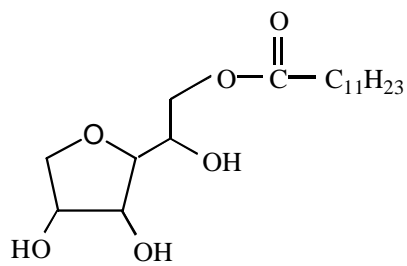

Figure 2. Molecular structure of SPAN 20 surfactant, Sorbitan Monolaurate.

A variation is the TWEEN product line of surfactants<smiles>CCCCCCCCCCCCCCCC(=O)OCC(OCC)C1OCC(OCC)C1OCC</smiles>

Figure 3. Molecular structure of TWEEN 20 surfactant. Polyoxyethylene (20) Sorbitan Monolaurate, $\mathrm{X}+\mathrm{Y}+\mathrm{Z}=20$

In this study alkyl polyglycosides (APG) surfactants were formulated with various Sorbitan surfactants in aqueous salt solutions, with the objective that this mixture has a low interfacial tension (IFT) versus n-octane. Such aqueous surfactant formulations are potential as EOR candidates.

\section{Experimental}

We included 3 different commercial APG products supplied by Cognis Corporation in this study (see Figure 1):

\begin{tabular}{lcccc} 
Pverage & & & \\
Product & Alkyl Chain & Average n & HLB & Activity \\
\hline PG 2067 & 9.1 & 1.7 & 13.6 & $70 \%$ \\
PG 2069 & 10.1 & 1.6 & 13.1 & $50 \%$ \\
PG 2062 & 12.5 & 1.6 & 11.6 & $50 \%$
\end{tabular}

The Sorbitan SPAN and TWEEN surfactants are commercial products; we obtained our samples from Aldrich.

\begin{tabular}{lcr} 
Product & Alkyl Chain & Average HLB \\
\hline SPAN 20 & C12 & 8.6 \\
SPAN 40 & C16 & 6.7
\end{tabular}


SPAN 60

SPAN 80

C18

C18 (one double bond)

4.7

SPAN 85

3 C18 (each has double bond)

\begin{tabular}{lccc} 
Product & $\begin{array}{c}\text { Number } \\
\text { EO Groups }\end{array}$ & $\begin{array}{c}\text { Average } \\
\text { Alkyl Chain }\end{array}$ & HLB \\
\hline TWEEN 20 & 20 & C12 & 16.7 \\
TWEEN 21 & 4 & C12 & 13.3 \\
TWEEN 40 & 20 & C16 & 15.6 \\
TWEEN 80 & 20 & C18 & 15.0 \\
TWEEN 81 & 5 & C18 & 10.0 \\
TWEEN 85 & 20 & 3 C18 chains & 11.0
\end{tabular}

The HLB (hydrophile-lipophile balance) of a surfactant refers to its behavior in creating emulsions and is related to its oil/water solubility. Higher HLB values indicate greater water solubility.

For the hydrocarbon phase n-octane was used (Aldrich) as a model compound. Other studies have shown that IFT and phase behavior of crude oils often is represented well by nalkanes ranging from $n$-hexane to $n$-decane. Thus, this current study has selected n-octane as a "typical" representative hydrocarbon. That is, surfactant formulations that are effective in reducing IFT versus n-octane are likely good candidates also for mobilizing crude oils.

Test tube samples were prepared with $5 \mathrm{ml}$ of aqueous surfactant/cosurfactant salt formulations and $5 \mathrm{ml}$ of n-octane. After mixing well for several hours, they were allowed to stand for a few weeks to allow the fluids to come to phase equilibrium at ambient conditions. The physical appearance of the phases was noted, such as the relative volumes of the aqueous and oleic phases, and if any third, so-called middlephase forms.

The interfacial tension (IFT) was determined for selected phase equilibrated test tube samples by using a spinning drop tensiometer (from Temco, Inc.) as detailed elsewhere. ${ }^{7}$ For our samples we loaded the glass tube with the aqueous phase, followed by injection of a few microliters of the uppermost oleic phase. The glass tube was spun in the instrument and the IFT determined from the oil drop shape. Because the samples already come from fluids at phase equilibrium, typically it required less than 2 hours for the measured IFT to stabilize to a final value.

This investigation also included oil displacement tests in porous media. Specifically, we injected APG/SPAN mixtures in salt water into sand packs containing n-octane and measured the capability of such surfactant solutions to mobilize the hydrocarbon that could not be removed by flooding with a $2 \mathrm{wt} \% \mathrm{NaCl}$ brine.

\section{Results and Discussion}

Sorbitan co-surfactants evaluated cover a spectrum of hydrophobic alkyl chain lengths, and in the case of the TWEEN products, a range of number of EO groups.

The aqueous phase has $2 \mathrm{wt} \%$ combined APG/Cosurfactant concentration and has a default brine salinity of $2 \mathrm{wt} \% \mathrm{NaCl}$. The oil and aqueous surfactant solutions are mixed at a 1/1 volume ratio and equilibrated at ambient temperature. The graph below shows IFT results with the PG 2069 APG surfactant and SPAN surfactants.

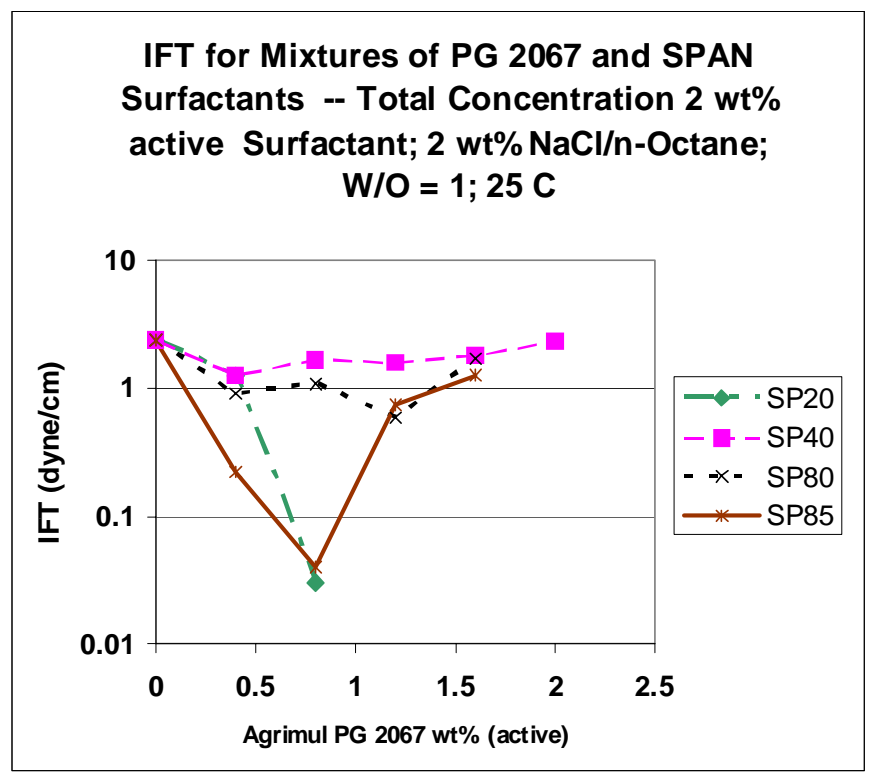

Figure 4. IFT measured for equilibrated samples containing PG 2067 and selected SPAN Sorbitan surfactants.

Note that the IFT for PG 2067 alone and these SPAN products by themselves is about $2 \mathrm{dyne} / \mathrm{cm}$. In some cases there is an obvious strong synergistic effect, with the IFT attaining very low values. One explanation for this synergistic action of the added surfactants is that they pack at the interface so as to decrease the curvature of the interfacial layer and thereby reduce the IFT. Perhaps the notion of a "hydrophobic linker" is a good physical model for the action of these surfactant combinations. ${ }^{8}$ That is, the second surfactant may improve performance by linking the oil and surfactant molecules better at the interface.

It is odd that the two "end members" of the SPAN series, SPAN $20(\mathrm{HLB}=8.6)$ and the SPAN $85(\mathrm{HLB}=1.8)$ can create a low IFT when used in these APG formulations. In contrast, mixtures employing SPAN $40(\mathrm{HLB}=6.7)$ and SPAN $80(\mathrm{HLB}=4.3)$ never exhibit this synergistic, lower IFT effect. Also, preliminary data suggest a low IFT may occur with PG 2067 / SPAN 60 mixtures (data not shown).

One available relevant set of data in the literature describes IFT for APG solutions mixed with SPAN 20 and equilibrated versus n-dodecane as the oil phase. ${ }^{6}$ Low IFT occurrs, but only over a narrow range of APG / SPAN 20 mixture ratios. We observed this same general effect, with for example the PG 2067/SPAN 85 having a sharp minimum in IFT at a ratio of $0.75 \% / 1.25 \%$., but significantly greater IFT at other mixing ratios.

Table 1 lists a sample of the IFT results for different combinations of the longer alkyl chain APG products, PG 2069 and PG 2062, and various SPAN products.

Table 1. Measured IFT for APG / SPAN surfactant mixtures in $2 \% \mathrm{NaCl}$ versus $\mathrm{n}$-octane as the hydrocarbon phase. 


\begin{tabular}{|c|c|c|c|c|}
\hline & SPAN & weight & weight & IFT \\
\hline APG & Product & \% APG & \% SPAN & (dyne/cm) \\
\hline PG 2069 & 20 & 0.80 & 1.20 & 0.0035 \\
\hline PG 2069 & 40 & 0.40 & 1.60 & 1.40 \\
\hline PG 2069 & 60 & 0.40 & 1.60 & 0.33 \\
\hline PG 2069 & 85 & 0.40 & 1.60 & 1.55 \\
\hline PG 2069 & 85 & 1.50 & 0.50 & 0.8 \\
\hline PG 2069 & 85 & 1.60 & 0.40 & 1.2 \\
\hline & & & & \\
\hline PG 2062 & 20 & 0.80 & 1.20 & 0.90 \\
\hline PG 2062 & 20 & 1.20 & 0.80 & 0.75 \\
\hline PG 2062 & 40 & 0.40 & 1.60 & 0.85 \\
\hline PG 2062 & 60 & 0.40 & 1.60 & 1.00 \\
\hline PG 2062 & 60 & 0.80 & 1.20 & 0.73 \\
\hline PG 2062 & 80 & 0.40 & 1.60 & 1.20 \\
\hline PG 2062 & 85 & 0.40 & 1.60 & 0.68 \\
\hline PG 2062 & 85 & 0.80 & 1.20 & 0.25 \\
\hline PG 2062 & 85 & 1.20 & 0.80 & 0.40 \\
\hline
\end{tabular}

Most other combinations of these APG products and the SPAN surfactants created stiff gels in the oil phase. Those combinations exhibiting such unfavorable phase behavior are not viable as an EOR formulation. The combinations (shown above) where all of the phase (aqueous, microemulsion, and oleic) appear to be fluid, the measured IFT results cover a sizable range of values. The IFT value is especially low (0.0035 dyne/cm) for the first sample shown (the PG 2069 / SPAN 20 blend at $0.8 / 1.2 \mathrm{wt} \%$,), but the IFT exceeds 0.1 dyne/cm for all of the others in Table 1.

Table 2. Measured IFT for APG / TWEEN surfactant mixtures in $2 \% \mathrm{NaCl}$ versus n-octane hydrocarbon phase.

\begin{tabular}{|c|c|c|c|c|}
\hline APG & TWEEN & & & IFT \\
\hline PG67 & 21 & 1.20 & 0.80 & \% \\
\hline PG67 & 21 & 1.60 & 0.40 & 1.07 \\
\hline PG67 & 85 & 0.80 & 1.20 & 0.76 \\
\hline PG67 & 85 & 1.00 & 1.00 & 0.38 \\
\hline PG67 & 85 & 1.20 & 0.80 & 0.9 \\
\hline PG67 & 85 & 1.60 & 0.40 & 0.82 \\
\hline PG69 & 21 & 1.60 & 0.40 & 1.25 \\
\hline PG69 & 40 & 1.60 & 0.40 & 1.7 \\
\hline PG69 & 81 & 1.00 & 1.00 & 9.6 \\
\hline PG62 & 21 & 0.40 & 1.60 & 0.05 \\
\hline PG62 & 81 & 0.40 & 1.60 & 1.3 \\
\hline PG62 & 81 & 0.80 & 1.20 & 6.10 \\
\hline PG62 & 85 & 0.40 & 1.60 & 0.76 \\
\hline
\end{tabular}

Similar to the observations of the APG / SPAN phase behavior test tubes, most combinations of the APG and TWEEN surfactants created gels in the oil phase. The combinations (shown above) where all of the phases (aqueous, microemulsion, and oleic) are fluid, allowed a meaningful measurement of the IFT. None of these combinations employing the TWEEN surfactant appeared to be interesting as an EOR candidate except perhaps for the PG 2062 / TWEEN 21 at $0.4 / 1.6 \mathrm{wt} \%$ (IFT of 0.05 dyne/cm).

Figure 5 below shows there is, as expected, a large increase in oil recovery in our laboratory experiment with the PG 2069 / SPAN 20, 0.8/ $1.2 \mathrm{wt} \%$ formulation (measured IFT reported in Table 1 is 0.003 dyne/cm). The brine by itself could mobilize only $55 \%$ of the n-octane (oil) in place, whereas the surfactant formulation displaced almost all oil.

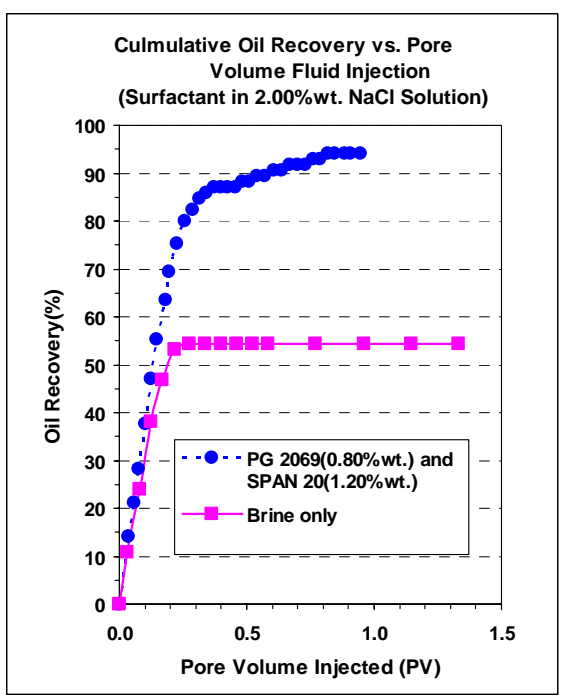

Figure 5. Increase in oil recovered in sand pack experiment with a PG 2067 / SPAN 20 formulation versus brine only.

\section{Conclusions}

This study demonstrates that alkyl polyglycosides (APG) and sorbitan-based surfactants may be combined to create chemical formulations useful for purposes of enhanced oil recovery (EOR). Suitable formulations for generating low IFT, however, occurs only with specific structure combinations of these two surfactants, and only over a narrow range of mixture ratios.

Acknowledgement. We wish to acknowledge the financial support from the Department of Energy for this study, and assistance from ChevronTexaco and Akzo Nobel.

\section{References}

(1) Balzer, D. Tenside Surf. Det., 1991, 38, 419 - 427.

(2) Hill, K. and Rhode, O. Fett/Lipid, 1999, 10, 25 - 33.

(3) Balzer, D. 1991, U.S. Patent 4,985,154.

(4) Balzer, D., and Luders, H., editors.: Nonionic Surfactants, Alkyl Polyglycosides, 1996, Marcel Dekker, New York, 1996; pp. 228 - 243.

(5) Kutschmann, E.M. Colloid Polym. Sci., 1995, 273, $565-571$.

(6) Forster, T. Progr. Colloid Polym. Sci., 1996, 101, $105-112$.

(7) Cayais, J.L., Schechter, R.S., and Wade, W.H. 1977, Surfactant Applications, Section 17.

(8) Sabatini, D.A., Acosta, E., and Harwell, J.H. 2003, submitted to Current Opinion in Col. and Int. Sci. 


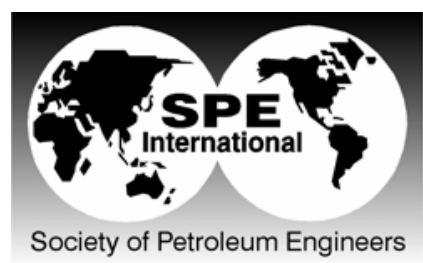

\title{
SPE/DOE 89472
}

\author{
Alkyl Polyglycoside Surfactants for Improved Oil Recovery \\ S. Iglauer, Y. Wu, P.J. Shuler, SPE, M. Blanco, Y. Tang, and W. A. Goddard III, California Institute of Technology
}

Copyright 2004, Society of Petroleum Engineers Inc.

This paper was prepared for presentation at the 2004 SPE/DOE Fourteenth Symposium on Improved Oil Recovery held in Tulsa, Oklahoma, U.S.A., 17-21 April 2004.

This paper was selected for presentation by an SPE Program Committee following review of information contained in a proposal submitted by the author(s). Contents of the paper, as presented, have not been reviewed by the Society of Petroleum Engineers and are subject to correction by the author(s). The material, as presented, does not necessarily reflect any position of the Society of Petroleum Engineers, its officers, or members. Papers presented at position of the Society of Petroleum Engineers, its officers, or members. Papers presented at Petroleum Engineers. Electronic reproduction, distribution, or storage of any part of this paper for commercial purposes without the written consent of the Society of Petroleum Engineers is prohibited. Permission to reproduce in print is restricted to a proposal of not more than 300 words; illustrations may not be copied. The proposal must contain conspicuous acknowledgment of where and by whom the paper was presented. Write Librarian, SPE, P.O. Box 833836, Richardson, TX 75083-3836, U.S.A., fax 01-972-952-9435.

\section{Abstract}

Many surfactants have been evaluated for their ability to recover incremental oil; this paper focuses on alkyl polyglycosides (APG) as candidates for this IOR application. These nonionic carbohydrate-based surfactants have become in recent years a large, significant volume (over 80,000 tons/year) commercial product that sees widespread use in household and agricultural products. This laboratory study determined the characteristics of many APG surfactant formulations, in particular for their capability to create low interfacial tensions (IFT) with n-alkane hydrocarbons. Formulations explored included a wide range of alcohol and Sorbitan-based surfactants as cosurfactants with these APG surfactants. Some APG-cosurfactant combinations did exhibit low IFT values of $0.01 \mathrm{dyne} / \mathrm{cm}$ or less versus n-octane. Laboratory testing did confirm the useful properties that the IFT for these APG formulations can be largely independent of both salinity and temperature. Preliminary studies also suggest some APG products will have only modest adsorption onto kaolinite clay.

\section{Introduction}

Background. Surfactant enhanced oil recovery (EOR) has been for many years, particularly in the 1970's and 1980's when the technology was put on a sound scientific basis.

Unfortunately, the economic reality of the process

performance in field trials has precluded widespread deployment of this technology, at least in the United States.

This study considers alkly polyglycosides (APG), one class of surfactants largely ignored as candidates for EOR applications. This is due at least in part because APG surfactants were not available as a large volume commercial product during this earlier period of intense interest in chemical EOR in the United States.

APG were described initially over 100 years ago, first recognized as a potentially useful surfactant type in 1936, and then largely ignored until the 1980's. APG has gained favor as economical processes were developed to manufacture them on a large scale, ands also because there has been an increased drive to use surfactants with favorable, low toxicity characteristics like APG for many purposes. This surfactant now sees widespread use in household detergents, cosmetics, and agricultural products. ${ }^{1}$ A recent (1999) estimate for worldwide capacity for APG surfactants is 80,000 tons/year. ${ }^{2}$ APG has been considered only briefly for EOR applications, with one U.S. patent issued on this topic. ${ }^{3}$

Potential Advantages of APG Formulations. The APG formulations have some interesting and potentially useful properties as EOR agents; literature reports state for APG formulations in contact with a hydrocarbon phase: ${ }^{4}$

1. When mixed with a hydrophobic cosurfactant (e.g. an alcohol or some other surfactants), a middle-phase microemulsion may appear, and in some cases it can create a low IFT ( $0.01 \mathrm{dyne} / \mathrm{cm}$ or less). Some encouraging phase behavior/IFT data are reported with simple n-alkanes as the oil phase ${ }^{1,3,4,5,6}$

2. A remarkable property for these APG formulations is that they are reported to have a phase behavior and IFT that is largely independent of temperature and salinity. Surfactant formulations that create a low IFT irrespective of temperature and salinity would be a very useful property for oilfield EOR applications.

Theoretical/modeling aspects also indicate that having this large head group and the nonionic character of the APG molecule is consistent with the observation of the phase behavior and IFT being largely indifferent to changes in the temperature and the salinity.

Other motivations for focusing on APG surfactants:

- They are available already as commercial products and used already in significant quantities for other industrial applications.

- These are manufactured from renewable resources and so their cost is largely uncoupled from the current price of crude oil.

- APG surfactants serve as a practical example of a sugar-based surfactant; there are thousands of other possible sugar-based surfactant structures. A related point is that the behavior of the APG products may offer insights into better applying bio-surfactants. ${ }^{7}$ This work may suggest then improvements in microbial enhanced oil recovery technology.

- The APG surfactants are non-toxic. 


\section{Experimental Methods}

Materials. The study focused on the behavior of 3 different commercial alkyl polyglycoside surfactant products. These products supplied by the Cognis Corporation (PG 2067, PG 2069, and PG 2062) are shown in Figure 1. The difference among these 3 products is in their distribution in number of head groups and the alkyl chain length (Table 1).

We obtained from Aldrich the various alcohols and SPAN and TWEEN products used as cosurfactants. Examples of these SPAN and TWEEN sorbitan surfactants are shown in Figures 2 and 3, respectively. Table 2 has more details. The HLB (hydrophile-lipophile balance) values of all of these surfactants are given by the suppliers. The HLB is a measure of the molecules ability in creating emulsions and is related to its oil/water solubility. Higher HLB indicates greater water solubility.

For the hydrocarbon phase n-octane was used (Aldrich) as a model compound. Other studies have shown that IFT and phase behavior of crude oils often can be represented well by $\mathrm{n}$-alkanes ranging from $\mathrm{n}$-hexane to $\mathrm{n}$-decane. Thus, this current study has selected n-octane as a "typical" representative hydrocarbon. That is to say, surfactant formulations that are effective in reducing IFT versus n-octane are likely good candidates also for mobilizing crude oils.

Interfacial Tension (IFT) and Phase Behavior. Test tube samples were prepared with $5 \mathrm{ml}$ of aqueous surfactant/cosolvent/salt formulations and $5 \mathrm{ml}$ of n-octane. After mixing well for several hours, they were allowed to stand for a few weeks to allow the fluids to come to phase equilibrium at ambient conditions. The physical appearance of the phases was noted, such as the relative volumes of the aqueous and oleic phases, and if any third, so-called middle-phase forms.

The interfacial tension (IFT) was determined for selected phase equilibrated test tube samples by using a spinning drop tensiometer (from Temco, Inc.). A glass tube was loaded with the aqueous phase, followed by injection of a few micro-liters of the uppermost, oleic phase. The glass tube was spun in the instrument at a know speed and the IFT determined from the oil drop geometry. Because the samples already come from fluids at phase equilibrium, typically it required less than 2 hours for the measured IFT to stabilize to a final value.

Surfactant Solid Adsorption. APG surfactant adsorption from $2 \mathrm{wt} \% \mathrm{NaCl}$ brines was measured onto kaolinite clay. All of these tests were conducted at $25{ }^{\circ} \mathrm{C}$ with a weight ratio of liquid/solid of 20, and for a mixing exposure period of 8 hours. Kaolinite is selected (obtained from the University of Missouri) as the adsorbant of choice because 1) it is among the most common clays found in oil reservoirs, 2) it may be obtained in a fairly reproducible form, and 3 ) it is a stable material (e.g., will not swell when immersed in water).

The composition provided by the supplier for the kaolinite has the following major components (weight percents): $\mathrm{SiO}_{2}$ 44.2, $\quad \mathrm{Al}_{2} \mathrm{O}_{3}$ 39.7, $\mathrm{TiO}_{2} \quad 1.39, \quad \mathrm{Fe}_{2} \mathrm{O}_{3} 0.13$ with trace amounts of sodium, manganese, calcium, potassium, phosphorous, and fluorine. The specific surface area is about 10 square meter/gram.

After the 8-hour exposure period, the sample is centrifuged and the supernatant analyzed for residual surfactant concentration via a gravimetric method. Knowing the activity of the starting surfactant material and brine salinity, one can calculate the mass of surfactant that is left in the supernatant solution after evaporating off the water solvent.

\section{Theoretical Methods}

Hansen Parameters. The relative solubility among the system components (brine, surfactant, co-surfactant, and hydrocarbon) is one determining factor in the phase behavior exhibited in these multi-phase liquid systems. Because the IFT is in turn related to the phase behavior, it would seem that having values of parameters that quantify the relative solubility of each component could be useful.

In 1936 Joel H. Hildebrand proposed a simple definition for a "solubility parameter" that would provide a systemic description of the miscibility behavior of solvents and which subsequently has found multiple uses in chemistry. ${ }^{8}$ System components with similar solubility parameters are expected to be miscible with one another. This solubility parameter $\delta$ is defined as square root of the cohesive energy density, that is, the heat of vaporization divided by the molar volume.

$$
\delta=\left[\left(\Delta \mathrm{H}_{\mathrm{v}}-\mathrm{RT}\right) / \mathrm{V}_{\mathrm{m}}\right]^{1 / 2}
$$

where $\quad 1$ hildebrand $=1 \mathrm{cal}^{1 / 2} \mathrm{~cm}^{-3 / 2}=2.0455 \mathrm{x} \mathrm{MPa}^{1 / 2}$

Hansen went one step further, and developed individual solubility parameters that when taken together their squares ad up to the Hildebrand parameter squared:

$$
\delta^{2}=\delta_{\mathrm{d}}^{2}+\delta_{\mathrm{p}}^{2}+\delta_{\mathrm{h}}^{2}
$$

where $\delta_{\mathrm{d}}, \delta_{\mathrm{p}}$, and $\delta_{\mathrm{h}}$ are the dispersion, electrostatic (or polar), and hydrogen bond components of $\delta$, respectively. ${ }^{9}$.

Solvents with similar Hansen solubilities are miscible in most proportions; dissimilar values yield limited solubilities. Hildebrand and Hansen solubility parameters are useful for selecting solvents and additives in chemical formulations. We are testing the idea that these parameters when taken together could provide some insights related to the miscibility/phase behavior and thereby the IFT of aqueous based chemical mixture formulations versus hydrocarbons. One caveat is that these parameters, or their equivalent measurements i.e., heats of vaporization and molar volumes, are usually available at a single temperature. Another potential problem relates to the large number of experimental techniques used to estimate the Hansen solubility parameters. These values are often inconsistent and spread a wide range of values. By estimating these values by temperature and pressured controlled (NPT) Molecular Dynamics computer simulations we hope to overcome all of these shortcomings.

The Hildebrand parameter can be calculated easily for compounds with known heats of vaporization and densities. The Hansen parameters may be measured, only with great difficulty. Often the hydrogen bond Hansen parameters are not measured but fitted to reproduce a broad range of miscibility and solubility data, losing its physical meaning. Thus, our strategy is to calculate these Hansen parameters for the relevant compounds from first principles MD calculations. Recent work at the California Institute of Technology has developed such molecular modeling approaches to calculate 
Hansen parameters. ${ }^{10}$ A Cohesive Energy Density (CED) computational method was used that offers consistency (precision) throughout the various organic compounds of interest in formulation work. CED is a multiple sampling Molecular Dynamics method that estimates Hildebrand and Hansen solubility parameters with good precision (ca. 0.44 hildebrands). The CED method, when combined with a generic force field and quantum mechanically determined atomic charges yields first-principles hildebrand parameter predictions in good agreement with experiment (accuracy is 1 . hildebrand or better).

\section{Results and Discussion}

Interfacial Tension (IFT) versus Cosurfactant Type.

Alcohol co-solvents being evaluated include several nalcohols ranging from $\mathrm{C} 3$ to $\mathrm{C} 20$. The aqueous phase has 2 $\mathrm{wt} \%$ combined APG/Co-solvent concentration and has a default brine salinity of $2 \mathrm{wt} \% \mathrm{NaCl}$. The oil and aqueous surfactant solutions are mixed at a $1 / 1$ volume ratio and equilibrated at ambient temperature. Figure 4 shows IFT results with the PG 2062 APG surfactant and the smaller nalcohols investigated.

Note that the IFT for PG 2062 alone is about 2 dyne/cm, and for an alcohol alone the IFT is over several dynes $/ \mathrm{cm}$, perhaps even greater than 30 dynes $/ \mathrm{cm}$. One explanation for the synergistic action of the added alcohols is that they pack at the interface so as to decrease the curvature of the interfacial layer and thereby reduce the IFT. Perhaps the notion of a "hydrophobic linker" is a good physical model for the action of these cosurfactants ${ }^{11}$ That is, an additive may work by linking the oil and surfactant molecules better at the interface. Our general observation is that virtually all alcohol cosolvents act to decrease the IFT of the main, APG surfactant.

Some other comments about these results:

- There is an "optimal" alcohol cosurfactant (in this system n-octanol) that creates the lowest IFT condition (less than 0.01 dyne $/ \mathrm{cm}$.). Larger nalcohols than n-octanol as co-solvents (data not shown here) created only a higher IFT.

- Almost all of the samples indicated in Figure $\mathbf{4}$ had a third, middle-phase, if only a small volume. Even the samples with PG 2062 mixed with the most unfavorable cosurfactant (n-propanol) has at least a small middle phase.

- The IFT behavior versus the amount of APG and nalcohol are fairly constant. This suggests the desirable result that the low IFT condition may be attained with low concentrations of APG surfactant.

Figure 5 summarizes data that compares the IFT measured among 3 different commercial APG surfactants. The trend is that increasing the alkyl chain length of the APG surfactant decreases the IFT, all other things being equal.

Closer inspection of the data in Figure 5 indicates that the IFT for PG 2067 and PG 2069 (average alkyl chain lengths of 9.1 and 10.1, respectively) also have a lower IFT as the cosurfactant alcohol chain length increases from n-propanol to $\mathrm{n}$-hexanol. (This is the same trend as shown for the PG 2062 surfactant in Figure 4.)

Experiments examine the effect of other alcohols as cosolvents focused on the PG 2062 APG product because its formulations with small $\mathrm{n}$ - alcohols gave the lowest IFT values (Figure 4 and 5). These other alcohol co-solvents included larger n-alcohols, with the result showing n-octanol still as the best co-solvent. Another series of tests (Figure 6) examined a series of $\mathrm{C} 6$ alcohols as co-solvents, with the variation being the alcohol structure as a straight chain aliphatic (n-hexanol), branched chain alcohol (4-methyl-2pentanol), saturated ring (cyclohexanol), and as an aromatic ring structure (phenol). Results show the straight chain (nhexane) structure provides the lowest IFT among this group of co-solvents.

Figure 7 presents measured IFT results for surfactant formulations containing PG 2067 and different SPAN products. Note that the IFT for PG 2067 alone and these SPAN products by themselves is about 2 dyne $/ \mathrm{cm}$. In some cases there is an obvious strong synergistic effect, with the IFT attaining very low values.

It seems odd that the two "end members" of the SPAN series, SPAN $20(\mathrm{HLB}=8.6)$ and the SPAN $85(\mathrm{HLB}=1.8)$ can create a low IFT when used in these APG formulations. In contrast, mixtures employing SPAN $40(\mathrm{HLB}=6.7)$ and SPAN $80(\mathrm{HLB}=4.3)$ never exhibit this synergistic, lower IFT effect. Also, preliminary data suggest a low IFT may occur with PG 2067 / SPAN 60 mixtures (data not shown).

One available relevant set of data in the literature describes IFT for APG solutions mixed with SPAN 20 and equilibrated versus n-dodecane as the oil phase. ${ }^{6}$. Low IFT occurrs, but only over a narrow range of APG / SPAN 20 mixture ratios. We observe this same general effect, with for example the PG 2067/SPAN 85 having a sharp minimum in IFT at a ratio of $0.75 \% / 1.25 \%$, but significantly greater IFT at other mixing ratios.

Other IFT results for APG and sorbitan surfactant mixtures are shown in Tables 3 and 4 . Most other combinations of these APG products and the SPAN or TWEEN surfactants created stiff gels in the oil phase. Those combinations exhibiting such unfavorable phase behavior are not viable as an EOR formulation. The combinations (shown above) where all of the phases (aqueous, microemulsion, and oleic) appear to be fluid, the measured IFT results encompass a wide range of values. The IFT value is especially low $(0.0035$ dyne $/ \mathrm{cm})$ for the first sample shown (the PG 2069 / SPAN 20 blend at $0.8 / 1.2 \mathrm{wt} \%$,), and a moderately low IFT of 0.05 dyne/cm Otherwise, the IFT exceeds $0.1 \mathrm{dyne} / \mathrm{cm}$ for the other combinations presented in Tables 3 and 4.

Interfacial Tension (IFT) versus Temperature and Salinity. Measured IFT results in this study confirm that APG/alcohol formulations may be largely indifferent to both changes in the temperature (Figure 8) and the salinity (Figure 9). This is desirable because in oil reservoirs, the temperature will vary from zone to zone, with higher temperatures occurring in deeper subsurface depths. This behavior means that one may formulate just a single aqueous based surfactant solution that is able to mobilize the crude oil as efficiently in spite of these temperature differences.

The salinity in the brine in the subsurface oil reservoir of course may vary both in an areal and vertical extent. Mature fields that have been subjected to years of waterflood (the primary targets for surfactant EOR) often have substantial differences in salinity, for example, due to contrasts between 
the injected and original formation brine.

A common approach in surfactant EOR is the so-called "salinity-gradient" design whereby the salinity is reduced stepwise from the formation water, surfactant slug, and polymer/water drive. The motivation for this design is to generate a low IFT, middle-phase microemulsion condition insitu, with the following drive solutions designed to put the surfactant back into the aqueous phase in order to avoid excessive chemical loss by phase trapping. One problem with this approach is that it may be difficult to locate sufficient fresh water sources to cause this gradual reduction in salinity. Also, maintaining target salinities both in-situ and in the chemical solutions becomes more difficult as the chemical project continues. This is important because the performance of most (anionic) surfactant systems is sensitive to salinity.

Having a single surfactant formulation that is indifferent to salinity should be an advantage in the EOR design. With APG/cosurfactant formulations, one might accomplish the same objectives of having low IFT in-situ and avoiding surfactant phase trapping by changing the ratio of the APG/cosurfactant during chemical injection. In addition, per Figure 9, there is the possibility of formulating for a low IFT for reservoirs containing high salinity, high hardness brines. . Surfactant Adsorption. Maximum adsorption measured for the 3 commercial APG surfactants (PG 2067, PG 2069, and PG 2062) are shown, left to right in Figure 10. Other surfactant retention tests onto kaolinite clay were performed with APG mixed with alcohol and a SPAN product (Table 5). Tests were in $2 \mathrm{wt} \% \mathrm{NaCl}$ and a ratio of solution/solid of $20: 1$.

Some trends evident from these data:

- Low adsorption for APG product with shorter alkyl chains, but significant adsorption for the PG 2062

- Increased total surfactant adsorption when mixing with the SPAN 20 sorbitan surfactant.

- The adsorption levels with mixtures of PG 2062 and 1 -alcohols are almost independent of the specific alcohol cosurfactant selected.

The anticipated surfactant adsorption in a sandstone rock would be less (estimate by an order of magnitude) because the clay content would be only a few percent in a typical reservoir. Roughly speaking, adsorption levels of $10 \mathrm{mg} / \mathrm{gram}$ kaolinite (perhaps $0.1-1 \mathrm{mg} / \mathrm{gram}$ sandstone) are typical for alkyl aryl sulfonate surfactants used for EOR. This suggests the adsorption of the PG 2062 may be greater than that for common EOR surfactants, but that the PG 2067 and PG 2069 adsorption levels are much less.

Hansen Parameters. We compare the 3 Hansen parameters for some of the components of the APG/alcohol formulations discussed in the previous section. Figures $\mathbf{1 1}$ is a plot of normalized values for the 3 Hansen parameters for several pure substances. These values for the PG 2062 APG surfactant, water, n-octane, and several alcohol cosurfactants are calculated as described earlier.

These plots have a notation about the measured IFT value underneath each alcohol. This IFT is for a PG $2062(0.8 \%)$ and alcohol cosurfactant (1.2\%) formulation in a $2 \mathrm{wt} \% \mathrm{NaCl}$ brine versus n-octane at room temperature.

From the observed pattern of component Hansen parameters that makes a low IFT, one may gain guidance with respect to creating new formulations for low IFT. Our future approach would be to calculate the Hansen parameters for a number of new compounds, and focus on those with follow-up experimental studies that exhibit the observed successful pattern of Hansen values.

For these results we do find that the IFT is lower for PG 2062/ alcohol formulations when the alcohol Hansen dispersion parameter increases, polarization parameter decreases, and hydrogen bonding parameter decreases. As the Hansen parameters for this alcohol series become more similar to the values for n-octane, the model oil phase, the

PG 2062/alcohol formulation reduces the interfacial tension to its lowest measured values in this study.

\section{Conclusions}

Key findings from this study include:

1. Alkyl polyglycoside surfactants may be formulated in brine solutions that can create IFT (interfacial tension) approaching $0.01 \mathrm{dyne} / \mathrm{cm}$, or less, versus simple alkane hydrocarbons.

2. Some of these APG formulations may generate a low IFT that is largely independent of both salinity and temperature effects.

3. Some APG surfactants (shorter alkyl chain products) exhibit relatively low adsorption on kaolinite clay.

4. Solubility parameters estimated from first principles offer another perspective to guide the formulation of APG with cosurfactants that will create low IFT.

\section{Acknowledgments}

The authors wish to thank the Department of Energy for their financial support for this project, and the technical assistance of ChevronTexaco and Akzo Nobel. We also wish to thank the Cognis Corporation for supplying APG surfactant samples.

\section{References}

1. Balzer, D.: "Alkylpolyglcosides, their Physico-chemical Properties and their Uses", Tenside Surf. Det., 38, 419 - 427, 1991.

2. Hill, K. and Rhode, O.: "Sugar-based surfactants for consumer products and technical applications", Fett/Lipid, 10, 25-33, 1999.

3.. Balzer,D.: "Process for the extraction of crude oil from an underground deposit using surfactants", U.S. Patent 4,985,154, 1991.

4. Balzer, D., and Luders, H., editors.: Nonionic Surfactants, Alkyl Polyglycosides, Marcel Dekker, New York, p. 228 - 243, 1996.

5. Kutschmann, E.M., et. al.: "Interfacial tension of alkylglucosides in different APG/oil/water systems", Colloid Polym. Sci. 273, $565-571,1995$.

6. Forster, T., et. al.: "Physico-chemical basics of microemulsions with alkyl polyglycosides", Progr. Colloid Polym. Sci., 101, $105-$ 112, 1996.

7. Peypoux, F., Bonmatin, J.M., and Wallach, J.: "Recent trends in biochemistry of surfactin", Appl. Microbial. Biotechno.l, 51, 553 563, 1999.

8. Hildebrand, J.H., The Solubility of Non-Electrolytes, New York, Reinhold, 1936. 
9. Hansen, C.M., Hansen Solubility Parameters, A User's Handbook, New York, CRC Press, 2000. .

10. Belmares, M., Blanco, M., Goddard III, W.A.et. al.: "Hildebrand and Hansen Solubility P:arameters from Molecular Dynamics with Applications to Electronic Nose Polymer Sensors", submitted 2003

11. Sabatini, D.A., Acosta, E., and Harwell, J.H.: "Linker Molecules in Surfactant Mixtures", submitted to Current Opinion in Colloid and Interface Science, February, 2003.

Table 1. Chemical structure information for Alkyl Polyglycosides (APG) investigated

\begin{tabular}{lccccc} 
Product & Alkyl Chains & Distribution & $\begin{array}{c}\text { Average } \\
\text { Chain Length }\end{array}$ & Average n & HLB \\
\hline PG 2067 & $8 / 10$ & $(45: 55)$ & 9.1 & 1.7 & 13.6 \\
PG 2069 & $9 / 10 / 11$ & $(20: 40: 40)$ & 10.1 & 1.6 & 13.1 \\
PG 2062 & $12 / 14 / 16$ & $(68: 26: 6)$ & 12.5 & 1.6 & 11.6
\end{tabular}

Table 2. Chemical structure information for SPAN and TWEEN surfactants.

\begin{tabular}{|c|c|c|c|c|c|c|}
\hline Product & $\begin{array}{l}\text { Length } \\
\text { Alkyl Chain }\end{array}$ & $\begin{array}{c}\text { verage } \\
\text { HLB }\end{array}$ & Product & $\begin{array}{c}\text { Number } \\
\text { EO Groups }\end{array}$ & $\begin{array}{c}\text { Length } \\
\text { Alkyl Chain }\end{array}$ & $\begin{array}{c}\text { Average } \\
\text { HLB }\end{array}$ \\
\hline SPAN 20 & $\mathrm{C} 12$ & 8.6 & TWEEN 20 & 20 & $\mathrm{C} 12$ & 16.7 \\
\hline SPAN 40 & $\mathrm{C} 14$ & 6.7 & TWEEN 40 & 5 & $\mathrm{C} 12$ & 13.3 \\
\hline SPAN 60 & $\mathrm{C} 18$ & 4.7 & TWEEN 80 & 20 & $\mathrm{C} 18$ & 15.0 \\
\hline SPAN 80 & C18 (one double bond) & 4.3 & TWEEN 81 & 5 & $\mathrm{C} 18$ & 10.0 \\
\hline SPAN 85 & $3 \mathrm{C} 18$ (each has double bond) & 1.8 & TWEEN 85 & 20 & $3 \mathrm{C} 18$ chains & 11.0 \\
\hline
\end{tabular}

Table 3. Selected measured IFT for formulations of APG / SPAN surfactants versus n-octane.

\begin{tabular}{|c|c|c|c|c|}
\hline & SPAN & weight & weight & IFT \\
\hline APG & Product & \% APG & \% SPAN & (dyne/cm) \\
\hline PG 2069 & 20 & 0.80 & 1.20 & 0.0035 \\
\hline PG 2069 & 40 & 0.40 & 1.60 & 1.40 \\
\hline PG 2069 & 60 & 0.40 & 1.60 & 0.33 \\
\hline PG 2069 & 85 & 0.40 & 1.60 & 1.55 \\
\hline PG 2069 & 85 & 1.50 & 0.50 & 0.8 \\
\hline PG 2069 & 85 & 1.60 & 0.40 & 1.2 \\
\hline & & & & \\
\hline PG 2062 & 20 & 0.80 & 1.20 & 0.90 \\
\hline PG 2062 & 20 & 1.20 & 0.80 & 0.75 \\
\hline PG 2062 & 40 & 0.40 & 1.60 & 0.85 \\
\hline PG 2062 & 60 & 0.40 & 1.60 & 1.00 \\
\hline PG 2062 & 60 & 0.80 & 1.20 & 0.73 \\
\hline PG 2062 & 80 & 0.40 & 1.60 & 1.20 \\
\hline PG 2062 & 85 & 0.40 & 1.60 & 0.68 \\
\hline PG 2062 & 85 & 0.80 & 1.20 & 0.25 \\
\hline PG 2062 & 85 & 1.20 & 0.80 & 0.40 \\
\hline
\end{tabular}


Table 4. Selected measured IFT for formulations of APG / TWEEN surfactants versus n-octane.

\begin{tabular}{|c|c|c|c|c|}
\hline & TWEEN & & & IFT \\
\hline APG & Product & \% APG & \%TWEEN & (dyne/cm) \\
\hline PG67 & 21 & 1.20 & 0.80 & 1.07 \\
\hline PG67 & 21 & 1.60 & 0.40 & 1.42 \\
\hline PG67 & 85 & 0.80 & 1.20 & 0.76 \\
\hline PG67 & 85 & 1.00 & 1.00 & 0.38 \\
\hline PG67 & 85 & 1.20 & 0.80 & 0.9 \\
\hline PG67 & 85 & 1.60 & 0.40 & 0.82 \\
\hline PG69 & 21 & 1.60 & 0.40 & 1.25 \\
\hline PG69 & 40 & 1.60 & 0.40 & 1.7 \\
\hline PG69 & 81 & 1.00 & 1.00 & 9.6 \\
\hline PG62 & 21 & 0.40 & 1.60 & 0.05 \\
\hline PG62 & 81 & 0.40 & 1.60 & 1.3 \\
\hline PG62 & 81 & 0.80 & 1.20 & 6.10 \\
\hline PG62 & 85 & 0.40 & 1.60 & 0.76 \\
\hline
\end{tabular}

(Note IFT measurements in Table 3 and 4 performed at ambient temperature)

Table 5. Selected adsorption results for APG/cosurfactant formulations

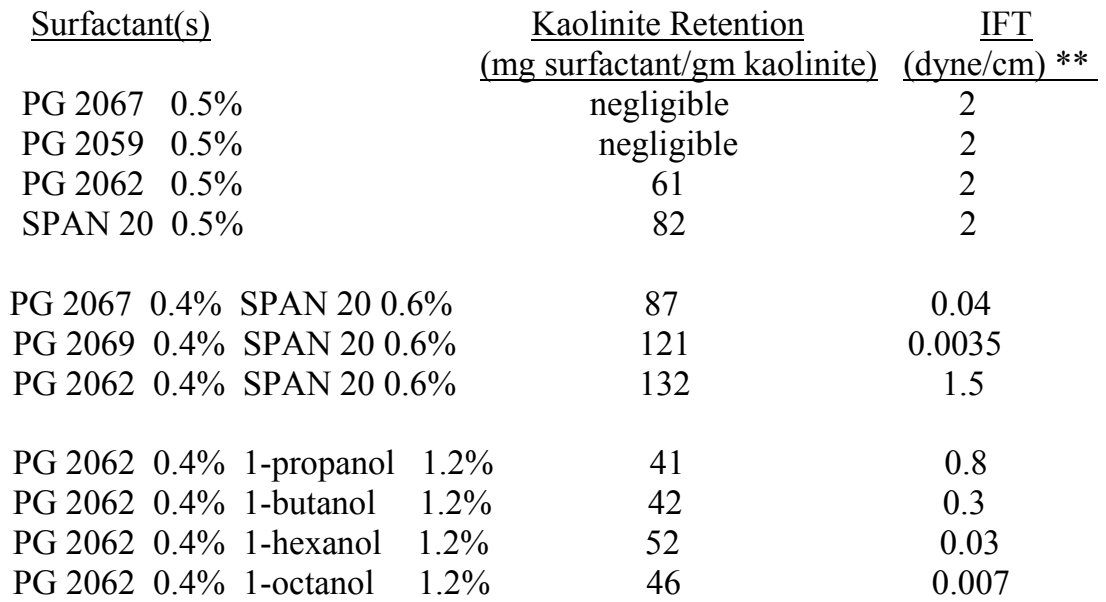

** IFT measured in separate experiment. IFT for surfactant formulation made up in a $2 \mathrm{wt} \% \mathrm{NaCl}$ brine after phase equilibration reached with n-octane at $25 \mathrm{C}$. 


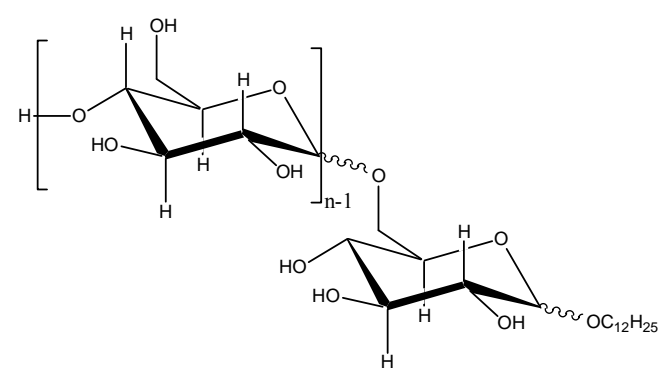

Figure 1. Molecular structure of an example alkyl polyglycoside (dodecylglucopyranoside)<smiles>CCCCCCCCC(=O)OCC(O)C1OCC(O)C1O</smiles>

Figure 2. Molecular structure of SPAN 20 surfactant, sorbitan monolaurate

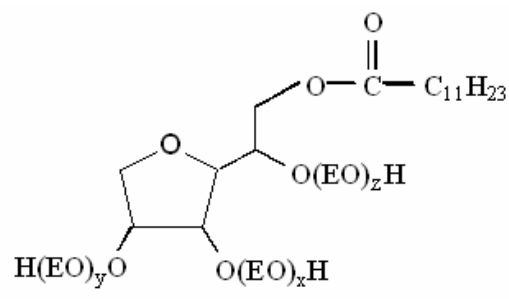

Figure 3. Molecular structure of TWEEN 20 surfactant, Polyoxyethylene (20) sorbitan monolaurate, $\mathrm{X}+\mathrm{Y}+\mathrm{Z}=\mathbf{2 0}$

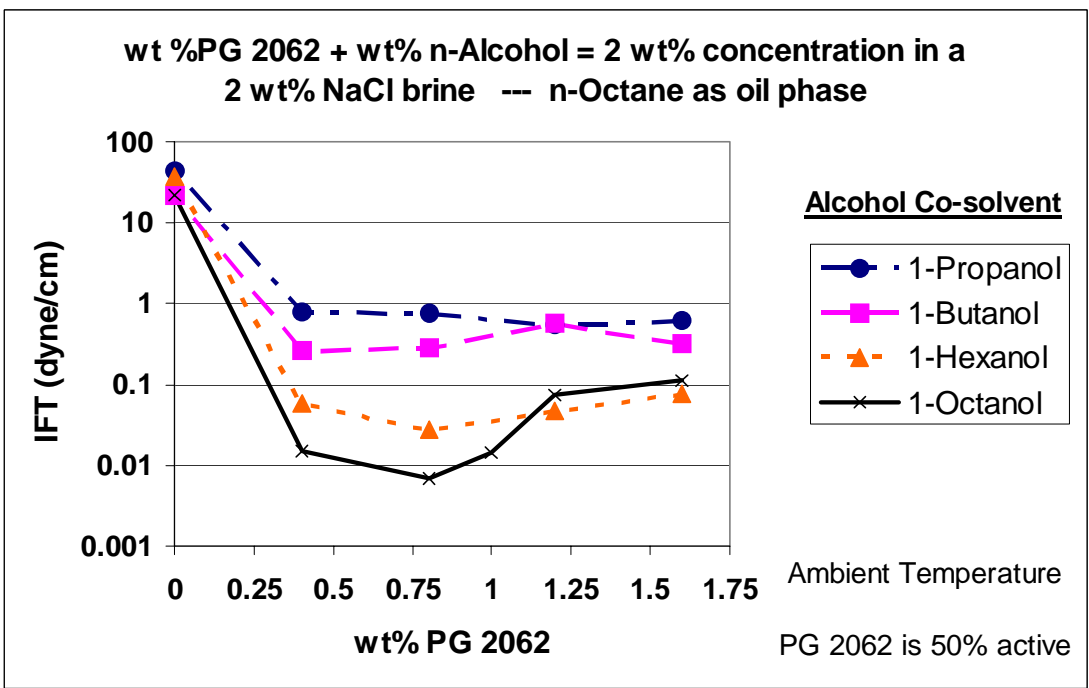

Figure 4. IFT measured for equilibrated samples containing PG 2062 mixed with small $n$-alcohol formulations versus n-octane.

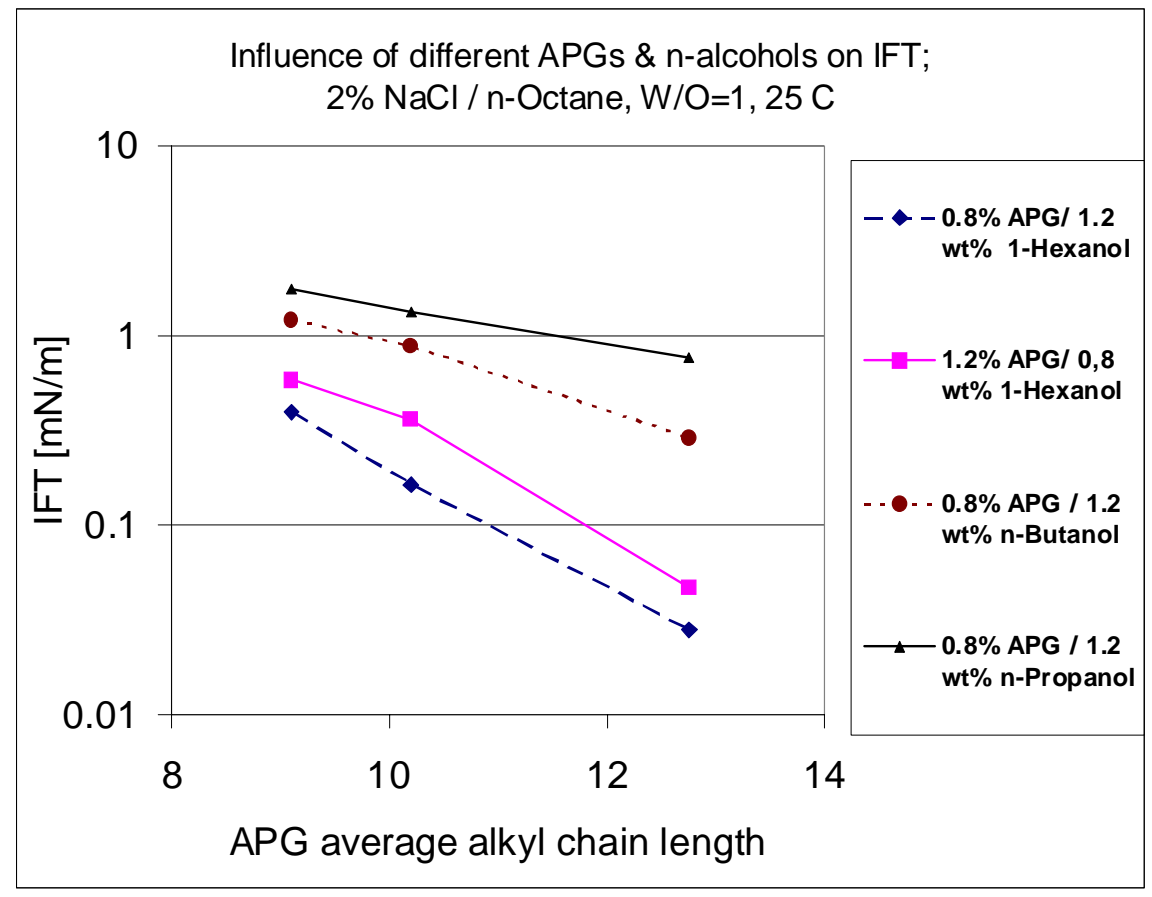

Figure 5. Increase of APG average alkyl chain length decreases the measured IFT. 


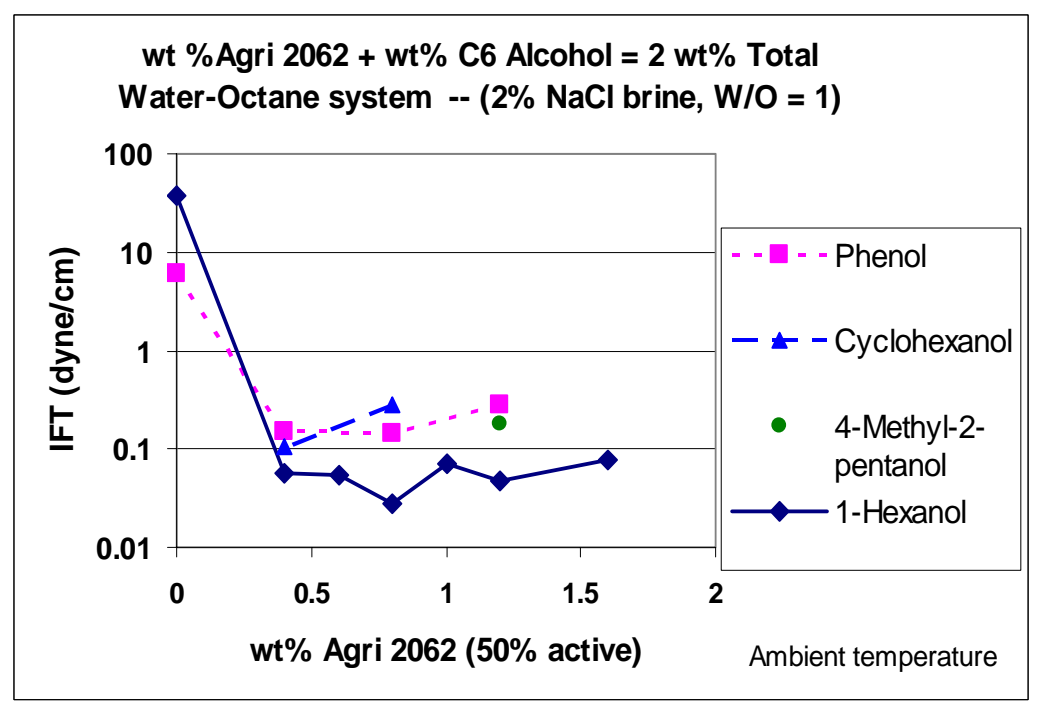

Figure 6. Comparison of IFT behavior for different alcohol cosurfactants that all have 6 carbons and are mixed with PG 2062.

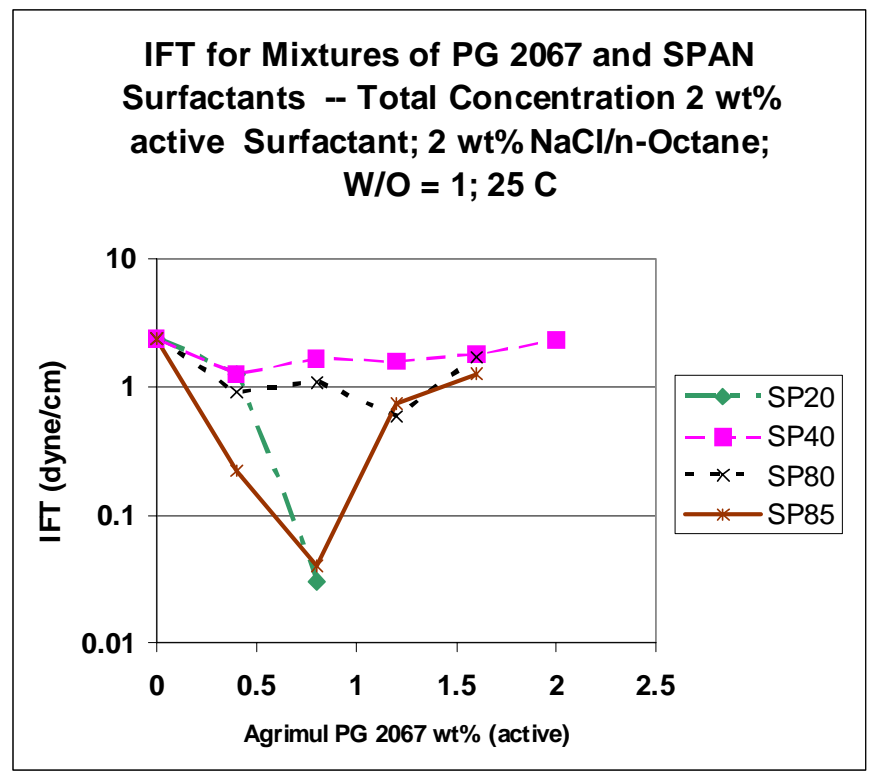

Figure 7. Measured IFT for mixtures of PG 2067 and selected SPAN surfactant products versus $n$-octane.

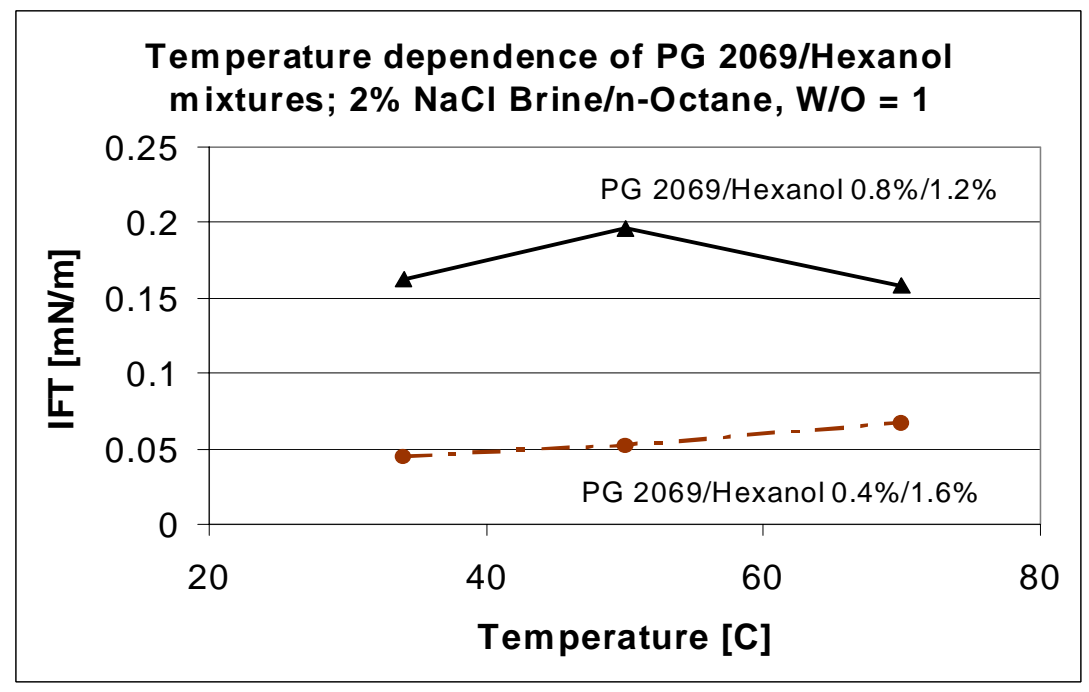

Figure 8. IFT is nearly independent of temperature for these PG 2069/1-hexanol formulations versus n-octane.

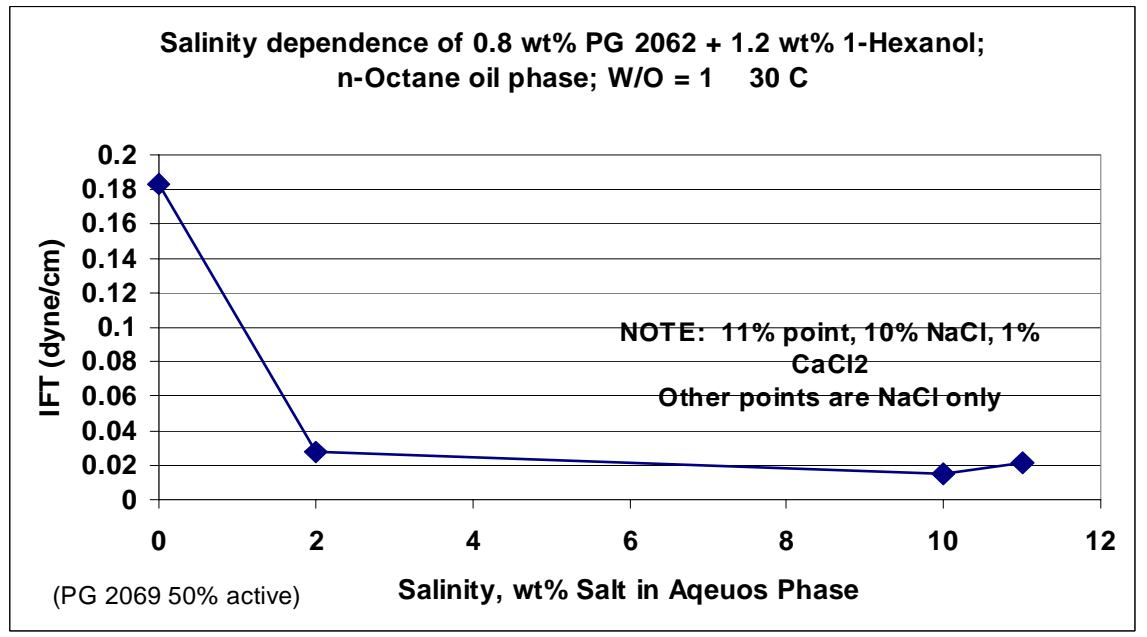

Figure 9 IFT is nearly independent of salinity for this PG 2062/1-hexanol formulation versus n-octane. 


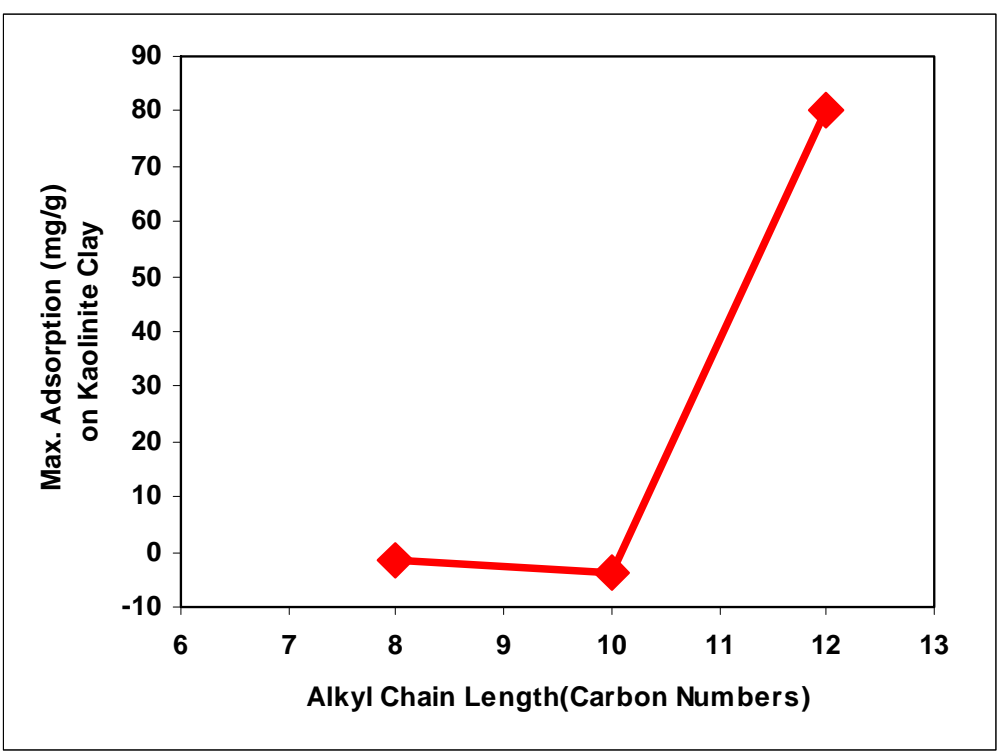

Figure 10. Measured plateau adsorption of APG surfactants. 20:1 ratio of solution:sand, $25^{\circ} \mathrm{C}$.

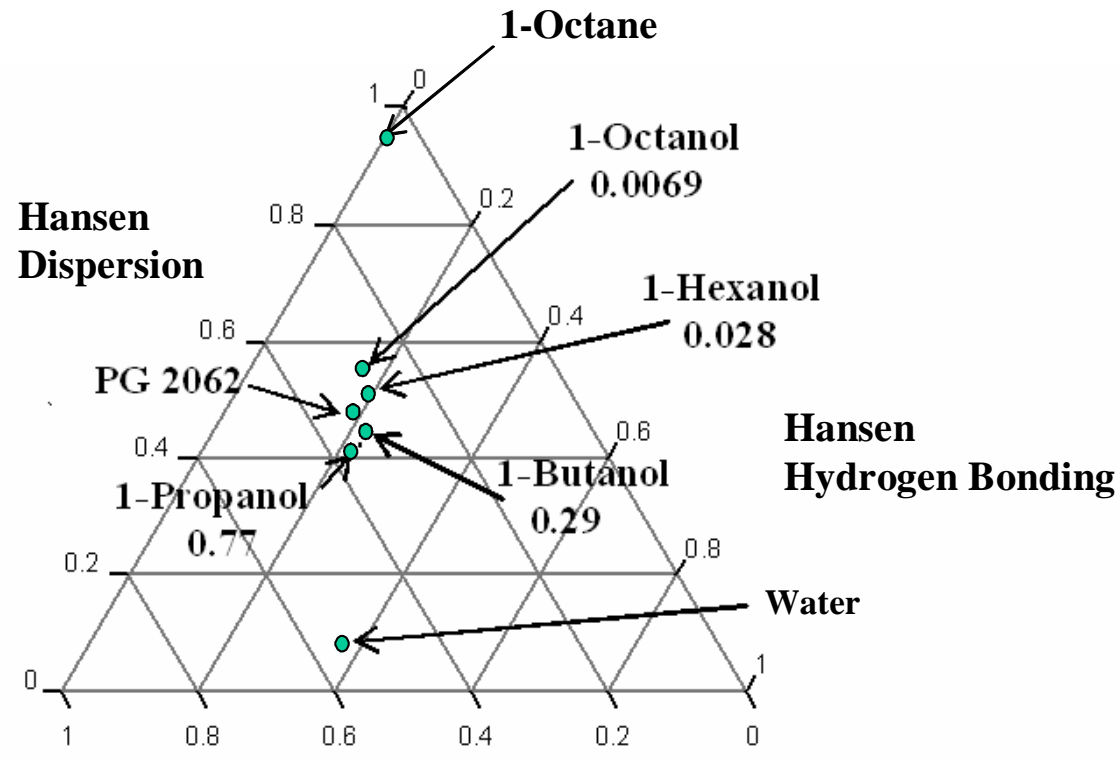

Hansen Polarization

Figure 11. Normalized Hansen parameters calculated for PG 2062, water, n-octane, and several alcohols. IFT values (dyne/cm) indicated for each alcohol. Measured IFT for PG 2062/alcohol $0.8 \% / 1.2 \%$ mixtures in $2 \% \mathrm{NaCl}$ brine versus $\mathrm{n}$-octane at $25^{\circ} \mathrm{C}$. 


\title{
Molecular Dynamics Study of a Surfactant-Mediated Decane-Water Interface: Effect of Molecular Architecture of Alkyl Benzene Sulfonate
}

\author{
Seung Soon Jang, Shiang-Tai Lin, Prabal K. Maiti, Mario Blanco, and \\ William A. Goddard III* \\ Materials and Process Simulation Center, Beckman Institute, California Institute of Technology, \\ Pasadena, California 91125 \\ Patrick Shuler and Yongchun Tang \\ Power, Energy \& Environmental Research Center, California Institute of Technology, \\ Covina, California 91722
}

Received: March 18, 2004; In Final Form: May 25, 2004

\begin{abstract}
The effect of molecular architecture of a surfactant, particularly the attachment position of benzene sulfonate on the hexadecane backbone, at the decane-water interface was investigated using atomistic MD simulations. We consider a series of surfactant isomers in the family of alkyl benzene sulfonates, denoted by $m$-C16, indicating a benzene sulfonate group attached to the $m$ th carbon in a hexadecane backbone. The equilibrated model systems showed a well-defined interface between the decane and water phases. We find that surfactant 4-C16 has a more compact packing, in terms of the interfacial area and molecular alignment at the interface, than other surfactants simulated in this study. Furthermore, surfactant 4-C16 leads to the most stable interface by having the lowest interface formation energy. The interfacial thickness is the largest in the case of surfactant 4-C16, with the thickness decreasing when the benzene sulfonate is located farther from the attachment position of 4-C16 (the 4th carbon). The interfacial tension profile was calculated along the direction perpendicular to the interface using the Kirkwood-Buff theory. From the comparison of the interfacial tension obtained from the interfacial tension profile, we found that surfactant 4-C16 induces the lowest interfacial tension and that the interfacial tension increases with decreasing interfacial thickness as a function of the attachment position of benzene sulfonate. Such a relationship between the interfacial thickness and interfacial tension is rationalized in terms of the miscibility of the alkyl tail of surfactant $m$-C16 with decane by comparing the "effective" length of the alkyl tail with the average end-to-end length of decane. Among the surfactants, the effective length of the 4-C16 alkyl tail $(9.53 \pm 1.36 \AA)$ was found to be closest to that of decane $(9.97 \pm 1.03 \AA)$, which is consistent with the results from the density profile and the interfacial tension profile.
\end{abstract}

\section{Introduction}

Surfactants are amphiphilic molecules which have one part that is more soluble in water and another part that is more soluble in oil. When added to an oil-water mixture, surfactant molecules are preferentially adsorbed into the interface, leading to a modification of the interfacial properties such as a decrease of interfacial tension. Surfactants are widely employed in household uses such as detergents, food, and cosmetic technology and in large-scale operations in petroleum recovery. ${ }^{1-4}$ It is important to understand the underlying principle governing interfacial properties of a given system and, thereby, to design the system or the molecular architecture of surfactants for the purpose of optimizing the performance in which we are interested.

Most thermodynamic models based on the Langmuir adsorption have been developed to describe the equilibrium adsorption of surfactants at the oil-water interface..$^{5-19}$ In particular, there have been efforts made to link the given thermodynamic models and molecular information, such as the conformation and intermolecular interaction. Fainerman and co-workers ${ }^{11,12}$ have studied the influence of the molecular reorientation of surfactants on the adsorption isotherms by taking into account the conformational dependency of surface area. Blankschtein and co- workers developed a molecular thermodynamic theory for predicting the interfacial behavior of surfactant mixtures that are adsorbed at the air-water ${ }^{17,18}$ or the oil-water interface ${ }^{19}$ by combining the two-dimensional nonideal gas model ${ }^{20}$ with specific molecular properties such as the number of carbons in the surfactant hydrocarbon tail and the molecular cross-sectional area. In these models, knowledge of the molecular structure and interaction is essential for the quantitative prediction and description of the properties of the interface.

Molecular modeling approaches such as the molecular dynamics (MD) and Monte Carlo (MC) simulations have been extensively used for studying the liquid- vapor $^{21-34}$ and liquidliquid $^{35-51}$ interfaces. These methods, performed on the basis of the molecular interaction and molecular structures, provide atomistic or molecular details of the interface that are potentially useful for the thermodynamic models mentioned previously. However, among these studies, only a few ${ }^{40,43,47,49,50}$ have specifically considered the role of the surfactant at the oilwater interface. Although there have been studies attempting to investigate the dependence of dynamics and morphology of surfactant aggregates on the surfactant structure using coarsegrained modeling techniques, ${ }^{43,52-56}$ to our knowledge, there has been no systematic study investigating the effect of 

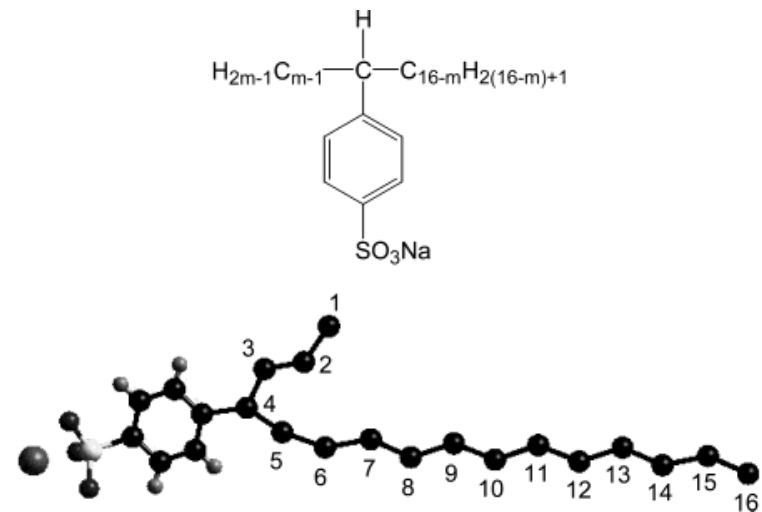

Figure 1. Hexadecane benzene sulfonate surfactant $(m=2,4,6$, and 8 ) which has a benzene sulfonate group attached at the $m$ th carbon in the backbone; for convenience, it is denoted as $m$-C16. The example shown is 4-C16. The benzene sulfonate part adopts the explicit allatom model and the alkyl part the united atom model.

molecular architecture of surfactants on the interfacial properties, such as interfacial tension and structure, of the oil-water interface.

In this paper, we present a molecular dynamics study on the changes in the decane-water interfacial properties as a function of the structural variable of surfactants. The surfactant molecules used here are a family of hexadecane benzene sulfonate groups denoted by $m$-C16 (Figure 1), which indicates a benzene sulfonate group attached to the $m$ th carbon in the hexadecane backbone. It was reported previously that the interfacial tension (IFT) between water and decane decreases with the addition of surfactants $m-\mathrm{C} 16$ and that the extent of IFT reduction changes with $m$. The maximum IFT reduction is found with 4-C16. ${ }^{57}$ Our goal in this study is to analyze how the variation in the molecular architecture of $m$-C16 surfactants affects interfacial properties such as the local density profile and IFT of the decane-water interface. For this purpose, we characterized the conformation of surfactants, the density profile, and the interfacial tension profile using surfactants 2-C16, 4-C16, 6-C16, and 8-C16.

\section{Model and Simulation Methods}

In this simulation, decane was described using the united atom model developed by Smit and co-workers, ${ }^{58-61}$ and water using an $\mathrm{F} 3 \mathrm{C}$ model. ${ }^{62}$ These force fields were extensively tested and also successfully used in our previous studies. ${ }^{63,64}$ For the surfactant, the benzene sulfonate part was described by the explicit all-atom model using the Dreiding force field, ${ }^{65}$ and the alkyl tail part was described by the same united atom model used for decane. The total potential energy is given as follows:

$$
E_{\text {total }}=E_{\mathrm{vdW}}+E_{\mathrm{Q}}+E_{\text {bond }}+E_{\text {angle }}+E_{\text {torsion }}
$$

where $E_{\mathrm{total}}, E_{\mathrm{vdW}}, E_{\mathrm{Q}}, E_{\mathrm{bond}}, E_{\text {angle }}$, and $E_{\text {torsion }}$ are the total energy and the van der Waals, electrostatic, bond-stretching, angle-bending, and torsion-energy components, respectively. The chemical structures of water, decane, and the surfactants are shown in Figure 2, and their force-field parameters used to calculate the intra- and intermolecular interactions are summarized in Table 1.

For all of the cases (2-C16, 4-C16, 6-C16, and 8-C16), we simulated model systems consisting of the decane and water phases having two decane-water interfaces (Figure 3a), which

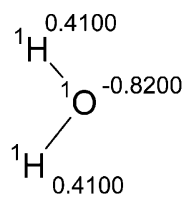

(a) water

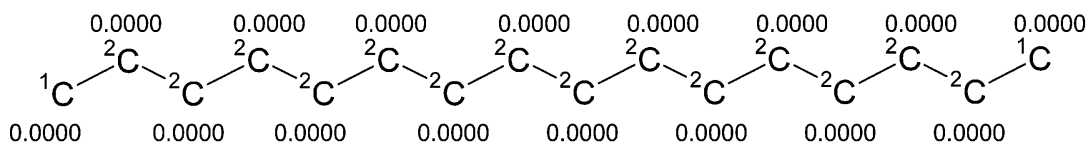

(b) decane

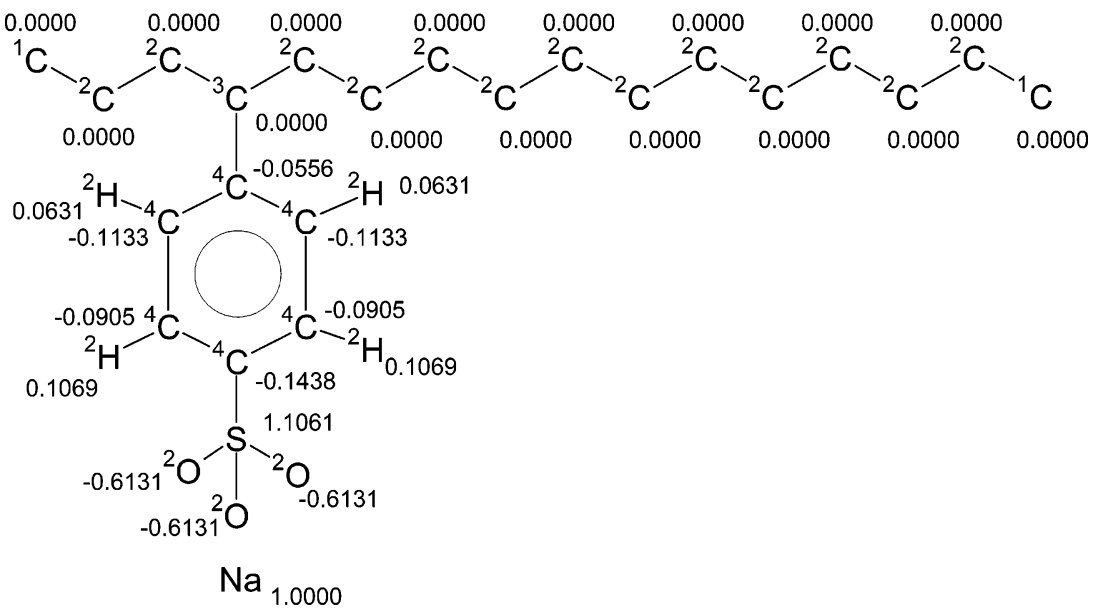

(c) surfactant

Figure 2. Chemical structure and partial charges of (a) water, (b) decane, and (c) surfactant (4-C16). The superscripts to the left and above the atoms denote the atom types used in Table 1 . 
TABLE 1: Force-Field Parameters Used for the Decane-Water Interface with a Surfactant

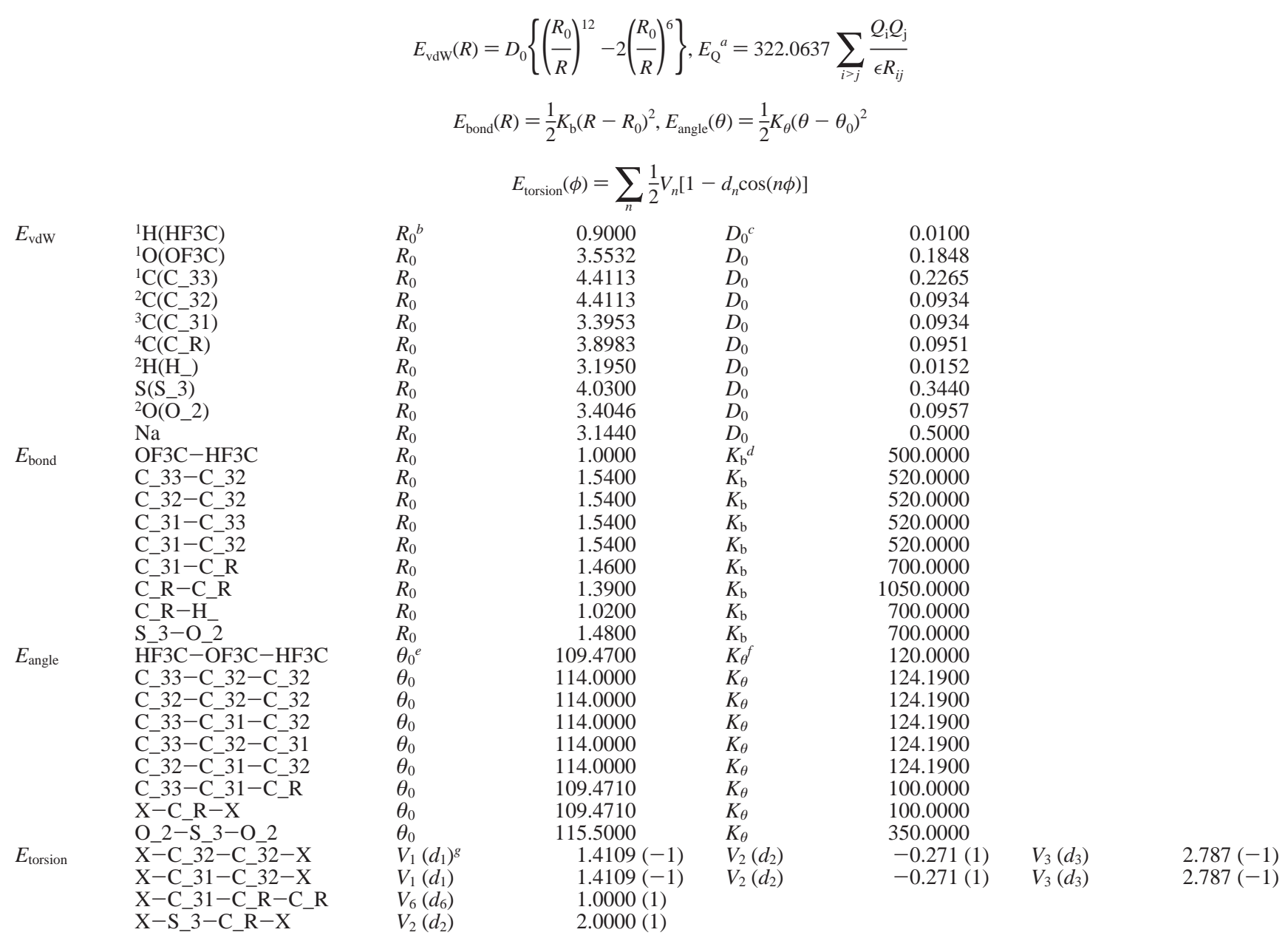

${ }^{a} Q_{\mathrm{i}}$ and $Q_{\mathrm{j}}$ are atomic charge of atoms i and j, respectively. Except for water, all atomic charges were calculated from QM Mulliken populations at the level of $6-31 \mathrm{G}^{* *} / \mathrm{B} 3 \mathrm{LYP}$. The atomic charges for water molecules and for decane are from the F3C model in ref 62 and from the united atom model of hydrocarbon in refs 58-61, respectively. ${ }^{b} \AA$ for $R_{0} .{ }^{c} \mathrm{kcal} / \mathrm{mol}$ for $D_{0} .{ }^{d} \mathrm{kcal} \mathrm{mol}{ }^{-1} \AA^{-2}$ for $K_{\mathrm{b}} .{ }^{e}$ Deg for $\theta_{0} .{ }^{f} \mathrm{kcal}^{\mathrm{mol}}{ }^{-1} \mathrm{deg}^{-2}$ for $K_{\theta}$. ${ }^{g} \mathrm{kcal} / \mathrm{mol}$ for $V_{n}$.

have been widely used for the studies of liquid-liquid interfaces. ${ }^{35,39,42,44,50,51}$ All of the systems have the same composition: 120 decane molecules, 800 water molecules, and 32 surfactants. To construct this configuration, first, we prepared the monolayer consisting of 16 surfactants on an assumption of hexagonal closed packing in an orthorhombic simulation box with the periodic boundary condition applied to all three spatial directions (Figure 3b). Then, we carried out an energy minimization to relax this monolayer of surfactants with the fixedcell dimensions $\left(L_{x}, L_{y}\right.$, and $\left.L_{z}\right)$. The next step was to prepare the decane and water phases separately using NVT MD simulations based on the experimental densities (at $300 \mathrm{~K}$ and 1 atm, $0.725 \mathrm{~g} / \mathrm{cm}^{3}$ for decane $\mathrm{e}^{6-69}$ and $0.997 \mathrm{~g} / \mathrm{cm}^{3}$ for water ${ }^{70-72}$ ). The cell parameters of the simulation box were set to have the same $L_{x}$ and $L_{y}$ dimensions as the orthorhombic simulation box with the surfactant monolayer. As the final step, we integrated these three phases into one simulation box. Before applying MD simulation to equilibrate this integrated system, we performed an energy minimization to relax the system during which the cell parameters $L_{x}, L_{y}$, and $L_{z}$ of the orthorhombic simulation box were adjusted to obtain better interaction between the newly jointed phases. Once the initial system was prepared, NVT and NPT MD simulations were sequentially carried out to equilibrate the system. First, a NVT MD simulation was performed for $200 \mathrm{ps}$ at $300 \mathrm{~K}$ as a pretreatment for overcoming local minima by imposing thermal energy in a constant volume condition. Then, a final equilibration was done by a NPT MD simulation for $400 \mathrm{ps}$ at $300 \mathrm{~K}$ and $1 \mathrm{~atm}$ to adjust the system to a more realistic density. To obtain good statistics, we simulated two independent samples for each surfactant case (from 2-C16 to 8-C16) with the NPT MD simulation for $2 \mathrm{~ns}$ at $300 \mathrm{~K}$ and $1 \mathrm{~atm}$. In addition, we prepared the bare decanewater interface consisting of 120 decane molecules and 800 water molecules without surfactants as a reference for comparison. Here, the kind of concentration with which our simulations are working should be addressed. From the general consensus saying that surfactant concentration at the interface is a function of the concentration in the bulk phase below the CMC (critical micelle concentration) but saturated above the $\mathrm{CMC}$, it seems to be clear that we are dealing with a case in which the bulk concentration is above the CMC, although the finite system size of our simulation does not allow free surfactant in the bulk phases. Therefore, the surfactant concentration and the interfacial properties in our simulations are insensitive to the variation in the bulk phase.

Throughout this study, all MD simulations were performed with the LAMMPS (large-scale atomic/molecular massively parallel simulator) code from Plimpton at Sandia (modified to handle our force fields). ${ }^{73,74}$ The equations of motion were integrated using the Verlet algorithm ${ }^{75}$ with a time step of 1.0 


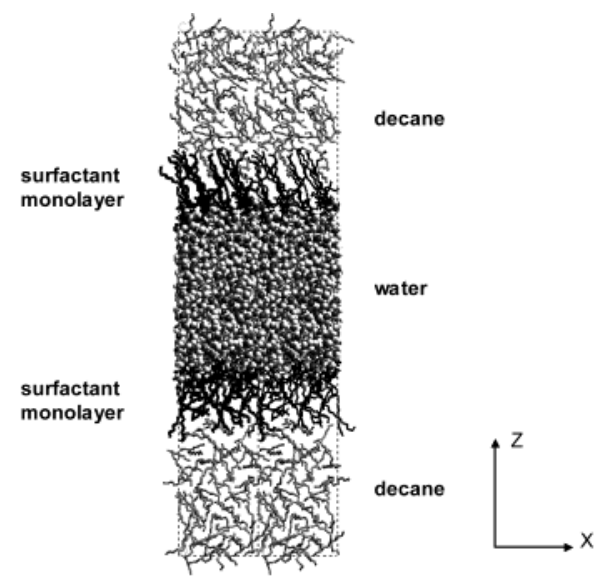

(a)

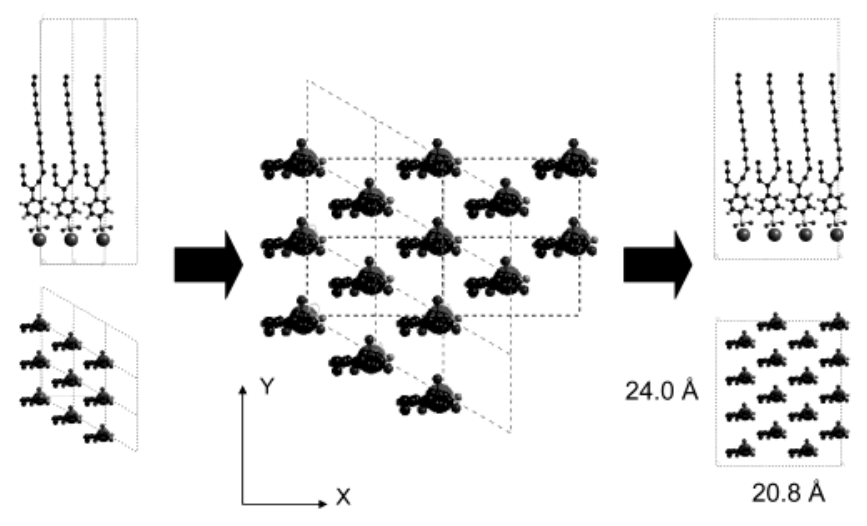

(b)

Figure 3. Simulated configuration of the decane-water interface in the presence of a surfactant monolayer (a) and the initial hexagonal packing of the surfactant monolayer (b).

fs. A Nose-Hoover-type thermostat ${ }^{76-78}$ with a relaxation time of 0.1 ps was used to control the temperature, and the pressure was controlled isotropically. ${ }^{79}$ The Lennard-Jones potential parameters for the van der Waals interaction of heterogeneous atomic pairs were calculated from the geometric mean of parameters of each atom. The particle-particle particle-mesh Ewald (PPPM) method $^{80}$ (accuracy criterion was set to $1.0 \times$ $10^{-5}$ and the near-field cutoff to $15.0 \AA$ ) was used for the longrange correction of electrostatic interactions.

\section{Results and Discussion}

3.1. Equilibrated System: Surfactant Conformation and Interface Formation Energy. Figure 3a illustrates a snapshot of the equilibrated structure of the decane-4-C16-water system after a 400 ps equilibration. The volumetric properties were converged from the equilibration since the volume fluctuation from a subsequent $2 \mathrm{~ns}$ NPT MD simulation for data collection is less than $1 \%$ of the average value. The structures of the other cases are quite similar, and the fluctuations of the cell in each dimension are summarized in Table 2. A point of interest is that surfactant 4-C16 has the smallest interfacial area $\left(L_{x} \times L_{y}\right)$, and this area increases when the benzene sulfonate is attached to a position farther from the 4th carbon, which correlates with the lowest IFT value for the decane-water interface using surfactant 4-C16.

To characterize the conformations of surfactants at the interface, we calculated the principal moments of inertia of each individual surfactant and the tilt angle between the largest principal moment and the interface normal vector. The results listed in Table 3 are averaged values from the equilibrated $2 \mathrm{~ns}$ MD trajectories. A common feature in all of the cases is that the surfactant molecules are packed like cylinders (having one long principal axis of moment of inertia, $I_{3}$, and two smaller, similar valued ones, $I_{1}$ and $I_{2}$ ) with a tilt angle ranging over $20-27^{\circ}$. It should be noted, however, that surfactant 4-C16 has the largest $I_{3} / I_{1,2}$ ratio and the smallest tilt angle, indicating that 4-C16 surfactants are aligned more vertically with the smaller molecular cross-sectional area at the interface, while the other surfactants have a 2-dimensionally dispersed conformation with a larger cross-sectional area. This is consistent with the results of the interfacial area in Table 2. From these results, we conclude that 4-C16 has more compact packing than the other cases have at the decane-water interface.

Next, to compare the energetic stability of each system, we calculated the interface formation energy (IFE) defined as follows:

$$
\mathrm{IFE}=\frac{E_{\text {total }}-\left(n E_{\text {surfactant,single }}+E_{\text {decane-water }}\right)}{n}
$$

where $E_{\text {total }}, E_{\text {surfactant,single }}$, and $E_{\text {decane-water }}$ denote the energies of whole system, the single surfactant molecule that is calculated from a separate $100 \mathrm{ps}$ MD simulation in vacuum at the same temperature, and a bare decane-water system, respectively. The variable $n$ is the number of surfactant molecules (32 in this study). The value of IFE is a measure of the average intermolecular interaction per surfactant molecule arising from the insertion of one surfactant molecule into the decane-water interface. The components necessary for this calculation and the results are summarized in Table 4. Although each surfactant has almost the same value for the single molecular energy ( $\left.E_{\text {surfactant,single }}\right), 4-\mathrm{C} 16$ has the lowest $E_{\text {total }}$ and thereby the lowest IFE, implying that the 4-C16-mediated interface is the most stable in terms of energetics. The results also show that molecular interactions between surfactants themselves and between surfactants and solvents are affected by the surfactant molecular architecture.

3.2. Density Profiles. Figure 4 shows the density profiles of each system along the $z$-axis direction of the simulation box, which were obtained by dividing the system into $1.5 \AA$ thick slabs parallel to the $x y$ plane. From the density profile, it is clear that the system consists of two phases (invariant density with $z$ ) with two well-defined interfaces (varying density with $z)$. It should be noted here that the densities of each phase in the decane-surfactant-water system $(0.723 \pm 0.005$ for decane and $0.994 \pm 0.005$ for water) agree well with those of the pure bulk phase $\left(0.725 \mathrm{~g} / \mathrm{cm}^{3}\right.$ for decane $\mathrm{e}^{66-69}$ and $0.997 \mathrm{~g} / \mathrm{cm}^{3}$ for water ${ }^{70-72}$ ). This shows that our simulation is sufficiently large for studying a realistic interface between two bulk phases. Another noteworthy point in Figure 4 is that most of the sodium ions stay between the water and the surfactant monolayer (within the average distance of $\sim 4.0 \AA$ from the sulfur atoms) during a 2 ns MD simulation. The binding of a counterion to an ionic surfactant at the interface has been well-characterized over a wide range of surfactant concentrations, especially above the CMC in experiment ${ }^{81}$ as well as theory. ${ }^{9}$ Thus, we believe that such a distribution of sodium ions in our simulation in the absence of a background salt concentration is in good agreement with the previous studies.

On the basis of this density profile, we calculated the interfacial thickness between decane and water. As shown in Figure 5, the density profile obtained from our simulation was fitted using the following hyperbolic tangent function that has 
TABLE 2: Equilibrated Cell Parameters of the Simulation Box ${ }^{a}$

\begin{tabular}{lccrc}
\hline \multicolumn{1}{c}{ system } & $L_{x}(\AA)$ & $L_{y}(\AA)$ & $L_{z}(\AA)$ & area/molecule $\left(\AA^{2}\right)$ \\
\hline decane-water & $28.90 \pm 0.08$ & $28.90 \pm 0.08$ & $77.27 \pm 0.22$ & \\
2-C16 & $21.96 \pm 0.05$ & $28.72 \pm 0.07$ & $133.55 \pm 0.31$ & $39.42 \pm 0.13$ \\
4-C16 & $21.86 \pm 0.06$ & $26.72 \pm 0.07$ & $143.95 \pm 0.18$ & $36.51 \pm 0.13$ \\
6-C16 & $21.93 \pm 0.05$ & $29.51 \pm 0.07$ & $131.10 \pm 0.31$ & $0.88 \pm 0.01$ \\
8-C16 & $26.91 \pm 0.05$ & $34.89 \pm 0.07$ & $90.05 \pm 0.18$ & $0.88 \pm 0.01$ \\
\end{tabular}

${ }^{a}$ During NPT MD simulation, the shape of the simulation box was retained as orthorhombic.

TABLE 3: Principal Axis Lengths of the Moment of Inertia for Surfactant Molecules and the Tilt Angle of the Longest Principal Axis to the Normal Vector to the Planar Interface

\begin{tabular}{ccccc}
\hline surfactant & $\begin{array}{c}I_{1,2}=\left(I_{1}+I_{2}\right) / 2 \\
(\AA)\end{array}$ & $\begin{array}{c}I_{3} \\
(\AA)\end{array}$ & $I_{3} / I_{1,2}$ & $\begin{array}{c}\text { tilt angle } \\
(\mathrm{deg})\end{array}$ \\
\hline 2-C16 & $1.61 \pm 0.60$ & $5.50 \pm 0.56$ & $3.42 \pm 1.32$ & $27.02 \pm 11.78$ \\
4-C16 & $1.27 \pm 0.53$ & $5.90 \pm 0.54$ & $4.64 \pm 1.96$ & $20.16 \pm 9.45$ \\
6-C16 & $1.55 \pm 0.78$ & $4.99 \pm 0.46$ & $3.23 \pm 1.66$ & $20.20 \pm 9.92$ \\
8-C16 & $2.04 \pm 1.21$ & $3.93 \pm 0.52$ & $1.93 \pm 1.18$ & $26.31 \pm 11.93$
\end{tabular}

TABLE 4: Interface Formation Energy

\begin{tabular}{lccc}
\hline \multicolumn{1}{c}{ system } & $\begin{array}{c}E_{\text {total }} \\
(\mathrm{kcal} / \mathrm{mol})\end{array}$ & $\begin{array}{c}E_{\text {surfactant,single }} \\
(\mathrm{kcal} / \mathrm{mol})\end{array}$ & $\begin{array}{c}\text { IFE } \\
(\mathrm{kcal} / \mathrm{mol})\end{array}$ \\
\hline 2-C16 & $-13341.01 \pm 66.04$ & $-92.66 \pm 4.25$ & $-73.909 \pm 0.004$ \\
4-C16 & $-13355.22 \pm 74.57$ & $-92.44 \pm 4.06$ & $-74.573 \pm 0.004$ \\
6-C16 & $-13338.12 \pm 64.25$ & $-93.09 \pm 4.42$ & $-73.388 \pm 0.004$ \\
8-C16 & $-13045.97 \pm 70.53$ & $-92.61 \pm 4.23$ & $-64.739 \pm 0.003$ \\
Edecane-water & & $-8010.81 \pm 62.37$ &
\end{tabular}

been used for the liquid-vapor interface..$^{51,82-85}$

$$
\rho_{\mathrm{i}}(z)=0.5 \rho_{\mathrm{i}, \text { bulk }}-0.5 \rho_{\mathrm{i}, \text { bulk }} \tanh \left(\frac{2\left(z-z_{0}\right)}{d}\right)
$$

where $\rho_{\mathrm{i}}$ is the density, $z_{0}$ is the position of the Gibbs dividing surface, and $d$ is the adjustable parameter related to the interfacial thickness. A common practice for defining the interfacial thickness for the liquid-vapor interface is the "1090 " criterion, ${ }^{51,82,84,85}$ which defines the interfacial thickness to be the distance between two positions where the density varies from 10 to $90 \%$ of the density of the bulk phase. However, defining the thickness for a complicated interface such as the oil-water interface in the presence of a surfactant is not a trivial matter. Although the density profiles of the oil-surfactantwater interfaces shown in Figure 5 suggest the consideration of two subinterfaces (one between water and the surfactant and the other between the surfactant and oil), the bulk density of the surfactant layer (normally monolayer) is not defined, so it is ambiguous to characterize these two subinterfaces. Thus, we suggest a "90-90" interfacial thickness $\left(t_{\text {total }}\right)$ criterion, which is the distance between two positions where the densities of decane and water are $90 \%$ of their own bulk densities. Figure 5 illustrates this idea. The interfacial thickness consists of the three components $t_{\text {oil }}, t_{\text {water }}$, and $t_{\text {surfactant }}: t_{\text {oil }}$ and $t_{\text {water }}$ are defined as the 10-90 thickness of the decane and water phases, respectively, and $t_{\text {surfactant }}$ is calculated as $t_{\text {surfactant }}=t_{\text {total }}-\left(t_{\text {oil }}\right.$ $\left.+t_{\text {water }}\right)$. Thus, the bare decane-water interface does not have $t_{\text {surfactant }}$.

The results of the interfacial thickness analysis are summarized in Table 5. For the bare decane-water interface (in the absence of a surfactant), we determined the interfacial thickness to be $3.90 \AA$, which is in good agreement with the measured thickness $(4.6 \pm 0.2 \AA)$ observed from the synchrotron $\mathrm{X}$-ray reflectivity experiment, ${ }^{86}$ the prediction from the capillarywave theory $(3.41 \AA),{ }^{86}$ and the other MD simulation results $(1.99 \AA) .{ }^{51}$ From the results in Table 5 as well as Figures 4 and 5 , it is clear that the interfaces of components $t_{\text {oil }}$ and $t_{\text {water }}$ are broadened with the addition of a surfactant because decane and water penetrate into hydrophobic alkyl tails and hydrophilic sulfonate groups, respectively. Interestingly, this interface broadening occurred mainly in the decane side and is strongly dependent on the attachment position of benzene sulfonate in the hexadecane backbone, whereas the water interface was insensitive to the variation of the attachment position. This is attributed to the fact that, while all of the surfactants have the same polar group (benzene sulfonate), the different attachment positions in the backbone give rise to the different effective alkyl tail lengths, which may affect the intermolecular interaction with decane molecules. It is important to note that the interfacial thickness (Table 5) does not vary monotonically as a function of the attachment position: surfactant 4-C16 results in the maximum broadening of the interface of the decane side $\left(t_{\mathrm{oil}}\right)$, implying that the alkyl tail part of surfactant 4-C16 has the best miscibility with decane among the other surfactants. Interestingly, it is observed that 4-C16 induces the thickest interface for both $t_{\text {total }}$ and $t_{\text {surfactant }}$. This can be explained by considering the packing and conformation of surfactant molecules at the interface as mentioned in section 3.1. In Table 2, it is shown that 4-C16 has the smallest interfacial area $\left(L_{x} \times L_{y}\right)$ and the most extended conformation, which clearly shows that it has the most compact packing at the interface.

On the basis of this definition of the interface and its thickness, the conformation of the alkyl tail of the surfactant as well as that of decane was characterized by investigating their torsion angles. In Figure 6, we present the ratio of trans to gauche for the torsion angle in the alkyl tail of the surfactant and decane. Note, surfactant 4-C16 has the largest trans/gauche ratio among the cases, indicating that the conformation of the alkyl tail of 4-C16 is more extended than that of the other surfactants. Again, this result is consistent with our previous analysis, concluding that 4-C16 has more compact packing with a small tilt angle and small cross-sectional area. In addition, it is clearly noticeable in Figure 6 that the value of the trans/ gauche ratio of the decane at the interface where the decane phase contacts the surfactant molecules is larger than that of decane belonging to its bulk phase. In particular, it is observed that 4-C16 has the largest ratio for the decane at the interface, and the trans/gauche ratio for the decane at the interface as a function of surfactant architecture is very similar to that for the alkyl tail of the surfactant. It is thought, therefore, that a larger trans/gauche ratio for the decane at the interface rather than at the bulk phase is induced by the conformation of an alkyl tail of the surfactant since the interface is a coexisting phase where the decane molecules are mixed with the surfactants. We infer that the largest ratio for the decane at the interface in the case of 4-C16 also indicates that the 4-C16 surfactant has better miscibility than the other surfactants.

3.3. Interfacial Tension. We calculated the interfacial tension $(\gamma)$ in our surfactant-mediated decane-water interface normal to the $z$-axis using its mechanical definition ${ }^{87,88}$

$$
\gamma=\frac{1}{2} \int_{0}^{L_{z}} \mathrm{~d} z\left[P_{\mathrm{N}}(z)-P_{\mathrm{T}}(z)\right]
$$




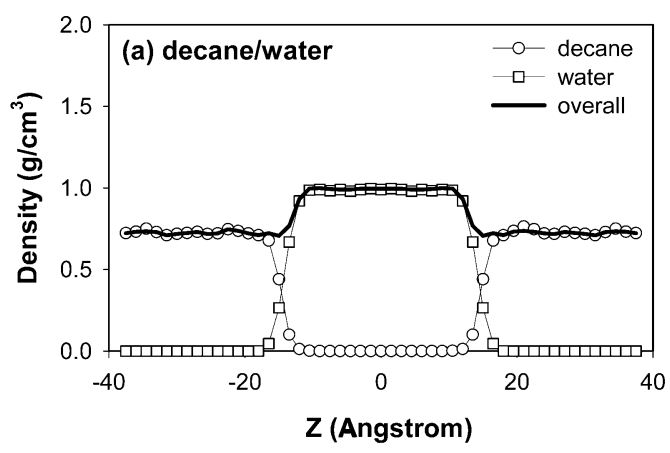

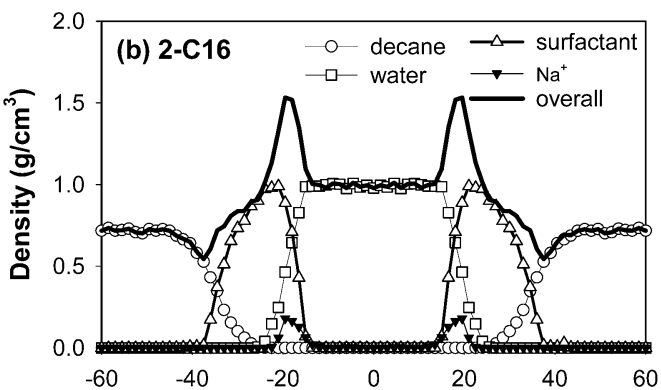

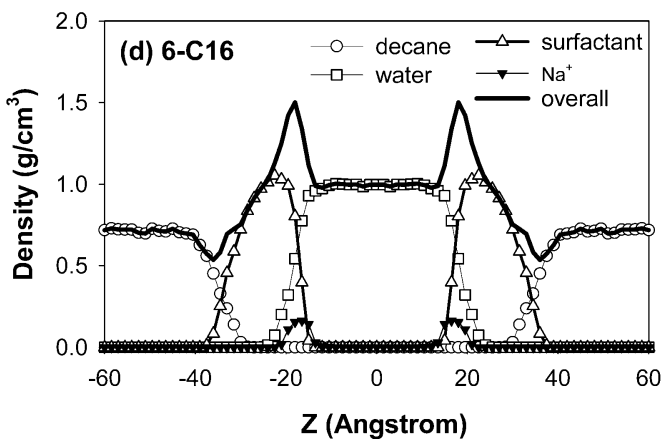

Figure 4. Density profiles along the $z$-axis direction.

where $P_{\mathrm{N}}$ and $P_{\mathrm{T}}$ are the normal and tangential components of the pressure with respect to the planar interface, respectively. $P_{\mathrm{N}}$ and $P_{\mathrm{T}}$ are the same in the bulk phase because the structure is isotropic in any direction, and they are different from each other only near the interface because the structure can be very anisotropic (e.g., Figures 3 and 4). $P_{\mathrm{N}}$ and $P_{\mathrm{T}}$ were calculated from each slab of the simulation box during the simulation as a time average by the Kirkwood-Buff theory ${ }^{89}$ that has been successfully used for liquid-vapor ${ }^{21,23-25,61,82-84,90}$ and liquidliquid $^{35,37,38,40,42,44,47,50,51}$ interfaces.

$$
\begin{gathered}
P_{\mathrm{N}}(z)=\rho(z) k_{\mathrm{B}} T-\frac{1}{V_{\mathrm{slab}}}\left(\sum_{\mathrm{i}, \mathrm{j}} \frac{z_{\mathrm{ij}}^{2}}{r_{\mathrm{ij}}} \frac{\mathrm{d} u\left(r_{\mathrm{ij}}\right)}{\mathrm{d} r_{\mathrm{ij}}}\right) \\
\left.P_{\mathrm{T}}(z)=\rho(z) k_{\mathrm{B}} T-\frac{1}{V_{\mathrm{slab}}} \mid \sum_{\mathrm{i}, \mathrm{j}} \frac{x_{\mathrm{ij}}^{2}+y_{\mathrm{ij}}^{2}}{2 r_{\mathrm{ij}}} \frac{\mathrm{d} u\left(r_{\mathrm{ij}}\right)}{\mathrm{d} r_{\mathrm{ij}}}\right)
\end{gathered}
$$

where $\rho(z)$ denotes the density of the slab at $z$ and $V_{\text {slab }}$ denotes the slab volume. $k_{\mathrm{B}}$ and $T$ are the Boltzmann constant and the absolute temperature, respectively. Angle brackets represent an ensemble average of all atoms located in the slab at $z . r_{\mathrm{ij}}, x_{\mathrm{ij}}$, $y_{\mathrm{ij}}$, and $z_{\mathrm{ij}}$ are the distances between the atoms and its components, and $u\left(r_{\mathrm{ij}}\right)$ is the potential energy of the atomic pair $\mathrm{i}$ and $\mathrm{j}$. If atom $\mathrm{i}$ belongs to the slab $z_{1}$, the virial contribution of $\mathrm{i}$ is added to the $P_{\mathrm{N}}\left(z_{1}\right)$ or $P_{\mathrm{T}}\left(z_{1}\right)$. Similarly, the virial
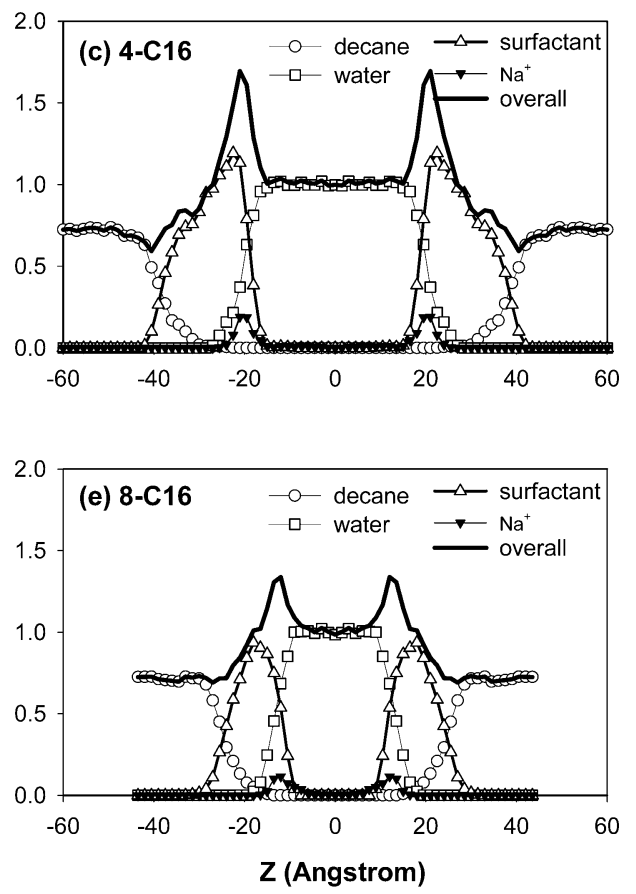

contribution of $\mathrm{j}$ is added to the virial sum of slab $z_{2}$ to which atom $\mathrm{j}$ belongs.

Figure 7 shows the equilibrated behavior of the interfacial tension as a function of simulation time for the typical case of surfactant 4-C16. The interfacial tension profiles for the systems studied here are shown in Figure 8. Compared to the interfacial tension profile of the decane-water interface (Figure $8 \mathrm{a}$ ) which shows a single peak, there are two distinct peaks at the interface (panels $\mathrm{b}-\mathrm{e}$ of Figure 8) with the presence of surfactant molecules. This indicates that two kinds of subinterfaces exist at the molecular level: one is between oil and the surfactant and the other is between the surfactant and water, as observed in the density profile analysis above. At positions far from the interface, the bulk phase has an interfacial tension value of zero on average. These two peaks are located within the 90-90 interface (between the dashed line and the solid line), determined from the density profile. Furthermore, surfactant 4-C16 has the greatest distance between these two peaks $(\sim 7.5 \AA)$ compared with the other cases $(\sim 4.5 \AA)$, which is similar to the feature of $t_{\text {surfactant }}$ (Table 5). From these results, we believe that the interfacial tension description of the interface with a surfactant is consistent with that of the 90-90 interface from the density profile.

By integrating these profiles, we obtained the interfacial tensions (Table 6). First, to validate our calculation of the interfacial tension, we simulated the water-vacuum and the decane-vacuum interfaces which consist of the same number 

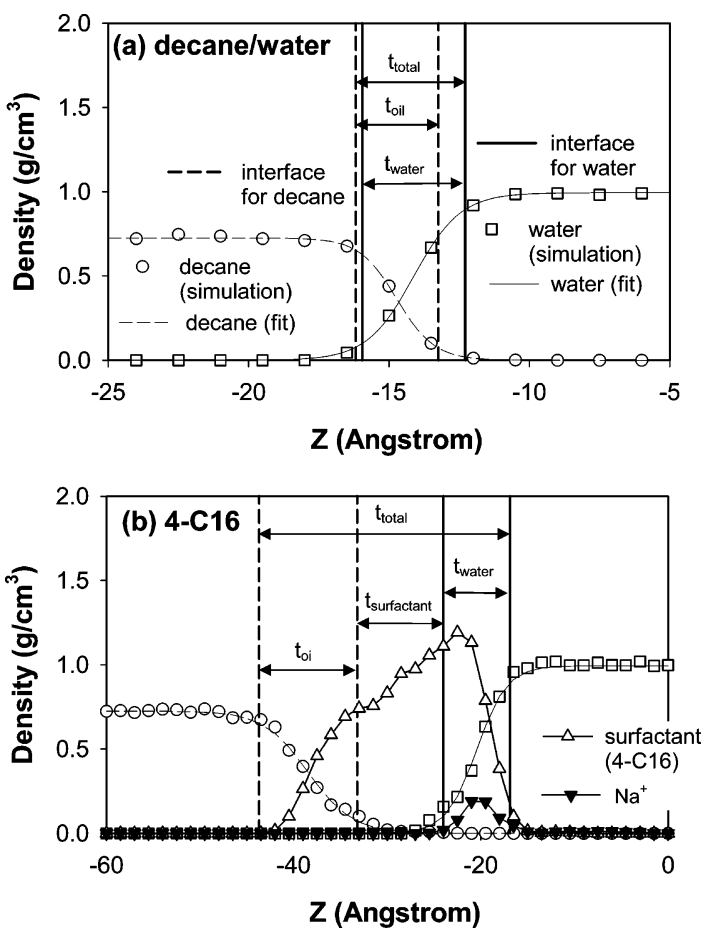

Figure 5. Definition of interfacial thickness.

TABLE 5: Interfacial Thickness

\begin{tabular}{|c|c|c|c|c|c|c|}
\hline system & & $z_{0}(\AA)$ & $d(\AA)$ & $t_{\mathrm{i}}(\AA)^{a}$ & $t_{\text {surfactant }}^{b}(\AA)$ & $t_{\text {total }}(\AA$ \\
\hline \multirow{2}{*}{ decane-water } & decane & \pm 14.71 & 1.34 & 2.94 & & \multirow{2}{*}{3.90} \\
\hline & water & \pm 14.12 & 1.67 & 3.67 & & \\
\hline \multirow{2}{*}{ 2-C16 } & decane & \pm 34.96 & 4.63 & 10.18 & \multirow{2}{*}{6.73} & \multirow{2}{*}{23.98} \\
\hline & water & \pm 19.23 & 3.22 & 7.07 & & \\
\hline \multirow{2}{*}{ 4-C16 } & decane & \pm 38.45 & 4.76 & 10.47 & \multirow{2}{*}{9.20} & \multirow{2}{*}{26.80} \\
\hline & water & \pm 20.44 & 3.25 & 7.13 & & \\
\hline \multirow{2}{*}{ 6-C16 } & decane & \pm 34.86 & 4.19 & 9.21 & \multirow{2}{*}{8.34} & \multirow{2}{*}{24.48} \\
\hline & water & \pm 18.45 & 3.15 & 6.93 & & \\
\hline \multirow[t]{2}{*}{$8-\mathrm{C} 16$} & decane & \pm 24.35 & 3.95 & 8.69 & \multirow{2}{*}{3.35} & \multirow{2}{*}{18.83} \\
\hline & water & \pm 13.26 & 3.09 & 6.79 & & \\
\hline
\end{tabular}

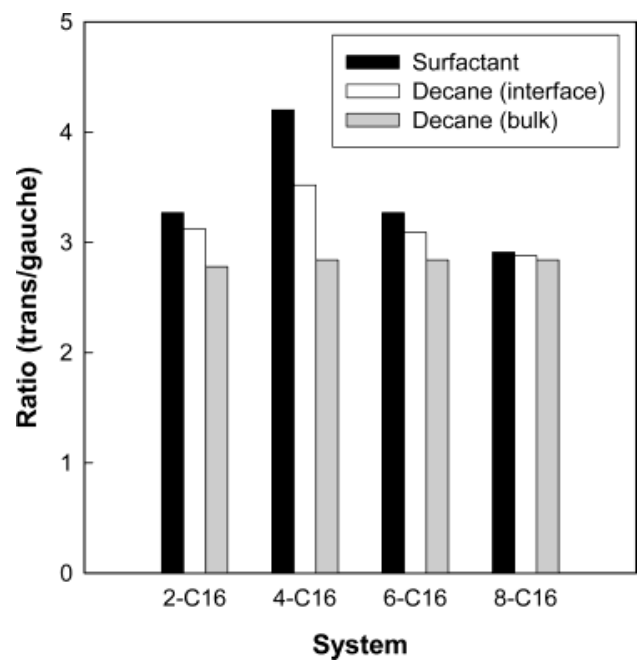

Figure 6. Trans/gauche ratio of the alkyl tail of a surfactant, decane at interface, and decane in bulk phase.

of water or decane molecules as used in each phase of the decane-water and decane-surfactant-water interfaces. The calculated interfacial tension at $300 \mathrm{~K}$ was $21.77 \pm 2.31 \mathrm{dyn} /$ $\mathrm{cm}$ for the decane-vacuum interface and $70.94 \pm 2.25 \mathrm{dyn} /$ $\mathrm{cm}$ for the water-vacuum interface, which agree very well with

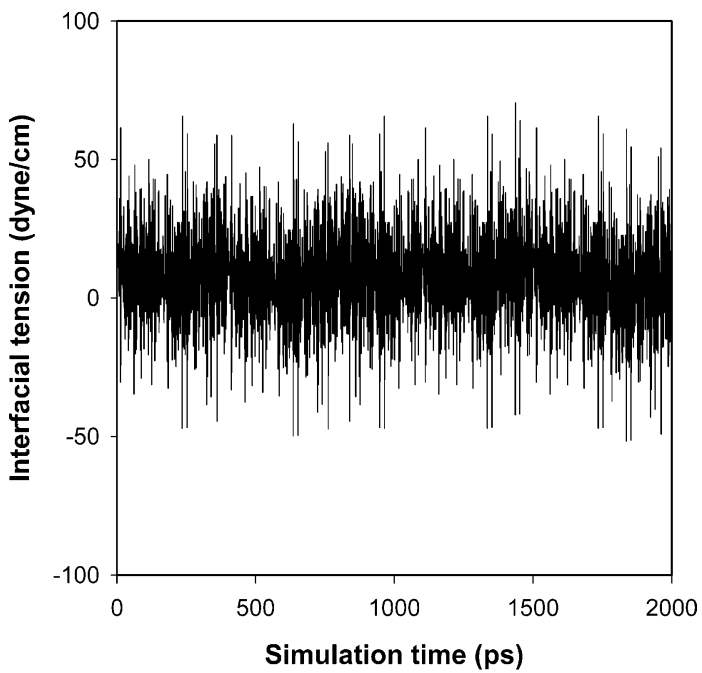

Figure 7. Typical equilibrated behavior of the interfacial tension as a function of the simulation time for the case of surfactant 4-C16.

the experimental values $\left(23.20 \mathrm{dyn} / \mathrm{cm}\right.$ for the decane $\mathrm{e}^{91}$ and $71.72 \mathrm{dyn} / \mathrm{cm}$ for the water ${ }^{72,92}$ ). In addition, the interfacial tension of the decane-water interface without a surfactant was $54.70 \pm 3.62 \mathrm{dyn} / \mathrm{cm}$, which is also in good agreement with the experimental value $(51.72 \mathrm{dyn} / \mathrm{cm}) .{ }^{93}$ Please note that these values were obtained without adjusting the given force fields (the united atom force field ${ }^{58-61}$ for decane and the $\mathrm{F} 3 \mathrm{C}$ force field $^{62}$ for water). We believe that our calculated results for the interfacial tension imply that the current force field provides acceptable accuracy for describing the interfacial systems in which we are interested. Figure 9 shows the comparison of the interfacial tension calculated from our simulations with the experimental values ${ }^{57}$ in which we found a qualitative agreement: the interfacial tension of the surfactant-mediated decanewater interface is changed as a function of the attachment position of the benzene sulfonate group, and the lowest interfacial tension is observed in the surfactant 4-C16 case. It should be addressed, however, that the interfacial tension reported from the experiment was measured in the presence of $2 \mathrm{wt} \%$ isopentanol as a cosurfactant and $0.003 \mathrm{~g} / \mathrm{cm}^{3}$ of $\mathrm{NaCl}$, which is generally known to have the effect of lowering the interfacial tension of the system dramatically. As the effect of other components such as cosurfactants and salt is out of the scope of this study, we focus on capturing the trend of the interfacial tension along the molecular architectural variation of the surfactant.

3.4. Effective Alkyl Tail Length. The consistent results from the analysis of the density profile and the interfacial tension profile indicate that the decane-water interface in the presence or absence of a surfactant was successfully described in our simulation. Nonetheless, one important question still remains to be answered: why does the 4-C16 surfactant result in the lowest interfacial tension among other surfactants? As a first step toward answering this question, we find that the 4-C16 surfactant induces the maximum interfacial thickness broadening compared to the other surfactants. This interesting feature is summarized in Figure 10, showing that there is strong correlation between the interfacial thickness and the interfacial tension. Since all the surfactants have the same architecture of benzene sulfonate as a polar pendant group, we may infer that the maximum broadening (between decane and the alkyl tail $\left(t_{\mathrm{oil}}\right)$ and, thereby, between decane and water $\left.\left(t_{\text {total }}\right)\right)$ induced by 4-C16 is a result of the better miscibility of the alkyl tail of 4-C16 with decane compared to other surfactants. In this situation, the 

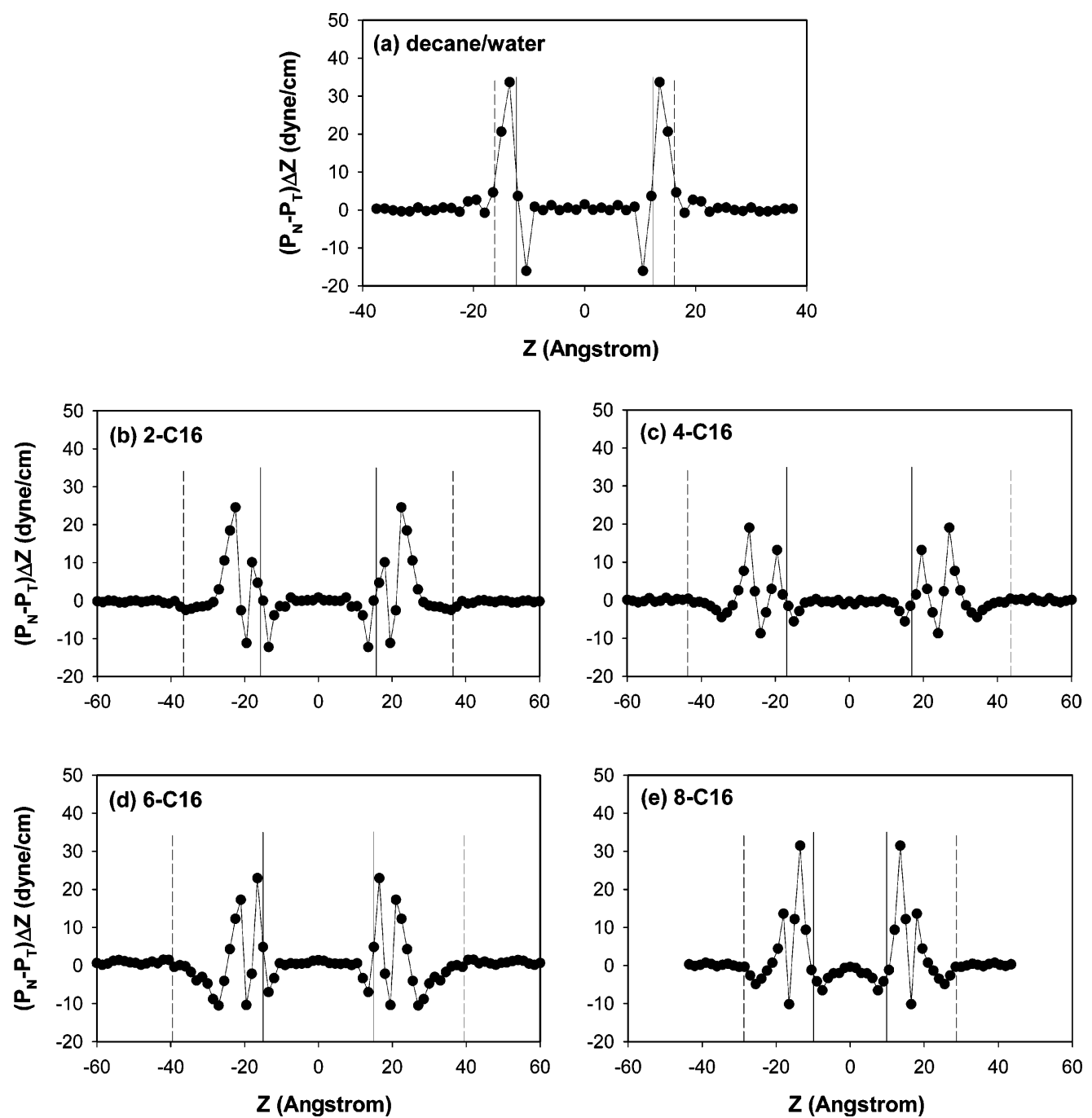

Figure 8. Interfacial tension profile along the $z$-axis direction. The dashed and solid lines indicate the $90-90$ interface defined by the two positions ranging from $90 \%$ of decane bulk density to $90 \%$ of water bulk density.

TABLE 6: Interfacial Tension

\begin{tabular}{lcc}
\hline \multirow{2}{*}{ system } & \multicolumn{2}{c}{ interfacial tension $(\mathrm{dyn} / \mathrm{cm})^{a}$} \\
\cline { 2 - 3 } & \multicolumn{1}{c}{ simulation } & experiments \\
\hline decane & $21.77 \pm 2.31$ & $23.20^{b}$ \\
water & $70.94 \pm 2.25$ & $71.71^{c}$ \\
decane-water & $54.70 \pm 3.62$ & $51.72^{d}$ \\
2-C16 & $23.19 \pm 4.94$ & \\
4-C16 & $8.02 \pm 4.12$ & \\
6-C16 & $18.12 \pm 4.39$ & \\
8-C16 & $30.21 \pm 4.41$ &
\end{tabular}

${ }^{a}$ The standard deviations were calculated from the average values of five 400 ps-long trajectories. ${ }^{b}$ From ref $91 .{ }^{c}$ From refs 72 and 92. ${ }^{d}$ From ref 93.

better miscibility of 4-C16 (with decane at the interface) does not seem to be explained only by the intermolecular interaction (since all of the surfactants have the same kind of alkyl moiety as the decane does), and the only difference among the surfactants, from 2-C16 to $8-\mathrm{C} 16$, is the attachment position of the benzene sulfonate group. Instead, the more plausible explanation is to consider the size similarity of surfactant with decane, which was inspired by the simple idea that decane has better miscibility with itself than it does with any other alkane homologues such as hexane, octane, and so forth.

For this purpose, first, we defined the alkyl tail length as the distance between the backbone ends and the carbon on which

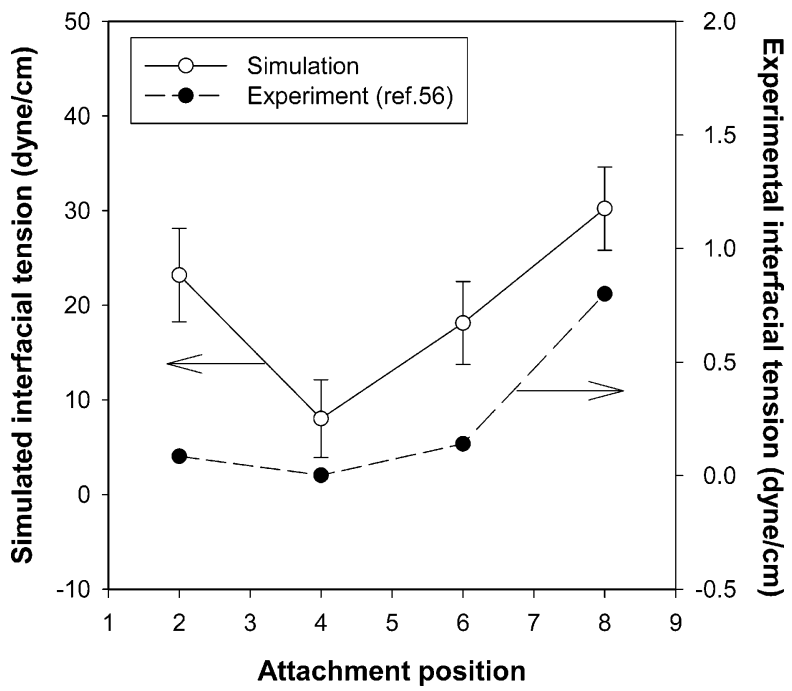

Figure 9. Comparison of interfacial tension between our simulation and the experiment (ref 56).

the benzene sulfonate group is attached. For each surfactant molecule, there are two tail lengths in one surfactant molecule: one is $r_{\text {long }}$, and the other is $r_{\text {short }}$, as shown in Figure 11 . The different attachment position gives rise to the different alkyl tail lengths (e.g., 2-C16 has two asymmetric tails, whereas 8-C16 

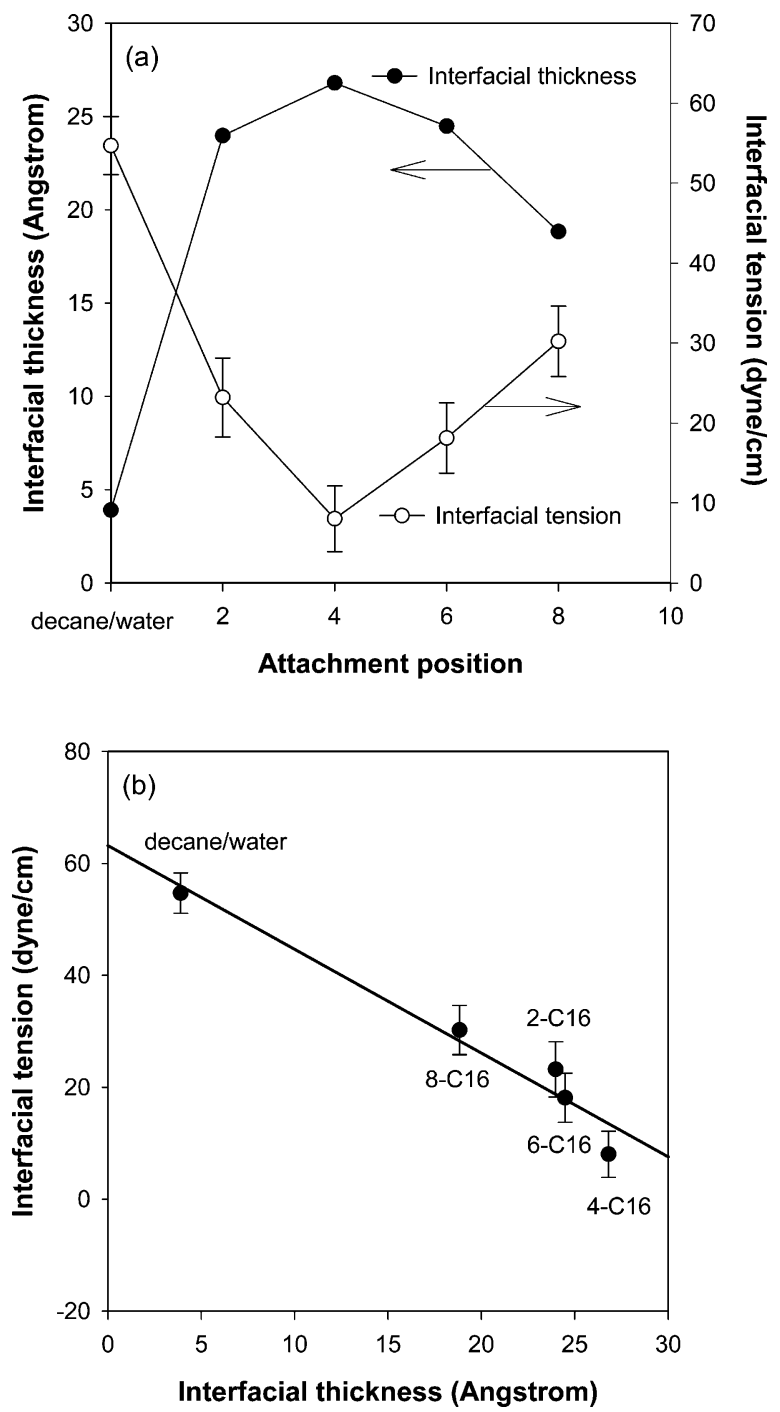

Figure 10. Relationship between the interfacial thickness and the interfacial tension. The solid line in (b) is the least-squares fit of the given results.

has almost symmetric ones). Next, we define the "effective" length (Figure 11) to be the difference between $r_{\text {long }}$ and $r_{\text {short }}$. This is because the effective tail length of the surfactant which contacts the decane molecules may not be necessarily the same as $r_{\text {long }}$ or $r_{\text {short }}$. In other words, in the surfactant layer at the interface, a certain space of longer alkyl tails close to the attachment point $\left(C_{\text {attach }}\right.$ in Figure 11$)$ may not be accessible due to the steric hindrance from the shorter tail. Therefore, the effective tail length $\left(r_{\text {effective }}\right)$ is closer to the true length of the alkyl tail that is accessible for decane (solvent) although its degree of freedom is not completely the same as the free alkane molecule.

Table 7 summarizes the statistical values of the alkyl tail lengths. It should be mentioned that for the case of 8-C16 which has nearly symmetric tail lengths, $r_{\text {long }}$ and $r_{\text {short }}$ are suitable for comparison with other cases because the two tails have similar length and neither of them has a dominant role for mixing with decane. Remarkably, $r_{\text {effective }}$ of surfactant 4-C16 is almost the same as the end-to-end length of decane, and all of the other lengths are farther from the length of decane. We believe that this argument rationalizes the reason that surfactant 4-C16 has the lowest interfacial tension and the largest interfacial thickness. This argument seems to be related to the

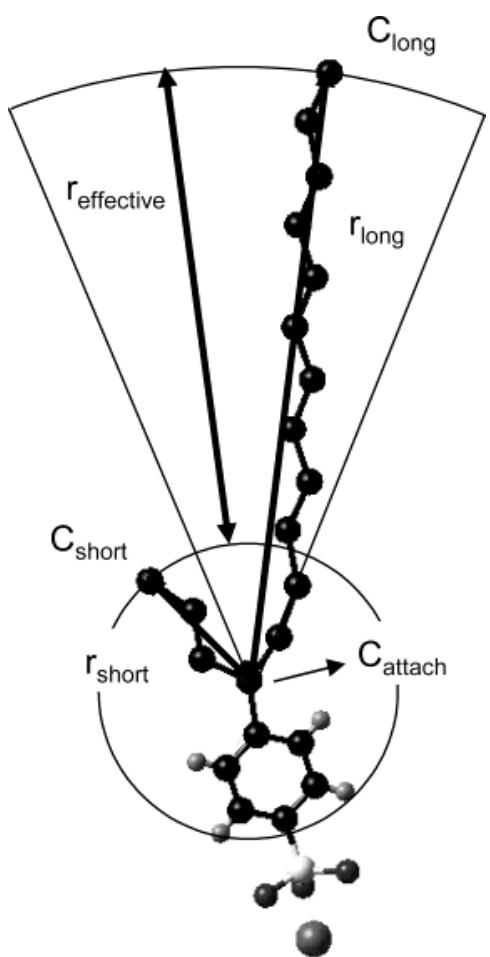

Figure 11. Effective length of the alkyl tail ( $\left.r_{\text {effective }}\right)$ of the surfactant that is defined as the difference between the average length of the long tail ( $\left.r_{\text {long }}\right)$ and that of the short tail $\left(r_{\text {short }}\right)$.

TABLE 7: Alkyl Tail Length of the Surfactant

\begin{tabular}{lrcc}
\hline \multicolumn{2}{l}{ system } & \multicolumn{2}{c}{ end-to-end length $(\AA)$} \\
\hline \multicolumn{2}{l}{ decane } & \multicolumn{2}{c}{$9.97 \pm 1.03$} \\
\hline & \multicolumn{1}{c}{$r_{\text {long }}(\AA)$} & $r_{\text {short }}(\AA)$ & $r_{\text {effective }}\left(=r_{\text {long }}-r_{\text {short }}\right)(\AA)$ \\
\hline $2-C 16$ & $14.79 \pm 1.43$ & $1.54 \pm 0.03$ & $13.25 \pm 1.43$ \\
$4-C 16$ & $13.37 \pm 1.35$ & $3.84 \pm 0.19$ & $9.53 \pm 1.36$ \\
$6-C 16$ & $10.92 \pm 0.96$ & $5.75 \pm 0.46$ & $5.17 \pm 1.07$ \\
$8-C 16$ & $8.97 \pm 0.76$ & $8.01 \pm 0.60$ & $0.96 \pm 0.97$
\end{tabular}

influence of the alkyl tail length on the mixing entropy at the interface, and we leave its quantitative assessment for future study.

\section{Summary}

Using MD simulations, we studied the effect of molecular architecture of a surfactant at the decane-water interface as a function of the attachment position of benzene sulfonate on the hexadecane backbone. For this purpose, we prepared the equilibrated model systems which consist of decane, water bulk phases, plus a surfactant layer at the interface.

The system with surfactant 4-C16 is found to have the smallest interfacial area $\left(L_{x} \times L_{y}\right)$ compared with the other surfactants, and the equilibrated molecular conformation of 4-C16 was aligned more vertically with the largest ratio of principal axis length of moments of inertia $\left(I_{3} / I_{1,2}\right)$. These results show that 4-C16 has more compact packing at the decanewater interface than do the other cases. The interface formation energy was the lowest for the 4-C16-mediated interface.

The density profiles show that the decane and water bulk phases have their own bulk density, indicating that the system size is fairly large enough to describe the interface between bulk phases. Using the 90-90 interface, the interfacial thickness of the bare decane-water interface is found to be in good agreement with experimental observation. It is also observed that the interface thickness of decane $\left(t_{\mathrm{oil}}\right)$ varies as a function 
of the attachment position of benzene sulfonate, while the water interface was not affected by such structural variation. From such thickness analysis, we found that surfactant 4-C16 results in the maximum interfacial broadening effect among the surfactants, and the interfacial thickness decreases as the attachment position is located farther from the fourth carbon. The reason might be that the alkyl tail of 4-C16 has better miscibility with decane than the other surfactants since the interfacial thickness is broadened as the miscibility increases.

The interfacial tension profile was calculated along the $z$-axis direction (perpendicular to the interface) using the KirkwoodBuff theory. In each bulk phase for decane and water, the interfacial tension profile showed a value of zero, indicating that the pressure difference $\left(P_{\mathrm{N}}-P_{\mathrm{T}}\right)$ exists only at the interface because of the structural anisotropy. On the contrary, where the decane-water interface has a single peak, the surfactantmediated interface has double peaks, which means that the actual interface consists of two subinterfaces at the molecular level: one for the decane-surfactant and the other for the surfactantwater. The values of interfacial tension were calculated by integrating the profiles along the $z$-axis direction. Through the comparison among the surfactants, we found that surfactant 4-C16 induces the lowest interfacial tension, and the IFT increases as the attachment position is located farther from the fourth carbon. Therefore, there is a correlation between the interfacial thickness and the interfacial tension: the interfacial tension decreases as the interfacial thickness (or the miscibility) increases.

To rationalize the difference in the miscibility of $m$-C16 with decane in terms of the size similarity, we introduced the effective length of the alkyl tail of each surfactant as the difference between the average length of the long tail and of the short tail and compared with the end-to-end length of decane. The effective length of the 4-C16 alkyl tail $(9.53 \pm 1.36 \AA)$ was remarkably closer to that of decane $(9.97 \pm 1.03 \AA)$ than to those of the other surfactants, which is consistent with the results from the density profile and the IFT profile.

Acknowledgment. This research was supported in part by the Department of Energy (DE-FC26-01BC15362, Paul West). The facilities of the Materials and Process Simulation Center used for these studies are supported by DURIP-ARO, DURIPONR, IBM-SUR, and NSF (MRI), and other support for the MSC comes from MURI-ARO, MURI-ONR, DOE, ONR, NSFCSEM, NIH, General Motors, ChevronTexaco, Seiko-Epson, Beckman Institute, and Asahi Kasei.

\section{References and Notes} 1946.

(1) Myers, D. Surfactant Science and Technology; VCH: New York,

(2) Dickinson, E.; Euston, S. R.; Woskett, C. M. Prog. Colloid Polym. Sci. 1990, 82,65 .

(3) Interfacial Phenomena in Petroleum Recovery; Morrow, N. M., Ed.; Dekker: New York, 1991.

(4) Liquid-Liquid Interface: Theory and Methods; Volkov, A. G., Deamer, D. W., Eds.; CRC: Boca Raton, FL, 1996.

(5) Rosen, M. J.; Murphy, D. S. Langmuir 1991, 7, 2630.

(6) Liggieri, L.; Ravera, F.; Passerone, A. J. Colloid Interface Sci. 1995, $169,238$.

(7) Ferrari, M.; Liggieri, L.; Ravera, F.; Amodio, C.; Miller, R. J. Colloid Interface Sci. 1997, 186, 40.

(8) Hansen, F. K.; Fagerheim, H. Colloids Surf., A 1998, 137, 217.

(9) Kalinin, V. V.; Radke, C. J. Colloids Surf., A 1996, 114, 337.

(10) Vaughn, M. W.; Slattery, J. C. J. Colloid Interface Sci. 1997, 195,

(11) Fainerman, V. B.; Miller, R.; Wuestneck, R.; Makievski, A. V. J. Phys. Chem. 1996, 100, 7669.

(12) Fainerman, V. B.; Miller, R.; Wuestneck, R. J. Phys. Chem. B 1997, 101,6479 .
(13) Fainerman, V. B.; Zholob, S. A.; Miller, R. Langmuir 1997, 13, 283.

(14) Fainerman, V. B.; Makievski, A. V.; Miller, R. Rev. Chem. Eng. 1998, 14, 373.

(15) Ravera, F.; Ferrari, M.; Liggieri, L.; Miller, R.; Passerone, A. Langmuir 1997, 13, 4817.

(16) Ravera, F.; Ferrari, M.; Liggieri, L. Adv. Colloid Interface Sci. 2000 88,129 .

(17) Mulqueen, M.; Blankschtein, D. Langmuir 1999, 15, 8832.

(18) Mulqueen, M.; Blankschtein, D. Langmuir 2000, 16, 7640.

(19) Mulqueen, M.; Blankschtein, D. Langmuir 2002, 18, 365.

(20) Nikas, Y. J.; Puvvada, S.; Blankschtein, D. Langmuir 1992, 8, 2680.

(21) Freeman, K.-S. C.; McDonald, I. R. Mol. Phys. 1973, 26, 529.

(22) Liu, K. S. J. Chem. Phys. 1974, 60, 4226.

(23) Rao, M.; Levesque, D. J. Chem. Phys. 1976, 65, 3233.

(24) Miyazaki, J.; Barker, J. A.; Pound, G. M. J. Chem. Phys. 1976, 64, 3364 .

(25) Rao, M.; Berne, B. J. Mol. Phys. 1979, 37, 455.

(26) Croxton, C. A. Statistical Mechanics of the Liquid Surface;

Wiley: New York, 1980.

(27) Lee, C. Y.; Scott, H. L. J. Chem. Phys. 1980, 73, 4591.

(28) Townsend, R. M.; Gryko, J.; Rice, S. A. J. Chem. Phys. 1985, 82, 4391.

(29) Wilson, M. A.; Pohorille, A.; Pratt, L. R. J. Phys. Chem. 1987, 91, 4873

(30) Brodskaya, E. N.; Rusanov, A. I. Mol. Phys. 1987, 62, 251.

(31) Matsumoto, M.; Kataoka, J. J. Chem. Phys. 1988, 88, 3233.

(32) Benjamin, I. J. Chem. Phys. 1991, 94, 662.

(33) Pohorille, A.; Benjamin, I. J. Chem. Phys. 1991, 94, 5599.

(34) Harris, J. G. J. Phys. Chem. 1992, 96, 5077.

(35) Linse, P. J. Chem. Phys. 1987, 86, 4177.

(36) Gao, J.; Jorgensen, W. L. J. Phys. Chem. 1988, 92, 5813.

(37) Smit, B. Phys. Rev. A 1988, 37, 3431.

(38) Meyer, M.; Mareschal, M.; Hayoun, M. J. Chem. Phys. 1988, 89, 1067.

(39) Carpenter, I. L.; Hehre, W. J. J. Phys. Chem. 1990, 94, 531.

(40) Smit, B.; Hilbers, P.-A. J.; Esselink, K.; Rupert, L.-A. M.; van Os,

N. M.; Schlijper, A. G. J. Phys. Chem. 1991, 95, 6361.

(41) Benjamin, I. J. Chem. Phys. 1992, 97, 1432.

(42) van Buuren, A. R.; Marrink, S.-J.; Berendsen, H.-J. C. J. Phys. Chem. 1993, 97, 9206.

(43) Esselink, K.; Hilbers, P.-A. J.; van Os, N. M.; Smit, B.; Karaborni, S. Colloids Surf., A 1994, 91, 155.

(44) Zhang, Y.; Feller, S. E.; Brooks, B. R.; Pastor, R. W. J. Chem. Phys. 1995, 103, 10252.

(45) Michael, D.; Benjamin, I. J. Phys. Chem. 1995, 99, 1530.

(46) Benjamin, I. Annu. Rev. Phys. Chem. 1997, 48, 407.

(47) Panhuis, M.-I. H.; Karaborni, S. Philos. Mag. B 1999, 79, 9.

(48) Diaz-Herrera, E.; Alejandre, J.; Ramirez-Santiago, G.; Forstmann, F. J. Chem. Phys. 1999, 110, 8084.

(49) Kuhn, H.; Rehage, H. Colloid Polym. Sci. 2000, 278, 114.

(50) Nicolas, J. P. Biophys. J. 2003, 85, 1377.

(51) Rivera, J. L.; McCabe, C.; Cummings, P. T. Phys. Rev. E 2003, 67, 011603

(52) Karaborni, S.; Esselink, K.; Hilbers, P.-A. J.; Smit, B.; Karthauser, J.; van Os, N. M.; Zana, R. Science 1994, 266, 254.

(53) Maiti, P. K.; Chowdhury, D. Europhys. Lett. 1998, 41, 183.

(54) Maiti, P. K.; Chowdhury, D. J. Chem. Phys. 1998, 109, 5126.

(55) Maiti, P. K.; Kremer, K.; Flimm, O.; Chowdhury, D.; Stauffer, D. Langmuir 2000, 16, 3784.

(56) Maiti, P. K.; Lansac, Y.; Glaser, M. A.; Clark, N. A.; Rouault, Y. Langmuir 2002, 18, 1908.

(57) Doe, P. H.; Wade, W. H.; Schechter, R. S. J. Colloid Interface Sci. 1977, 59, 525 .

(58) Siepmann, J. I.; Karaborni, S.; Smit, B. Nature 1993, 365, 330.

(59) Smit, B.; Karaborni, S.; Siepmann, J. I. J. Chem. Phys. 1995, 102, 2126.

(60) Martin, M. G.; Siepmann, J. I. J. Am. Chem. Soc. 1997, 119, 8921.

(61) Nicolas, J. P.; Smit, B. Mol. Phys. 2002, 100, 2471.

(62) Levitt, M.; Hirshberg, M.; Sharon, R.; Laidig, K. E.; Daggett, V.

J. Phys. Chem. B 1997, 101, 5051.

(63) Jang, S. S.; Cagin, T.; Goddard, W. A., III J. Chem. Phys. 2003, $119,1843$.

(64) Jang, S. S.; Molinero, V.; Cagin, T.; Goddard, W. A., III J. Phys. Chem. B 2004, 108, 3149.

(65) Mayo, S. L.; Olafson, B. D.; Goddard, W. A., III J. Phys. Chem. 1990, 94, 8897 .

(66) Calvo, E.; Brocos, P.; Bravo, R.; Pintos, M.; Amigo, A.; Roux, A. H.; Roux-Desgranges, G. J. Chem. Eng. Data 1998, 43, 105.

(67) Sastry, N. V.; Valand, M. K. J. Chem. Thermodyn. 1998, 30, 929.

(68) Trenzado, J. L.; Matos, J. S.; Segade, L.; Carballo, E. J. Chem.

Eng. Data 2001, 46, 974. 
(69) Bjola, B. S.; Siddiqi, M. A.; Fornefeld-Schwarz, U.; Svejda, P. J. Chem. Eng. Data 2002, 47, 250.

(70) Kell, G. S.; Haar, L.; Gallagher, J. S. NBS/NRC Steam Tables; Hemisphere Publishing Corp.: Washington, DC, 1984.

(71) Pai, Y. H.; Chen, L. J. J. Chem. Eng. Data 1998, 43, 665.

(72) Tsierkezos, N. G.; Molinou, I. E. J. Chem. Eng. Data 1998, 43, 989.

(73) Plimpton, S. J. J. Comput. Phys. 1995, 117, 1.

(74) Plimpton, S. J.; Pollock, R.; Stevens, M. The Eighth SIAM Conference on Parallel Processing for Scientific Computing; Minneapolis, MN, 1997.

(75) Verlet, L. Phys. Rev. 1967, 159, 98.

(76) Nose, S.; Klein, M. L. J. Chem. Phys. 1983, 78, 6928-6939.

(77) Nose, S. J. Chem. Phys. 1984, 81, 511-519.

(78) Hoover, W. G. Phys. Rev. A 1985, 31, 1695.

(79) Melchionna, S.; Ciccotti, G.; Holian, B. L. Mol. Phys. 1993, 78, 533.

(80) Hockney, R. W.; Eastwood, J. W. Computer Simulation using Particles; McGraw-Hill: New York, 1981.

(81) Yoshida, T.; Taga, K.; Okabayashi, H.; Matsushita, K.; Kamaya, H.; Ueda, I. J. Colloid Interface Sci. 1986, 109, 336.
(82) Chapela, G. A.; Saville, G.; Thompson, S. M.; Rowlinson, J. S. J Chem. Soc., Faraday Trans. 2 1977, 73, 1133-1144.

(83) Walton, J. P. R. B.; Tildesley, D. J.; Rowlinson, J. S.; Henderson, J. R. Mol. Phys. 1983, 48, 1357.

(84) Alejandre, J.; Tildesley, D. J.; Chapela, G. A. J. Chem. Phys. 1995, $102,4574$.

(85) Rivera, J. L.; Predota, M.; Chialvo, A. A.; Cummings, P. T. Chem Phys. Lett. 2002, 357, 189

(86) Mitrinovic, D. M.; Tikhonov, A. M.; Li, M.; Huang, Z.; Schlossman, M. L. Phys. Rev. Lett. 2000, 85, 582.

(87) Ono, S.; Kondo, S. In Encyclopedia of Physics; Flugge, S., Ed.; Springer: Berlin, 1960; Vol. 10

(88) Hill, T. L. Introduction to Statistical Mechanics; Dover: New York, 1986.

(89) Kirkwood, J. G.; Buff, F. P. J. Chem. Phys. 1949, 17, 338

(90) Chen, L.J. J. Chem. Phys. 1995, 103, 10214

(91) Jasper, J. J. J. Phys. Chem. Ref. Data 1972, 1, 841.

(92) Alvarez, E.; Rendo, R.; Sanjurjo, B.; Sanchez-Vilas, M.; Navaza, J. M. J. Chem. Eng. Data 1998, 43, 1027-1029.

(93) Zeppieri, S.; Rodriguez, J.; de Ramos, A.-L. L. J. Chem. Eng. Data 2001, 46, 1086 
C A L I F O R N I A I N S T I T U T E O F T E C H N O L O G Y
DOE CONTRACT DE-FC26-01B15362 DR. WILLIAM GODDARD III, PRINCIPAL IN VESTIGATOR, C A L T E C H

\section{LOWER COST METHODS FOR IMPROVED OIL RECOVERY (IOR) VIA SURFACTANT FLOODING}

DOE Project: DE-FC26-01BC15362

\section{ATTACHMENT 2 -- FOR FINAL PROJECT REPORT LITERATURE REVIEW}

(Originally Issued as a Supplement to $2^{\text {nd }}$ Annual Report)

PI: William A. Goddard III Co-PI: Yongchun Tang

Senior Staff: Patrick Shuler, Mario Blanco

Postdoctoral Scholars: Seung Soon Jang, Shiang-Tai Lin, Prabal Maiti,

Yongfu Wu, Stefan Iglauer

- Sections written by Mario Blanco, Seung Soon Jang, Shiang-Tai Lin, and Patrick Shuler.

- Originally issued September, 2003.

California Institute of Technology

September, 2004 


\section{DOE SURFACTANT PROJECT -- DE-FC26-01BC15362 \\ ATTACHMENT 2 -- LITERATURE REVIEW}

\section{OUTLINE}
A. Introduction to Literature Study
B. Background/Historical Overview of Surfactant EOR Processes
C. General Comments About Phase Behavior/IFT (Interfacial Tension)
D. General Comments About Surfactant Adsorption
E. Background on Different Types of Surfactant Candidates
F. Selected Literature Data Concerning Chemical Structure and IFT
G. Selected Literature Data and Trends About Chemical Structure and Solid Adsorption
H. References

APPENDIX A. FURTHER LITERATURE IFT DATA

APPENDIX B. DETAILS OF ASSEMBLED LITERATURE SURFACTANT

ADSORPTION DATA (done as a separate electronic file) 


\section{A. Introduction to Literature Study}

This study supported by the Department of Energy is an integrated theoretical and experimental program that seeks to identify chemicals to improve the economics of surfactant flooding enhanced oil recovery. The objective is to identify surfactant chemical formulations that have one or more of these desirable characteristics:

- Need only low concentration to produce ultra-low interfacial tension (IFT), and/or maintain low IFT over a wide span of surfactant concentrations and salinities

- Have lower adsorption to clay/mineral surfaces

- Cost less that conventional petroleum sulfonates and work as well

- Require little or no co-surfactants

- Are "robust" and maintain high efficiency if injected into multiple zones that vary a bit in temperature, oil properties, brine composition. etc.

We focus on the generation of low IFT and having low solid adsorption in these studies as the primary surfactant performance parameters of interest. The overall study approach is to use both literature and generated laboratory experimental IFT and solid adsorption data to provide test cases to tune and challenge the theoretical models being development. The literature review also serves as a guide for systems already investigated, and may suggest some surfactant types not yet considered actively for EOR, but perhaps have attributes that warrant further study for this application.

\section{B. Background/Historical Overview of Surfactant EOR Processes}

\section{Standard Micellar Flood Process}

"Traditional" surfactant flooding involves the injection of a chemical solution that has water, surfactant, co-surfactant, and salt. Some surfactant formulations include a polymer to build viscosity in the surfactant slug. Normally polymer is included at least as a tapered polymer solution pushing the surfactant slug in order to maintain favorable mobility control and hence achieve good sweep efficiency. One variation of this process was the so-called "Uniflood" process developed by Unocal in the 1960's and 70's added oil to the surfactant formulation so to create an oil-external microemulsion.

The most common practice in the late 1970's and early 1980's was to inject waterexternal micro-emulsions (no added oil). Results from several field tests have demonstrated that in fact the designed surfactant formulations could produce incremental oil beyond that from a mature waterflood (for example, Borah, 1988 and Cole 1988). This experience has validated laboratory experiments where waterflood residual oil is recovered in controlled conditions. The most common design for these micellar floods to use the so-called "salilnity gradient" approach in which the salinity of the surfactant slug is somewhat lower than that of the oil reservoir, and the salinity of the following polymer drive solution is tapered to be even less. The rationale for this design (discussed further in the next section with reference to phase behavior) is to guarantee that the injected 
surfactant solution is forced to go through its minimum IFT condition. The lower salinity in the polymer drive slugs aids in remobilizing any surfactant that may be trapped in the reservoir as a higher-salinity microemulsion. Care must be exercised in selecting the make-up brines for the various chemical solutions in order to maintain compatibility and not have any unintended side-effects. For example, if the salinity of the injected polymer solutions is very low, there is the risk of having the reservoir clays swell or migrate and cause some near wellbore plugging (Shuler et. al., 1987)

However, the overall economics of this process as first implemented in that time period was not sufficient to warrant wide implementation by the industry, even with crude oil process well over $\$ 20 /$ barrel. With the collapse of oil prices in 1986 , the interest in surfactant flooding (and most all EOR methods) greatly diminished for some time.

\section{More Advanced Variations on the Standard Micellar Flooding Process}

One interesting and a potentially significant variation in the "traditional" surfactant flooding design is to add alkali to the surfactant formulation. This process has been called ASP (Alkaline-Surfactant-Polymer) flooding, among other things, and came into prominence beginning in the early 1980's (Shuler, et al., 1989). Increasing the $\mathrm{pH}$ of the injected slug(s) can be beneficial towards increasing surfactant efficiency by 1) extracting acidic components (e.g. aliphatic or naphthenic acids) from the crude oil that then act as "natural" surfactants that supplement the injected surfactants, and/or 2) reducing the adsorption of the injected surfactant. The net effect, when properly designed, is to recover as much oil as with the traditional micellar process, but now with less surfactant required. Most commonly, for ASP projects having been implemented in recent field applications, the injected surfactant is a petroleum sulfonate or an alkyl aryl sulfonate surfactant (Meyers, 1992). As explained in more detail later, the increase in $\mathrm{pH}$ from adding an alkali creates a net negative charge on the clay surfaces thereby reducing the electrostatic attraction of the main anionic surfactant to be adsorbed onto the clays.

There are several possible disadvantages of operating with now a more complicated chemical system because of the addition of an alkaline agent, including: 1) reduced thickening efficiency of (polyacrylamide) polymers, 2) requirement for make-up water to have essentially no hardness (expensive softening needed for injection water), 3) increased chance of inorganic scale deposition for the higher $\mathrm{pH}$ conditions, especially calcium carbonate scale, and 4) safety/environmental concerns for handling and applying the alkali chemical, especially if $\mathrm{NaOH}$ is selected.

Currently, and in the last few years, the most significant (largest volume) chemical flooding projects have been associated with countries outside of the U.S., namely China and Indonesia. Small chemical flooding field projects (predominantly ASP process) have been performed and continue to be applied in the Mid-Continent and Rocky Mountain areas of the U.S. and Canada (Pitts, 2001).

One other approach to reduce the cost of chemical EOR has been to consider dilute surfactant flooding. For example, a process developed for North Sea reservoirs has the 
design of using only low concentrations of surfactant and not include polymer for mobility control (Taugbol et. al., 1995, Austad, 1993). The philosophy is to have a design that will be much less expensive as compared to a conventional surfactant flood process, with the realization that the oil recovery efficiency also will be less. Skauge and Palgren (1989) discuss a series of different types of ethoxylated anionic surfactants that they claim would be suitable for formulation in sea water as a dilute surfactant solution and be suitable for enhanced oil recovery from typical North Sea reservoirs.

The more recent application of surfactants for remediation of oil-contaminated soils (removal of NAPL -- nonaqueous phase liquids) may offer some new ideas for EOR applications. Different surfactants have been studied and developed for this particular environmental application (SEAR - Surfactant Enhanced Aquifer Remediation). For example, Jayanti (2001) report research on developing branched alcohol propoxy sulfate surfactants to remove gasoline and diesel from contaminated groundwater.

Another approach investigated in recent years has been the development of anionic surfactant formulations that have included a small amount of cationic surfactant, not only for EOR (Wellington, 1993), but also to improve the performance of systems for groundwater clean up (Jayanti, 2001). The paper by Wellington reports significant oil recovery in laboratory tests with much less than the customary amounts of surfactant used in formulations developed up to the mid-1980's. Kalpakci (1990) is another author who claims obtaining very good efficiency in their surfactant flood designs. Ruan, et. al. (2002) discuss how low IFT may be achieved with a mixture of cationic and anionic s surfactants. They considered DoTEAB/SDS and OTEAB/SDS systems (showed the dodecyltriethylammonium bromide, octyltriethylenammonium bromide, and sodium dodecylsulfate, respectively). The behavior was explained in terms of the change of the mixed micelles composition and symmetry of hydrophobic chains of cationic and anionic surfactants.

In a similar vein, there is the suggestion for the addition of a so-called linker molecule to the surfactant formulation (Sabatini, et. al., 2003). Linker molecules are amphiplies that segregate near the microemulsion membrane near the surfactant tail (lipophilic linkers) or the surfactant head group (hydrophilic linkers). The concept of lipophilic linkers was first introduced by Graciaa et al. in 1993. They observed that isooctane microemulsions formulated with ethoxylated octyl phenols mixtures containing 1 or 2 ethoxy groups showed significantly higher oil solubilization capacity than those formulated with more homogeneous mixtures of octylphenols. They proposed that octylphenols with one or two ethoxy groups did not participate at the interface, but rather segregated near the oil/water interface and between the tails of the nonylpnenol surfactants with a higher number of ethoxy groups. The thought is that by these components "linking" the oil molecules and the surfactant tails, that this promotes better solubilization of the excess oil by the main surfactant. Benefits from this action are lower IFT and improved microemulsion fluidity.

In addition, Sabatini et. al. (2003) discuss hydrophilic linkers, especially for SEAR type applications. These linkers (for example shorter alkyl chain surfactants) are thought to 
coadsorb with the surfactant at the oil/water interface, thereby promoting the surfactantwater interaction, but have a poor interaction with the oil phase. The thought is that the hydrophilic linker may open "holes" in the interface (Acosta, 2002). They observed a synergistic effect when combining the lipophilic and hydrophilioc linkers, which further allows an increase in oil solubilization by the surfactant.

Yet another tact is to investigate more creative and more cost-effective ways to provide necessary mobility control for surfactant flooding. For example, the concept of using foam for mobility control is attractive. It is conceivable to have a surfactant blend that can provide both improved oil displacement and foam. More fundamental understanding of foam flow would be required to exploit this approach (Radke, 2001) to combine improved water-soluble polymers may become available as well. Yet another idea is to combine the function of a polymer with a surfactant. For example, Cao (2002) reports about polymeric surfactants based on carboxymethyl cellulose and alkyl poly(etheroxy) acrylate were synthesized by ultrasonic irradiation. They report that these solutions can achieve low IFT (approaching $0.001 \mathrm{dyne} / \mathrm{cm}$ ) if alkali is added, presumably mostly by the action versus the crude oil.

\section{General Comments About Phase Behavior/IFT (Interfacial Tension)}

The basics of surfactant phase behavior and IFT is well established, at least for “conventional” EOR surfactants (Morgan 1977). Previous studies (described below) establish several general trends.

(1). Pseudoternary Phase Diagrams (Nelson, 1978), Borrel and Schechter, 1988, Kahlweit, et. al., 1989) - Surfactant systems are conveniently displayed on ternary diagrams, with the three compositions considered as brine, oil, and surfactant. Following the nomenclature of Nelson, there may be a Type (II-), Type (III), or Type (II+) phase behavior. For Type (II-) systems the surfactant is predominantly in the water phase in equilibrium with oil (water external microemulsion), whereas in Type (II + ) systems, the surfactant partitions predominantly into the oil phase in equilibrium with the brine (oil external microemulsion). The Type (III) condition refers to adding a third, surfactant-rich middle phase that is in equilibrium with external water and oil phases.

The lowest overall IFT occurs usually in the Type (III) phase environment. Furthermore, to a first approximation, the minimum overall IFT will occur where there is an equal volume of brine and oil solubilized into this middle phase. This minimum IFT attained is expected to decrease with an increase in the ratio of the volume of solubilized water and oil to the volume of surfactant. That is, all things being equal, the more the uptake volume of water and oil into the middle-phase per volume of surfactant, the lower we expect the IFT. One model envisions the middle phase to consist of alternating layers of oil and water with surfactant at the interfaces (Huh 1979, 1983).

Huh offers a theoretical relationship between the solubilization parameter (SP) and the IFT for a middle-phase microemulsion. In particular, there is an expression at "optimal 
conditions" where the IFT is the same between the microemulsion and water phase, and the microemulsion and the oil phase.

$$
\mathrm{IFT}=\mathrm{a}_{\mathrm{H}} \cos (\pi / 4) /\left(\mathrm{SP}^{2}\right)
$$

where

$$
\begin{aligned}
& \mathrm{a}_{\mathrm{H}}=\mathrm{a} \text { constant } \\
& \mathrm{SP}^{*}=\text { volume of water (or oil)/volume of surfactant in middle phase }
\end{aligned}
$$

This condition of equal solubilization of oil and water, or minimum IFT is referred to as an "optimum" condition. For example, if salinity is a variable whose impact on phase behavior/IFT is being examined, when we reach this condition it is called the "optimal salinity" (Lake, 1984). Per above, the larger the SP (the more excess oil and water solubilized by the surfactant), the lower is the IFT.

(2). EACN (Equivalent Alkane Carbon Number, Cash 1976) - If one measures the IFT for a surfactant system (at fixed salinity and temperature) versus the chain length of a series of alkanes as the hydrocarbon phase, there is only one hydrocarbon that exhibits desirable ultra-low IFT (of the order of $0.001 \mathrm{dyne} / \mathrm{cm}$ ). The EACN principle is that molar mixtures of hydrocarbons that produce that same average chain length will also have this very low IFT behavior. The EACN concept is especially useful in surfactant flood design because most crude oils have a unique EACN. One way to use this knowledge, for example, is that one may then screen a number of surfactants using a simple n-alkane (or appropriate mixture) as a good proxy for that crude oil.

\section{General Comments About Surfactant Adsorption}

Surfactant retention in the reservoir is perhaps the most significant barrier to the commercial application of this IOR technique (Wu 1996, Taber 1996). The basic problem is that surfactants require good selectivity at oil-water interfaces to generate required IFT, but it is difficult to find the same molecules that then have poor affinity to move to fluid-solid interfaces. There is at least one encouraging field example of low surfactant adsorption; Reppert et. al. (1990) claim less than $0.08 \mathrm{mg} / \mathrm{g}$ surfactant retention in the second Ripley surfactant flood test.

Four important mechanisms for surfactant adsorption include (Lake, 1989 p. 400): (1). On metal oixide surfaces the surfactant monomer will physically adsorb through hydrogen bonding and ionically bond with cationic surface sites. At and above the CMC the supply of monomer becomes constant, as does the retention. This produces a Langmuir-type isotherm shape and should be reversible with surfactant concentration.

(2) In hard brines, the prevalence of divalent cations creates surfactant-divalent complexes. Some complexes such as calcium and sulfonate have a relatively low solubility in the brine and may cause surfactant retention via precipitation (Somasundran 1979). 
(3) At levels below those required for precipitation, the multivalent cations can complex with surfactant and become a monovalent aqueous complex cation. This monovalent surfactant complex can chemically exchange with cations already bound to the reservoir clays (Hill 1978).

(4). If the phase behavior becomes Type (II+), then the surfactant will partition preferentially into the oil phase. Because this is above optimal salinity, the IFT will be relatively high and this surfactant-rich oil phase can become trapped in the pore spaces. The "salinity gradient" design is the common approach to overcome this potential problem; the salinity of the chase polymer is low enough to shift the phase behavior to Type (II-) in the reservoir, thus preventing the phase trapping loss of surfactant.

Various factors such as $\mathrm{pH}$, temperature, brine composition, and the type of clay or mineral and surfactant structure all impact adsorption. Increasing $\mathrm{pH}$, for example, can change the surface charge from positive to a negative charge. Thus adding a base to a sulfonate (anionic) surfactant solution, for example, can be beneficial by reducing the adsorption due to electrostatic attraction (mechanism (1)) for sulfonate surfactants. Increasing salinity increases adsorption for all of these mechanisms.

Not surprisingly, the surfactant retention on mineral surfaces in sandstone reservoirs is roughly proportional to the weight fraction of clay. In addition, the difference in lab- and field-measured mineral retention is not significant (Goldburg 1983). Thus, there is the prospect that a molecular model (tuned with the right laboratory data) can provide a firstorder calculated estimate of retention for surfactant candidates for a specific field condition.

Section $\mathrm{G}$ has some further discussion about the factors that control surfactant adsorption in conjunction with some examples of literature data.

\section{E. Background on Diferent Types of Surfactant Candidates}

\section{Overview of Types of Surfactant Chemistries Evaluated for EOR}

A wide variety of surfactants already have been studied as prospects for surfactant flooding. Examples include petroleum sulfonates (Knaggs 1976), alpha-olefin sulfonates (Barakat 1982), dodecyl-o-xylene sulfonate (Austad 1996), diphenyl ether sulfonate (Mannhardt 1987), alkyl propoxy ethoxy glyceryl sulfonate (Michels 1996), alkyl ethoxylated sulfates and sulfonates (Bansal 1978), sulfonated fatty acid esters (Baviere 1991), alkyl aryl ether sulfate and alkyl aryl ether acetate (Andrews 1981), alkylpropoxy-ethoxy sulfate (Austad 1997), nonyl phenol carboxymethylates, and betain and sulfo-betaine. More recently researchers report propoxylated tridecyl alcohol sodium sulfates and guebert alcohol hydrophobes (Wu 1996).

Some further details about different classes of surfactant for improved oil recovery are discussed below. 


\section{Crude Oil and Petroleum Sulfonates}

The predominant type of surfactant investigated for EOR has been sulfonated hydrocarbons. "Crude oil sulfonates" refer to products created when a lighter fraction of a crude oil is sulfonated (minimal separation of the crude). The term "petroleum sulfonates" are sulfonates produced when an intermediate molecular-weight refinery stream is sulfonated, whereas "synthetic sulfonates" refers to products when a relatively pure organic compound is sulfonated. Crude oil and petroleum sulfonates have been used for low-salinity situations (less than say sea water salinity, $<3 \%$ salt). Of course a particular advantage of these rough cut petroleum-based feedstock materials are that they are lower cost versus using a custom-designed synthetic organic molecule. In addition, petroleum sulfonates certainly can attain low IFT versus common crude oils, and are reported to be chemically stable (Salter 1986). Disadvantages of using these "natural" feedstocks is that quality control becomes a major issue as the detailed composition of the crude oil or refinery cut may change with time. In most particular, for a large EOR project, it would be difficult to supply a large volume of consistent surfactant material.

Reasonable EOR candidates have some water solubility, which is imparted by the ionic sulfonate group. The hydrocarbon tail also affects the solubility. As a rough approximation, compounds with 16 or fewer carbons on the hydrocarbon chain are soluble, whereas those with even longer alkyl chains have marginal or no water solubility (Ottewill 1984). Of course the solubility depends not only upon the total salinity, but also its ionic composition. In particular, "hard water" (calcium and magnesium) creates oil soluble components.

The characteristics and structure of petroleum sulfonates suitable for EOR depends upon the chemical composition of the feedstock, degree of sulfonation, and the average number of sulfonate groups attached to each molecule. Example feedstocks present in refineries and then sulfonated to create EOR surfactants include white oil, fractionated gas oil, vacuum gas oil, and lube-oil extract. Typical petroleum sulfonates contain unreacted oil, the sodium salt of the sulfonated hydrocarbon (provided it is neutralized with sodium hydroxide), and sodium sulfate from the neutralization of the excess sulfuric acid used in the sulfonation process (Salter 1986).

Molecular weights of the feedstocks range from 350 to 450, but may vary from these values, with significant outliers on both ends. When the feedstock contain polyaromatics (more than one ring per molecule), these materials often are polysulfonated. The parameters of interest to characterize these materials include the equivalent weight (molecular weight divided by the number of sulfonate groups), and the percentage of the sulfonated material that is polysulfonate. As a rule of thumb, petroleum sulfonates with equivalent weights above 450 are not easily water soluble, whereas those smaller are water soluble (Gale and Sandvik 1973). 


\section{Alkyl Benzene Sulfonates (ABS)}

A step beyond the petroleum sulfonates is to use feedstocks that have perhaps a fairly narrow range of alkyl chain lengths and known structure. For example, consider the general structure for an alkyl benzene sulfonate surfactants shown below:

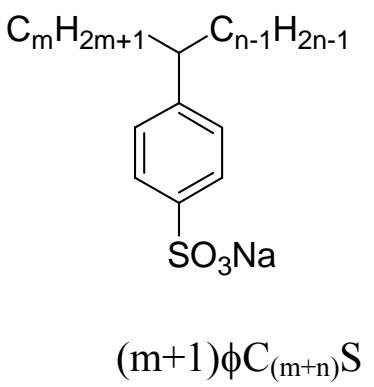

Other variations are to have another or even two R (alkyl) groups attached to the benzene ring; that is, so-called dialkyl or tri-alkyl benzene sulfonates.

There have been a number of studies performed with well-defined ABS materials (Doe 1977a, 1977b, 1977c, 1978a, 1978b). These results are discussed in more detail in the Section $\mathrm{F}$ that covers IFT literature data and trends. Some main points:

The equivalent weight found to be effective for the petroleum sulfonates is validated by the ABS materials. Typically, examples that create ultra-low IFT's have an alkyl chain length that totals between $\mathrm{C} 12$ and $\mathrm{C} 16$. Beyond that, it seems that the ABS surfactants with regards to IFT performance may be divided into 3 groups:

Group I - these are the preferred types of ABS surfactants for creating low IFT for most crude oils. This is because they can attain a minimum in IFT against hydrocarbons of size that typifies most crude oils (EACN between 6 and 10). The Group I surfactants are modified linear alkyl benzene sulfonates (LABS), having also a short (C3 or less) alkyl group ortho to the SO3 group, plus one or two other alkyl groups, the major one of which is meta to the sulfonate and has at least 12 carbon atoms.

Group II - These surfactants are matched best only with very short hydrocarbons, and hence not of much practical interest for crude oil EOR. In addition, the $\gamma_{\text {min }}$ values never achieve very low values. This group has modified LAB's where the group ortho to the sulfonate is $\mathrm{C} 4$ and/or the major chain meta to the SO3 is $\mathrm{C} 10$ or less.

Group III - Surfactants in this group can achieve acceptably low IFT values, but are suited best for hydrocarbons that have on average a larger EACN than found in most crude oils. LAB's in the molecular weight range from C11 up to C18 typify the surfactants in this group. 
More recently, Bolsman and Daane (1986) report a systematic study of the effect surfactant structure for a series of alkylxylenesulfonates on phase behavior. These materials are from the Enordet LXS series of surfactants manufactured by Shell, with products containing linear alkylxylenesulfonates with a 1,2,-dimethyl-4alkylbenzenesulfonate isomer content of approximately $93 \%$. They noted several parameters all contributed to the phase behavior: especially the MWD (molecular weight distribution), the aromatic substitution pattern, and the presence of a particular cosurfactant, Enordet 3ES. The Enordet 3ES was included to boost salt tolerance, and is represented by:

$$
\mathrm{C}_{\mathrm{n}} \mathrm{H}_{2 \mathrm{n}+1}(\mathrm{O}-\mathrm{CH} 2-\mathrm{CH} 2)_{3} \mathrm{OSO}_{3} \mathrm{Na}
$$

Where $n=12$ through 15 .

They found that the SP (solubilization parameter) can be increased by making the MWD of the surfactant wider and by adding the Enordet 3ES cosurfactant. (Recall that increasing the SP is expected to decrease the IFT, and hence is a desirable outcome). Another desirable feature is the reduced sensitivity of the optimal salinity to changes in the temperature. A disadvantage of this strategy, however, is that the very low IFT region may occur only a narrow range of conditions.

They conclude that while certain phase behavior/IFT performance parameters can be influenced more or less independently of each other, translation into the efficiency of oil recovery with such blends required further study.

Adsorption losses of the surfactant of course is another important operating parameter. One of the generalizations is that the adsorption of the sodium alkylbenzenesulfonates is dependent upon the molecular structure; it is a common observation that the plateau in adsorption can be roughly related to the molecular weight. For example, Li (1996) presents data showing the greater maximum adsorption for a series of alkylbenzenesulfonates for those surfactants having a longer alkyl chain length.

\section{Apha-Olefin Sulfonates}

One variation from the more common surfactants alkylbenzenesulfonates (ABS) are alpha-olefin sulfonates which do not contain an aromatic ring (Barakat, 1982). One investigation considered the use of alpha-olefins at high temperatures and in brines with elevated salinity (Baviere, 1988). The use of cosolvent enables aqueous solutions to be prepared with concentrated brine, even at high divalent cation levels. But the chemical stability of some solutions can be affected by their sensitivity to the oxidation of unsaturated components, resulting in a decrease of the $\mathrm{pH}$. Precautionary measures to stabilize the solutions are stressed - i.e. maintain an anaerobic environment, provide an alkaline $\mathrm{pH}$, or addition of the correct alcohol. These surfactants can achieve low interfacial tensions (IFT's) and high solubilization parameters at high salinity and divalent cation content. Properties of optimal formulations have been investigated as a function of surfactant and cosolvent molecular weight and brine composition. 
The study by Baviere also inclided measurements of adsorption of these surfactants onto $\mathrm{Na}$ - and $\mathrm{Ca}$ - kaolinite. In $\mathrm{NaCl}$ solutions, the amount of sulfonate adsorbed increased slightly with salinity. Preliminary measurements in hard water are shown to bring out the specific effects of calcium ions. From these results the authors concluded that the alphaolefin sulfonates are viable candidates for enhanced oil recovery applications.

\section{Alkene Sulfonic Acids -- One-Step Alkylation and Sulfonation}

Sulfonated alkyl aromatics have been reported as being suitable for EOR just recently, and in fact these have seen use in some small field projects (Berger, 2000 and 2002). These are different structures in that the sulfonate group is attached to the alkyl chain as opposed to the benzene ring. In contrast, the usual alkyl benzene sulfonates, the alkyl group is coupled to an aromatic ring which now has an attached sulfonate group. One advantage claimed for this newer type of surfactant is that it is easier and therefore perhaps cheaper to make, and still can achieve low IFT (U.S. Patent 6,043,391).

This newer family of anionic surfactants are derived from the reaction of benzene or its alkyl substituted forms, with olefin sulfonic acid. This becomes a one step process to accomplish the alkylation and sulfonation without using conventional alkylation processes and catalysts. This approach also has the advantage of allowing manufacture of some different molecules such as disulfonated alkylaromatics of the gemini-type structure can be produced. In addition, sulfonates of very viscous, high molecular weight aromatics may be prepared.

\section{Ethoxylated and Propoxylated Anionic Surfactants}

\section{Alkylbenzene- Ethoxylated Sulfonates::}

Skauge and Palgren (1989) describe detailed phase behavior and solution properties of 46 different ethoxylated anionic surfactants. In particular, they conduct these tests in mixtures of $\mathrm{NaCl}$ brine and heptane at a water/oil ratio of 2. The influence of changes in the lipophilic structure or degree of ethoxylation on the formation of multi-phase microemulsions was studied. Specifically, they studied alkyl- and alkylbenzene- ethoxy sulfonates with different chain length, branching and degree of ethoxylation. Nonylbenzene and dodeylbenzene surfactants are tested with both ethoxy sulfonate and ethyl methylcarboxylate as the hydrophilic head group.

They focused on the solubility of the ethoxylated anionics in brine equivalent to seawater salinity, is characterized at $22 \mathrm{C}$ (of interest since typical of injection water conditions for a North Sea application). Phase behavior studies were performed at $90 \mathrm{C}$, again with the idea of mimicking a North Sea application. 
Some key findings include:

1) the width of the salinity window and the gel free area are inversely proportional to the solubilization parameter,

2) branching of the alkyl chain reduces the optimal salinity and also the solubilization parameter,

3) sulfonates show higher solubilization than the methylcarcoxylates with a similar lipophilic group,

4) at a low degree of ethoxylation the solubilization parameter increases, while the optimal salinity decreases. Further ethoxylation lowers the degree of solubiliozation and increases the optimal salinity.

\section{Alcohol Ethoxylated Sulfonates:}

One study (Michels, 1996) examined the possibility of using sulfonate instead of sulfate versions of propoxy ethoxy types of surfactants. These have shown promise for EOR applications (Gale, 1981, 1992). Their study had the objective to demonstrate the viability of surfactant systems for application for North Sea reservoirs. In this case the requirements are a bit different; namely the objective was to develop low IFT with a low concentration of surfactant in order to decrease the cost, and also perform at North Sea elevated reservoir temperatures and seawater salinities.

This requirement for stability at elevated temperatures in these reservoirs (perhaps $100 \mathrm{C}$ or even more). Thus Michels et. al. investigated changing from a sulfate to a sulfonate head group. This change required adding a spacer in the molecule between the ethoxy and the sulfonate group for which a glycerol was selected, thus ending up with an alkyl propoxy ethoxy glycerol sulfonate surfactant. The strategy they further developed was not to include a polymer for mobility, but include some sacrificial agents to mitigate against excess adsorption losses. A theoretical economic analysis indicated that this process potentially could have favorable economics.

Another study (Austad, 1993) focused on the retention of ethoxylated sulfonate surfactants during a Norwegian supported research program about enhanced oil recovery. Thus, the focus of these studies are for applications for North Sea reservoirs. In particular, they considered nonylphenyl-type surfactants

$$
\mathrm{C}_{9}-\mathrm{Ph}-(\mathrm{EO})_{\mathrm{x}}-\mathrm{SO}_{3}^{-} \quad \text { where } \mathrm{x}=2-9
$$

Another loss mechanism considered is the stability of these surfactants to exposure to typical reservoir conditions for some months. Tests indicated no significant degradation at $80 \mathrm{C}$ during a period of 150 days in a anaerobic sea-water solution. The plateau adsorption on kaolinite was found to decrease as the degree of ethoxylation increases, except at very low concentrations where that trend is reversed (Austad, 1991). Another observation is a decrease in the plateau adsorption level with an increase in the $\mathrm{pH}$. 
Wang et. al.(2001) propose the use of a surfactant structure with both nonionic and anionic hydrophilic groups. Specifically, they examined the characteristics of ether caxhoxylated surfactants. The structure formula is as follows:

$$
\mathrm{C}_{9} \mathrm{H}_{19}-\mathrm{Ph}-\mathrm{O}-\left(\mathrm{CH}_{2} \mathrm{CH}_{2} \mathrm{O}\right)_{8}-\mathrm{CH}_{2} \mathrm{COONa}
$$

The notion is that this combination will overcome some of the deficiencies of either surfactant type by itself. Nonionic surfactants and anionic surfactants will salt out because of cloud point phenomena and high salinity, respectively. They claim this surfactant type is soluble in $30 \% \mathrm{NaCl}$ brine and shows good surface activity in brine with $100,000 \mathrm{mg} / 1$ salinity that includes $5,000 \mathrm{mg} / 1$ of calcium.

These workers found that by itself, the ether caxhoxylated surfactants (LF) was not able to create a low IFT. Similarly, the petroleum sulfonates tried did not make a low IFT with a brine/n-decane test system. By adjusting the proportion of the LF and petroleum sulfonate in a combined system, water/n-decane IFT's could be low, even with high salinity systems.

Luciani et. al.(2001) studied the adsorption tendencies for three anionic surfactants of the sodium nonylphenolpolyethoxy sulfate type: nonylphenol ethoxy(4) sulfate (NPE4S), nonylphenol ethoxy(10) sulfate (NPE10S), and nonylphenol ethoxy(25) sulfate (NPE25S). Three adsorbents examined were alumina, kaolinite, and a natural, crushed quartz. As would perhaps be expected, the adsorption decreases with an increase in the number of ethoxy groups (as increase water solubility/HLB). For kaolinite, as the $\mathrm{pH}$ increases above the $4-5$ range for $\mathrm{NaCl}$ the adsorption is quite low because the kaolinite has a net negative charge. In contrast in the presence of calcium chloride solutions, the adsorption occurs at even these lower $\mathrm{pH}$ conditions because it changes the zeta potential of the kaolinite surfaces. The adsorption of these anionic surfactants seem to be mainly driven by the interaction of the anionic group with the surface; adsorption occurs only if the surface charge is positive. On the other hand, the final structure of the adsorbed phase at saturation is governed by the ethoxy chain length, as for non-ionic surfactants. In particular, the apparent area per molecule at the plateau adsorption level follows the same trends (as a function of ethoxy number) as that followed by non-ionic surfactants.

\section{Alcohol Ethoxylated and Propoxylated Sulfates:}

Sumwoo and Wade (1992) report about a series of alkyl ethoxylate surfactants that they claim are superior with respect to the requirement for minimal cosurfactant, or even the elimination of the need for a cosurfactant. This points out of the general advantages of was the alkyl ethoxylated (EO) or propoxylated (PO) anionic surfactants is that the same molecule with multiple hydrophobic and hydrophilic groups has some chance of being effective as a stand alone surfactant.

In particular, Sunwoo and Wade examined C14 and C16 Guerbet alcohol sulfates. These investigators found the $\mathrm{C} 14$ Guerbet alcohol sulfates in fact functioned well without 
cosurfactant whereas the C16 type was more restrictive in its use by itself. The Guerbet alcohols (the $\mathrm{R}$ alkyl chain portion of the surfactant) are branched approximately at the mid-carbon location. Note the expected trends with regards to hydrophobe structure (Abe, 1986, and Graciaa, 1981):

Twin tail surfactants 1) prefer to micellize in the oil phase.

2) produce low values of SP at optimum salinity.

3) do not have particularly low IFT at optimum conditions

4) have minimal cosurfactant requirements.

Single tail surfactants 1) prefer to micellize in the aqueous phase

2) produce high values of SP at optimum conditions

3) can have low values of IFT at optimal conditions

4) have high cosurfactant requirements to avoid forming liquid crystal phases in the microemulsion phase.

Another interesting feature with these surfactants is that their anionic character seems to control the phase with shorter EO chains, whereas with perhaps 3 or more EO groups, the nonionic behavior begins to dominate. In addition, for the $\mathrm{C}_{14}(\mathrm{PO})$ sulfate surfactant evaluated, these molecules all showed more of anionic character. Thus EO units function as hydrophiles and PO units function as hydrophobes.

The difference between linear and branched hydrocarbon chains carries over to other surfactant systems as well. For example, Chattopadhyay (1993) considers surfactant molecules that have similar head groups and chain lengths of which one is comprised of linear alkyl chains and the other of branched hydrocarbon chains (diethanolamine derivatives of n-alkyl as well as polyisobutylene succinic anhydride). Measurements of a deep channel surface viscometer showed that contrary to the surfactants with linear hydrocarbon chains, the monolayers of the surfactants with branched hydrocarbon chains were more liquid like. The surfactants with linear hydrocarbon chains exhibited increased viscosity in the presence of $\mathrm{NaCl}$ in the aqueous sub-phase whereas a significant drop in viscosity was observed with the surfactant comprising branched hydrocarbon chains. This is consistent with the general idea that using surfactants that are more branched are less likely to have problems with regards to forming undesirable liquid crystals and thus viscous microemulsion phases that are difficult to transport in porous media.

One study (Osterioh, 1992) reports the results of laboratory experiments designed to test the efficacy of polyethylene glycols, PEG's, in reducing the adsorption of optimized PO/EO surfactants onto clays. Their results show the addition of PEG-1000 to such a microemulsion could reduce the measured surfactant adsorption onto Berea sandstone by a factor of four. PEG-1000 is a polyethylene glycol with an average molecular weight of 1000 manufactured by Union Carbide. In addition, oil recoveries were the same or improved with smaller volumes of injected surfactant. Another interesting feature is that this was designed for a high salinity connate brine (almost 200,000 mg/l total dissolved 
solids). The authors claim that the economics for including the polyethylene glycol is a net favorable approach.

Branched alcohol propoylated sulfates have emerged as an effective type of surfactant for the removal of nonaqueous phase liquids (NAPLs) from near surface, contaminated sites (Jayanti et. al., 2002). This application to remediate shallow subsurface aquifers (socalled SEAR -- surfactant-enhanced aquifer remediation) by injecting a surfactant solution is a relatively recent spin-off technology from conventional surfactant EOR. These propoxylated sulfate surfactants also may be tailored to achieve middle-phase micoemulsions versus crude oils, and presumably low interfacial tension (Aoudia, 1995). Minana-Perez et. al. (1995) found they could custom design surfactants as mixtures of ethoxylated and propoxylated products to provide optimum performance versus a given oil and process conditions. The addition of an intermediate polarity polypropylene oxide chain inserted between conventional lipophilic and hydrophilic groups makes an "extended" surfactant that may improve solubilization of water and oil.

Jayanti et. al. (2002) claims there are special advantages of the alcohol propoxylated sulfates in terms of having acceptable environmental properties, low viscosity middlephases, excellent solubilization of the NAPL contaminants, and fast coalescence times. In addition, the starting materials such as the branched alcohols may be fairly common, relatively low cost materials. These authors also recommend branched chain molecules versus straight chain ones. Straight chain surfactants are more prone to form liquid crystals and thus require higher cosolvent concentrations in order to break these relatively rigid structures. In contrast, branched chain molecules require less cosolvent and also increase the range of electrolyte concentrations over which Type III, middle-phase microemulsion behavior is observed.

\section{Carboxymethyl Ethoxylates}

Carboxymethyl ethoxylate (CME) surfactants have the general structure of

\section{R-O- $\left(\mathrm{CH}_{2} \mathrm{CH}_{2} \mathrm{O}\right)$ n- $\mathrm{CH}_{2} \mathrm{COONa}$}

It is expected that the proper choice of $\mathrm{R}$ and $\mathrm{n}$ would result in good surfactant performance in enhanced oil recovery. Huls Chemical Co. of Germany is one commercial source of these surfactants. These surfactant generally have good thermal stability and high electrolyte tolerance, and have been demonstrated to have moderate potential for use in EOR (Balzer and Kosswig, 1979, and Balzer 1982)

One DOE report (Stryker, 1990) discusses an investigation of six ethoxylated methylcarboxylates in which the salinities tested were at the high range $(10-30 \%$ sodium chloride) of what is expected in oil reservoirs. Temperatures in this study ranged from about $25-100 \mathrm{C}$, and hydrocarbon phases ranged from hexane to dodecane. Only one product (code name J-6) was found that could produce consistent three-phase behavior at these test conditions without including a cosurfactant. It created oil/water IFT values as low as $0.1 \mathrm{dyne} / \mathrm{cm}$. Other surfactants where a co-surfactant was included 
managed to make somewhat lower IFT values, with the lowest reported value of about $0.05 \mathrm{dyne} / \mathrm{cm}$. One interesting and useful feature is that if the $\mathrm{pH}$ is near the $\mathrm{pKa}$, of the CME surfactant, it may maintain its lower IFT value over a wider salinity range.

One study (Chiu and Hwang, 1987) indicated that oil solubilization (an indicator of IFT is proportional to the surfactant micellar size. An electrode measurement showed significant interaction between the surfactant and counter-ions, that did in fact indicate a large surfactant micelle. A commercial sample from Huls with an R group of C9H19(C6H4) and $n=4$ ethoxy groups showed good oil recovery capability.

One important parameter affecting the properties of the behavior of these carboxylatecontaining surfactants is the $\mathrm{pH}$. At low $\mathrm{pH}$, these surfactants will be as a carboxylic acid, whereas at slightly acid conditions or higher $\mathrm{pH}$, this becomes an anionic carboxylate surfactant. Phase behavior studies with branched tail ethoxylated carboxylated surfactants did show a considerable effect of $\mathrm{pH}$ on the salinity requirement to create middle-phase microemulsions (Masahiko, 1987). At 60 and $70 \mathrm{C}$, alcohol-free optimal three-phase microemulsion systems could be formed with all of alkanes studied over the entire $\mathrm{pH}$ range of $6-12$. At lower temperatures and higher $\mathrm{pH}$ values, liquid crystals were found to form in systems which contained lower molecular weight alkanes. For example, with branched tail EO sulfonates at $40 \mathrm{C}$, liquid crystals form with tetradecane and hexadecane, but are absent with the presence of octane, decane, and dodecane.

The shift in optimal salinity observed with a $\mathrm{pH}$ of below 9 is due to the change in the ratio of the acid form to salt form of the carboxylate head group increases with a decrease in $\mathrm{pH}$, especially at $\mathrm{pH}$ values close to $\mathrm{pKa}$ of the acid. The acid form being more oil soluble, while the salt form is more water soluble, causes the mixture to alter dramatically the a greater overall water solubility of the surfactant. This has the effect of increasing the salinity required to create a middle phase microemulsion. The solubilization parameters are almost independent of the $\mathrm{pH}$, although slight increases are observed with decreases in $\mathrm{pH}$.

\section{$\underline{\text { Ligonsulfonates }}$}

Ligonsulfonates are a broad class of wood-based chemicals made from the spent liquor generated in the sulfite pukping process. Thermal degradation and sulfonmation reactions that take place during the cooking of wood convert high molecular weight native lignion into a water-soluble polydisperse anionic polyelectrolyte with molecular weight varying from 1000 to 20,000. The functional groups that provide a high content of different polar groups make it miscible in all proportions with water.

Experiments have demonstrated that ligonsulfonates either alone (Bansel, 1979) or with petroleum sulfonates (Kumar, 1984) can improve oil recovery. Further improvements in the performance of these products has been explored, such as modifying the ligonsulfonates by copolymerization with propylene oxide (Hornof, 1990). They demonstrated a marked increase in interfacial activity with increasing degree of propxylation, to the order of $0.1 \mathrm{dyne} / \mathrm{cm}$. One useful feature is the increase in the 
optimal salinity with the propxylated products versus a ligonsulfonate/petroleum sulfonate synergistic mixture (Son, 1982).

\section{N-acyl-N-methylamide and Carboxylate Groups}

The simultaneous presence of $N$-acyl- $N$-methylamide and carboxylate groups in the surfactant molecule produces some unique interfacial properties in aqueous media (Tsubone, 2001). They are ascribed to the steric effect of hydrogen bonding between the two groups. Evidence for this includes trend of increasing $\mathrm{pH}$ in the vicinity of the $\mathrm{cmc}$ and observing greater cmc values by conductance than by surface tension. The motivation for this study is that there some surfactants with both groups that are used in cosmetic products because of their good foaming power and mildness to skin.

\section{Phosphate Surfactants}

Other types of potential surfactant would be those containing phosphorous. In particular, some work already has been done to examine the inclusion of phosphate ester surfactants (Halbert, 1971) for EOR applications. This author was able to make some formulations containing $4-8$ percent surfactant concentration solutions (using BASF Wyandotte, KLEARFAC AA-420 phosphate ester). One advantage of this anionic product is that it is a biodegradable liquid.

Other experiments also have examined phosphate ester surfactants as possible cosurfactants to increase the salt tolerance of petroleum sulfonate surfactants (Shankar, 1982). These authors considered a petroleum sulfonate blend (Stepan Chemical Petrostep 465/420) then combined with commercially available ethoxylated and other cosurfactants. The petroleum sulfonates may exhibit a salinity tolerance of only about $1-2 \% \mathrm{NaCl}$. Adding phosphate esters (Wayfos systems) in creased significantly the maximum allowable salinity for clear microemulsion solutions.

Another potential advantage of phosphate ester or similar chemistries for an EOR surfactant is that the adsorption may be decreased. Previous studies point in particular to the decrease in petroleum sulfonates and "normal" surfactants in the presence of phosphate ions (adding phosphate salts) can be beneficial (Somasundaran, 1979).

Bis(ethylhexyl) hydrogen phosphate (HDEHP) and sodium bis(2-ethylhexyl)phosphate (SDEHP) have been recently as agents to create microemulsions (Steytler, 1996 and Kurumada,1995). These authors note that this phosphate has some similarity to AOT (sodium bis(2-ethylhexyl) sulfosuccinate), a surfactant that is known to be very efficient in stabilizing water-in-oil microemulsions (Tapas, 1995). The ammonium salt of HDEHP has been found to stabilize water-in-oil emulsions producing, for most oils, a single-phase region which is more extended than that usually obtained with AOT. NH4DEHP is more stable than AOT and may be a better choice for applications at elevated $\mathrm{pH}$. The studies by Steytler and Kurumada focused mostly on the structure of the microemulsions created and did not consider the potential application of these phosphate surfactants as EOR agents. 


\section{$\underline{\text { Sorbitan Ester Surfactants }}$}

These surfactants have been investigated as emulsifiers, but not as agents for the improved recovery of oil (Peltonen, 2001). These authors describe the phase behavior and the interfacial tension of various sorbitan surfactants and several n-alkanes. They found trends such as the shorter chain length surfactant increased slightly the interfacial tension at the CMC, similar to that seen for ethyloxide surfactants. The longer the alkyl chain of the hydrocarbon phase, the higher the interfacial tension at the CMC. For this series of tests, the interfacial tensions reported for these aqueous surfactant solutions (in fresh water) never reach low levels (not less than 14 dyne/cm). However, as will be mentioned later, these surfactants could be quite potent when mixed with alkyl polyglycosides.

\section{Sulfobetaines}

Sulfobetaines, an amphoteric surfactant, have been examind as a possible family of surfactants suitable for enhanced oil recovery. One patent (U.S. 4,704,229) presents the method for their
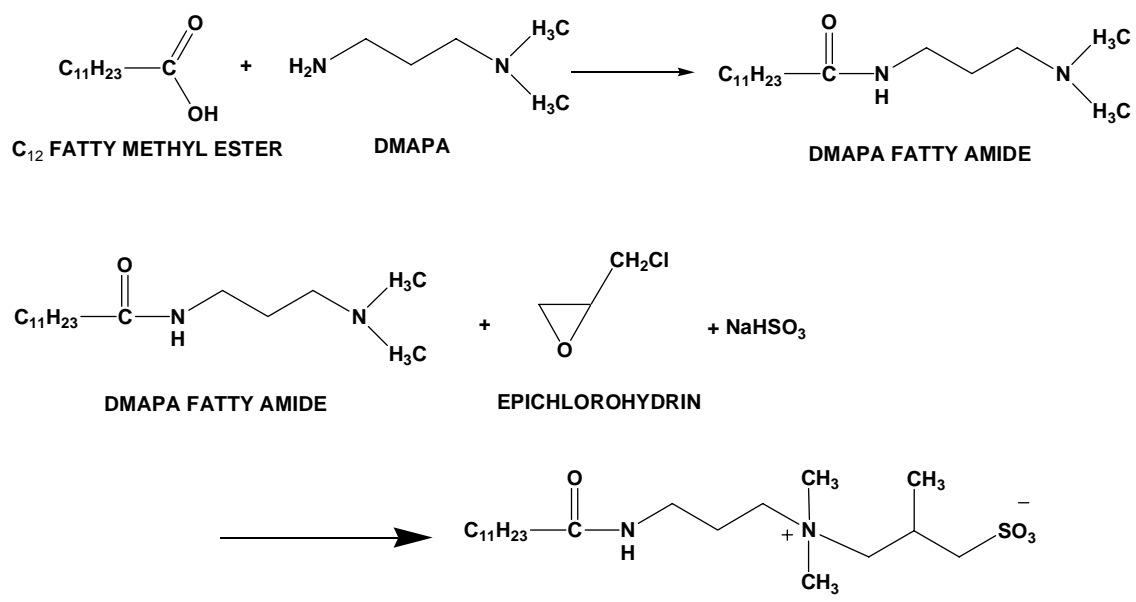

SULFOBETAINE

manufacture and use. The inventors from Institut Francais du Petrole claim that these chemistries may function well for enhanced oil recovery, having perhaps for lower adsorption and interfacial tension (U.S. 4,704,229).

One study that offered a comparison of adsorption levels onto different rock types (Berea sandstone, Indiana limestone, dolomite, and Baker dolomite (Mannhardt, 1992), and also included different surfactant types: sulfo-betaine, betaine, and an anionic surfactant. 
Varian CAS, an alkyl amido sulfobetaine, was supplied by Sherex Chemical Company and had an average molecular weight of $440 \mathrm{~g} /$ mole, meaning an average alkyl chain of approximately C16. Empigen BT, an alkyl amido betaine was supplied by Albright \& Wilson, and averaged a molecular weight of $365 \mathrm{~g} /$ mole. The anionic surfactant was DOW XS84321.05, a mixture of a C10 diphenyl ether disulfonate and a C14-C16 alphaolefin sulfoinate. Of the two amphoteric surfactants tested, the plateau adsorption the sulfobetaine is less sensitive to divalent cations. The anionic surfactant appears to have the lower adsorption in sandstone and dolomite rock.

Adsorption mechanism for the anionic surfactant is consistent with the electrostatic mechanisms, the adsorption of the anionic surfactants increases whenever the rock surfaces become less negative or by adding divalent cations to the solution. With both amphoteric surfactants, electrostatic interactions of both cationic and the anionic group in the surfactant molecule with the solids, and the complexation of the surfactant with divalent ions in solution or at the carbonate surfaces are likely to contribute to adsorption.

\section{Sulfosuccinates}

Examples of these are the Aerosol series of surfactants, such as the Aerosol OT product (butanedioic acid, Sulfo, 1,4-ditridecyl ester, sodium salt.). One virtue of the OT product is that it may be used to form cosurfactant-free microemulsions, especially revcerse $\mathrm{W} / \mathrm{O}$ mincroemulsions (Eicke and Markovic, 1981). The AOT and similar products are interesting because of their dichain structure (see below).

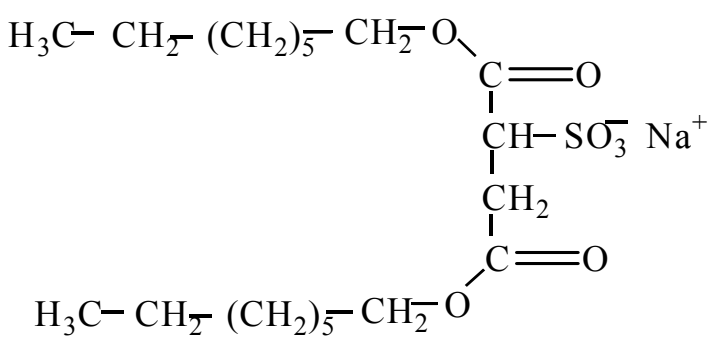

Mixtures of AOT and sodium mono- and dimethylnaphthalene sulfonate (SMDNS) have been used in the formation of middle phase microemulsions; binary and ternary mixtures of chlorinated solvents could be solubilized (Solans, 1997). Acosta et. al. (2002) describe this combination system and ascribe the improved performance of the AOT/SMDNS to be from the SMDNS acting as a hydrophilic linker. Their data show that a hydrophilic linker is an amphiphile that coadsorbs with the surfactant at the oil/water interface but has negligible interaction with the oil phase. Sodium octonate was found to be an alternative candidate hydrophilic linker to the SMDNS. The interest in this application is for remediation of contaminated ground water. Another common system studied with AOT is as mixtures with didodecyldimethyl ammonium bromide (DDAB). 


\section{Sulfonated Fatty Acid Esters}

Baviere et al. (1991) present a study of various sulfonated fatty esters as candidates for EOR. Their general formula are

$$
\mathrm{CH}_{3}-\left(\mathrm{CH}_{2}\right)_{\mathrm{n}-3}-\mathrm{CH}\left(\mathrm{SO}_{3} \mathrm{Na}\right)-\mathrm{COOR}
$$

where $\mathrm{n}=16$ (palmitic acid), 18 (stearic acid) or 22 (behenic acid) and $\mathrm{R}$ is usually a short alkyl group. The particular emphasis is on designing micellar chemical systems that are suited for oil recovery in reservoirs typical of the North Sea conditions.

One limitation of these surfactants is due to their chemical structure, as these are subject to hydrolysis, which increases rapidly above $50 \mathrm{C}$. The $\mathrm{pH}$ sensitivity of the reaction is much less pronounced around neutral $\mathrm{pH}$. In addition, the rate of hydrolysis is perhaps slower in sea water because of the buffering effect of its substantial bicarbonate concentration.

As expected, increasing the molecular weight of both the hydrophobic part and the alcohol group creates a decrease of optimal salinity. The highest solubilization of excess oil and water at optimal conditions is associated with a combination of the longest fatty acid chains with the shortest alcohol groups. The IFT follows the relationship of Huh (1979 and 1983), Equation (1), whereby the IFT is inversely proportion to the solubilization ratio. The constant calculated by Baviere (1991) for this relationship is $0.41 \mathrm{mN} / \mathrm{m}$, which is very close to the value of $0.48 \mathrm{mN} / \mathrm{m}$ reported for alkylbenzene sulfonates, $0.34 \mathrm{mN} / \mathrm{m}$ for ethoxlated alkylphenols, and $0.40 \mathrm{mN} / \mathrm{m}$ for alpha-olefin sulfonates (Barakat, 1982).

Adsorption studies with various sulfonated fatty esters were conducted on kaolinite clay (Baviere, 1991). The plateau value of surfactant adsorption is moderate with low and medium $\mathrm{NaCl}$ salinities, but increases in the presence of calcium and magnesium ions. Assuming a linear variation of adsorption with salinity, the influence of the divalent ion cannot be explained by the ionic-strength effect alone. The adsorption at the plateau has been shown to increase with the surfactant hydrophilicity, expressed in terms of optimal salinity or critical micelle concentration, decreases.

\section{$\underline{\text { Taurates }}$}

These products have appeared in the literature for use in enhanced oil recovery, at least at 


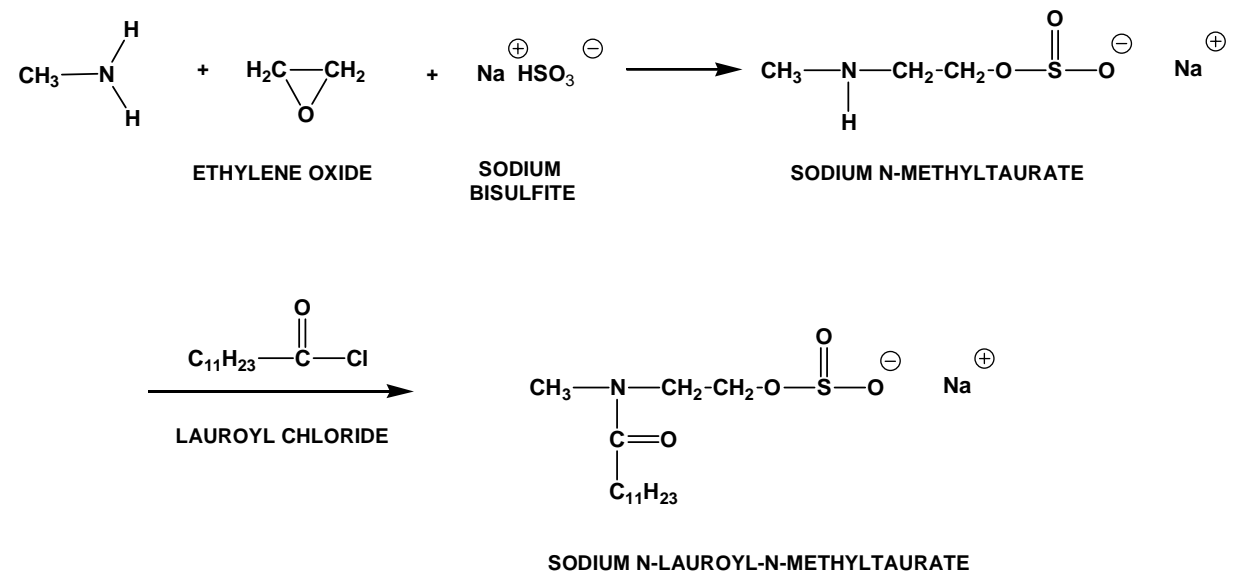

as supplemental agents. For example, one patent (U.S. 4,976,315) claims that taurine additives with other anionic surfactants may increase the salt and divalent ion tolerance of the anionic surfactant.

\section{Gemini Surfactants}

One newer trend in creating surfactants is to consider creating so-called "dimeric" or "gemini" molecules. These are defined as surfactants made up of two identical amphiphillic moieties connected at the level of the head groups, by a spacer group which can be hydrophobic or hydrophilic, flexible, or rigid. The terms "dimeric" or "gemini" are reserved for surfactants where the spacer, whichever its nature, be located very close to the head groups. Indeed, when a fully hydrophobic spacer connects the amphiphilic moieties far form the head groups, somewhere in the second half of the alkyl chains, the dimeric surfactant is then simply considered to be a bolaform surfactant containing a branched alkyl chain. These bolaform surfactants are characterized by a high critical micelle concentration $(\mathrm{cmc})$, and even poorer performance is expected if the bolaform surfactant has a partly hydrophilic group between the two head groups (Yiv, 1980).

Some of the potential advantages of these gemini surfactants are 1) lower surface tension, 2), lower cmc, so that the minimum surface tension occurs at a lower concentration, 3) better solubilizing, wetting, foaming properties. Besides the Kraft temperatures of dimeric surfactants with hydrophilic spacers are generally low, giving these surfactants the capacity to be used in cold water. The gemini version of surfactants may have a tendency for less adsorption as compared to the monomeric version of the same surfactant molecule. Finally, some dimeric surfactants show remarkable rheological properties (viscoelasticity, gelification, shear thickening) at relatively low concentrations, which is not observed with comparable, conventional surfactants having the same alkyl chain (Zana, 1998). 
Thus in a perfect scenario, a gemini surfactant could proved perhaps the advantageous properties of:

- Low IFT (there some evidence for increased oil solubilization with a dimeric surfactant versus its conventional version (Zana, 2002).

- This low IFT occurs at a low concentration (consistent with a low cmc)

- The solid adsorption perhaps can be made less than conventional surfactants

- Some systems may achieve some noticeable viscosity at modest concentrations. If that feature can be controlled, it could mean that the surfactant solution could meet a target solution viscosity to meet mobility control requirements.

One example of a common surfactant that is nearly a gemini structure are the so-called Aerosol series of sulfosuccinates (for example, the Aerosol AOT-B product)

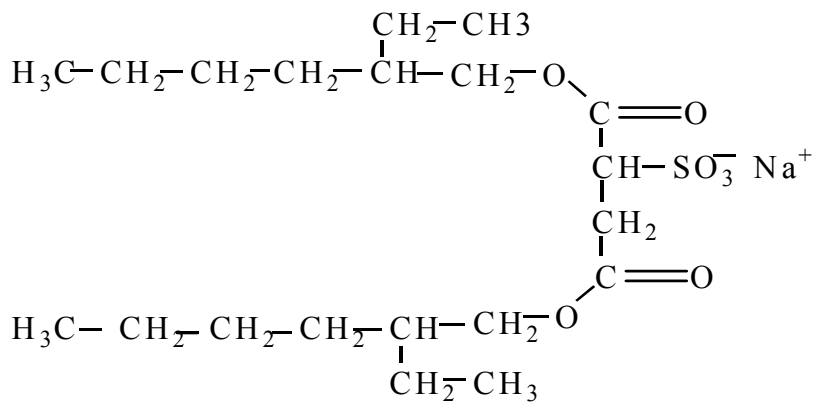

As an alternative to AOT some new double-chain compounds with one head and two tails (with a lower cmc than AOT) have been derived from glutamine and lactone (Hait, 2002).

Some of the other features that are reported for gemini surfactants include:

The CMC valus are sensitive to polarity of short spacers ( $2-8$ atoms).

A long hydrocarbon spacer (say 16 methylene groups) reduces the $\mathrm{CMC}$ almost ten-fold relative to a shorter spacer of $3-8$ methylene groups.

Gemini surfactants are more responsive to tail length than conventional surfactants.

Anionic gemini surfactants have somewhat lower $\mathrm{cmc}$ values than their cationic counterparts.

As mentioned above, these gemini surfactants can have interesting rhelogical properties, associated with their ability to form organized aggregates at a fairly low concentration (Oliviero, 2002, Hait, 2002). Some gemini surfactants have shown the ability to form vesicles similar to that observed for lecithin and phospholipids. Dimeric surfactants may form worm-like micelles at higher concentrations, thereby building substantial aqueous solution viscosity. This transition in micelle structure may be related in some cases to the molecular satructure. For example, for a series of gemini surfactants that have two quaternary amine heads and two identical alkyl tails, the micelle structure (such as sheriodal micelles or vesicles) is influenced strongly by the length of the spacer. Molecular dynamic simulations accounted for the change of structure (Zana 1998). They also predicted the formation of thread-like micelles. 
Forming some built-in viscosity/mobility control could be an advantage for gemini surfactant systems in that it would reduce the requirement for adding a polymer to thicken the aqueous phase. However, if the viscosity is based upon "liquid crystal", rigid structures that would not transport well in porous media, then that is not desirable.

The surface aggregation is one important factor in the adsorption of gemini surfactants onto solid surfaces (Zana, 2002). Adsorption is greater when their is formation of a continuous bilayer structure or micelle-like aggregates on the substrate. In one study of zwitterionic gemini surfactants, adsorption was significantly higher on a hydrophilic than the hydrophobic surface (Seredyuk, 2002). This same trend was observed for a series of "heterogeminis" (HG's), which refer to surfactant molecules with different polar headgroups, but identical tail groups. These investigators also observed that the adsorption tendency decreased with an increase in the number of ethoxylated groups; a trend we will mention below with regards to the behavior of surfactants such as ethoxylated nonylphenols. Fro some cationic gemini surfactants, the solid adsorption decreased with an increase in the spacer number. The thought is that increasing the specer component of the gemini surfactant makes the adsorbed structure to go form a flat bilayer to parallel cylinders and to spheres (Zana, 2002). This suggests it may be able to make a gemini surfactant with less adsorption that conventional surfactants.

\section{Polymeric Surfactants}

One interesting concept is to combine the function of a surfactant with a polymer. One molecule that can both reduce IFT and build some viscosity into the aqueous phase could have some operational advantage. Namely, this could help insure that the mobility control is maintained at the point where IFT is reduced significantly and the residual oil is being mobilized and banked up. One polymeric surfactant based on carboxymethyl cellulose and alkyl poly(etheroxy) acrylate was synthesized by ultrasonic irradiation.

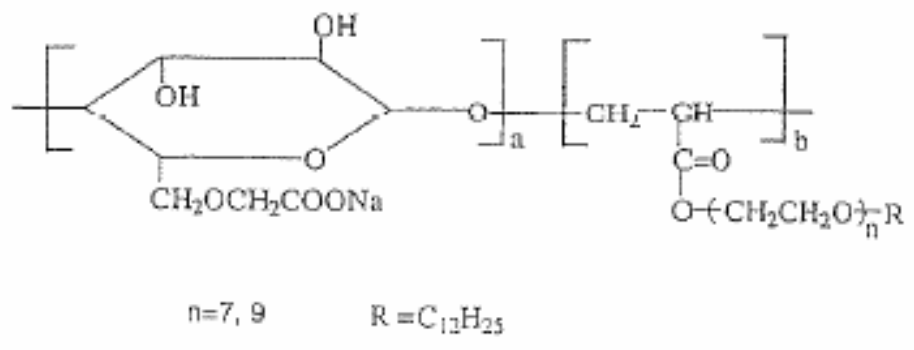

It is claimed that solutions of this chemical can attain low IFT (certainly less than 0.01 dyne $/ \mathrm{cm}$ ) if alkali is added. This low IFT may be due to more by the alkali rwacting with the organic acids in the crude oil than by the polymeric surfactant.

There are also polymerizable surfactants that are applied typically to combine with another monomer and create a latex type of polymer particle. A reactive surfactant is an amphiphilic molecule with an additional functionality that provides it with chemical 
reactivity. SURFMERS are one class, and have the property that they act in radical polymerization as a comonomer (Tauer, 1997). These can be used in emulsion polymerization to create a latex product; SURFMERS are designed to stabilize the polymer dispersion throughout the reaction, and to be bound covalently to the particle at the end of the polymerization.

The combination of a SURFMER with another reacting component offers several possibilities in the design of the generated polymer particles. In particular, this offers more parameters to control the size distribution of the created particles. The individual surfactant units may or may not offer the possibility of creating low IFT.

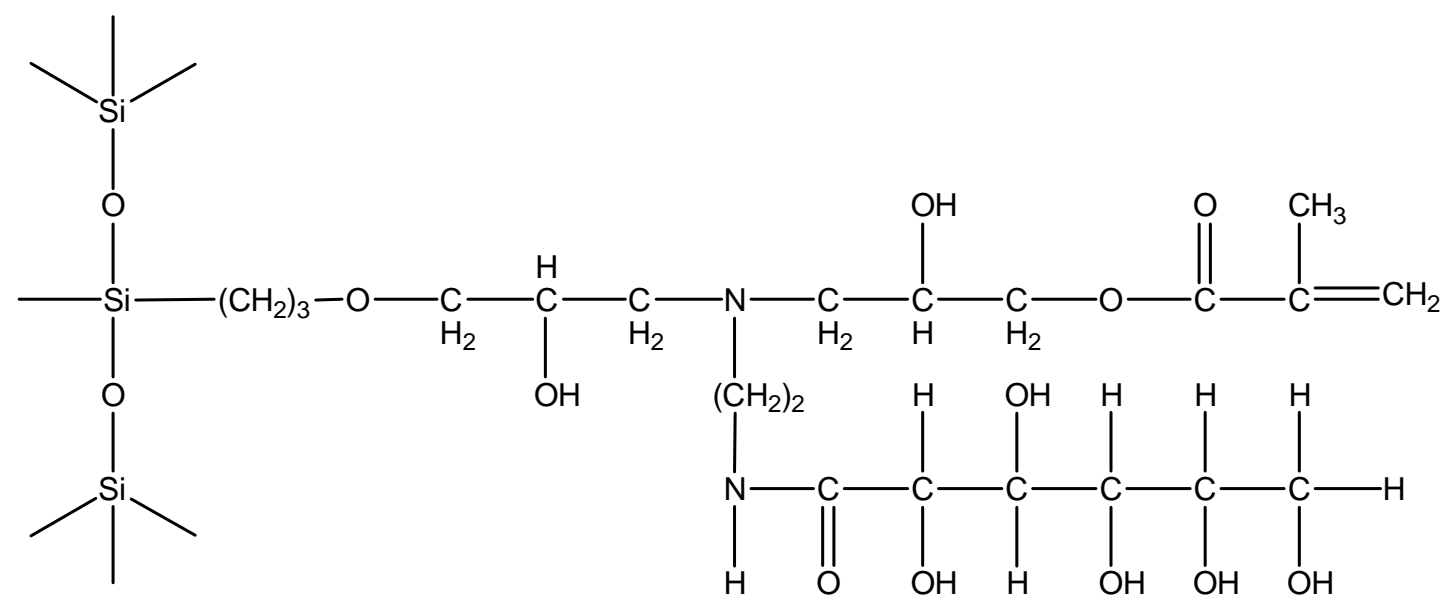

This siloxane sugar surfactant has the polymerizable methacrylate group located between the hydrophobic and hydrophilic part (Wagner, in press). Using a SURFMER containing silica perhaps can lead to a surface active oligomer or a polymer with better thermal stability. (Perhaps even further improvement is possible by increasing the size of the dialkyl-siloxane group.). This compound forms multi-lamellar vesicles in aqueous solution, and can self-polymerize if an uncharged initiator is used (PEGA200, poly(ethylene glycol) isobutryate), (Tauer, 1994). When used in emulsion polymerization with styrene, other phenomena include the co-polymerization within the growing copolymer particles, and surface interactions.

A variation of the above would be to explore using so-called INSURFS. These are also reactive surfactants, but now their special property is having a radical generating group as well as being a surfactant (Guyot, 1998). These systems are less complex than the SURFMER recipes, as a water, monomer, and INSURF mixture could be sufficient ingredients to generate copolymer latex particles. Again, it is possible to use the INSURF surfactant by itself, and allowing it to polymerize to some extent.

One approach is to use polymeric surfactants in conjunction with another surfactant. Ghaicha (1995) describe the monolayer behavior of a sorbitan monooleate and diethanolamine derivatives of polyisobutylene succinic anhydride of three molecular weights $(500,700$, and 1050$)$ as single as well as binary mixtures at the oil-water 
interface. Two oils were used in the study, heptane and paraffin oil. The observed differences in packing features revealed the significance of oil penetration and cohesive interactions in the aliphatic layer of the surfactant film. At the condition of the chain length compatibility, the mixed surfactant system produced emulsions with maximum stability. Chattopadhyay (1992) reported about similar chemistry, with a focus on double tailed surfactants. The primary hydrocarbon chain of the surfactants comprised polyisobutylene of approximately 34 backbone carbon number and the secondary hydrocarbon chain comprises n-alkyl groups from C8 to C20. The results indicate the surface properties at the water-oil interface correlate well with chain length compatibility effects in the mixed surfactant and cosurfactant systems.

\section{Polymers as Efficiency Boosters for Microemulsions}

Recently, there are study results where the addition of amphiphilic block copolymers are reported to provide a boost in the efficiency of microemulsions (see for example, Enco (2002), Jakobs (1999), and Mihailescu (2001). Specifically, block copolymers with the structure $\left(\mathrm{PEP}_{\mathrm{x}}-\mathrm{PEO}_{\mathrm{y}}\right)$ have this effect; that is, poly(ethylenepropylene)-copoly(ethylene oxide). The surfactant-oil system studied is an alkyl ethoxylate surfactant $\left(\mathrm{C}_{\mathrm{x}} \mathrm{E}_{\mathrm{y}}\right)$ and simple alkanes as the hydrocarbon phase.

The observations are that the "efficiency" of the surfactant is improved with respect to an increase in the volume of oil solubilized into a middle-phase microemulsion. Consistent with that behavior is in fact a decrease in the IFT with the inclusion of the block copolymer. For example, Jakobs (1999) reports that adding the polymer can decrease the IFT by a factor of 5; in one particular formulation they found it possible to go from a 13.2 $\mathrm{wt} \%$ surfactant concentration to create a single-phase microemulsion to just requiring $3.08 \%$ and $0.42 \mathrm{wt} \$$ of polymer.

The observation that the efficiency increase can be accomplished by increasing either block size or both block sizes symmetrically points to the direction that the origin of the efficiency boosting can not be from regular mixing effects. The effect is felt to be related to the ability of the block copolymer to extend further into the adjacent subphases.

Furthermore, analysis of high-precision neutron scattering data and theoretical calculation of the phase diagram researchers (Endo (2002)) deduce that these block copolymers are incorporated into the surfactant layer where they form mushroom conformations. One speculated beneficial effect is that the copolymer modifies the elastic moduli of the layer such that the formation of passages between neighboring membranes is hindered.

\section{$\underline{\text { Nonionic Surfactants }}$}

One option is to consider nonionic surfactants as the main agent for IFT reduction. One advantage of this choice is that these may have superior tolerance to very saline water. 
On the other hand, many of these surfactants can be sensitive to temperature; as the temperature increases the solution can change from a clear to turbid. This phenomenon is termed the "cloud point' for a given surfactant, and can limits is application.

\section{Alcohol Ethoxylated Ethers}

One common series of surfactants are based on the general formula of

$$
\mathrm{R}-\mathrm{EO}_{\mathrm{n}}-\mathrm{OH} \quad \text { where } \mathrm{R} \text { is usually a linear alkyl chain }
$$

Commercial examples would include the Neodol series of surfactants manufactured by Shell. Another common abbreviation is $\mathrm{C}_{\mathrm{i}} \mathrm{E}_{\mathrm{j}}$ where $\mathrm{i}$ refers to the alkyl chain length and $\mathrm{j}$ refers to the number of ethyoxlated groups.

Aveyard (1998) report on a study of the aggregate structures with microemulsions containing these surfactants, water, and an oil phase (heptane and tetradecane). They interpret solubilization phase boundaries in terms of spontaneous curvature of the surfactant monolayer and a critical concentration of surfactant required for microemulsion droplet formation. The measured droplet sizes are proportional to the molar ratio of dispersed component to surfactant within the droplets.

Aveyard (1998) also presents IFT data for these systems, where they focused on a $\mathrm{C}_{12} \mathrm{E}_{5}$ surfactant (dodeecyl pentaethylene glycol ether). They observed the IFT going to a deep minimum as the system approaches the PIT (Phase Inversion Temperature) from either a colder or a hotter temperature. The PIT (and the minimum IFT condition) occurs at a lower temperature for the case of a hexane (IFT $=10-3$ dyne $/ \mathrm{cm}$ ) than the larger, tetradecane hydrocarbon phase (IFT = 10-2 dyne/cm).

This transition from hydrophilic to lipophilic with a rise in temperature produces a middle-phase to appear. Furthermore the HLB (hydrophile-lipophile-balancd) temperature is defined as the temperature at which the middle-phase contains equal weights of water and oil. This is a condition of maximum solubilization of excess phases, and note that is analogous to the concept of an "optimal salinity" for the case of using anionic surfactants. Kunieda $(1993,1995)$ examined this behavior where there are mixtures of these alkyl polyethylene glycol ethers involved, each with its own HLB temperature. They conclude that the weight additivity of the three-phase (HLB) temperatures of each surfactant holds for the mixed surfactant systems.

Sottmann (1996) reports there is a similarity in the shape of the IFT curves for these surfactants versus the temperature. (They used only one oil phase, n-octane in this studyt.) There is a sharp minimum in the IFT at the PIT. The difference is that as the molecular weight increases, the minimum IFT achieved becomes lower and the PIT increases. These authors were able to normalize the results versus a reduced temperature, and further, could arrive at a single IFT versus T curve by normalizing the IFT by the interfacial surfactant volume squared. 


\section{Ethoxylated Alkyl Phenols}

The common commercial examples of this type of nonionic surfactants include the Igepal, Tritons, and Tergitol series of products. These are different from the above section, where now the molecule includes a phenyl group.

$$
\mathrm{R}-\mathrm{Ph}-\mathrm{EO}_{(\mathrm{n}-1)}-\mathrm{CH}_{2} \mathrm{CH}_{2} \mathrm{OH}
$$

where $\mathrm{R}$ is an alkyl chain, $\mathrm{Ph}$ represents a phenyl group, and there are $\mathrm{n} \mathrm{EO}$ ethoxylate groups.

Graciaa et. al. (1981) performed a rather detailed study of the Igepal series of surfactants for their phase behavior and ability to create a low IFT. The phase behavior of these nonionic surfactants having the same HLB but differing molecular weights was studied. It is shown that the optimum ACN (Alkane Carbon Number, the equivalent carbon length of the hydrocarbon phase) depends on the HLB, but that increasing the hydrophobe molecular weight narrows the middle phase region, increases the solubilization parameter and decreases the interfacial tension. That is to say that increasing the alkyl chain length can provide a lower IFT at its optimal conditions, but that the optimal conditions will occur over a more narrow range of process conditions. The width of the three phase region is found to be in simple inverse proportion to the solubilization parameter at optimum salinity and the multiple of the interfacial tension times the square of the solubilization is constant. In addition, the solubilization (minimum achievable IFT) is diminished by increasing the size of the hydrocarbon (increasing ACN) and diminished surfactant molecular weight. .

Besides the commercial type products, the authors found it was also found possible to synthesize nonionics which rival anionics in the above mentioned properties. Results form this study indicate it may be possible to create low IFT values and solubilization performance.

A separate study (Nevskaia, 1996) measured the adsorption of several polyxyethylenic surfactants. In general, the plateau adsorption became less with an increase in the number of ethoxylate groups (an increase in the surfactant HLB). The analogous surfactants which are sulfates and thus anionic in nature, show less adsorption than the nonionic (Triton series) onto silica. On the other hand, the adsorbed amounts of Tritons and sulfated Tritons on kaolin are similar, probably due to the positive changes on the edges of this material.

\section{Alkyl Polyglycosides}

Alkyl polyglycosides (APG) are a relatively untested surfactant idea with respect to creating a formulation for enhanced oil recovery. An example structure is shown below: 


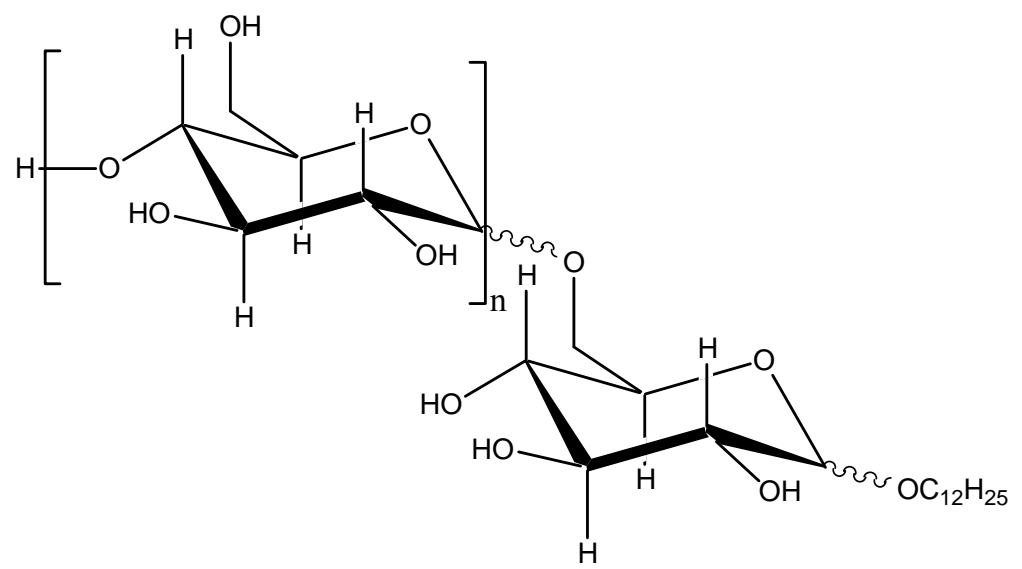

The degree of polymerization is usually small; $\mathrm{n}$ may vary from $0-2$ and is more often in the range of $0.2-0.8$. The alkyl chain shown here is a C12; commercial APG products have a mixture of chain lengths and can vary from $\mathrm{C} 8$ to $\mathrm{C} 16$. The length of this alkyl chain depends upon the fatty alcohol selected for making the molecule. The relative water/oil solubility depends upon the number of sugar units and the length of the alkyl chain. Specifically, larger $\mathrm{n}$ and a shorter alkyl tail promote water solubility (increases the HLB). Commercial products typically are water soluble with a HLB ranging from around $13-16$.

As discussed further below, there are examples in the literature where the alkyl polyglycosides can form middle-phase microemulsions with very low IFT, and hence seem a candidate for EOR. However, the bulk of the literature focuses on their use in other industries (von Rybinski, 1998).

For example, the alkyl polyglycosides are in cleansing formulations for personal care products. It is classified to belong to the group of very-mild surfactants for body cleansing. In a related cleaning application, is in surface cleaners and laundry detergents. Desirable properties in that regard include synergistic performance with anionic surfactants, good foaming ability, low toxicity concerns, and it is completely derived from renewable resources. Note that one route to make alkyl polyglycosides is the acid catalysis of a fatty alcohol and about any carbohydrate source (like starch).

Also alkyl polyglycosides are reported to be used for agricultural applications. Particular benefits for this industry include 1) their excellent wetting and penetrating properties, 2) it has tolerance for high concentrations of electrolytes, 3 ) within certain limits of alkyl chain length, they do not exhibit the inverse solubility with increasing temperature or "cloud point" phenomenon characteristic of alkene oxide-based nonionic surfactants, and 4) the ecotoxicity profiles of alkyl polyglycosides are among the most environmentally friendly that are known.

For the above reasons of tolerance to salt, temperature, and being nontoxic, the alkyl polyglycosides become an intriguing choice to be a key part of a surfactant flood formulation. This offers the potential advantage of developing a formulation for a 
particular reservoir application where the performance is largely indifferent to changes in reservoir temperature or salinity. One system discussed (Forster, 1996) shows that the IFT approaches $0.001 \mathrm{dyne} / \mathrm{cm}$ versus dodecane as the hydrocarbon phase. The formulation also included sorbitan monolaurate, SML as well as an APG with alkyl chain length of $\mathrm{C} 12-\mathrm{C} 14$ (ratio of SML:APG is 1:1 at the optimum conditions). These authors attribute the high interfacial activity to the fact that the hydrophilic alkyl polyglycoside with its large polyglcoside head group is present in exactly the right mixing ratio with the hydrophobic coemulsifier SML with its small head group at the oilwater interface. In contrast to ethoxylated nonionic surfactants, hydration and hence the effective size of the head group are hardly dependent on the temperature (Fukuda, 1993).

Data do suggest that by themselves, the common glycosides do not have ability to reduce the IFT to ultra-low levels (Nickel, 1996). For example, the IFT is a bit less than 1 dyne/cm with a $\mathrm{C}_{12}$ monoglycoside aqueous solution versus n-decane, but the lowest IFT is greater than 1 dyne/cm for the $\mathrm{C}_{10}$ monoglycoside, and is about 3 dyne/cm for the $\mathrm{C}_{8}$ monoglycoside. The trend in the decrease in IFT and the cmc with an increase in alkyl chain length is not unusual. One interesting feature is that the IFT appears to be independent of temperature (or at least IFT measurements are almost the same at 40 and $60 \mathrm{C}$. This study also includes data about solid adsorption of these type of surfactants. One interesting benchmark is that the adsorption levels on graphitized carbon black of $\mathrm{C}_{8}$ monglycoside is comparable with a $\mathrm{C}_{10}$ ethoxylated surfactant.

Another example reported is for the decane-water-alkyl polyglcoside system (Balzer, 1991). By adding iso-butanol to the formulation it was possible to generate a third-phase and a drastic reduction in the IFT. In addition, the range where the middle-phase microemulsion is observed is only slightly dependent on the temperature and the electrolyte concentration. Yet one other example is the decyl beta- D-glucoside in hydrocarbon/salt water (Aveyard, 1988). These authors report how toluene and cyclohexane as the hydrocarbon phase can produce a normal Winsor phase behavior, which includes the formation of a middle-phase microemulsion. One patent claims alkyl polyglucosides as a useful component for a micellar/flood. The particular advantage claimed in a U.S patent from 1991 for using these surfactants is to provide good performance over a wide range of both temperature and salinity (U.S. patent 4,985,154).

Due to the inherent high water solubility of the alkyl polyglcosides, it is expected that in fact another surfactant or co-surfactant (more oil soluble) component needs to be added in order to form a three phase microemulsion (Solans, 1997). This is particularly true for hydrocarbons like n-alkanes, but perhaps less so for polar oils. The quite different partitioning between the alkyl polyglycosides and a co-surfactant leads to the invariance of the three phase region with temperature. Besides simple alcohols as co-surfactants, diols are another choice which have the advantage of being a nontoxic alternative (Kahlweit, 1996).

A presentation about sugar-based surfactants (n-octyl- $\beta$-D-glucoside and n-dodecyl- $\beta$-Dmaltoside) also provides some encouragement to investigate the APG surfactants further as EOR candidates (Somansundaran, 2001). Laboratory measurements of the surface 
tension is fairly indifferent, hence indicating tolerance to multivaltent aluminum and calcium ions. The adsorption of these sugar-surfactants exhibits no sharp rise in surface density, which is an encouraging sign.

\section{Bacteria-Based Surfactants}

Two types of bio-surfactants that have possible application as EOR materials include surfactin and rhammolipids. Extensive studies have been carried out to reveal the molecular mechanisms of the regulation of rhamnolipid (a member of glycolipids) in $P$. aeruginosa and surfactin production (a member of lipopeptides) by Bacillus subtilis. Both the rhamnolipids and surfactin are controlled by a quorum sensing system, a process bacteria use to monitor cell density. Sullivan (1998) and Rahlm (2001) provide the mechanisms in very great detail; the interested reader is referred to these papers. Peypoux (1999) and Desai and Banat (1997) provide useful other useful background on these surfactants.

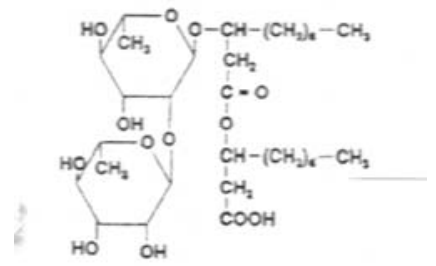

rhamnolipid 1

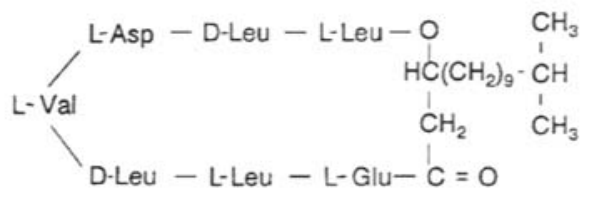

surfactin

Figure E-1. Structure of two candidate bio-surfactants

Hung and Shreve (2001) provide a detailed study of the surface active properties of the rhammolipid type of biosurfactant with known structures. The figure above shows the Rhamolipid 1 structure. Their so-called Rhamolipid 2 structure is the same, except it has a single ring structure as opposed to the two-ring structure shown above. The rhamnolipids of their study were produced by Pseudomonas strains Dyna 270 and PG201 (called Dyna270 and PG201, respectively). The Dyna 270 is a 1:3 mixture of R1 and R2.

Some of the IFT data generated by Hung and Shreve (2001) versus some pure hydrocarbons are reported in more detail in Section F. It is encouraging that they observed IFT values even below $0.01 \mathrm{dyne} / \mathrm{cm}$. It is conceivable that with further optimization that these biosurfactants could be able to mobilize trapped waterflood residual oil.

The major conclusions and observations from their study include: 1) lowest IFT's found versus alkanes such as hexadecane and dodecane, the IFT's were higher versus aromatic hydrocarbons, and 2) the Dyna270 functioned best when it had a buffered, $\mathrm{pH} 5.7$ salt system, whereas the PG201 seemed to perform better in fresh water. 
The rhamnolipids are even more interesting considering that they are somewhat similar in structure to the alkyl polyglucosides (APG). Perhaps lessons learned from the studies with APG may help tune the application of the bio-surfactants.

\section{F. Some Selected Literature Data/Trends Concerning Surfactant Structure and IFT}

This section summarizes some IFT literature data that are the subject of the computational chemistry modeling, and some other interesting situations. Appendix A. has yet more surfactant IFT data.

\section{$\underline{\text { Alkyl Benzene Sulfonates (ABS) }}$}

Several systematic studies of surfactant structure versus IFT have been reported for some alkyl benzene sulfonate surfactants (Doe 1977a, 1977b, 1978a, 1978b, and 1978c).

One test series included linear alkyl benzene sulfonate surfactants (LABS) with the same molecular weight (a 16 carbon length alkyl chain attached to the benzene (para- to the sulfonate). IFT values are reported for different isomers, each with a measured IFT versus a number of different n-alkanes as the hydrocarbon phase. See Figure F-1.

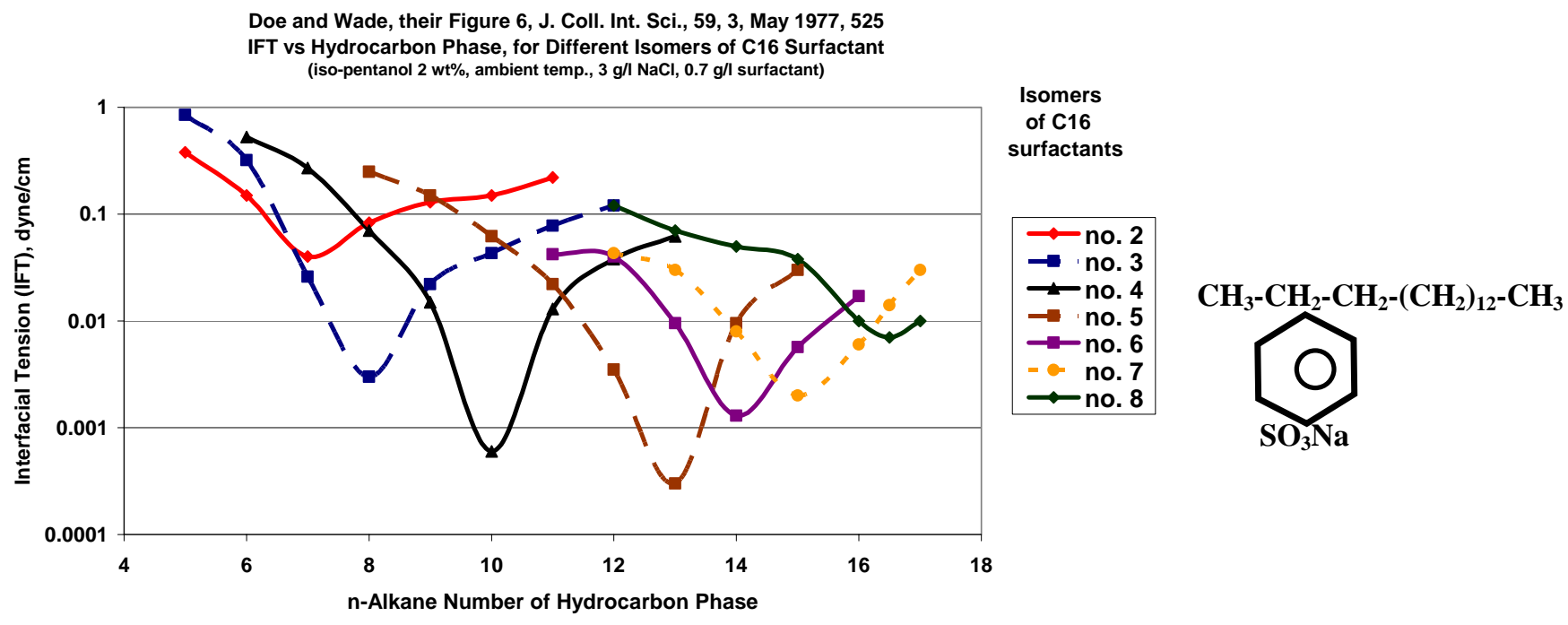

Figure F-1. Reported IFT values for different isomers of the C16 linear alkyl benzene sulfonate (LABS) surfactants

The nomenclature is \# phiC16SO3Na, where \# refers to the isomer number. That is the carbon in the alkyl chain that is at the point of attachment to the benzene ring. Results are not shown for the number 1 isomer (the no branching case) because it is too water insoluble. The larger isomer numbers (those with more even branching of the alkyl chain) produce their minimum IFT with a larger n-alkane as the hydrocarbon phase. 
If these data are cross-plotted, we can uncover other trends such as shown below (Figure F-2). This illustrates that for a given n-alkane hydrocarbon phase and a constant set of other conditions (salinity, temperature, co-surfactant, etc.), there is an "optimum" isomer number with regards to generating the lowest IFT condition. For example, in this case, the number 4 isomer is the one that produces the lowest IFT versus n-decane as the oil phase.

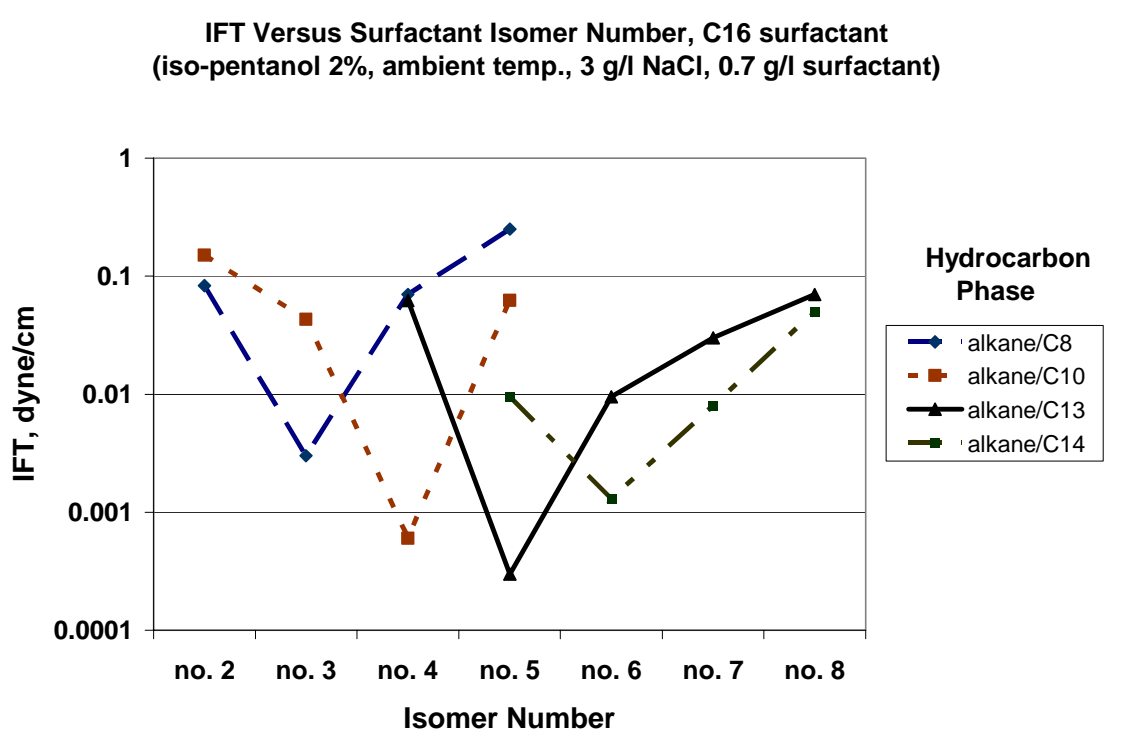

Figure F-2. Reported IFT versus the isomer number for $\mathrm{C} 16$ linear alkyl benzene sulfonate surfactants.

The Figure F-3 below illustrates that the same trend holds for the C12 isomer of LABS as the $\mathrm{C} 16$ isomer (see Figure F-1). The more evenly branched (6 isomer) achieves its lowest IFT condition with a larger n-alkane hydrocarbon phase than the lower, number 5 isomer. 
IFT Versus Alkane Hydrocarbon Phase -- Isomers of C12 Surfactant

(iso-pentanol $2 \mathrm{wt} \%$, ambient temperature, $3 \mathrm{~g} / \mathrm{l} \mathrm{NaCl}, 0.7 \mathrm{~g} / \mathrm{l}$ surfactant)

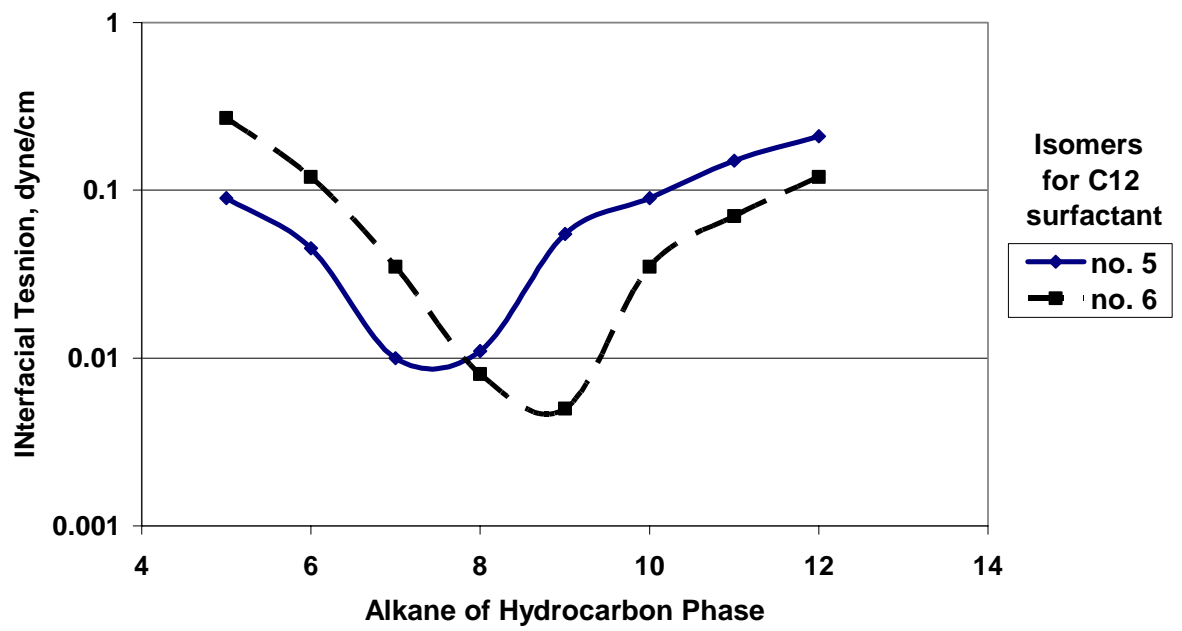

Figure F-3. Reported IFT values for different isomers of the $\mathrm{C} 12$ linear alkyl benzene sulfonate (LABS) surfactants

Finally, consider Figure F-4, that shows the IFT scan for the number 6 isomer, going from a total alkyl chain length of $\mathrm{C} 12, \mathrm{C} 14$, to $\mathrm{C} 16$. This shows directly the increase in the most compatible oil with increasing total chain length.

IFT vs. Hydrocarbon for no. 6 Isomers of Different Linear Alkyl Benzene Sulfonates

$0.7 \mathrm{~g} / \mathrm{l}$ surfactant, $3 \mathrm{~g} / \mathrm{N} \mathrm{NaCl}$, $2 \%$ iso-pentanol, ambient temperature

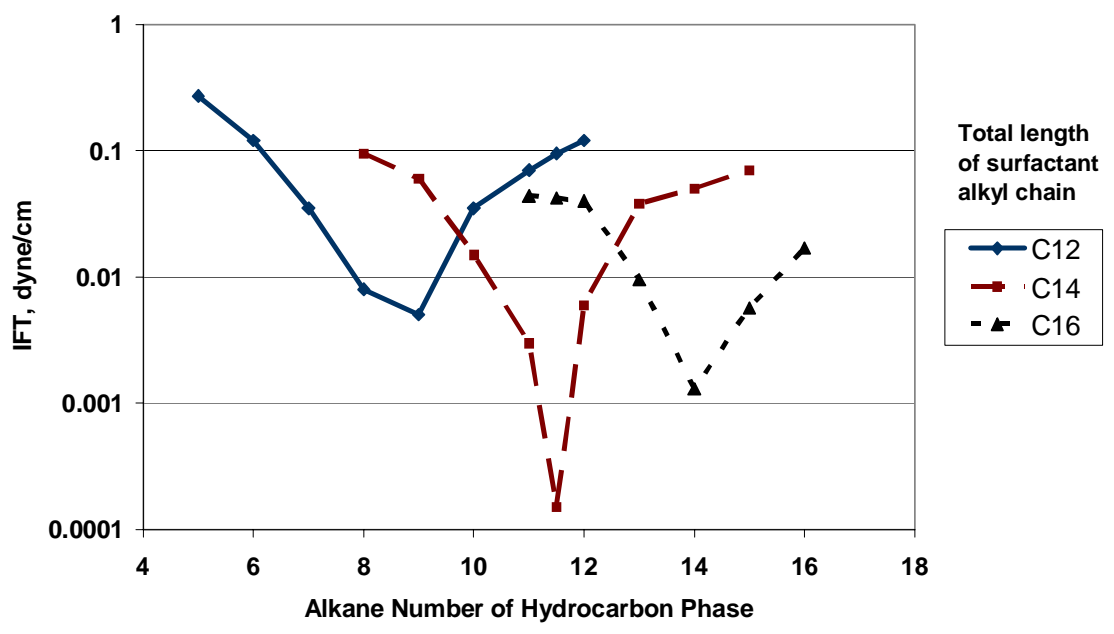

Figure F-4. IFT versus the n-alkane making up the hydrocarbon phase, all for the number 6 isomer. 
Best alkane oil

\begin{tabular}{|c|c|c|}
\hline \multirow{2}{*}{\multicolumn{3}{|c|}{$\begin{array}{l}\text { Surfactant } \\
\text { Total Length }\end{array}$}} \\
\hline & & \\
\hline C12 & 7 & 9 \\
\hline $\mathrm{C} 14$ & & 12 \\
\hline C16 & 13 & 14 \\
\hline
\end{tabular}

Increasing the surfactant alkyl chain increases the most compatible hydrocarbon phase by 5 or 6 carbons. These observed shifts in most compatible surfactant/oil combinations may aid in interpreting simulations comparing $\mathrm{C} 12$ and $\mathrm{C} 16$ surfactants. Also, roughly speaking, for each increase in the isomer number, the alkane hydrocarbon that makes the lowest IFT goes up by 2 carbons.

That is, these studies show there is a fairly systematic (increasing) shift in the most compatible n-alkane hydrocarbon phase as one adds more alkyl groups to either the shorter or longer LABS tail. A simple argument for this behavior is that increasing the relative oil-solubility of the surfactant then makes it more prone to reach its minimum IFT with larger hydrocarbons, all other things being equal.

Calculation tools that predict such trends and shifts successfully will be very useful in design and selection of surfactants. One reason is that these relative changes in surfactant structure/oil compatibility are likely insensitive to other factors (e.g. salinity, co-surfactant).

Follow-up studies considered more complicated structures where instead of a simple LABS type of structure, the surfactant is a di- or even tri-alky benzene sulfonate (ABS). Doe et.al. (1978b, 1978c) developed rules of thumb, using their concept of an "alkane preference curve". This refers to a plot of the minimum IFT, $\gamma_{\min }$, versus the n-alkane compound used as the oil phase, $\mathrm{n}_{\min }$. The hydrocarbon phase, $\mathrm{n}_{\mathrm{o}}$, where that surfactant achieves its absolute minimum IFT is of particular interest. They identified for the ABS surfactants three classes with different behaviors. These are:

Group I, $\mathrm{n}_{\mathrm{o}}$ is about 10 - these are the preferred types of ABS surfactants for creating low IFT for most crude oils. This is because for most real oils, they behave in a phase behavior and IFT sense most like n-alkanes from n-hexane to n-decane. That is, the EACN for most crude oils falls between 6 and 10. The Group I surfactants are modified LABS, having a short (C3 or less) alkyl group ortho to the SO3 group, plus one or two other alkyl groups, the major one of which is meta to the sulfonate and has at least 12 carbon atoms.

Group II, $\mathrm{n}_{\mathrm{o}}<5$. These surfactants are matched best only with very shot hydrocarbons, and hence not of much practical interest for crude oil EOR. In addition, the $\gamma_{\min }$ values never achieve very low values. This group has modified LAB's where the group ortho to the sulfonate is $\mathrm{C} 4$ and/or the major chain meta to the SO3 is C10 or less. 
Group III, $\mathrm{n}_{\mathrm{o}}$ about 12.5. Surfactants in this group can achieve acceptably low IFT values, but are suited best for hydrocarbons that have on average a larger EACN than found in most crude oils. The LAB's in the molecular weight range from C11 up to C18 typify the surfactants in this group.

\section{$\underline{\text { Biosurfactants }}$}

Hung and Shreve (2001) provide some IFT measured data for rhammolipid type of biosurfactants with known structures. The Figure E-1 above shows the Rhamolipid 1 structure. Their so-called Rhamolipid 2 structure is the same, except it has a single ring structure as opposed to the two-ring structure shown above. The rhamnolipids of their study were produced by Pseudomonas strains Dyna 270 and PG201 (called Dyna270 and PG201, respectively). The Dyna 270 is a 1:3 mixture of R1 and R2.

The Table below provides some of their measured IFT data.

Table F-1 Interfacial Tensions of Dyna 270 and PG201 at 25 C

\begin{tabular}{|c|c|c|}
\hline Hydrocarbon & $\underline{\text { Surfactant }}$ & $\underline{\text { IFT (dyne } / \mathrm{cm} \text { ) }}$ \\
\hline \multirow[t]{2}{*}{ Hexacdecane } & PG201 & 0.26 \\
\hline & Dyna 270 & 0.54 \\
\hline \multirow[t]{2}{*}{ Dodecane } & PG201 & 0.076 \\
\hline & Dyna 270 & 0.12 \\
\hline \multirow[t]{2}{*}{ Benzene } & PG201 & 5.4 \\
\hline & Dyna 270 & 0.94 \\
\hline \multirow[t]{2}{*}{ Toluene } & PG201 & 5.5 \\
\hline & Dyna 270 & 0.72 \\
\hline \multirow[t]{2}{*}{ Air } & PG201 & 26.0 \\
\hline & Dyna 270 & 27.1 \\
\hline
\end{tabular}

\section{G. Some Selected Literature Data/Trends Concerning Solid Adsorption}

\section{$\underline{\text { Introduction }}$}

The adsorption of surfactants onto the surfaces of rock is one of the important factors governing the economics of chemical flooding in tertiary oil recovery. Therefore, reduction of the amount of surfactant required can turn a marginal project into a good one. 
The emphasis here I on kaolinite clay as the adsorbant because 1) it is a common reservoir clay, 2) its structure is more well-defined than other clays, and 3) we focus on kaolinite in the experimental adsorption work for this project.

Composition of Kaolinite Clay(wt\%)

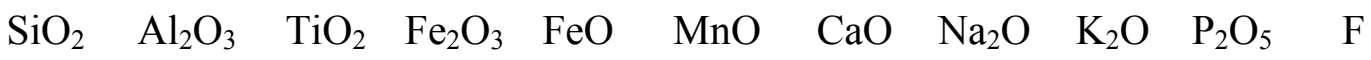

$\begin{array}{llllllllll}44.2 & 39.7 & 1.39 & 0.13 & 0.08 & 0.002 & \text { trace } & 0.013 & 0.05 & 0.034\end{array}$

0.013

Loss on heating: $\quad 12.6 \%\left(\sim 550{ }^{\circ} \mathrm{C}\right) ; \quad 1.18 \%\left(550 \sim 1100{ }^{\circ} \mathrm{C}\right)$.

\section{Mechanism of the Adsorption}

Since 1980, the mechanism of surfactant adsorption at the kaolinite clay solid/liquid interface has been intensively studied and a great deal of literature has been published. It is now admitted that two steps, more or less separated, can describe the formation of the adsorption layer along the adsorption isotherm. In the case of minerals, adsorption at low equilibrium concentration is generally due to the interaction between the polar head of the amphiphile molecule and some specific sites of the surface, e.g., H-bonds, electrostatic forces. For higher concentration, aggregates are formed at the interface as a result of lateral interactions between hydrophobic chains. This aggregation is due to the same forces as those responsible for bulk micelle formation. The concentration at which aggregation appears on the surface, their lateral extent and their thickness depends on: 1.) the normal interaction between the polar head and the surface; 2.) the lateral interaction between hydrophobic chains; 3 .) the spatial and energetic distribution of adsorbing sites. Cases and Villieras (1992) have classified systems as a function of the strength of the normal adsorbent/adsorbate bond. The formation of hydrophobic monolayers at low concentration (useful for example in floatation) followed by bilayers at higher concentration would occur in the case of a strong bond. When the bond is weak, the formation of surface micelles (aggregates of finite size) would be more frequently observed, such conditions are interesting for enhanced oil recovery applications. Nevertheless, recent studies by ellipsometry (Tiberg, 1994, Luciani, 1997), neutron reflectivity (Thomas, 1997), or atomic force microscopy (Manne, 1995) on model substrates suggeste that surfactants with the same polar group but different hydrophobic chain length can form either bilayers or surface micelles.

\section{Main Factors Affecting the Adsorption}

Several physicochemical processes can be expected to take place when clays are contacted with ionic surfactant aqueous solutions and to contribute toward determining the overall behavior of the resulting suspensions. Major among such processes are recognized to be hydrolysis of surface species, ion-exchange, electrostatic adsorptions and dissolution of the clay constitute, and adsorption or precipitation of resultant complexes. Each of the factors described above is discussed below. 


\section{Hydrolysis:}

The process of equilibration of dry kaolinite with the aqueous phase involves at least two steps. The fast step can partly be accounted for by hydrolysis reactions involving surface species on kaolinite. Past studies (Reid, 1967 and 1968, Lloyd, 1970, Mukherjee, 1953, Grim, 1968, Conley, 1971) have suggested the source of acidity on kaolinite to be the terminal bonds and structural coordination across the edge faces either due to the silanol groups or due to aluminol at the strained gibbsite layer. In aqueous solutions, the broken bonds at the edge surface of dry kaolinite will be hydrated and converted to a hydroxylated surface. Bronsted acid generation, which is likely under these conditions can result from one of the following deprotonation processes (fast) (Conley, 1971):

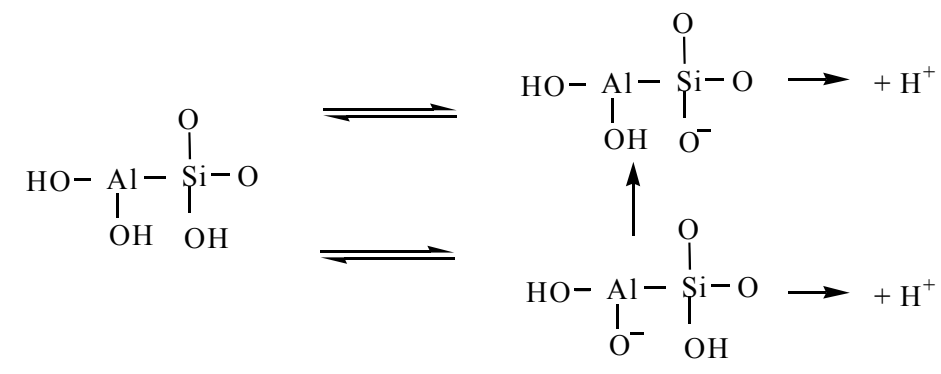

\section{Ion-Exchange:}

This process is characteristic of clay minerals since exchangeable cations are present as counterions in the clay (Swarten-Allen, 1974, Martin, 1964). This possibility is supported by the fact that increased acidity resulted upon adding $\mathrm{NaCl}$ to the system (Hanna, 1979).

$$
\mathrm{H}-\text { Kaolinite }+\mathrm{Na}^{+} \rightleftharpoons \mathrm{Na} \text { - Kaolinite }+\mathrm{H}^{+}
$$

The hydrogen ions released under such conditions should be related to the ion- exchange capacity of the clay. Such an ion-exchange process is expected to be fast and therefore can partly explain the fast $\mathrm{pH}$ drop observed ${ }^{13]}$. In addition, the resulting decrease in $\mathrm{pH}$ will have its own effect on the adsorption of surfactant on the clay. This indirect effect can in fact predominate the direct effect of changes in ionic strength.

\section{Dissolution of Surface Species:}

Hanna and Somasundaran investigated acidity of aqueous solution of kaolinite (Hanna, 1979) and found that $\mathrm{pH}$ value of the solutions decreases with time to a constant. They attributed the decrease in the acidity of kaolinite suspension with time to the release of exchangeable aluminum ions into the system. The highly charged $\mathrm{Al}^{3+}$ species could readsorb on the mineral surface in different forms after a series of hydrolysis reactions that produce complex ions such as $\mathrm{Al}(\mathrm{OH})^{2+}, \mathrm{Al}_{8}(\mathrm{OH})_{20}{ }^{4+}, \mathrm{Al}(\mathrm{OH})_{3}$ and $\mathrm{Al}(\mathrm{OH})_{4}{ }^{-}$. Such a process can be expected to be very slow in comparison to the dissociation of the weakly acidic group on the kaolinite surface. However, at longer contact times, the slow release 
of increasing amounts of charged aluminum species from the basal kaolinite surfaces into the solution followed by their adsorption on the kaolinite surface will be an important process particularly in the acidic and basic $\mathrm{pH}$ ranges where concentration of dissolved aluminum species is considerably higher than in the neutral range. This will increase the affinity of the negatively charged sulfonate toward kaolinite and hence an increase in adsorption, especially at low $\mathrm{pH}$ values.

\section{Ionic Strength:}

In tertiary oil recovery, there are many kinds of cations in the aqueous solution. So it is necessary to understand the effect of ionic strength on adsorption of surfactants on kaolinite clay. For example, adsorption of petroleum sulfonate, e.g., TRS-80, increases with ionic strength (Baviere, 1991).

This can be explained by a salting-out effect on the surfactant, and also because the thickness of the electric double layer at the surface of the kaolinite diminishes with salt, hence reducing the repulsion against sulfonate anions.

\section{Effect of $p H$ :}

As discussed earlier, exchange between $\mathrm{Na}^{+}$and $\mathrm{H}^{+}$ions is an important process that may affect adsorption of surfactant on kaolinite clay. Therefore, it is expected that adsorption of dodecyl benzenesulfonate on Na-kaolinite is highly sensitive to hydrogen ion concentration (Hanna, 1979) . It was found that sulfonate adsorption increases markedly with increase in hydrogen ion concentration, particularly in the acidic $\mathrm{pH}$ range. In addition, a maximum adsorption of sodium dodecyl benzenesulfonate (SDDBS) on kaolinite was found in the acidic $\mathrm{pH}$ range, which corresponds to a compact monolayer with a molecular area of $33.7 \AA^{2}$ per SDDBS molecule. However, the adsorption densities in the neutral and alkaline $\mathrm{pH}$ range were found to be only $25 \%$ and $12 \%$ of close packed monolayer, respectively.

\section{Ionic Environment:}

To compare effect of ionic environment on adsorption of surfactant on kaolinite clay, Bavière and co-workers investigated the effects of sodium carbonate and sodium hydroxide in $\mathrm{NaCl}$ brines on the adsorption of petroleum sulfonate on kaolinite clay (Baviere, 1991). It was found that at a constant ionic strength $(\mathrm{I}=0.448 \mathrm{M})$, from pure $\mathrm{NaCl}$ brine $(\mathrm{pH}=7)$ to pure $\mathrm{Na}_{2} \mathrm{CO}_{3}$ brine $(\mathrm{pH}=11.3)$, the effect of carbonates is pronounced only above $\mathrm{pH}=11$. At low carbonate concentration, the $\mathrm{pH}$ increases strongly but does not decrease adsorption. The decrease of adsorption above a $\mathrm{pH}$ of about 10.8 seems to be due to the quantitative replacement of $\mathrm{Cl}^{-}$ions by $\mathrm{CO}_{3}{ }^{2-}$ ions. This replacement at constant ionic strength also contributes to a decrease in sodium ions concentration, and then to a decrease in sulfonate activity, that may partially explain the decrease in the adsorption. On the other hand, it was found that sodium hydroxide is much less effective than carbonate in decreasing adsorption of petroleum sulfonate on kaolinite. It is worth noting that adsorption in the presence of carbonate $(\mathrm{pH}=11.3)$ is about only one tenth of that in the presence of sodium hydroxide $(\mathrm{pH}=12.2)$. 


\section{Effect of Adsorption Temperature:}

$\mathrm{Xu}$ and co-workers investigated the effect of temperature on adsorption of sodium oleate on kaolinite. It was found that there are adsorption plateaus at different temperatures $(25$, 35 and $\left.40{ }^{\circ} \mathrm{C}\right)(\mathrm{Xu}, 2001)$. Although adsorbed amount of the first plateau are almost constant at different temperatures, a decrease of adsorbed amounts of the second plateau and absorbed equilibrium concentrations of sodium oleate at the surface of kaolinite is observed with increase of the adsorption temperature under the same experimental conditions.

Nevshaia and co-workers (1996) determined adsorption of octylphenoxypoly(ethyleneoxy) ethanol (TX-114) on kaolinite at different temperatures $(273,298$ and $308 \mathrm{~K})$. It was found that, at low $C_{\text {eq. }}$ or a low degree of coverage $\theta\left(\theta=\Gamma / \Gamma_{\max }\right)$, the monomers are adsorbed on the solid surface, displacing water molecules. This kind process has an exothermic nature. When the adsorption temperature increases, the adsorbed surfactant amount decreases. When the majority of the adsorption sites are covered by surfactant molecules, interactions take place between the adsorbed surfactant molecules to form micelles at the solid surface. This process is endothermic. Thus, at high $C_{e q}$. or a high degree of coverage, when the adsorption temperature is increased, the adsorption amount also rises. According to modern polymer adsorption theory (Scheutjens, 1979), this kind behavior is expected since the Flory-Huggins parameter $\chi$ for the oxyethylenic (EO) part increases with temperature (T) and it can be calculated by the equation:

$$
\chi(T)=\left(5.879 \times 10^{-3}\right) T-1.6538
$$

It means that an increase in adsorption temperature favors contacts between polyoxyethylenic chains over water-polyoxyethylenic chain interactions. Or, on other words, micelle formation is favored when the temperature increases.

\section{Effect of the Ratio of Liquid/Solid:}

$\mathrm{Xu}$ and co-workers (2001) measured adsorption amount of sodium oleate at the kaolinite/aqueous solution interface at different liquid/solid ratio(w/w) under the same experimental conditions. It was found that the adsorbed amount increases with increase of the ratio until it reached a steady and constant value, $\Gamma_{\max }$, i.e. the plateau was attained within 24 hours when the ratio was no less than 9.

Theoretically, the ratio of liquid/solid should not affect adsorption isotherm of a disperse system. However, in some cases, the ratio may affect the adsorbed amount at water/solid interface. For some $\mathrm{Al}^{3+}$ ions of the $\mathrm{Al}-\mathrm{O}$ bond in kaolinite, substituted by some $\mathrm{Si}^{4+}$ ions, under natural condition, the surface of kaolinite shows a positive charge determined by the structure of kaolinite. However, surfactant solution may affect the charge on the surface. And the charged ions of kaolinite are ion-exchanged with the cations and anions in sodium oleate solution. Thus, the concentration of ions in the solution would change 
when increasing the ratio of liquid/solid. Another cause is that the ion-exchange occurs easily between the solid surface and the solution phase due to the positive charge on the surface of kaolinite. Consequently, the concentration of ions would change when increasing the ratio of liquid/solid. The smaller the ratio is, the stronger is the interaction iof double layers at the interface of solution/kaolinite, and the less the adsorption amount.

The general observation is that for anionic surfactants that the solid adsorption will decrease with an increase in temperature. In contrast, for nonionic surfactants the adsorption increases with an increase in temperature (Lewis, 1986). The key to understanding the adsorption of nonionic surfactants is related to the phase behavior. In particular, the adsorption increases markedly as the cloud point is approached. In fact, these authors show a correlation of adsorption to the difference between the use temperature and the cloud point. Generally, they recommend using nonionic molecules with larger molecular weight (both a larger lipophilic and a larger hydrophilic group); they found such molecules had less adsorption and

\section{Interpretation of Adsorption Maxima}

In studies of surfactant adsorption from aqueous solutions onto kaolinite clay surface, a number of investigators have reported finding of adsorption maxima. Hanna et al. (1976) suggested that the maximum may be an experimental artifact, arising from precipitation accompanying adsorption, surfactant trapping in dead end pores, or loss of adsorbed surfactant during the washing step sometimes taken to remove nonadsorbed surfactant. It seems unlikely that any one of these mechanisms could be the general explanation for all of the observations. Mukerjee and Anavil (19) have both suggested that micellar exclusion may be the cause of the maximum. This basic concept, not supported by quantitative arguments, proposes that a double layer reples micelles from pores in the substrate, thereby reducing the total adsorption at high concentration.

Recently, Yang and coworkers (2002) proposed a mechanism responsible for the adsorption maximum. When aqueous surfactant solution contacts with kaolinite clay, the concentration of $\mathrm{Ca}^{2+}$ and $\mathrm{Mg}^{2+}$ in the solution increases due to the ion-exchange process. At low surfactant concentration, surfactant molecules exist in the solution as monomers. Meanwhile, the adsorption of surfactant increases with increase in the concentration. At high concentration, when the adsorption density of surfactant on kaolinite surface is high enough to make the concentration product of adsorbed surfactant anion piece and $\mathrm{Ca}^{2+}$

and $\mathrm{Mg}^{2+}$ greater than their solubility products, precipitate will be formed on the kaolinite surface. However, for further increase in surfactant concentration in aqueous solution, surfactant molecules aggregate and micelles are formed in the bulk solution, which have a strong ability to enhance the solubility of the precipitate. Consequently, the micelles compete with the surface of kaolinie for the adsorbed molecules and dissolve some of the precipitate on the surface. As a result, the adsorption decreases after a maximum. 


\section{$\underline{\text { Selected Literature Data about Surfactant Adsorption onto Kaolinite }}$}

Below is a summary table of literature surfactant adsorption data results:

\begin{tabular}{|c|c|c|c|c|c|c|c|c|}
\hline \multirow{2}{*}{ Surfactant } & \multirow{2}{*}{$\begin{array}{l}\text { Soln./Solid } \\
\text { (w/w) }\end{array}$} & \multirow{2}{*}{$\begin{array}{c}\text { Temp } \\
\left({ }^{\circ} \mathrm{C}\right)\end{array}$} & \multirow{2}{*}{$\begin{array}{l}\text { Time } \\
\text { (hour) }\end{array}$} & \multirow{2}{*}{$\mathrm{pH}$} & \multirow{2}{*}{$\begin{array}{l}\text { Additives } \\
(\mathrm{g} / \mathrm{L})\end{array}$} & \multirow{2}{*}{$\begin{array}{l}\text { Ionic } \\
\text { Strength } \\
\text { (M) }\end{array}$} & \multicolumn{2}{|c|}{ Maximum Adsorption } \\
\hline & & & & & & & $(\mathrm{mg} / \mathrm{g})$ & $\left(\mathrm{mol} / \mathrm{cm}^{2}\right)$ \\
\hline $\begin{array}{l}\text { Petroleum Sulfonate } \\
\text { (TRS 10-80) } \\
\text { (Witco Co.) } \\
\text { Ave. M.W.=405 }\end{array}$ & 10.0 & 30.0 & 4.0 & $>7$ & $\begin{array}{cc}\mathrm{NaCl} & 10.0 \\
\mathrm{Na}_{2} \mathrm{CO}_{3} & 10.0 \\
\text { Butanol } & 30.0\end{array}$ & 0.448 & 10.0 & $1.07 \times 10^{-10 *}$ \\
\hline \multirow{3}{*}{$\begin{array}{l}\text { Petroleum Sulfonate } \\
\text { (TRS 10-80) } \\
\text { (Witco Co.) } \\
\text { Ave. M.W. }=405\end{array}$} & 10.0 & 30.0 & 4.0 & 7.0 & $\begin{array}{cc}\mathrm{NaCl} & 26.2 \\
\text { Butanol } & 30.0\end{array}$ & 0.448 & 54.0 & $5.80 \times 10^{-10}$ \\
\hline & 10.0 & 30.0 & 4.0 & 7.0 & $\begin{array}{cc}\mathrm{NaCl} & 21.0 \\
\text { Butanol } & 30.0\end{array}$ & 0.360 & 39.8 & $4.27 \times 10^{-10}$ \\
\hline & 10.0 & 30.0 & 4.0 & 11.2 & $\begin{array}{ll}\mathrm{NaCl} & 10.0 \\
\mathrm{Na}_{2} \mathrm{CO}_{3} & 10.0 \\
\text { Butanol } & 30.0\end{array}$ & 0.448 & 8.8 & $0.94 \times 10^{-10}$ \\
\hline \multirow{3}{*}{$\begin{array}{l}\text { Petroleum Sulfonate } \\
\text { (TRS 10-80) } \\
\text { (Witco Co.) } \\
\text { Ave. M.W. }=405\end{array}$} & 10.0 & 30.0 & 4.0 & 11.2 & $\begin{array}{cc}\mathrm{NaCl} & 10.0 \\
\mathrm{Na}_{2} \mathrm{CO}_{3} & 10.0 \\
\text { Butanol } & 30.0\end{array}$ & 0.448 & 12.1 & $1.29 \times 10^{-10^{*}}$ \\
\hline & 10.0 & 30.0 & 4.0 & 11.1 & $\begin{array}{cc}\mathrm{NaCl} & 10.0 \\
\mathrm{Na}_{2} \mathrm{CO}_{3} & 5.0 \\
\text { Butanol } & 30.0\end{array}$ & 0.310 & 5.5 & $0.59 \times 10^{-10}$ \\
\hline & 10.0 & 30.0 & 4.0 & 10.9 & $\begin{array}{lc}\mathrm{NaCl} & 10.0 \\
\mathrm{Na}_{2} \mathrm{CO}_{3} & 2.0 \\
\text { Butanol } & 30.0\end{array}$ & 0.226 & 2.4 & $0.26 \times 10^{-10}$ \\
\hline \multirow{2}{*}{$\begin{array}{l}\text { Petroleum Sulfonate } \\
\text { (TRS 10-80) } \\
\text { (Witco Co.) } \\
\text { Ave. M.W. }=405\end{array}$} & 10.0 & 30.0 & 4.0 & 12.2 & $\begin{array}{ll}\mathrm{NaCl} & 24.3 \\
\mathrm{NaOH} & 1.32 \\
\text { Butanol } & 30.0\end{array}$ & 0.448 & 42.4 & $4.53 \times 10^{-10}$ \\
\hline & 10.0 & 30.0 & 4.0 & 11.3 & $\begin{array}{ll}\mathrm{NaCl} & 24.3 \\
\mathrm{Na}_{2} \mathrm{CO}_{3} & 15.8 \\
\text { Butanol } & 30.0\end{array}$ & 0.448 & 4.53 & $0.49 \times 10^{-10}$ \\
\hline \multirow{4}{*}{$\begin{array}{l}\text { Petroleum Sulfonate } \\
\text { (TRS 10-80) } \\
\text { (Witco Co.) } \\
\text { Ave. M.W. }=405\end{array}$} & 10.0 & 30.0 & 4.0 & 7.0 & $\begin{array}{ll}\mathrm{NaCl} & 26.2 \\
\mathrm{Na}_{2} \mathrm{SO}_{4} & 0.00 \\
\text { Butanol } & 30.0\end{array}$ & 0.448 & 54.8 & $5.88 \times 10^{-10}$ \\
\hline & 10.0 & 30.0 & 4.0 & 7.0 & $\begin{array}{ll}\mathrm{NaCl}_{1} & 13.1 \\
\mathrm{Na}_{2} \mathrm{SO}_{4} & 10.7 \\
\text { Butanol } & 30.0\end{array}$ & 0.448 & 39.6 & $4.24 \times 10^{-10}$ \\
\hline & 10.0 & 30.0 & 4.0 & 7.0 & $\begin{array}{ll}\mathrm{NaCl} & 6.55 \\
\mathrm{Na}_{2} \mathrm{SO}_{4} & 15.9 \\
\text { Butanol } & 30.0\end{array}$ & 0.448 & 17.4 & $1.87 \times 10^{-10}$ \\
\hline & 10.0 & 30.0 & 4.0 & 7.0 & $\begin{array}{ll}\mathrm{NaCl} & 0.00 \\
\mathrm{Na}_{2} \mathrm{SO}_{4} & 21.2 \\
\text { Butanol } & 30.0\end{array}$ & 0.448 & 12.2 & $1.20 \times 10^{-10}$ \\
\hline
\end{tabular}




\begin{tabular}{|c|c|c|c|c|c|c|c|c|}
\hline \multirow{2}{*}{ Surfactant } & \multirow{2}{*}{$\begin{array}{c}\text { Soln./Solid } \\
\text { (w/w) }\end{array}$} & \multirow{2}{*}{$\begin{array}{c}\text { Temp } \\
\left({ }^{\circ} \mathrm{C}\right)\end{array}$} & \multirow{2}{*}{$\begin{array}{l}\text { Time } \\
\text { (hour) }\end{array}$} & \multirow{2}{*}{$\mathrm{pH}$} & \multirow{2}{*}{$\begin{array}{l}\text { Additives } \\
(\mathrm{g} / \mathrm{L})\end{array}$} & \multirow{2}{*}{$\begin{array}{l}\text { Ionic } \\
\text { Strength } \\
\text { (M) }\end{array}$} & \multicolumn{2}{|c|}{ Maximum Adsorption } \\
\hline & & & & & & & $(\mathrm{mg} / \mathrm{g})$ & $\left(\mathrm{mol} / \mathrm{cm}^{2}\right)$ \\
\hline \multirow{3}{*}{$\begin{array}{c}\text { Sodium Oleate } \\
\mathrm{C}_{18} \mathrm{H}_{33} \mathrm{O}_{2}^{-} \mathrm{Na}^{+} \\
\mathrm{M} . \mathrm{W} .=304 \\
\mathrm{CMC}=4.8 \times 10^{-3} \mathrm{M}\end{array}$} & 9.0 & 25.0 & 24 & 7.0 & N/A & & 36.1 & $5.76 \times 10^{-10}$ \\
\hline & 9.0 & 35.0 & 24 & 7.0 & N/A & & 27.5 & $4.39 \times 10^{-10}$ \\
\hline & 9.0 & 45.0 & 24 & 7.0 & N/A & & 21.5 & $3.43 \times 10^{-10}$ \\
\hline \multirow{3}{*}{$\begin{array}{c}\text { Sodium Oleate } \\
\mathrm{C}_{18} \mathrm{H}_{33} \mathrm{O}_{2} \mathrm{Na}^{+} \\
\mathrm{M} . \mathrm{W} .=304 \\
\mathrm{CMC}=4.8 \times 10^{-3} \mathrm{M}\end{array}$} & 9.0 & 35.0 & 24 & 7.0 & $\mathrm{NaCl} 1.0$ & & 37.6 & $6.01 \times 10^{-10}$ \\
\hline & 9.0 & 35.0 & 24 & 7.0 & $\mathrm{NaCl} \quad 0.1$ & & 29.3 & $4.84 \times 10^{-10}$ \\
\hline & 9.0 & 35.0 & 24 & 7.0 & $\mathrm{NaCl} 0.0$ & & 28.6 & $4.56 \times 10^{-10}$ \\
\hline \multirow{5}{*}{$\begin{array}{c}\text { Sodium Oleate } \\
\mathrm{C}_{18} \mathrm{H}_{33} \mathrm{O}_{2} \mathrm{Na}^{+} \\
\mathrm{M} . \mathrm{W} .=304 \\
\mathrm{CMC}=4.8 \times 10^{-3} \mathrm{M}\end{array}$} & 9.0 & 35.0 & 24 & 7.0 & HPAM 1.0 & & 11.5 & $1.88 \times 10^{-10}$ \\
\hline & 9.0 & 35.0 & 24 & 7.0 & $\begin{array}{c}\text { n-Butanol } \\
30.0 \mathrm{~g} / \mathrm{L}\end{array}$ & & 13.9 & $2.22 \times 10^{-10}$ \\
\hline & 9.0 & 35.0 & 24 & 7.0 & $\begin{array}{c}\text { n-Pentanol } \\
30.0 \mathrm{~g} / \mathrm{L}\end{array}$ & & 16.2 & $2.58 \times 10^{-10}$ \\
\hline & 9.0 & 35.0 & 24 & 11.3 & $\mathrm{C}_{12} \mathrm{EO}_{6} \mathrm{OH} \quad 1.0$ & & 28.1 & $4.49 \times 10^{-10}$ \\
\hline & 9.0 & 35.0 & 24 & 12.2 & $\begin{array}{ll}\mathrm{NaCl} & 0.0\end{array}$ & & 28.6 & $4.56 \times 10^{-10}$ \\
\hline 4- $\Phi \mathrm{C}_{10} \mathrm{ABS}$ & 10.0 & 30.0 & 24 & 7.0 & $\mathrm{NaCl} 10.0$ & 0.171 & 5.73 & $1.06 \times 10^{-10}$ \\
\hline 4- $\Phi \mathrm{C}_{11} \mathrm{ABS}$ & 10.0 & 30.0 & 24 & 7.0 & $\mathrm{NaCl} 10.0$ & 0.171 & 7.96 & $1.48 \times 10^{-10}$ \\
\hline 4- $\Phi \mathrm{C}_{12} \mathrm{ABS}$ & 10.0 & 30.0 & 24 & 7.0 & $\mathrm{NaCl} 10.0$ & 0.171 & 12.76 & $2.45 \times 10^{-10}$ \\
\hline $3-\Phi \mathrm{C}_{11} \mathrm{ABS}$ & 10.0 & 30.0 & 24 & 7.0 & $\mathrm{NaCl} 10.0$ & 0.171 & 6.17 & $1.22 \times 10^{-10}$ \\
\hline $4-\Phi \mathrm{C}_{11} \mathrm{ABS}$ & 10.0 & 30.0 & 24 & 7.0 & $\mathrm{NaCl} 10.0$ & 0.171 & 7.34 & $1.46 \times 10^{-10}$ \\
\hline $5-\Phi \mathrm{C}_{11} \mathrm{ABS}$ & 10.0 & 30.0 & 24 & 7.0 & $\mathrm{NaCl} 10.0$ & 0.171 & 8.41 & $1.67 \times 10^{-10}$ \\
\hline $3-\Phi \mathrm{C}_{12} \mathrm{ABS}$ & 10.0 & 30.0 & 24 & 7.0 & $\mathrm{NaCl} 10.0$ & 0.171 & 9.34 & $1.78 \times 10^{-10}$ \\
\hline 4- $\Phi \mathrm{C}_{12} \mathrm{ABS}$ & 10.0 & 30.0 & 24 & 7.0 & $\mathrm{NaCl} 10.0$ & 0.171 & 12.68 & $2.41 \times 10^{-10}$ \\
\hline 5- $\Phi \mathrm{C}_{12} \mathrm{ABS}$ & 10.0 & 30.0 & 24 & 7.0 & $\mathrm{NaCl} 10.0$ & 0.171 & 22.53 & $4.29 \times 10^{-10}$ \\
\hline $6-\Phi \mathrm{C}_{12} \mathrm{ABS}$ & 10.0 & 30.0 & 24 & 7.0 & $\mathrm{NaCl} 10.0$ & 0.171 & 24.35 & $4.63 \times 10^{-10}$ \\
\hline
\end{tabular}




\begin{tabular}{|c|c|c|c|c|c|c|c|c|}
\hline \multirow{2}{*}{ Surfactant } & \multirow{2}{*}{$\begin{array}{c}\text { Soln./Solid } \\
\text { (w/w) }\end{array}$} & \multirow{2}{*}{$\begin{array}{c}\text { Temp } \\
\left({ }^{\circ} \mathrm{C}\right)\end{array}$} & \multirow{2}{*}{$\begin{array}{l}\text { Time } \\
\text { (hour) }\end{array}$} & \multirow{2}{*}{$\mathrm{pH}$} & \multirow{2}{*}{$\begin{array}{l}\text { Additives } \\
(\mathrm{g} / \mathrm{L})\end{array}$} & \multirow{2}{*}{$\begin{array}{l}\text { Ionic } \\
\text { Strength } \\
\text { (M) }\end{array}$} & \multicolumn{2}{|c|}{ Maximum Adsorption } \\
\hline & & & & & & & $(\mathrm{mg} / \mathrm{g})$ & $\left(\mathrm{mol} / \mathrm{cm}^{2}\right)$ \\
\hline $\begin{array}{c}\text { Cetyl Prydinium } \\
\text { Chloride (CPC) } \\
\text { M.W. }=339.5\end{array}$ & 15.0 & 25.0 & 24 & 7.0 & N/A & & 30.8 & $7.56 \times 10^{-10}$ \\
\hline $\begin{array}{c}\text { Sodium } \\
\text { Dodecylbenzene } \\
\text { Sulfonate (SDDBS) } \\
\text { Ave. M.W.=348 }\end{array}$ & 15.0 & 25.0 & 24 & 7.0 & N/A & & 2.30 & $5.51 \times 10^{-11}$ \\
\hline \multirow{6}{*}{$\begin{array}{c}\text { Sodium } \\
\text { Dodecylbenzene } \\
\text { Sulfonate (SDDBS) } \\
\text { Ave. M.W.=348 }\end{array}$} & $\begin{array}{l}9.0 \text { (Untreated } \\
\text { Kaolinite) }\end{array}$ & 30.0 & 24 & 4.6 & $\mathrm{NaCl}: 0.59$ & $0.01 \mathrm{M}$ & 8.68 & $2.55 \times 10^{-10}$ \\
\hline & $\begin{array}{c}9.0 \text { (Treated } \\
\text { Kaolinite) }\end{array}$ & 30.0 & 24 & 4.6 & $\mathrm{NaCl}: 0.59$ & $0.01 \mathrm{M}$ & 16.7 & $4.89 \times 10^{-10}$ \\
\hline & $\begin{array}{l}9.0 \text { (Untreated } \\
\text { Kaolinite) }\end{array}$ & 30.0 & 24 & 6.6 & $\mathrm{NaCl}: 0.59$ & $0.01 \mathrm{M}$ & 3.34 & $0.98 \times 10^{-10}$ \\
\hline & $\begin{array}{c}9.0 \text { (Treated } \\
\text { Kaolinite) }\end{array}$ & 30.0 & 24 & 6.6 & $\mathrm{NaCl}: 0.59$ & $0.01 \mathrm{M}$ & 4.22 & $1.24 \times 10^{-10}$ \\
\hline & $\begin{array}{l}9.0 \text { (Untreated } \\
\text { Kaolinite) }\end{array}$ & 30.0 & 24 & 10.2 & $\mathrm{NaCl}: 0.59$ & $0.01 \mathrm{M}$ & 1.20 & $0.35 \times 10^{-10}$ \\
\hline & $\begin{array}{c}9.0 \text { (Treated } \\
\text { Kaolinite) }\end{array}$ & 30.0 & 24 & 10.2 & $\mathrm{NaCl}: 0.59$ & $0.01 \mathrm{M}$ & 2.28 & $0.67 \times 10^{-10}$ \\
\hline \multirow{5}{*}{$\begin{array}{c}\text { Sodium } \\
\text { Dodecylbenzene } \\
\text { Sulfonate (SDDBS) } \\
\text { Ave. M.W.=348 }\end{array}$} & $\begin{array}{c}4.0 \text { (Untreated } \\
\text { Kaolinite) }\end{array}$ & 30.0 & 72 & 4.5 & $\mathrm{NaCl}: 0.59$ & $0.01 \mathrm{M}$ & 16.5 & $4.83 \times 10^{-10}$ \\
\hline & $\begin{array}{c}4.0 \text { (Treated } \\
\text { Kaolinite) }\end{array}$ & 30.0 & 72 & 4.5 & $\mathrm{NaCl}: 0.59$ & $0.01 \mathrm{M}$ & 17.1 & $5.00 \times 10^{-10}$ \\
\hline & $\begin{array}{c}4.0 \text { (Treated } \\
\text { Kaolinite) }\end{array}$ & 30.0 & 72 & 5.8 & $\mathrm{NaCl}: 0.59$ & $0.01 \mathrm{M}$ & 6.52 & $1.91 \times 10^{-10}$ \\
\hline & $\begin{array}{c}4.0 \text { (Treated } \\
\text { Kaolinite) }\end{array}$ & 30.0 & 72 & 6.6 & $\mathrm{NaCl}: 0.59$ & $0.01 \mathrm{M}$ & 4.94 & $1.45 \times 10^{-10}$ \\
\hline & $\begin{array}{c}4.0 \text { (Treated } \\
\text { Kaolinite) }\end{array}$ & 30.0 & 72 & 10.0 & $\mathrm{NaCl}: 0.59$ & $0.01 \mathrm{M}$ & 2.26 & $0.66 \times 10^{-10}$ \\
\hline
\end{tabular}


Figure G-1 shows some of the literature kaolinite adsorption data. In this case, specifically for different isomers of a linear alkyl beneze sulfonate (Barakat, et. al., 1995). Note only the maximum adsorption levels are reported in the summary table.

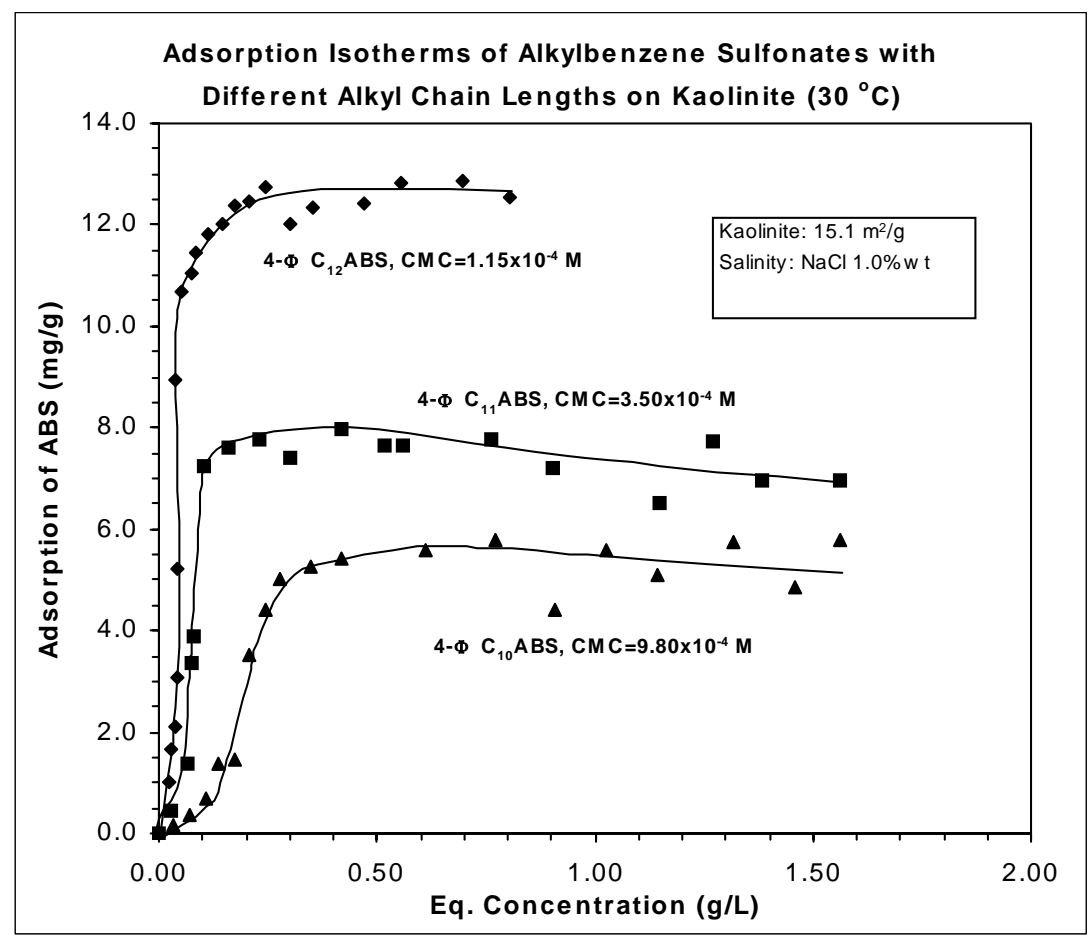

Figure G-1. Plot of alkylbenzene sulfonates adsorption onto kaolinite. These surfactants all have the $4^{\text {th }}$ carbon on their alkyl chain attached to the benzene ring. The total alkyl chain length is 10,11 , and 12 carbons, and the sulfonate group is para- to the alkyl chain.

The graph below uses the results from Figure G-1 to illustrate that the maximum, plateau adsorption increases with the alkyl chain length. This is consistent with the general rule of thumb that increasing the molecular weight of alkyl aromatic sulfonates will increase their tendency to adsorb onto clays. 


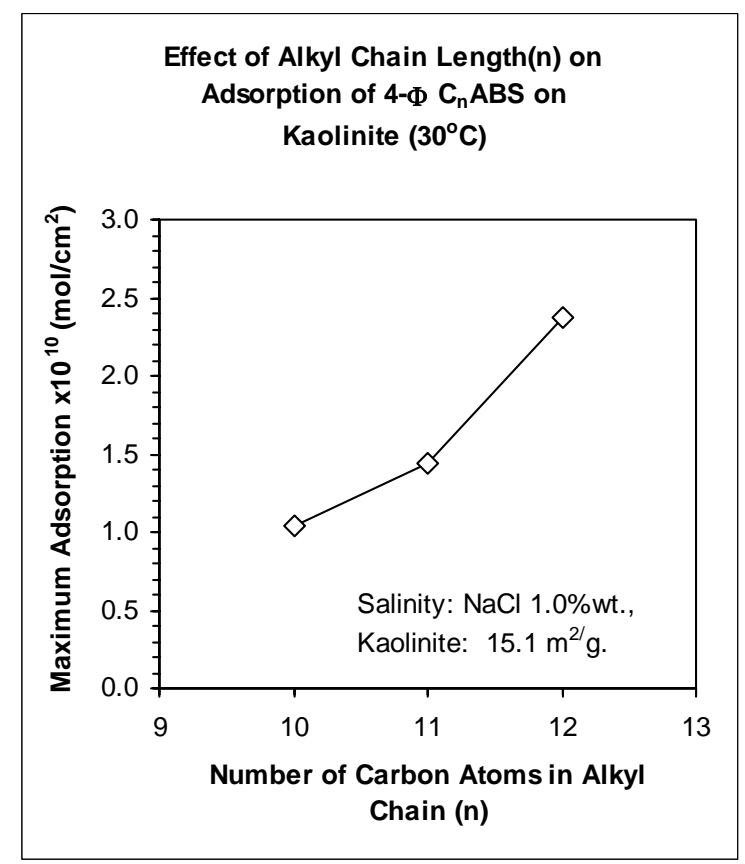

Figure G-2. Maximum, plateau adsorption levels for a series of linear alkyl benzene sulfonates. All have the $4^{\text {th }}$ carbon on the alkyl chain attached to the benzene ring.

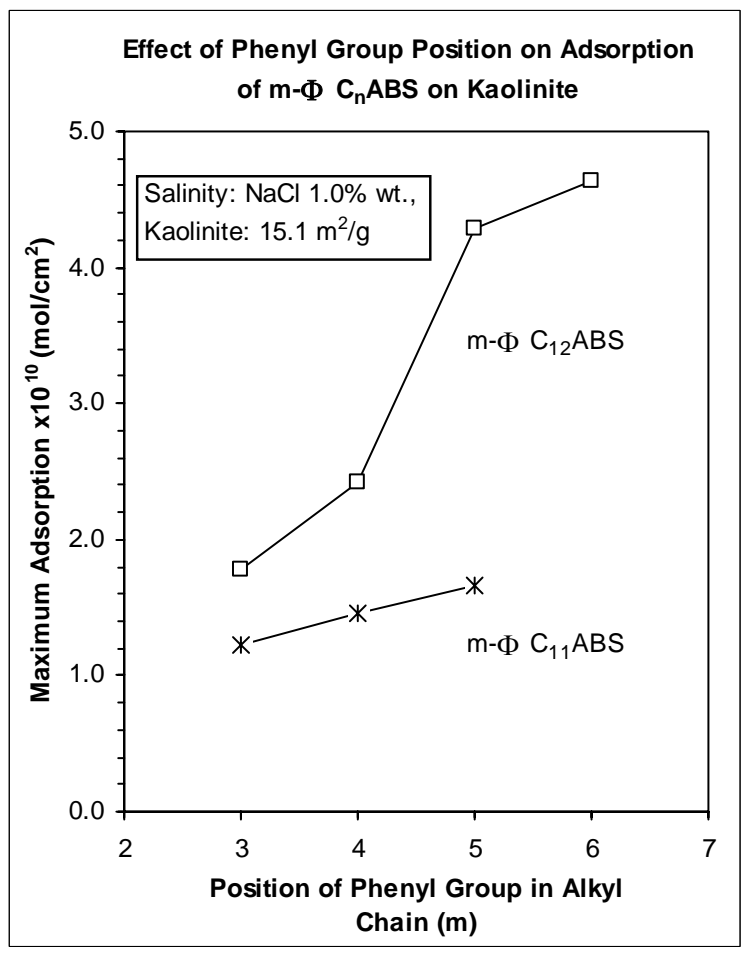

Figure G-3. Maximum, plateau adsorption levels for an akyl beneze sulfonate as a function of which carbon on the chain is attached to the benzene ring. 
Appendix B presents graphs of the surfactant adsorption data taken from various literature sources.

\section{H. References}

Abe, M., et. al. J. Colloid Interface Sci., 119 422, 1987.

Acosta, E., Uchiyama, H., Sabatini, D.A., and Harwell, J.H.: "The role of hydrophilic linkers", J. Surfactants and Detergents, 5, 151-157, April 2002.

Alami, E., Holmberg, K., and Eastoe, J.: "Adsorption Properties of Novel Gemini Surfactants with Nonidentical Head Groups”, J. Coll. Inter. Sci., 247, 447 - 455, 2002.

Andrews, C., et. al.: "Preliminary Studies of the Behavior of Some Commercially Available Surfactants in Hydrocarbon-Brine-Mineral Systems", Proceedings of the third European Symposium on Enhanced Oil Recovery, in Bournemouth, U.K., edited by F. John Fayers 62-80, 1981.

Aoudia, M., Wade, W., and Weerasooriya, J. Dispersion Sci. Technol., 16, 115-135, 1996.

Austad, T., et. al.: "Adsorption I. Competitive Adsorption of Mixtures of Ethoxylated Surfactants onto Kaolinite and Quartz”, J. Pet. Sci. Eng., 6, 107-124, 1991.

Austad, T.: "A Review of Retention Mechanisms of Ethoxylated Sulfonates in Reservoir Cores", paper SPE 25174, presented at SPE Symposium on Oilfield Chemistry, New Orleans, 2-5 March 1993.

Austad, T. and Strand, S.: "Chemical Flooding of Oil Reservoirs 4. Effects of temperature and pressure on the middle phase solubilization parameters close to optimum flood conditions", Colloids and Science A. Physiochemical and Engineering Aspects, 108, 243-252, 1996.

Austad, T., et. al.: "Chemical Flooding of Oil Reservoirs Part 9. Dynamic Adsorption of Surfactant onto Sandstone cores from Injection Water with and Without Polymer Present", Colloids and Science A. Physiochemical and Engineering Aspects, 127, 69-82, 1997.

Aveyard, R., et. al.: "Surface and colloid chemistry of systems containing pure sugar surfactant”, Langmuir, 14, 4699-4709, August 18, 1998.

Aveyard, R., Binks, B.P., and Fletcher, P.D.: "Interfacial Tension and Aggregate Structure in C12E5/Oil/Water Microemulsion Systems", Langmuir, 5, 1210-1217 (1989).

Balzer, D. and Kosswig, Tenside Surf. Det., 162561979. 
Balzer, D., Proceedings $2^{\text {nd }}$ Eurpoean Meeting, p.21, 1982.

Balzer, D., Tenside Surf. Det., 28, 419, 1991.

Bansel, V.K. and Shah, D.O., J. Colloid Interface Science, 85, 451, 1978.

Bansel, B.B., Hornof, V., and Neale, G.H., Can. J. Chem. Eng., 57, 203-210, 1979.

Barakat, Y, et.al.: "Apha Olefin Sulfonates for Enhanced Oil Recovery", $2{ }^{\text {nd }}$ European Symposium on EOR”, Paris, 5-10 November, 1982.

Barakat, Y., et. al., Indian J. of Chem. Tech., 2, May 1995.

Baviěre, M.; Ruaux, E.; Defives, D., paper SPE 21031, 327, 1991.

Baviěre, M, Bazin, B., and Mileo, J.C.: "Physicochemical propertie of sulfonated fatty acid esters for oil recovery by surfactant flooding", Coll. and Sci., 52, 301-313, 1991.

Baviere, M., Bazin, B., Nolk, C.: "Surfactants for EOR: Olefin Sulfonate Behavior at High Temperature and Hardness", paper 14933, Soc. Pet. Eng. J., 1988.

Baviere, M., Bazin, B., Mileo, J.C.: "Physicochemical Properties of Sulfonated Fatty Acid Ester for Oil Recovery of Surfactant Flooding", Colloids and Surfaces, 52, 301-313, 1991.

Berger, P. and Lee, C.H.: "Ultra-low Concentration Surfactants for Sandstone and Limestone Floods", paper SPE 75186, presented at SPE/DOE Symposium on Improved

Oil Recovery, Tulsa, 13-17 April 2002.

Berger, P.D. and C.H. Lee: "New Anionic Alkylaryl Surfactants Based on Olefin Sulfonic Acids", J. Surfactants, 45, 345, 2000.

Boek, E.S.; Coveney, P.V.; Skipper, N.T.; Langmuir, 11, 4629, 1995 a.

Boek, E.S.; Coveney, P.V.; Skipper, N.T.; J. Am. Chem. Soc., 117,12608, 1995 b.

Bolsman, T.A.B.M. and Daane, G.J.R.: "Effect of Surfactant Structure on Phase Behavior of Alkylxylenesulfonate/Crude-Oil/Brine Systems", SPE Res. Eng., 53-60, January 1986.

Borah, M.T. and Gregory, M.D.: "A Summary of the Big Muddy Field Low-Tension Flood Demonstration Project", paper SPE 17536 presented at the Rocky Mountain Regional Meeting, Casper, WY, 11-13 May, 1988.

Bou-Mikael, Sami, et. al.: "Minas Surfactant Field Trial Tests Two Newly Designed Surfactants with High EOR Potential", paper 62488, presented at SPE Asia Pacific Oil Conference, Brisbane, Australia, 16-18 October, 2000. 
Bourrel, M., and Schechter, R.S.: Microemulsions and Related Systems, Marcel Dekker, New York, 1988.

Cao, Y. and Huilin, L.: "Interfacial activity of a novel family of polymeric surfactants", European Polymer J., 38, 1457-1463, 2002.

Cases, J. M.; Villieras, F. $\quad$ Langmuir, 8, 1251, 1992.

Cash, R.L., et. al.: "Modeling Crude Oils for Low Interfacial Tension", paper SPE 5813, presented at the SPE Symposium on Improved Oil Recovery, 22-24 March, 1976.

Chattopadahyay, A.K., Shah, D.O., and Ghaicha, L.: "Double-Tailed Surfactants and Their Chain-Length Compatibility in Water-in-Oil Emulsions”, Langmuir, 8, 27-30, January 1992.

Chattopadahyay, A.K., Ghaicha, L, Oh, S.G., Knight, J., Moaddel, T., and Frieberg, S.E.: "Some Anomalies Between the Surfactants Comprising Linear and Branched Hydrocarbon Chains”, J. Indian Chemical Society, 70, 353-364, April-May 1993.

Chiu, Y.C. and Hwang, H.J.: "The Use of Carboxymethyl Ethoxylates in Enhanced Oil Recovery", Colloids and Surfaces, 28, 53-65, 1987.

Cole, E.L.: "An Evaluation of the Robinson M-1 Commercial Scale Demonstration Project of Enhanced Oil Recovery by Micellar-Polymer Flood", Report DOE/BC/1083010, U.S. DOE, Bartlesville, OK, December, 1988.

Conley, R. F.; Althoff, A. C., J. Colloid \& Interface Sci., 37(1), 186, 1971.

Delville, A.; Langmuir, 7, 547, 1997.

Desai, J.D. and Banat, I.M.: "Microbial Production of Surfactants and Their Commercial Potential”, Microbiology and Molecular Biology Reviews, 47-64, March 1997.

Doe, P.H. and Wade, W.H.: "Alkyl Benzene Sulfonates for Producing Low Interfacial Tensions Between Hydrocarbons and Water”, J. Colloid Interface Sci. 59, 525-531, $1977 \mathrm{a}$.

Doe, P.H., El-Emary, M., Wade, W.H., and Schechter, R.S.: "Surfactants for producing Low Interfacial Tensions I: Linear Alkyl Benzene Sulfonates", J. Colloid Interface Sci. 59, 570-577 1977b.

Doe, P.H., El-Emary, M., and Wade, W.H.: "Surfactants for Producing Low Interfacial Tensions II: Linear Alkyl Benzene Sulfonates with Additional Alkyl Substituents", J. Am. Chem Oil Chem Soc, 55, 505-512, 1978a. 
Doe, P.H., El-Emary, M., Wade, W.H., and Schechter, R.S.: "Surfactants for Producing Low Interfacial Tensions III: Di and Tri n-Alkylbenzenesulfonates”, J. Am. Chem Oil Chem Soc, 55, 513-520, 1978 b.

Doe, P.H., El-Emary, M., Wade, W.H., and Schechter, R.S.: Chapter 2 "The Influence of Surfactant Structure on Low Interfacial Tensions", from Chemistry of Oil Recovery, Johansen and Berg, ed., American Chemical Society, 1978c.

Donaldson, E.C., Chilingarian, G.V., and Yen, T.F., enhanced oil recovery, II processes and operations, Elsevier, New York, 1989.

Dowd, Timothy, Improved Oil Recovery, Interstate Oil Compact Commission, March, 1983.

Eicke, H. and Markovic, Z., J. Colloid Interface Sci., 76, 151, 1981.

Endo, H., et. al.: "Amphiphilic block copolymers as efficiency boosters in microemulsions: a SANS investigation of the role of polymers", Appl. Phys. A [Suppl.], S392 - S395, 2002.

Forster, T., Gucjenbiehl, B., Hensen, H., and von Rybinski, W., Progr, Colloid Polym. Sci. 101, 105, 1996.

Fukuda, K., Soderman, O., Lindman, B., and Shinoda, Langmuir, 9, 2921, 1993.

Gale, W.W. and Sandvik, E.I.: "Tertiary Surfactant Flooding: Petroleum Sulfonate Composition Efficacy Studies”, SPEJ 191-199, August, 1973.

Gale, W.W., Puerto, M.C., and Aschcraft, T.L.: "Propoxylated ethoxylated surfactants and method of recovery oil therewith", Exxon, U.S. Patent, No. 4,293,4289, October.6, 1981.

Gale, Maerker, J.W. and Gale, W.W.: “Design for Loudon”, SPE Res. Eng., 36, Feb. y

Gao, S., et. al.: "Alkaline/Surfactant/Polymer Pilot Performance of the West Central Daqing Oil Field”, SPE Reservoir Engineering, August 1996.

Ghaicha, L., et. al.: "Monolayers of Mixed Surfactants at the Oil-Water Interface, Hydrophobic interactions, and Stability of Water-in-Oil Emulsions", Langmuir, 11, 585$590,1995$.

Goldburg, A., Price, H., and Paul, G.W.: "Selection of Reservoirs Ameneable to Micellar Flooding”, EngDOE/BC/00048 and 00051-29, August, 1985. 
Graciaa A, et. al.: "Criteria for Structuring Surfactants to Maximize Solubilization of Oil and Water I: Commercial Non-ionics”, SPE/DOE paper 9815, presented at the Joint Symposium on EOR, Tulsa, OK, April 5-8, 1981.

Graciaa A, Lachaise J, Cucuphat C, Bourrel M, Salager JL.: "Improving Solubilization in Microemulsions with Additives.1. The Lipophilic Linker Role.” Langmuir, 9, 669, 1993.

Graciaa A, Lachaise J, Cucuphat C, Bourrel M, Salager JL.:” Improving Solubilization in Microemulsions With Additives.2. Long chain alcohol as lipophilic linkers:, Langmuir. 9, $3371,1993$.

Grim, R. E. "Clay Mineralogy” McGraw-Hill, New York, $2^{\text {nd }}$ Ed. p. 193, 1968.

Guyot, A., "Polymerizable Surfactants", Chapter 10 from Novel Surfactants, ed. K. Holmberg, Marcel Dekker, New York, 1998.

Hait, S.K. and Moulik, S.P.: "Gemini surfactants: a distinct class of self-assembling molecules”, Current Science, 82, 1101 - 1111, May, 2002.

Hanna, H. S.; Goyal, A.; Somasundaran, P. Paper No. 239, Presented at VII International Congress on Surface Active Substrances, Moscow, Russia, Sept. 12-18, 1976.

Hanna, H. S.; Somasundaran, P. $\quad$ J. Colloid \& Interface Sci., 70(1), 181, 1979.

Halbert, W.G.: "Miscible Waterflooding Using a Phosphate Ester Solubilizer", paper SPE 3697, presented at SPE the California Regional Meeting, Los Angeles, 4-5 November, 1971.

Hill, H.J. and Lake, L.W.: "Cation Exchange in Chemical Flooding: Part 3 Experimental”, Soc. Pet. Eng. J., 18, 445-456 (1978).

Hirasaki, G.J., et. al.: "Evaluation of the Salinity Gradient Concept in Surfactant Flooding”, Soc. Pet. Eng. J., 23, 486-500 (1983).

Holmberg, K., Novel Surfactants, Marcel Dekker, New York, 1998.

Hornof, V. and R. Hombek, R.: "Surface Active Agents Based on Propoxylated Ligonsulfonate”, J. App. Polym. Sci., 41, 2391-2398, 1990.

Hung, H. and Shreve, G.S.: "Effect of Hydrocarbon Phase on interfacial and Thermodynamic Properties of Two Anionic Glyycolipid Biosurfactants in Hydrocarbon/Water Systems", J. Phys. Chem B 105, 12596-12600, 2001.

Huh, C.: "Interfacial Tensions and Stabilizing Ability of a Microemulsion Phase that Coexists with Oil and Brine", J. Coll. Interface Sci., 71, 408-426, May 1979. 
Huh, C.: "Equilibrium of a Microemulsion That Coexists With Oil or Brine", Soc. Pet. Eng. J., 829 - 860, October 1983.

Jacobson, J.K., et. al.: "Low Interfacial Tensions Involving Mixtures of Surfactants", Paper SPE 6002, SPE Annual Meeting, New Orleans, LA, 3-6 October, 1976.

Jakobs, B., et. al.: "Amphiphillic Block Copolymers as Efficiency Boosters for Microemulsions", Langmuir, 15, 6707-6711, 1999.

Jayanti, Shekhar, et. al.: "Use of Surfactants to Recover Oils from Groundwater", SPE 66753, presented, SPE/EPA/DOE E\&P Environmental Conference, San Antonio, TX, 2628 February 2001.

Jayanti, S., et. al.: "Laboratory Evaluation of Custom-Designed Surfactants to Remediate NAPL Source Zones”, Environ. Sci. Technol., 36, 5491-5497, 2002.

Kalpakci, B., et. al., paper SPE/DOE 20220, presented at SPE/DOE Symposium on EOR, Tulsa, 1990.

Kahlweit, M.,: "General Patterns of the Phase Behavior of Mixtures of Water, Nonpolar Solvents, Amphiphiles, and Electrolytes, 2", Langmuir, 5, 305-315, 1989.

Kahlweit, M., Busse, G., and Faulhaber, B: "Preparing Nontoxic Microemulsions with Alkyl Monoglucosides and the Role of Alkanediols as Cosolvents", Langmuir, 12, 861$862,1996$.

Knagga, E.A., et. al.: "Petroleum Sulfonate Utilization in Enhanced Oil Recovery Systems”, SPE Paper 6006, New Orleans, LA, 3-6 October, 1976.

Kumar, A., Neale, G.H., and Hornof, V., Pulp Paper Can., 85, T180-T183, 1984.

Kunieda, H. and Yamagata, M.: "Mixing of Nonionic Surfactants at Water-Oil Interfaces in Miocroemulsions", Langmuir, 9, 3345-3351, 1993.

Kunieda, H., Nakano, A., and Akimaru, M.: "The Effect of Mixing of Surfactants on Solubilization in a Microemulsion System", J. Coll. and Int. Sci., 170, 78-84, 1995.

Kurumanda, K., Shoi, A., Harada, M.: "Dynamical behavior and structure of coincentrated water-in-oil microemulsions in the sodium bis(2-ethylhexyl)phosphate systems", J. Phys. Chem.., 99, 16982-16990, 1995.

Lake, L.W.: “A Technical Survey of Micellar Polymer Flooding”, presented at Enhanced Oil Recovery, Southern Methodist University, Dallas, Texas, November, 1984.

Lake, L.W.: Enhanced Oil Recovery, Prentice-Hall, Inc., 1989. 
Lewis, S.J.,Verkruyse, L.A., and Salter, S.J.: "Selection of Nonionic Surfactants for Minimal Adsorption and Maximized Solubilization", paper SPE/DOE 14910, presented at SPE/DOE Symposium on EOR, Tulsa, OK, April 20-23, 1986.

Li, B. and Ruckenstein, E.: "Adsorption of Ionic Surfactants on Charged Solid Surfaces from Aqueous Solutions”, Langmuir, 12, 5052-5063, 1996.

Lloyd, M. K.; Conley, R. F. Clays Clay Miner., 18, 37, 1970.

Luciani, L.; Denoyel, R. J. Colloid \& Interface Sci., 88, 75, 1997.

Luciani, L., Denoyel, R., and Rouquerol, J.: "Poly(ethoxy) anionic surfactants: micellization and adsorption at the solid/liquid interface", Colloids and Surfaces A: Physiochemical and Engineering Aspects, 178, 297-312, 2001.

Manne, S.; Gaub, H. E. Science, 270, 1480, 1995.

Martin, R. T. Proc. $9^{\text {th }}$ Natl. Conf. On Clay and Clay Mineralogy, Pergamon Press, New York, p.36, 1964.

Mannhardt, K, Schramm, L.L., and Novosad, J.J.: "Adsorption of anionic and amphoteric foam-forming surfactants on different rock types", Coll. and Surf., 68, 37-53, 1992.

Masahiko, A., et. al.: "Phase Behavior of Branched Tail Ethoxylated Carboxylate Surfactant/Electrolyte/Alkane Systems", J. Disp. Sci. and Tech., 8, 157-172, 1987.

Maurice, P.A.: "Application of atomic-force microscopy to environmental colloid and surface chemistry", Colloids and Science A: Physiochemical and Engineering Aspects, 107, 57-75 (1996).

Meyers, J.J., Pitts, M.J., and Wyatt, K.: "Alkaline-Surfactant-Polymer Flood of the West/Kiehl, Minnelusa Unit", paper SPE 24144 presented at the SPE/DOE Enhanced Oil Recovery Symposium, Tulsa, OK, 22-24 April, 1992.

Michels, A.M., et. al.: "Enhanced Waterflooding Design with Dilute Surfactant Concentration for North Sea Conditions", SPE Reservoir Engineering, (August 1996).

Mihailescu, M., et. al.: "Dynamics of bicontinuous microemulsion phases with and without amphiphilic block-coplymers", J. Chem. Phys., 115, 9563 - 9577, 2001.

Minana-Perez, M., Graciaa, A., Lachaise, J., and Salager, J., J. Colloids Surf. A, 100, 217-224, 1995.

Morgan, J.C., Schechter, R.S., and W.H. Wade: "Improved Oil Recovery by Surfactant and Polymer Flooding", D.O. Shan and R.S. Schechter, editors, Acad. Press, Inc, N.Y. (1977). 
Mukherjee, S. K.; Gangully, A. K. Ind. J. Phys., 24, 223, 1953.

Mukerjee, P.; Anavil, A. in “Adsorption at Interfaces” Mittal, K. L. Ed. P.107, Amer. Chem. Soc. Symp. Ser. No. 8, ACS, Washington, D. C. 1975

Nelson, R.C., and Pope, G.A.: "Phase Relationships in Chemical Flooding", Transactions, AIME, 265, 325-338 (1976).

Nevskaia, D. M.; Guerrero-Ruĩz, A.; "Adsorption of Polyoxyethylenic Surfactants on Quartz, Kaolin, and Dolomite: a Correlation between Surfactant Structure and Solid Surface Nature”, López-GonzÁlez, J. D., Colloid \& Interface Sci., 181, 571, 1996.

Nickel, D., von Rybinski, W., Kutschmann, E.M., Stubenrauch, C., and Findenegg, G.H.: "The Importance of the Emulsifying and Dispersing Capacity of Alkyl Polyglcosides for Applications in Detergent and Cleaning Agents", Fett/Lipid, 98, 363-369, 1996.

Osterioh, W.T. and Jante, M.J.: "Surfactant-Polymer Flooding with Anionic PO/EO Surfactant Microemulsions Containing Polyethylene Glycol Additives", paper SPE/DOE 24151, presented the SPE/DOE Enhanced Oil Recovery Symposium, Tulsa, 22-24 April, 1992.

Ottewill, R.H.: "Introduction" Surfactants. T.F. Tadros (ed.), Academic Press, San Francisco, 1-18 (1984).

Peltonen, L., Hirvonen, J., and Yliruusi, J.:, "The Behavior of Sorbitan Surfactants at the Water-Oil Interface: Straight-Chained Hydrocarbons from Pentanes to Dodecane as an Oil Phase", J, Coll. Interface Sci., 240, 272-276, 2001.

Pitts, M.J.: "Recent Field Work in the United States with Alkali-Surfactant", presented at the NSF Workshop, Use of Surfactants for Improved Petroleum Recovery, 22-23 October, 2001.

Peypoux, F., et. al.: "Recent trends in the biochemistry of surfactin", Appl. Microbiol Biotechanol (51) 533-563 (1999).

Radke, C..: "Fundamentals of Foam Flow in Porous Media", NSF Workshop report, Use of Surfactants for Improved Petroleum Recovery”, Houston, October 22-23, 2001.

Rahlm, R., Ochesner, U.A., Olvera, C., Braninger, M., Messner, P., Lam, J.S., and Soberon-chavez, G., "Cloning and function characterization of the Pseudomonas aeruginosa rhlC gene that encodes rhamnosyltransferase 2, an enzyme responsible for dithamnolipid biosynthesis", Mol. Microbio., 40:708-718 (2001)

Reppert, T.R.: "Second Ripley Surfactant Flood Pilot Test, SPE/DOE 20219, presented at SPE/DOE $7^{\text {th }}$ Symposium on Enhanced Oil Recovery, Tulsa, OK, 22-25 April, 1990. 
Reid, V. M.; Longman, G. E.; Heinech, E. Tenside, 4(9), 292, 1967; 5(3), 90, 1968.

Ruan, K., et. al.: "Interfacial properties of aqueous surfactant two-phase systems", Acta Chimica Sinica”, 60, 961-963, June 2002.

Russell, S.T. and A. Warshel, J. Mol. Biol., 185, 389, 1985

Sabatini, D.A., Acosta, E., and Harwell, J.H.: "Linker Molecules in Surfactant Mixtures", submitted to Current Opinion and Interface Science, February 15, 2003.

Salter, S.J.: “Criteria for Surfactant Selection in Micellar Flooding”, paper SPE 14106, SPE Intl. Meeeting on Petroleum Engineering, Beijing, March 17-20, 1986.

Scheutjens, J. M. H. M.; Fleer, G. J., J. Phys. Chem., 83, 1619, 1979.

Seredyuk, V., et. al., : "Adsorption of zwitterionnic gemini surfactants at the air-water and solid-water interfaces”, Coll. and Surf. A; Physiochem. Eng. Aspects, 203, 245 - 258, 2002.

Seright. R.: "Mobility Control by Polymers", NSF Workshop report, Use of Surfactants for Improved Petroleum Recovery", Houston, October 22-23, 2001.

Shah, D.O. and Schechter, R.S.: Improved Oil Recovery by Surfactant and Polymer Flooding, Academic Press, New York (1977).

Shankar, P.K., Bae, J.H., and Enick, R.M.: "Salinity Tolerance and Solution Property Correlations of Petroleum Sulfonate-Cosurfactant Blends", paper SPE 10600, presented at SPE Symposium on Oilfield and Geothermal Chemistry, Dallas, 25-27 January 1982.

Shuler, P.J., et. al.: "Improving Polymer Injectivity at West Coyote Field, California", SPE Reservoir Engineering, 271-280 (August 1987).

Shuler, P.J., Kuehne, D.L., and Lerner, R.M.: "Improving Chemical Flood Efficiency with Micellar/Alkaline/Polymer Processes", J. Pet. Tech., 41, 80-88 (January, 1989).

Skauge, A. and Palmgren, O.: "Phase Behavior and Solution Properties of Ethoxylated Anionic Surfactants", paper SPE 18499, presented at SPE Oilfield Chemistry Symposium, Houston, February 8-10, 1989.

Solans, C. and Garcia-Celma, M.J.: "Surfactants for Microemulsions", Current Opinion in Coll. \& Int. Sci., 2, 464-471, 1997.

Somasundaran, P. and Hanna, H.S.: "Adsorption of Sulfonates on Reservoir Rocks", paper SPE 7059, Soc. Pet. Eng. J., 1979. 
Somasundran, M.C., et. al.: "The Role of Surfactant Precipitation and Redissolution in the Adsorption of Sulfonate on Minerals", SPE 8263, SPE Annual Meeting, Las Vegas, NV, (1979)

Somasundaran, P., :"Adsorption of Surfactant Mixtures in IOR: Elucidation of Mechanism for Minimum Loss", NSF Workshop report, Use of Surfactants for Improved Petroleum Recovery”, Houston, October 22-23, 2001.

Son, J.E., Neale, G.H., and Hornof, V., Can. J. Chem. Eng., 60, 684-691, 1982.

Sottmann, T. and Stry, R.: "Shape Similarities of Ultra-Low Interfacial Tension Curves in Ternary Microemulsion Systems of the Water-Alkane- $\mathrm{C}_{\mathrm{i}} \mathrm{E}_{\mathrm{j}}$ Type", Ber. Bunsemges Phys. Chem., 100, 237-241, 1996.

Steytler, D.C., Sargeant, L., Welsh, G.E., Robinson, B.H., and Heenan, R.K.:

"Ammonium bis(ethylhexyl) phosphate: a new surfactant for microemulsions", Langmuir, 12, 5312-5318, 1996.

Strker, A.: "Seelction and Design of Ethoxylated Carboxylates for Chemical Flooding", work performed for DOE under agreement FC22-83FE60149, NIPER-449, DE90000213, January, 1990.

Sullivan, E.R., "Molecular genetics of biosurfactant production", Current Opinion in Biotechnology, 9:263-269 (1998).

Sunwoo, C.K. and Wade, W.: "Optimal Surfactant Structures for Cosurfactant-Free Microemulsion Systems”, J. Dispersion Sci. and Tech., 13 (5), 491-514, 1992.

Swartzen-Allen, S. L.; Matijević, E, Chem. Rev., 74(3), 385, 1974.

Taber, J.J., Martin, F.D., and Seright, R.S.: "EOR Screening Criteia Revisited - Part 2. Application and Impact of Oil Prices", SPE Reservoir Engineering (August 1996).

Tapas, K.D., Adv. Colloid Interface Sci., 59, 95-98, 1995.

Tauer, K.: "Reactive Surfactants", Polymeric Dispersions: Principles and Applications, 463-476, Klaver Academic Publishers, Netherlands, 1997.

Tauer, K.: "Block copolymers prepared by emulsion polymerization with poly(ethylene oxide azo-initiators", Polym. Adv. Technol. 6, 435-440, 1994.

Taugbol, K., van Ly, T., and Austad, T.: "Chemical flooding of oil reservoirs. 2. Dissociative surfactant-polymer interaction with a positive effect on oil recovery", Colloids and Science A, 103, 83, 1995.

Teppen, B.J.; Rasmussen, K.; Bertsch, P.M.; Miller, D.M.; Schafer, L.; J. Phys. Chem.B, 
101, 1579, 1997.

Teppen, B.J.; Yu, C.H.; Miller, D.M.; Schafer, L.; J. Comp. Chem.; 19, 144, 1998.

Thomas, R. K.; Progre. Colloid \& Polymer Sci.,103, 216, 1997.

Tiberg, F.; Jősson, B.; Tang, J. A.; Lindman, B. Langmuir, 10, 2294, 1994.

Tsubone, K. and Rosen, M.: "Structural Effect on Surface Activities of Anionic

Surfactants Having $N$-acyl- $N$-methylamide and Carboxylate Groups", J. Coll. Inter. Sci., 244, 394-398, 2001.

U.S. Patent 4,704,229, "Surface active compounds of the sulfobetaines family, their manufacture and use, particularly for enhanced oil recovery", November 3, 1987.

U.S. Patent 4,976,315, "Use of taurine additives in enhanced oil recovery with anionic surfactants", December 11, 1990.

U.S. Patent 4,985,154, "Process for the extraction of crude oil from an underground deposit using surfactants", January 15, 1991.

U.S. Patent 6,043, 391: "Anionic surfactants based on alkene sulfonic acid", March 28, 2000.

von Rybinski, W. and Hill, K.: "Alkyl Polyglycosides", from Novel Surfactants, ed. K. Holmberg, Mercel Dekker, 1998.

von Rybinski, W., Guckenbiehl, W., and Tesmann, H.: "Influence of co-surfactants on microemulsions with alkyl polyglycosides", Coll. And Surf. A, 142, 333-342, 1998..

Warner, R., et. al., Appl. Organaomet. Chem., in press.

Wang, D., et. al.: "An Alkaline/Surfactant/Polymer Field Test in a Reservoir with a Long-Term 100\% Water Cut", SPE 49018, presented at SPE Annual Meeting, New Orleans, 27-30, September (1998).

Wang, Y., et. al.: "Surfactants Oil Displacement System in High Salinity Formations: Research and Application", paper 70047, presented at SPE Permian Basin Oil and Gas Recovery Confernece, Midland, Texas, 15-16 May 2001.

Warshel, A. and S.T. Russell, Quart. Rev. Biophy., 17, 3, 1984

Warshel, A. . Aquist, Ann. Rev., Biophys. Chem., 20, 267, 1991

Wellington, S.L. and Richardson, E.A.: "Low Surfactant Concentration Waterflooding", paper SPE 30748, presented at SPE Fall Meeting, Dallas, 22-25 October 1995. 
Wu, W., et.al.: "Design and Optimization of Low-Cost Chemical Flooding", paper SPE/DOE 35355, presented at Symposium on Improved Oil Recovery, Tulsa, OK, 21-24 April, 1996.

Xu, G.-Y.; Yuan, S.-L.; Wang, Y.-B. J. Dispersion Sci. \& Tech., 22(4), 355, 2001.

Yalamanchili, M.R., et. al.: "Use of atomic force microscopy in particle science and technology research", Colloids and Science A: Physiochemical and Engineering Aspects, 133, 77-88 (1998).

Yang, C.-Z.; Yan, H.-K.; Li, G.-Z.; Cui, G.-Z.; Yuan, H. in "Fundamental and Advances in Combined Chemical Flooding" (in Chinese), p. 174, Yu, G.-Y. et al. Ed. China Petroleum Press, Beijing, 2002.

Yiv, S. and Zana, R., J. Colloid Interface Sci., 77, 449, 1980.

Zana, R., "Dimeric (Gemini) Surfactants", Chapter 8, Novel Surfactants, ed. K. Holmberg, Marcel Dekker, New York, 1998.

Zana, R.: "Dimeric and oligomeric surfactants. Behavior at interfaces and in aqueous solution: a review”, Adv. Coll. Interface Sci., 97, 205-253, 2002. 
LITERATURE REVIEW

APPENDIX A. FURTHER LITERATURE IFT DATA

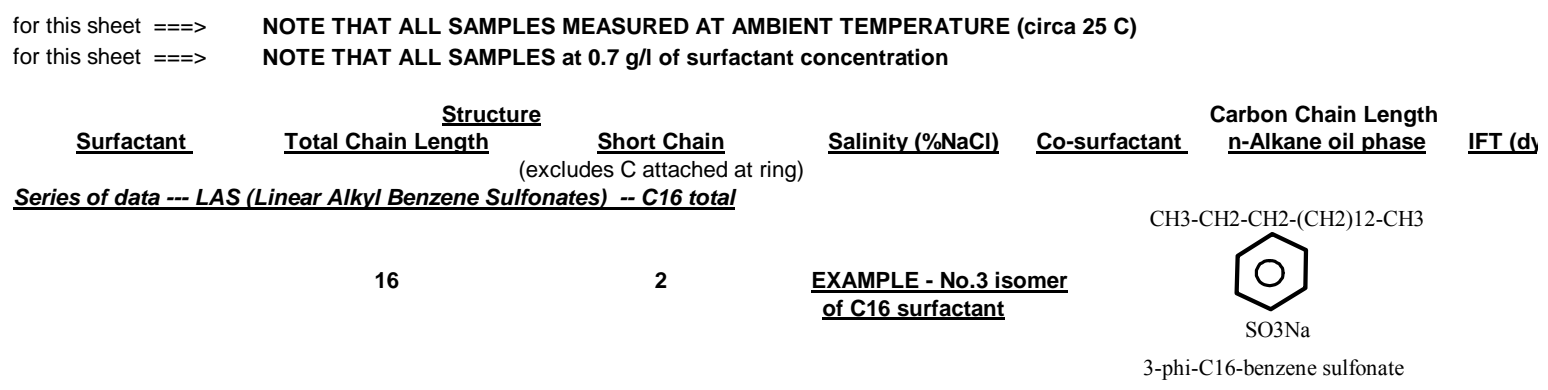

2-phi-C16 LAS

2-phi-C16 LAS

2-phi-C16 LAS

2-phi-C16 LAS

2-phi-C16 LAS

2-phi-C16 LAS

2-phi-C16 LAS

3-phi-C16 LAS

3-phi-C16 LAS

3-phi-C16 LAS

3-phi-C16 LAS

3-phi-C16 LAS

3-phi-C16 LAS

3-phi-C16 LAS

3-phi-C16 LAS

4-phi-C16 LAS

4-phi-C16 LAS

4-phi-C16 LAS

4-phi-C16 LAS

4-phi-C16 LAS

4-phi-C16 LAS

4-phi-C16 LAS

4-phi-C16 LAS

5-phi-C16 LAS

5-phi-C16 LAS

5-phi-C16 LAS

5-phi-C16 LAS

5-phi-C16 LAS

5-phi-C16 LAS

5-phi-C16 LAS

5-phi-C16 LAS

6-phi-C16 LAS

6-phi-C16 LAS

6-phi-C16 LAS

6-phi-C16 LAS

6-phi-C16 LAS

6-phi-C16 LAS

7-phi-C16 LAS

7-phi-C16 LAS

7-phi-C16 LAS

7-phi-C16 LAS

7-phi-C16 LAS

7-phi-C16 LAS

8-phi-C16 LAS

8-phi-C16 LAS

8-phi-C16 LAS

8-phi-C16 LAS

8-phi-C16 LAS

8-phi-C16 LAS
$2 \%$ iso-pentanol

$2 \%$ iso-pentano

$2 \%$ iso-pentanol

$2 \%$ iso-pentanol

$2 \%$ iso-pentanol

$2 \%$ iso-pentanol

$2 \%$ iso-pentanol

$2 \%$ iso-pentanol

$2 \%$ iso-pentanol

$2 \%$ iso-pentanol

$2 \%$ iso-pentanol

$2 \%$ iso-pentanol

$2 \%$ iso-pentanol

$2 \%$ iso-pentanol

$2 \%$ iso-pentanol

$2 \%$ iso-pentanol

$2 \%$ iso-pentanol

$2 \%$ iso-pentanol

$2 \%$ iso-pentanol

$2 \%$ iso-pentanol

$2 \%$ iso-pentanol

$2 \%$ iso-pentanol

$2 \%$ iso-pentanol

$2 \%$ iso-pentanol

$2 \%$ iso-pentanol

$2 \%$ iso-pentanol

$2 \%$ iso-pentanol

$2 \%$ iso-pentanol

$2 \%$ iso-pentanol

$2 \%$ iso-pentanol

$2 \%$ iso-pentanol

$2 \%$ iso-pentanol

$2 \%$ iso-pentanol

$2 \%$ iso-pentanol

$2 \%$ iso-pentanol

$2 \%$ iso-pentanol

$2 \%$ iso-pentanol

$2 \%$ iso-pentanol

$2 \%$ iso-pentanol

$2 \%$ iso-pentano

$2 \%$ iso-pentanol

$2 \%$ iso-pentano

$2 \%$ iso-pentanol

$2 \%$ iso-pentanol

$2 \%$ iso-pentanol

$2 \%$ iso-pentanol

$2 \%$ iso-pentanol

$2 \%$ iso-pentanol

$2 \%$ iso-pentanol

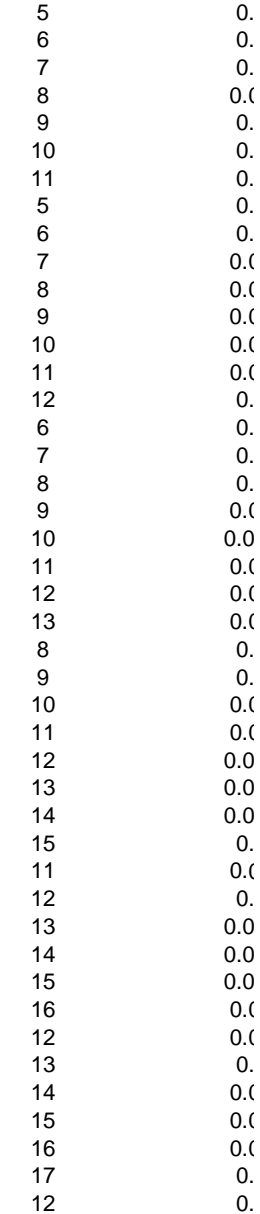

0 .

$\begin{array}{cc}13 & 0 . \\ 14 & 0 . \\ 15 & 0.1 \\ 16 & 0 . \\ 17 & 0 .\end{array}$


EXAMPLE - No.3 isomer

of C12 surfactant
응

Carbon Chain Length Salinity (\%NaCl) $\underline{\text { Co-surfactant }}$ n-Alkane oil phase

$2 \%$ iso-pentanol $2 \%$ iso-pentanol $2 \%$ iso-pentanol

$2 \%$ iso-pentanol

$2 \%$ iso-pentanol $2 \%$ iso-pentano $2 \%$ iso-pentano $2 \%$ iso-pentanol $2 \%$ iso-pentanol $2 \%$ iso-pentano $2 \%$ iso-pentanol $2 \%$ iso-pentanol $2 \%$ iso-pentano $2 \%$ iso-pentano $2 \%$ iso-pentano $2 \%$ iso-pentano $2 \%$ iso-pentano $2 \%$ iso-pentanol $2 \%$ iso-pentano $2 \%$ iso-pentano $2 \%$ iso-pentanol $2 \%$ iso-pentanol $2 \%$ iso-pentanol $2 \%$ iso-pentano $2 \%$ iso-pentanol $2 \%$ iso-pentanol $2 \%$ iso-pentano $2 \%$ iso-pentano $2 \%$ iso-pentanol $2 \%$ iso-pentano $2 \%$ iso-pentano $2 \%$ iso-pentanol
IFT (dyne/cm)

$$
\begin{gathered}
0.09 \\
0.045 \\
0.01 \\
0.011 \\
0.055 \\
0.09 \\
0.15 \\
0.21 \\
0.27 \\
0.12 \\
0.035 \\
0.008 \\
0.005 \\
0.035 \\
0.07 \\
0.12 \\
0.1 \\
0.06 \\
0.015 \\
0.0002 \\
0.006 \\
0.04 \\
0.15 \\
0.038 \\
0.0035 \\
0.018 \\
0.45 \\
0.09 \\
0.25 \\
0.03 \\
0.05 \\
0.1
\end{gathered}
$$

2-phi-C18 LAS

2-phi-C18 LAS

Series of data --- LAS (Linear Alkyl Benzene Sulfonates) -- NO co-surfactant and 1\% NaCl

$$
\begin{aligned}
& \text { 6-phi-C14 LAS } \\
& \text { 6-phi-C14 LAS } \\
& \text { 6-phi-C14 LAS } \\
& \text { 6-phi-C14 LAS } \\
& \text { 6-phi-C14 LAS } \\
& \text { 6-phi-C14 LAS } \\
& \text { 7-phi-C16 LAS } \\
& \text { 7-phi-C16 LAS } \\
& \text { 7-phi-C16 LAS } \\
& \text { 7-phi-C16 LAS } \\
& \text { 7-phi-C16 LAS } \\
& \text { 7-phi-C16 LAS } \\
& \text { 8-phi-C16 LAS } \\
& \text { 8-phi-C16 LAS } \\
& \text { 8-phi-C16 LAS } \\
& \text { 8-phi-C16 LAS } \\
& \text { 8-phi-C16 LAS } \\
& \text { 8-phi-C16 LAS }
\end{aligned}
$$

NONE
NONE
NONE
NONE
NONE
NONE
NONE
NONE
NONE
NONE
NONE
NONE
NONE
NONE
NONE
NONE
NONE

NONE

0.1
0.06
0.015
0.0002
0.006
0.04
0.015
0.009
0.0005
0.001
0.0025
0.004
0.017
0.005
0.0005
0.0009
0.002
0.003 
DIALKYL OR TRIALKYL BENZENE SULFONATES

for this sheet $===>$ for this sheet $===>$

Surfactant

1,4-Dibutyl-2-ethylbenzene 1,4-Dibutyl-2-ethylbenzene 1,4-Dibutyl-2-ethylbenzene 1,4-Dibutyl-2-propylbenzene 1,4-Dibutyl-2-propylbenzene 1,4-Dibutyl-2-propylbenzene 1,4-Dibutyl-2-propylbenzene 1,4-Dibutyl-2-propylbenzene 1,4-Dibutyl-2-propylbenzene 1,2,4-Tributylbenzene 1,2,4-Tributylbenzene 1,2,4-Tributylbenzene 1,2,4-Tributylbenzene 1,2,4-Tributylbenzene

1,4-Dibutyl-2-propylbenzene 1,4-Dibutyl-2-propylbenzene 1,4-Dibutyl-2-propylbenzene 1,2,4-Tributylbenzene 1,2,4-Tributylbenzene 1,2,4-Tributylbenzene 1,2,4-Tributylbenzene

1,2 Diethyl-4-heptylbenzene 1,2 Diethyl-4-heptylbenzene 1,2 Diethyl-4-heptylbenzene 1,2 Diethyl-4-heptylbenzene 1,2 Diethyl-4-heptylbenzene 1,2 Diethyl-4-heptylbenzene
NOTE THAT ALL SAMPLES MEASURED AT AMBIENT TEMPERATURE (circa $25 \mathrm{C}$ ) NOTE THAT ALL SAMPLES at $0.7 \mathrm{~g} / \mathrm{l}$ of surfactant concentration

Structure

\begin{tabular}{|c|c|c|c|}
\hline \multicolumn{2}{|c|}{$\underline{\text { Structure }}$} & \multirow{2}{*}{ Chain 2} & \multirow[b]{2}{*}{ Chain 3} \\
\hline Abbreviation & Chain 1 & & \\
\hline di bu et phi-S & 4 & 4 & 2 \\
\hline di bu et phi-S & 4 & 4 & 2 \\
\hline di bu et phi-S & 4 & 4 & 2 \\
\hline di bu prop phi-S & 4 & 4 & 3 \\
\hline di bu prop phi-S & 4 & 4 & 3 \\
\hline di bu prop phi-S & 4 & 4 & 3 \\
\hline di bu prop phi-S & 4 & 4 & 3 \\
\hline di bu prop phi-S & 4 & 4 & 3 \\
\hline di bu prop phi-S & 4 & 4 & 3 \\
\hline tri bu phi-S & 4 & 4 & 4 \\
\hline tri bu phi-S & 4 & 4 & 4 \\
\hline tri bu phi-S & 4 & 4 & 4 \\
\hline tri bu phi-S & 4 & 4 & 4 \\
\hline tri bu phi-S & 4 & 4 & 4 \\
\hline di bu prop phi-S & 4 & 4 & 3 \\
\hline di bu prop phi-S & 4 & 4 & 3 \\
\hline di bu prop phi-S & 4 & 4 & 3 \\
\hline tri bu phi-S & 4 & 4 & 4 \\
\hline tri bu phi-S & 4 & 4 & 4 \\
\hline tri bu phi-S & 4 & 4 & 4 \\
\hline tri bu phi-S & 4 & 4 & 4 \\
\hline di et hept phi-S & 2 & 2 & 7 \\
\hline di et hept phi-S & 2 & 2 & 7 \\
\hline di et hept phi-S & 2 & 2 & 7 \\
\hline di et hept phi-S & 2 & 2 & 7 \\
\hline di et hept phi-S & 2 & 2 & 7 \\
\hline di et hept phi-S & 2 & 2 & 7 \\
\hline
\end{tabular}

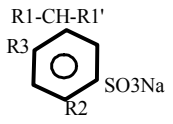

5 (p ethyl phenyl) dodecane 5 (p ethyl phenyl) dodecane 5 (p ethyl phenyl) dodecane 5 (p ethyl phenyl) dodecane 5 (p i-propyl henyl) dodecane 5 (p i-propyl henyl) dodecane 5 (p i-propyl henyl) dodecane 5 (p i-propyl henyl) dodecane 5(p i-propyl henyl) dodecane 5 ( $\mathrm{p}$ i-propyl henyl) dodecane 5(p i-propyl henyl) dodecane 5(p me phi) $\mathrm{C} 12$ 5 (p me phi) $\mathrm{C} 12$ 5(p me phi) C12 5(p me phi) C12 5(p i-prop phi) $\mathrm{C} 12$ 5(p i-prop phi) C12 5(p i-prop phi) C12 5(p i-prop phi) C12 5(p i-prop phi) C12 5(p i-prop phi) C12 5(p i-prop phi) C12
Salinity (\% $\mathrm{NaCl})$

Co-surfactant

$2 \%$ iso-pentanol

\% iso-pentanol

$2 \%$ iso-pentanol

$2 \%$ iso-pentanol

$2 \%$ iso-pentanol

$2 \%$ iso-pentano

$2 \%$ iso-pentanol

$2 \%$ iso-pentanol

$2 \%$ iso-pentanol

$2 \%$ iso-pentanol

$2 \%$ iso-pentanol

$2 \%$ iso-pentano

$2 \%$ iso-pentano

$2 \%$ iso-pentanol

NONE

NONE

NONE

NONE

NONE

NONE

NONE

$1 \%$ iso-pentanol

$1 \%$ iso-pentanol

$1 \%$ iso-pentanol

$1 \%$ iso-pentanol

$1 \%$ iso-pentanol

$1 \%$ iso-pentano
Carbon Chain Length

n-Alkane oil phase IFT (dyne/cm)

$\begin{array}{cc}5 & 1.5 \\ 7 & 3.5 \\ 9 & 5 \\ 5 & 0.1 \\ 6 & 0.06 \\ 7 & 0.004 \\ 8 & 0.008 \\ 9 & 0.09 \\ 10 & 0.2 \\ 5 & 0.15 \\ 7 & 0.08 \\ 8 & 0.04 \\ 9 & 0.003 \\ 10 & 0.06 \\ & \\ 5 & 0.4 \\ 7 & 0.7 \\ 10 & 1 \\ 5 & 0.09 \\ 7 & 0.15 \\ 8 & 0.3 \\ 9 & 1 \\ & \\ 8 & 0.15 \\ 10 & 0.06 \\ 11 & 0.015 \\ 12 & 0.005 \\ 13 & 0.1 \\ 16 & 0.15\end{array}$

NONE

NONE

NONE

NONE

NONE

NONE

NONE

NONE

NONE

NONE 
$0.7 \mathrm{~g} /$ 8-isomer $\mathrm{Cl} 6$ surfactant IFT

Room Temperature Saturated with $\mathrm{n}$-butanol

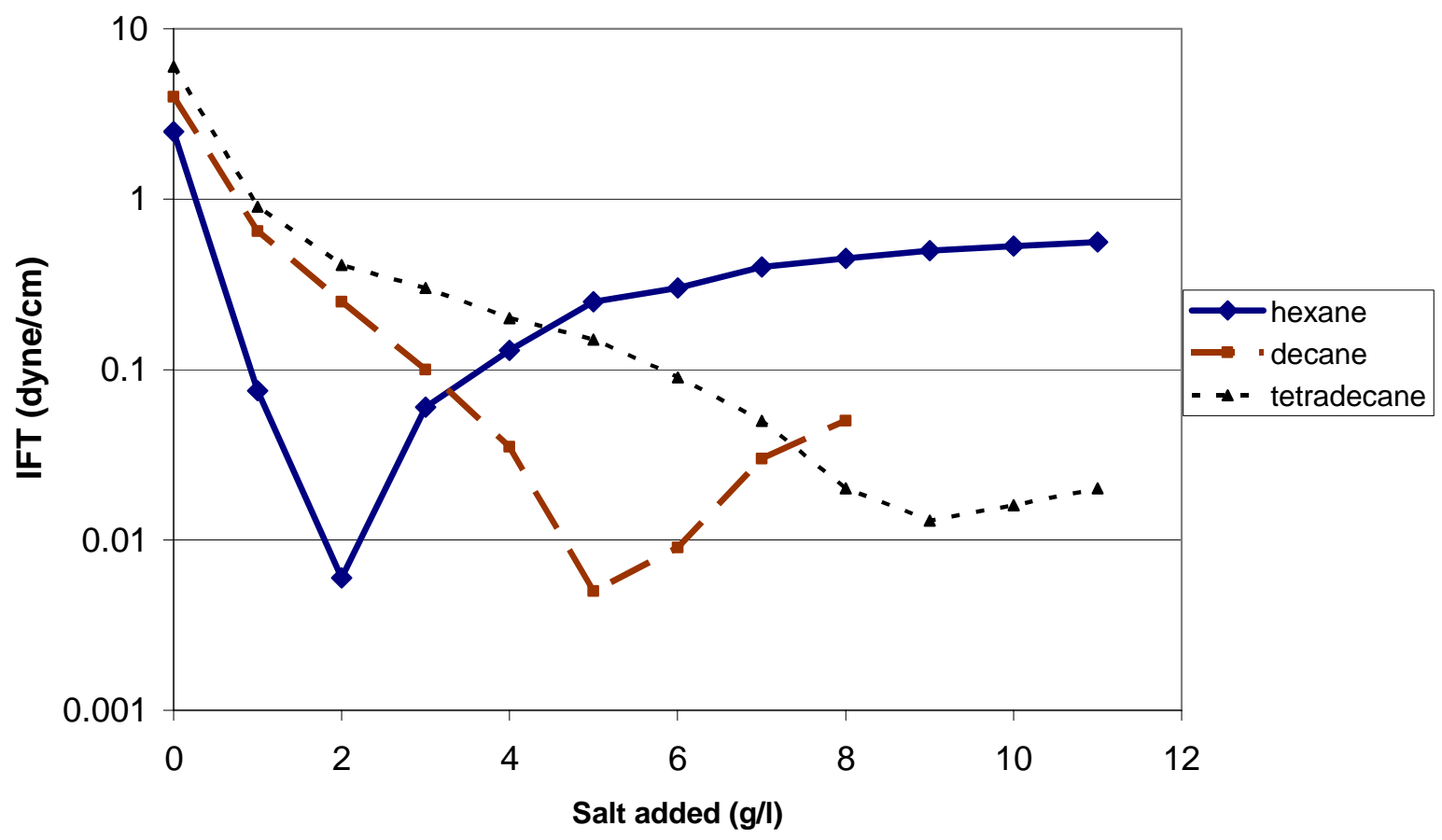

0.7 g/l 8-isomer C16 surfactant IFT

Room Temperature No Co-surfactant

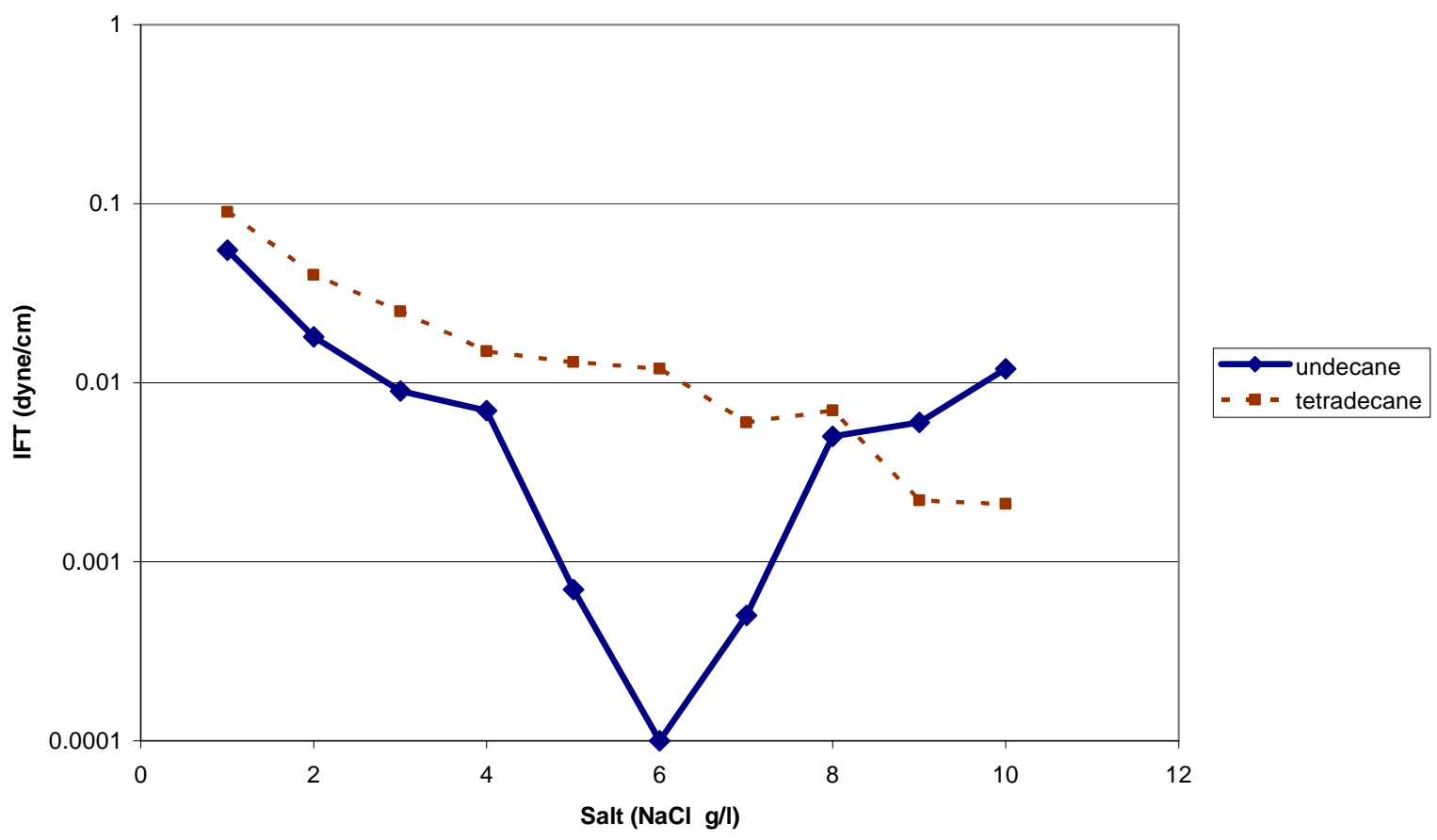




\section{LITERATURE REVIEW \\ APPENDIX B. \\ DETAILS OF ASSEMBLED LITERATURE SURFACTANT ADSORPTION DATA}

\begin{tabular}{|c|c|}
\hline \multicolumn{2}{|c|}{$\begin{array}{l}\text { Adsorption of TRS } 10-80 \text { (Deoiled) on Na-Kaolinite (Solid/Soln. }=0.1 \text { ) } \\
\text { This paper was presented at the SPE International Symposium on Oilfield }\end{array}$} \\
\hline \multicolumn{2}{|c|}{ Composition of the petroleum sulfonate TRS $10-80$ (Witco Co.) } \\
\hline 1. Surfonate(active material) & $80 \%$ wt \\
\hline 2. Unsulfonated oil & $11 \% w t$ \\
\hline 3. Water & $8 \% w t$ \\
\hline 4. Inorganic salts & $1 \%$ wt \\
\hline Average Molecular Weight & 405 \\
\hline
\end{tabular}

\begin{tabular}{|ccc|}
\hline \multicolumn{3}{|c|}{$\mathrm{CMC}=10 \mathrm{E}-2.1771=6.65 \mathrm{E}-03(\mathrm{~g} / \mathrm{L})$} \\
\hline $\mathrm{Log}\left(\mathrm{C}_{\mathrm{eq}}\right)$ & $\mathrm{C}_{\text {eq }} \cdot(\mathrm{g} / \mathrm{L})$ & $\mathrm{Ads}(\mathrm{mg} / \mathrm{g})$ \\
\hline-2.69 & $2.04 \mathrm{E}-03$ & 0.0446 \\
-2.69 & $2.04 \mathrm{E}-03$ & 0.0782 \\
-2.39 & $4.07 \mathrm{E}-03$ & 0.1660 \\
-2.05 & $8.91 \mathrm{E}-03$ & 0.3532 \\
-1.66 & $2.19 \mathrm{E}-02$ & 0.6808 \\
-1.17 & $6.76 \mathrm{E}-02$ & 1.5066 \\
-0.83 & $1.48 \mathrm{E}-01$ & 1.8535 \\
-0.66 & $2.19 \mathrm{E}-01$ & 3.2961 \\
-0.26 & $5.50 \mathrm{E}-01$ & 3.8815 \\
0.64 & $4.37 \mathrm{E}+00$ & 5.4702 \\
0.89 & $7.76 \mathrm{E}+00$ & 5.4702 \\
1.26 & $1.82 \mathrm{E}+01$ & 10.0000 \\
\hline
\end{tabular}
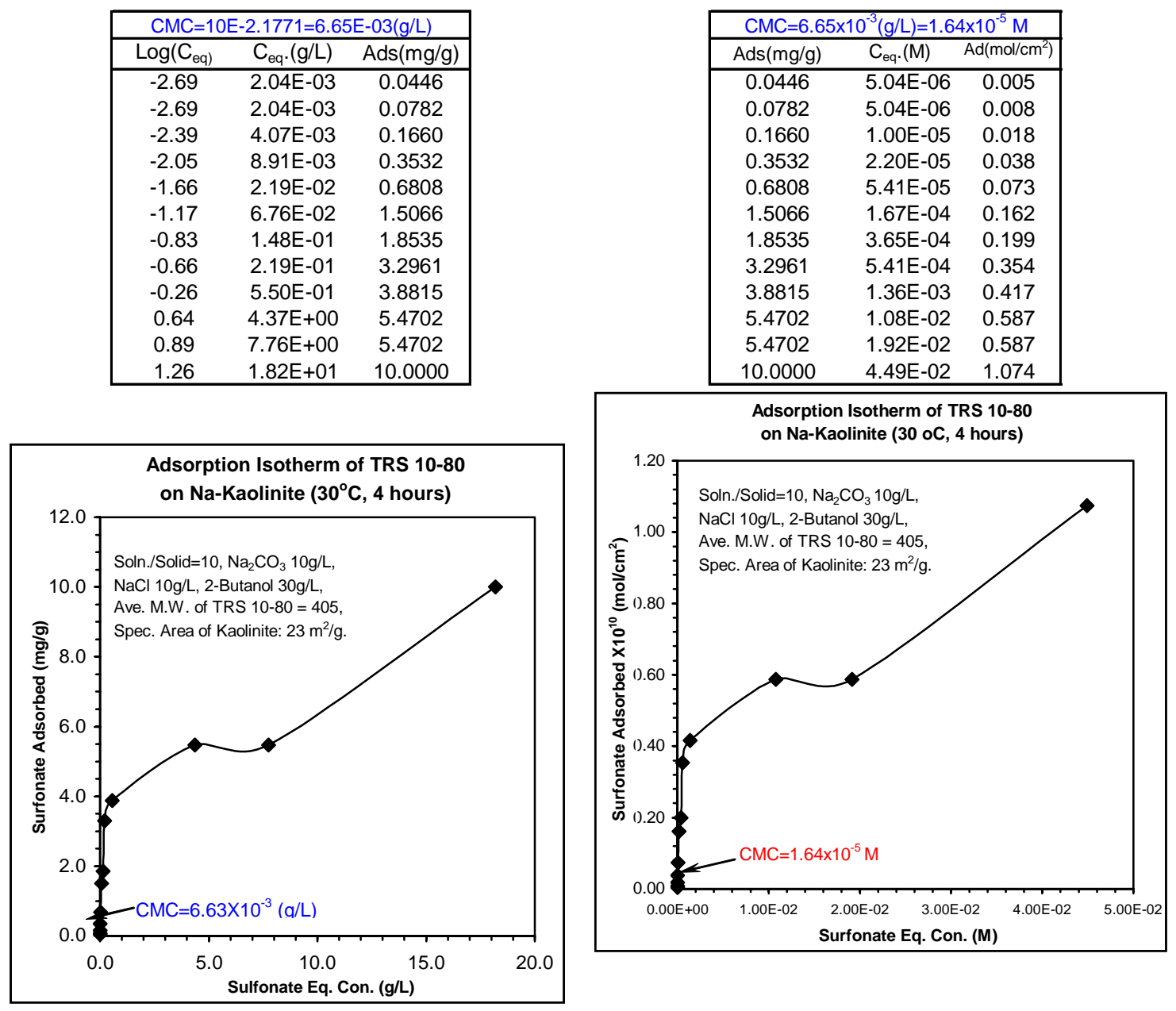

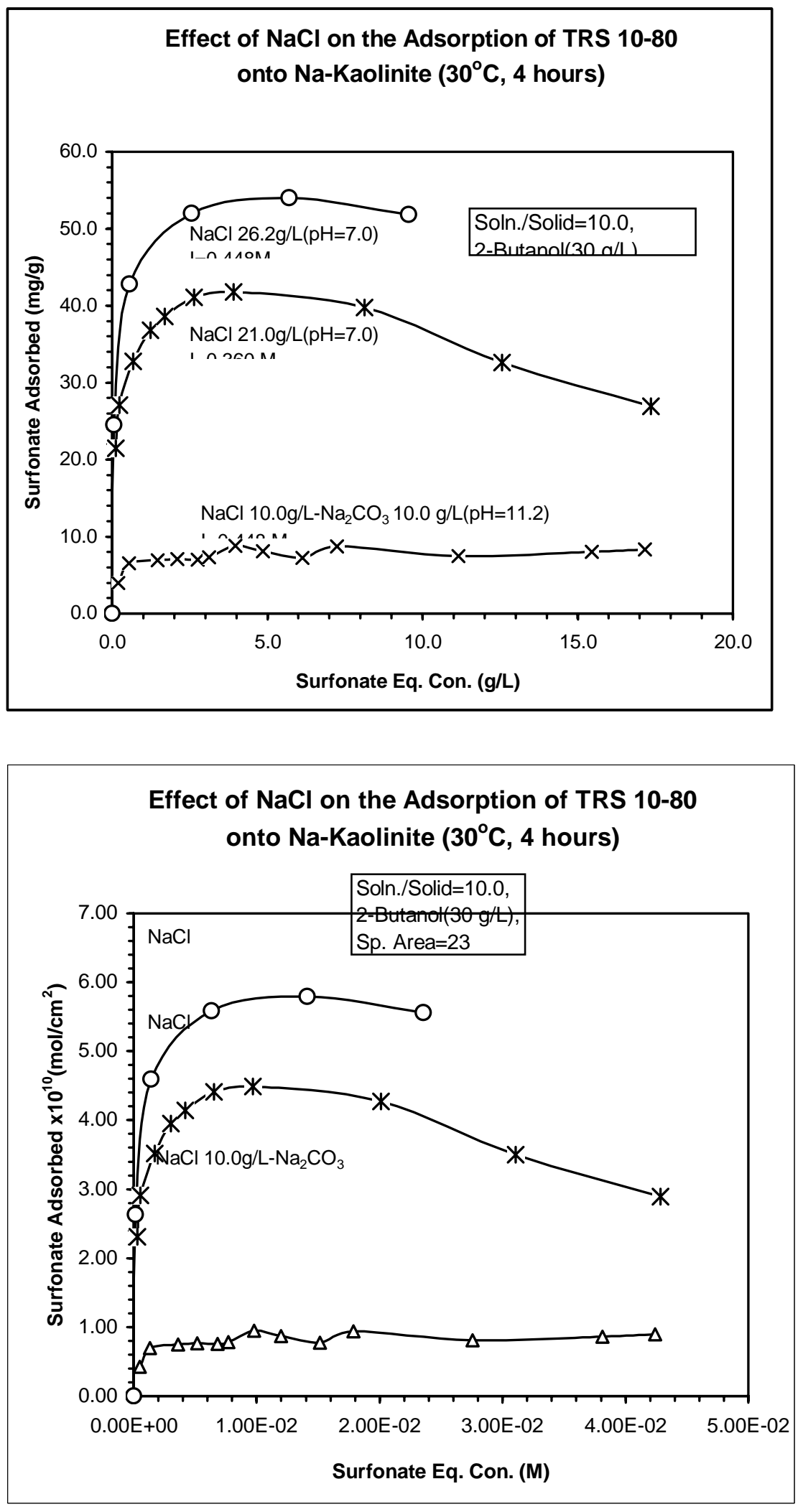

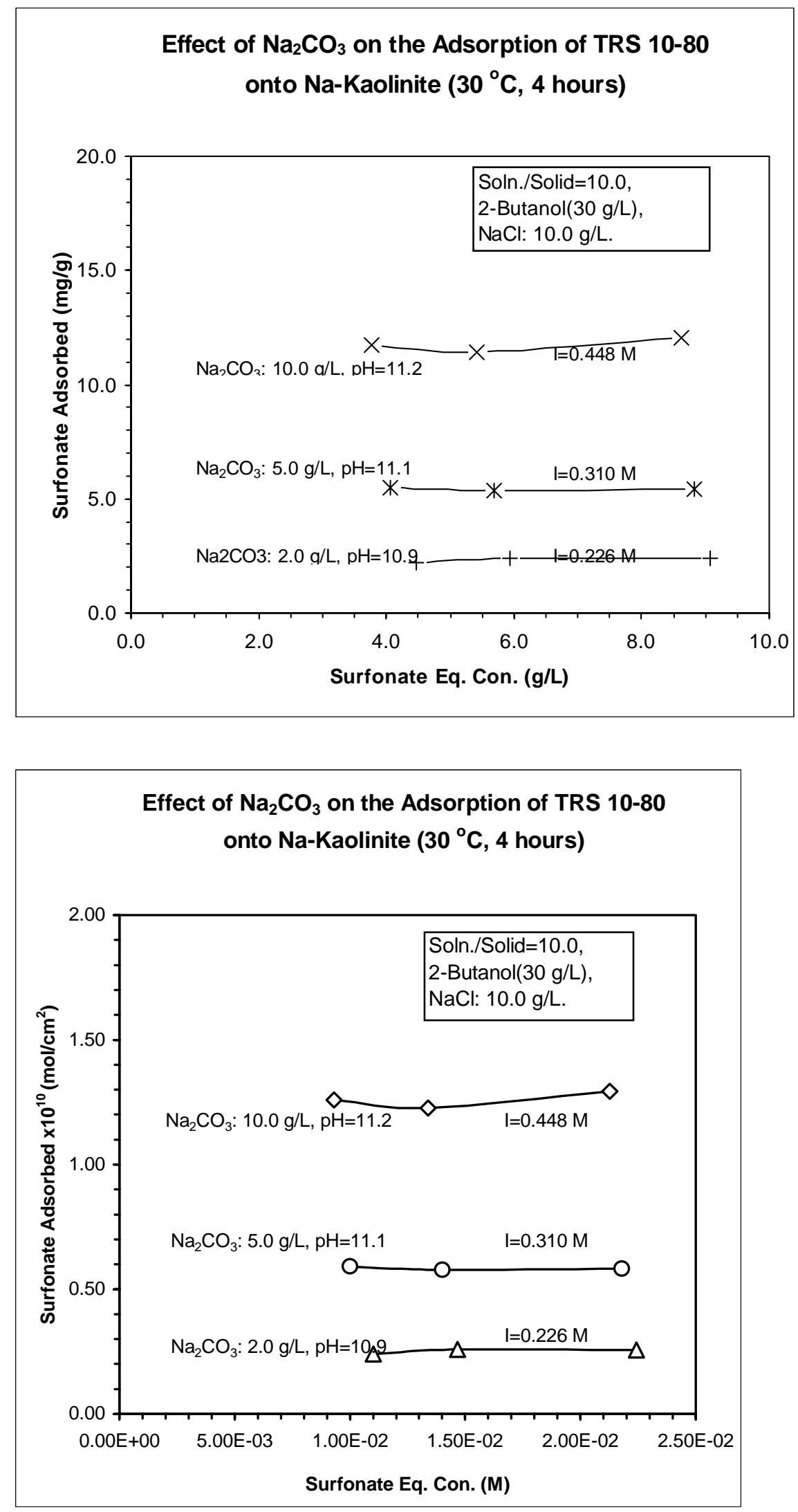

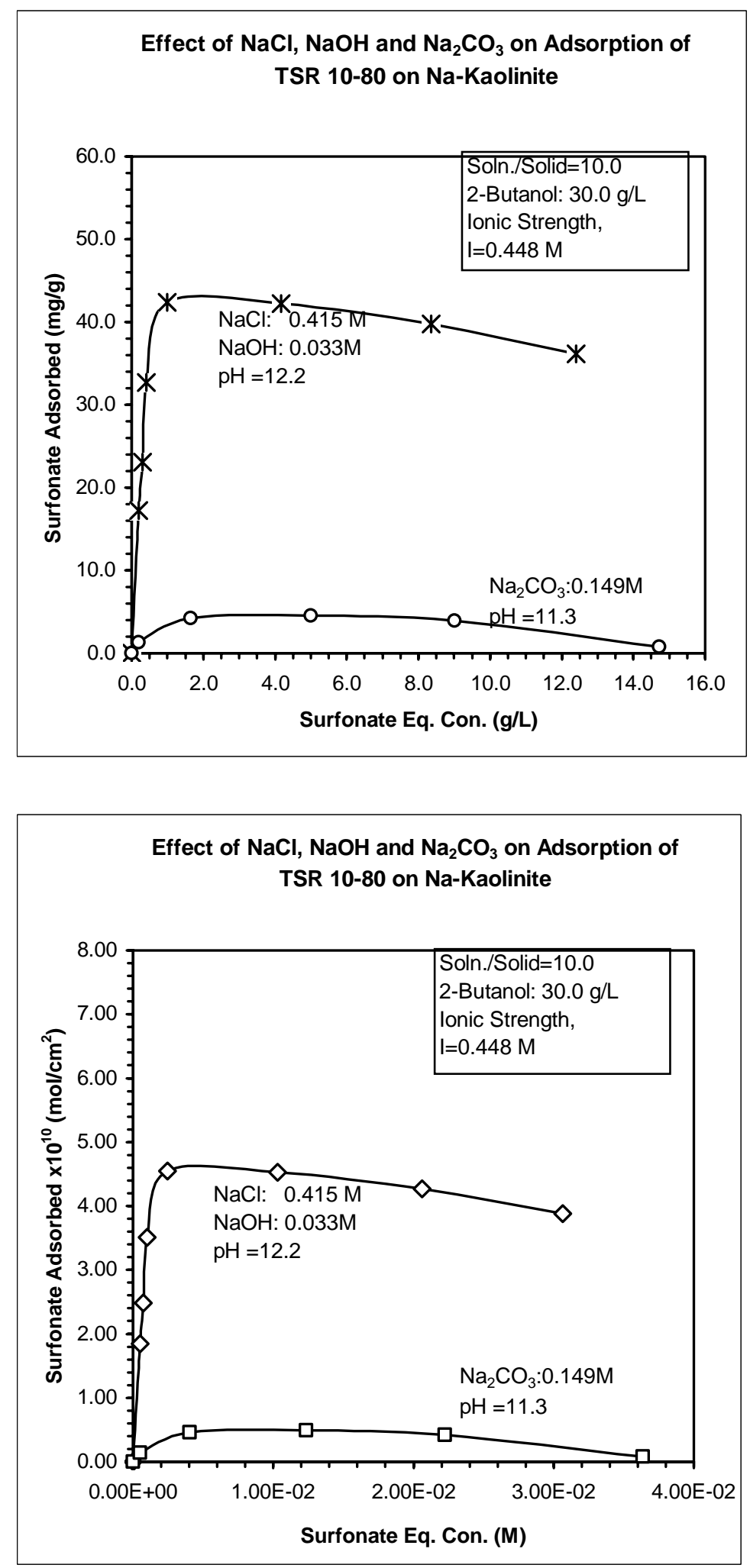

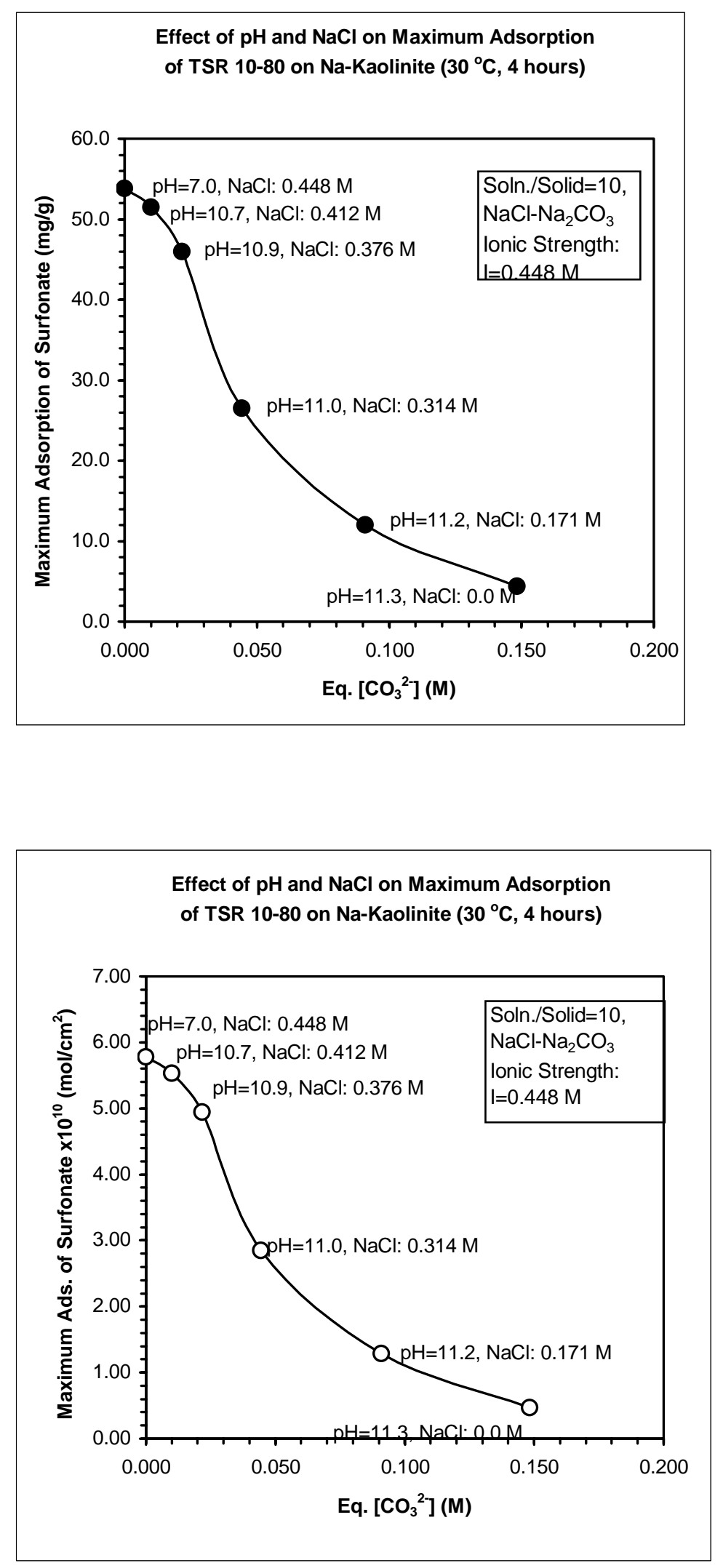

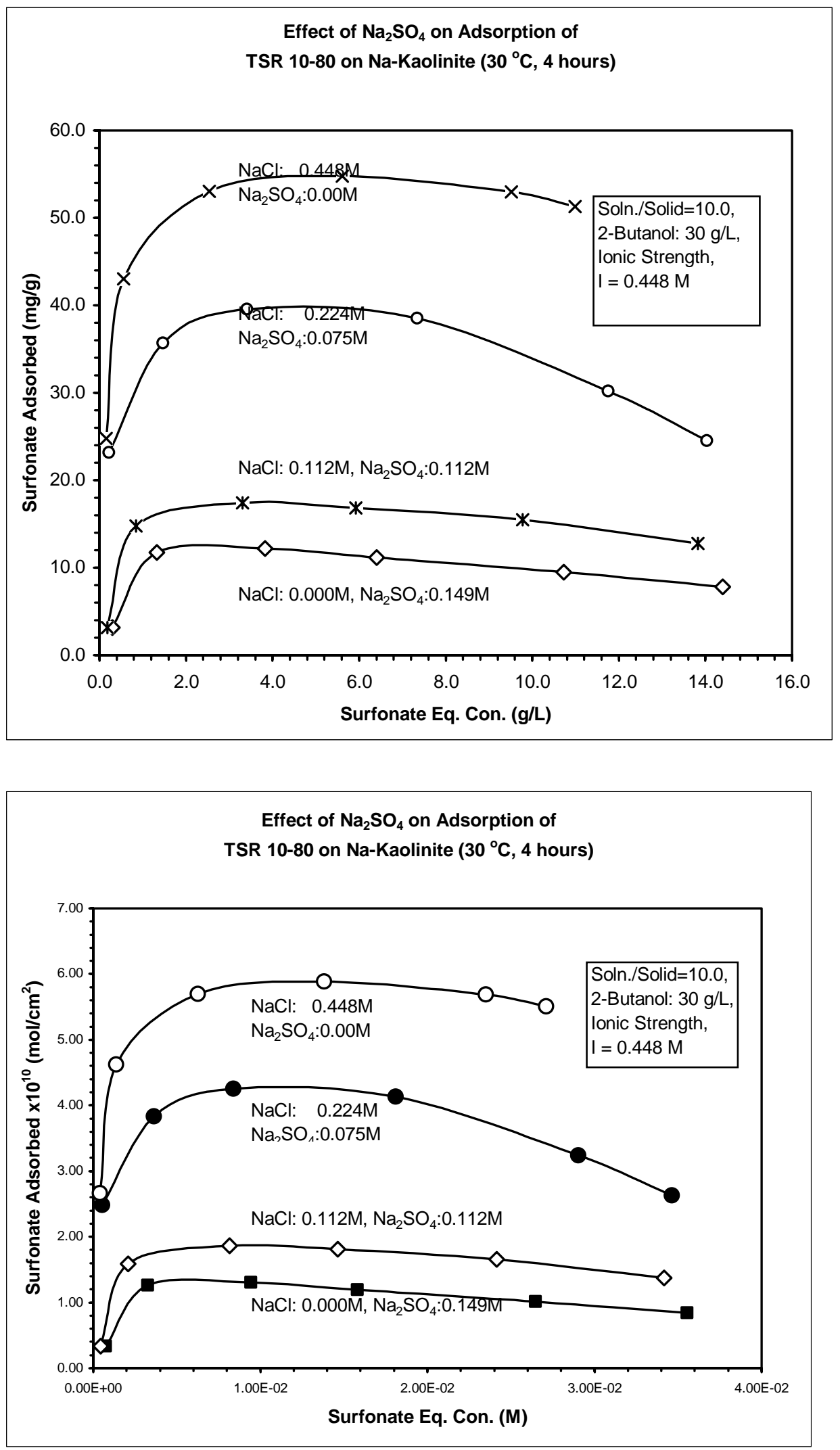


\begin{tabular}{|cc|cc|cc|}
\hline \multicolumn{2}{|c|}{ Adsorption of Sodium Oleate on Kaolinite at Various Temperatc } \\
\hline \multicolumn{2}{|c|}{ Xu, G. et al, J. Dispersion Sci.\& Tech, 22(4), 355-362(2001) } \\
\hline \multicolumn{2}{|c|}{$\mathbf{2 5}^{\circ} \mathbf{C}$} & \multicolumn{2}{c|}{$\mathbf{3 5}^{\circ} \mathbf{C}$} & \multicolumn{2}{c|}{$\mathbf{4 5}{ }^{\circ} \mathbf{C}$} \\
\hline $\mathrm{Ceq}(\mathrm{g} / \mathrm{L})$ & $\mathrm{Ad}(\mathrm{mg} / \mathrm{g})$ & $\mathrm{Ceq}(\mathrm{g} / \mathrm{L})$ & $\mathrm{Ad}(\mathrm{mg} / \mathrm{g})$ & $\mathrm{Ceq}(\mathrm{g} / \mathrm{L})$ & $\mathrm{Ad}(\mathrm{mg} / \mathrm{g})$ \\
\hline 0.240 & 1.00 & 0.000 & 0.00 & 0.235 & 1.16 \\
0.360 & 3.75 & 0.233 & 1.25 & 0.400 & 3.33 \\
0.496 & 2.57 & 0.400 & 3.33 & 0.664 & 2.08 \\
0.612 & 2.75 & 0.832 & 1.67 & 1.160 & 1.67 \\
0.840 & 2.08 & 1.240 & 1.58 & 1.360 & 5.33 \\
0.960 & 2.42 & 1.760 & 7.58 & 1.568 & 10.16 \\
1.560 & 1.67 & 2.431 & 24.15 & 1.825 & 17.41 \\
1.825 & 3.95 & 2.496 & 25.32 & 2.000 & 18.49 \\
2.400 & 16.66 & 2.896 & 28.32 & 2.120 & 19.91 \\
2.496 & 19.49 & 3.840 & 28.07 & 2.672 & 20.40 \\
2.784 & 24.15 & 4.688 & 25.82 & 3.024 & 20.99 \\
3.464 & 35.48 & 5.096 & 27.48 & 3.392 & 20.91 \\
3.640 & 35.81 & & & 4.384 & 21.57 \\
4.871 & 36.05 & & & 4.616 & 21.49 \\
\hline
\end{tabular}

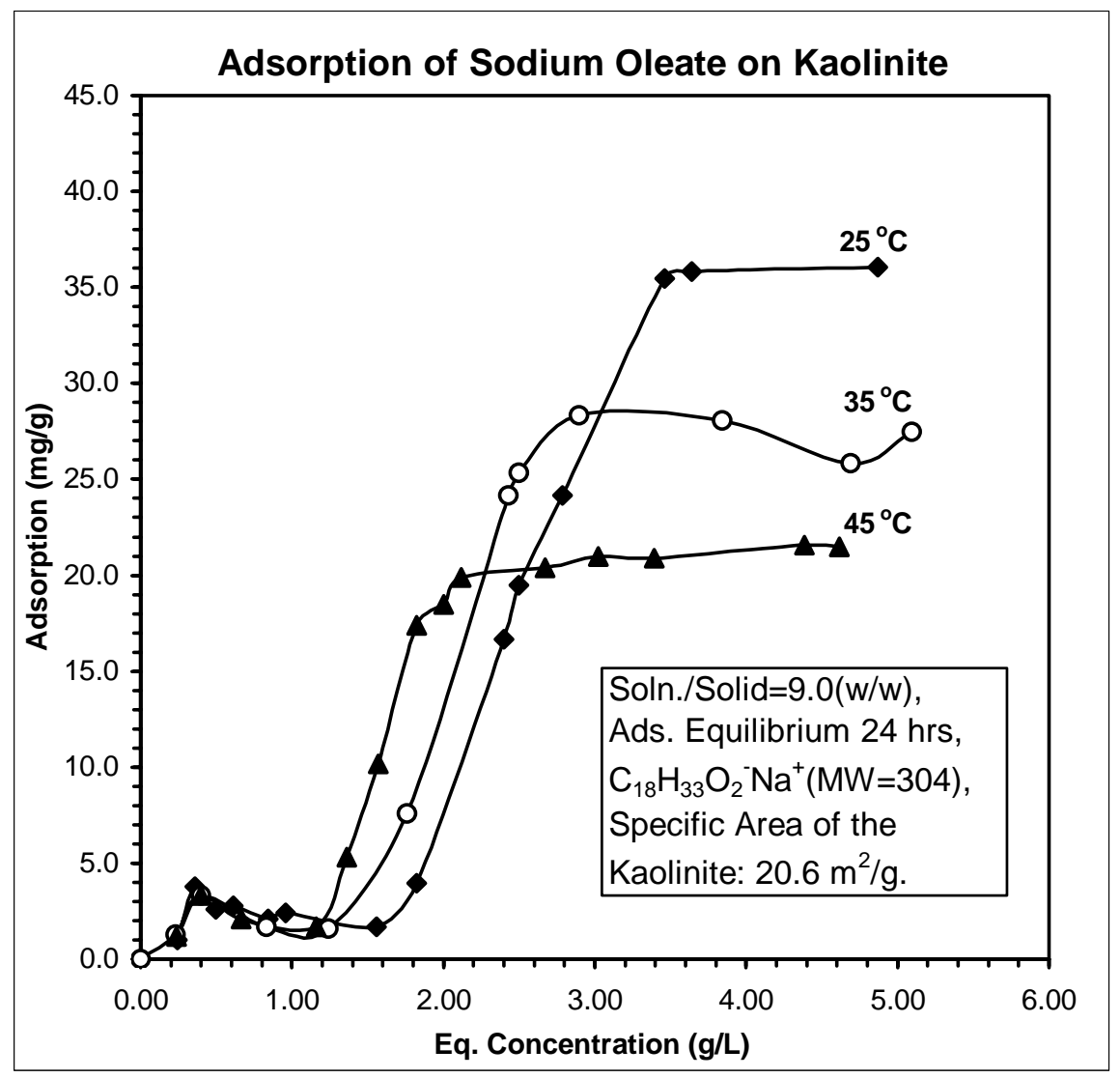




\begin{tabular}{|cc|cc|cc|}
\hline \multicolumn{6}{|c|}{ Adsorption of Sodium Oleate on Kaolinite at Various Temperature } \\
\hline \multicolumn{2}{|c|}{$\mathrm{Xu}$, G. et al, J. Dispersion Sci.\& Tech, 22(4), 355-362(2001) } \\
\hline \multicolumn{2}{|c|}{$\mathbf{2 5} \mathbf{~}^{\circ} \mathbf{C}$} & \multicolumn{2}{c|}{$\mathbf{3 5}{ }^{\circ} \mathbf{C}$} & \multicolumn{2}{c|}{$\mathbf{4 5}{ }^{\circ} \mathbf{C}$} \\
\hline $\mathrm{Ceq}(\mathrm{M})$ & $\mathrm{Ad}\left(\mathrm{mol} / \mathrm{cm}^{2}\right)$ & $\mathrm{Ceq}(\mathrm{M})$ & $\mathrm{Ad}\left(\mathrm{mol} / \mathrm{cm}^{2}\right.$ & $\mathrm{Ceq}(\mathrm{M})$ & $\mathrm{Ad}\left(\mathrm{mol} / \mathrm{cm}^{2}\right)$ \\
\hline $7.89 \mathrm{E}-04$ & 0.160 & $0.00 \mathrm{E}+00$ & 0.000 & $7.74 \mathrm{E}-04$ & 0.186 \\
$1.18 \mathrm{E}-03$ & 0.599 & $7.65 \mathrm{E}-04$ & 0.200 & $1.32 \mathrm{E}-03$ & 0.532 \\
$1.63 \mathrm{E}-03$ & 0.410 & $1.32 \mathrm{E}-03$ & 0.532 & $2.18 \mathrm{E}-03$ & 0.333 \\
$2.01 \mathrm{E}-03$ & 0.439 & $2.74 \mathrm{E}-03$ & 0.266 & $3.82 \mathrm{E}-03$ & 0.266 \\
$2.76 \mathrm{E}-03$ & 0.332 & $4.08 \mathrm{E}-03$ & 0.253 & $4.47 \mathrm{E}-03$ & 0.851 \\
$3.16 \mathrm{E}-03$ & 0.386 & $5.79 \mathrm{E}-03$ & 1.210 & $5.16 \mathrm{E}-03$ & 1.622 \\
$5.13 \mathrm{E}-03$ & 0.266 & $8.00 \mathrm{E}-03$ & 3.857 & $6.00 \mathrm{E}-03$ & 2.780 \\
$6.00 \mathrm{E}-03$ & 0.631 & $8.21 \mathrm{E}-03$ & 4.043 & $6.58 \mathrm{E}-03$ & 2.952 \\
$7.90 \mathrm{E}-03$ & 2.660 & $9.53 \mathrm{E}-03$ & 4.522 & $6.97 \mathrm{E}-03$ & 3.179 \\
$8.21 \mathrm{E}-03$ & 3.112 & $1.26 \mathrm{E}-02$ & 4.482 & $8.79 \mathrm{E}-03$ & 3.258 \\
$9.16 \mathrm{E}-03$ & 3.857 & $1.54 \mathrm{E}-02$ & 4.123 & $9.95 \mathrm{E}-03$ & 3.351 \\
$1.14 \mathrm{E}-02$ & 5.665 & $1.68 \mathrm{E}-02$ & 4.389 & $1.12 \mathrm{E}-02$ & 3.338 \\
$1.20 \mathrm{E}-02$ & 5.718 & & & $1.44 \mathrm{E}-02$ & 3.445 \\
$1.60 \mathrm{E}-02$ & 5.757 & & & $1.52 \mathrm{E}-02$ & 3.431 \\
\hline
\end{tabular}

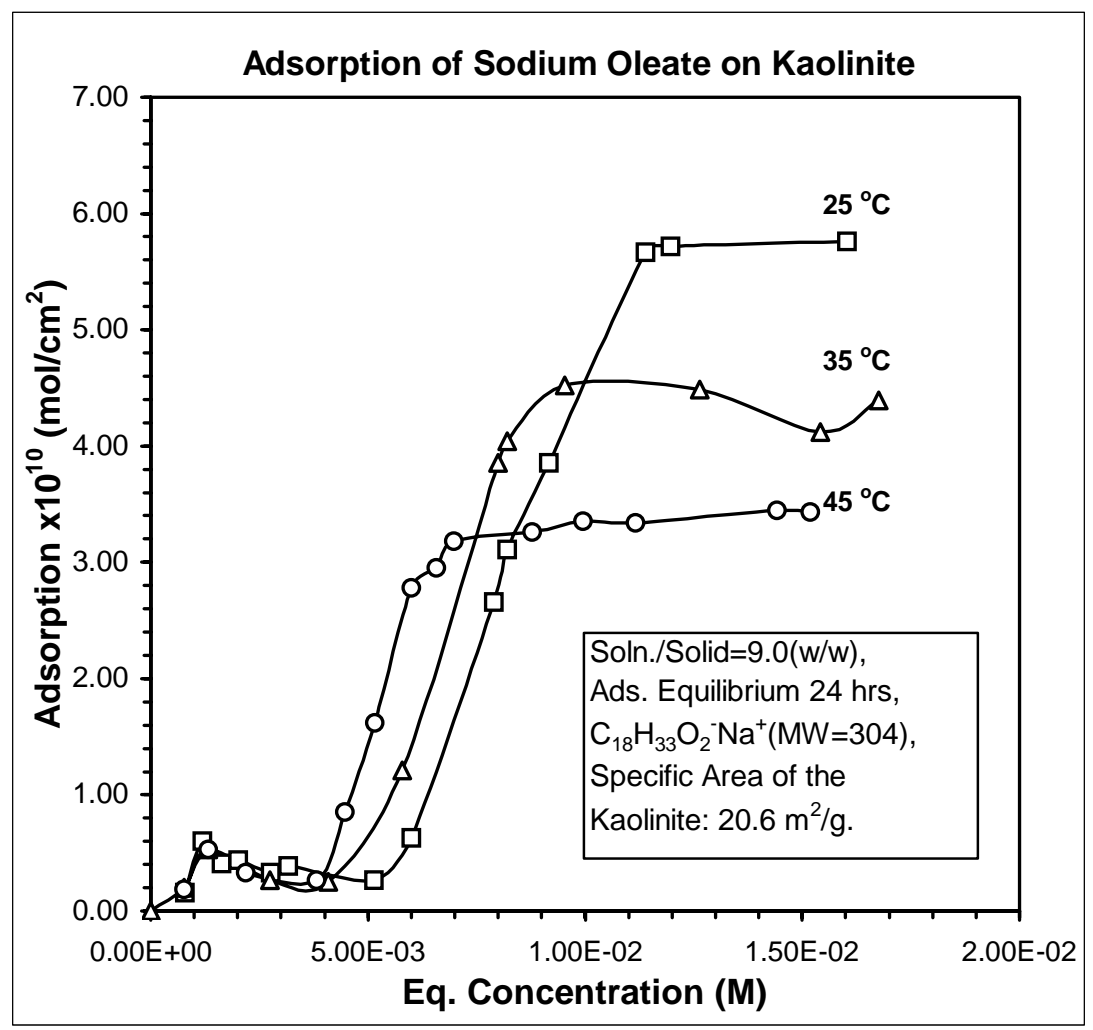



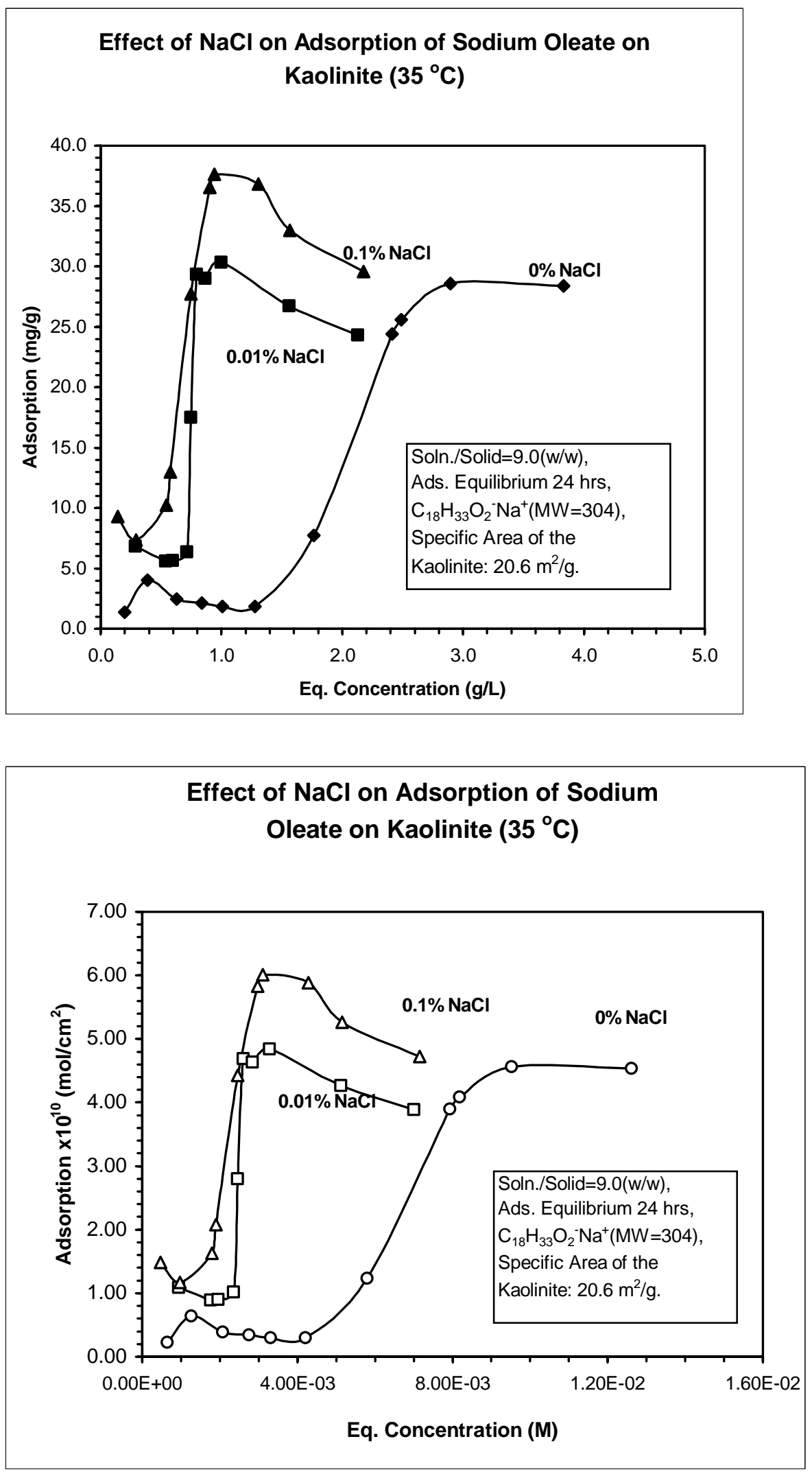

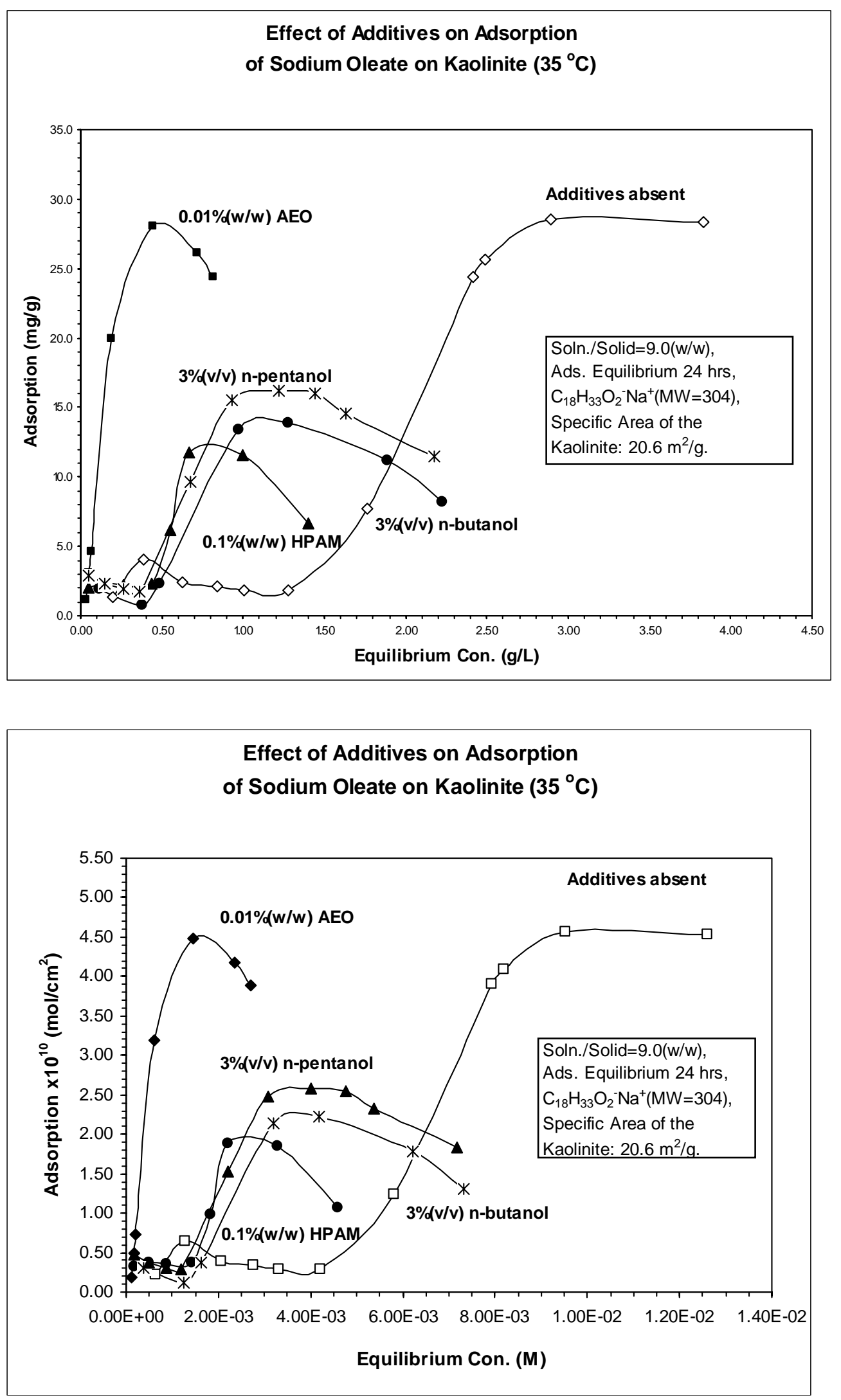


\begin{tabular}{|c|c|c|c|c|c|}
\hline Adsorptio & of Alkylb & nzene Si & fonates o & o Minera & rfaces \\
\hline & 3arakat, Y. et a & Indian J. of & hemical Tech & oll. 2, May, 1 & \\
\hline $4-\Phi C$ & ${ }_{2} \mathrm{ABS}$ & $4-\Phi$ & ${ }_{11} \mathrm{ABS}$ & 4- $\Phi$ & ${ }_{0} \mathrm{ABS}$ \\
\hline Ceq(g/L) & Ads $(\mathrm{mg} / \mathrm{g})$ & Ceq(g/L) & Ads $(\mathrm{mg} / \mathrm{g})$ & $\mathrm{Ceq}(\mathrm{g} / \mathrm{L})$ & Ads $(\mathrm{mg} / \mathrm{g})$ \\
\hline 0.024 & 1.020 & 0.000 & 0.000 & 0.033 & 0.164 \\
\hline 0.026 & 1.670 & 0.030 & 0.463 & 0.069 & 0.348 \\
\hline 0.036 & 2.088 & 0.064 & 1.392 & 0.107 & 0.696 \\
\hline 0.042 & 3.087 & 0.077 & 3.365 & 0.135 & 1.392 \\
\hline 0.040 & 5.227 & 0.082 & 3.873 & 0.172 & 1.462 \\
\hline 0.038 & 8.954 & 0.102 & 7.238 & 0.206 & 3.504 \\
\hline 0.050 & 10.673 & 0.159 & 7.611 & 0.243 & 4.409 \\
\hline 0.074 & 11.042 & 0.231 & 7.771 & 0.279 & 5.036 \\
\hline 0.083 & 11.439 & 0.299 & 7.423 & 0.350 & 5.265 \\
\hline 0.113 & 11.808 & 0.418 & 7.959 & 0.421 & 5.404 \\
\hline 0.145 & 12.016 & 0.520 & 7.656 & 0.611 & 5.568 \\
\hline 0.173 & 12.364 & 0.561 & 7.656 & 0.771 & 5.801 \\
\hline 0.205 & 12.458 & 0.763 & 7.771 & 0.906 & 4.409 \\
\hline 0.247 & 12.761 & 0.904 & 7.193 & 1.026 & 5.568 \\
\hline 0.301 & 12.016 & 1.147 & 6.497 & 1.145 & 5.105 \\
\hline 0.351 & 12.344 & 1.272 & 7.726 & 1.317 & 5.732 \\
\hline 0.471 & 12.413 & 1.386 & 6.960 & 1.458 & 4.872 \\
\hline 0.554 & 12.806 & 1.560 & 6.960 & 1.560 & 5.801 \\
\hline 0.698 & 12.876 & & & & \\
\hline 0.804 & 12.535 & & & & \\
\hline
\end{tabular}

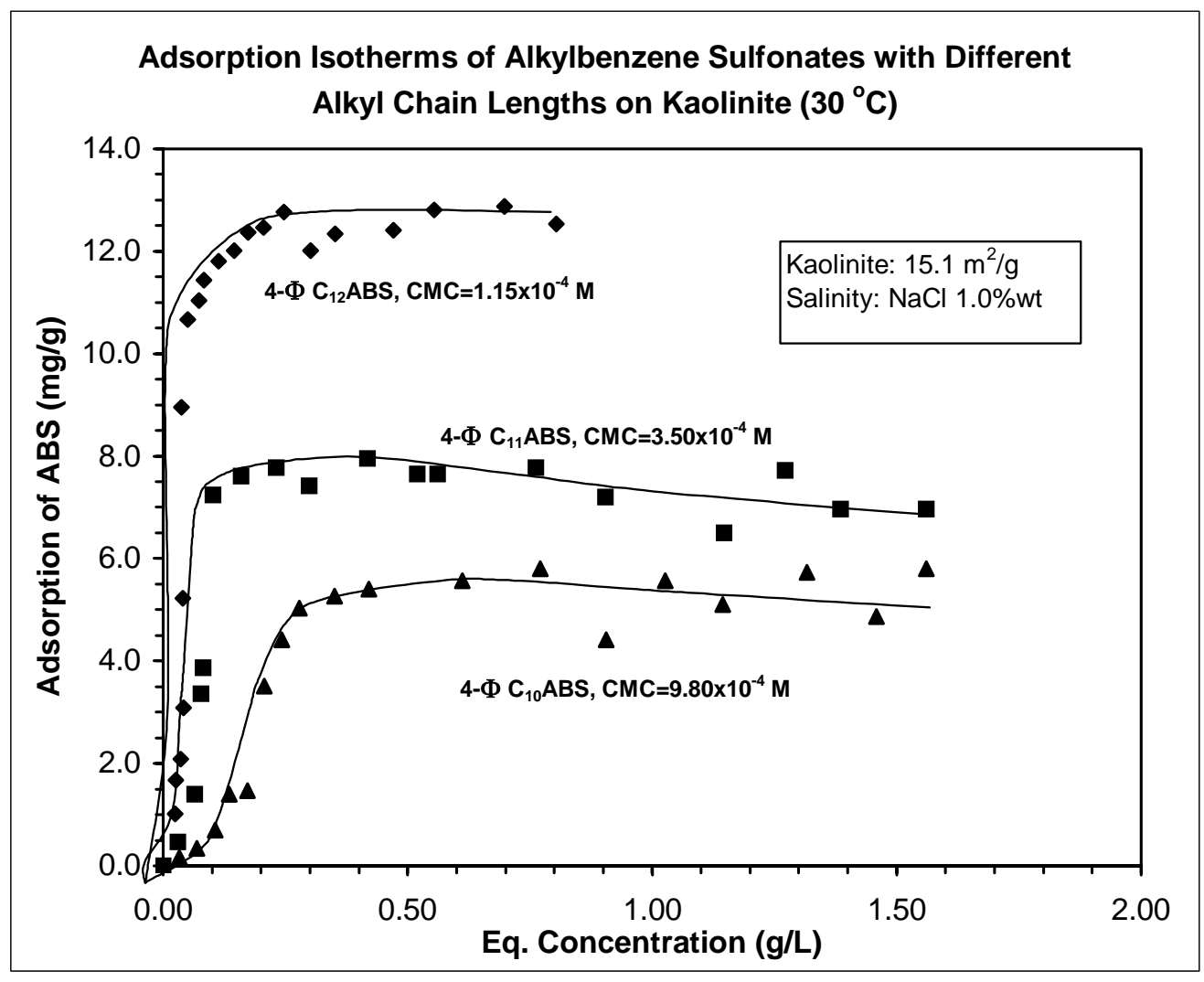




\begin{tabular}{|c|c|c|c|c|c|}
\hline \multicolumn{6}{|c|}{ Adsorption of Alkylbenzene Sulfonates onto Mineral Surfaces } \\
\hline \multicolumn{6}{|c|}{ Barakat, Y. et al, Indian J. of Chemical Tech. Vol. 2, May, 1995} \\
\hline \multicolumn{2}{|c|}{$4-\Phi \mathrm{C}_{12} \mathrm{ABS}$} & \multicolumn{2}{|c|}{$4-\Phi \mathrm{C}_{11} \mathrm{ABS}$} & \multicolumn{2}{|c|}{$4-\Phi \mathrm{C}_{10} \mathrm{ABS}$} \\
\hline Ceq(M) & Ads $\left(\mathrm{mol} / \mathrm{cm}^{2}\right)$ & Ceq(M) & Ads $\left(\mathrm{mol} / \mathrm{cm}^{2}\right)$ & Ceq(M) & Ads $\left(\mathrm{mol} / \mathrm{cm}^{2}\right)$ \\
\hline $6.82 \mathrm{E}-05$ & 0.194 & $0.00 \mathrm{E}+00$ & 0.000 & 9.55E-05 & 0.031 \\
\hline 7.50E-05 & 0.318 & $8.52 \mathrm{E}-05$ & 0.088 & 1.98E-04 & 0.066 \\
\hline $1.02 \mathrm{E}-04$ & 0.397 & 1.84E-04 & 0.265 & 3.07E-04 & 0.132 \\
\hline 1.19E-04 & 0.587 & 2.22E-04 & 0.640 & 3.89E-04 & 0.265 \\
\hline 1.16E-04 & 0.995 & 2.35E-04 & 0.737 & 4.94E-04 & 0.278 \\
\hline 1.09E-04 & 1.704 & 2.93E-04 & 1.377 & 5.93E-04 & 0.667 \\
\hline 1.43E-04 & 2.031 & 4.57E-04 & 1.448 & 6.99E-04 & 0.839 \\
\hline $2.11 \mathrm{E}-04$ & 2.101 & 6.65E-04 & 1.479 & 8.01E-04 & 0.958 \\
\hline 2.39E-04 & 2.177 & 8.59E-04 & 1.413 & $1.01 E-03$ & 1.002 \\
\hline 3.24E-04 & 2.247 & 1.20E-03 & 1.515 & $1.21 \mathrm{E}-03$ & 1.028 \\
\hline 4.16E-04 & 2.287 & 1.49E-03 & 1.457 & 1.76E-03 & 1.060 \\
\hline 4.98E-04 & 2.353 & 1.61E-03 & 1.457 & $2.22 \mathrm{E}-03$ & 1.104 \\
\hline 5.90E-04 & 2.371 & 2.19E-03 & 1.479 & 2.60E-03 & 0.839 \\
\hline 7.09E-04 & 2.428 & 2.60E-03 & 1.369 & 2.95E-03 & 1.060 \\
\hline 8.66E-04 & 2.287 & 3.30E-03 & 1.236 & $3.29 E-03$ & 0.972 \\
\hline 1.01E-03 & 2.349 & $3.65 E-03$ & 1.470 & 3.78E-03 & 1.091 \\
\hline 1.35E-03 & 2.362 & 3.98E-03 & 1.325 & 4.19E-03 & 0.927 \\
\hline 1.59E-03 & 2.437 & 4.48E-03 & 1.325 & 4.48E-03 & 1.104 \\
\hline 2.00E-03 & 2.450 & & & & \\
\hline $2.31 \mathrm{E}-03$ & 2.385 & & & & \\
\hline
\end{tabular}

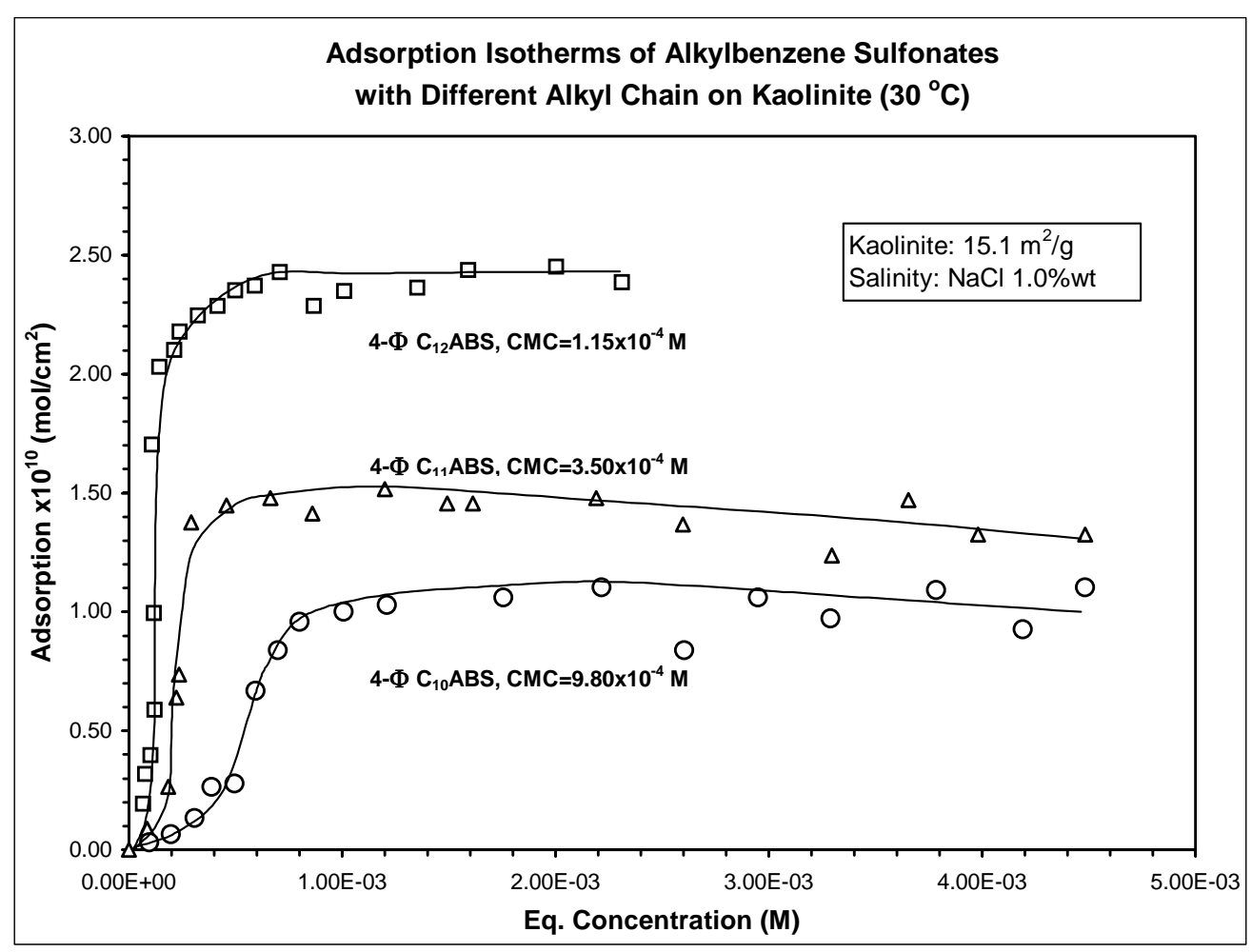



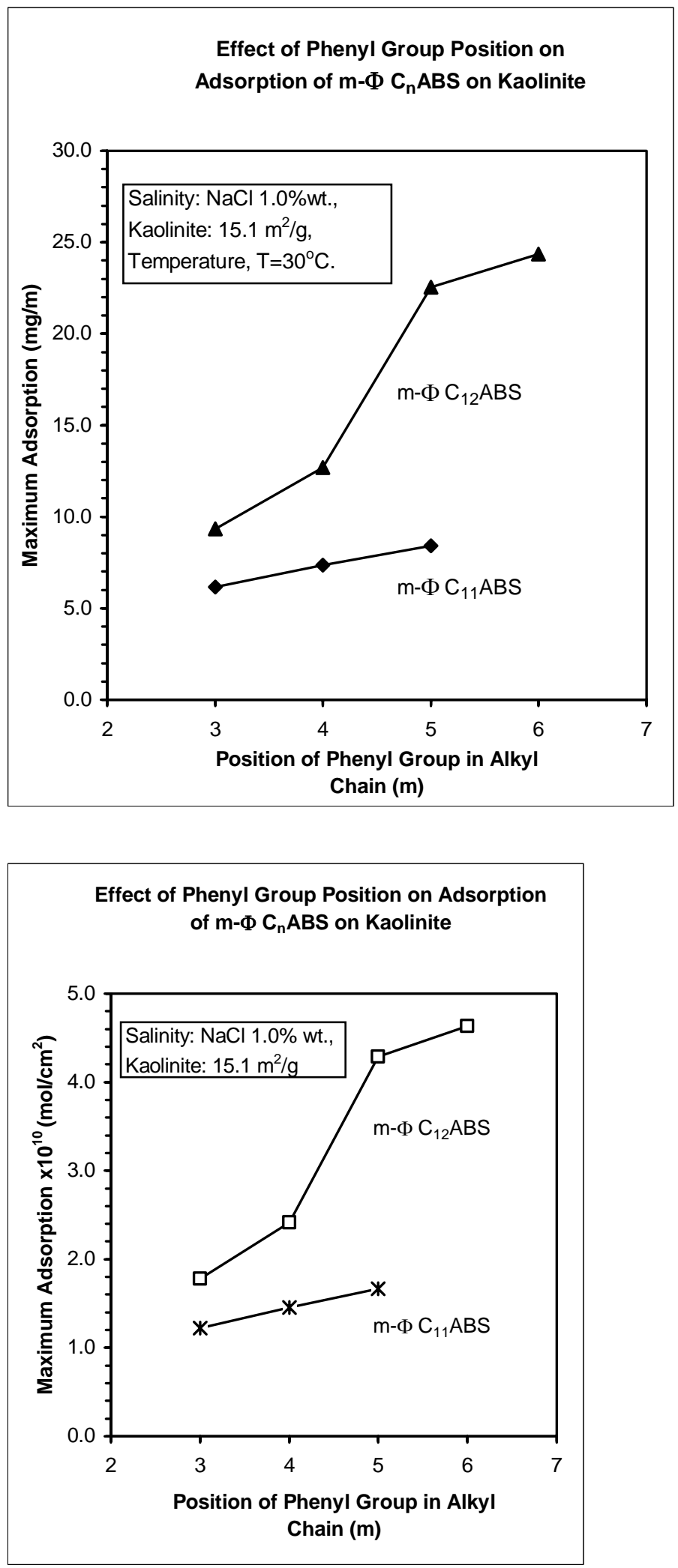

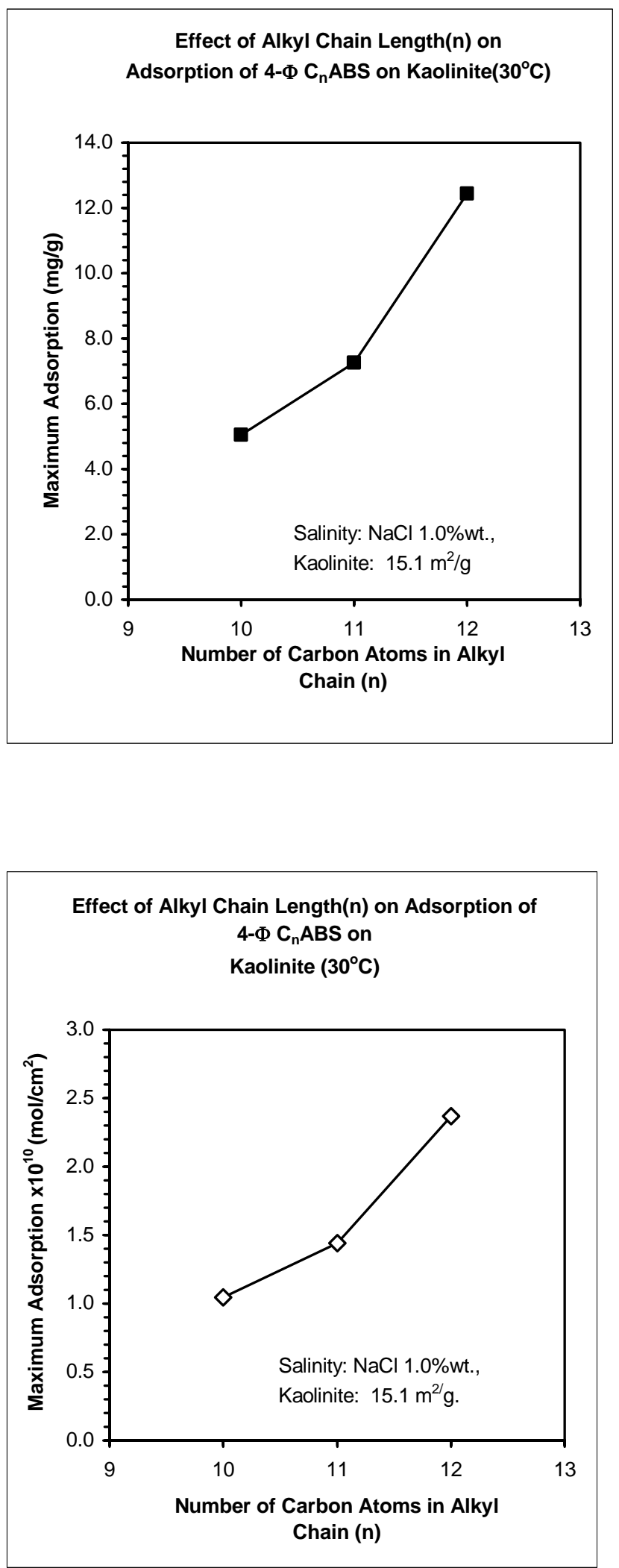


\begin{tabular}{|c|c|c|c|c|c|c|c|}
\hline Ads & tion 0 & at Differ & t pH $(\mathrm{T}=$ & $\begin{array}{l}\text { ne sultor } \\
{ }^{\circ} \mathrm{C}, \mathrm{I}=0.0\end{array}$ & M NaCl) & ont & \\
\hline H. S. Hanr & and P. Sc & רasundaran, & Colloid 8 & nterface Sc & nce Vol. 7 & No. 1,181 & 191(1979) \\
\hline $\mathrm{pH}=4$ & Ionic StI & ngth $=0.01$ & $\mathrm{NaCl}$ & $\mathrm{pH}=6$. & Ionic Str & ggth $=0.01 \mathrm{I}$ & $\mathrm{NaCl}$ \\
\hline Original & aolinite & $\mathrm{Na}-\mathrm{Ka}$ & linite & Original & aolinite & $\mathrm{Na}-\mathrm{Ka}$ & linite \\
\hline $\mathrm{Ceq}(\mathrm{mg} / \mathrm{L})$ & $\Gamma(\mathrm{mg} / \mathrm{g})$ & $\mathrm{Ceq}(\mathrm{mg} / \mathrm{L})$ & $\Gamma(\mathrm{mg} / \mathrm{g})$ & $\mathrm{Ceq}(\mathrm{mg} / \mathrm{L})$ & $\Gamma(\mathrm{mg} / \mathrm{g})$ & $\mathrm{Ceq}(\mathrm{mg} / \mathrm{L})$ & $\Gamma(\mathrm{mg} / \mathrm{g})$ \\
\hline 0.00 & 6.82 & 0.00 & 14.88 & 0.00 & 2.01 & 0.00 & 2.81 \\
\hline 1.63 & 8.68 & 0.38 & 16.66 & 1.70 & 3.34 & 2.51 & 4.22 \\
\hline 6.22 & 4.13 & 3.76 & 16.53 & 6.41 & 2.41 & 6.24 & 3.82 \\
\hline 10.02 & 2.03 & 7.52 & 13.56 & 10.74 & 0.88 & 9.93 & 2.78 \\
\hline 17.33 & 0.16 & 15.03 & 10.42 & 17.56 & 0.00 & 17.35 & 1.33 \\
\hline & & 16.24 & 10.11 & & & & \\
\hline & & 16.54 & 7.32 & & & & \\
\hline
\end{tabular}

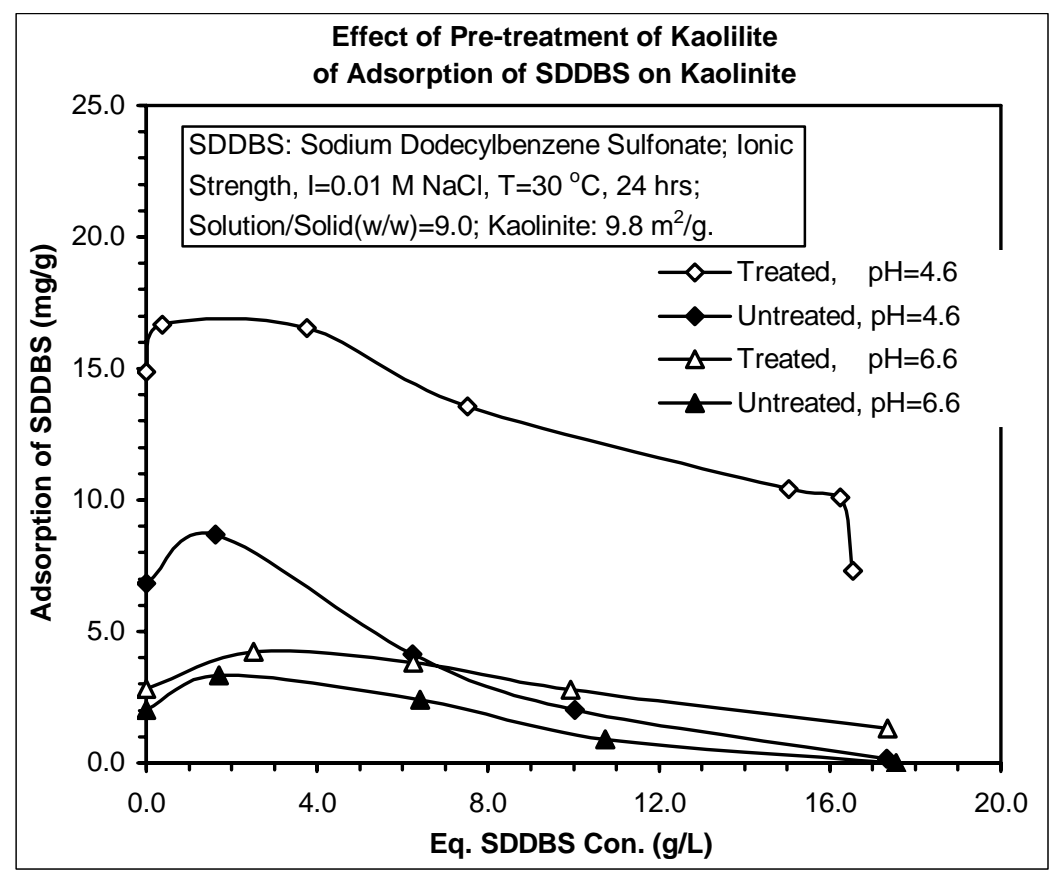




\begin{tabular}{|c|c|c|c|c|c|c|c|}
\hline \multicolumn{8}{|c|}{$\begin{array}{c}\text { Adsorption of Sodium Dodecyl Benzene Sulfonate (SDDBS) on Kaolinite } \\
\text { at Different } \mathrm{pH}\left(\mathrm{T}=25^{\circ} \mathrm{C}, \mathrm{I}=0.01 \mathrm{M} \mathrm{NaCl}\right)\end{array}$} \\
\hline \multicolumn{8}{|c|}{ H. S. Hanna and P. Somasundaran, J. Colloid \& Interface Science Vol. 70, No. 1, 181-191(1979) } \\
\hline \multicolumn{4}{|c|}{$\mathrm{pH}=4.6$, Ionic Strength $=0.01 \mathrm{M} \mathrm{NaCl}$} & \multicolumn{4}{|c|}{$\mathrm{pH}=6.6$, Ionic Strength $=0.01 \mathrm{M} \mathrm{NaCl}$} \\
\hline \multicolumn{2}{|c|}{ Original Kaolinite } & \multicolumn{2}{|c|}{ Na-Kaolinite } & \multicolumn{2}{|c|}{ Original Kaolinite } & \multicolumn{2}{|c|}{ Na-Kaolinite } \\
\hline Ceq(M) & $\Gamma\left(\mathrm{mol} / \mathrm{cm}^{2}\right)$ & Ceq(M) & $\Gamma\left(\mathrm{mol} / \mathrm{cm}^{2}\right)$ & Ceq(M) & $\overline{\Gamma\left(\mathrm{mol} / \mathrm{cm}^{2}\right)}$ & Ceq(M) & $\Gamma\left(\mathrm{mol} / \mathrm{cm}^{2}\right)$ \\
\hline $0.00 \mathrm{E}+00$ & 2.00 & $0.00 \mathrm{E}+00$ & 4.36 & $0.00 \mathrm{E}+00$ & 0.59 & $0.00 \mathrm{E}+00$ & 0.83 \\
\hline 4.68E-03 & 2.55 & $1.08 \mathrm{E}-03$ & 4.89 & 4.89E-03 & & $7.22 \mathrm{E}-03$ & 1.24 \\
\hline 1.79E-02 & 1.21 & 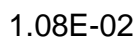 & 4.85 & 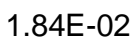 & & E-02 & 1.12 \\
\hline $2.88 \mathrm{E}-02$ & 0.59 & $2.16 \mathrm{E}-02$ & 3.98 & 3.09E-( & 0.2 & $2.85 \mathrm{E}-02$ & 0.81 \\
\hline \multirow[t]{3}{*}{$4.98 \mathrm{E}-02$} & 0.05 & 4.32E-02 & 3.06 & 5.05E-02 & 0.00 & 4.99E-02 & 0.39 \\
\hline & & $4.67 \mathrm{E}-\mathrm{C}$ & 2.96 & & & & \\
\hline & & 4.75E-02 & 2.15 & & & & \\
\hline
\end{tabular}

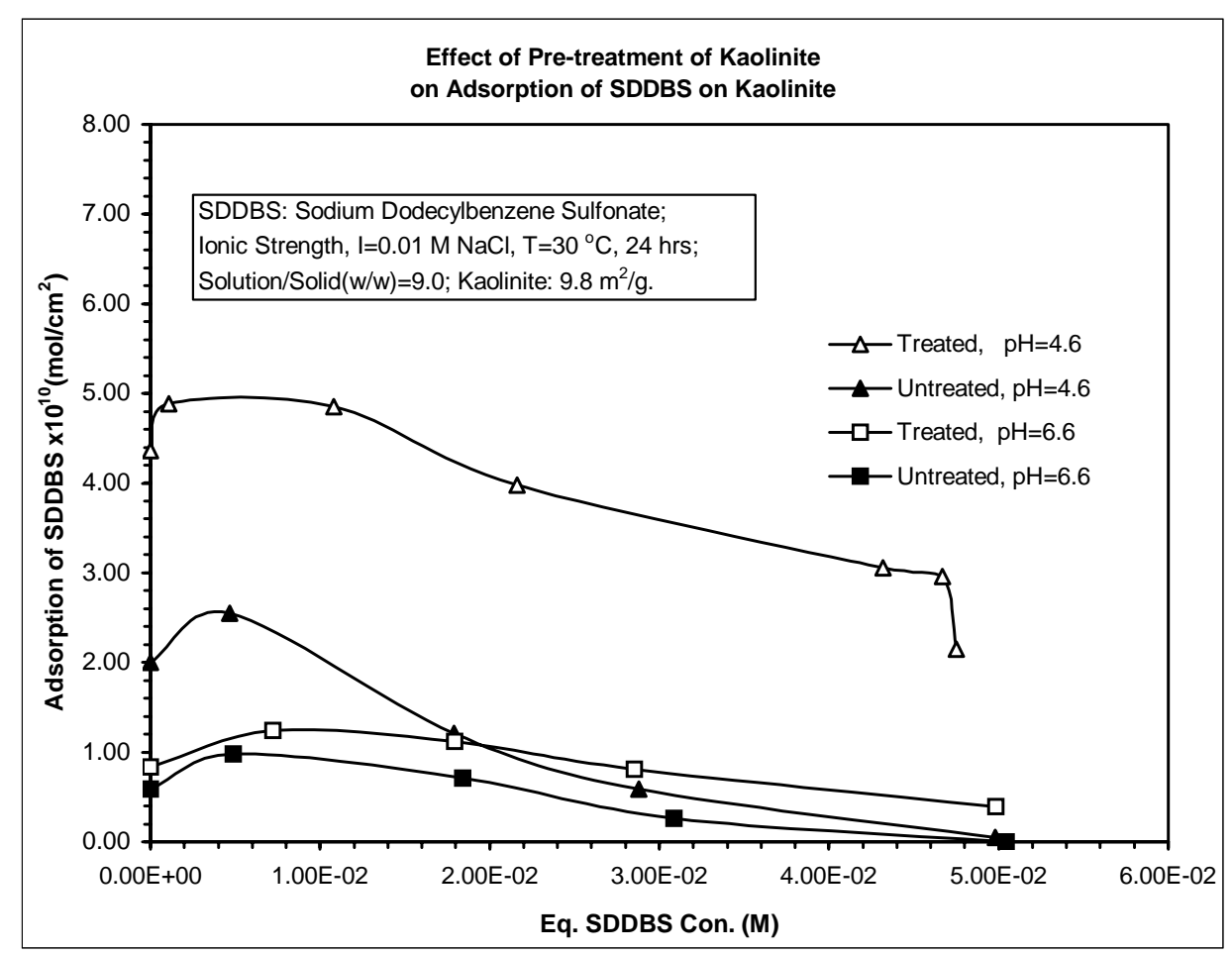



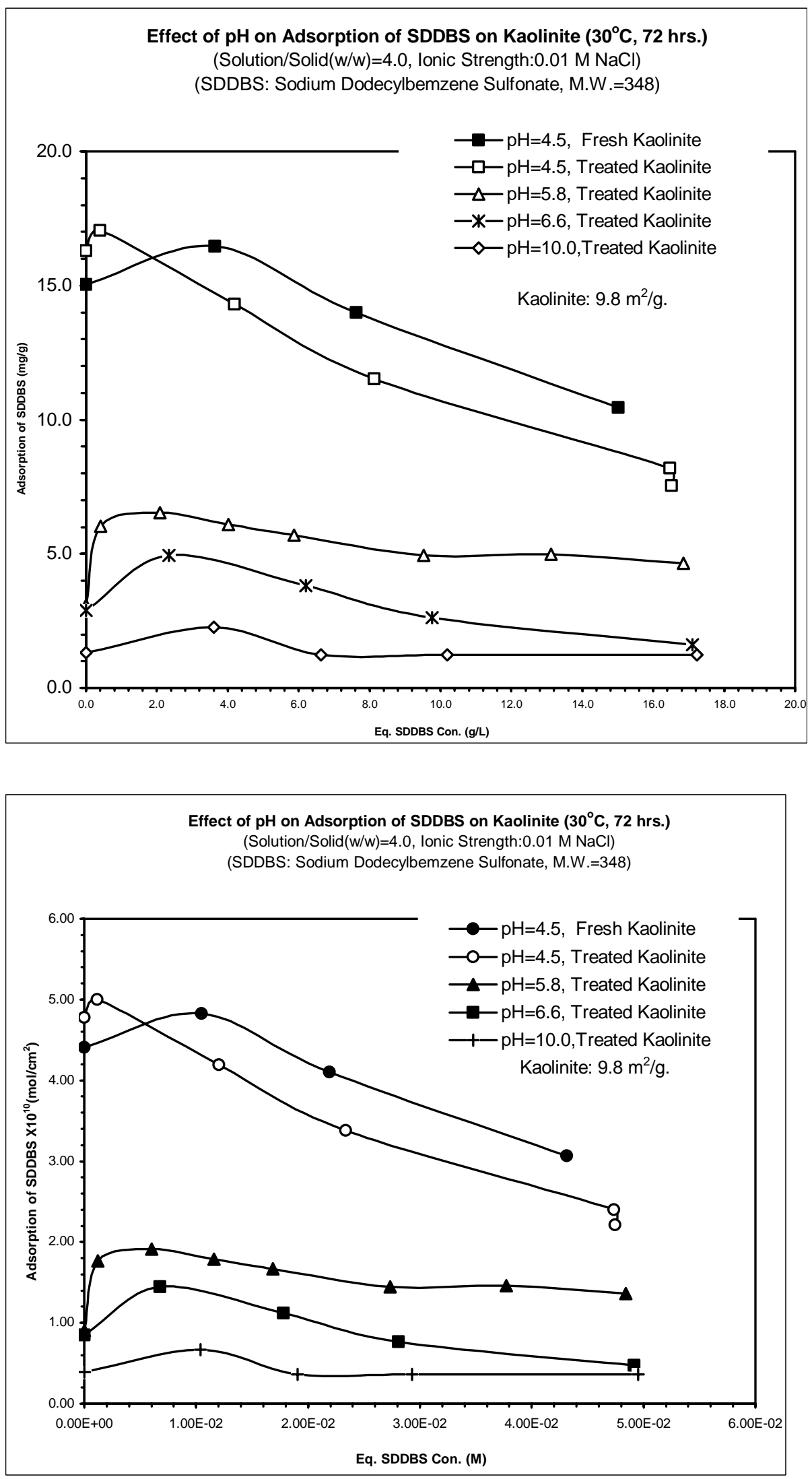

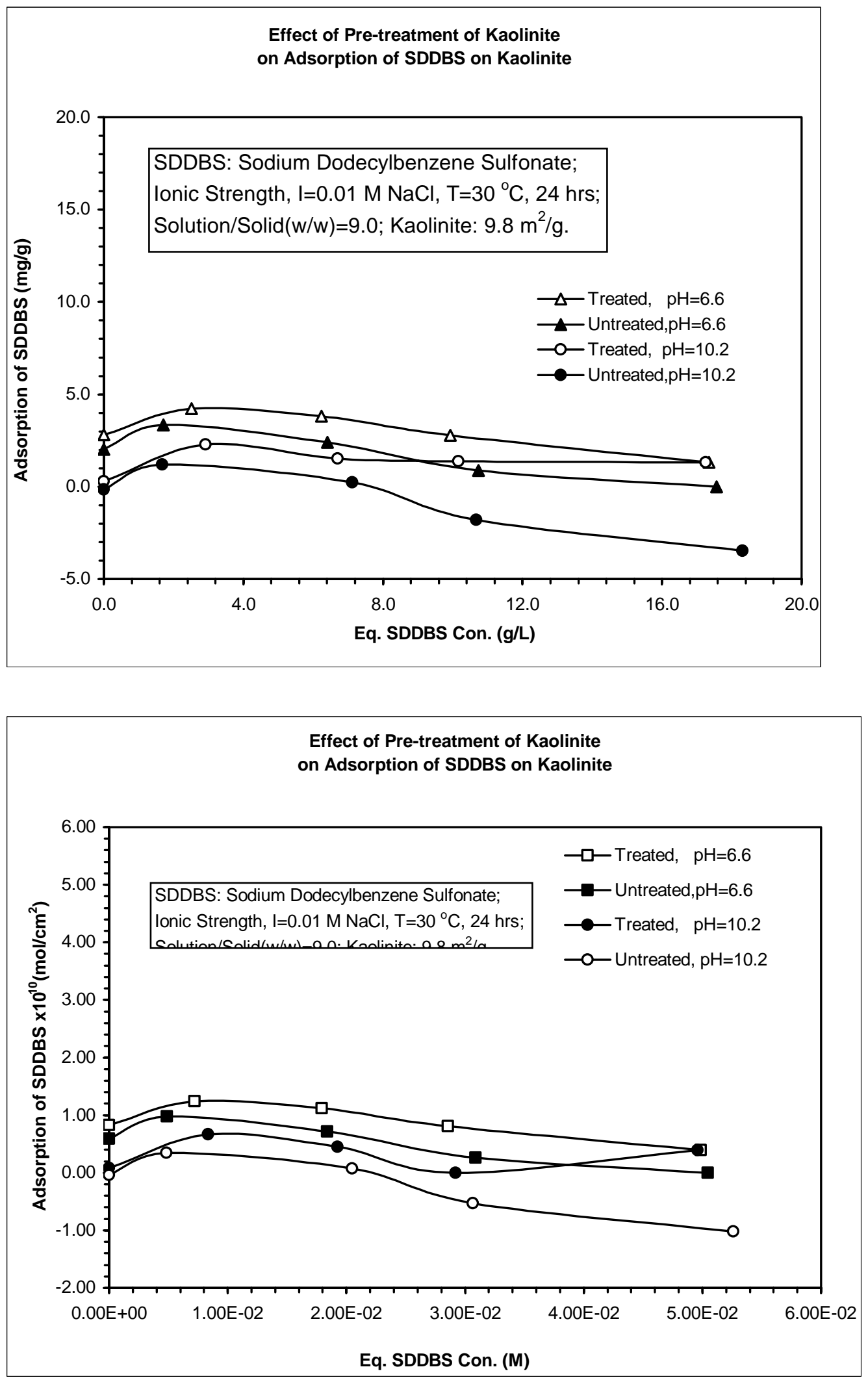


\begin{tabular}{|ccc|ccc|}
\hline \multicolumn{6}{|c|}{$\begin{array}{c}\text { Adsorption of CPC and DBS on Kaolinite } \\
\text { Aita,A.A. et al Ads. Sci. \& Tech. Vol.5(8),1997 }\end{array}$} \\
\hline \multicolumn{6}{|c|}{ CPC: Cetyl Pyridinium Chloride, M.W.=339.5, $\mathrm{A}_{\min }=0.38 \mathrm{~nm}^{2}$} \\
DBS: Dedecyl Benzene Sulfonate, M.W. $=348, \mathrm{~A}_{\min }=0.49 \mathrm{~nm}^{2}$ \\
\hline CPC & \multicolumn{5}{c|}{ DBS } \\
\hline LogC $(\mathrm{g} / \mathrm{L})$ & $\mathrm{C}(\mathrm{g} / \mathrm{L})$ & Ads $(\mathrm{mg} / \mathrm{g})$ & $\mathrm{LogC}(\mathrm{g} / \mathrm{L})$ & $\mathrm{C}(\mathrm{g} / \mathrm{L})$ & $\mathrm{Ads}(\mathrm{mg} / \mathrm{g})$ \\
\hline-2.00 & 0.010 & 3.424 & -1.06 & 0.087 & 2.301 \\
-1.47 & 0.034 & 8.370 & -0.76 & 0.174 & 2.301 \\
-1.17 & 0.067 & 11.795 & -0.46 & 0.348 & 2.301 \\
-0.63 & 0.236 & 13.317 & -0.16 & 0.696 & 2.301 \\
-0.32 & 0.481 & 14.458 & 0.14 & 1.392 & 2.301 \\
-0.03 & 0.929 & 22.828 & 0.32 & 2.088 & 2.301 \\
0.12 & 1.309 & 27.622 & 0.44 & 2.784 & 2.301 \\
0.34 & 2.187 & 30.666 & 0.54 & 3.480 & 2.301 \\
0.53 & 3.356 & 30.438 & 0.72 & 5.220 & 2.301 \\
0.81 & 6.499 & 30.818 & 0.84 & 6.960 & 2.301 \\
\hline
\end{tabular}

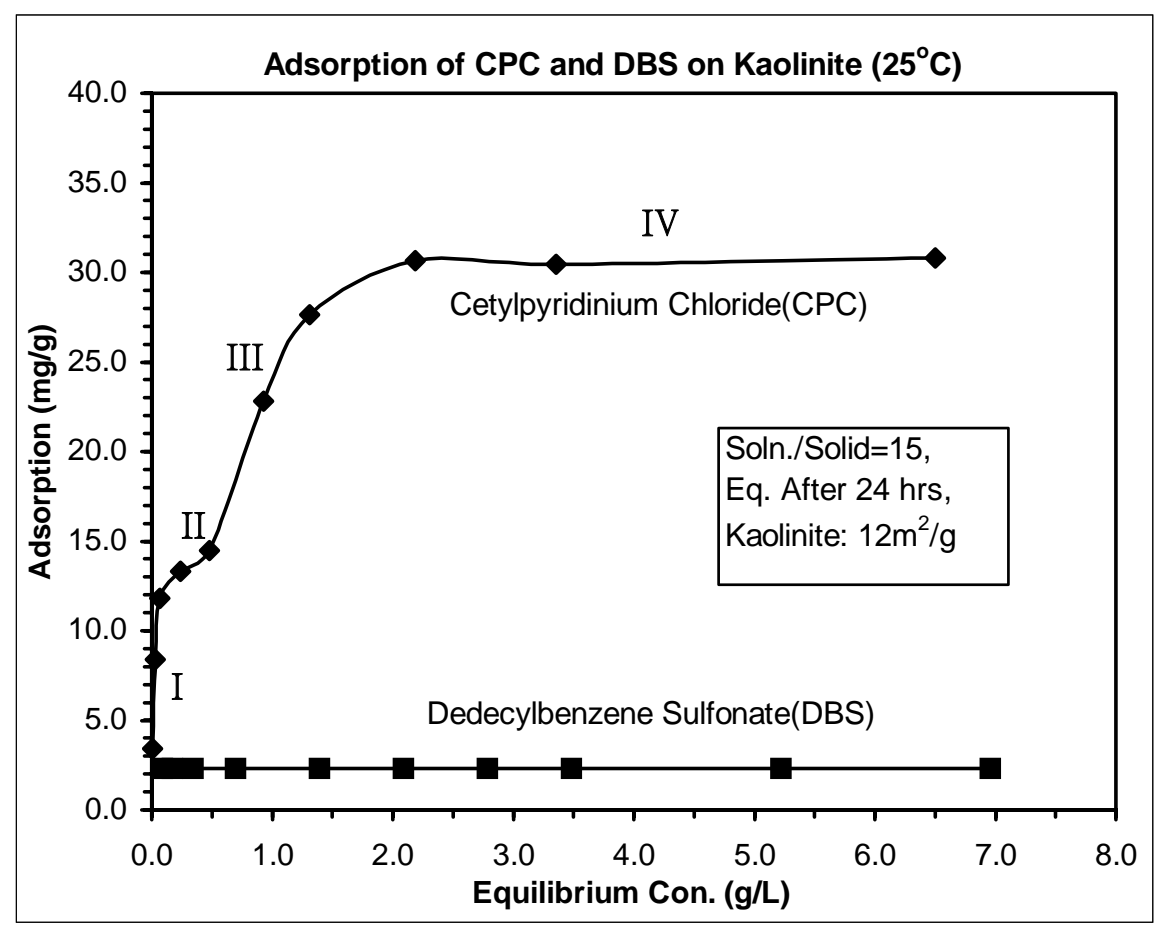




\begin{tabular}{|c|c|c|c|c|c|}
\hline \multicolumn{6}{|c|}{$\begin{array}{l}\text { Adsorption of CPC and DBS on Kaolinite } \\
\text { Aita,A.A. et al Ads. Sci. \& Tech. Vol.5(8),1997 }\end{array}$} \\
\hline \multirow{2}{*}{\multicolumn{6}{|c|}{$\begin{array}{l}\text { CPC: Cetyl Pyridinium Chloride, M.W. }=339.5, A_{\min }=0.38 \mathrm{~nm}^{2} \\
\text { DBS: Dedecyl Benzene Sulfonate, M.W. }=348, A_{\min }=0.49 \mathrm{~nm}^{2}\end{array}$}} \\
\hline & & & & & \\
\hline \multicolumn{3}{|c|}{ CPC } & \multicolumn{3}{|c|}{ DBS } \\
\hline $\log C(M)$ & $C(M)$ & $\mathrm{Ads}\left(\mathrm{mol} / \mathrm{cm}^{2}\right)$ & $\log C(M)$ & $\mathrm{C}(\mathrm{M})$ & Ads $\left(\mathrm{mol} / \mathrm{cm}^{2}\right)$ \\
\hline-4.53 & 2.96E-05 & 0.84 & -3.60 & $2.50 \mathrm{E}-04$ & 0.551 \\
\hline-4.00 & 9.91E-05 & 2.05 & -3.30 & 5.00E-04 & 0.551 \\
\hline-3.71 & 1.97E-04 & 2.90 & -3.00 & 1.00E-03 & 0.551 \\
\hline-3.16 & 6.95E-04 & 3.27 & -2.70 & 2.00E-03 & 0.551 \\
\hline-2.85 & $1.42 \mathrm{E}-03$ & 3.55 & -2.40 & 4.00E-03 & 0.551 \\
\hline-2.56 & 2.74E-03 & 5.60 & -2.22 & 6.00E-03 & 0.551 \\
\hline-2.41 & 3.85E-03 & 6.78 & -2.10 & 8.00E-03 & 0.551 \\
\hline-2.19 & 6.44E-03 & 7.53 & -2.00 & 1.00E-02 & 0.551 \\
\hline-2.01 & 9.89E-03 & 7.47 & -1.82 & 1.50E-02 & 0.551 \\
\hline-1.72 & 1.91E-02 & 7.56 & -1.70 & 2.00E-02 & 0.551 \\
\hline
\end{tabular}

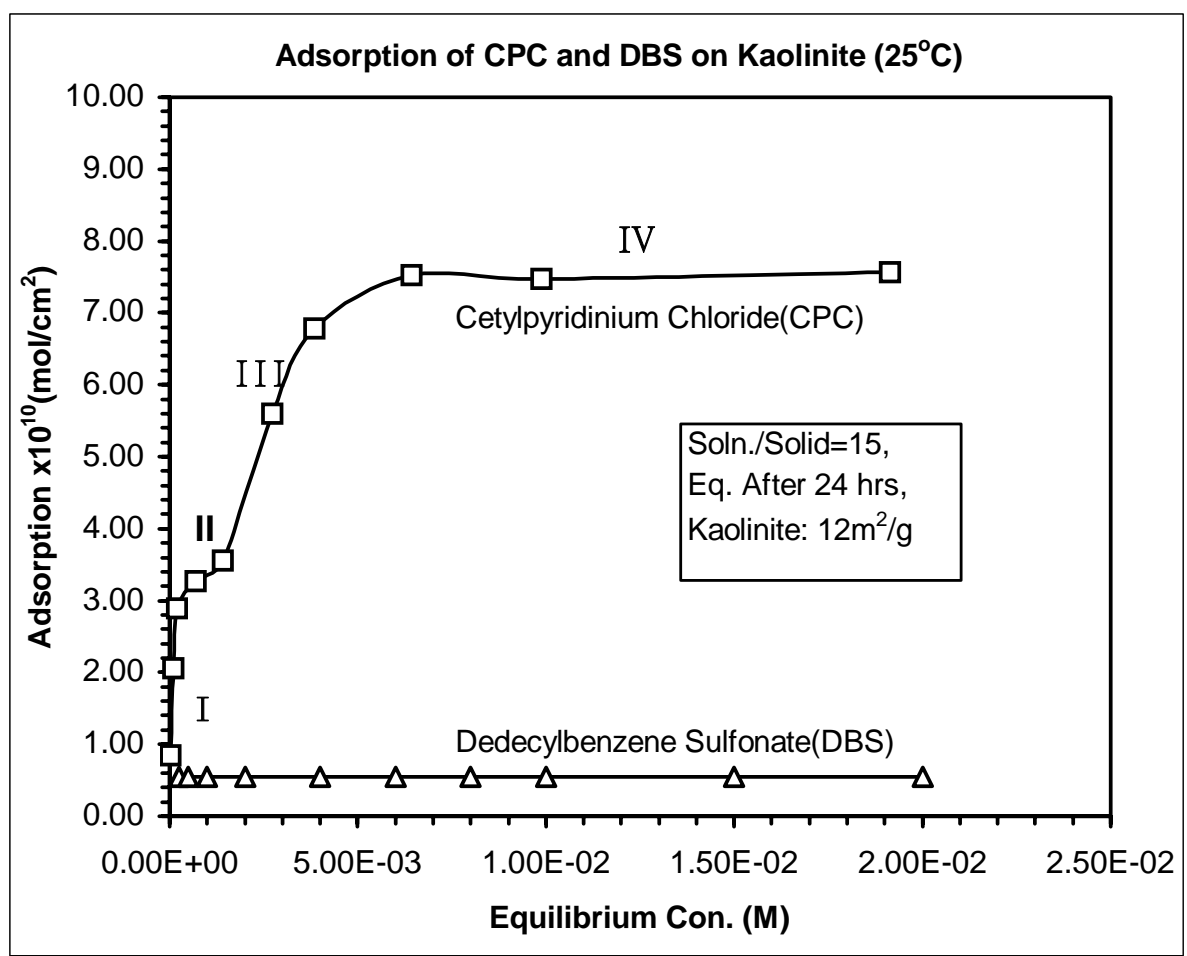

